\title{
\%ै: Battelle
}

Pacific Northwest Laboratories

Richland, Washington 99352

\section{AEC Research and Development Report}
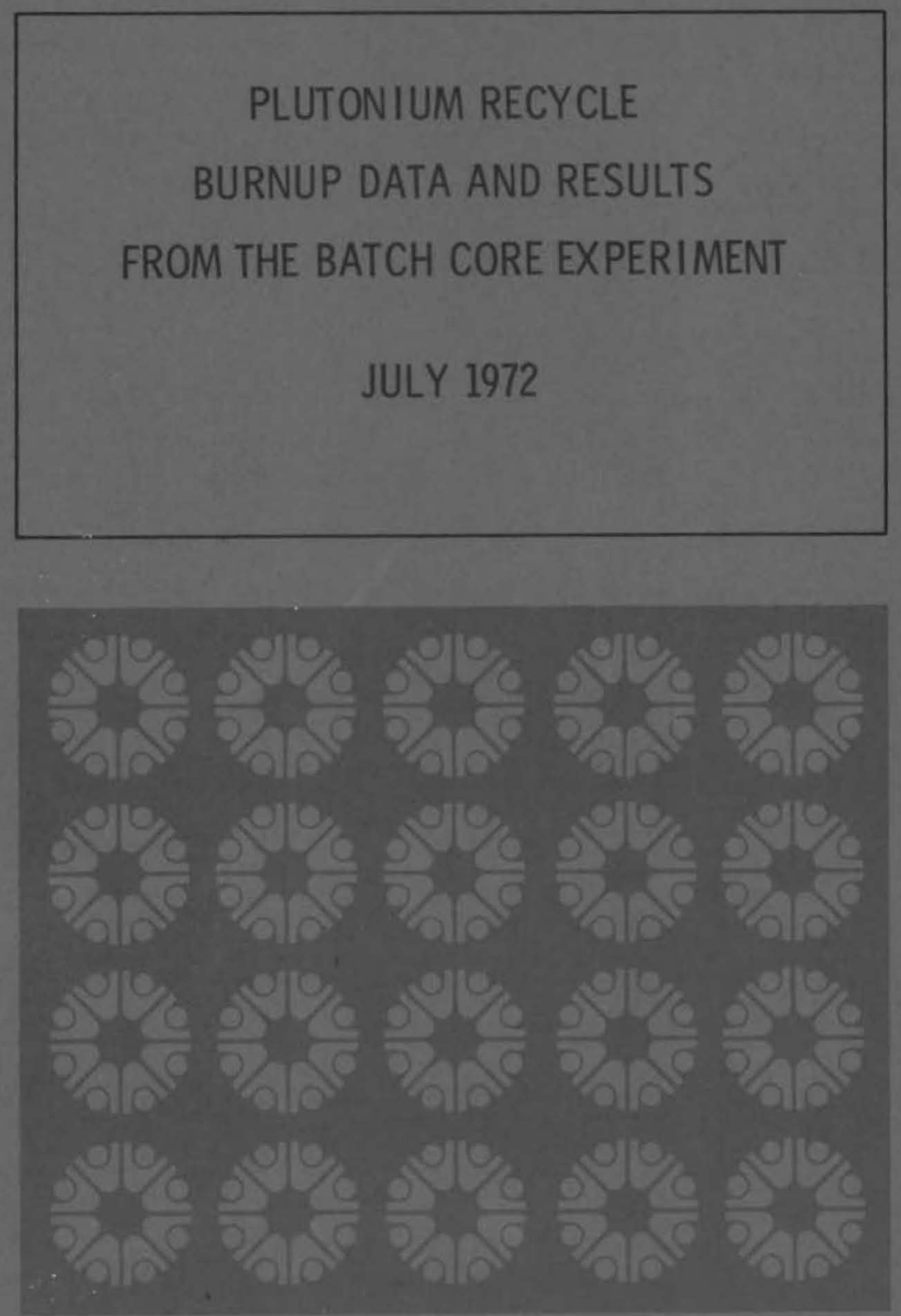


\title{
NOTICE
}

This report was prepared as an account of work sponsored by the United States Covernment. Neither the United States nor the United States Atomic Energy Commssion, nor any of their employees, makes any warranty. capress or implied, or assumes any legal liability or responsibility for the accuracy. completeness or usefulness of any information, apparatus, product, or process disclosed, of represents that its use woold not infringe privately-owned rights.

\author{
PACIFIC NORTHWEST LABORATORY \\ operaled by \\ BATtELE \\ torthe \\ US. ATOMIC ENERGV COMMISSION \\ Under Contract AT(45.1h-19:0
}

*

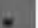


BNWL-1674

Special Distribution

\section{PLUTONIUM RECYCLE \\ BUPNUP DATA AND RESULTS FROM THE \\ BATCH CORE EXPERIMENT}

\section{By}

D. E. Christensen

D. A. Kottwitz

D. P. Oden

R. P. Matsen

Reactor Physics Department

July 1972

BATTELLE

PACIFIC NORTHWEST LABORATORIES

RICHLAND, WASHINGTON 99352 


\title{
PLUTONIUM RECYCLE \\ BURNUP DATA AND RESULTS FROM THE \\ BATCH CORE EXPERIMENT
}

\begin{abstract}
$\underline{\text { ABSTRACT }}$
As a result of the Batch Core Experiment which was conducted in the Plutonium Recycle Test Reactor, a large amount of burnup data and gammaray data was made available for analysis. The data represented plutonium recycle fuel in that the fuel composition was initially $\mathrm{UO}_{2}-2 \mathrm{wt} \% \mathrm{PuO}_{2}$.

A maximum burnup of 9485 MWD/MTM was determined from the fuel samples analyzed. The burnup data were then used to derive effective cross section ratios utilizing a least-squares fitting code. The results indicate exposure averaged values of $0.2240 \pm 0.0009$ and $0.5159 \pm 0.0106$ for $\hat{\alpha}^{25}$ and $\hat{\alpha}^{49}$, respectively (capture to fission ratio of ${ }^{235} \mathrm{U}$ and ${ }^{239} \mathrm{Pu}$ ) for the mixed oxide fuel.

The gamma-ray data were analyzed to determine power distributions and effects of fission product migration. The results indicated localized regions of fission product concentrations due to the migrations of ${ }^{106} \mathrm{Ru}$ ${ }^{106} \mathrm{Rh},{ }^{134} \mathrm{Cs}$ and ${ }^{137} \mathrm{Cs}$.
\end{abstract}




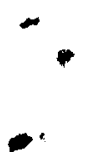

• 
TABLE OF CONTENTS

Page No.

LIST OF TABLES . . . . . . . . . . . . . . . . $i_{i \dagger}$

LIST OF FIGURES . . . . . . . . . . . . . . iv

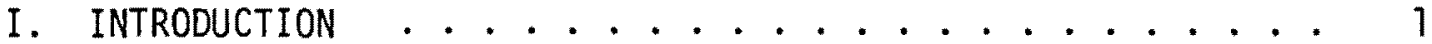

II. BATCH CORE EXPERIMENT DESCRIPTION . . . . . . . . . 2

A. Core Configurations ................ 2

B. Orientation of Assemblies and Removable Rods . . . . . 6

III. BATCH CORE BURNUP DATA FROM DESTRUCTIVE ANALYSIS . . . . . 16

A. Burnup Sample Identification ........... 16

B. Tabulated Burnup Sample Results . . . . . . . . . 20

C. Plotted Results ................ 23

IV. DERIVATION OF EFFECTIVE CROSS-SECTION RATIOS . . . . . 36

A. Least-Squares Method: Program DBUFIT-I . . . . . 36

B. Previous Reactor Burnup Calculations .......... 38

C. Least-squares Fitting to Burnup Data . . . . . . . . 38

D. Sensitivity Tests .............. 41

E. Discussion of Cross-Section Ratios . . . . . . . . . 48

V. BATCH CORE DATA FROM NONDESTRUCTIVE ANALYSIS . . . . . . 54

A. Procedures................... 61

B. Relative Measurement Results .. . . . . . . . 62

C. Gamma Ray Spectra Results. . . . . . . . . . . 69

D. Photopeak Areas Normalized to Burnup . . . . . . . . . 73 
TABLE OF CONTENTS (continued)

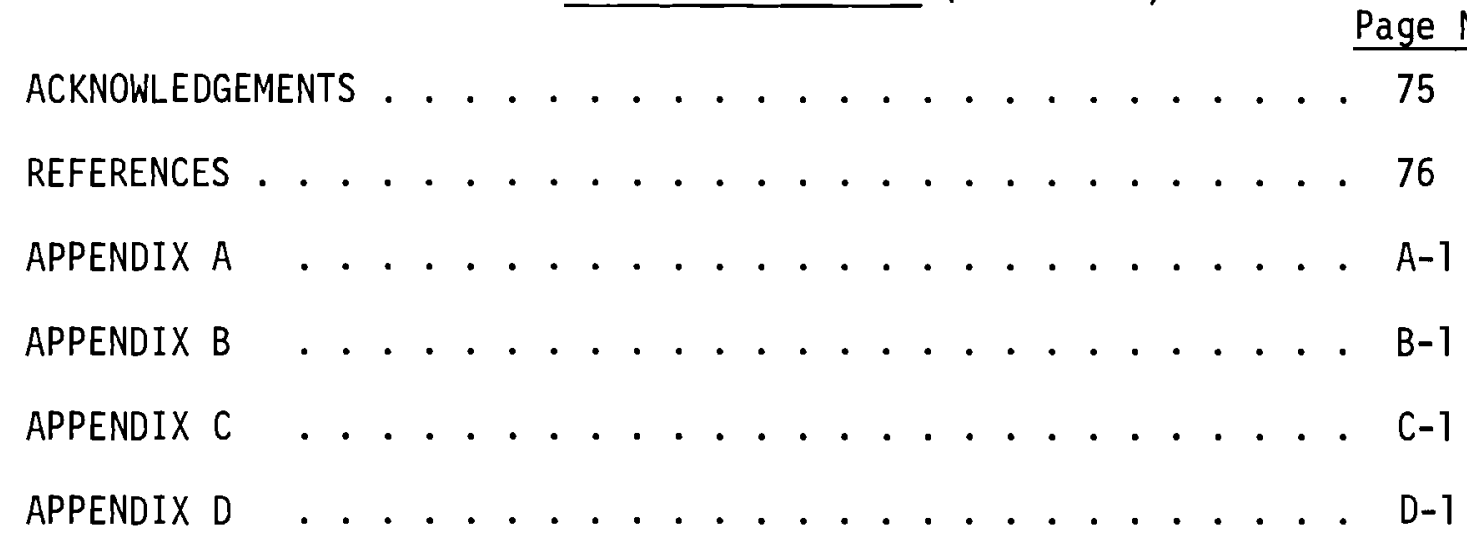


Page No.

I. Exposure and History Summary of Batch Core Assemblies

II. Location and Irradiation History of Batch Core Samples

III. Initial Compositon $(a / 0)$ of Plutonium Fuel Lots

19

IV. Typical Listing of Results Obtained from the Analyses of a Burnup Sample

V. Cross-Section Ratios from Reactor Burnup Calculations 39

VI. Sets of Sample Data used in Least-Squares Fits 40

VII. Cross-Section Ratios Determined by Least-Squares Fits for Fuel Rods in Three Positions in a 19-Rod Assembly

VIII. Results of Sensitivity Tests on Cross-Section Ratios for the 12-Rod Ring Rods

IX. Results of Least-Squares Fits of Partial Sets of Data for the 12-Rod Ring Rods.

$X$. Comparison of Calculated and Fitted Values of CrossSection Ratios

XI. Summary of PRTR Fuels and Hardware Analyzed by Gamma Scanning Since Start of Batch Core Experiment

XII. Summary of High Resolution Gamma-Ray Spectra Collected from Batch Core Fuel Rods and Photopeak Areas Determined from the Spectra

A-I. Burnup Data and Results as Listed by Program ISODIL A-2

B-I. Integrated Activity Expressed as Counts per Second from Continuous Scans of FERTF Rods

C-I. Gamma-Ray Activity Measurements from Intact Fuel Assemblies

D-I. Gamma-Ray Spectra Collected from Batch Core Fuel Rods D-2 


\section{LIST OF FIGURES}

Page No.

1 PRTR Core Loading as of April 14, 1967

2 PRTR Core Loading as of January 2, 1968

3 PRTR Core Loading as of March 8, 1968

7

4 PRTR Core Loading as of July 1, 1968

5 Orientation of 19-Rod Fuel Assemblies

$6 \quad$ Ring Number PRTR Loading Chart

$7 \quad 236 \mathrm{U} /{ }^{238} \mathrm{U}$ Atom Ratio Versus ${ }^{235} \mathrm{U} /{ }^{238} \mathrm{U}$ Atom Ratio

$8 \quad 238 \mathrm{Pu} /{ }^{239} \mathrm{Pu}$ Atom Ratio Versus ${ }^{235} \mathrm{U} /{ }^{238} \mathrm{U}$ Atom Ratio

$9 \quad 240 \mathrm{Pu} /{ }^{239} \mathrm{Pu}$ Atom Ratio Versus ${ }^{235} \mathrm{U} /{ }^{238} \mathrm{U}$ Atom Ratio

$10 \quad 241 \mathrm{Pu} /{ }^{239} \mathrm{Pu}$ Atom Ratio Versus ${ }^{235} \mathrm{U} /{ }^{238} \mathrm{U}$ Atom Ratio

$11{ }^{242} \mathrm{Pu} /{ }^{239} \mathrm{Pu}$ Atom Ratio Versus ${ }^{235} \mathrm{U} /{ }^{238} \mathrm{U}$ Atom Ratio

12 Pu/U Ratio Versus ${ }^{235} \mathrm{U} /{ }^{238} \mathrm{U}$ Atom Ratio

13 Burnup (GWD/MTM) Versus ${ }^{235} \mathrm{U} /{ }^{238} \mathrm{U}$ Ratio

$14 \quad 238 \mathrm{Pu} / 239 \mathrm{Pu}$ Atom Ratio Versus ${ }^{240} \mathrm{Pu} /{ }^{239} \mathrm{Pu}$ Atom Ratio

$15 \quad 241 \mathrm{Pu} / 239 \mathrm{Pu}$ Atom Ratio Versus ${ }^{240} \mathrm{Pu} /{ }^{239} \mathrm{Pu}$ Atom Ratio

$16 \quad{ }^{242} \mathrm{Pu} /{ }^{239} \mathrm{Pu}$ Atom Ratio Versus ${ }^{240} \mathrm{Pu} /{ }^{239} \mathrm{Pu}$ Atom Ratio

$17 \mathrm{Pu} / \mathrm{U}$ Ratio Versus ${ }^{240} \mathrm{Pu} /{ }^{239} \mathrm{Pu}$ Atom Ratio

18 Burnup (GWD/MTM) Versus ${ }^{240} \mathrm{Pu} /{ }^{239} \mathrm{Pu}$ Atom Ratio

$19{ }^{238} U$ Versus ${ }^{235} U$ and Least-Squares Fitting Result for 12-Rod Ring Data

$20 \quad 236_{U}$ Versus ${ }^{235} U$ and Least-Squares Fitting Result for 12-Rod Ring Data

$21{ }^{239} \mathrm{Pu},{ }^{240} \mathrm{Pu},{ }^{241} \mathrm{Pu},{ }^{242} \mathrm{Pu}$ and ${ }^{239} \mathrm{Pu}$ Fissions Versus ${ }^{235} U$ and Least-Squares Fitting Results for 12-Rod Ring Data 


\section{LIST OF FIGURES (continued)}

Page No.

$22 \quad 239 \mathrm{Pu},{ }^{240} \mathrm{Pu},{ }^{241} \mathrm{Pu},{ }^{242} \mathrm{Pu}$ and ${ }^{239} \mathrm{Pu}$ Fissions Versus Burnup and Least-Squares Fitting Results for 12-Rod Ring Data

$23 \quad \hat{\sigma}_{a}^{40} / \hat{\sigma}_{a}^{25}$ Cross Section Ratio Versus Burnup for 12-Rod Ring Data

24 Gamma-Ray Activity Profiles of Rods from Position $r$, FS16, Position c, F079, and Position a, FE77, of Assembly 6066 which was Irradiated in Core Ring Position 3.

25 Gamma-Ray Activity Profiles of Fuel Rods FSO1, FS23, FSO8 and FR64 from the 12-Rod Ring Positions of Assemblies $6063,6067,6058$ and 6516 which Were Irradiated in Core Ring Positions $1,3,5$ and 7 , Respectively.

26 Gamma-Ray Activity Profiles of Rods from the 12-Rod Ring, FS07, the 6-Rod Ring, F017 and the Center Rod, FE74, of Assembly 6065 which was Irradiated in the Third Ring of Fuel Channels

B-1 FERTF Fuel Assembly $9035 \quad$ B-2

B-2 Gamma-Ray Activity Profiles for Rod FS43

(A) Over 6-Inch Length after 29 Days Cooling,

(B) Over Total Length after 233 Days Cooling and

(C) Over 6-Inch Length after 240 Days Cooling

B-3 Normalized Activity of Eight Rods from Fuel Assembly 9035 as a Function of Rod Position. The Values Shown are Listed in Table B-I

C-1 Typical Gamma Ray Spectra Obtained with NaI(T1) $\quad$ C-4 Detector and MCA

C-2 Radial Power Distribution for Batch Core Prior to C-6 Shutdown on January 28, 1968 



\section{PLUTONIUM RECYCLE \\ BURNUP DATA AND RESULTS FROM THE \\ BATCH CORE EXPERIMENT}

\section{INTRODUCTION}

Fuel assembly irradiation tests were conducted during 1967 and 1968 in the Plutonium Recycle Test Reactor (PRTR) ${ }^{(1)}$ through the use of a high power density core. $(2,3,4)$ The average power density of the PRTR was increased above previous core loadings by shortening the fuel assemblies and reducing the total number of fueled tubes. The tests were designated as the Batch Core Experiment and were designed to determine the excess reactivity, fuel isotopic composition and fuel operational characteristics as functions of time for a mixed uranium oxidetwo weight percent plutonium oxide $\left(\mathrm{UO}_{2}-2 \mathrm{wt} \% \mathrm{PuO}_{2}\right)$ fueled reactor as an example of plutonium recycle.

The purpose of this report is to present burnup data collected from several $\mathrm{UO}_{2}-2 \mathrm{wt} \% \mathrm{PuO}_{2}$ fuel rods and assemblies irradiated during the Batch Core Experiment. The data represent fuel isotopic composition data as a function of increasing exposure (to 9485 MWD/MTM) determined by destructive sampling of the fuel rods and nondestructive gamma-ray data collected to indicate the relative burnup distributions of various rods and assemblies of the Batch Core. The analysis of the burnup sample data included determining effective cross-section ratios from the measured isotopic data. To aid the presentation of data and results, a description of the Batch Core is given after which the isotopic composition data and analysis are presented. The nondestructive data are presented last. 
II. BATCH CORE EXPERIMENT DESCRIPTION

The Batch Core Experiment was a physics-fuels experiment which involved the operation of the PRTR loaded with 66 new 19-rod fuel assemblies containing about 2.3 tons of pneumatically impacted $\mathrm{UO}_{2}$ 2 wt.\% $\mathrm{PuO}_{2}$, a plutonium recycle fuel. $(4,5)$ Assembly dimensions are given in Reference 4 and 5 . For normal power operation, the central channel of the reactor, see Reference 6 , pp 5, was connected to the Fuel Element Rupture Test Facility (FERTF). The central channel and 54 assemblies surrounding the central channel constituted the basic core. Twelve additional $\mathrm{UO}_{2}-2$ wt.\% $\mathrm{PuO}_{2}$ assemblies formed a buffer region separating the basic 55 assembly core and selected assemblies previously irradiated in the PRTR which were charged as special irradiation tests in the peripheral positions of the core. Some of the special test assemblies were also $\mathrm{UO}_{2}-2$ wt.\% $\mathrm{PuO}_{2}$ assemblies and a listing of the various assembly types charged in the periphery of the PRTR is given in Reference 2, pp 7.

\section{A. Core Configurations}

The above assemblies are identified by number and their core positions as shown in Figure 1. The core configuration diagram represents the core loading as of April 14, 1967, at the beginning of power operation of the Batch Core. Significant events and daily MWd operation to June 30, 1967, are given in Reference 7, pp 5.4. An operation power histogram covering the entire operation of the PRTR to July 14, 1968, is given in Reference 8, pp. 15 . 


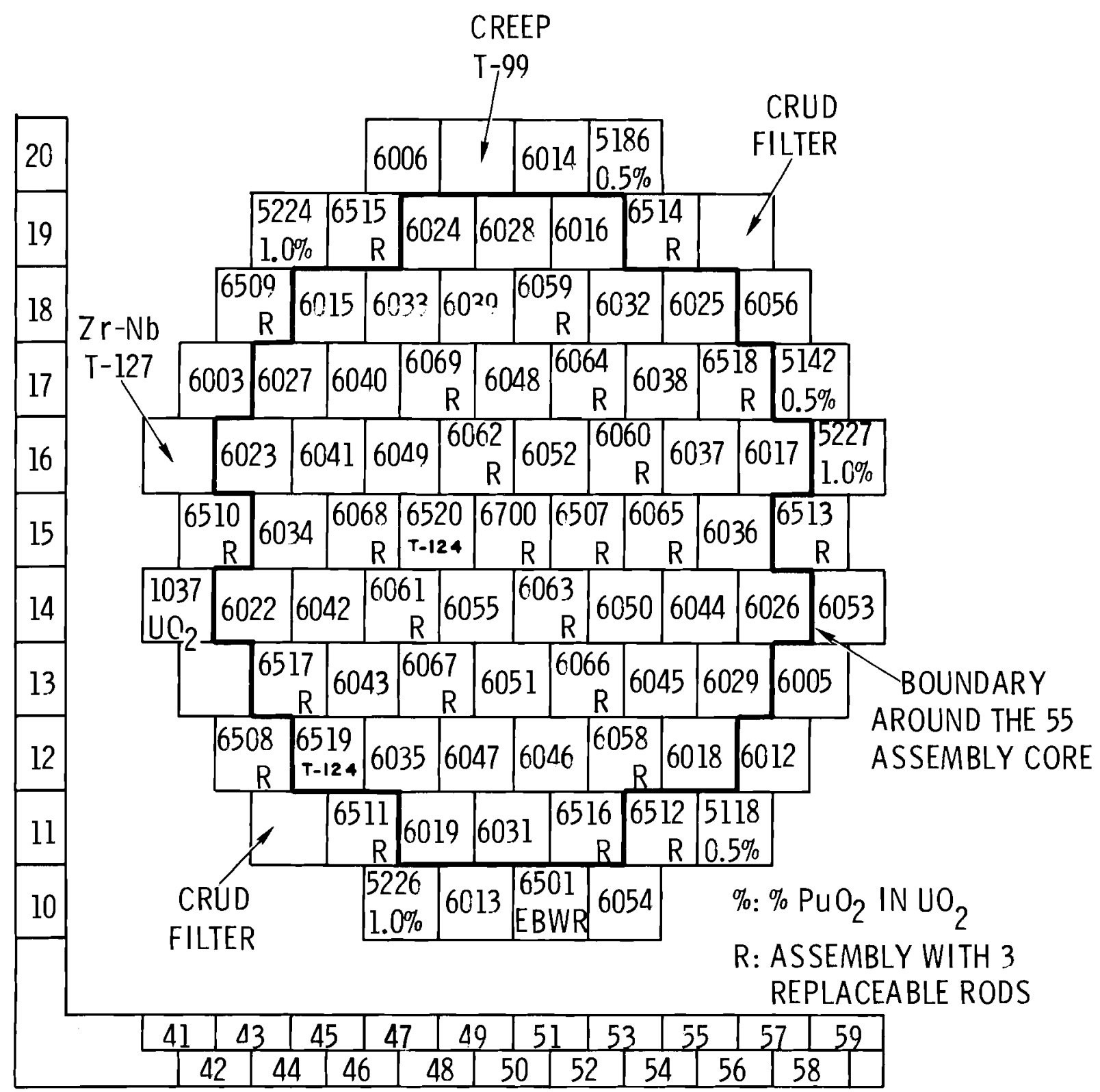

FIGURE 1. PRTR CORE LOADING AS OF APRIL 14, 1967 
Every effort was made to minimize fuel shuffling in the Batch Core and the configuration given in Figure 1 was generally maintained until January, 1968. Adjustments occurred during June-July, 1967, when assembly 6700 in process channel 1550 was discharged, assembly 6508 from channel 1243 was charged to channel 1550 and assemblies 6052 in channel 1651 and 6063 in channel 1451 were interchanged (assembly 6021 was charged to channel 1243). However, assembly 6700 was recharged to channel 1550 during August, 1967, and assemblies 6508, 6052 and 6063 were returned to their original positions. During September, 1967, permanent changes were made in that assembly 6701 was charged to channel 1542, displacing assembly 6510 which was charged to channel 1459, displacing assembly 6053 which was in turn charged to channel 1051, displacing assembly 6501 which was dischared. An additiona) assembly, 6521, was charged to channel 1342 in December, 1967. These changes all occurred in the periphery of the core.

The first permanent change in the center of the core occurred in January, 1968, when assembly 6700 was moved to channel 1649 to permit an 8-rod FERTF assembly to be charged in channel 1550 . This move displaced assembly 6062 which was recharged in channel 1051 and assembly 6053 was subsequently discharged. These changes are reflected in the core configuration as of January 2, 1968, as shown in Figure 2.

Only one assembly, 6066, from the basic 55 assembly core was discharged prior to termination of the Batch Core for the purpose of cutting burnup samples from fuel rods in the assembly. Assembly 6066 was discharged in March, 1968, after the 2nd interim Batch Core critical tests $^{(9)}$ (the 1 st interim critical tests were done during 


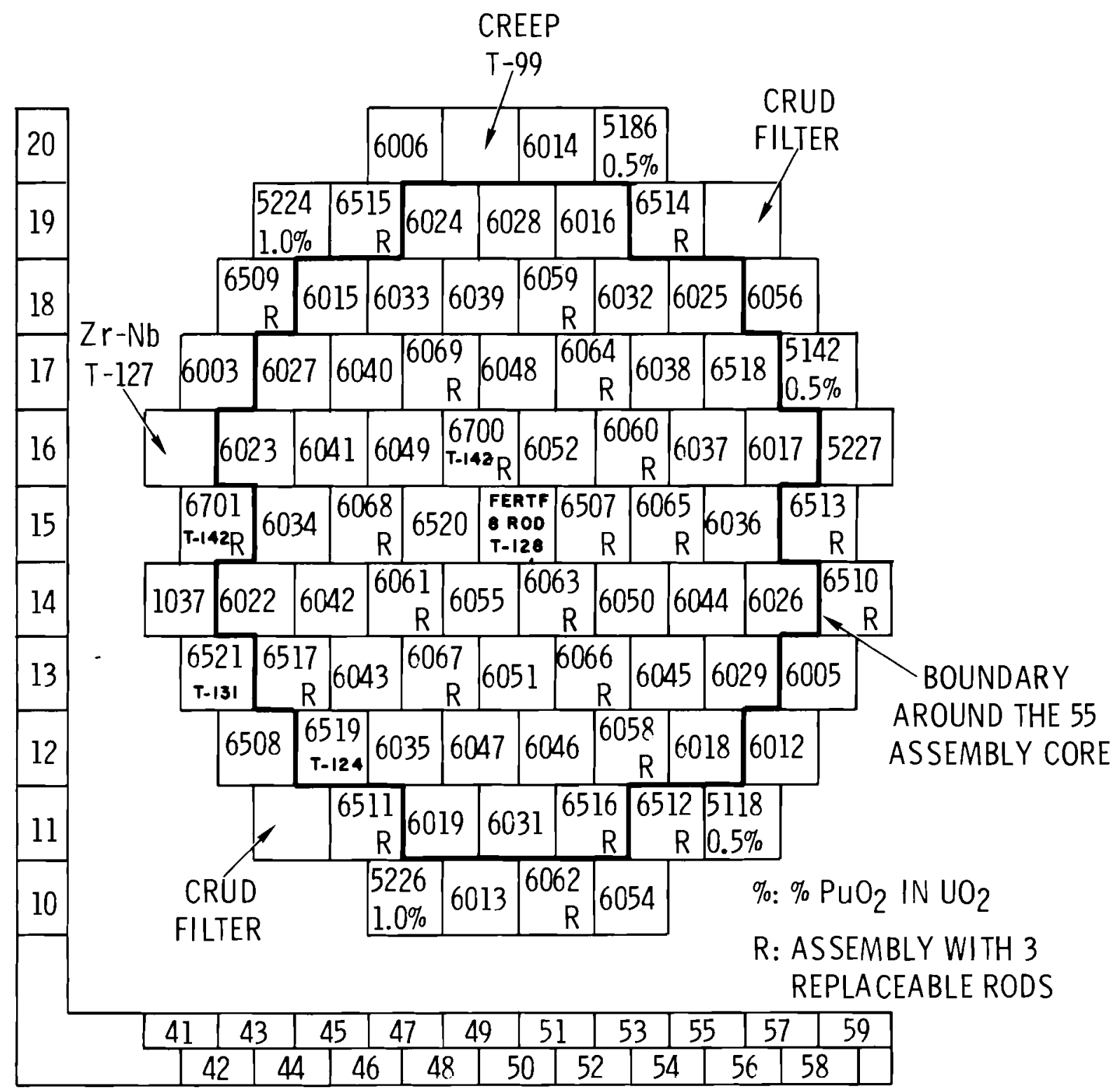

FIGURE 2. PRTR LOADING AS OF JANUARY 2, 1968 
September, 1967). This change was compensated for by charging assembly 6035 in channel 1352 in place of 6066, and subsequently assemblies 6511 , 6062 and 6053 were removed from their original positions and were charged in channels 1247,1146 and 1051 , respectively, to minimize any perturbations to the basic core caused by the removal of assembly 6066 . These changes are reflected in the core configuration diagram as of March 8, 1968, shown in Figure 3. The last changes occurred in June and July when assemblies 6519 and 5227 were discharged (June 6, 1968), and assembly 6700 failed (June 27, 1968). Assembly 6508 was charged in channel 1245 in place of 6519 and 6007 was charged to channel 1243. Assembly 5222 was charged to channel 1659 in place of assembly 5227. When assembly 6700 was discharged from channel 1649, assembly 6061 was charged in its place. Subsequently, assemblies $6065,6043,6062,6013$ and 6008 were charged to channels 1447 , 1554, 1346, 1146 and 1049, respectively, to minimize any perturbations due to the failure of assembly 6700. These changes are reflected in the core configuration diagram shown in Figure 4 . The power operation of the Batch Core was terminated two week later on July 14, 1968.

\section{B. Orientation of Assemblies and Removable Rods}

A specified orientation of each assembly within its process channel was done when each assembly was charged to the PRTR during the Batch Core Experiment. The orientation is shown in Figure 5. Also, certain assemblies of the basic core and buffer region were equipped with removable fuel rods. Those assemblies so designed have been indicated in Figures 1 through 4 and the positions of the removable rods within an assembly are shown in Figure 5. 


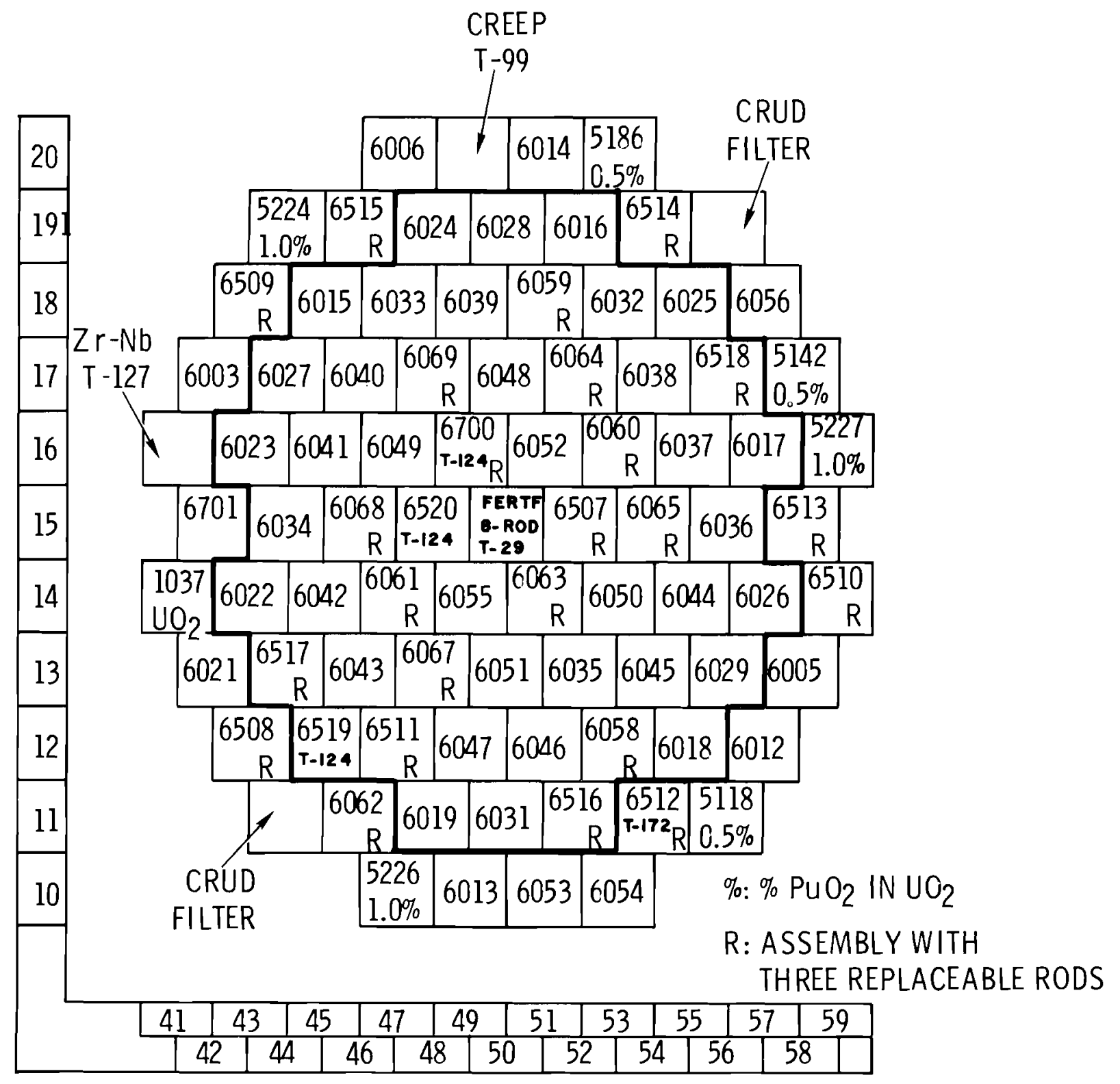

FIGURE 3. PRTR CORE LOADING AS OF MARCH 8, 1968 


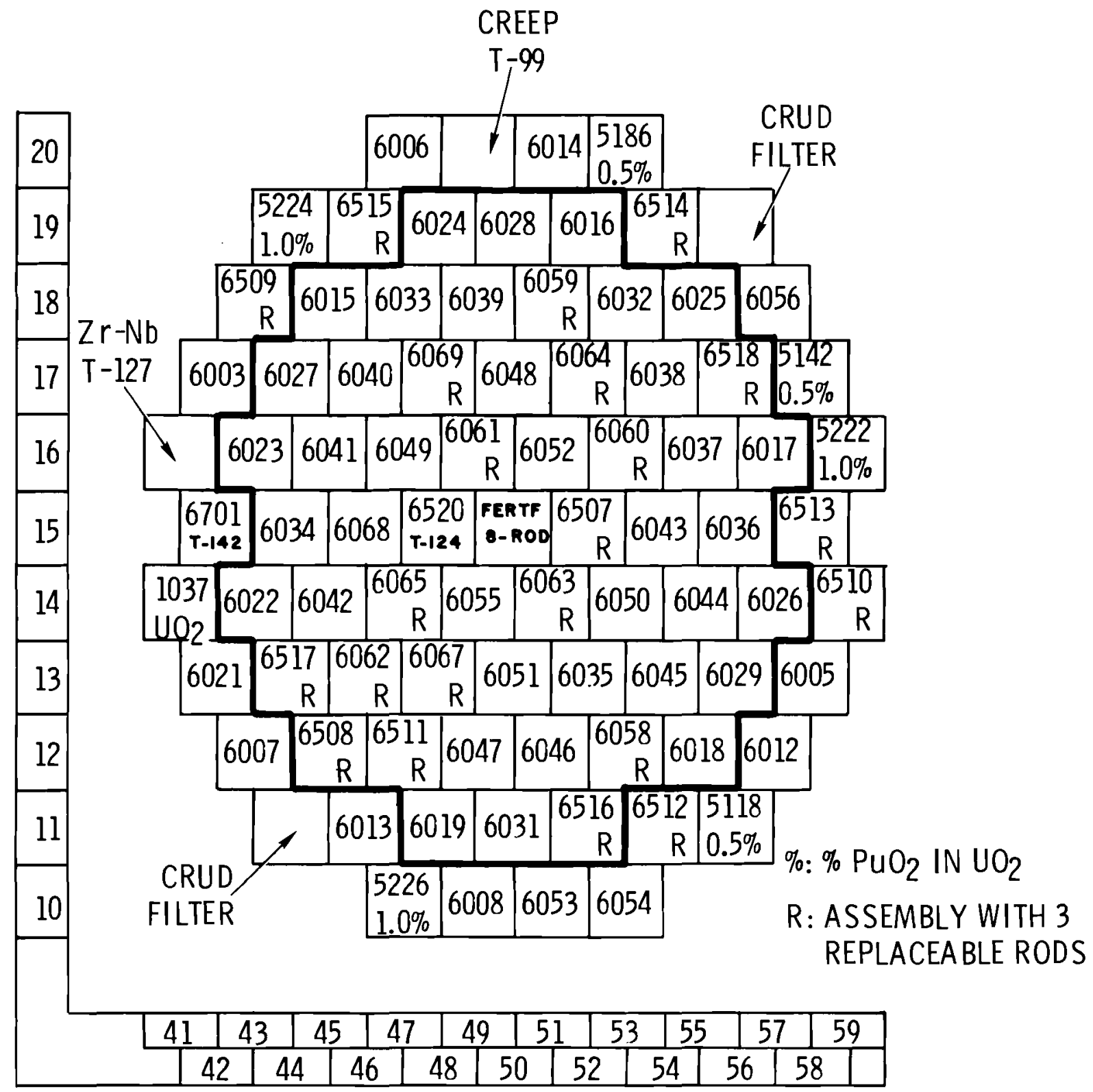

FIGURE 4. PRTR CORE LOADING AS OF JULY 1,1968 


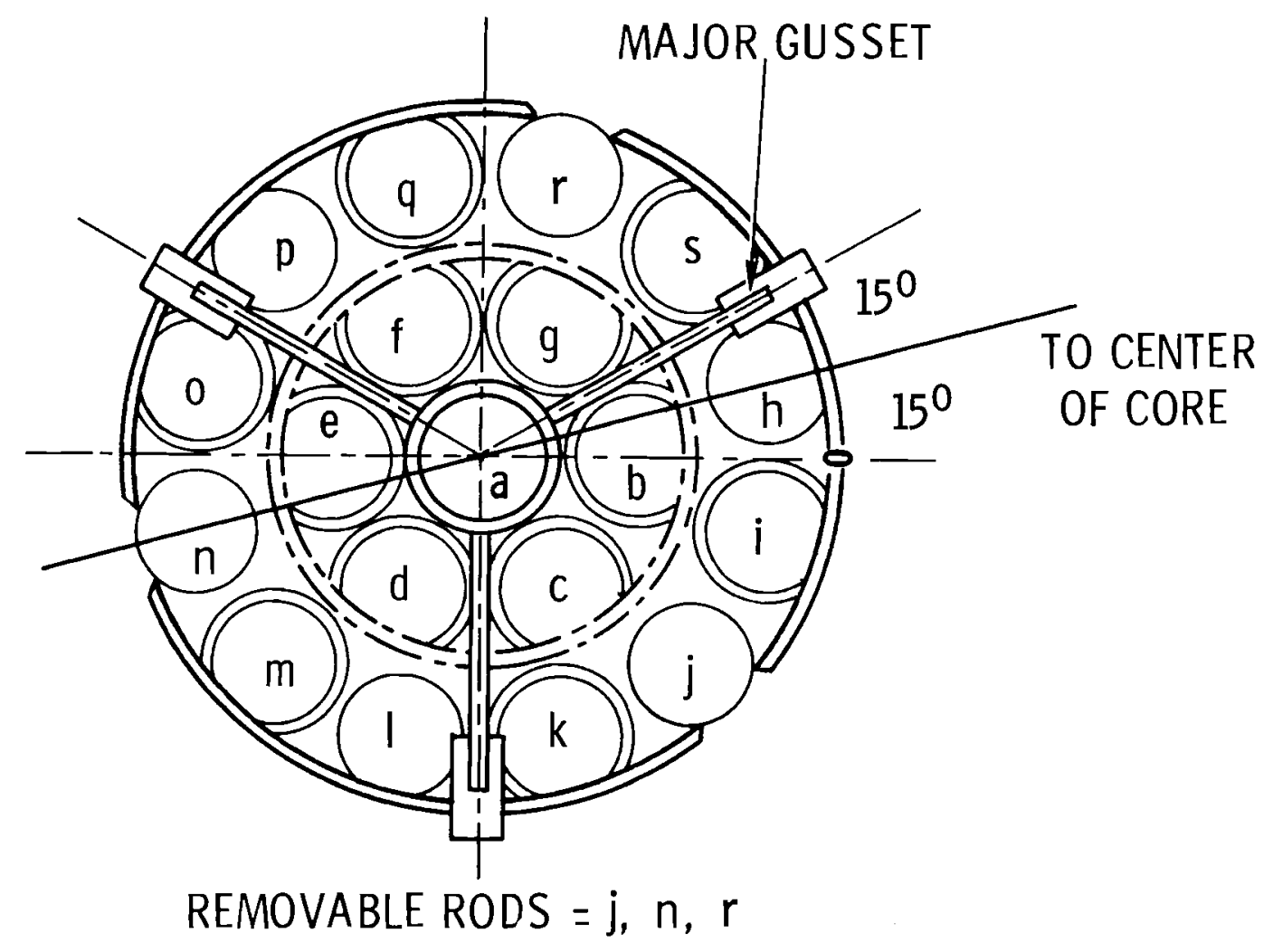

FIGURE 5. ORIENTATION OF 19-ROD FUEL AS SEMBLIES WHICH MADE UP THE BATCH CORE IN THE PRTR. ALL BATCH CORE ASSEMBLIES WERE ORIENTED ACCORDING TO THIS DIAGRAM. 
To meet the need of obtaining burnup samples as a function of increasing exposure, several rods were removed from various assemblies as the experiment progressed.

A summary of position and rod changes for all Batch Core assemblies is given in Table $I$. The ring designation in Table $I$ is the ring of fuel assemblies in the core as shown in Figure 6 (see Reference 8 pp. 14 for the radius of each ring). The ring designation in the reactor core is not to be confused with the rings of rods in each fuel assembly, see Figure 5. The rods from which burnup samples have been cut are described as coming from the center position or "a" position, the 6-rod ring or " $b$ " through "g" positions and the 12-rod ring or " $h$ " through "s" positions of the fuel assembly. Removable rods were available from the 12-rod ring at positions " $j$ ", " $n$ " and " $r$ ". Not all the replaceable rods indicated as having been removed from the core in Table I were removed for the purpose of obtaining isotopic composition data from burnup samples. However, only those that were removed for that purpose ${ }^{(5)}$ are considered in the next section of this paper. Assemblies from which rods were taken both during operation and after shutdown to obtain burnup samples from were $6520,6063,6065,6066,6067,6058,6516$, and 6519 .

The total MWd exposure accumulated by each assembly during the Batch Core operation is listed in Table I. Where the position of the assembly remained unchanged during its residence time in the core is also indicated. As mentioned earlier, several assemblies were moved and the accumulated exposure in MWd for each position occupied in the core by these assemblies is listed. When a rod was removed from an assembly, the history of the rod replacing it is also given. 


\section{TABLE I}

EXPOSURE AND HISTORY SUMMARY OF BATCH CORE ASSEMBLIES

\begin{tabular}{|c|c|c|c|c|}
\hline Ring & $\mathrm{PC}$ & $\mathrm{FE}$ & MWd & Position and Rod Changes \\
\hline 0 & 1550 & 6700 & 194.7 & $\begin{array}{r}122.8 \text { MWd in PC } 1550 \\
71.9 \text { MWd in PC } 1649\end{array}$ \\
\hline \multirow[t]{6}{*}{1} & 1548 & 6520 & 215.5 & Fission gas sensing assembly. Unchanged \\
\hline & 1649 & $6061^{R}$ & 202.0 & $\begin{array}{r}194.8 \text { MWd in PC-1447 (Ring 2) } \\
7.2 \text { MWd in PC-1649 (Ring 1). }\end{array}$ \\
\hline & 1651 & 6052 & 206.1 & Had 2.7 MWd in PC-1451 \\
\hline & 1552 & $6507^{\mathrm{R}}$ & 204.7 & $\left\{\begin{array}{lll}1 & \text { new rod } 6-26-67 & (28.1 \mathrm{MWd}) \\
2 & \text { new rods } 7-16-67 & (37.1 \mathrm{MWd})\end{array}\right.$ \\
\hline & 1451 & $6063^{R}$ & 207.7 & $\left\{\begin{array}{l}\text { Had } 2.7 \text { MWd in } P C-1651 \\
1 \text { rod } 7-17-67(36.0 \text { MWd }) \text { brought from } \\
\text { FE } 6508(19.6 \text { MWd: } \\
9.6 \text { in } P C-1243,10.0 \text { in } P C-1550) . \\
2 \text { rods } 6-5-68(192.0 \text { MWd }) \text { brought from } \\
\text { FE } 6067(165.9 \text { MWd in PC-1348). }\end{array}\right.$ \\
\hline & 1449 & 6055 & 212.2 & Unchanged \\
\hline \multirow[t]{6}{*}{2} & 1647 & 6049 & 189.8 & Unchanged \\
\hline & 1750 & 6048 & 189.9 & Unchanged \\
\hline & 1653 & $6060^{R}$ & 195.3 & Unchanged \\
\hline & 1453 & 6050 & 208.0 & Unchanged \\
\hline & 1350 & 6051 & 204.9 & Unchanged \\
\hline & 1447 & $6065^{R}$ & 197.3 & $\left\{\begin{aligned} & 189.6\text { MWd in } P C-1554 \text { (Ring } 3) \\
& 7.7 \text { MWd in } P C-1447 \text { (Ring 2) } \\
& 1 \text { rod } 9-14-67(61.2 \text { MWd) brought from } \\
& \text { FE } 6514(23.6 \text { MWd in } P C-1954)\end{aligned}\right.$ \\
\hline \multicolumn{5}{|c|}{$\begin{array}{l}\text { PC - Process Channel } \\
\text { FE - Fuel Assembly } \\
\text { MWd - Total Exposure } \\
\text { R - Assembly with } 3\end{array}$} \\
\hline
\end{tabular}


TABLE I (Continued)

\begin{tabular}{|c|c|c|c|c|}
\hline Ring & $\mathrm{PC}$ & $\mathrm{FE}$ & MWd & Position and Rod Changes \\
\hline \multirow[t]{7}{*}{3} & 1546 & $6068^{R}$ & 191.0 & Unchanged \\
\hline & 1748 & $6069^{R}$ & 191.7 & Unchanged \\
\hline & 1752 & $6064^{R}$ & 172.5 & Unchanged \\
\hline & 1554 & 6043 & 181.9 & $\left\{\begin{array}{r}174.3 \text { MWd in PC-1346 (Ring 4) } \\
7.6 \text { MWd in PC-1554 (Ring } 3 \text { ) }\end{array}\right.$ \\
\hline & 1352 & 6035 & 188.5 & $\left\{\begin{array}{r}122.0 \text { MWd in PC-1247 (Ring 5) } \\
66.5 \text { MWd in PC-1352 (Ring 3) }\end{array}\right.$ \\
\hline & 1348 & $6067^{R}$ & 181.3 & $\left\{\begin{array}{c}1 \text { rod } 6-5-68(165.9 \text { MWD) brought } \\
\text { from FE } 6514 \text { (75.2 MWd in PC-1954) } \\
2 \text { rods 6-5-68 }(165.9 \text { MWd) brought } \\
\text { from FE } 6515 \text { (81.9 MWd in PC-1946) }\end{array}\right.$ \\
\hline & 1352 & $6066^{R}$ & 121.9 & Unchanged (Discharged Feb. 6, 1968) \\
\hline \multirow[t]{12}{*}{4} & 1645 & 6041 & 160.5 & Unchanged \\
\hline & 1746 & 6040 & 164.8 & Unchanged \\
\hline & 1849 & 6039 & 170.6 & Unchanged \\
\hline & 1851 & $6059^{R}$ & 155.6 & Unchanged \\
\hline & 1754 & 6038 & 165.3 & Unchanged \\
\hline & 1655 & 6037 & 167.9 & Unchanged \\
\hline & 1455 & 6044 & 170.4 & Unchanged \\
\hline & 1354 & 6045 & 184.6 & Unchanged \\
\hline & 1251 & 6046 & 156.1 & Unchanged \\
\hline & 1249 & 6047 & 156.9 & Unchanged \\
\hline & 1346 & $6062^{R}$ & 179.1 & $\left\{\begin{aligned} 129.7 \text { MWd in PC-1649 (Ring 1) } \\
7.2 \text { MWd in PC-1051 (Ring 9) } \\
35.2 \text { MWd in PC-1146 (Ring 8) } \\
7.0 \text { MWd in PC-1346 (Ring 4) } \\
1 \text { rod } 6-26-67 \text { ( } 30.7 \text { MWd) which had } \\
21.8 \text { MWd in this assembly } \\
\text { previously } \\
1 \text { new rod } 7-17-67 \text { (39.7 MWd). }\end{aligned}\right.$ \\
\hline & 1445 & 6042 & 163.4 & Unchanged \\
\hline
\end{tabular}


TABLE I (Cont inued)

\begin{tabular}{|c|c|c|c|c|}
\hline Ring & $\mathrm{PC}$ & $\mathrm{FE}$ & MWd & Position and Rod Changes \\
\hline \multirow[t]{6}{*}{5} & 1544 & 6034 & 160.7 & Unchanged \\
\hline & 1847 & 6033 & 146.9 & Unchanged \\
\hline & 1853 & 6032 & 132.7 & Unchanged \\
\hline & 1556 & 6036 & 158.5 & Unchanged \\
\hline & 1253 & $6058^{R}$ & 142.7 & $\left\{\begin{array}{c}1 \text { rod } 6-4-68 \text { (128.4 MWd) brought from } \\
\text { FE 6515 (81.9 MWd in PC-1946). }\end{array}\right.$ \\
\hline & 1247 & $6511^{R}$ & 130.6 & $\left\{\begin{array}{l}73.9 \text { MWd in PC-1146 (Ring 8) } \\
56.7 \text { MWd in PC-1247 (Ring 5) }\end{array}\right.$ \\
\hline \multirow[t]{6}{*}{6} & 1744 & 6027 & 129.5 & Unchanged \\
\hline & 1950 & 6028 & 109.1 & Unchanged \\
\hline & 1756 & $6518^{R}$ & 127.6 & Unchanged \\
\hline & 1356 & 6029 & 143.5 & Unchanged \\
\hline & 1150 & 6031 & 144.3 & Unchanged \\
\hline & 1344 & $6517^{\mathrm{R}}$ & 128.5 & Unchanged \\
\hline \multirow[t]{10}{*}{7} & 1643 & 6023 & 114.4 & Unchanged \\
\hline & 1845 & 6015 & 112.3 & Unchanged \\
\hline & 1948 & 6024 & 117.8 & Unchanged \\
\hline & 1952 & 6016 & 110.7 & Unchanged \\
\hline & 1855 & 6025 & 110.9 & Unchanged \\
\hline & 1657 & 6017 & 108.3 & Unchanged \\
\hline & 1456 & 6026 & 129.6 & Unchanged \\
\hline & 1255 & 6018 & 131.6 & Unchanged \\
\hline & 1152 & $6516^{R}$ & 130.3 & 1 new rod 6-3-68 (119.1 MWd). \\
\hline & 1148 & 6019 & 121.5 & Unchanged \\
\hline
\end{tabular}


TABLE I (Continued)

\begin{tabular}{|c|c|c|c|c|}
\hline Ring & PC & $\mathrm{FE}$ & MWd & Position and Rod Changes \\
\hline \multirow[t]{2}{*}{7} & 1245 & $6508^{R}$ & 99.0 & $\left\{\begin{array}{l}9.6 \text { MWd in PC-1243 (Ring 9) } \\
10.0 \text { MWd in PC-1550 (Ring 0) } \\
68.4 \text { MWd in PC-1243 (Ring 9) } \\
11.0 \text { MWd in PC-1245 (Ring 7) } \\
2 \text { new rods } 7-17-67 \text { (19.6 MWd) }\end{array}\right.$ \\
\hline & 1443 & 6022 & 119.2 & Unchanged \\
\hline \multirow[t]{6}{*}{8} & 1542 & 6701 & 78.1 & Pellet assembly, unchanged \\
\hline & 1946 & $6515^{R}$ & 90.5 & 3 new rods $6-4-68$ (81.9 MWd) \\
\hline & 1954 & $6514^{R}$ & 83.3 & $\left\{\begin{array}{l}1 \text { rod } 9-14-67 \text { (23.6 MWd) brought } \\
\text { from FE 6512 (31.0 MWd in PC-1154) } \\
1 \text { new rod 6-3-68 (75.2 MWd) }\end{array}\right.$ \\
\hline & 1558 & $6513^{R}$ & 98.4 & Unchanged \\
\hline & 1154 & $6512^{R}$ & 108.8 & Zr coupons in place of one fuel rod \\
\hline & 1146 & 6013 & 86.2 & $\left\{\begin{array}{r}81.3 \text { MWd in PC-1049 (Ring 9) } \\
4.9 \text { MWd in PC-1146 (Ring 8) }\end{array}\right.$ \\
\hline \multirow[t]{5}{*}{$9 *$} & 1843 & $6509^{R}$ & 83.7 & Unchanged \\
\hline & 2051 & 6014 & 88.2 & Unchanged \\
\hline & 1857 & 6056 & 90.5 & Unchanged \\
\hline & 1257 & 6012 & 97.4 & Unchanged \\
\hline & 1051 & 6053 & 86.1 & $\left\{\begin{array}{l}0.2 \text { MWd in PC-1552 (Ring 1) } \\
22.9 \text { MWd in PC-1459 (Ring 10) } \\
63.0 \text { MWd in PC-1051 (Ring 9) }\end{array}\right.$ \\
\hline \multirow[t]{2}{*}{$10^{*}$} & 1459 & $6510^{R}$ & 88.6 & $\left\{\begin{array}{l}28.7 \text { MWd in PC-1542 (Ring 8) } \\
59.9 \text { MWd in PC-1459 (Ring 10) }\end{array}\right.$ \\
\hline & 1053 & 6054 & 89.8 & $\left\{\begin{array}{r}0.2 \text { MWd in PC-1548 (Ring 1) } \\
89.6 \text { MWd in PC-1053 (Ring 10) }\end{array}\right.$ \\
\hline
\end{tabular}

* No assemblies listed were in PRTR prior to Batch Core. 
15

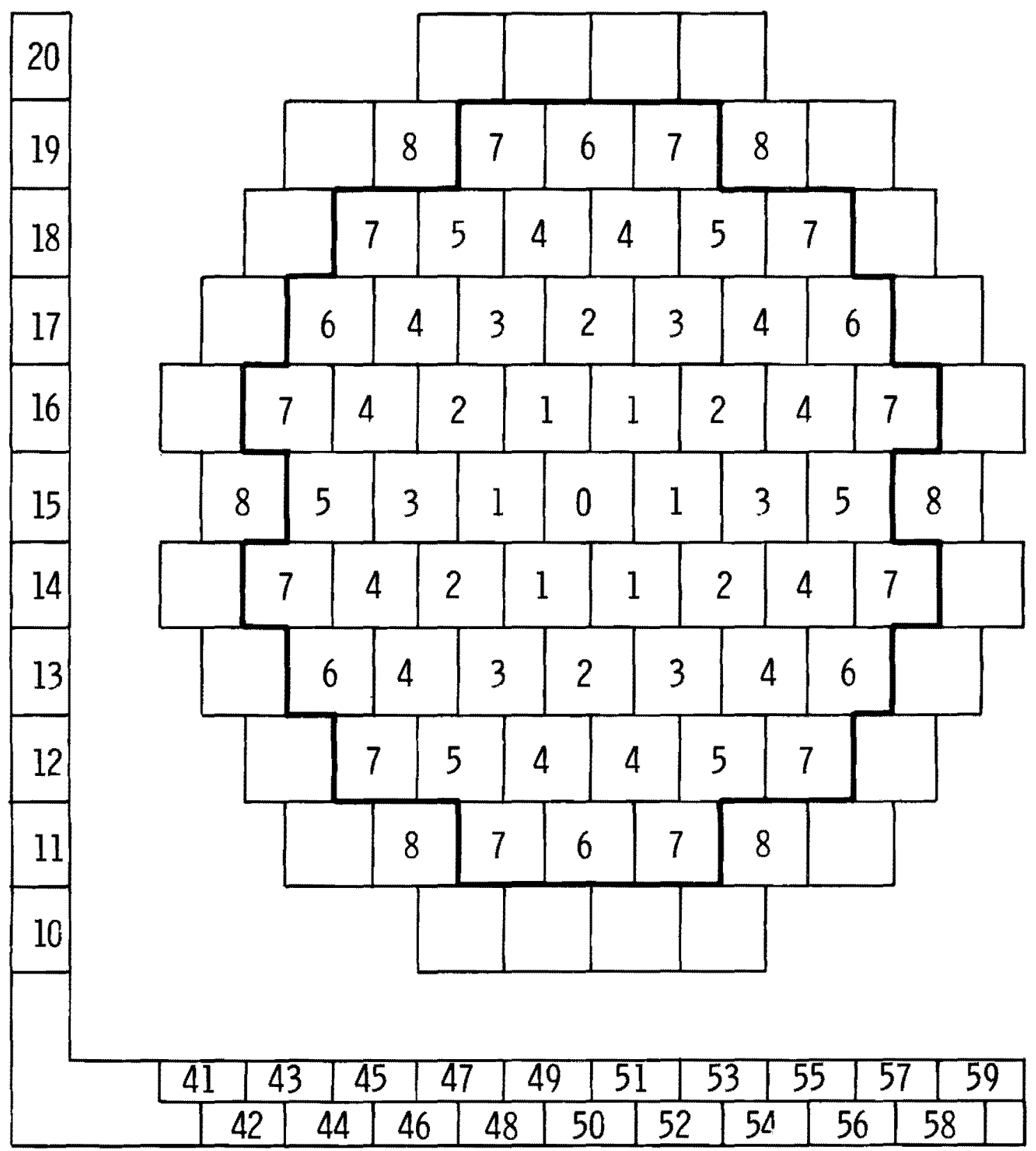

FIGURE 6. RING NUMBER PRTR LOADING CHART 
III. BATCH CORE BURNUP DATA FROM DESTRUCTIVE ANALYSIS

In this section the results of the isotopic dilution measurements made on 36 samples taken throughout the core in the PRTR Batch Core Experiment are presented. The location and irradiation history of each of the samples are provided first followed by a tabulation of the fuel isotopic compositions, burnup, and other pertinent results from the analyses of the isotopic dilution data. Plots of selected fuel isotopic ratios are also presented to affort a basis for a judgement on the internal consistency of the data and to aid in determining which data points should be fit as a set for the purposes of determining cross section ratios.

\section{A. Burnup Sample Identification}

The location and irradiation history of the samples from the PRTR Batch Core Experiment are provided in Table II. The assembly numbers from which the rods were obtained and the primary ring designation (assembly 6065 resided most of its times in ring 3, see Table I) of the assembly in the core are listed. Within an assembly, positions $j, n$, and $r$ listed in Table II are from the 12-rod or exterior ring, positions $c$ and $f$ are from the 6-rod or middle ring, and position a is the center rod of the 19-rod assembly. The date irradiation was terminated and the location of each sample with respect to the top of the fuel (including the $\mathrm{UO}_{2}$ pellet) are also indicated. The plutonium fuel lots from which the rods were fabricated are listed in Table II and the initial composition of the various fuel lots are shown in Table III. Several 
TABLE II

LOCATION AND IRRADIATION HISTORY OF

BATCH CORE SAMPIES

\begin{tabular}{|c|c|c|c|c|c|c|c|c|}
\hline $\begin{array}{l}\text { Fuel } \\
\text { Assembly No. }\end{array}$ & $\begin{array}{l}\text { Position } \\
\text { in Reactor } \\
\text { (Ring) }\end{array}$ & Rod No. & $\begin{array}{l}\text { Position } \\
\text { in } \\
\text { Assembly }\end{array}$ & $\begin{array}{l}\text { Plutonium } \\
\text { Fuel } \\
\text { Lot }\end{array}$ & $\begin{array}{l}\text { Irradiation } \\
\text { Terminated } \\
\text { on } \\
\end{array}$ & $\begin{array}{l}\text { Removal } \\
\text { During }\end{array}$ & $\begin{array}{l}\text { Position* } \\
\text { in Rod, in. }\end{array}$ & $\begin{array}{l}\text { Approximate } \\
\text { Date that } \\
\text { Samples Analyzed }\end{array}$ \\
\hline 6065 & 3 & FS12 & $r$ & $62-23$ & $9-10-67$ & $\begin{array}{l}\text { Interim } \\
\text { Test } 1 \\
\mid\end{array}$ & $\begin{array}{r}6.5 \\
10.4 \\
29.4 \\
41.3 \\
47.5\end{array}$ & $\begin{array}{l}9-10-68 \\
9-10-68 \\
9-10-68 \\
9-10-68 \\
9-10-68\end{array}$ \\
\hline 6066 & 3 & FSl6 & $r$ & $62-23$ & $1-28-68$ & $\begin{array}{c}\text { Interim } \\
\text { Test } 2 \\
\mid\end{array}$ & $\begin{array}{r}6.5 \\
9.6 \\
28.9 \\
43.6 \\
48.1\end{array}$ & $\begin{array}{l}6-15-69 \\
6-15-69 \\
6-15-69 \\
6-15-69 \\
6-15-69\end{array}$ \\
\hline & & F079 & c & $62-22$ & $1-28-68$ & & $\begin{array}{r}6.5 \\
10.4 \\
30.9 \\
47.3\end{array}$ & $\begin{array}{l}6-15-69 \\
6-15-69 \\
6-15-69 \\
6-15-69\end{array}$ \\
\hline . & & $\mathrm{FE} 77$ & a & $62-23$ & $1-28-68$ & 1 & $\begin{array}{r}6.5 \\
30.3 \\
47.6\end{array}$ & $\begin{array}{l}6-15-69 \\
6-15-69 \\
6-15-69\end{array}$ \\
\hline
\end{tabular}

* Length of Fuel $=58.675 \pm 0.125 \mathrm{in}$. which includes $57.837 \pm 0.125 \mathrm{in}$. of $\mathrm{UO}_{2}-\mathrm{PuO}_{2}$ and a $\mathrm{UO}_{2}$ pellet on each end. 
TABLE II (Continued)

LOCATION AND IRRADIATION HISTORY OF BATCH CORE SAMPLES

\begin{tabular}{|c|c|c|c|c|c|c|c|c|}
\hline $\begin{array}{c}\text { Fuel } \\
\text { Assembly No. }\end{array}$ & $\begin{array}{l}\text { Position } \\
\text { in Reactor } \\
\text { (Ring) }\end{array}$ & Rod No. & $\begin{array}{l}\text { Position } \\
\text { in } \\
\text { Assembly }\end{array}$ & $\begin{array}{l}\text { Plutonium } \\
\text { Fuel } \\
\text { Lot }\end{array}$ & $\begin{array}{l}\text { Irradiation } \\
\text { Terminated } \\
\text { on } \\
\end{array}$ & $\begin{array}{l}\text { Rernoval } \\
\text { During } \\
\end{array}$ & $\begin{array}{l}\text { Position } \\
\text { in Rod, in. }\end{array}$ & $\begin{array}{l}\text { Approximate } \\
\text { Date That } \\
\text { Samples Analyzed }\end{array}$ \\
\hline 6063 & 1 & FSOI & $n$ & $62-23$ & $6-1-68$ & $\begin{array}{l}\text { Interim } \\
\text { Test } 3\end{array}$ & 28.9 & $6-15-69$ \\
\hline \multirow[t]{5}{*}{6067} & \multirow[t]{5}{*}{3} & \multirow[t]{5}{*}{ FS23 } & \multirow[t]{5}{*}{$r$} & \multirow[t]{5}{*}{$62-23$} & \multirow[t]{5}{*}{$6-1-68$} & \multirow{7}{*}{$\left.\right|^{3}$} & 6.5 & $6-15-69$ \\
\hline & & & & & & & 9.7 & $6-15-69$ \\
\hline & & & & & & & 29.1 & $6-15-69$ \\
\hline & & & & & & & 43.5 & $6-15-69$ \\
\hline & & & & & & & 48.5 & $6-15-69$ \\
\hline 6058 & 5 & FS08 & $j$ & $62-23$ & $6-1-68$ & & 29.9 & $6-15-69$ \\
\hline 6516 & 7 & F'R64 & $j$ & $62-6$ & $6-1-68$ & & 30.5 & $6-15-69$ \\
\hline 6520 & 1 & FR78 & $\mathrm{n}$ & $62-18$ & $7-14-68$ & Interim & 30.4 & $4-1-70$ \\
\hline \multirow[t]{5}{*}{6065} & \multirow[t]{5}{*}{3} & FS07 & $j$ & $62-23$ & $7-14-68$ & Test 4 & 30.4 & $4-1-70$ \\
\hline & & \multirow[t]{2}{*}{$\mathrm{F} 017$} & \multirow[t]{2}{*}{$c$} & \multirow[t]{2}{*}{$62-22$} & \multirow[t]{2}{*}{$7-14-08$} & \multirow{2}{*}{$\begin{array}{l}\text { (Reactor } \\
\text { Shutdown) }\end{array}$} & 10.0 & $4-1-70$ \\
\hline & & & & & & & 30.4 & $4-1-70$ \\
\hline & & FE74 & a & $62-23$ & $7-14-68$ & & 10.0 & $4-1-70$ \\
\hline & & & & & & & 30.4 & $4-1-70$ \\
\hline \multirow[t]{4}{*}{6519} & \multirow[t]{5}{*}{7} & FN86 & $f$ & $62-18$ & $7-14-68$ & & 7.8 & $4-1-70$ \\
\hline & & & & & & & 30.4 & $4-1-70$ \\
\hline & & FE69 & a & $62-10$ & $7-14-68$ & & 7.8 & $4-1-70$ \\
\hline & & & & & & & 30.4 & $4-1-70$ \\
\hline 6519 & & $\# 4$ & $\mathrm{r}$ & $62-18$ & $7-14-68$ & 1 & 30.0 & $6-18-70$ \\
\hline
\end{tabular}


TABLE III

INITIAL COMPOSITION $(\mathrm{a} / 0)$ OF PLUTONIUM FUEL LOTS

\begin{tabular}{|c|c|c|c|c|c|}
\hline Fuel Lot & ${ }^{238} \mathrm{Pu}$ & ${ }^{239} \mathrm{Pu}$ & ${ }^{240} \mathrm{Pu}$ & ${ }^{241} \mathrm{Pu}$ & ${ }^{242} \mathrm{Pu}$ \\
\hline $62-23$ & 0.026 & 90.518 & 8.228 & 1.148 & 0.080 \\
\hline $62-22$ & unknown & 91.128 & 8.086 & 0.745 & 0.041 \\
\hline \multicolumn{6}{|l|}{$62-6$} \\
\hline $62-10$ & unknown & 91.050 & 8.087 & 0.811 & 0.052 \\
\hline $62-18$ & & & & & \\
\hline
\end{tabular}


samples of unirradiated fuel were submitted for analysis in order to ensure that the data for the zero burnup point was as reliable as that from the other samples.

\section{B. Tabulated Burnup Sample Results}

Results of the isotopic dilution measurements made on the burnup samples are tabulated in Appendix A. For the purpose of explaining these results, the tabulated results of one sample is given in Table IV. The table is a reproduction of computer output from program ISODIL $(10)$ which processes data from the isotopic dilution measurements for each irradiated fuel sample to provide information descriptive of the nuclear transformations that have occurred within the sample. The equations used in the ISODIL code as well as references to the isotopic dilution technique are given in Reference 10 and will not be duplicated here. The format and contents of the ISODIL output (Appendix A and Table IV) are described below.

The first line of Table IV provides information descriptive of the fuel samples location in the reactor. This information includes the fuel assembly number, the rod identification number and its location in the 19-rod assembly. The distance from the top of the fuel at which the sample was cut is also indicated.

The next line of output lists the input values required for corrections for radioactive decay occurring during and after the irradiation. Neodymium isotopic ratios for the spike, sample and mixture are given on the next line. Throughout the table, values in parentheses following a table entry are standard deviation uncertainties based on propagation of error techniques. 


\section{TABLE IV}

\section{TYPICAL LISTING OF RESULTS OBTAINED FROM THE ANALYSES OF A BURNUP SAMPLE}

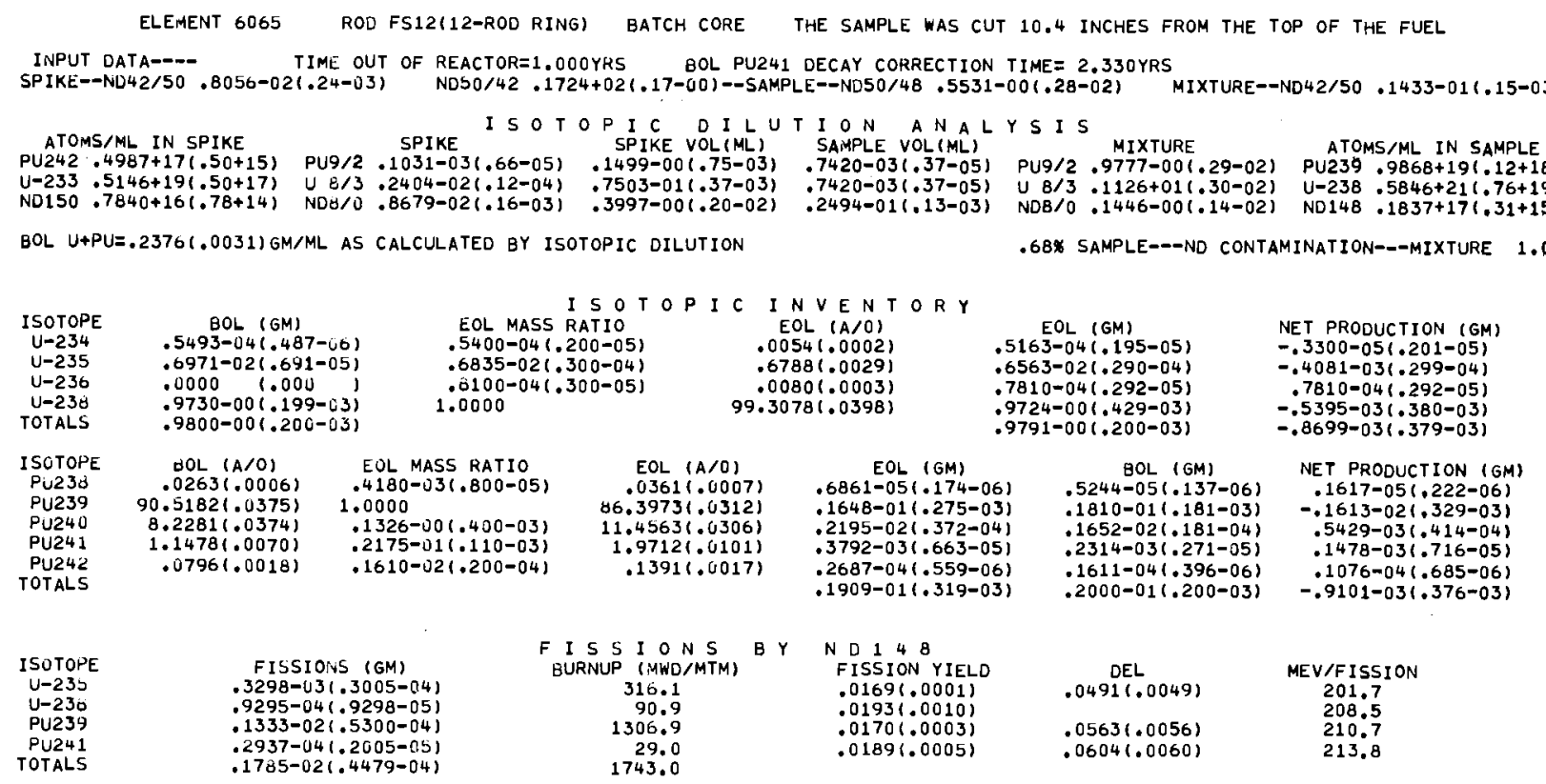

U235/U235BOL $=.941(.004)$ ALPHA235 $=.2372(.0241)$ U238 INTO PU239 $=.4484-03(.38-03)$ GM U236 INTO NP237 $=.476-06 G M$

------GUANTITIES EATCLOSED BY PARENTHESES ARE ONE STANDARD DEVIATION ERRORS-------

ELEMENT 6065 ROO FS12(12-ROD RING) BATCH CORE THE SAMPLE WAS CUT 6.5 INCHES FROM THE TOP OF THE FUEL

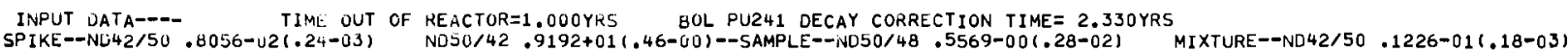
ATOMSML IN SPIKE SPIKE I S O T O P I C DILUT I ON ANALYS IS ATOMSAML IN SPIKE SPIKE ISO T O IPIKE VOL (ML) SAMPLE VOL (ML)

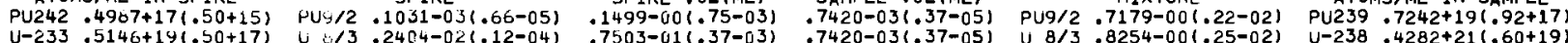

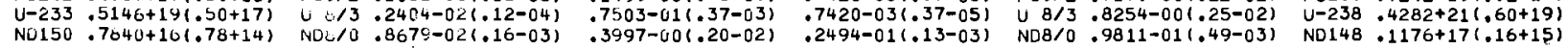
BOL U+PU $=.1740(.0024)$ GM/NiL. AS CALCULATED BY ISOTOPIC OILUTION 1.30\% SAMPLE---ND CONTAMINATION---MIXTURE $1.10 \%$

\begin{tabular}{|c|c|c|c|c|c|c|c|c|}
\hline $\begin{array}{c}\text { ISCTOPE } \\
U-234 \\
U-230 \\
U-230 \\
U-230 \\
\text { TOTALS }\end{array}$ & \multicolumn{5}{|c|}{ 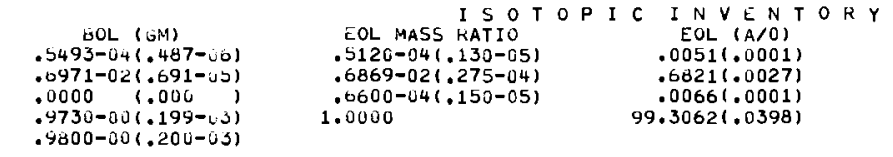 } & \multicolumn{2}{|c|}{$\begin{array}{l}\text { EOL }(G M) \\
.4897-04(.127-05) \\
.6598-02(.266-04) \\
.6366-04(.146-05) \\
.9728-00(.437-03) \\
.9795-00(.200-03)\end{array}$} & $\begin{array}{l}\text { NET PRODUCTION } \\
-.5961-051.136 \\
-.3732-031.276 \\
.6366-041.146 \\
-.2151-031.389 \\
-.5250-031.388\end{array}$ \\
\hline $\begin{array}{l}\text { ISOTOPC } \\
\text { PU230 } \\
\text { PU239 } \\
\text { PUE4U } \\
\text { PU24I } \\
\text { PL24L } \\
\text { TOTALS }\end{array}$ & $\begin{array}{r}\text { JOL }(A / 0) \\
.0263(.0006) \\
90.0182(.0375) \\
8.0281(.0374) \\
1.1478(.0070) \\
.0796(.0018)\end{array}$ & $\begin{array}{l}\text { EOL MASS RATIO } \\
.3840-03(.800-05) \\
1.0000 \\
.1252-00(.400-03) \\
.1953-01(.100-03) \\
.1450-02(.200-04)\end{array}$ & $\begin{array}{r}E O L(A / 0) \\
.0335(.000 \\
87.14441 .031 \\
10.91651 .031 \\
1.78531 .009 \\
.12641 .001\end{array}$ & $\begin{array}{l}\text { (2) } \\
\text { 15) } \\
\text { 11) } \\
\text { 93) }\end{array}$ & $\begin{array}{r}\text { EOL }(G M) \\
.6317-0 S 1.172- \\
.1652-011.288- \\
.2077-021.368- \\
.3413-031.622- \\
.2425-041.539- \\
.1897-011.331-\end{array}$ & & $\begin{array}{l}\text { BOL }(G M) \\
.5244-05(.137-06) \\
.1810-01(.181-03) \\
.1652-02(.181-04) \\
.2314-03(.271-05) \\
.1611-04(.396-06) \\
.2000-01(.200-03)\end{array}$ & $\begin{array}{l}\text { NET PRODUCTI } \\
.1073-051.2 \\
-.1576-021.3 \\
.4251-031.4 \\
.1099-031.6 \\
.8148-051.6 \\
-.1032-021.3\end{array}$ \\
\hline $\begin{array}{l}\text { ISOTOHE } \\
\text { U-23: } \\
\text { U-230 } \\
\text { PU234 } \\
\text { PU241 } \\
\text { TOTALS }\end{array}$ & $\begin{array}{r}\text { FISS1 } \\
.3095-0 \\
.0111-0 \\
.1148-0 \\
.2223-0 \\
.1560-0\end{array}$ & $\begin{array}{l}5(G M) \\
(.2761-04) \\
(.8097-05) \\
(.4542-04) \\
(.1906-05) \\
(.3688-04)\end{array}$ & $\begin{array}{c}=\text { I S S I ON S } \\
\text { BURNUP (MWD/MTM) } \\
296.6 \\
79.3 \\
1125.1 \\
21.9 \\
1523.0\end{array}$ & $B Y$ & $\begin{array}{l}\text { ND } 14{ }^{4} 8 \\
\text { FISSIUN YIEL } \\
.0169(.0001) \\
.0193(.0010) \\
.0170(.0003) \\
.0189(.0005)\end{array}$ & & $\begin{array}{c}\text { DEL } \\
.0491(.0049)\end{array}$ & $\begin{array}{l}\text { MEV/FISSION } \\
201.7 \\
208.5 \\
210.7 \\
213.8\end{array}$ \\
\hline
\end{tabular}


The next three lines list the results of the isotopic dilution analysis. The beginning of life $(B O L) U+P u$, in grams per $\mathrm{ml}$ of sample, is given next as calculated either by isotopic dilution or by fuel weight and solution volume (where input). The Nd contamination of the sample and mixture is also provided on this line.

The isotopic inventory for the uranium and plutonium isotopes and their respective totals are tabulated next. Table entries with the units of grams are normalized to one gram of the $U+$ Pu initially in the sample. Results are also quoted in terms of mass ratios and atom percentages. The mass ratios are relative to ${ }^{238} \mathrm{U}$ and ${ }^{239} \mathrm{Pu}$ for the uranium and plutonium isotopes respectively.

The next section of the table indicates the number of fissions which have occurred in each isotope and the total fissions as determined by ${ }^{148} \mathrm{Nd}$. Here again the entries in units of grams are relative to one gram of $U+P u$ initially. The fission yields of ${ }^{148} \mathrm{Nd}$ used for each of the fuel isotopes in this determination are listed. The DEL values shown are the calculated (input) ratios of fissions in $U^{238}$ per fission in the other fuel isotopes and are used to determine the number of fissions in ${ }^{238} \mathrm{U}$. The burnup by isotope, the total burnup, and the energy release per fission used to determine these quantities are also tabulated.

The last line in the table indicates the ratio of final to initial atoms of ${ }^{235} U$ in the fuel, the average capture to fission ratio for ${ }^{235} U$ (ALPHA 235), the grams of ${ }^{238} U$ converted to ${ }^{239} \mathrm{Pu}$ per initial gram of $U+\mathrm{Pu}$, and the grams of ${ }^{236} U$ converted to ${ }^{237} \mathrm{~Np}$ per initial gram of $U+P u$. 


\section{Plotted Results}

Results of the isotopic dilution measurements are also shown in Figures 7 through 18 which are plots of the atom ratios for the various fuel isotopes as a function of the $\mathrm{U}^{235} / \mathrm{U}^{238}$ and $\mathrm{Pu}^{240} / \mathrm{Pu}^{239}$ atom ratios. The error bars associated with each data point are indicated on the figures and the plotting symbols used define both rod position in an assembly and plutonium fuel lot numbers from which the rods were fabricated.

The major data sets for the central $\operatorname{rod}(\diamond), 6$-rod ring $(x)$ and 12-rod ring (o) locations in an assembly contain 5,6 and 18 data points respectively. An eyeball fit of these three sets of data in Figures 7 through 18 indicates that the three sets should be fitted separately for purposes of determining cross section ratios. These three apparently different burnup paths for the central rod, 6-rod and 12-rod ring locations may be due, in part, to the differences in plutonium lots as well as to differences in neutronic characteristics attributable to the three different locations in the assembly. The fitted cross section ratios will aid in determining the cause of this apparent difference in burnup paths. As a matter of interest in comparison to slightly enriched $235 \mathrm{U}$ systems and as seen in Figures 13 and 18 , the $U^{235} / U^{238}$ atom ratio is essentially linear with burnup while the $\mathrm{Pu}^{240} / \mathrm{Pu}^{239}$ atom ratio shows a second order dependence. 


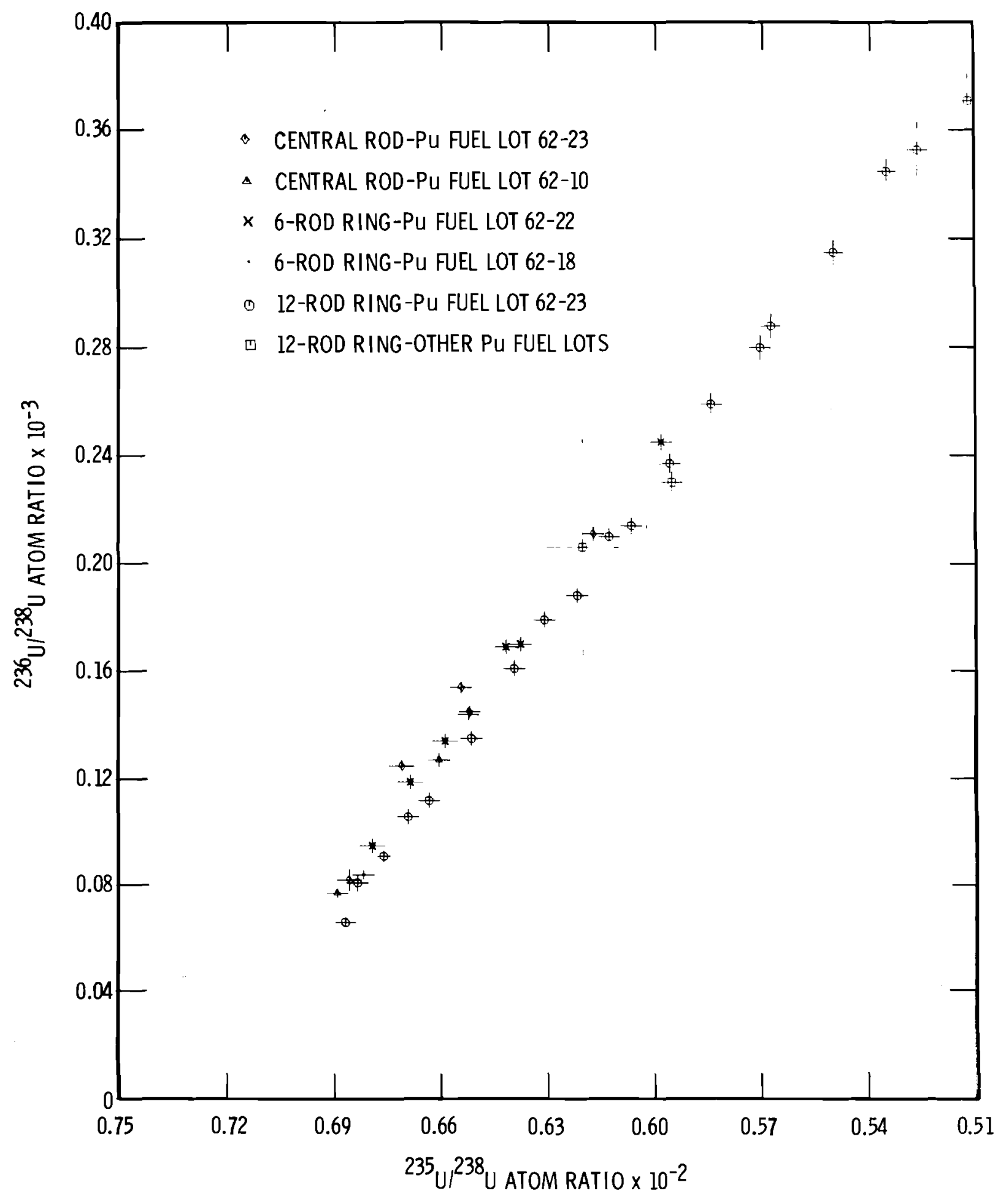

FIGURE $7 .{ }^{236} \mathrm{U} /{ }^{238} \mathrm{U}$ ATOM RATIO VERSUS ${ }^{235} \mathrm{U} /{ }^{238} \mathrm{U}$ ATOM RATIO 


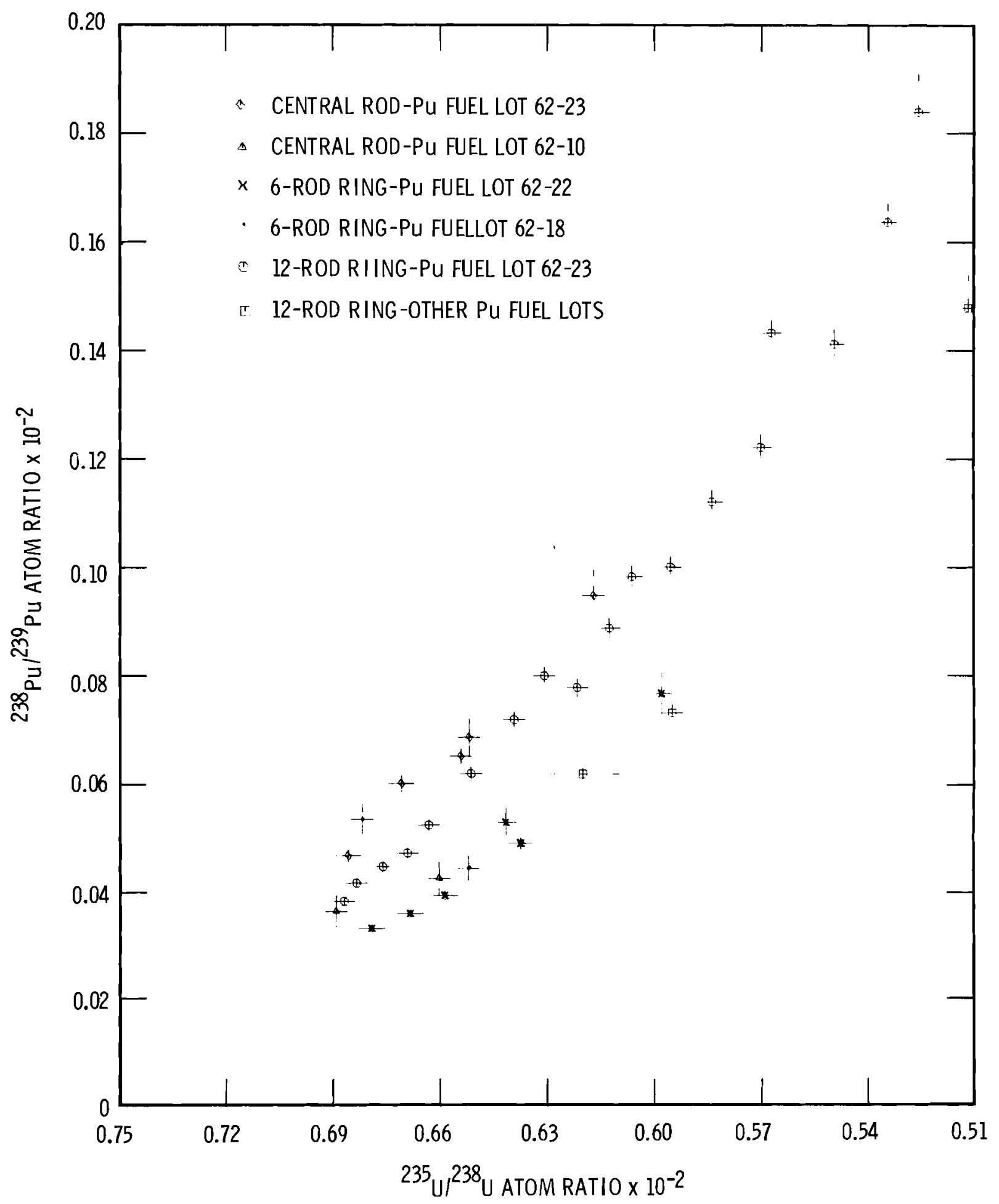

FIGURE $8 .{ }^{238} \mathrm{Pu} /{ }^{239} \mathrm{Pu}$ ATOM RATIO VERSUS ${ }^{235} \mathrm{U} /{ }^{238} \mathrm{U}$ ATOM RATIO 


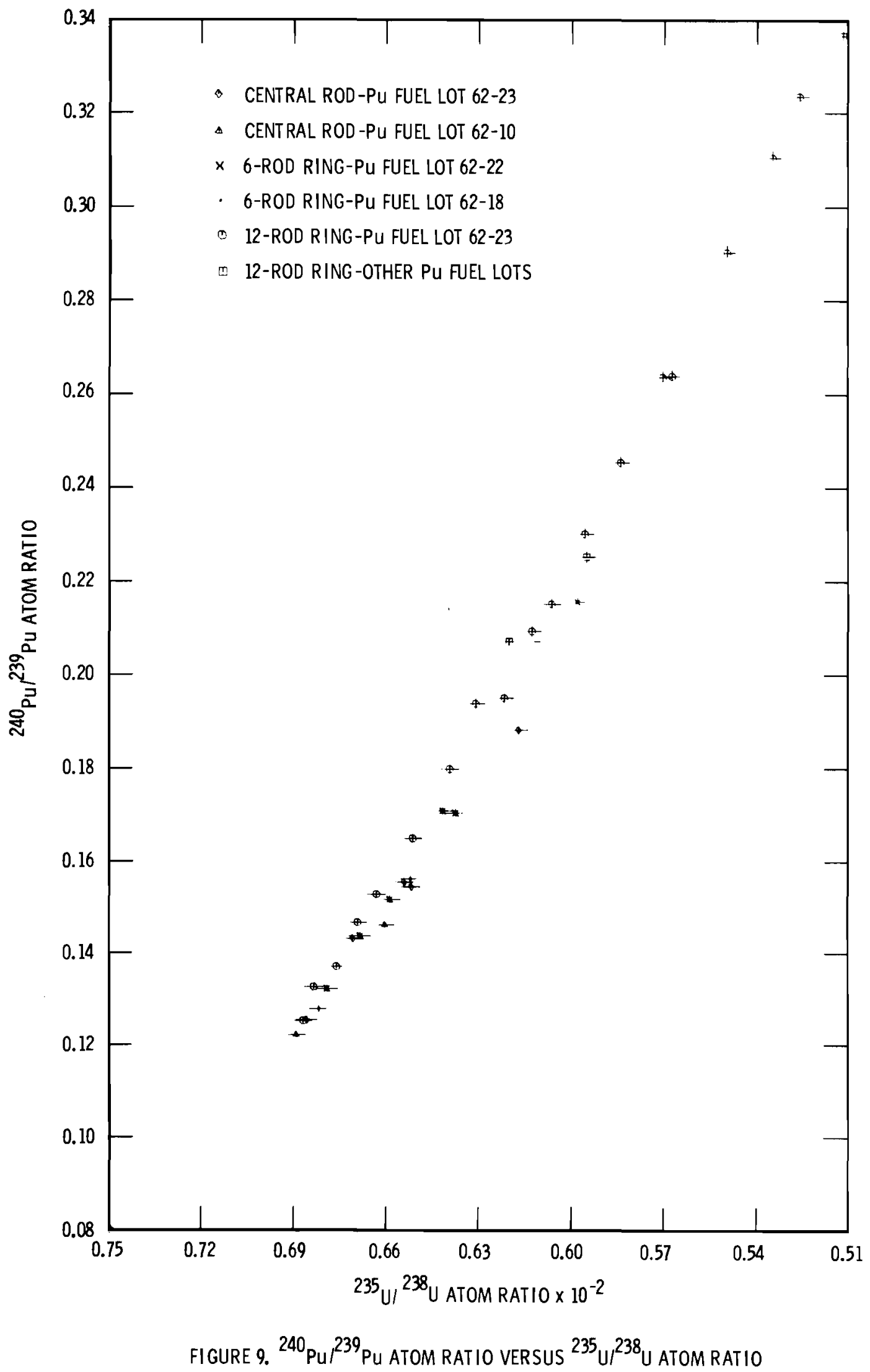




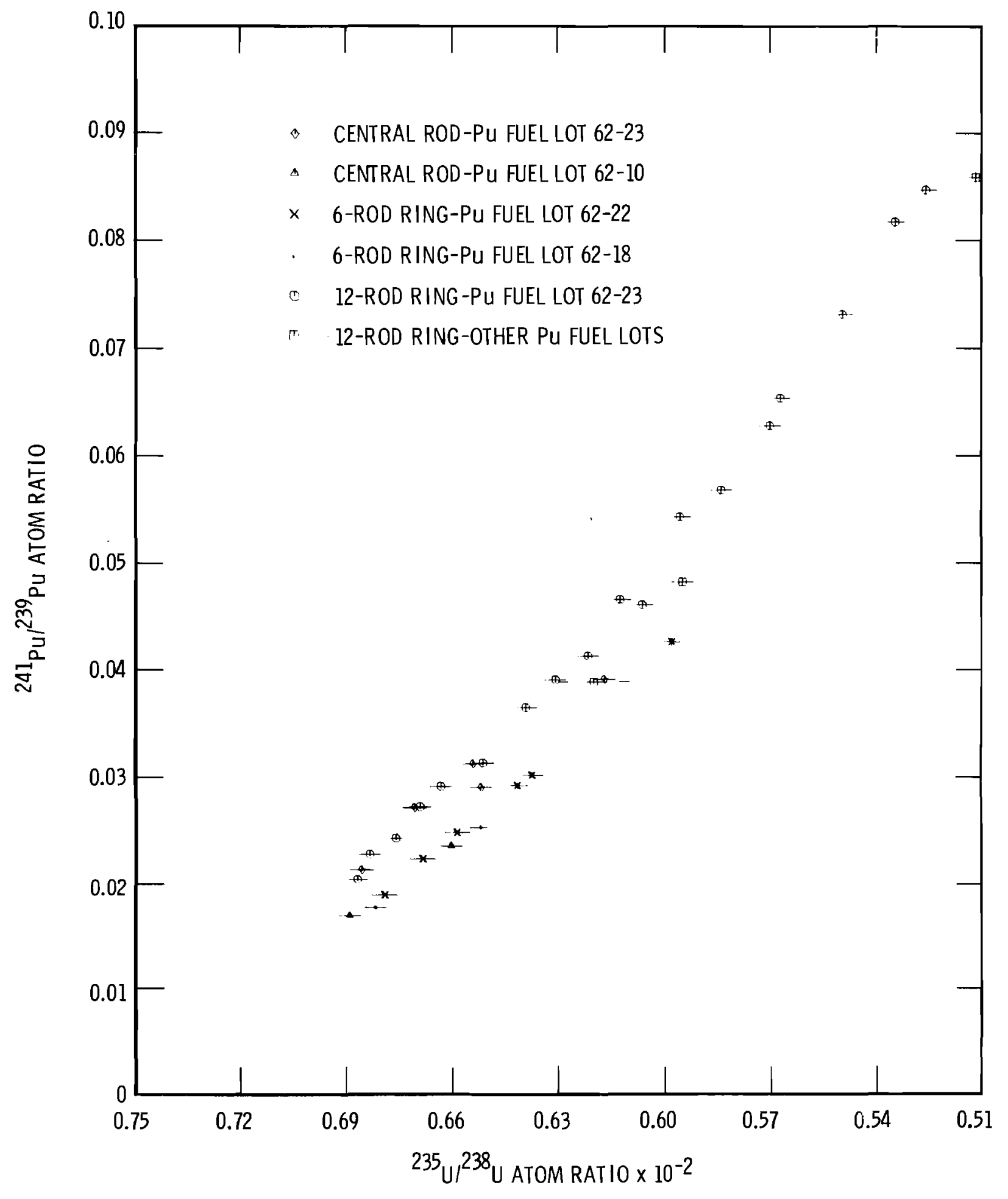

FIGURE $10 .{ }^{241} \mathrm{Pu} /{ }^{239} \mathrm{Pu}$ ATOM RATIO VERSUS ${ }^{235} \mathrm{U} /{ }^{238} \mathrm{U}$ ATOM RATIO 


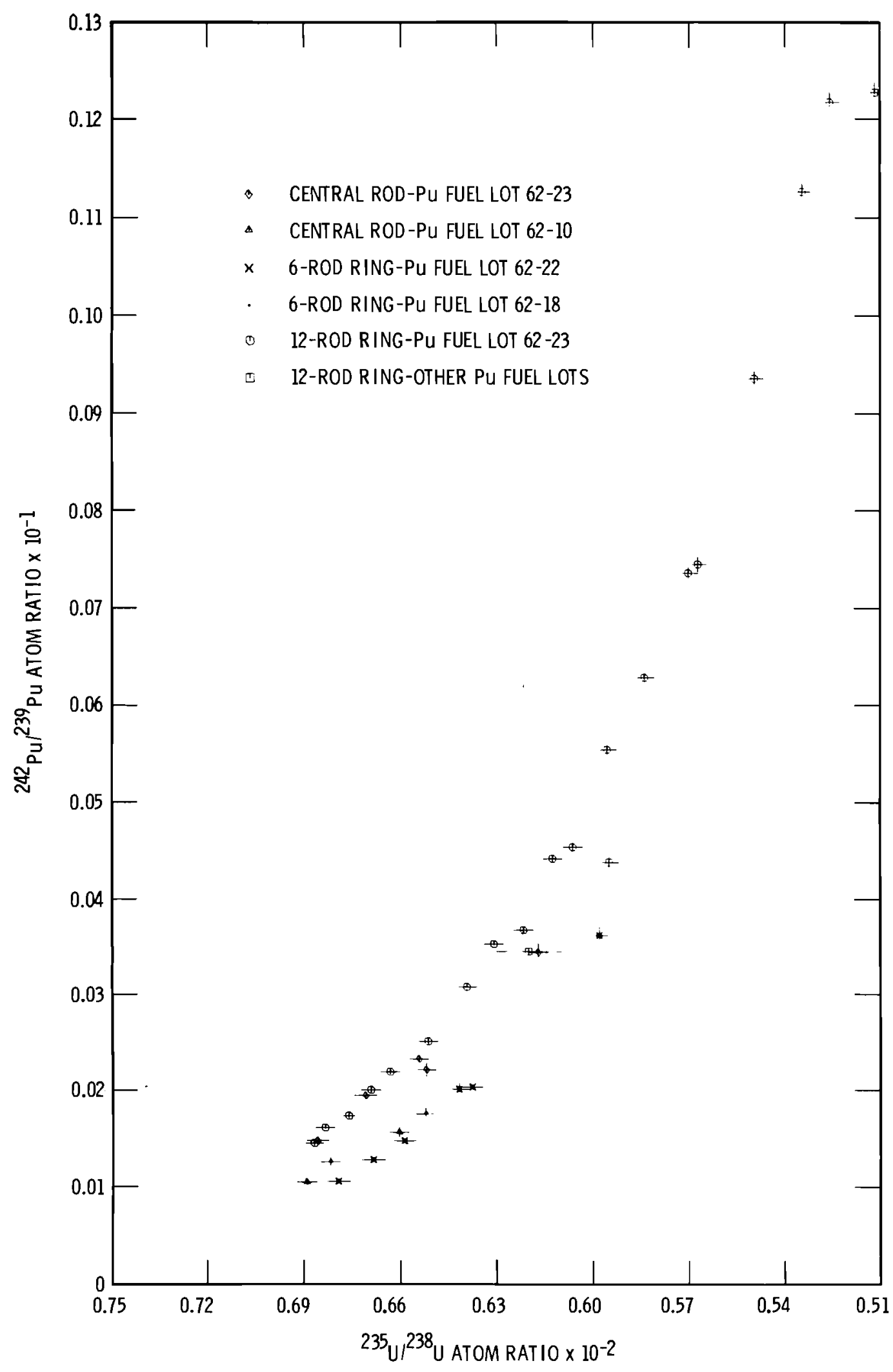

Figure $11 .{ }^{242} \mathrm{Pu} /{ }^{239} \mathrm{Pu}$ ATOM RATIO VERSUS ${ }^{235} \mathrm{U} /{ }^{238} \mathrm{U}$ atOM RATIO 


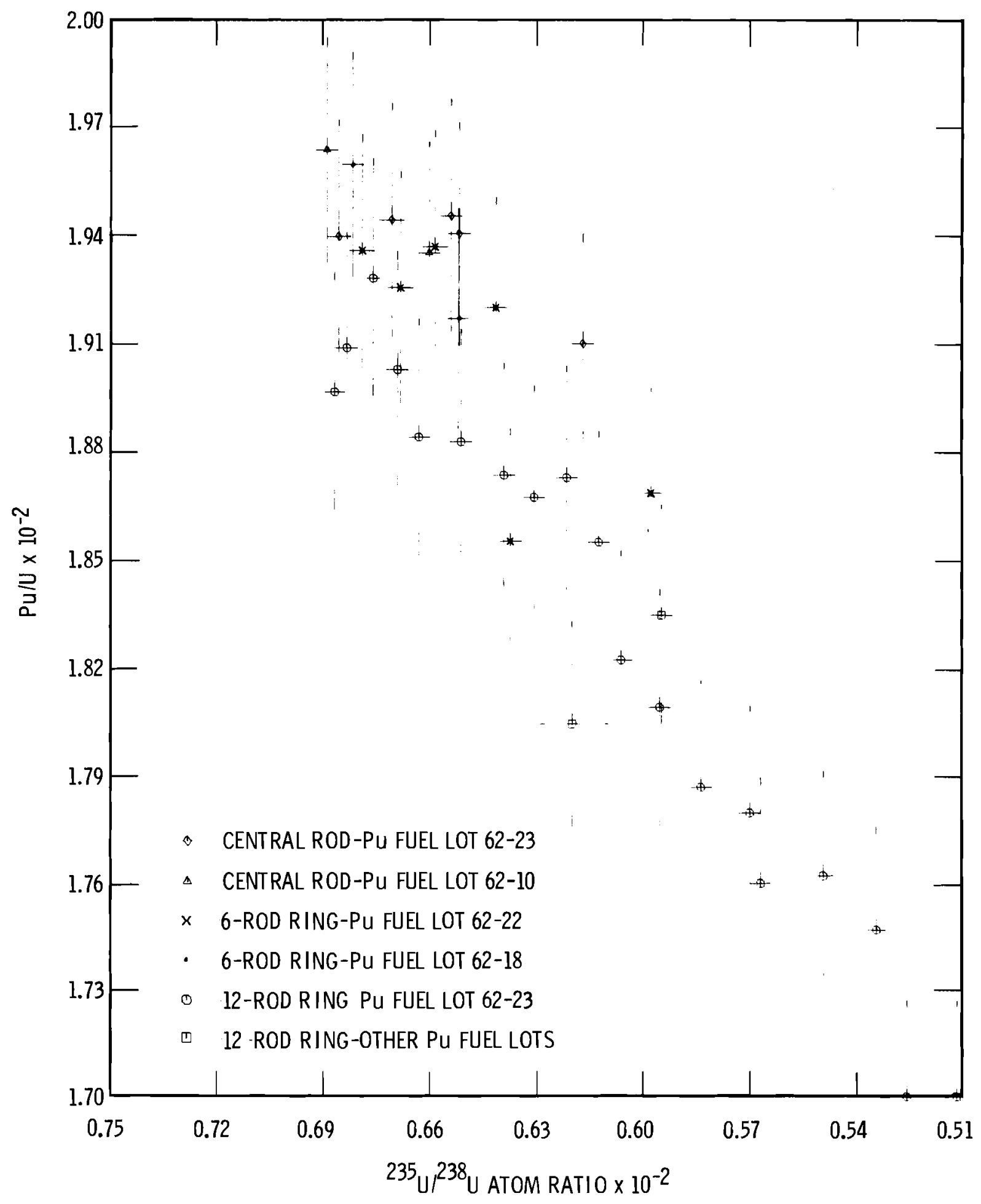

FIGURE 12. PU/U RATIO VERSUS ${ }^{235} \mathrm{U} /{ }^{238} \mathrm{U}$ ATOM RATIO 


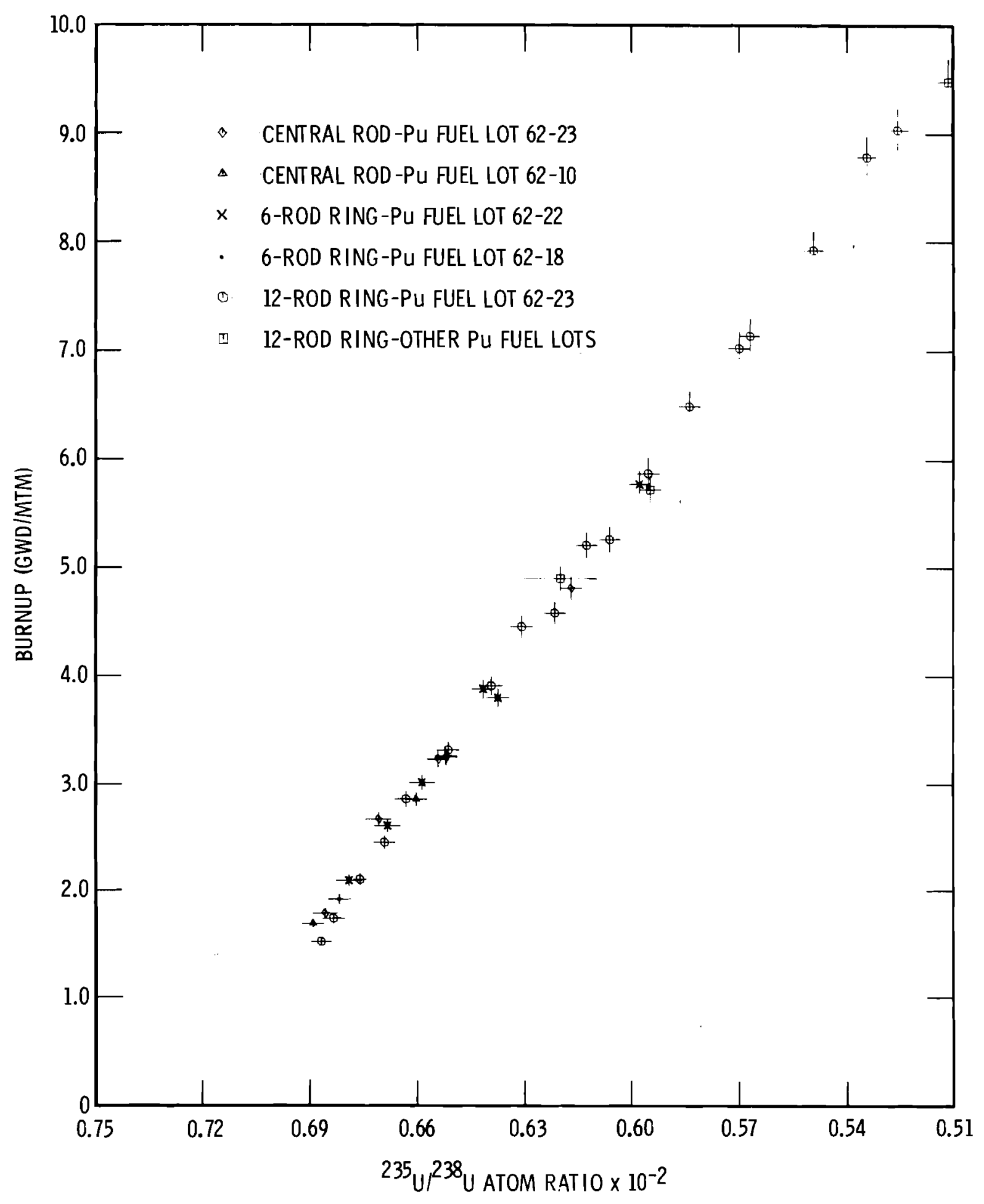

FIGURE 13. BURNUP (GWD/MTM) VERSUS ${ }^{235} \mathrm{U}^{238} \mathrm{U}$ RATIO 


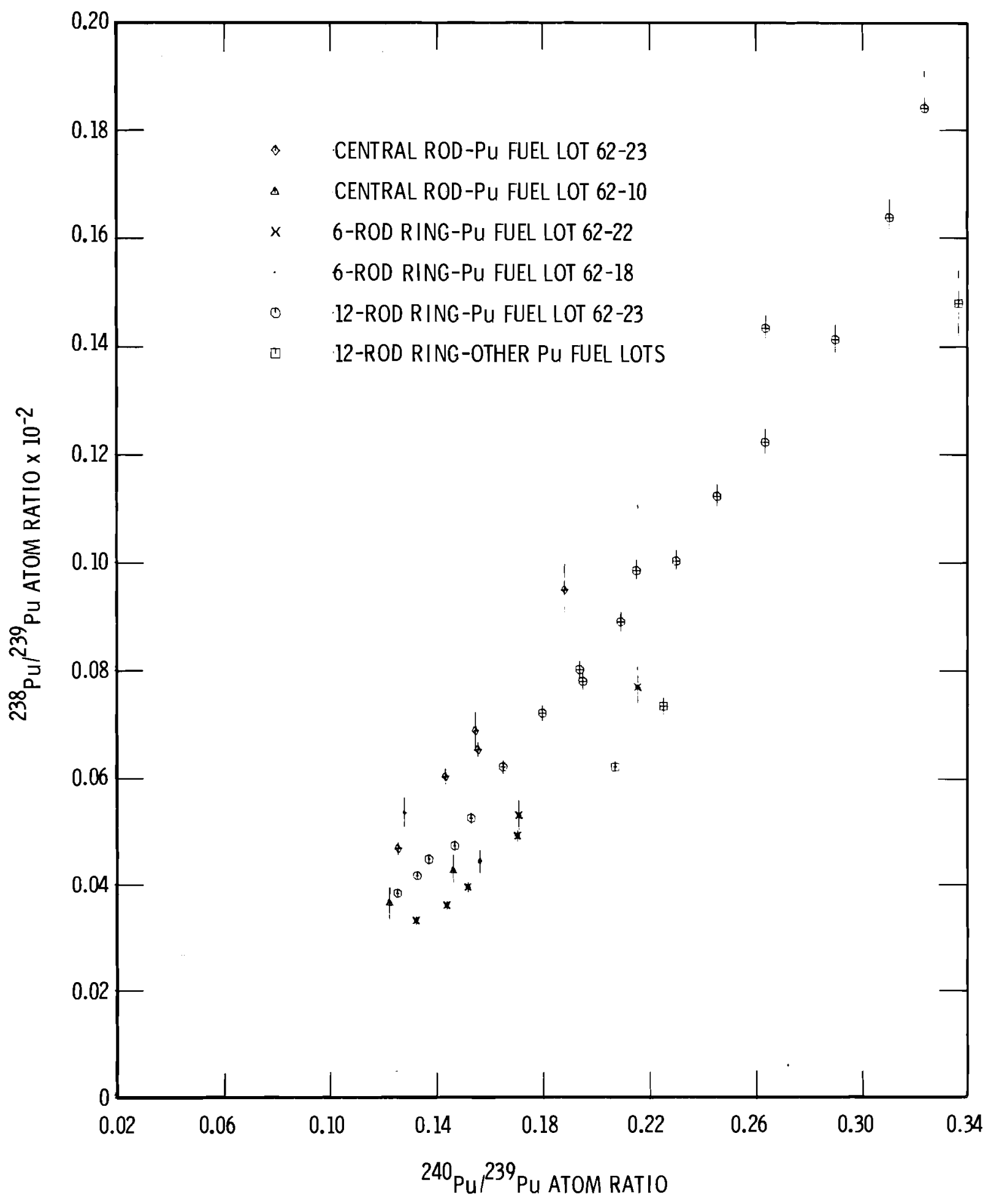

Figure $14 .{ }^{238} \mathrm{Pu} /{ }^{239} \mathrm{Pu}$ ATOM RATIO VERSUS ${ }^{240} \mathrm{Pu} /{ }^{239} \mathrm{Pu}$ AtOM RATIO 


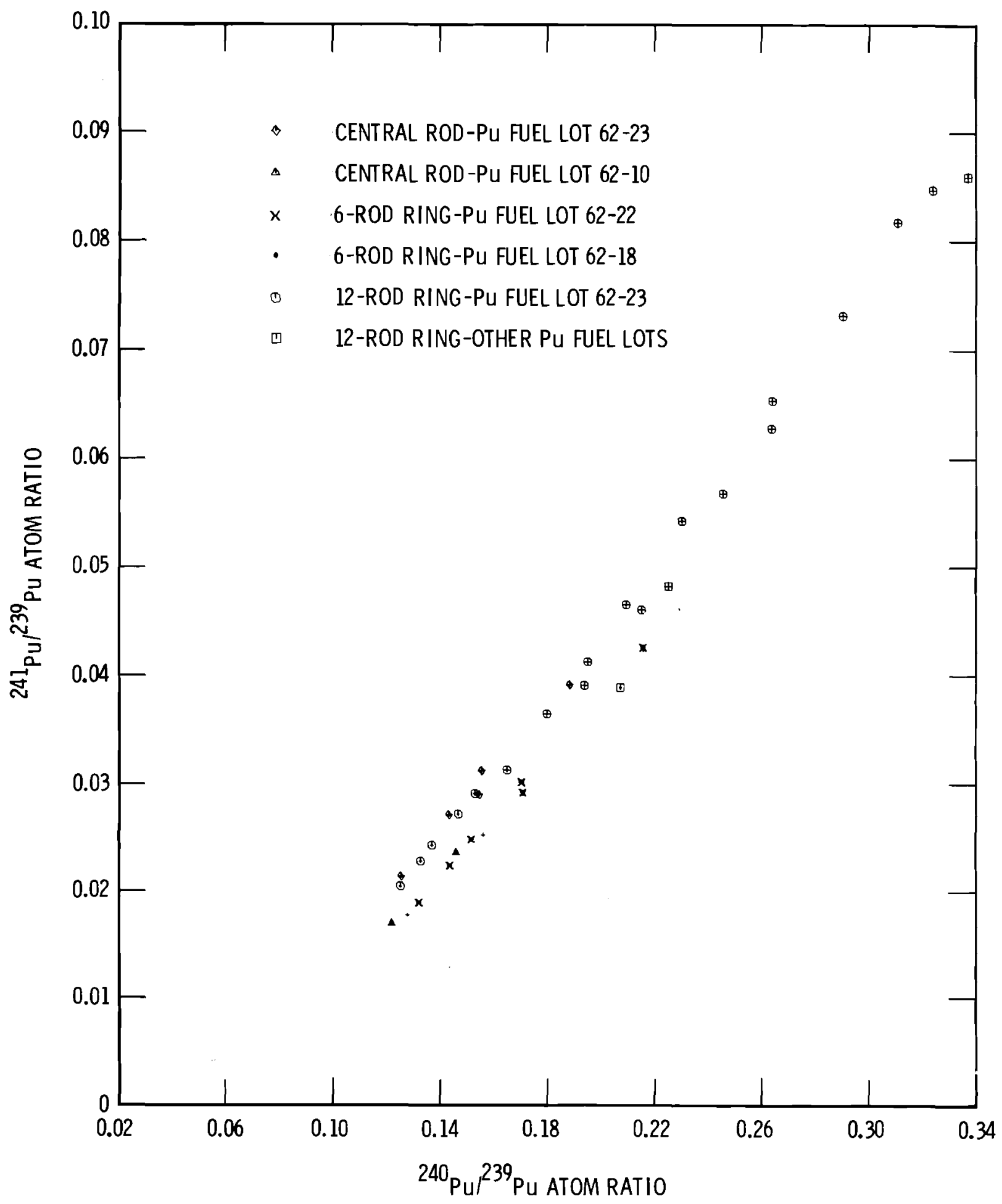

FIGURE 15. ${ }^{241} \mathrm{Pu} / 239 \mathrm{Pu}$ ATOM RATIO VERSUS ${ }^{240} \mathrm{Pu} /{ }^{239} \mathrm{Pu}$ ATOM RATIO 


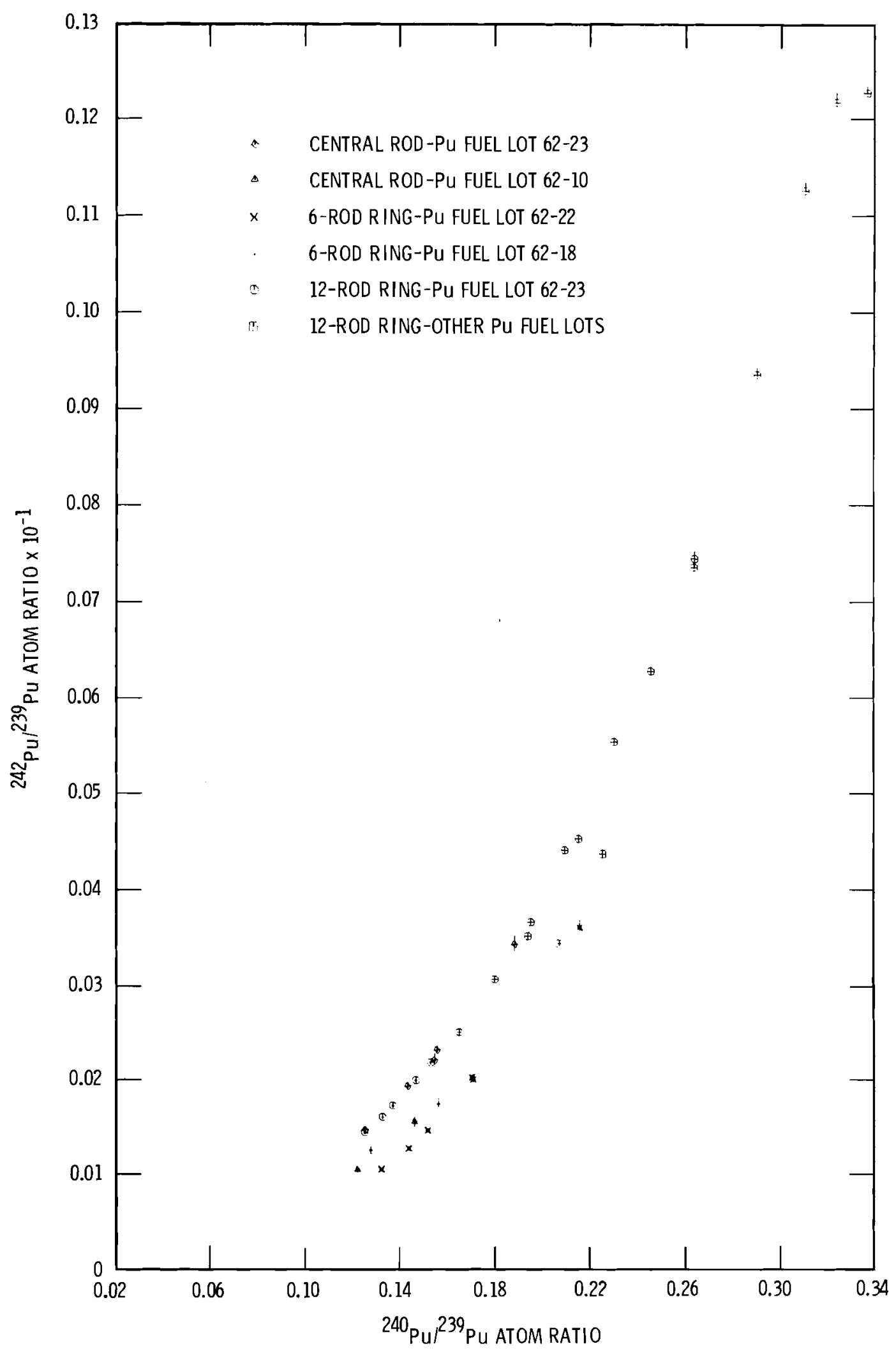

FIgURE 16. ${ }^{242} \mathrm{Pu} /{ }^{239} \mathrm{Pu}$ ATOM RATIO VERSUS ${ }^{240} \mathrm{Pu} /{ }^{239} \mathrm{Pu}$ ATOM RATIO 


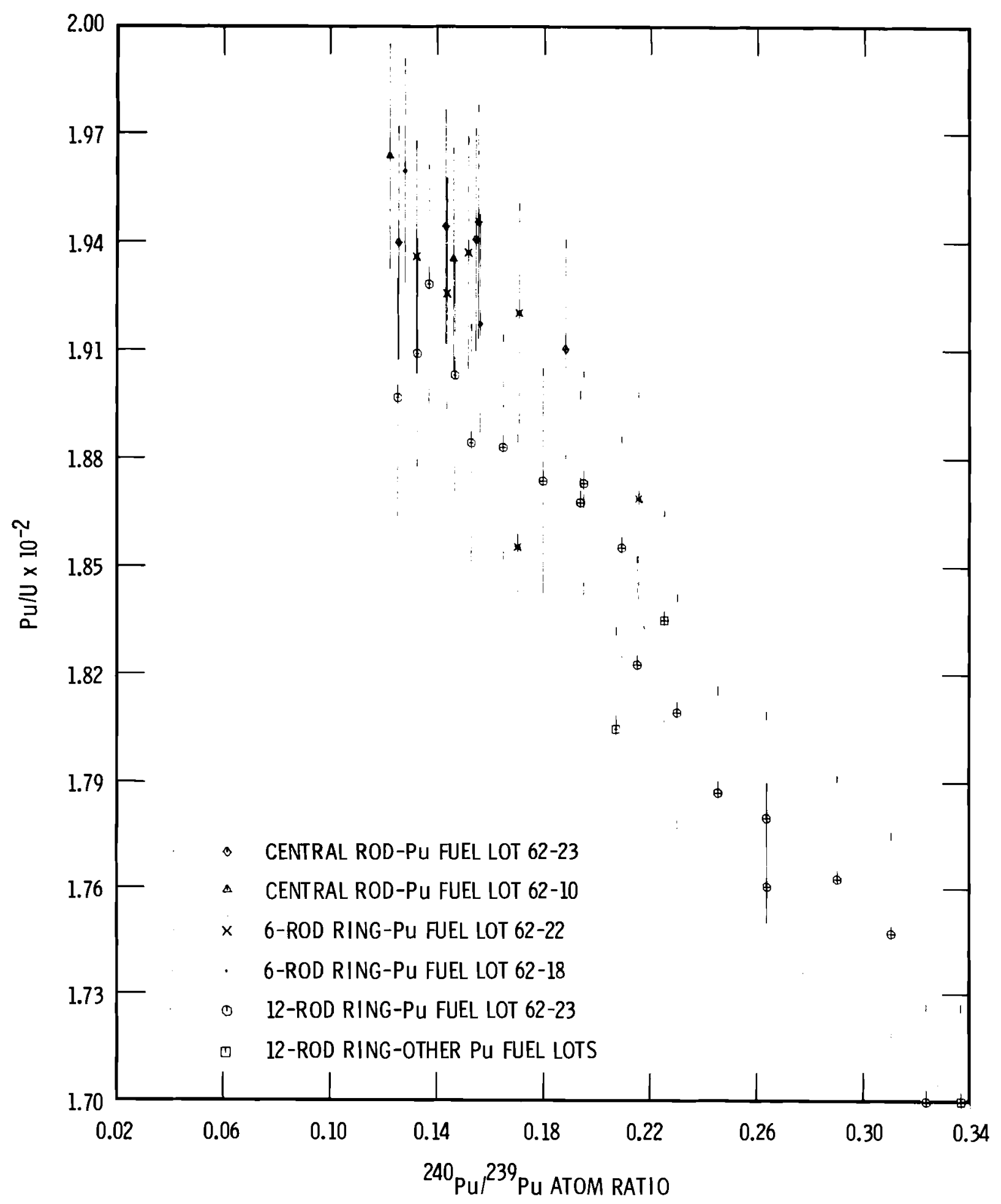

FIgURE 17. Pu/U RATIO VERSUS ${ }^{240} \mathrm{Pu} /{ }^{239} \mathrm{Pu}$ ATOM RATIO 


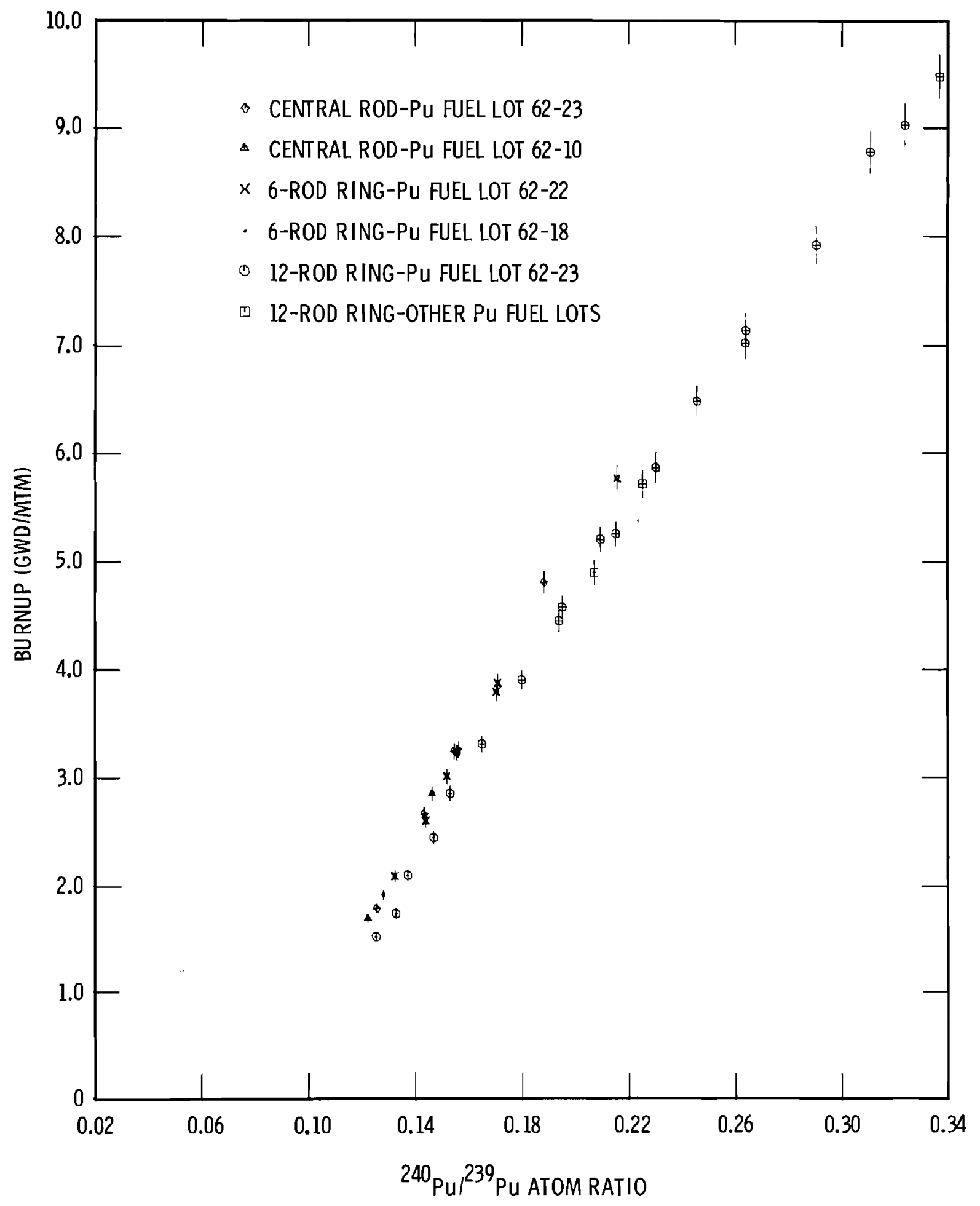

FIGURE 18. BURNUP (GWD/MTM) VERSUS ${ }^{240} \mathrm{Pu} /{ }^{239} \mathrm{PU}$ ATOM RATIO 
$-36-$

IV. DERIVATION OF EFFECTIVE CROSS SECTION RATIOS

A. Least-Squares Method: Program DBUFIT-I

The Batch Core isotopic concentration data have been fitted by means of the nonlinear least-squares method embodied in computer program DBUFIT-I. (11) The principles and characteristics of the method have been discussed at length by Matsen in earlier reports. $(12,13)$ We shall confine ourselves to a brief description only.

By assuming a particular functional form for the ratios of effectfive cross sections, it is possible to integrate the coupled differential equations of the burnup chain in closed form with the time variable eliminated. (11) For the isotopes of interest here - ${ }^{235} \mathrm{U}$, ${ }^{238} \mathrm{U},{ }^{236} \mathrm{U},{ }^{239} \mathrm{Pu},{ }^{240} \mathrm{Pu},{ }^{241} \mathrm{Pu}$ and ${ }^{242} \mathrm{Pu}$ - these integrated equations are

$$
\begin{aligned}
& N^{28}-K_{8}\left(N^{25}\right)^{A}=0 \\
& N^{26}+\frac{B}{1-b} N^{25}+\frac{b b}{b}-K_{6}\left(N^{25}\right)^{b}=0 \\
& N^{49}+\frac{B K_{8}}{A-C}\left(N^{25}\right)^{A}-K_{9}\left(N^{25}\right)^{C}+\frac{C C}{C}=0 \\
& N_{f}^{49}-\frac{(1-\varepsilon) B C K_{8}}{(A-C) A}\left(N^{25}\right)^{A}+(1-\varepsilon) K_{9}\left(N^{25}\right)^{C}-K_{9 f}=0 \\
& N^{40}-\frac{\varepsilon B C K_{8}}{(A-C)(A-D)}\left(N^{25}\right)^{A}+\frac{E C K_{g}}{C-D}\left(N^{25}\right)^{C}-K_{0}\left(N^{25}\right)^{D}+\frac{D D}{D}=0
\end{aligned}
$$




$$
\begin{aligned}
& N^{41}+\frac{\varepsilon B C D K_{8}}{(A-C)(A-D)(A-E)}\left(N^{25}\right)^{A}-\frac{\varepsilon C D K_{9}}{(C-D)(C-E)}\left(N^{25}\right)^{C}+\frac{D K_{0}}{D-E}\left(N^{25}\right)^{D}-K_{1}\left(N^{25}\right)^{E}=0 \\
& N^{42}-\frac{\varepsilon \gamma B C D E K_{8}}{(A-C)(A-D)(A-E)(A-F)}\left(N^{25}\right)^{A}+\frac{\varepsilon \gamma C D E K_{9}}{(C-D)(C-E)(C-F)}\left(N^{25}\right)^{C}-\frac{\gamma D E K_{0}}{(D-E)(D-F)}\left(N^{25}\right)^{D} \\
& +\frac{\gamma E K_{1}}{E-F}\left(N^{25}\right)^{E}-K_{2}\left(N^{25}\right)^{F}=0,
\end{aligned}
$$

where the cross section ratios are represented by

$$
\begin{aligned}
& \hat{\sigma}_{a}^{28} / \hat{\sigma}_{a}^{25}=A, \hat{\sigma}_{c}^{25} / \hat{\sigma}_{a}^{25}=B, \hat{\sigma}_{a}^{26} / \hat{\sigma}_{a}^{25}=b+b b / N^{26}, \\
& \hat{\sigma}_{c}^{28} / \hat{\sigma}_{a}^{25}=B, \hat{\sigma}_{a}^{49} / \hat{\sigma}_{a}^{25}=C+C C / N^{49}, \hat{\sigma}_{c}^{49} / \hat{\sigma}_{a}^{49}=\varepsilon, \hat{\sigma}_{a}^{40} / \hat{\sigma}_{a}^{25}=D+D D / N^{40}, \\
& \left(\hat{\sigma}_{a}^{41} / \hat{\sigma}_{a}^{25}\right)\left(1+\lambda^{41} / \hat{\sigma}_{a}^{41} \phi\right)=E,\left(\hat{\sigma}_{c}^{41} / \hat{\sigma}_{a}^{41}\right) /\left(1+\lambda^{41} / \hat{\sigma}_{a}^{41} \phi\right)=\gamma, \hat{\sigma}_{a}^{42} / \hat{\sigma}_{a}^{25}=F .
\end{aligned}
$$

The $\mathrm{K}$ 's are constants of integration, the N's are isotopic concentrations (where the superscript 25 represents ${ }^{235} \mathrm{U}, 49$ represents ${ }^{239} \mathrm{Pu}$, etc.), $\lambda^{41}$ is the radioactive decay constant of ${ }^{241} \mathrm{Pu}$, and $\phi$ is the neutron flux. Note that $\mathrm{N}_{f}^{49}$ is the concentration of fissioned nuclei of ${ }^{239} \mathrm{Pu}$. The factor $\lambda^{41} / \hat{\sigma}_{a}^{41} \phi$ is included to correct for the radioactive decay of ${ }^{241} \mathrm{Pu}$ during burnup. It is independently calculated prior to the least-squares fit.

The values of the 7 initial concentrations, which are related to the constants of integration, and the 13 parameters defining the cross-section ratios are in principle determinable by the least-squares fitting process. 
However, as we shall see later, it is usually necessary to fix one or more of the parameters by means of independent information. Note that 3 of the 10 cross-section ratios depend formally on isotopic concentrations and thus indirectly on exposure.

\section{B. Previous Reactor Burnup Calculations}

Burnup calculations for the PRTR Batch Core Experiment have been done previously by Matsuura and Prezbindowski. (14) In the course of that work, they calculated ratios of effective one-group cross sections for two reactor regions: region \#2, a non-central high-temperature section containing 18 fuel clusters, and region \#3, a non-central low-temperature section containing 36 fuel clusters. Their results (previously unpublished) for region $\# 2$, which is the region from which most of the isotopic samples were taken, are shown in Table $v$. Note that the ratio displayed for the fissile isotopes is $\hat{\alpha} \equiv \hat{\sigma}_{c} / \hat{\sigma}_{f}=\left(\hat{\sigma}_{c} / \hat{\sigma}_{a}\right) /\left(1-\hat{\sigma}_{c} / \hat{\sigma}_{a}\right)$.

\section{Least-Squares Fitting to Burnup Data}

In the fitting of burnup data it is important that all of the data being fitted at the same time lie along the same burnup path (see Figures 7 through 18). In particular, the initial isotopic composition and the neutron spectrum should be identical for all samples included in a single fit. For this reason, data from only 29 of the 36 exposed samples were used in least-squares fits. These data were grouped into 3 sets according to both the position of the fuel rod in its assembly and the fuel lot number, as shown in Table VI. 


\section{TABLE V}

CROSS-SECTION RATIOS FROM REACTOR BURNUP

CALCULATIONS BY MATSUURA AND PREZBINDOWSKI (unpublished)

\begin{tabular}{|c|c|c|c|c|}
\hline Ratio & $\begin{array}{c}0 \\
\text { (MWD/MTM) }\end{array}$ & $\begin{array}{c}3060 \\
\text { (MWD/MTM) }\end{array}$ & $\begin{array}{c}6490 \\
\text { (MWD/MTM) }\end{array}$ & $\begin{array}{l}\text { Percent } \\
\text { Change }\end{array}$ \\
\hline$\hat{\alpha}^{25}$ & 0.2433 & 0.2388 & 0.2340 & -3.8 \\
\hline$\hat{\sigma}_{a}^{26} / \hat{\sigma}_{a}^{25}$ & 0.2167 & 0.2024 & 0.1871 & -14.0 \\
\hline$\hat{\sigma}_{a}^{28} / \hat{\sigma}_{a}^{25}$ & 0.01238 & 0.01184 & 0.01125 & -9.1 \\
\hline$\hat{\sigma}_{c}^{28} / \hat{\sigma}_{a}^{25}$ & 0.01192 & 0.01140 & 0.01085 & -9.0 \\
\hline$\hat{\sigma}_{a}^{49} / \hat{\sigma}_{a}^{25}$ & 2.0082 & 2.022 & 2.039 & 1.5 \\
\hline$\alpha^{49}$ & 0.4865 & 0.4853 & 0.4843 & -0.45 \\
\hline$\hat{\sigma}_{a}^{40} / \hat{\sigma}_{a}^{25}$ & 1.426 & 1.2106 & 1.0605 & -26.0 \\
\hline$\hat{\sigma}_{a}^{41} / \hat{\sigma}_{a}^{25}$ & 2.349 & 2.3586 & 2.3701 & 0.90 \\
\hline$\hat{\alpha}^{41}$ & 0.3782 & 0.3788 & 0.3795 & 0.34 \\
\hline$\hat{\sigma}_{a}^{42} / \hat{\sigma}_{a}^{25}$ & 0.6817 & 0.6378 & 0.5905 & -13.0 \\
\hline
\end{tabular}




\section{$\underline{\text { TABLE VI }}$}

\section{SETS OF SAMPLE DATA USED IN LEAST-SQUARES FITS}

\begin{tabular}{|c|c|c|c|}
\hline Set & $\begin{array}{c}\text { Number of } \\
\text { Samples }\end{array}$ & $\begin{array}{l}\text { Rod Assembly } \\
\text { Position } \\
\end{array}$ & $\begin{array}{c}\text { Fuel Lot } \\
\text { Number }\end{array}$ \\
\hline 1 & 18 & 12-Rod Ring & $62-23$ \\
\hline 2 & 6 & 6-Rod Ring & $62-22$ \\
\hline 3 & 5 & Central & $62-23$ \\
\hline
\end{tabular}

Since burnup data are usually not extensive or accurate enough to permit a unique least-squares determination of all the cross-section ratios, it is necessary to constrain the parameters by means of independent information. In program DBUFIT-I this may be done by holding any subset of the parameters fixed at appropriate values. For this purpose, the calculated values in Table $V$ have been extremely useful.

In all of the fits reported here, three of the cross-section ratios have been held fixed: $\hat{\sigma}_{a}^{26} / \hat{\sigma}_{a}^{25}, \hat{a}^{41}$, and $\hat{\sigma}_{a}^{42} / \hat{\sigma}_{a}^{25}$.

For the first two quantities we have chosen values corresponding to the intermediate exposure (3060 MWD/MTM, Table V) while for the last we have chosen a value more strongly weighted toward the highest exposure at which calculations were done (6490 MWD/MTM).

The fitting of data for each set of samples is done in three stages. The single equations for ${ }^{238} U$ and ${ }^{236} U$ are each fitted separately, and then the remaining 5 equations for ${ }^{239} \mathrm{Pu},{ }^{239} \mathrm{Pu}$ fissions, ${ }^{240} \mathrm{Pu},{ }^{241} \mathrm{Pu}$ and ${ }^{242} \mathrm{Pu}$ are all fitted simultaneously. The results for the fuel rods 
in the three positions are given in Table VII, where the uncertainty for each adjusted quantity is the computed estimate of the standard deviation. It should be noted that the data for the 6-rod ring and central rods are not extensive enough to determine an exposure dependence of the ratio $\hat{\sigma}_{a}^{49} / \hat{\sigma}_{a}^{25}$. A satisfactory solution is obtained by fixing the parameter $\mathrm{CC}$ at zero, thus forcing the ratio to be constant (but still adjustable). Plots of the burnup data and the fitted curves for the 12-rod ring data are given in Figures $19,20,21$ and 22 . The error flags on the data points indicate one-standard-deviation estimates, while the one-standarddeviation uncertainty error bands of the fitted curves are indicated by the outer two lines that are shown with the least-squares fitted curve (middle). The fitted curves are seen to represent the data points adequately.

\section{Sensitivity Tests}

Since the values of the least-squares adjusted cross-section ratios depend on the input values of the fixed ratios, it is important to determine the sensitivity of the results to the assumed values. For this purpose the three fixed ratios $\hat{\sigma}_{a}^{26} / \hat{\sigma}_{a}^{25}, \hat{\sigma}_{c}^{41} / \hat{\sigma}_{a}^{41}$ and $\hat{\sigma}_{a}^{42} / \hat{\sigma}_{a}^{25}$ were each separately perturbed by $10 \%$ for the case of the exterior rods, and three additional runs were made. The results are given in Table VIII.

One of the most significant features of the calculated cross-section ratios in Table $V$ and the fitted ratios in Table VII is the great dependence of $\hat{\sigma}_{a}^{40} / \hat{\sigma}_{a}^{25}$ on exposure. Since there is some question about the adequacy of the assumed functional form of this ratio, $(11,12,13)$ it is 
$\underline{\text { TABLE VII }}$

CROSS-SECTION RATIOS DETERMINED BY LEAST-SQUARES

FITS FOR FUEL RODS IN THREE POSITIONS IN A 19-ROD ASSEMBLY

\begin{tabular}{|c|c|c|c|}
\hline Ratio & $\begin{array}{l}\text { 12-Rod Ring } \\
\left(\begin{array}{l}\text { Max. Exposure } \\
9200 \text { MWD/MTM }\end{array}\right)\end{array}$ & $\begin{array}{l}\text { 6-Rod Ring } \\
\left(\begin{array}{l}\text { Max. } \\
570 x \text { Exposure } \\
\text { MWD/MTM }\end{array}\right)\end{array}$ & $\begin{array}{c}\text { Central } \\
\left(\begin{array}{c}\text { Max. Exposure } \\
4800 \text { MWD/MTM }\end{array}\right)\end{array}$ \\
\hline$\hat{\alpha}^{25}$ & $0.2240 \pm 0.0009$ & $0.2442 \pm 0.0032$ & $0.2536 \pm 0.0086$ \\
\hline$\hat{\sigma}_{a}^{26} / \hat{\sigma}_{a}^{25}$ & 0.2020 (fixed) & 0.2020 (fixed) & 0.2020 (fixed) \\
\hline$\hat{\sigma}_{a}^{28} / \hat{\sigma}_{a}^{25}$ & $0.01586 \pm 0.00052$ & $0.01823 \pm 0.00144$ & $0.02042 \pm 0.00094$ \\
\hline$\hat{\sigma}_{c}^{28} / \hat{\sigma}_{a}^{25}$ & $0.01399 \pm 0.00119$ & $0.01635 \pm 0.00218$ & $0.01818 \pm 0.00543$ \\
\hline 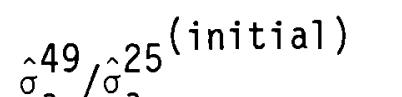 & $2.120 \pm 0.039$ & $2.056 \pm 0.038$ & $2.008 \pm 0.086$ \\
\hline a a (final) & $2.281 \pm 0.067$ & (constant) & (constant) \\
\hline$\alpha^{49}$ & $0.5159 \pm 0.0106$ & $0.4976 \pm 0.0173$ & $0.4830 \pm 0.0388$ \\
\hline$\hat{\sigma}^{40} \hat{\sigma}^{25}($ initial $)$ & \pm 0.042 & \pm 0.046 & $1.863 \pm 0.120$ \\
\hline$a^{10} a$ (final) & \pm 0.029 & \pm 0.046 & $1.215 \pm 0.125$ \\
\hline$\hat{\sigma}_{a}^{41} / \hat{\sigma}_{a}^{25}$ & $2.4885 \pm 0.0735$ & $2.2247 \pm 0.0781$ & $2.1129 \pm 0.1226$ \\
\hline$\hat{\alpha}^{41}$ & 0.3788 (fixed) & 0.3787 (fixed) & 0.3787 (fixed) \\
\hline$\hat{\sigma}_{a}^{42} / \hat{\sigma}_{a}^{25}$ & (fixed) & (fixed) & $0.600 \quad$ (fixed) \\
\hline
\end{tabular}




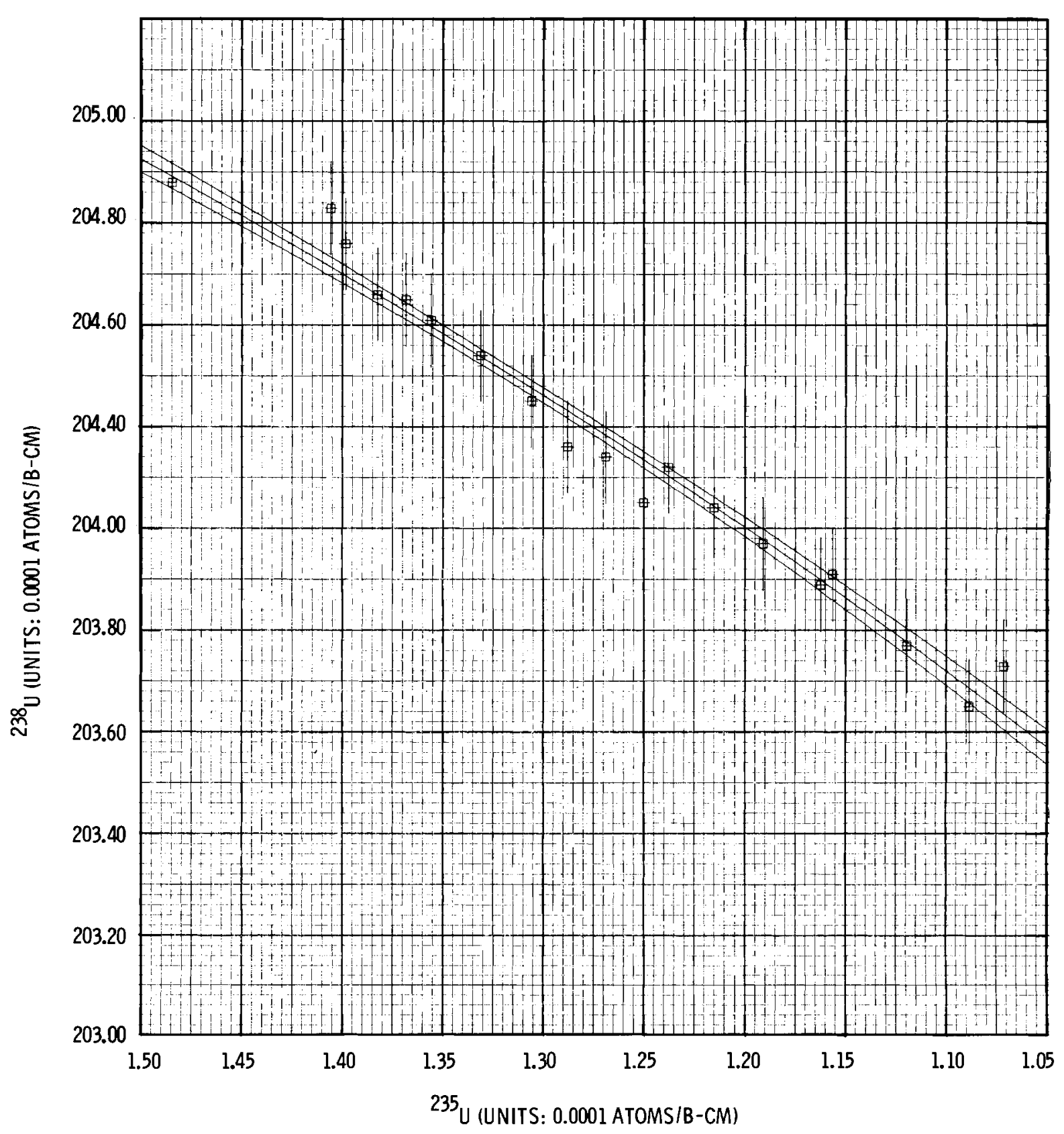

FIGURE $19 .{ }^{238} \mathrm{U}$ VERSUS ${ }^{235} \mathrm{U}$ AND LEAST-SQUARES FITIING RESULT FOR 12-ROD RING DATA 


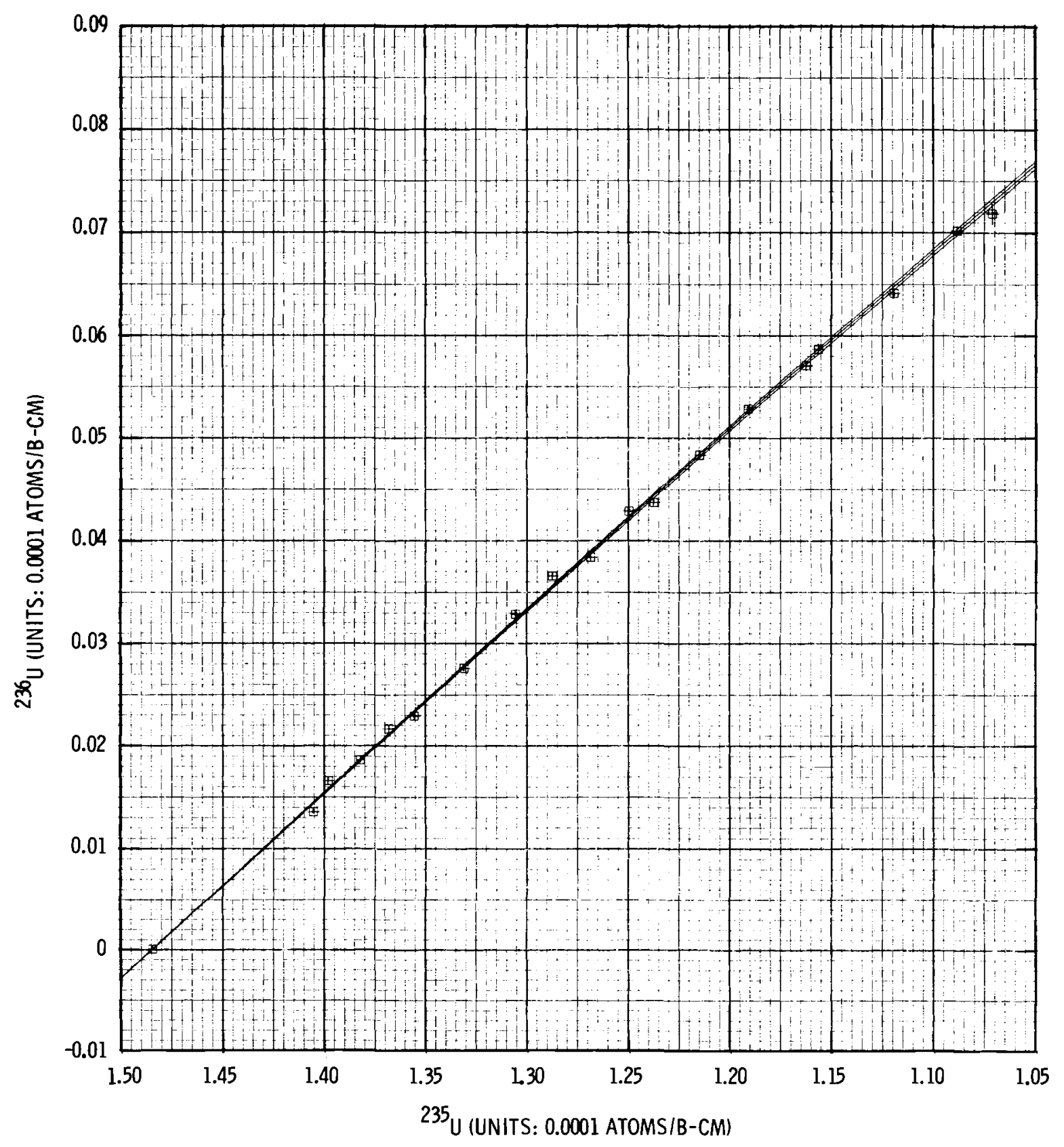

FIGURE $20 .{ }^{236} \mathrm{U}$ VERSUS ${ }^{235} \mathrm{U}$ AND LEAST-SQUARES FITING RESULT FOR 12-ROD RING DATA 


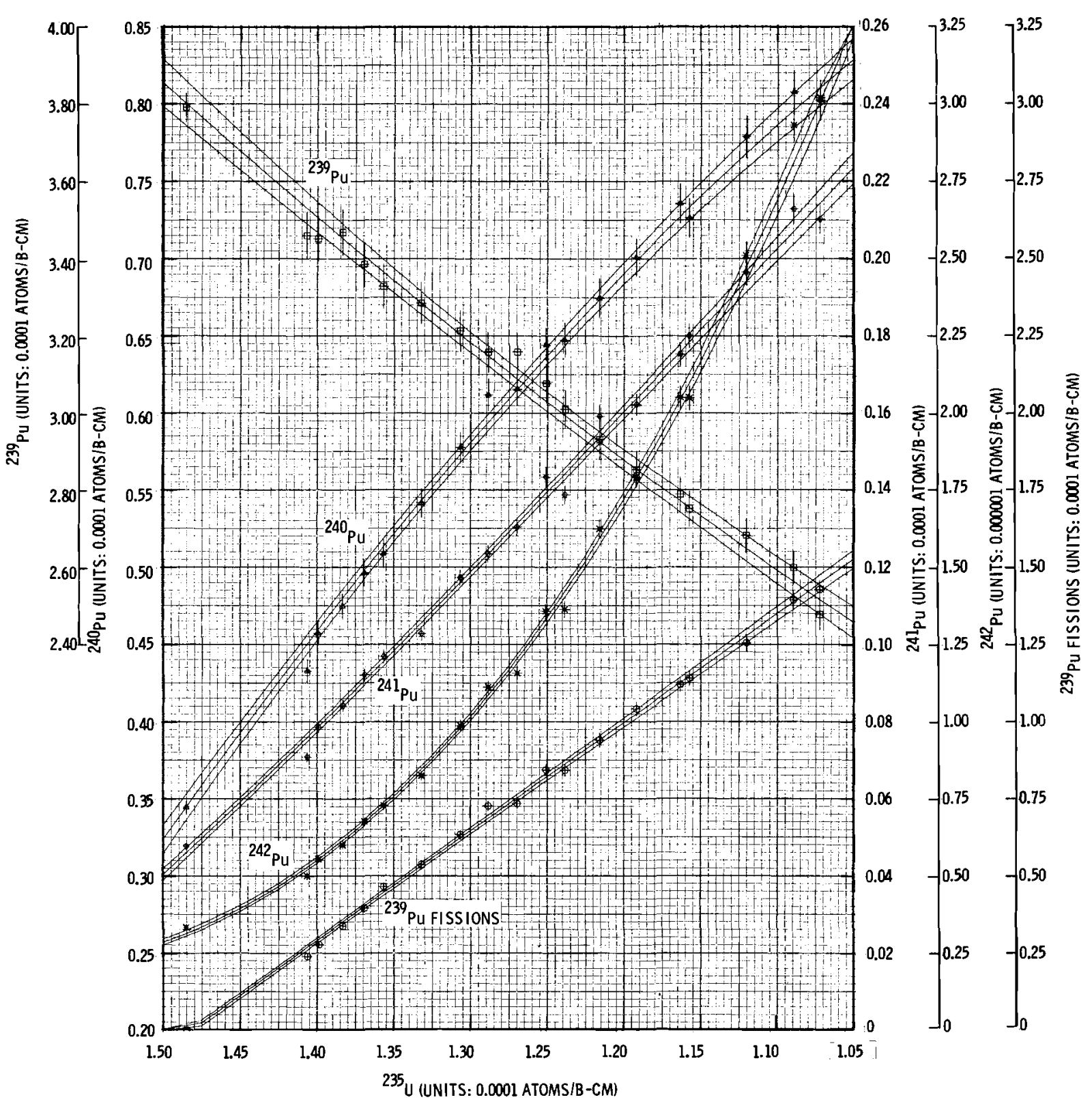

FIGURE 21. ${ }^{239} \mathrm{Pu},{ }^{240} \mathrm{Pu}_{,}{ }^{241} \mathrm{Pu}_{\mathrm{P}}{ }^{242} \mathrm{Pu}$ AND ${ }^{239} \mathrm{Pu}$ FISSIONS VERSUS
FITING RESULTS FOR 12 -ROD RING DATA 

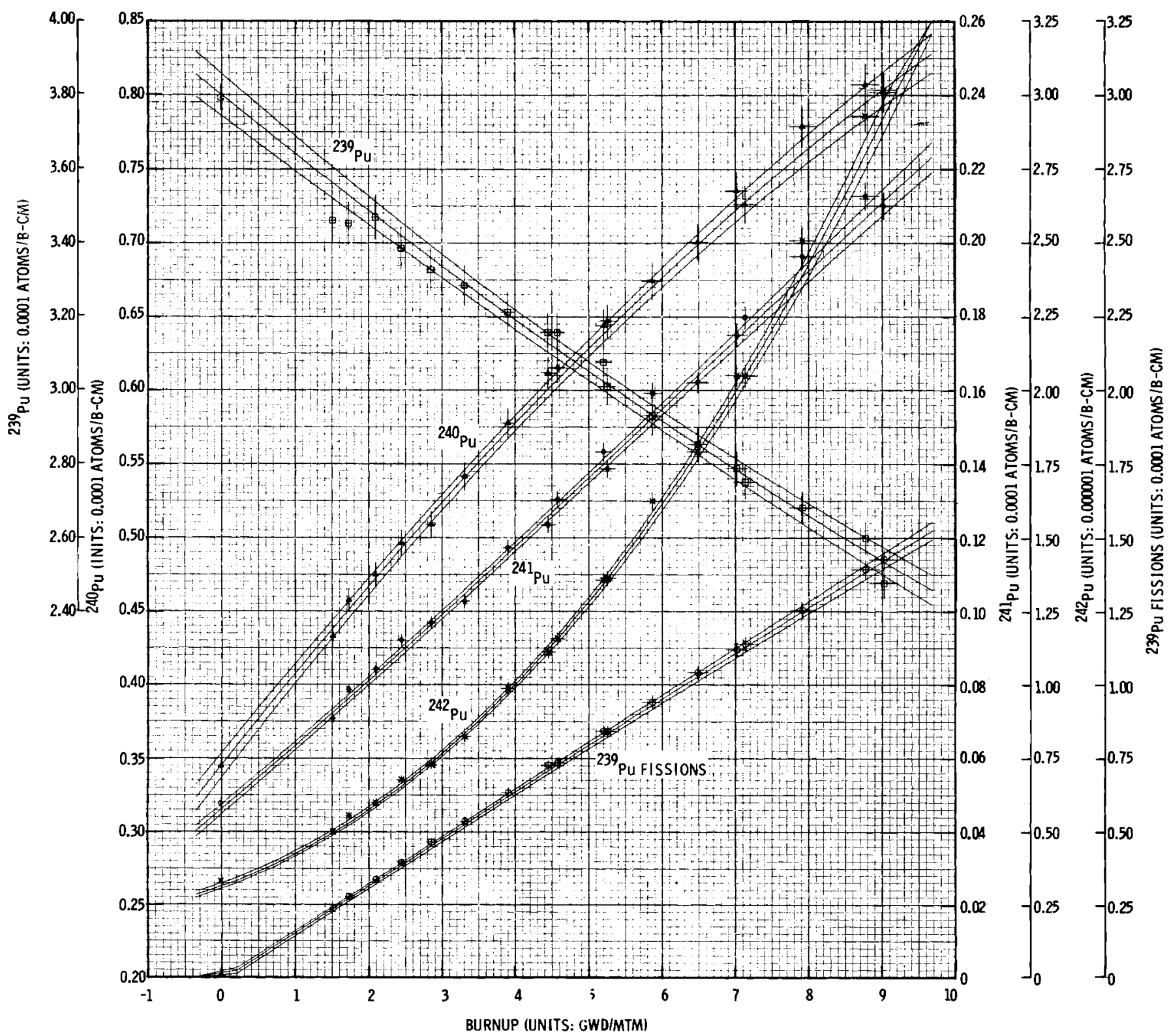

FIGURE $22 .{ }^{239} \mathrm{PU},{ }^{240} \mathrm{PU},{ }^{241} \mathrm{Pu},{ }^{242} \mathrm{PU}$ AND ${ }^{239} \mathrm{PU}$ FISSIONS VERSUS BURNUP AND LEAST-SQUARES FITTING RESULTS FOR 12-ROD RING DATA 


\section{TABLE VIII}

RESULTS OF SENSITIVITY TESTS ON CROSS-SECTION

RATIOS FOR THE 12-ROD RING RODS

\begin{tabular}{l|c|c|c}
\multicolumn{1}{c|}{$\begin{array}{c}\text { Perturbed } \\
\text { Ratio }\end{array}$} & $\hat{\sigma}_{a}^{26} / \hat{\sigma}_{a}^{25}$ & $\hat{\sigma}_{c}^{41} / \hat{\sigma}_{a}^{41}$ & $\hat{\sigma}_{a}^{42} \hat{\sigma}_{a}^{25}$ \\
\hline$\hat{\alpha}^{25}$ & $0.2234 \pm 0.0009$ & $0.2240 \pm 0.0009$ & $0.2240 \pm 0.0009$ \\
$\hat{\sigma}_{a}^{26} / \hat{\sigma}_{a}^{25}$ & 0.1820 (fixed) & 0.2020 (fixed) & 0.2020 (fixed) \\
$\hat{\sigma}_{a}^{28} / \hat{\sigma}_{a}^{25}$ & $0.01586 \pm 0.00052$ & $0.01586 \pm 0.00052$ & $0.01586 \pm 0.00052$ \\
$\hat{\sigma}_{c}^{28} / \hat{\sigma}_{a}^{25}$ & $0.01399 \pm 0.00119$ & $0.01385 \pm 0.00119$ & $0.01400 \pm 0.00118$ \\
$\hat{\sigma}_{a}^{49} / \hat{\sigma}_{a}^{25}$ (finitial) & $2.120 \pm 0.039$ & $2.121 \pm 0.039$ & $2.117 \pm 0.038$ \\
$\hat{\alpha}^{49}$ & $2.281 \pm 0.067$ & $2.253 \pm 0.067$ & $2.283 \pm 0.067$ \\
$\hat{\sigma}_{a}^{40} / \hat{\sigma}_{a}^{25}$ (finitial) & $1.963 \pm 0.042$ & $1.932 \pm 0.042$ & $1.956 \pm 0.041$ \\
$\hat{\sigma}_{a}^{41} / \hat{\sigma}_{a}^{25}$ & $1.284 \pm 0.029$ & $1.223 \pm 0.029$ & $1.280 \pm 0.029$ \\
$\hat{\alpha}^{41}$ & $2.4885 \pm 0.0735$ & $2.2630 \pm 0.0668$ & $2.4682 \pm 0.0729$ \\
$\hat{\sigma}_{a}^{42} / \hat{\sigma}_{a}^{25}$ & 0.3787 (fixed) & 0.4330 (fixed) & 0.3787 (fixed) \\
& 0.600 (fixed) & 0.600 (fixed) & 0.540 (fixed)
\end{tabular}


worthwhile to investigate this point further. For this purpose the extensive data for the 12 -rod ring rods were grouped into three parts covering approximately equal intervals of exposure. The three partical sets of data for the plutonium isotopes were then refit separately, with all exposure dependence suppressed by fixing the parameters CC and DD at zero. The results of these runs are given in Table IX and are discussed at the end of the following subsection.

E. Discussion of Cross-Section Ratios

Inspection of Table $V$ shows that the calculated cross-section ratios depend approximately linearly on the exposure. Three of them $-\hat{\sigma}_{a}^{26} / \hat{\sigma}_{a}^{25}$, $\hat{\sigma}_{a}^{40} / \hat{\sigma}_{a}^{25}$, and $\hat{\sigma}_{a}^{42} / \hat{\sigma}_{a}^{25}$ - change by more than $10 \%$ during an exposure of $6490 \mathrm{MWD} / \mathrm{MTM}$. The first and third of these are constant in the leastsquares fits and, in fact, must be fixed at input values to get satisfactory iterative convergence. The fitted values of $\hat{\sigma}_{a}^{40} / \hat{\sigma}_{a}^{25}$ in Table VII show an even greater exposure dependence than the calculated values for all types of fuel rods. Similarly, the fitted values of $\hat{\sigma}_{a}^{49} / \hat{\sigma}_{a}^{25}$ for the 12-rod ring rods have a somewhat greater exposure dependence than the calculated values.

The fitted ratios in Table VII indicate systematic differences among the rods in different positions, although the picture is confused by the various exposure intervals and the large uncertainties for the central rod. Nevertheless, there appear to be real differences in $\hat{\alpha}^{25}$, $\hat{\sigma}_{a}^{28} / \hat{\sigma}_{a}^{25}, \hat{\sigma}_{a}^{49} / \hat{\sigma}_{a}^{25}, \hat{\alpha}^{49}, \hat{\sigma}_{a}^{40} / \hat{\sigma}_{a}^{25}$, and $\hat{\sigma}_{a}^{41} / \hat{\sigma}_{a}^{25}$. 
TABLE IX

RESULTS OF LEAST-SQUARES FITS OF PARTIAL

SETS OF DATA FOR THE 12-ROD RING RODS

\begin{tabular}{|c|c|c|c|}
\hline 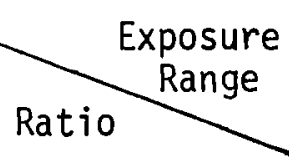 & $\begin{array}{c}0-3200 \\
(\text { MWD/MTM) }\end{array}$ & $\begin{array}{l}3900-5900 \\
\text { (MWD/MTM) }\end{array}$ & $\begin{array}{l}6500-9000 \\
\text { (MWD/MTM) }\end{array}$ \\
\hline$\hat{\sigma}_{c}^{28} / \hat{\sigma}_{a}^{25}$ & $0.01736 \pm 0.00673$ & $0.01222 \pm 0.01005$ & $0.01227 \pm 0.00452$ \\
\hline$\hat{\sigma}_{a}^{49} / \hat{\sigma}_{a}^{25}$ & $2.101 \pm 0.088$ & $2.115 \pm 0.274$ & $2.188 \pm 0.185$ \\
\hline$\hat{\alpha}^{49}$ & $0.5302 \pm 0.0362$ & $0.5226 \pm 0.1199$ & $0.5523 \pm 0.0837$ \\
\hline$\hat{\sigma}_{a}^{40} / \hat{\sigma}_{a}^{25}$ & $1.655 \pm 0.070$ & $1.493 \pm 0.188$ & $1.367 \pm 0.099$ \\
\hline$\hat{\sigma}_{a}^{41} / \hat{\sigma}_{a}^{25}$ & $2.3698 \pm 0.1265$ & $2.5034 \pm 0.2632$ & $2.6109 \pm 0.1603$ \\
\hline$\hat{\alpha}^{41}$ & 0.3787 (fixed) & 0.3787 (fixed) & 0.3787 (fixed) \\
\hline$\hat{\sigma}_{a}^{42} / \hat{\sigma}_{a}^{25}$ & 0.600 (fixed) & 0.600 (fixed & 0.600 (fixed) \\
\hline
\end{tabular}


There is, of course, considerable interest in a comparison of the calculated and fitted ratios. Unfortunately, a straightforward comparison of the calculated values in Table $V$ and the fitted values in Table VII is not possible. The reactor calculations were done for smeared cells, while the least-squares fits were done for separate fuel rods. Furthermore, the exposure intervals do not coincide. Nevertheless, we shall present comparisons for the purpose of example. The calculated ratios in the second column of Table $V$ (3060 MWD/MTM) may be considered to be average values appropriate over an exposure interval of 0-6000 MWD/MTM. To get comparable fitted values we must do averaging over the same exposure and mass-weighted averaging over the fuel rod positions. The results of this process are given in Table $x$. The differences in 4 of the ratios appear to be reasonably small $(<5 \%)$. However, 3 of them $-\hat{\sigma}_{a}^{28} / \hat{\sigma}_{a}^{25}, \hat{\sigma}_{c}^{28} / \hat{\sigma}_{a}^{25}$, and $\hat{\sigma}_{a}^{40} / \hat{\sigma}_{a}^{25}$ - differ by $22 \%$ to $30 \%$. All of these ratios involve cross sections having substantial resonance contributions. This suggests the possibility of difficulties in the calculation of resonance integrals or the ratio of fast to thermal flux components.

The sensitivity test results in Table VIII show that the fitted cross-section ratios are insensitive to the assumed values of $\hat{\sigma}_{a}^{26} / \hat{\sigma}_{a}^{25}$ and $\hat{\sigma}_{a}^{42} / \hat{\sigma}_{a}^{25}$. In particular the adjusted values for the 12 -rod ring change by less than $1 \%$ when the fixed values are perturbed by $10 \%$. However, there is some sensitivity to the assumed value of $\hat{\sigma}_{c}^{41} / \hat{\sigma}_{a}^{41}$. When it changes by $10 \%$, the value of $\hat{\sigma}_{a}^{41} / \hat{\sigma}_{a}^{25}$ and the final value of 
TABLE $X$

COMPARISON OF CALCULATED AND FITTED VALUES

OF CROSS-SECTION RATIOS

\begin{tabular}{|c|c|c|c|}
\hline Ratio & $\begin{array}{l}\text { Average } \\
\text { Fitted } \\
\text { Values }\end{array}$ & $\begin{array}{c}\text { Central } \\
\text { Calculated } \\
\text { Values }\end{array}$ & $\begin{array}{l}\text { Percent } \\
\text { Difference }\end{array}$ \\
\hline$\hat{\alpha}$ & 0.2319 & 0.2388 & 3.0 \\
\hline$\hat{\sigma}_{a}^{26} / \hat{\sigma}_{a}^{25}$ & $\begin{array}{r}0.2020 \\
\text { (fixed) }\end{array}$ & 0.2024 & -- \\
\hline$\hat{\sigma}_{a}^{28} / \hat{\sigma}_{a}^{25}$ & 0.01685 & 0.01184 & -30.0 \\
\hline$\hat{\sigma}_{\mathrm{c}}^{28} / \hat{\sigma}_{\mathrm{a}}^{25}$ & 0.01496 & 0.01140 & -24.0 \\
\hline$\hat{\sigma}_{a}^{49} / \hat{\sigma}_{a}^{25}$ & 2.121 & 2.022 & -4.7 \\
\hline$\hat{\alpha}^{49}$ & 0.5084 & 0.4853 & -4.5 \\
\hline$\hat{\sigma}_{a}^{40} / \hat{\sigma}_{a}^{25}$ & 1.560 & 1.2106 & -22.0 \\
\hline$\hat{\sigma}_{a}^{41} / \hat{\sigma}_{a}^{25}$ & 2.385 & 2.3586 & -1.1 \\
\hline$\hat{\alpha}^{41}$ & $\begin{array}{r}0.3787 \\
\text { (fixed) }\end{array}$ & 0.3788 & -- \\
\hline$\hat{\sigma}_{a}^{42} / \hat{\sigma}_{a}^{25}$ & $\begin{array}{c}0.600 \\
\text { (fixed) }\end{array}$ & 0.6378 & -- \\
\hline
\end{tabular}


$\hat{\sigma}_{a}^{40} / \hat{\sigma}_{a}^{25}$ change by $9 \%$ and $5 \%$, respectively. Note that the preceding discussion of relatively large discrepancies in some of the cross sections is not sensitive to the assumed values of the fixed ratios. Finally, we come to a discussion of the functional form of the exposure dependence of the ratio $\hat{\sigma}_{a}^{40} / \hat{\sigma}_{a}^{25}$. In Figure 23 the values of $\hat{\sigma}^{40} / \hat{\sigma}^{25}$ obtained from the fits of the partial sets of 12 -rod ring data (Table IX) have been plotted in histogram form as a function of exposure. The dashed straight line may then be drawn as a smooth approximation to the discontinuous histogram. Also plotted is the curved function (and its error band) for $\hat{\sigma}_{a}^{40} / \hat{\sigma}_{a}^{25}$, which is used by DBUFIT-I. Note that within the limits of its shape, the curve is a rough approximation of the dashed line. Its most serious weakness is to overestimate the initial value of the cross-section ratio by about $16 \%$. Of course, it must be remembered that the curved shape of the cross-section ratio is the price that has been paid to obtain closed form solutions involving only a few elementary functions. $(11,12,13)$

In addition to the comparison of calculated and measured cross section ratios shown above, Matsuura and Prezbindowski compared calculated and measured isotopic data for the 12-rod ring position. These results are given in Reference 14, pp 30-37. 


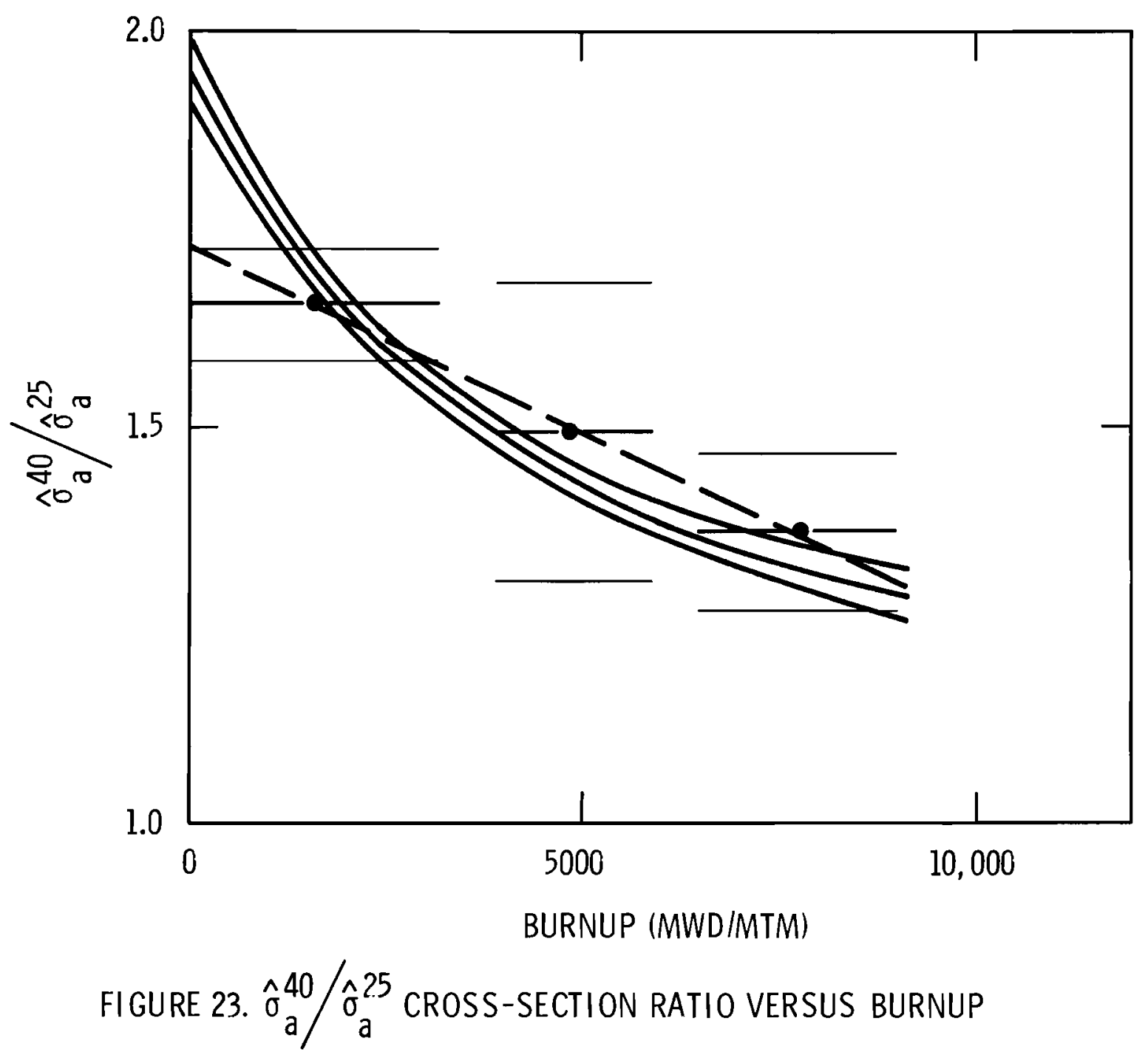




\section{BATCH CORE DATA FROM NONDESTRUCTIVE ANALYSIS}

Since October, 1967, a large amount of fission product gammaray data has been collected from fuel rods and intact fuel assemblies irradiated during the Batch Core Experiment. Fuel rods, sleeves and inserts comprising the FERTF fuel assemblies, $(4,9)$ irradiated assemblies and rods from the basic core, and rods from special irradiation tests in the periphery of the core have been gamma scanned. In general, measurements were made to determine the relative power generation profiles of fuel rods along their lengths or at a point as inferred from the measured gamma-ray activity levels. For several fuel rods, including those listed in Table II, activity profiles were then used to identify positions at which gamma-ray spectra were obtained to investigate fission product migration and to identify the location of fuel samples to be cut for radiometallurgical and radiochemical analyses.

A summary of the gamma-ray measurements accomplished is listed in Table XI. Many of the entries of Table XI pertain to the data collected from fuel rods, sleeves and inserts which were components of FERTF fuel assemblies. These tests and their purposes have been discussed in several reports. $(16-19,21)$ Summaries of the gamma scanning results have also been presented. However, the details of the measurements and how the results were arrived at have not been presented. These details for test 35 are included in this report in Appendix B and the details illustrate the type of measurements done and results obtained for FERTF fuel rods. 
TABLE XI

SUMMARY OF PRTR FUELS AND HARDWARE ANALYZED BY GAMMA SCANNING SINCE START OF BATCH CORE EXPERIMENT

\begin{tabular}{|c|c|c|c|c|}
\hline \multirow{2}{*}{ DATE } & \multirow{2}{*}{$\begin{array}{l}\text { MATERIAL } \\
\text { IDENTIFICATION }\end{array}$} & \multirow{2}{*}{$\begin{array}{l}\text { PURPOSE OF MEASUREMENTS AND REFERENCE } \\
\text { TO REPORTS PRESENTING RESULTS OF } \\
\text { MEASUREMENTS }\end{array}$} & \multicolumn{2}{|c|}{ DETECTOR USED } \\
\hline & & & 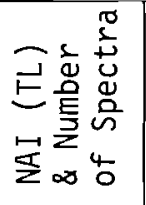 & 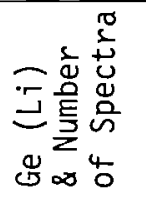 \\
\hline Oct 17, 1968 & PRTR Rod FS12 & $\begin{array}{l}\text { Data taken to indicate relative burnup distribution } \\
\text { in first rod removed from Batch Core to obtain burnup } \\
\text { data. }\end{array}$ & $x$ & \\
\hline Feb $21-23,1968$ & $\begin{array}{l}\text { Assemblies } 6047 \\
6019,6031,6035 \\
6067,6051,6055 \\
\& 6066\end{array}$ & $\begin{array}{l}\text { Data taken to indicate relative power profile of assem- } \\
\text { blies (15) in the Batch Core at its midplane during the } \\
\text { 2nd interim critical tests (Reactor shutdown, Jan 28,1968) }\end{array}$ & 23 & \\
\hline Mar 4,5,1968 & $\begin{array}{l}\text { FERTF Rods FR90 } \\
\text { FR93, FR91, FR94 } \\
\text { \& FR95 }\end{array}$ & $\begin{array}{l}\text { Data taken to indicate symmetry of power distribution } \\
\text { around the FERTF fuel assembly--FERTF test \#28 }(16,17)\end{array}$ & & \\
\hline $\operatorname{Mar} 8,1968$ & Rods 101 and B15 & $\begin{array}{l}\text { Data taken to determine whether either rod had a greater } \\
\text { burnup than the other. (18) }\end{array}$ & & \\
\hline Apri1 11, 1968 & $\begin{array}{l}\text { FERTF Rods F } 330 \\
\text { FR77, FR99, FR59 } \\
\text { FR75, FR28, FR98 } \\
\text { \& FS69 }\end{array}$ & $\begin{array}{l}\text { Data taken to indicate symmetry of power distribution } \\
\text { around the FERTF fuel assembly--FERTF test } \# 29 .(16,17)\end{array}$ & & \\
\hline
\end{tabular}


TABLE XI (CONTINUED)

SUMMARY OF PRTR FUELS AND HARDWARE ANALYZED BY GAMMA SCANNING SINCE START OF BATCH CORE EXPERIMENT IN APRIL, 1967

\begin{tabular}{|c|c|c|c|c|}
\hline \multirow[t]{2}{*}{ DATE } & \multirow{2}{*}{$\begin{array}{l}\text { MATERIAL } \\
\text { IDENTIFICATION }\end{array}$} & \multirow{2}{*}{$\begin{array}{l}\text { PURPOSE OF MEASUREMENTS AND REFERENCE } \\
\text { TO REPORTS PRESENTING RESULTS OF } \\
\text { MEASUREMENTS }\end{array}$} & \multicolumn{2}{|c|}{ DETECTOR USED } \\
\hline & & & 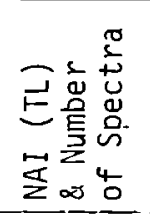 & 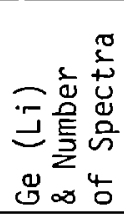 \\
\hline Apri1 20-22,1968 & $\begin{array}{l}\text { FERTF Rods FS31, } \\
\text { FS32, FS33, FS34, } \\
\text { FS35, FS36, FS37 } \\
\& \text { FS38 }\end{array}$ & $\begin{array}{l}\text { Data taken to indicate symmetry of power distribution } \\
\text { around the FERTF fuel assembly--FERTF test } \# 30(16,17)\end{array}$ & $x 2$ & \\
\hline Apri1 23, 1968 & $\begin{array}{l}\text { FERTF Rods FS30 } \\
\text { FR75, FR28, FS31 } \\
\text { FS33 \& FS34 }\end{array}$ & Data repeated from FERTF tests \#29 \& \#30. $(16,17)$ & $x$ & \\
\hline $\begin{array}{l}\text { Apri } 130- \\
\text { May } 1,1968\end{array}$ & FERTF Sleeves & $\begin{array}{l}\text { Data taken to indicate symmetry of stainless steel \& } \\
\text { Zircaloy gamma-ray activity around the stainless steel } \\
\text { and Zircaloy sleeves. }(16,17)\end{array}$ & $x \quad 2$ & \\
\hline May $17-21,1968$ & FERTF Rod FS34 & $\begin{array}{l}\text { Data taken to indicate distribution of individual fission } \\
\text { products. }(16,17)\end{array}$ & $x$ & 9 \\
\hline May $23-27,1968$ & $\begin{array}{l}\text { FERTF Rods FS34 } \\
\text { \& FR28 }\end{array}$ & Rods re-scanned to verify earlier data. $(16,17)$ & $x$ & \\
\hline $\begin{array}{l}\text { June } 10,1968 \\
\text { June } 11,1968\end{array}$ & $\begin{array}{l}\text { FERTF Sleeve } \\
\text { FERTF Insert }\end{array}$ & $\begin{array}{l}\text { The stainless steel sleeve \& insert were scanned } \\
\text { axially \& around their circumference to determine } \\
\text { gamma activity distributions in greater detail. }(16,17)\end{array}$ & $x$ & \\
\hline
\end{tabular}


TABLE XI (CONTINUED)

SUMMARY OF PRTR FUELS AND HARDWARE ANALYZED BY GAMMA SCANNING SINCE START OF BATCH CORE EXPERIMENT IN APRIL, 1967

\begin{tabular}{|c|c|c|c|c|}
\hline \multirow[t]{2}{*}{ DATE } & \multirow{2}{*}{$\begin{array}{l}\text { MATERIAL } \\
\text { IDENTIFICATION }\end{array}$} & \multirow{2}{*}{$\begin{array}{l}\text { PURPOSE OF MEASUREMENTS AND REFERENCE } \\
\text { TO REPORTS PRESENTING RESULTS OF } \\
\text { MEASUREMENTS }\end{array}$} & \multicolumn{2}{|c|}{ DETECTOR USED } \\
\hline & & & 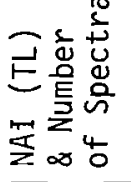 & 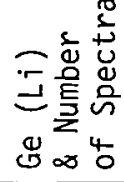 \\
\hline June 12, 1968 & $\begin{array}{l}\text { FERTF Rods } \text { FR91 } \\
\text { FR90, FR59, FR69 } \\
\text { FR92, FR77, FR98 } \\
\text { \& FR99 }\end{array}$ & $\begin{array}{l}\text { Data taken to indicate symmetry of power distribution } \\
\text { around the FERTF fuel assembly--FERTF test } \# 31(16,17)\end{array}$ & $x$ & \\
\hline June 13, 1968 & $\begin{array}{l}\text { FERTF Rods FS31 } \\
\text { FS32, FS33, FS34 } \\
\text { FS35, FS36, FS37 } \\
\text { FS38 }\end{array}$ & Re-scanned FERTF Test \#30 $(16,17)$ & $x$ & \\
\hline June 20, 1968 & PRTR Rod FR62 & Data taken to indicate relative burnup distribution $\delta 2^{20}$ rod. & $x$ & \\
\hline June 24, 1968 & FERTF Rod FR59 & Rod re-scanned to obtain data with Ge $(L i)$ detector. $(16,17)$ & $x$ & \\
\hline July $18,19,1968$ & $\begin{array}{l}\text { FERTF Rods FR91, } \\
\text { FR59, FR69, FR92 } \\
\text { FR90, FR77, FR98 } \\
\text { \& FR99 }\end{array}$ & Re-scanned FERTF Test \#31. $(16,17,19)$ & $x$ & \\
\hline July $22,23,1968$ & $\begin{array}{l}\text { FERTF Rods FS31, } \\
\text { FS32, FS33, FS34, } \\
\text { FS35, FS36, FS37 } \\
\& \text { FS38 }\end{array}$ & $\begin{array}{l}\text { Data taken to indicate symmetry of power distribution around } \\
\text { the FERTF fuel assembly--FERTF test } \# 33 \text {. }(17,19)\end{array}$ & $x$ & \\
\hline
\end{tabular}


TABLE XI (CONTINUED)

SUMMARY OF PRTR FUELS AND HARDWARE ANALYZED BY GAMMA SCANNING SINCE START OF BATCH CORE EXPERIMENT IN APRIL, 1967

\begin{tabular}{|c|c|c|c|c|c|}
\hline \multirow[b]{2}{*}{ DATE } & \multirow[b]{2}{*}{$\begin{array}{l}\text { MATERIAL } \\
\text { IDENTIFICATION }\end{array}$} & \multirow[b]{2}{*}{$\begin{array}{l}\text { PURPOSE OF MEASUREMENTS AND REFERENCE } \\
\text { TO REPORTS PRESENTING RESULTS OF } \\
\text { MEASUREMENTS }\end{array}$} & \multicolumn{3}{|c|}{ DETECTOR USED } \\
\hline & & & 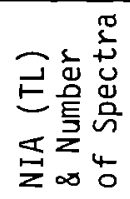 & & $\begin{array}{l}0 \\
0 \\
\vdots \\
0 \\
0 \\
0 \\
0 \\
4 \\
4 \\
0\end{array}$ \\
\hline Alxg 12, 1968 & $\begin{array}{l}\text { FERTF Rods FS41, } \\
\text { FS42, FS43, FS44, } \\
\text { FS45, FS46, FS47 } \\
\& \text { FS48 }\end{array}$ & $\begin{array}{l}\text { Data taken to indicate symmetry of power distribution around } \\
\text { the FERTF fuel assembly--FERTF test } \# 35 \text {. Continuous scans } \\
\text { were done over a } 6 \text { inch portion of each rod. }(17,19,21)\end{array}$ & $x$ & & \\
\hline Jan $14-24,1969$ & $\begin{array}{l}\text { Assemblies 6055, } \\
6051,6066,6067 \\
6047,6035,6031 \\
\& 6019\end{array}$ & $\begin{array}{l}\text { Data taken to indicate relative burnup profile of Batch } \\
\text { Core assemblies at the core midplane after the final } \\
\text { burnup step. (Core shutdown July 14, 1968) }\end{array}$ & & $x$ & \\
\hline Mar3, 1969 & $\begin{array}{l}\text { FERTF Rods FS41, } \\
\text { FS42, FS43, FS45, } \\
\text { FS46 \& FS48 }\end{array}$ & $\begin{array}{l}\text { Repeat of FERTF test } \# 35 \text { using } 6 \text { of the } 8 \text { rods and scanning } \\
\text { entire rod lengths. }(17,19,21)\end{array}$ & & $x$ & \\
\hline Mar 11, 1969 & $\begin{array}{l}\text { FERTF Rods FS41, } \\
\text { FS42, FS43, FS45, } \\
\text { FS46, \& FS48 }\end{array}$ & $\begin{array}{l}\text { Repeat of FERTF test \#35 using } 6 \text { of the } 8 \text { rods \& scanning } \\
\text { over the identical } 6 \text { inch portion of each rod as was done } \\
\text { on Aug } 12,1968 .(17,19,21)\end{array}$ & & $x$ & \\
\hline
\end{tabular}


TABLE XI (CONTINUEDD)

SUMMARY OF PRTR FUELS AND HARDWARE ANALYZED BY GAMMA SCANNING SINCE START OF BATCH CORE EXPERIMENT IN APRIL, 1967

\begin{tabular}{|c|c|c|c|c|}
\hline \multirow[t]{2}{*}{ DATE } & \multirow[t]{2}{*}{$\begin{array}{l}\text { MATERIAL } \\
\text { IDENT IFICATION }\end{array}$} & \multirow{2}{*}{$\begin{array}{l}\text { PURPOSE OF MEASUREMENTS AND REFERENCE } \\
\text { TO REPORTS PRESENTING RESULTS OF } \\
\text { MEASUREMENTS }\end{array}$} & \multicolumn{2}{|c|}{ DETECTOR USED } \\
\hline & & & 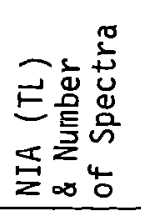 & 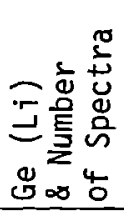 \\
\hline Sept 2-18, 1969 & $\begin{array}{l}\text { PRTR Rods FS07, } \\
\text { FE74, FE69, FR78, } \\
\text { FN86, \& F017 }\end{array}$ & $\begin{array}{l}\text { Data taken to indicate burnup profiles of Batch Core rods } \\
\text { removed from core after final burnup step. Reactor shut- } \\
\text { down July 14, 1968. (This report) }\end{array}$ & $\times 1$ & 13 \\
\hline $\begin{array}{l}\text { Aug 27- } \\
\text { Nov 13, } 1969\end{array}$ & $\begin{array}{l}\text { PRTR Rods A102, \#6, } \\
\text { A23, FR72, 5C27, } \\
\text { 5F36, DF80, ZZ7, } \\
\text { ZZ12, ZZ17, DB63 } \\
\text { CN5, CS27, } 1330 \\
1377 \& 1398 .\end{array}$ & $\begin{array}{l}\text { Data taken to indicate burnup profiles of Batch Core rods } \\
\text { \& PRTR peripheral rods removed from the core after final } \\
\text { burnup step. Reactor shutdown July 14, 1968. (21) }\end{array}$ & & 43 \\
\hline Jan $12,13,1970$ & $\begin{array}{l}\text { PRTR Rods } 1325 \& \\
1807\end{array}$ & $\begin{array}{l}\text { Data taken to determine which rod had the highest gamma ray } \\
\text { activity at the center of the fuel and therefore was an } \\
\text { outer ring rod of PRTR assembly } 1037 \text {. (Rod } 1325 \text { was the } \\
\text { outer ring rod.) (21) }\end{array}$ & & $X$ \\
\hline Sept $17,18,1970$ & $\begin{array}{l}\text { ETR Rods F10, } \\
\text { F11, \& F12 }\end{array}$ & Data taken to determine gamma activity profiles. (22) & & $x$ \\
\hline
\end{tabular}


Two entries of Table XI pertain to the scanning of intact 19-rod fuel assemblies to determine the midplane radial power distributions of the Batch Core at two different exposure levels. The first measurements were done when the assemblies had reached an average exposure of 4,000 MWD/MTM. Preliminary results from these measurements were summarized in Reference 15 and the detailed measurement procedures plus final results are given in Appendix $C$. The second measurements were taken after the termination of the Batch Core. These data have not been analyzed. The remaining entries of Table $X I$ pertain to the scanning of special irradiation test rods and Batch Core rods. Rods 101, B15, FR62, A102, A1330, A1377, A1398, A1325, DB63, ZZ17, ZZ7, ZZ12, CN5, CS27, DF80, 5C27, 5F36 and \#6 listed in Table XI were gamma scanned in support of either post irradiation measurements by the Fuels Research Section of the Materials Department or other burnup experiments. Summaries of the evaluations done by Fuels Research are given in References 18, 20, 21, 23, 24, and 25. Three of the rods, A1330, A1377, and Al398 were part of another burnup experiment and the gamma ray measurements made for these rods were reported in Reference 26 .

From the basic core, fuel rod FS12, (average assembly exposure at 2,000 MWD/MTM), rods FS16, F079, FE77 (average assembly exposure at 4,000 MWD/MTM), and rods FR78, FS07, F017, FE74, FN86, and FE69 (Batch Core termination) were gamma scanned to determine the gammaray activity profiles of each rod and to collect gamma-ray spectra at specified rod positions. These measurement procedures and results are discussed in the remaining subsections of this report. 


\section{A. Procedures}

A11 the gamma-ray measurements indicated in Table XI were done in the PNL Underwater Gamma Scan Facility. In the case of the Batch Core fuel rods (FS12 through FE69 above), the details of the facility have been reported previous $1 y^{(8,26)}$ and scanning procedures for the measurements discussed here were essentially the same as has been described. (8) An exception was the procedure used to scan rod FS12 which was done before a $G E(L i)$ detector was available to the facility and before the $x-y$ movement of the facility was automated. In the case of rod FS12, the scanning along the length of the rod to determine the relative activity profile was done by moving the detector housing and collimator at inch (or less) increments instead of the automated continuous movement of the detector housing and collimator as was the procedure for the rest of the rods scanned.

A11 rods were rotated before being scanned to determine the rod orientation of maximum activity since the rods did not rotate during measurement. Automated continuous scans to determine the relative activity profiles of each rod were made over the active fuel length using a $\mathrm{Ge}(\mathrm{Li})$ detector in conjunction with a single channel analyzer (SCA), a linear rate meter and a strip-chart recorder. Due to the long cooling times of the fuel and the low efficiency of a $G e(L i)$ detector, the SCA was set to accept all gamma-ray contributions with energies greater than $30 \mathrm{KeV}$ to drive the ratemeter in a range that would give statistical significance to any structure in the activity profiles. A rate of travel of approximately one inch per two minutes was used. 
As indicated by the last columns of Table XI, gamma-ray spectra were also collected using either a $\mathrm{NaI}(\mathrm{Tl})$ or a $\mathrm{Ge}(\mathrm{Li})$ detector and the number of spectra collected is listed in Table XI. When the activity profiles had been determined, they were used to identify rod positions at which the gamma-ray spectra were obtained. Also the activity profiles served to identify the rod locations of fuel samples to be cut for burnup and radio metallurgical analysis. The results from the burnup analyses were discussed earlier in this report and the final radiometallurgy results are presented in References 24 and 25. The gamma-ray data taken to indicate the relative activity profiles of the Batch Core rods are presented in the next section after which some of the results determined from the spectra are discussed. The $\mathrm{Ge}\left(L_{i}\right)$ spectra collected from the Batch Core fuel rods are tabulated in Appendix D.

\section{B. Relative Measurement Results}

The relative axial gamma-ray activity profiles obtained by automated continuous scans of fuel rods indicated several features of interest. In general, the profiles of all rods showed a continuous distribution of fission product activity peaked at the center and ends of the $\mathrm{UO}_{2}-\mathrm{PuO}_{2}$ fuel which was directly related to the axial power profile. The activity peaking at the ends of the $\mathrm{UO}_{2}-\mathrm{PuO}_{2}$ fuel was always more pronounced for fuel rods which were from the center or 6-rod ring positions of the 19-rod assembly. Also, activity peaking at the ends of the $\mathrm{UO}_{2}-\mathrm{PuO}_{2}$ fuel was more evident for fuel rods from the 12-rod ring of an assembly when the assembly had been 
positioned in the fifth and seventh ring of fuel channels of the core. A common feature of all rods was that the activity of the depleted $\mathrm{UO}_{2}$ pellet at each end of the $\mathrm{UO}_{2}-\mathrm{PuO}_{2}$ fuel evidenced much lower activity as well as evidencing some activity peaking at the pellet edge opposite to the pellet and $\mathrm{UO}_{2}-\mathrm{PuO}_{2}$ : fuel interface.

One feature noted from several of the gamma-ray activity profiles involved only those rods that were from the 12-rod ring of an assembly which had been positioned in the first and third ring of fuel channels of the core. These rods experienced higher power generation rates and as a result substantial localized concentrations of various fission products were formed. When the continuous scans were made, these localized high activity regions appeared as peaks superimposed upon the relative gamma-ray activity profiles. The width of these activity peaks indicated that the concentrations were generally less than $1 / 8$ of an inch long and probably due to fission product migration.

To illustrate these features, the activity profiles determined for rods FS16, F079 and FE77 are shown in Figure 24. The dimensions of each rod are given in the figure and the positions of the two $\mathrm{UO}_{2}$ pellets are also indicated. The zero on the $x$-scale is with reference to the beginning of the $\mathrm{UO}_{2}-\mathrm{PuO}_{2}$ fuel at the top of the rod. The three activity profiles shown are of rods which were from assembly 6066. Assembly 6066 was irradiated in the third ring of fuel channels in the core. High activity regions appeared superimposed on the relative gamma-ray activity profile in the case of rod FS16 which was from 

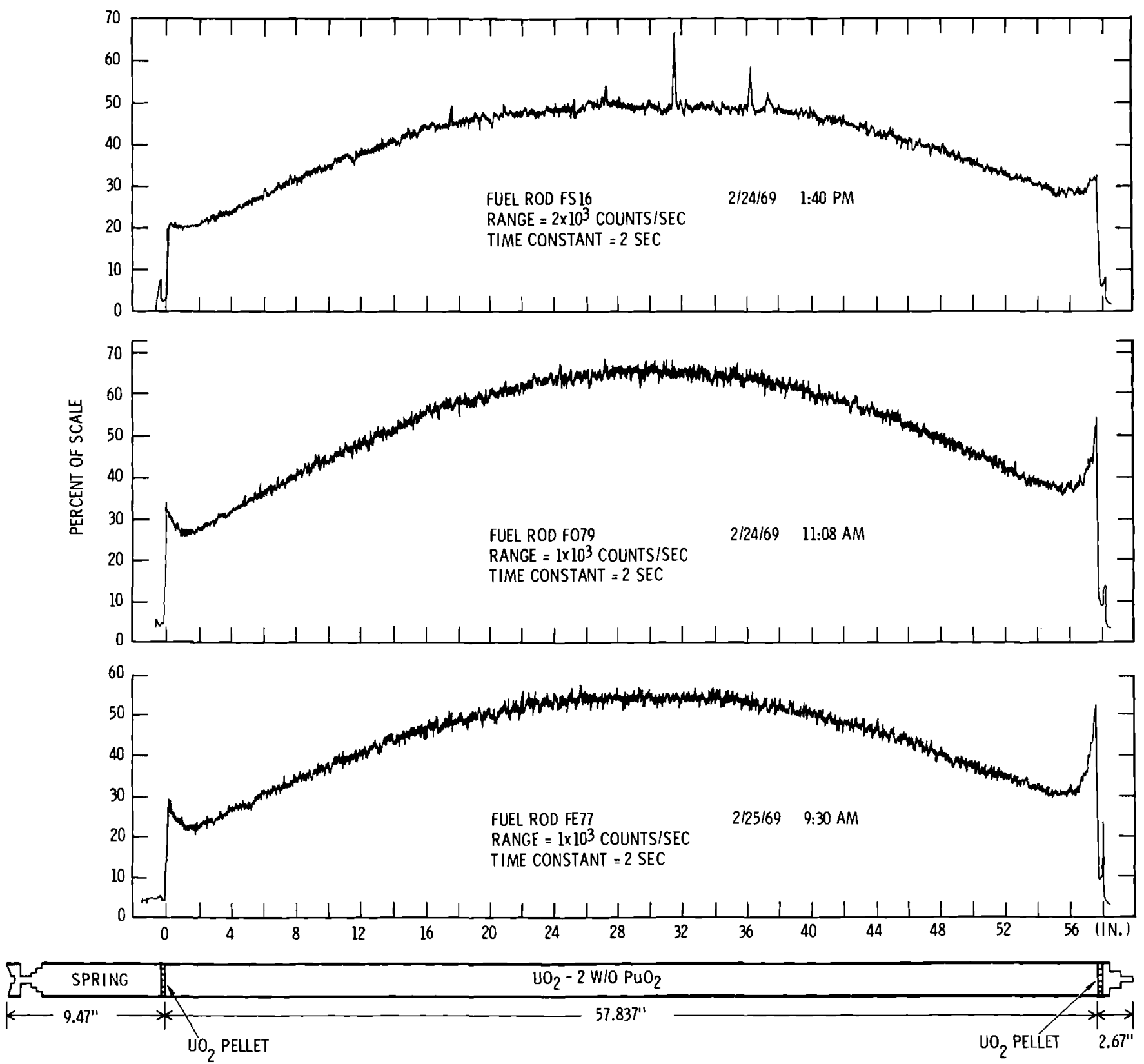

FIGURE 24. GAMMA-RAY ACTIVITY PROFILES OF ROOS FROM POSITION r, FS16, POSITION C, FO79 AND POSITION a, FE77, OF ASSEMBLY 6066 WHICH WAS IRRADIATED IN CORE RING POSITION 3. 
the 12-rod ring of assembly 6066. Rod F079 was from the 6-rod ring and rod FE77 was the center rod of the assembly. The peaking at the ends of the $\mathrm{UO}_{2}-\mathrm{PuO}_{2}$ fuel of these latter two rods can be seen to be more pronounced than for rod FS16.

Gamma-ray activity profiles of fuel rods from the 12-rod ring of four assemblies are shown in Figure 25. Each of the assemblies had been irradiated in a different ring of fuel channels in the core. Assembly 6063 from which rod FSO1 was taken was irradiated in the first ring of fuel channels. It is seen that several regions of high activity were evident in the rod over an approximate 15 inch length of the fuel which had experienced maximum power generation for the rod. A repeat scan over the 15 inch length gave identical results. Rod FSO1 was then rotated $90^{\circ}$ and rescanned over the 15 inches. This procedure was repeated three more times and except for a variation in activity as a function of rod orientation, all the structure seen in Figure 25 , for rod FSOI, was reproduced for each scan.

Assembly 6067 from which rod FS23 was taken was irradiated in the third ring of fuel channels and high activity regions were also evident from the gamma-ray activity profile of rod FS23. Rods FS08 and FR64 were from assemblies 6058 and 6516 which were irradiated in the fifth and seventh ring of fuel channels, respectively. The profiles for these rods showed continuous distributions of fission product activity, except for one region of low activity noted to be present in rod FR64. As indicated earlier, the activity peaking at 

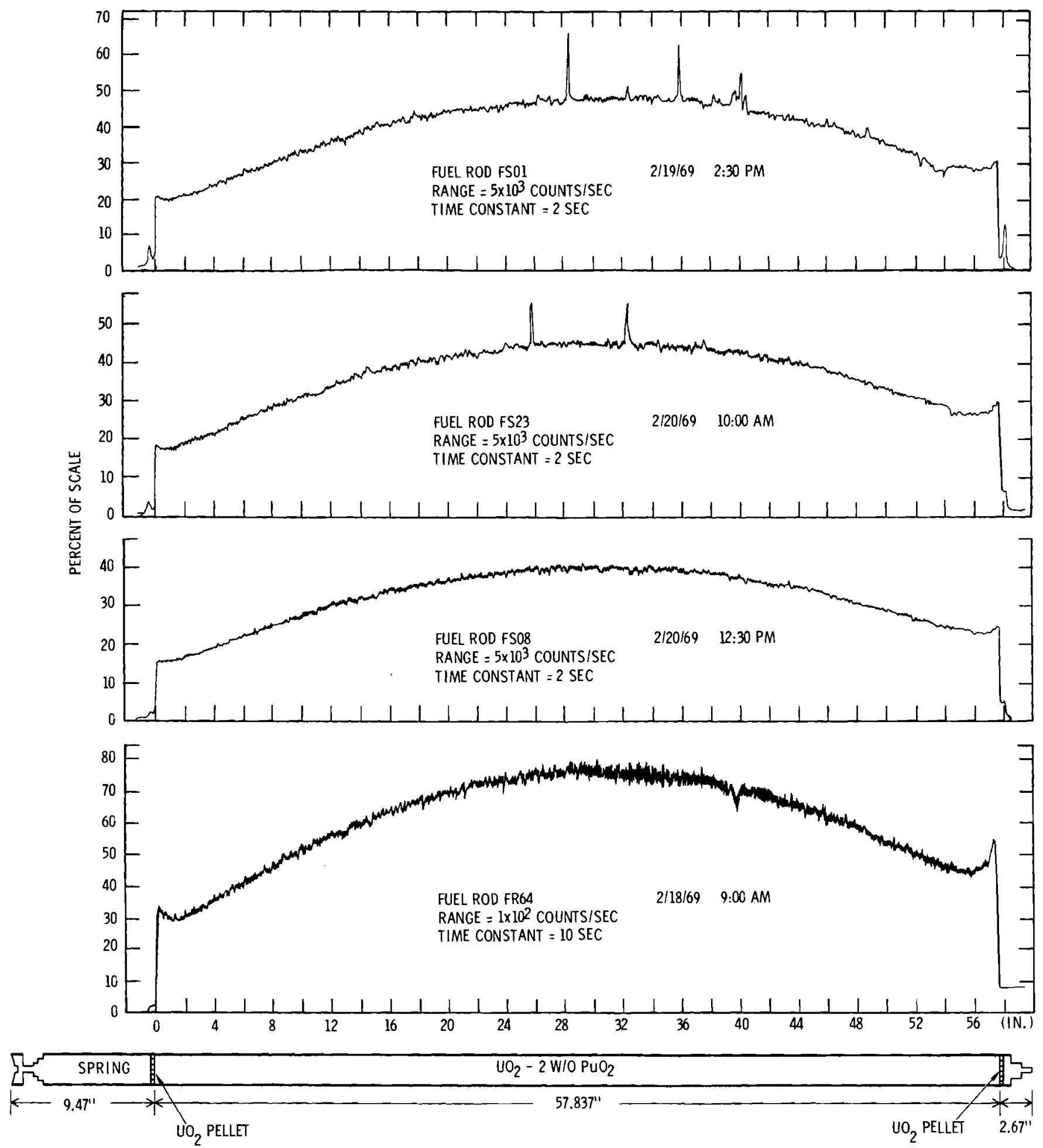

FIGURE 25. GAMMA-RAY ACTIVITY PROFILES OF FUEL RODS FS01, FS23, FS09 AND FR64 FROM THE 12-ROD RING POSITIONS OF ASSEMBLIES 6063, 6067, 6058 AND 6516 WHICH WERE IRRADIATED IN CORE RING POSITIONS 1, 3, 5 AND 7 RESPECTIVELY. 
the ends of the $\mathrm{UO}_{2}-\mathrm{PuO}_{2}$ fuel is seen to be more pronounced for fuel rods further removed from the center of the core as in the case of rod FR64.

The average assembly exposure was at 4,000 MWD/MTU in the case of the rods shown in Figure 24 and at 6,000 MWD/MTU for the rods shown in Figure 25. Gamma-ray activity profiles of three rods removed at the termination of the Batch Core are shown in Figure 26. Rod FSO7 from the 12-rod ring, rod F017 from the 6-rod ring and the center rod FE74 of assembly 6065 which was irradiated in the third ring of fuel channels of the core show the same features discussed above for rods from similar assembly and core positions.

The gamma-ray activity profile features of rod FN86 and FE69 (from the 6-rod ring and the center rod, respectively) of assembly 6519 were also consistent with the features described and are not presented. Assembly 6519 was removed from the seventh ring of fuel channels at the termination of the Batch Core. The profile for rod FR78 from the 12-rod ring of assembly 6520 has been presented in Reference 21, pp 3.6. Assembly 6520 was irradiated in the first ring of fuel channels of the core. All profile features of rod FR78 were consistent with those explained except that concentrations of migrating fission products were not substantial enough to result in high activity peaks superimposed upon the activity profile. Instead, the effect of migrating fission products can best be described as resulting in a nonuniform distribution over the length of the rod that experienced maximum power generation. 

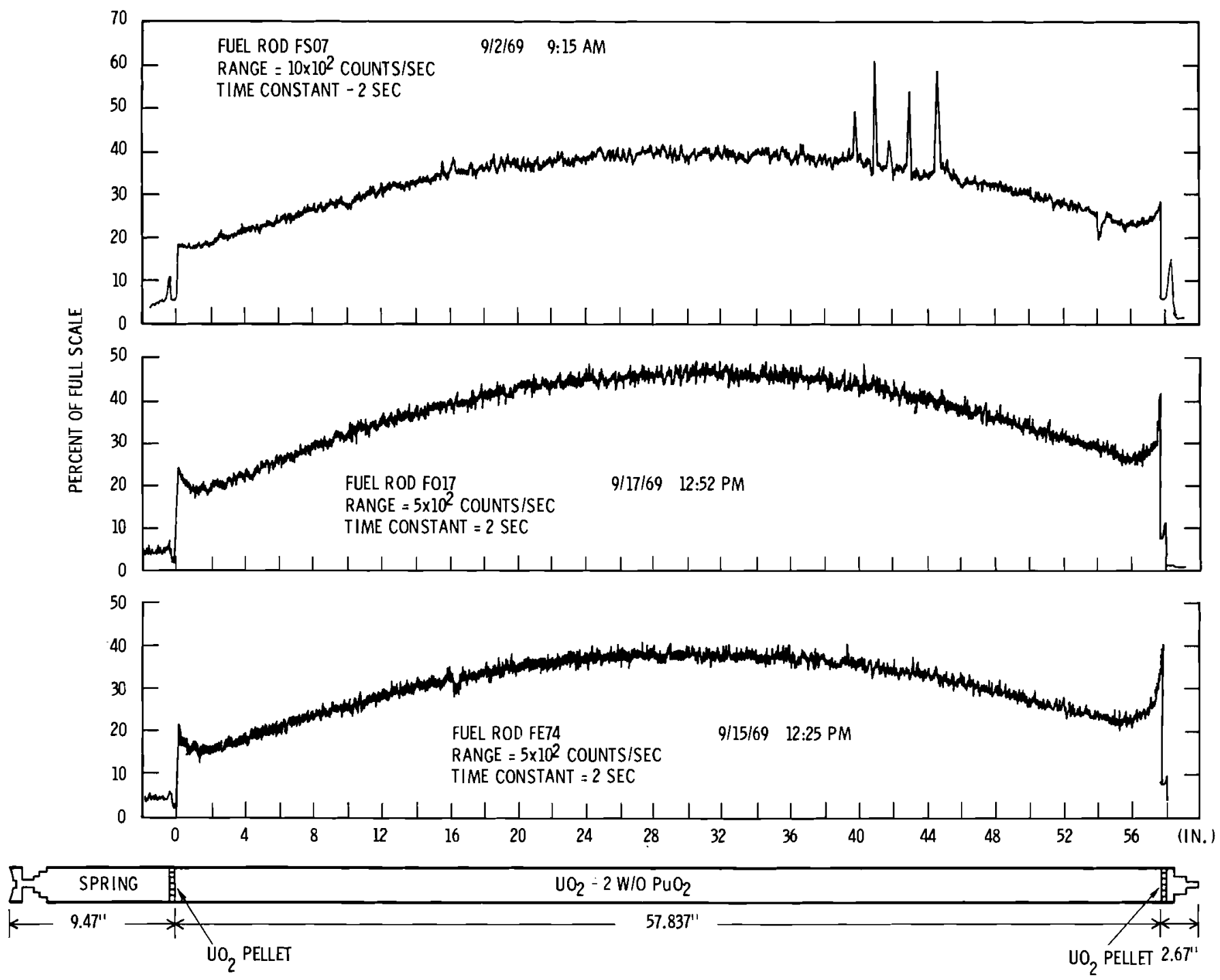

FIGURE 26. GAMMA-RAY ACTIVITY PROFILES OF RODS FROM THE 12-ROD RING, FS07, THE 6-ROD RING, F017 AND THE CENTER ROD, FE74, OF ASSEMBLY 6065 WHICH WAS IRRADIATED IN THE THIRD RING OF FUEL CHANNELS 


\section{Gamma-Ray Spectra Results}

High resolution gamma-ray spectra were collected from several of the fuel rods discussed above and these spectra are listed in Table XII. A total of $33 \mathrm{Ge}(\mathrm{Li})$ spectra were collected and the position of 18 of these coincided with the positions of burnup samples which were later cut from the rods. The exposure of these 18 samples are also listed in Table XII and were obtained from the data tabulated in Appendix $A$. The remaining gamma-ray spectra were obtained from positions selected either because burnup samples were cut from the rods (but were not analyzed) or to investigate the fission product composition of high (or low) activity points as noted from the gamma-ray activity profiles determined for each rod.

It was determined from the spectra collected that fission product isotopes ${ }^{95} \mathrm{Zr}-{ }^{95} \mathrm{Nb},{ }^{106} \mathrm{Ru}-{ }^{106} \mathrm{Rh},{ }^{133} \mathrm{Cs}$ (by neutron capture to $\left.{ }^{134} \mathrm{Cs}\right),{ }^{137} \mathrm{Cs}$ and ${ }^{144} \mathrm{Ce}-{ }^{144} \mathrm{Pr}$ contributed gamma rays of sufficient intensity to be readily observed. Due to the longer cooling times of the fuels involved, the short half-lived fission products had decayed away and low intensity long lived isotopes such as ${ }^{154}$ Eu were beginning to become discernable (only for those rods which had cooled 430 days).

of the above isotopes it was determined that ${ }^{106} \mathrm{Ru}-{ }^{106} \mathrm{Rh},{ }^{134} \mathrm{Cs}$ and ${ }^{137}$ Cs contributed to the high activity points which were observed from the activity profiles. Not all three isotopes were detected concentrated at every high activity point, however, as ${ }^{106} \mathrm{Ru}-{ }^{106} \mathrm{Rh}$ was generally concentrated at higher activity points by itself while 
TABLE XII

SUMMARY OF HIGH RESOLUTION GAMMA-RAY SPECTRA COLLECTED FROM BATCH CORE FUEL RODS AND PHOTOPEAK AREAS DETERMINED FROM THE SPECTRA

\begin{tabular}{|c|c|c|c|c|c|c|c|c|c|c|c|c|c|c|}
\hline $\begin{array}{c}\text { Fuel } \\
\text { Assembly } \\
\text { No. }\end{array}$ & $\begin{array}{l}\text { Ring } \\
\text { Position } \\
\text { in Reactor } \\
\text { (Channel No.) }\end{array}$ & $\begin{array}{l}\text { Fuel } \\
\text { Rod No. }\end{array}$ & $\begin{array}{l}\text { Position } \\
\text { in } \\
\text { Assembly }\end{array}$ & $\begin{array}{l}\text { Spectrum } \\
\text { No. }\end{array}$ & $\begin{array}{l}\text { Position } \\
\text { in Rod } \\
\text { (inch) }\end{array}$ & $\begin{array}{l}\text { Date } \\
\text { Taken }\end{array}$ & $\begin{array}{l}\text { Live Time } \\
\text { (hours) }\end{array}$ & $\begin{array}{l}\text { Cooling } \\
\text { Time } \\
\text { (Days) }\end{array}$ & $\begin{array}{l}\text { Exposure } \\
\text { (MWD/MTM) }\end{array}$ & $\begin{array}{c}106 \mathrm{Rh} \\
0512 \\
\mathrm{KeV}\end{array}$ & $\begin{array}{c}{ }^{137} \mathrm{Cs} \\
0662 \\
\mathrm{KeV}\end{array}$ & $\begin{array}{c}{ }^{95} \mathrm{Zr} \\
0 \mathrm{HeV} \\
\mathrm{KeV}\end{array}$ & $\begin{array}{c}{ }^{134} \mathrm{Cs} \\
\text { e } 796 \\
\mathrm{KeV}\end{array}$ & $\begin{array}{l}144 \mathrm{Pr} \\
\text { C } 2186 \\
\mathrm{KeV}\end{array}$ \\
\hline 6066 & $\begin{array}{c}3 \\
(1352)\end{array}$ & $\begin{array}{l}\text { FS16 } \\
\\
\\
\\
\text { F079 } \\
\text { FE77 }\end{array}$ & $\begin{array}{l}c \\
a\end{array}$ & $\begin{array}{r}14 \\
15 \\
13 \\
9 \\
10 \\
11 \\
12 \\
8 \\
7\end{array}$ & $\begin{array}{c}\left\{\begin{array}{c}\text { Top } \mathrm{UO}_{2} \\
\text { PeIl et }\end{array}\right. \\
1.40\end{array}$ & $\begin{array}{l}3-2-69 \\
3-2-69 \\
3-1-69 \\
2-27-69 \\
2-28-69 \\
2-28-69 \\
3-1-69 \\
2-27-69 \\
2-27-69\end{array}$ & $\begin{array}{r}12.217 \\
10.000 \\
11.667 \\
15.000 \\
5.500 \\
14.500 \\
10.833 \\
5.167 \\
16.000\end{array}$ & $\begin{array}{l}398 \\
398 \\
397 \\
395 \\
396 \\
396 \\
397 \\
395 \\
395\end{array}$ & $\begin{array}{l}3319 \\
5878 \\
4587 \\
\\
3800 \\
3238\end{array}$ & $\begin{array}{r}898 \\
2137 \\
32195 \\
43559 \\
75444 \\
59541 \\
43066 \\
47628 \\
38623\end{array}$ & $\begin{array}{r}709 \\
1960 \\
17899 \\
20999 \\
31551 \\
28059 \\
20487 \\
24666 \\
19905\end{array}$ & $\begin{array}{r}155 \\
730 \\
5516 \\
7681 \\
13140 \\
10340 \\
7596 \\
8741 \\
7299\end{array}$ & $\begin{array}{r}18274 \\
293 \\
1152 \\
2239 \\
5321 \\
3901 \\
1874 \\
3403 \\
2605\end{array}$ & $\begin{array}{r}-7 \\
42 \\
309 \\
429 \\
739 \\
565 \\
431 \\
483 \\
418\end{array}$ \\
\hline 6063 & $\begin{array}{c}1 \\
(1451)\end{array}$ & FS01 & $n$ & $\begin{array}{l}25 \\
26 \\
23 \\
24 \\
28 \\
27\end{array}$ & $\begin{array}{r}26.56 \\
26.75 \\
27.31 \\
29.40 \\
\left\{\begin{array}{l}\text { Bottom } \\
\text { U02 } \\
\text { Pe1let }\end{array}\right.\end{array}$ & $\begin{array}{l}3-7-69 \\
3-9-69 \\
3-7-69 \\
3-7-69 \\
3-9-69 \\
3-9-69\end{array}$ & $\begin{array}{r}18.783 \\
19.867 \\
3.500 \\
5.833 \\
1.500 \\
1.000\end{array}$ & $\begin{array}{l}279 \\
281 \\
279 \\
279 \\
281 \\
281\end{array}$ & 8789 & $\begin{array}{r}121257 \\
106984 \\
370534 \\
121828 \\
11043 \\
50090\end{array}$ & $\begin{array}{r}52786 \\
40263 \\
36932 \\
36042 \\
7637 \\
22355\end{array}$ & $\begin{array}{r}45282 \\
39210 \\
46279 \\
45809 \\
6247 \\
19252\end{array}$ & $\begin{array}{r}13460 \\
9262 \\
9026 \\
8917 \\
1843 \\
4880\end{array}$ & $\begin{array}{l}1181 \\
1119 \\
1206 \\
1254 \\
173 \\
504\end{array}$ \\
\hline 6067 & $\begin{array}{c}3 \\
(1348)\end{array}$ & FS23 & $t$ & $\begin{array}{l}22 \\
21 \\
18 \\
19 \\
20\end{array}$ & $\begin{array}{r}61.00 \\
9.20 \\
28.60 \\
43.00 \\
48.00\end{array}$ & $\begin{array}{l}3-6-69 \\
3-6-69 \\
3-5-69 \\
3-5-69 \\
3-5-69\end{array}$ & $\begin{array}{r}14.500 \\
5.500 \\
5.000 \\
5.167 \\
8.833\end{array}$ & $\begin{array}{l}278 \\
278 \\
277 \\
277 \\
277\end{array}$ & $\begin{array}{l}4461 \\
5268 \\
7932 \\
7148 \\
6496\end{array}$ & $\begin{array}{r}66142 \\
79571 \\
115414 \\
107040 \\
90227\end{array}$ & $\begin{array}{l}28261 \\
32316 \\
38882 \\
35321 \\
35238\end{array}$ & $\begin{array}{l}26342 \\
30900 \\
45740 \\
41193 \\
35468\end{array}$ & $\begin{array}{l}4154 \\
5555 \\
9700 \\
7966 \\
6752\end{array}$ & $\begin{array}{r}664 \\
826 \\
1138 \\
1077 \\
921\end{array}$ \\
\hline 6058 & $\begin{array}{c}5 \\
(1253)\end{array}$ & FS08 & $\mathrm{j}$ & 16 & 29.40 & $3-2-69$ & 4.000 & 274 & 7032 & 104035 & 30167 & 40864 & 6591 & 1014 \\
\hline 6520 & $\begin{array}{c}1 \\
(1548)\end{array}$ & FR78 & $n$ & 15 & 30.00 & $9-17-69$ & 3.000 & 430 & 9485 & 108285 & 44428 & 9453 & 10332 & 1008 \\
\hline 6065 & $\begin{array}{c}3 \\
(1554)\end{array}$ & $\begin{array}{l}\text { FS07 } \\
\\
\\
\text { F017 } \\
\text { FE74 }\end{array}$ & $\begin{array}{l}c \\
a\end{array}$ & $\begin{array}{r}2 \\
7 \\
3 \\
4 \\
6 \\
8 \\
5 \\
17 \\
12\end{array}$ & $\begin{array}{l}30.00 \\
38.80 \\
39.40 \\
40.60 \\
44.20 \\
44.80 \\
53.60 \\
30.00 \\
30.00\end{array}$ & $\begin{array}{l}9-8-69 \\
9-10-69 \\
9-9-69 \\
9-9-69 \\
9-10-69 \\
9-10-69 \\
9-9-69 \\
9-18-69 \\
9-15-69\end{array}$ & $\begin{array}{r}14.333 \\
2.000 \\
3.000 \\
2.000 \\
1.500 \\
2.000 \\
15.250 \\
8.000 \\
14.000\end{array}$ & $\begin{array}{l}421 \\
423 \\
422 \\
422 \\
423 \\
423 \\
422 \\
431 \\
428\end{array}$ & $\begin{array}{l}5778 \\
4817\end{array}$ & $\begin{array}{r}101532 \\
96563 \\
176515 \\
409386 \\
229588 \\
82477 \\
42494 \\
62120 \\
50693\end{array}$ & $\begin{array}{l}45170 \\
49994 \\
51341 \\
48111 \\
55877 \\
46784 \\
31543 \\
34804 \\
29541\end{array}$ & $\begin{array}{l}9397 \\
9495 \\
9033 \\
8827 \\
8597 \\
7641 \\
4300 \\
5883 \\
4916\end{array}$ & $\begin{array}{l}10614 \\
11470 \\
11190 \\
10338 \\
11765 \\
10294 \\
5518 \\
6724 \\
5223\end{array}$ & $\begin{array}{l}947 \\
987 \\
923 \\
913 \\
894 \\
803 \\
387 \\
638 \\
531\end{array}$ \\
\hline 6519 & $\begin{array}{c}7 \\
(1245)\end{array}$ & $\begin{array}{l}\text { FN86 } \\
\text { FE69 }\end{array}$ & $\begin{array}{l}f \\
a\end{array}$ & $\begin{array}{l}16 \\
13\end{array}$ & $\begin{array}{l}30.00 \\
30.00\end{array}$ & $\begin{array}{l}9-17-69 \\
9-17-69\end{array}$ & $\begin{array}{l}14.000 \\
14.000\end{array}$ & $\begin{array}{l}430 \\
430\end{array}$ & $\begin{array}{l}3266 \\
2860\end{array}$ & $\begin{array}{l}35860 \\
32300\end{array}$ & $\begin{array}{l}21862 \\
19666\end{array}$ & $\begin{array}{l}2600 \\
2362\end{array}$ & $\begin{array}{l}2232 \\
1963\end{array}$ & $\begin{array}{l}341 \\
306\end{array}$ \\
\hline
\end{tabular}


${ }^{134}$ Cs and ${ }^{137}$ Cs were sometimes detected concentrated together. To illustrate the localized concentrations of the various isotopes, photopeak areas of one gamma ray emitted by each of the five radioactive isotopes are tabulated in Table XII. The areas are given in counts/hour and the area for ${ }^{134} \mathrm{Cs}$ represents the sum of the 796 and the $801 \mathrm{KeV}$ gamma rays. Of the spectra collected to investigate higher activity regions, spectra 14 and 15 were measured immediately above the top $\mathrm{UO}_{2}$ pellet (at the activity peak observed from the activity profile, Figure 24) and on the $\mathrm{UO}_{2}$ pellet, respectively, (at the valley between the activity peak and the beginning of the $\mathrm{UO}_{2}-\mathrm{PuO}_{2}$ fuel) of rod FS16. The activity peak was determined to consist principally of ${ }^{134} \mathrm{Cs}$ which was concentrated by a factor of 3.4 over the highest ${ }^{134} \mathrm{Cs}$ activity in the $\mathrm{UO}_{2}-\mathrm{PuO}_{2}$ fuel of the rod (spectrum 10). Also, ${ }^{60}$ Co activity from neutron activation of the spring in the plenum contributed to the peak. The $1333 \mathrm{KeV}$ gammaray activity had increased by a factor of 15 over the background rate of $229 \pm 12$ counts/hour. The background value represents the average of a $11{ }^{60}$ Co determination at $1333 \mathrm{KeV}$ made from all spectra and the one standard deviation of the average.

Spectra 28 and 27 were measured on the bottom $\mathrm{UO}_{2}$ pellet of rod FSOI and it was determined that the bottom activity peak, see Figure 25, was contributed to in this case by all five isotopes (spectrum 27). The isotopes ${ }^{106} \mathrm{Ru}-{ }^{106} \mathrm{Rh},{ }^{95} \mathrm{Zr}$ and ${ }^{144} \mathrm{Ce}-{ }^{144} \mathrm{Pr}$ were 2.4 to 2.5 times less concentrated compared to the activity at the 
center of the fuel, spectrum 24 , and ${ }^{137} \mathrm{Ce}$ and ${ }^{134} \mathrm{Cs}$ were 1.7 and 1.8 times less concentrated than at the center of the rod. The presence of all five isotopes may have indicated the movement of particles of $\mathrm{UO}_{2}-\mathrm{PuO}_{2}$ fuel to the point while the larger concentrations of ${ }^{137} \mathrm{Cs}$ and ${ }^{134} \mathrm{Cs}$ relative to ${ }^{106} \mathrm{Ru}-{ }^{106} \mathrm{Rh},{ }^{95} \mathrm{Zr}$ and ${ }^{144} \mathrm{Ce}-{ }^{144} \mathrm{Pr}$ also indicated fission product migration. The lower activity of all five isotopes as seen from spectrum 28 which was measured in the valley between the bottom activity peak and the bottom of the $\mathrm{UO}_{2}-\mathrm{PuO}_{2}$ fuel was indicative of the expected activity of the $\mathrm{UO}_{2}$ pellet.

Spectrum 23 of rod FSO1 was measured at the center of the most prominent high activity peak (27.3 inches from the top of the $\mathrm{UO}_{2}-\mathrm{PuO}_{2}$ fuel) as seen from the activity profile of rod FSO1 in Figure 25 . The photopeak areas determined from spectrum 23 and listed in Table XII indicated that ${ }^{106} \mathrm{Ru}-{ }^{106} \mathrm{Rh}$ had concentrated at the point by a factor of 3.0 over its concentration two inches further down the rod as represented by spectrum 24. It appeared also, from spectrum 26 , that there may have been a slight decrease in fuel concentration at 26.75 inches and the decrease in ${ }^{106} \mathrm{Ru}-{ }^{106} \mathrm{Rh},{ }^{95} \mathrm{Zr}$ and ${ }^{144} \mathrm{Ce}-{ }^{144} \mathrm{Pr}$ activity was compensated for by the migration of ${ }^{137} \mathrm{Cs}$ and ${ }^{134} \mathrm{Cs}$. At 3/16 of an inch away (26.56), the concentrations of ${ }^{137} \mathrm{Cs}$ and ${ }^{134} \mathrm{Cs}$ had increased even more contributing to a small activity peak while the concentrations of ${ }^{106} \mathrm{Ru}-{ }^{106} \mathrm{Rh},{ }^{95} \mathrm{Zr}$ and ${ }^{144} \mathrm{Ce}-{ }^{144} \mathrm{Pr}$ were similar to their concentrations at 29.40 inches, spectrum 24 .

The migration of ${ }^{106} \mathrm{Ru}-{ }^{106} \mathrm{Rh}$ was determined to be the principle cause of three high activity peaks observed from the activity profile 
of fuel rod FSO7 (see Figure 26). The photopeak areas 1isted in Table XII for spectra 3, 4, and 6 show an increase in ${ }^{106} \mathrm{Ru}-{ }^{106} \mathrm{Rh}$ activity of $1.7,4.0$, and 2.2, respectively, over the ${ }^{106} \mathrm{Ru}-{ }^{106} \mathrm{Rh}$ activity at the center of the fuel as represented by spectrum 2. The variation in activity of ${ }^{137}$ Cs also evidenced a nonuniform distribution due to migration. However, the variations were not large as in the case of ${ }^{106} \mathrm{Ru}-{ }^{106} \mathrm{Rh}$. Spectrum 5 was measured at the point of low activity (53.6 inches) as observed from the activity profile of rod FSO7. The lower activity of all isotopes as indicated by the photopeak areas suggested a possible lower concentration of $\mathrm{UO}_{2}-\mathrm{PuO}_{2}$ fuel at that point. The migration of ${ }^{106} \mathrm{Ru}-{ }^{106} \mathrm{Rh},{ }^{137} \mathrm{Cs}$ and ${ }^{134} \mathrm{Cs}$ were thus seen to be the causes of the high activity peaks superimposed on the activity profiles of the $\mathrm{UO}_{2}-\mathrm{PuO}_{2}$ fuel of rods FS16, FSO1, and FSO7. The migration of ${ }^{106} \mathrm{Ru}-{ }^{106} \mathrm{Rh}$ was somewhat independent of the migration of ${ }^{137} \mathrm{Cs}$ and ${ }^{134} \mathrm{Cs}$ although the three isotopes were determined to be concentrated together at certain points. Two isotopes, ${ }^{134} \mathrm{Cs}$ and ${ }^{137} \mathrm{Cs}$, tended to follow the same distributions except that ${ }^{134}$ Cs was found concentrated at the top of the $\mathrm{UO}_{2}$ pellet while ${ }^{137} \mathrm{Cs}$ was not.

D. Photopeak Areas Normalized to Burnup

The normalization of the gamma-ray photopeak areas to measured burnup values for 18 of the spectra listed in Table XII remains to be done. The isotopes that appear applicable in the normalization are ${ }^{144} \mathrm{Ce}-{ }^{144} \mathrm{Pr}$ and ${ }^{95} \mathrm{Zr}-{ }^{95} \mathrm{Nb}$. These isotopes did not appear to migrate 
as did ${ }^{106} \mathrm{Ru}-{ }^{106} \mathrm{Rh},{ }^{134} \mathrm{Cs}$ and ${ }^{137} \mathrm{Cs}$. All the spectra are tabulated in Appendix $D$ and decay correction data are presented throughout the report in order that the normalization can be done should the opportunity arise in the future. 


\section{ACKNOWLEDGEMENTS}

The authors wish to acknowledge valuable discussions with D. L. Prezbindowski and to thank him for making available the unpublished calculations of Matsuura and Prezbindowski. We also acknowledge the help of Elizabeth B. Reppond who prepared the tabulated and plotted data given in the report. Arnie Penttila operated the gamma scan facility and collected the gamma-ray data and his assistance was appreciated.

The experiment was accomplished in close cooperation with M. D. Freshley and D. G. Carter of the Fuels Research Section. Fabrication data were provided by Harriet Wisely and core configuration information was provided by L. D. Turner. Several others contributed to the experiment through the handling, cutting and measurement of the burnup samples. J. W. Goffard, Radiometallurgy; W. Y. Matsumoto, A. C. Leaf, M. W. Goheen and C. R. Lagergren, Chemical Analysis and Mass Spectrometry, were among those people who assisted the program. 


\section{REFERENCES}

1. N. G. Wittenbrock, P. C. Walkup, and J. K. Anderson, Plutonium Recycle Test Reactor, Final Safeguards Analyses, HW-61236 Supl.1, General Electric Company, Richland, Washington (October 1, 1959).

2. J. R. Worden, W. L. Purcell and L. C. Schmid, Physics Experiment High Power Density Core of the PRTR, BNWL-221, Pacific Northwest Laboratory, Richland, Washington (January, 1966).

3. Program Analysis and Plans, Plutonium Utilization Program, FY-1967 through - 1970, Compiled by F. G. Dawson, Program Leader, BNWL-298, Pacific Northwest Laboratory, Richland, Washington (July, 1966).

4. M. D. Freshley, R. E. Sharp, and R. E. Skavdahl, Plutonium Utilization Program: PRTR Irradiation Plans, BNWL-314, Pacific Northwest Laboratory, Richland, Washington (August, 1966).

5. W. J. Bailey and M. D. Freshley, Irradiation Properties of High Energy Rate Pneumatically Impacted $\mathrm{UO}_{2}-\mathrm{PuO}_{2}$ Fuels, BNWL-356, Pacific Northwest Laboratory, Richland, Washington, (Apri1, 1967).

6. J. W. Kutcher and R. I. Smith, Power Tests with the $\mathrm{UO}_{2}-2$ wt\% $\mathrm{PuO}_{2}$ Batch Core in the PRTR, BNWL-1383, Pacific Northwest Laboratory, Richland, Washington (May 1970).

7. Plutonium Utilization Program Annual Report-Fiscal Year 1967, Compiled by F. G. Dawson, Program Leader, BNWL-624, Pacific Northwest Laboratory, Richland, Washington (January, 1968).

8. D. E. Christensen and R. P. Matsen, Destructive and Nondestructive Burnup Data from Natural UO, Fuel, BNWL-1568, Pacific Northwest Laboratory, Richland, Washington (May 1971).

9. R. I. Smith, J. W. Kutcher, J. H. Lauby and L. D. Williams, Critical Experiments with the $\mathrm{UO}_{2}-2$ wt.\% $\mathrm{PuO}_{2}$ Batch Core in the PRTR, BNWL-1553, Pacific Northwest Laboratory, Richland, Washington (February 1971).

10. R. P. Matsen, ISODIL: A Computer Code for Processing Isotopic Dilution Measurements from Spent Fuel Samples, BNWL-1555, Pacific Northwest Laboratory, Richland, Washington (January, 1971). 
11. R. P. Matsen, DBUFIT-I A Least Squares Analysis Code for Nuclear Burnup Data, BNWL-1396, Pacific Northwest Laboratory, Richland, Washington (May 1970).

12. R. P. Matsen, "A Technique for the Determination of Ratios of Effective Cross Sections from Reactor Fuel Burnup Data," Nucl. Sci. Eng., vol. 32, p. 267 (1968).

13. R. P. Matsen, An Analysis of Yankee-Rowe Burnup Data, BNWL-1122, Pacific Northwest Laboratory, Richland, Washington (July 1969).

14. S. Matsuura and D. L. Prezbindowski, Analytical Correlations of PRTR Batch Core Experiment, BNWL-B-113, Pacific Northwest Laboratory

15. D. E. Christensen and G. Manca, "Nondestructive Analyses of Irradiated Fuel," Plutonium Utilization Program Technical Activities Quarterly Report-December, 1967, January, February 1968. BNWL-739, pp 3.3 (Apri1 1968).

16. M. D. Freshley and J. L. Maryott, "FERTF Testing," Plutonium Utilization Program Technical Activities Quarterly Report-March, Apri1, May 1968 BNWL-828, pp 2.2 (September 1968).

17. M. D. Freshley and J. L. Maryott, "FERTF Testing," Plutonium Utilization Program Technical Activities Quarterly Report-June, July, August 1968, BNWL-907, pp 2.22 (November 1968).

18. M. D. Freshley and T. B. Burley, "High Power Density Fuel Irradiation in PRTR," Plutonium Utilization Program Technical Activities Quarterly Report - December, 1967, January, February, 1968, BNWL-739, pp 2.1, (April 1968).

19. M. D. Freshley, T. B. Burley and S. Goldsmith, Plutonium Recycle Fuel Evaluations at BNL, BNWL-SA-1979 (November 1968).

20. M. D. Freshley and T. B. Burley, "High Power Density Fuel Irradiations in PRTR," Plutonium Utilization Program Technical Activities ReportDecember 1968, January, February 1969, BNWL-1039, pp 2.6 (Apri1 1969). 
21. D. G. Carter, T. B. Burley and M. D. Freshley, "PRTR Fuel Evaluations," Plutonium Utilization Program Technical Activities Quarterly ReportSeptember, October, November 1969, BNWL-1289, pp 31., (January 1970).

22. M. D. Freshley and D. G. Carter, "Defect Testing," Technical Activities Quarterly Report - AEC Reactor Development and Technology Programs July, August, September 1970, BNWL-1522-1, pp 2.22 (October 1970).

23. D. G. Carter and M. D. Freshley, "PRTR Fuel Evaluations," Technical Activities Quarterly Report - AEC Reactor Development and Technology Programs - October, November, December 1970, January, February, March 1971, BNWL-1522-2, pp 2.20 (June 1971).

24. M. D. Freshley, "Mixed-0xide Fuel Irradiations in PRTR," BNWL-SA-3981, June 1971.

25. M. D. Freshley, "The Defect Behavior of $\mathrm{UO}_{2}-\mathrm{PuO}_{2}$ Thermal Reactor Fuel," BNWL-SA-4138, November 1971 .

26. E. S. Murphy, et a1., "PNL Gamma Scan Facility," Reactor Physics Quarterly Report July, August, September 1968, BNWL 921 (December 1968). 
APPENDIX A 
APPENDIX A

\section{TABULATION OF BURNUP SAMPLE DATA}

The data obtained from measurements made on 36 burnup samples are tabulated in Table A-I on the pages that follow. The data have been processed by program ISODIL and values which result from the data processing are tabulated also. An explanation of the entries for each sample is given in the body of the report. Table IV shown there is the first page of tabulated data in this appendix. The order of the burnup samples is the same order as the listing given in Table II. 


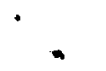

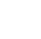

,

•

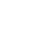


TABLE A-I

BURNUP DATA AND RESULTS AS LISTED BY PROGRAM ISODIL

pp. A-2 to $A-19$ 


\section{A-2}

ELEMENT GUGS ROO FS12(12-ROD RING) BATCH CORE THE SAMPLE WAS CUT 10.4 INCHES FROM THE TOP OF THE FUEL INPUT DATA---- TIME OUT OF REACTOR=1.000YRS BOL PU241 DECAY CORRECTION TIME= 2.33OYRS SPIKE--NU42/50 .8056-02(.24-U3) NDb0/42 .1724+02(.17-00)--SAMPLE--IVD50/48 .5531-00(.28-02) MIXTURE--ND42/50 .1433-01(.15-03)

ATOMS/PIL IN SPIKE SPIKE

IS O TOP I C O I LU T ION A IN A L Y S IS

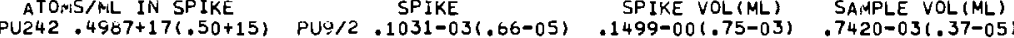

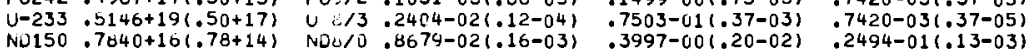
BOL U+PUE $=.2370(.0031) \mathrm{GM} / \mathrm{ML}$ AS CALCULATED BY ISOTOPIC DILUTION

PU9/2. $9777-00(.29-02)$ PUTOMS/ML IN SAMPLE $\cup 8 / 3: 1126+01(.30-02) \quad U-238: 5846+21(.76+19)$ NDB $/ 0: 1446-00(.14-02)$ ND148: $: 1837+17(: 31+15)$

$.68 \%$ SAMPLE---ND CONTAMINATION---MIXTURE $1.08 \%$

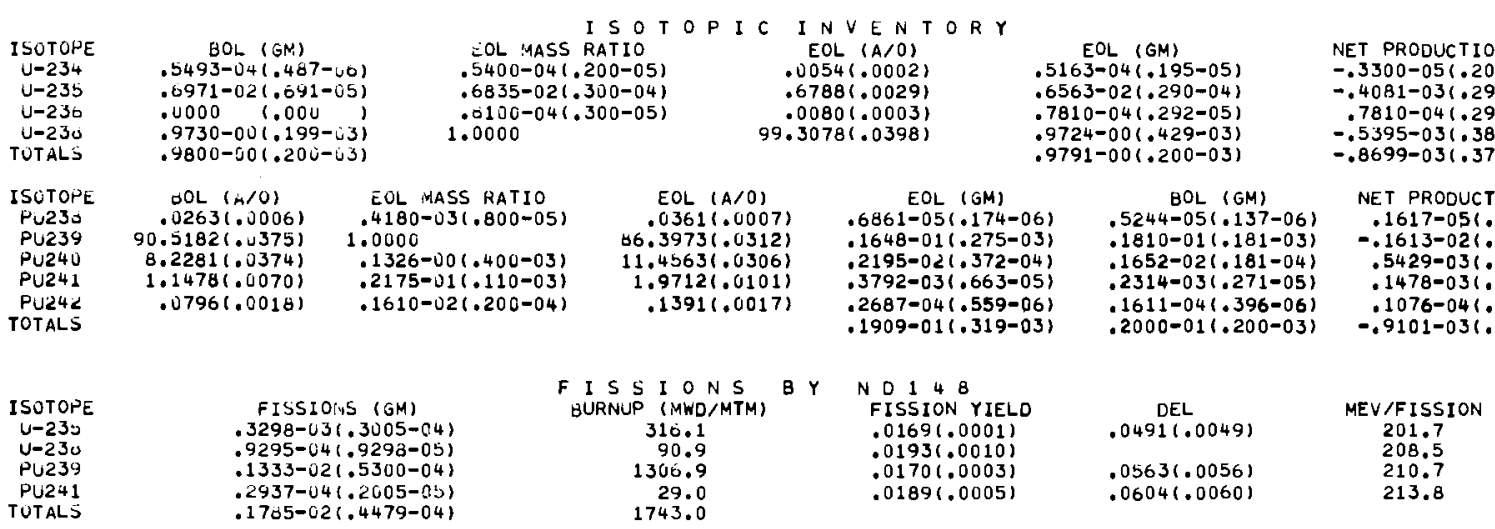

U2S5/U23560L $=.9411 .0041$ ALPHA235 $=.23721 .0241$ ) U238 INTO PU239=.4484-03(.38-03)GM U236 INTO NP237=.476-06GM

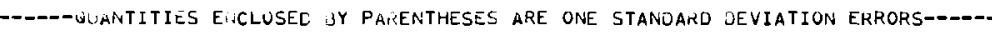

ELEENENT OUGS RUU FS1Z̈(12-RGD RING) BATCH CORE THE SAMPLE WAS CUT 6.5 INCHES FROM THE TOP OF THE FUEL

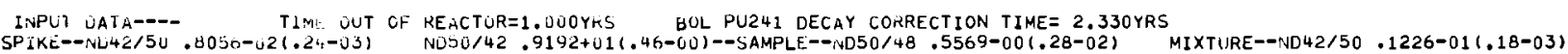
ISOTUPIC DILUTION ANALYSIS ATU,WS/ML IN SPIKE SPIKE SPIKE VOL (ML) SAMPLE VOL (ML)

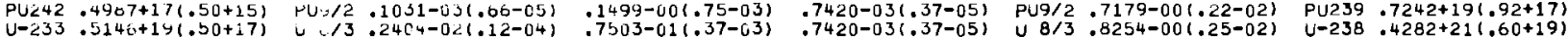

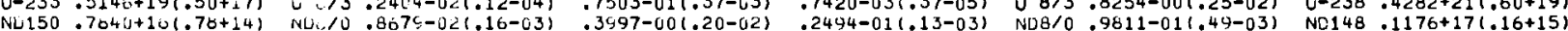
BUL UTPLI $=.1740(.0024) \mathrm{OM} / \mathrm{MLL}$ AS CALCLLATEO GY ISOTOPIC UILUTION

1.30\% SAMPLE---IND CONTAMINATION---MIXTURE $1.10 \%$

\begin{tabular}{|c|c|c|c|c|c|c|c|c|}
\hline $\begin{array}{l}\text { ISUTOFE } \\
U-234 \\
U-230 \\
U-230 \\
U-230 \\
\text { TUTALS }\end{array}$ & $\begin{array}{l}00 L(3,1) \\
.3493-0+(.467 \\
.0971-021.091 \\
.0000 \quad 1.000 \\
.9730-001.195 \\
.9000-006.200\end{array}$ & $\begin{array}{l}\text { COL MASS } \\
.5120-041 \\
.0669-021 \\
.0600-041 \\
1.0000\end{array}$ & $\begin{array}{l}\text { ISOT OPIC } \\
\text { KATIO } \\
(.150-65) \\
(.275-(4) \\
(.150-051\end{array}$ & $\begin{array}{r}\text { C IN } \\
\text { EO } \\
.005 \\
.582 \\
.006 \\
99.300\end{array}$ & $\begin{array}{l}V E N T O R \\
(A / 0) \\
1(.0001) \\
1(.0027) \\
6(.0001) \\
2(.0398)\end{array}$ & & $\begin{array}{l}\text { OL }(G M) \\
-04(.127-05) \\
-02(.266-04) \\
-04(.146-05) \\
-00(.437-03) \\
-00(.200-03)\end{array}$ & $\begin{array}{l}\text { NET PRODUCTIO } \\
-.5961-051.13 \\
-.3732-031.27 \\
.6366-041.14 \\
-.2151-031.38 \\
-.5250-031.38\end{array}$ \\
\hline $\begin{array}{l}\text { ISCTOHL } \\
\text { PU230 } \\
\text { PU23S } \\
\text { PUL4O } \\
\text { PU241 } \\
\text { PLE4L } \\
\text { TOTALS }\end{array}$ & $\begin{array}{c}\text { SUL }(A / 0) \\
.0203(.0005) \\
90.3180(.4375) \\
8.2281(.0574) \\
1.1470(.0070) \\
.0790(.0618)\end{array}$ & $\begin{array}{l}\text { CUL iNSS RATIO } \\
.5040-t 3(.200-05) \\
1.0000 \\
.1252-00(.400-03) \\
.1953-01(.100-03) \\
.1+50-02(.200-04)\end{array}$ & $\begin{array}{r}\text { EOL (A/O) } \\
.03351 .000 \\
67.14441 .031 \\
10.91051 .031 \\
1.76531 .009 \\
.12641 .001\end{array}$ & $\begin{array}{l}1 \\
07) \\
151 \\
113 \\
931 \\
17)\end{array}$ & $\begin{array}{c}\text { EOL }(G M) \\
.6317-051.172-0 \\
.1652-011.288-0 \\
.2077-021.368-0 \\
.3413-031.622-0 \\
.2425-041.539-0 \\
.1697-011.331-0\end{array}$ & & $\begin{array}{c}\text { BOL (GM) } \\
.5244-05(.137-06) \\
.1810-01(.181-03) \\
.1652-02(.181-04) \\
.2314-03(.271-05) \\
.1611-04(.396-06) \\
.2000-01(.200-03)\end{array}$ & $\begin{array}{l}\text { NET PRODUCT } \\
.1073-051 . \\
-.1576-021 \\
.4251-031 \\
.1099-031 \\
.8148-051 \\
-.1032-021 .\end{array}$ \\
\hline $\begin{array}{l}\text { ISUTUPL } \\
\text { U-235 } \\
\text { U-230 } \\
\text { PUESS } \\
\text { PU.241 } \\
\text { TUTALS }\end{array}$ & $\begin{array}{l}\text { FISS1 } \\
.2055-0 \\
0111-0 \\
.1140-0 \\
22223-0 \\
.1560-0\end{array}$ & $\begin{array}{l}.6(0 M) \\
(.2701-(04) \\
.6397-(5) \\
.4542-C 4) \\
(.1906-05) \\
(.3008-04)\end{array}$ & $\begin{array}{c}\text { F I S S I O N S } \\
\text { BURNUH (, (1WD/MTM) } \\
290.6 \\
79.3 \\
1125.1 \\
21.9 \\
1523.0\end{array}$ & BY & $\begin{array}{l}\text { NO } 148 \\
\text { FISSIUN YIELD } \\
.0169(.0001) \\
.0193(.0010) \\
.0170(.0003) \\
.0189(.0005)\end{array}$ & & $\begin{array}{c}\text { DEL } \\
.0491(.0049)\end{array}$ & $\begin{array}{c}\text { MEV/FISSION } \\
201.7 \\
208.5 \\
210.7 \\
213.8\end{array}$ \\
\hline
\end{tabular}

U2 $55 / U 2550 U L=.940(.004)$ ALPHA235 $=.2060(.0192)$ U238 INTO P.J239=.1346-031.39-03)GM U236 INTO NP237=.354-06GM 
ELEMENT GUGS ROU FSIZ̈(12-ROD RING) BATCH CORE THE SAMPLE WAS CUT 29.4 INCHES FROM THE TOP OF THE FUEL INPUT DATA---- TIME OUT OF REACTUR=1.000YRS BOL PU241 DECAY CORRECTION TIME= 2.330YRS SPIKE--NO42/50.8050-02(.24-03) NDS0/42.1518+02(.76-00) --SAMPLE--IND50/48.5490-00(.27-02) MIXTURE--ND42/50 .1814-01(.18-03) ATOMS/ML IN SPIKE SPIKE

$$
\text { I SOTOPIC DILUT I ON ANALYSIS }
$$

PU24 SPSTML IN SPIKE SPIKE VOL(ML) SAMPLE VOL(ML) PUट2 . 4987+17(.50+15) PUC/2 .1031-03(.66-05) .1499-00(.75-03) .7420-03(.37-05)

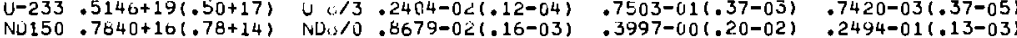
BOL U+PU $=.1757(.0024) \mathrm{GM} / \mathrm{ML}$ AS CALCULATEO BY ISOTOPIC DILUTION

\begin{abstract}
MIXTURE
PU9/2 $.6970-00(.21-02)$
ATOMS $/ M L$ IN SAMPLE
$.0239 .7034+19(.89+17)$ U $8 / 3: 8325-00(.25-02) \quad U-238: 4319+21(.60+19)$ .77\% SAMPLE---HO CONTAMINATION---MIXTURE $1.42 \%$
\end{abstract}

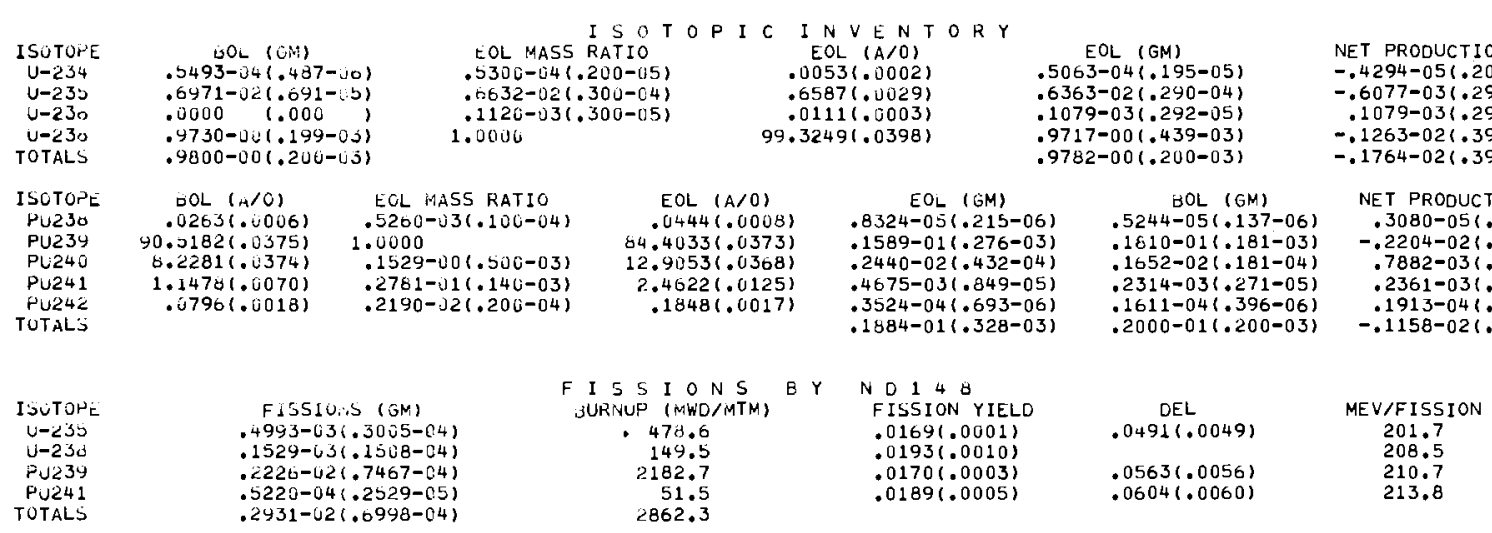

U235/U2350UL=.913(.004) ALPHA235=.2172(.01'8) U238 INTO PU239=.1115-02(.39-03)GM U236 INTO NP237=.999-06GM

-----idiantities EIHCLUSEL iY PARENTHESES ARE ONE STANDARD DEVIATION ERRORS------

ELEMENT DOGS ROE FS1Z112-ROO RINUG) BATCH CORE THE SAMPLE WAS CUT 41.3 INCHES FROM THE TOP OF THE FUEL

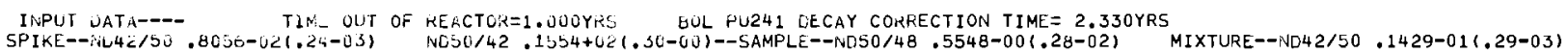

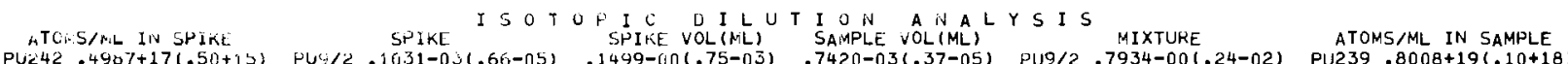

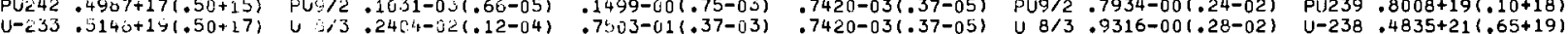

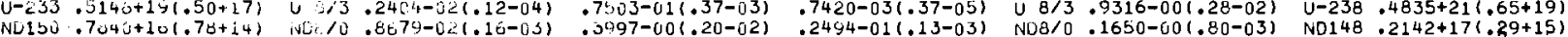

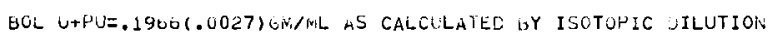

.76\% SAMPLE---ND CONTAMINATION---MIXTURE .94\%

\begin{tabular}{|c|c|c|c|c|c|c|c|c|}
\hline $\begin{array}{l}\text { ISUTOHL } \\
\text { U-234 } \\
\text { U-235 } \\
\text { U-230 } \\
\text { U-230 } \\
\text { TCTALS }\end{array}$ & 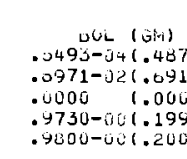 & $\begin{array}{l}002 \text { MASS } \\
.5400-041 \\
.0692-001 \\
.1060-031 \\
1.00100\end{array}$ & $\begin{array}{l}\text { ISC TOP I } \\
\text { RATIC } \\
(.200-65) \\
(.300-04) \\
(.300-05)\end{array}$ & $\begin{array}{r}11 \\
.00 \\
.00 \\
.66 \\
.010 \\
9.31\end{array}$ & $\begin{array}{l}V E N T O R Y \\
-(A / 0) \\
+(.0002) \\
5(.0029) \\
5(.0003) \\
5(.0398)\end{array}$ & $\begin{array}{l}.51 \\
.64 \\
.10 \\
.97 \\
.97\end{array}$ & $\begin{array}{l}O L(G M) \\
=04(.195-05) \\
=02(.290-04) \\
=03(.292-05) \\
=00(.436-03) \\
-00(.200-03)\end{array}$ & $\begin{array}{l}\text { NET PRODUCTION (GM) } \\
-.3329-05(.201-05) \\
-.5490-03(.299-04) \\
.1022-03(.292-05) \\
-.1090-02(.388-03) \\
-.1538-02(.387-03)\end{array}$ \\
\hline 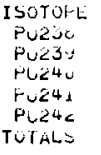 & $\begin{array}{r}\text { UOL }(a / 0) \\
.026 j(.0006) \\
90.5182(.2375) \\
2.2281(.0374) \\
1.1470(.0470) \\
.0790(.4012)\end{array}$ & $\begin{array}{l}\text { EOL WHSS RATIU } \\
.4740-03(.900-05) \\
1.0000 \\
.1467-00(.400-03) \\
.2599-01(.130-03) \\
.2600-02(.200-64)\end{array}$ & $\begin{array}{r}E O L(A / 0) \\
.6403(.000 \\
05.00251 .030 \\
12.46991 .029 \\
2.31741 .011 \\
.17001 .001\end{array}$ & & $\begin{array}{c}\text { EOL (GM) } \\
.7630-05(.195-0 \\
.1016-01(.277-0 \\
.2331-026.413-0 \\
.4444-03(.794-0 \\
.3274-041.649-0 \\
.1903-016.326-0\end{array}$ & & $\begin{array}{c}\Delta O L(G M) \\
.5244-05(.137-06) \\
.1810-01(.181-03) \\
.1652-02(.181-04) \\
.2314-03(.271-05) \\
.1611-04(.396-06) \\
.2000-01(.200-03)\end{array}$ & $\begin{array}{l}\text { NET PRODUCTION (GM) } \\
.2386-05(.238-06) \\
-.1931-02(.331-03) \\
.7295-03(.451-04) \\
.2130-03(.839-05) \\
.1663-04(.760-06) \\
-.9698-03(.382-03)\end{array}$ \\
\hline $\begin{array}{l}\text { ISUTGLL } \\
U-232 \\
U-230 \\
\text { PL235 } \\
\text { PL } 241 \\
\text { TOTALS }\end{array}$ & $\begin{aligned} F 1551 \\
.4444-0 \\
.1321-0 \\
.1893-0 \\
.4537-0 \\
.5510-0\end{aligned}$ & $\begin{array}{l}S(S M) \\
(.3 \cup(5-(24) \\
(.1295-04) \\
.6511-04) \\
.2357-06) \\
.5900-(4)\end{array}$ & 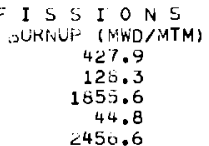 & $\begin{array}{ll}B Y \\
\end{array}$ & $\begin{array}{l}\text { ND14 } 4 \\
\text { FISSION YIELD } \\
.0169(.0001) \\
.0193(.0010) \\
.0170(.00003) \\
.0189(.0005)\end{array}$ & & $\begin{array}{c}\text { DEL } \\
.0491(.0049) \\
.0563(.0056) \\
.0604(.0060)\end{array}$ & $\begin{array}{c}\text { MEV/FISSION } \\
201.7 \\
208.5 \\
210.7 \\
213.8\end{array}$ \\
\hline
\end{tabular}

ULJS/U2350OL=.9:16.0(4) ALFHAZ3i5=.2297(.0174) U235 INTO PU239=.9629-03(.39-03)GM U236 INTO NP237=.849-06GM 


\section{A-4}

ELEMENT 6065 ROD FS12(12-ROD RING) BATCH CORE THE SAMPLE WAS CUT 47.5 INCHES FROM THE TOP OF THE FUEL 1NPUT DATA---- TIME OUT OF REACTOR=1.000YRS BOL PU241 OECAY CORRECTION TIME= $2.330 Y R S$
SPIKE--NO42/50 .8056-02(.24-03) ND50/42.1736+02(.17-60)--SAMPLE--ND50/48.5568-00(.28-02) MIXTURE--ND42/50 .1038-011.52-03) IS 0 TOPIC DILUTION A NAL YSIS

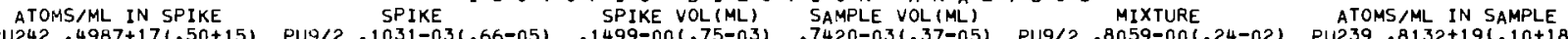

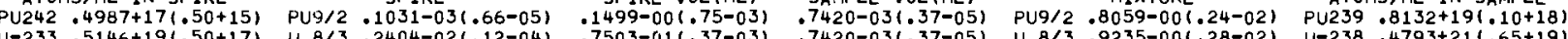

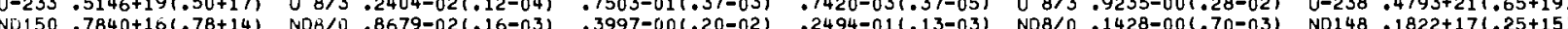
BOL U+PU $=.1949(.0026) \mathrm{GM} / \mathrm{ML}$ AS CALCULATED BY ISOTOPIC DILUTION

.68\% SAMPLE---ND CONTAMINATION---MIXTURE . .46\%

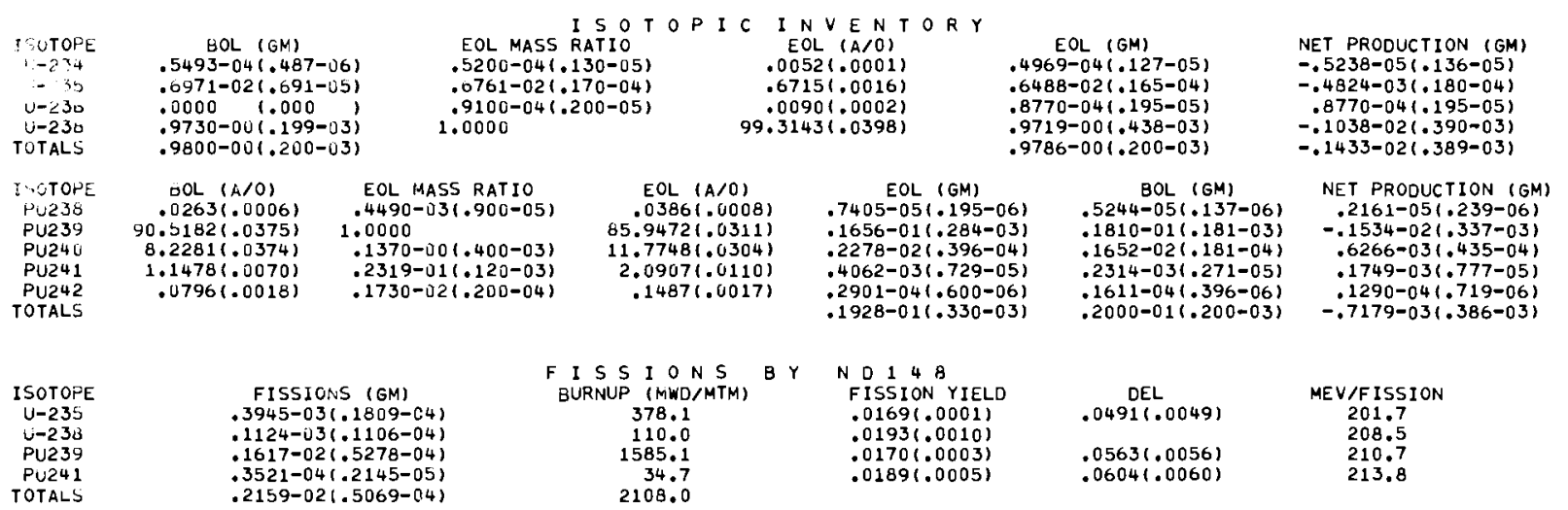

U235/U235BOL $=.931(.003)$ ALPHA235 $=.2230(.0118)$ U238 INTO PU239=.9296-03(.39-03)GM U236 INTO NP237=.637-06GM

------Q QUANTITIES ENCLOSED BY PARENTHESES ARE ONE STANDARD DEVIATION ERRORS------

ELEMENT 6066 ROD FS16(12-ROD RING) BATCH CORE THE SAMPLE WAS CUT 6.5 INCHES FROM THE TOP OF THE FUEL INPUT DATA--- TIME OUT OF REACTOR=1.378YRS BOL PU241 DECAY CORRECTION TIME= 2.330YRS OXIDE FUEL= .2909GM/ML SPIKE--ND42/50.8056-02(.24-03) ND50/42.4845+02(.24-00)--SAMPLE--ND50/48.5515-00(.20-02) MIXTURE--ND42/50.7980-021.40-04) ATUMS/ML IN SPIKE SPIKE ISOT O P I C D I L U T I O N A N A L PU242 $4967+17(050+15)$ PU9

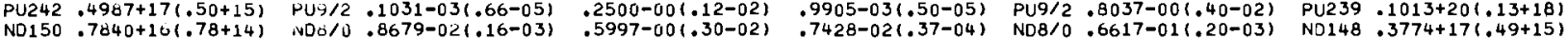
BOL U+PU $=.2564(.0026) \mathrm{GM} / \mathrm{ML}$ AS CALCULATED BY FUEL WEIGHT AND SOLUTION VOLUME .24\% SAMPLE---ND CONTAMINATION---MIXTURE .07\%

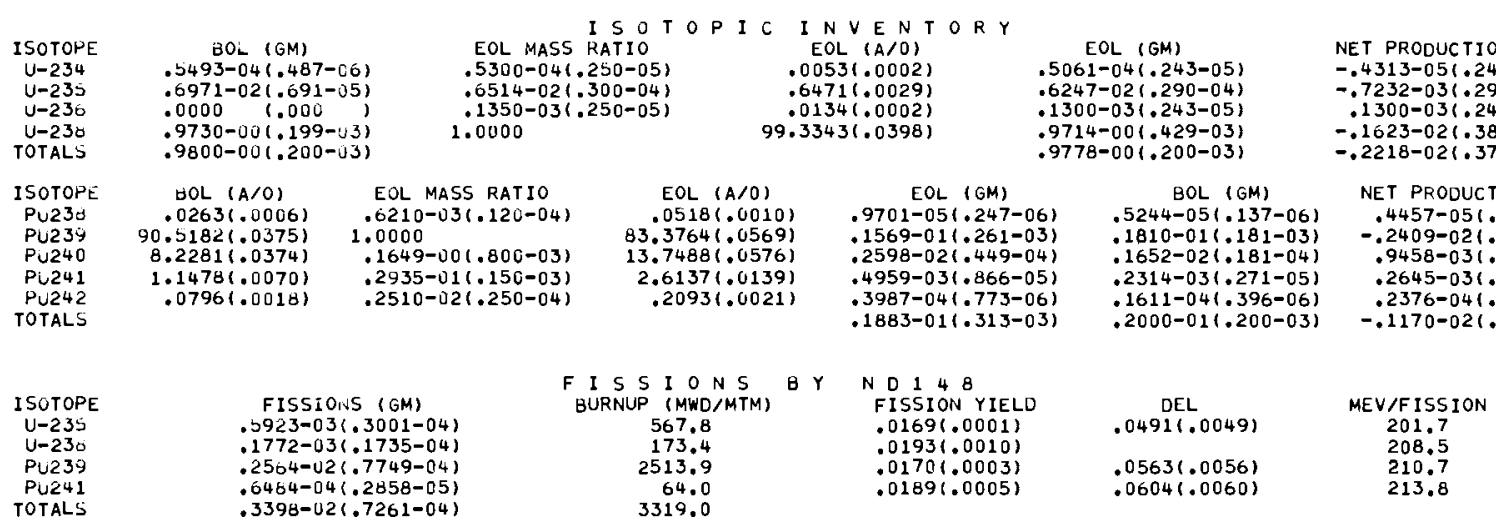

U235/L2J5EOL $=.896(.004)$ ALPHA235 $=.2210(.0122)$ U238 INTO PU239=.1452-02 $(.38-03)$ GM U236 INTO NP237=.145-05GM 


\section{A-5}

ELEMENT 6066 ROD FS16(12-ROD RING) BATCH CORE THE SAMPLE WAS CUT 9.6 INCHES FROM THE TOP OF THE FUEL INPUT DATA--1- TIME OUT OF REACTOR=1.378YRS BOL PU241 DECAY CORRECTION TIME= 2.330YRS OXIDE FUEL= .2731GM/ML SPIKE--ND42/50.8056-02(.24-03) ND50/42.4662+02(.23-00)--SAMPLE--ND50/48.5487-00 $(.20-02)$ MIXTURE--ND42/50.7720-02(.040-04)

\section{SOTOPIC DILUTION A NAL YSIS}

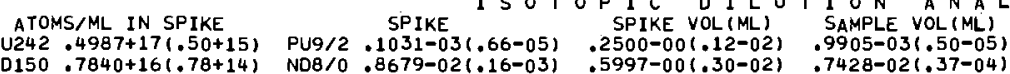
PUIXTURE ATOMS/ML IN SAMPLE

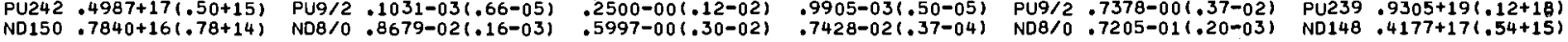
BOL U+PU $=.2407(.0024)$ GM/ML AS CALCULATEO BY FUEL WEIGHT ANO SOLUTION VOLUME .25\% SAMPLE---ND CONTAMINATION---MIXTURE -.02\%

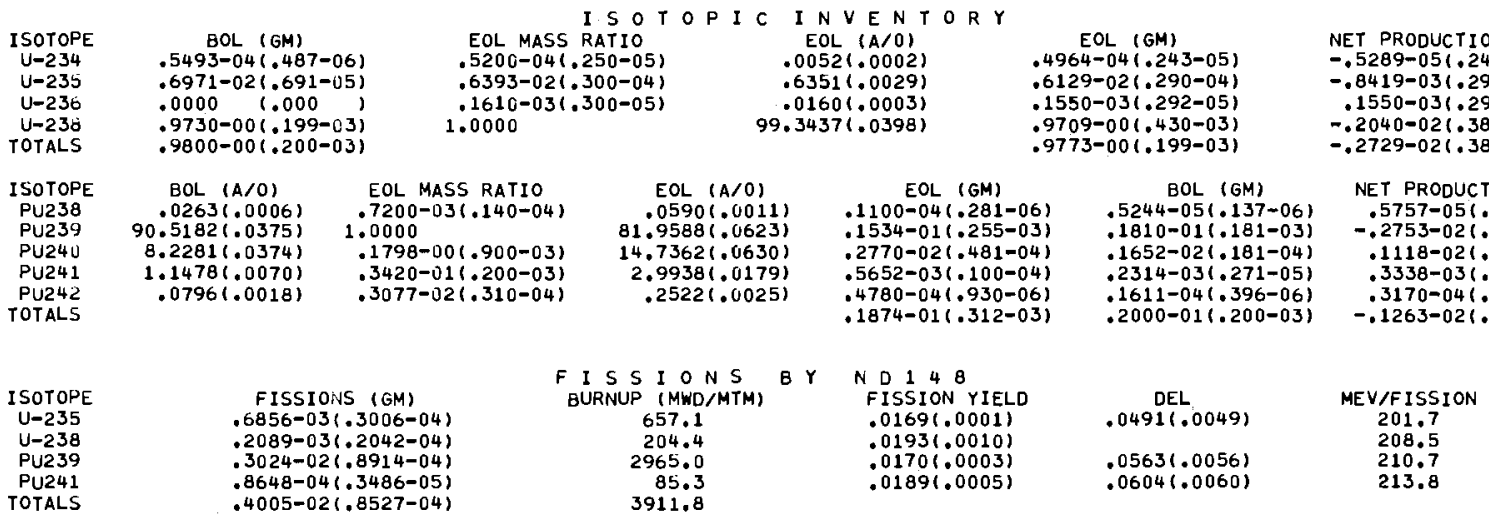

U235/U235BOL $=.879(.004)$ ALPHA235 $=.2281(.0112)$ U238 INTO PU239=.1839-02(.38-03)GM U236 INTO NP237=.204-05GM

-------QUANTITIES ENCLOSED BY PARENTHESES ARE ONE STANDARD DEVIATION ERRORS------

ELEMENT 6066 ROD FS16(12-ROD RING) BATCH CORE THE SAMPLE WAS CUT 28.9 INCHES FROM THE TOP OF THE FUEL INPUT DATA---- TIME OUT OF REACTOR=1.378YRS BOL PU241 DECAY CORRECTION TIME= 2.330YRS SPIKE--ND42/50 .8056-02(.24-03) ND50/42.4637+02(.23-00)--SAMPLE--ND50/48 .5511-00(.20-02) MIXTURE--ND42/50 .8090-02(.40-04)

ATOMS/ML IN SPIKE SPIKE

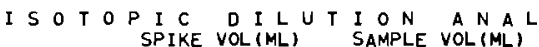
$\begin{array}{lllllll}\text { PU242 } .4987+17(.50+15) & \text { PU9/2 } & .1031-03(.66-05) & .2500-00(.12-02) & .9905-03(.50-05) & \text { PU9/2 .6654-001.33-02) PU239 } & .8404+19(.11+18\end{array}$

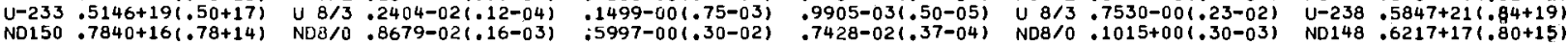
BOL U+PU $=.2383(.0034) \mathrm{GM} / \mathrm{ML}$ AS CALCULATED BY ISOTOPIC DILUTION . $25 \%$ SAMPLE---ND CONTAMINATION---MIXTURE . $10 x$

\begin{tabular}{|c|c|c|c|c|c|c|c|c|}
\hline $\begin{array}{c}\text { ISOTOPE } \\
U-234 \\
U-235 \\
U-236 \\
U-236 \\
\text { TOTALS }\end{array}$ & $\begin{array}{c}\text { BOL (GM) } \\
.5493-04(.487-06) \\
.6971-02(.691-05) \\
.0000 \quad(.000) \\
.9730-00(.199-03) \\
.9800-00(.200-03)\end{array}$ & \multicolumn{4}{|c|}{$\begin{array}{l}\text { ISOT } \\
\text { EOL MASS RATIO } \\
.5100-04(.250-05) \\
.5955-02(.300-04) \\
.2370-03(.350-05) \\
1.0000\end{array}$} & \multicolumn{2}{|c|}{$\begin{array}{c}\text { EOL }(\mathrm{GM}) \\
.4863-04(.243-05) \\
.5703-02(.291-04) \\
.2279-03(.341-05) \\
.9699-00(.457-03) \\
.9759-00(.199-03)\end{array}$} & $\begin{array}{l}\text { NET PRODUCTIOI } \\
-.6294-051.248 \\
-.1268-021.29 \\
.2279-031.34 \\
-.3051-021.41 \\
-.4096-021.41\end{array}$ \\
\hline $\begin{array}{l}\text { ISOTOPE } \\
\text { PU238 } \\
\text { PU239 } \\
\text { PU240 } \\
\text { PU241 } \\
\text { PU242 } \\
\text { TOTALS }\end{array}$ & $\begin{array}{c}D O L(A / 0) \\
.0263(.0006) \\
90.0182(.0375) \\
8.2281(.0374) \\
1.1478(.0070) \\
.0796(.0018)\end{array}$ & $\begin{array}{l}\text { EOL MASS RATIO } \\
.1002-02(.200-04) \\
1.0000 \\
.2301-00(.120-02) \\
.5088-01(.250-03) \\
.5543-02(.420-04)\end{array}$ & $\begin{array}{r}E O L(A / 0) \\
.07761 .0015 \\
77.4600(.0741 \\
17.8235(.0765 \\
4.2095(.0215 \\
.4294(.0033\end{array}$ & & $\begin{array}{c}\text { EOL }(\mathrm{GM}) \\
.1397-041.377-0 \\
.1400-01(.255-0 \\
.3235-021.612-0 \\
.7672-031.145=0 \\
.7857-041.155-0 \\
.1809-011.329-0\end{array}$ & $\begin{array}{l}06) \\
03) \\
04) \\
04) \\
05) \\
03)\end{array}$ & $\begin{array}{c}\text { BOL }(G M) \\
.5244-05(.137-06) \\
.1810-01(.181-03) \\
.1652-02(.181-04) \\
.2314-03(.271-05) \\
.1611-04(.396-06) \\
.2000-01(.200-03)\end{array}$ & $\begin{array}{l}\text { NET PRODUCT } \\
.8725-051 . \\
-.4096-021 . \\
.1583-021 . \\
.5358-031 . \\
.6247-041 . \\
-.1906-021 .\end{array}$ \\
\hline $\begin{array}{l}\text { ISOTOPE } \\
\text { U-23S } \\
\text { U-23U } \\
\text { PU239 } \\
\text { PU241 } \\
\text { TOTALS }\end{array}$ & $\begin{array}{r}\text { FISSI } \\
.1036-0 \\
.3141=0 \\
.4498-0 \\
.1704-0 \\
.6018-0\end{array}$ & 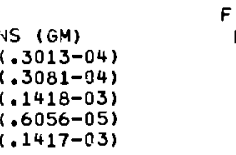 & $\begin{array}{l}\text { ISS S I O N S } \\
\text { BURNUP (MWD/MTM) } \\
993.1 \\
307.3 \\
4409.7 \\
168.2 \\
5878.3\end{array}$ & $\mathrm{~B} Y$ & $\begin{array}{l}\text { ND } 148 \\
\text { FISSION YIELD } \\
.0169(.0001) \\
.0193(.0010) \\
.0170(.0003) \\
.0189(.0005)\end{array}$ & & $\begin{array}{c}\text { DEL } \\
.0491(.0049) \\
.0563(.0056) \\
.0604(.0060)\end{array}$ & $\begin{array}{c}\text { MEV/FISSION } \\
201.7 \\
208.5 \\
210.7 \\
213.8\end{array}$ \\
\hline
\end{tabular}

U2J5/U2358OL $=.818(.004)$ ALPHA235 $=.2236(.0076)$ U238 INTO PU239 $=.2749-02(.41-03)$ GM U236 INTO NP237 $=.473-05$ GM 


\section{A-6}

ELEMENT 6066 HOD FS16(12-ROD RING) BATCH CORE THE SAMPLE WAS CUT 43.6 INCHES FRCM THE TOP OF THE FUEL INPUT DATA---- TIME OUT OF REACTOR=1.378YRS BOL PU241 DECAY CORRECTION TIME= 2.330YRS OXIDE FUEL= .2521GM/ML SPIKE--ND42/50.8056-02(.24-03) ND50/42.4589+02(.23-00)--SAMPLE--ND50/48.5490-00(.20-02) MIXTURE--ND42/50.8120-021.40-04)

$$
\text { I SOIOPIC DILUTION A NALYS I S }
$$

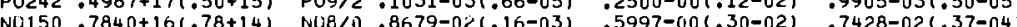
BOL U+PU $=.2222(.0022) \mathrm{GM} / M L$ AS CALCULATED BY FUEL WEIGHT AND SOLUTION VOLUME $.25 \%$ SAMPLE---ND CONTAMINATION---MIXTURE . $11 \%$

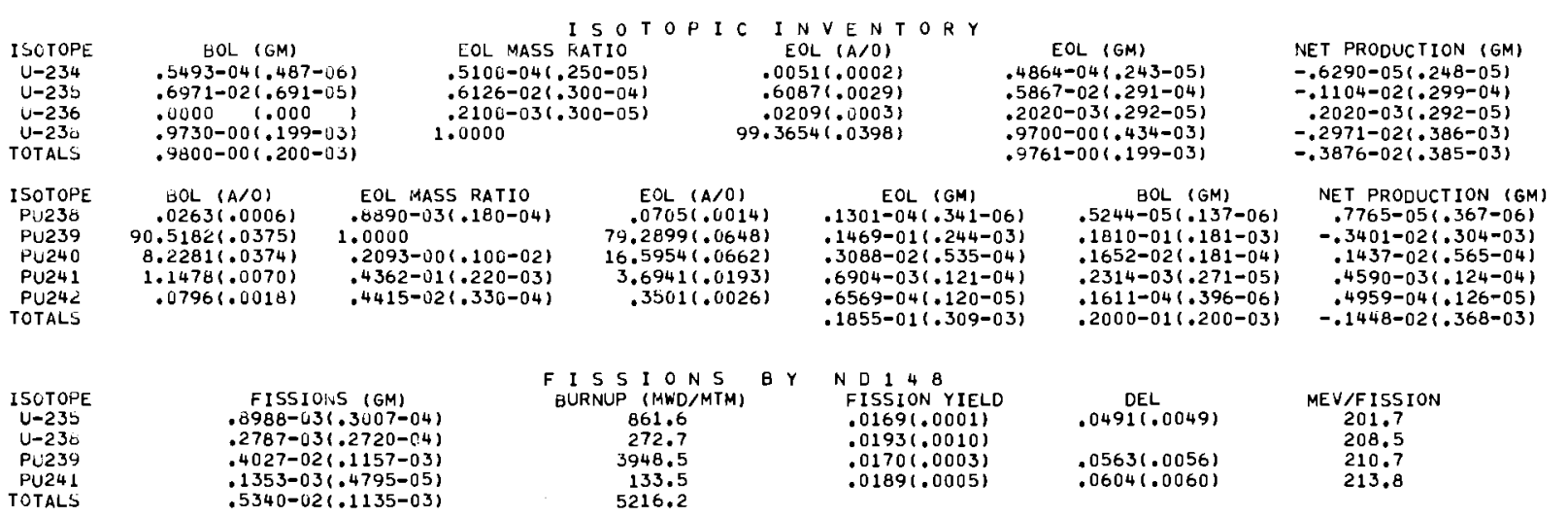

U235/U23580L $=.842(.004)$ ALPHA235 $=.2277(.0086)$ U238 INTO PU239 $=.2703-02(.39-03)$ GM U236 INTO NP237=.358-05GM

------QLANTITIES EINCLOSEO dY PARENTHESES ARE ONE STANDARD OEVIATION ERRORS------

ELEMENT 6066 ROD FS16(12-ROD RING) BATCH CORE THE SAMPLE WAS CUT 48.1 INCHES FROM THE TOP OF THE FUEL INPUT DATA---- TLME OUT OF REACTOR=1.378YRS EOL PU241 DECAY CORRECTION TIME $=2.330 Y R S$ OXIDE FUEL= .2638GM/ML SPIKE--NQ42/50. $8056-02(.24-03)$ ND50/42.4807+02(.24-00)--5AMPLE--ND50/48.5492-00(.20-02) MIXTURE--ND42/50 .8340-02(.60-04)

ATOMS/ML IN SPIKE IS OT T O I C ODILLU T IO N N A NALY Y IS

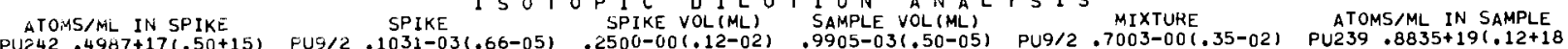

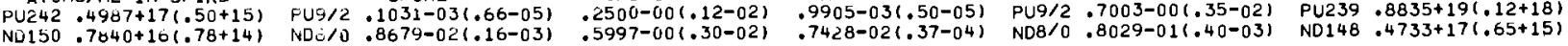
BOL U+PU $=.2325(.0023) \mathrm{GM} / M L$ AS CALCULATED BY FUEL WEIGHT AND SOLUTION VOLUME .24\% SAMPLE---ND CONTAMINATION---MIXTURE .18x

\begin{tabular}{|c|c|c|c|c|c|c|c|c|}
\hline $\begin{array}{l}\text { ISUTOPE } \\
\text { U-234 } \\
U-235 \\
U-230 \\
\text { U-23O } \\
\text { TOTALS }\end{array}$ & $\begin{array}{r}\text { BOL (GM) } \\
.5493-041.487 \\
.0971-02(.691 \\
.0000 \quad 1.000 \\
.97300001 .195 \\
.9800-001.200\end{array}$ & $\begin{array}{l}\text { EOL MASS } \\
.5300-041 \\
.0216-021 \\
.1880-031 \\
1.0000\end{array}$ & $\begin{array}{l}\text { ISOTOPI } \\
\text { RATIC } \\
(.250-05) \\
(.300-04) \\
(.250-05)\end{array}$ & $\begin{aligned} & 11 \\
& \mathrm{EC} \\
& .005 \\
& .61 \\
& .018 \\
& 9.35\end{aligned}$ & 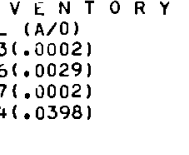 & $\begin{array}{l}.50 \\
.59 \\
.18 \\
.97 \\
.97\end{array}$ & $\begin{array}{l}\text { OL }(G M) \\
-04(.243-05) \\
-02(.291-04) \\
=03(.243-05) \\
-00(.433-03) \\
-00(.199-03)\end{array}$ & $\begin{array}{l}\text { NET PRODUCTION (GM) } \\
-.4362-05(.248-05) \\
-.1015-02(.299-04) \\
.1809-03(.243-05) \\
-.2576-02(.385-03) \\
-.3413-02(.384-03)\end{array}$ \\
\hline $\begin{array}{l}\text { ISOTOP } \\
\text { PU236 } \\
\text { PU239 } \\
\text { PU240 } \\
\text { PU241 } \\
\text { PU242 } \\
\text { TOTALS }\end{array}$ & $\begin{array}{r}d O L(A / O) \\
.0263(.0006) \\
90.5182(.0375) \\
8.2281(.0374) \\
1.1478(.0070) \\
.0796(.0018)\end{array}$ & $\begin{array}{l}\text { EOL MASS RATIO } \\
.7790-03(.160-04) \\
1.0000 \\
.1950-00(.100-02) \\
.3868-01(.190-03) \\
.3670-02(.280-04)\end{array}$ & $\begin{array}{r}\text { EOL }(\mathrm{A} / 0) \\
.06281 .001 \\
80.59561 .066 \\
10.71611 .068 \\
3.32971 .017 \\
.29581 .002\end{array}$ & & $\begin{array}{c}\text { EOL }(G M) \\
.1170-04(.309-0 \\
.1508-01(.251-0 \\
.2953-02(.514-0 \\
.6283-03(.109-0 \\
.5004-04(.103-c \\
.1873-016.312-c\end{array}$ & & $\begin{array}{c}\text { BOL }(G M) \\
.5244-05(.137-06) \\
.1810-01(.181-03) \\
.1652-02(.181-04) \\
.2314-03(.271-05) \\
.1611-04(.396-06) \\
.2000-01(.200-03)\end{array}$ & $\begin{array}{l}\text { NET PRODUCTION (GM) } \\
.6455-05(.338-06) \\
-.3015-02(.309-03) \\
.1301-02(.545-04) \\
.3969-03(.113-04) \\
.3994-04(.110-05) \\
-.1270-02(.370-03)\end{array}$ \\
\hline $\begin{array}{l}\text { ISOTOPE } \\
\text { U-235 } \\
\text { U-230 } \\
\text { PU23Y } \\
\text { PU241 } \\
\text { TOTALS }\end{array}$ & $\begin{aligned} \text { FISS } \\
.0319-1 \\
.2449-1 \\
.3512-1 \\
.1090-c \\
.4698-c\end{aligned}$ & 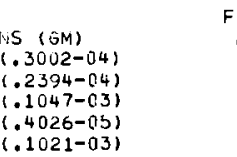 & $\begin{array}{c}\text { FISS I ONS } \\
\text { BURNUP (MWD/MTM) } \\
797.4 \\
239.6 \\
3443.4 \\
107.5 \\
4587.9\end{array}$ & $B Y$ & $\begin{array}{l}\text { ND } 148 \\
\text { FISSION YIELD } \\
.0169(.0001) \\
.0193(.0010) \\
.0170(.0003) \\
.0189(.0005)\end{array}$ & & $\begin{array}{l}\text { DEL } \\
.0491(.0049) \\
.0563(.0056) \\
.0604(.0060)\end{array}$ & $\begin{array}{l}\text { MEV/FISSION } \\
201.7 \\
208.5 \\
210.7 \\
213.8\end{array}$ \\
\hline
\end{tabular}

U235/U235SOL $=.854(.004)$ ALPHA235 $=.22001 .0087)$ U238 INTO PU239 $=.2341-02(.38-031$ GM U236 INTO NP237=.292-05GM 


\section{A-7}

ELEMENT 6066 ROD F079( 6-ROD RING) BATCH CORE THE SAMPLE WAS CUT 6.5 INCHES FROM THE TOP OF THE FUEL INPUT DATA--- $\quad$ TIME OUT OF REACTOR=1.378YRS BOL PU241 DECAY CORRECTION TIME= 2.330YRS OXIDE FUEL= .2601GM/ML SPIKE--ND42/50 .8056-02(.24-03) ND50/42 .5778+02(.30-00)--SAMPLE--ND50/48 .5530-00(.22-02) MIXTURE--ND42/50 .8200-02(.40-04)

SPIKE I S OTOPIC DILUTI ION A NALY 5 I S

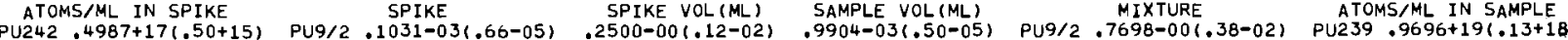

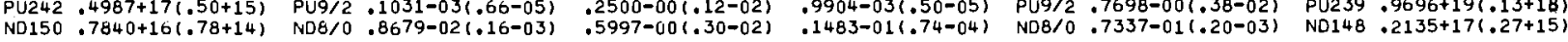
BOL U+PU $=.22931 .0023) \mathrm{GM} / M L$ AS CALCULATED BY FUEL WEIGHT AND SOLUTION VOLUME

.20\% SAMPLE--NO CONTAMINATION---MIXTURE . . $14 \%$

\begin{tabular}{|c|c|c|c|c|c|c|c|c|}
\hline $\begin{array}{l}\text { ISOTOPE } \\
U-234 \\
U-235 \\
U-236 \\
U-238 \\
\text { TOTALS }\end{array}$ & $\begin{array}{c}B O L(G M) \\
.5493-04(.487-06) \\
.6971-02(.691-05) \\
.0000 \quad(.000) \\
.9730-00(.199-03) \\
.9800-00(.200-03)\end{array}$ & \multicolumn{2}{|c|}{$\begin{array}{l}\text { ISO T } \\
\text { EOL MASS RATIO } \\
.5400-04(.250-05) \\
.6792-02(.350-04) \\
.9500-04(.250-05) \\
1.0000\end{array}$} & \multicolumn{2}{|c|}{$\begin{array}{c}\text { C INVEN T } \\
\text { EOL }(A / O) \\
.0054(.0002) \\
.6745(.0034) \\
.0094(.0002) \\
99.3107(.0399)\end{array}$} & \multicolumn{2}{|c|}{$\begin{array}{c}\text { EOL }(\mathrm{GM}) \\
.5160-04(.243-05) \\
.6517-02(.339-04) \\
.9155-04(.243-05) \\
.9718-00(.431-03) \\
.9785-00(.200-03)\end{array}$} & $\begin{array}{l}\text { NET PRODUCTION (GM) } \\
-.3332-05(.248-05) \\
-.4533-03(.346-04) \\
.9155-04(.243-05) \\
-.1135-02(.383-03) \\
-.1497-02(.381-03)\end{array}$ \\
\hline $\begin{array}{l}\text { ISOTOPE } \\
\text { PU236 } \\
\text { PU239 } \\
\text { PU240 } \\
\text { PU241 } \\
\text { PU242 } \\
\text { TOTALS }\end{array}$ & $\begin{array}{r}\text { BOL }(A / 0) \\
.0000(.0000) \\
91.1282(.0370) \\
8.0858(.0369) \\
.7450(.0065) \\
.0410(.0009)\end{array}$ & $\begin{array}{l}\text { EOL MASS RATIO } \\
.3320-03(.700-05) \\
1.0000 \\
.1322-00(.700-03) \\
.1773-01(.900-04) \\
.1052-02(.110-04)\end{array}$ & $\begin{array}{r}\text { EOL }(A / 0) \\
.02881 .000 \\
86.76631 .053 \\
11.47051 .053 \\
1.64316 .008 \\
.09131 .001\end{array}$ & & $\begin{array}{c}\text { EOL }(\mathrm{GM}) \\
.5549-051.149-0 \\
.1679-011.279-0 \\
.2228-021.388-0 \\
.3205-031.559-0 \\
.1788-041.351-0 \\
.1936-011.321-0\end{array}$ & & $\begin{array}{l}{ }^{\mathrm{BOL}(\mathrm{GM})} \\
.0000(1.000 \\
.1822-01(.182-03) \\
.1623-02(.178-04) \\
.1502-03(.199-05) \\
.8301-05(.200-06) \\
.2000-01(.200-03)\end{array}$ & $\begin{array}{l}\text { NET PRODUCTION (GM) } \\
.5549-05(.149-06) \\
-.1433-02(.333-03) \\
.6051-03(.427-04) \\
.1704-03(.594-05) \\
.9579-05(.404-06) \\
-.6420-03(.379-03)\end{array}$ \\
\hline $\begin{array}{l}\text { ISOTOPE } \\
\text { U-235 } \\
\text { U-238 } \\
\text { PU239 } \\
\text { PU241 }\end{array}$ & $\begin{array}{r}\text { FISS } \\
.3616- \\
.1472- \\
.1612- \\
.2614-\end{array}$ & $\begin{array}{l}S(G M) \\
(.3470-04) \\
(1437-04) \\
.5709-04) \\
.1277-05)\end{array}$ & $\begin{array}{c}\text { I S S I I O N S } \\
\text { BURNUP (MWD/MTM) } \\
346.6 \\
144.0 \\
1580.0 \\
25.8 \\
2006\end{array}$ & B Y & $\begin{array}{l}\text { ND } 148 \\
\text { FISSION YIELO } \\
.0169(.0001) \\
.0193(.0010) \\
.0170(.0003) \\
.0189(.0005)\end{array}$ & & $\begin{array}{l}.0766(.0077) \\
.0799(.0080)\end{array}$ & $\begin{array}{l}\text { MEV/FISSION } \\
201.7 \\
208.5 \\
210.7 \\
213.8\end{array}$ \\
\hline
\end{tabular}

U235/U235BOL $=.935(.005)$ ALPHA235 $=.2538(.0257)$ U238 INTO PU239=.9915-03(.38-03) GM U236 INTO NP237=.623-06GM

-----QUANTITIES ENCLOSED BY PARENTHESES ARE ONE STANDARD DEVIATION ERRORS-----

ELEIMENT 6066 ROD F079( 6-ROD RING) BATCH CORE THE SAMPLE WAS CUT 10.4 INCHES FROM THE TOP OF THE FUEL INPUT DATA-D- TIME OUT OF REACTOR=1.378YRS BOL PU241 DECAY CORRECTION TIME= 2.330YRS OXIDE FUEL= .2492GM/ML SPIKE--NU42/50.8056-02(.24-03) NDS0/42.5735+02(.30-00)--SAMPLE--ND50/48.5501-00(.20-02) MIXTURE--ND42/50 .7910-02(.60-04)

I SOTOPIC DILUTION A N L Y Y I I 5

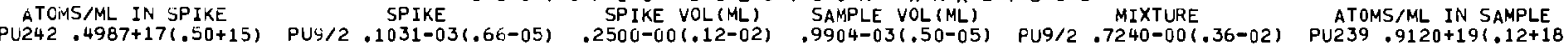
ND150 .7840+10(.78+14) ND8/0 .8679-02(.16-03) .5997-00(.30-02) $.1483-01(.74-04)$ ND8/0 .8534-01(.42-03) ND148 .2549+17(.35+15) BOL U+PU $=.2197(.0022) \mathrm{GM} / \mathrm{ML}$ AS CALCULATED BY FUEL WEIGHT AND SOLUTION VOLUME .20\% SAMPLE---ND CONTAMINATION---MIXTURE .05\%

\begin{tabular}{|c|c|c|c|c|c|c|c|c|}
\hline $\begin{array}{l}\text { ISOTOPE } \\
\text { U-234 } \\
U-235 \\
U-230 \\
U-233 \\
\text { TOTALS }\end{array}$ & $\begin{array}{r}\text { BOL }(6 M) \\
.5493-041.487 \\
.6971-021.691 \\
.0000 \quad 1.000 \\
.9730-001.199 \\
.9800-001.200\end{array}$ & $\begin{array}{l}\text { EOL MASS } \\
.5300-041 \\
.0684-021 \\
.1190-031 \\
1.0000\end{array}$ & 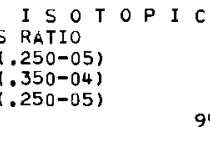 & $\begin{array}{r}\text { C I N } \\
.005 \\
.663 \\
.011 \\
99.315\end{array}$ & $\begin{array}{l}V E N T O R Y \\
L(A / 0) \\
3(.0002) \\
8(.0034) \\
B(.0002) \\
1(.0399)\end{array}$ & $\begin{array}{l}.50 \\
.64 \\
.11 \\
.97 \\
.97\end{array}$ & $\begin{array}{l}\text { OL }(G M) \\
-04(.243-05) \\
-02(.339-04) \\
-03(.243-05) \\
-00(.432-03) \\
-00(.200-03)\end{array}$ & $\begin{array}{l}\text { NET PRODUCTIO } \\
-.4305-051.24 \\
-.5592-031.34 \\
.1146-031.24 \\
-.1477-021.38 \\
-.1922-021.38\end{array}$ \\
\hline $\begin{array}{l}\text { ISOTOPE } \\
\text { PU238 } \\
\text { PU239 } \\
\text { PU240 } \\
\text { PU241 } \\
\text { PU242 } \\
\text { TOTALS }\end{array}$ & $\begin{array}{r}\text { OOL }(A / 0) \\
.0000(.0000) \\
91.1282(.0370) \\
8.0858(.0369) \\
.7450(.0065) \\
.0410(.0009)\end{array}$ & $\begin{array}{l}\text { EOL MASS RATIO } \\
.3610-03(.700-05) \\
1.0000 \\
.1437-00(.700-03) \\
.2098-01(.100-03) \\
.1275-02(.180-04)\end{array}$ & $\begin{array}{r}\text { EOL }(A / 0) \\
.03091 .000 \\
85.63521 .052 \\
12.30581 .052 \\
1.91901 .009 \\
.10921 .001\end{array}$ & $\begin{array}{l}1 \\
061 \\
201 \\
26) \\
971 \\
151\end{array}$ & $\begin{array}{c}E O L(G M) \\
.5924-051.151-0 \\
.1648-011.274-0 \\
.2378-02(.412-0 \\
.3724-031.647-0 \\
.2128-041.464-0 \\
.1926-011.320-0\end{array}$ & & $\begin{array}{c}\text { BOL (GM) } \\
.0000(.000) \\
.1822-01(.162-03) \\
.1623-02(.178-04) \\
.1502-03(.199-05) \\
.8301-05(.200-06) \\
.2000-01(.200-03)\end{array}$ & $\begin{array}{r}\text { NET PRODUC } \\
.5924-051 \\
-.1739-021 \\
.7547-031 \\
.2222-031 \\
.1297-041 \\
-.7432-031\end{array}$ \\
\hline $\begin{array}{l}\text { ISOTOPE } \\
U-235 \\
U-23 d \\
\text { PU239 } \\
\text { PU241 } \\
\text { TOTALS }\end{array}$ & $\begin{array}{r}\text { FISSI } \\
.4441-0 \\
.1835-0 \\
.2012-0 \\
.3540-0 \\
.2675=0\end{array}$ & $\begin{array}{l}S(G M) \\
(.3470-04) \\
(.1782-04) \\
(.6728-04) \\
(.1632-05) \\
(.5833-04)\end{array}$ & 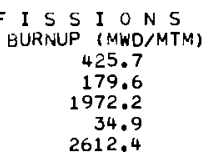 & BY & $\begin{array}{l}\text { ND } 148 \\
\text { FISSION YIELD } \\
.0169(.0001) \\
.0193(.0010) \\
.0170(.0003) \\
.0189(.0005)\end{array}$ & & $\begin{array}{c}\text { DEL } \\
.0609(.0061) \\
.0766(.0077) \\
.0799(.0080)\end{array}$ & $\begin{array}{l}\text { MEV/FISSION } \\
201.7 \\
208.5 \\
210.7 \\
213.8\end{array}$ \\
\hline
\end{tabular}

U235/U2358OL $=.920(.005)$ ALPHA235=.2592(.0213) U238 INTO PU239=.1298-02(.38-03)GM U236 INTO NP237=.972-06GM 


\section{A-8}

ELEMENT 6060 ROD F079( 6-ROD RING) BATCH CORE THE SAMPLE WAS CUT 30.9 INCHES FROM THE TOP OF THE FUEL INPUT DATA--- TIME OUT OF REACTOR=1.200YRS BOL PU241 DECAY CORRECTION TIME= 2.330YRS OXIDE FUEL= .2719GM/ML SPIKE--ND42/50.8056-02(.24-03) ND50/42.5678+02(.10+00)--SAMPLE--ND50/48.5481-00(.26-02) MIXTURE--ND42/50.8150-02(.80-04)

ISOTOPIC DILUUTION A N A L Y S I S

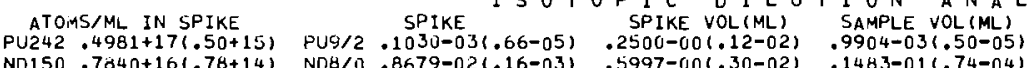

MIXTURE
ATOMS/ML IN SAMPLE ND8/0.1273-00(.60-03) ND148:4038+17(.54+15) BOL U+PU $=.2397(.0024)$ GM/ML AS CALCULATED BY FUEL WEIGHT AND SOLUTION VOLUME .20x SAMPLE---NO CONTAMINATION---MIXTURE .11\%

\begin{tabular}{|c|c|c|c|c|c|c|c|c|}
\hline $\begin{array}{l}\text { ISUTOPE } \\
U-234 \\
U-230 \\
U-230 \\
U-233 \\
\text { TOTALS }\end{array}$ & $\begin{array}{r}\text { DOL (GM) } \\
.3493-04(.48 \\
.5971-02(.69 \\
.0000 \quad 1.00 \\
.9730-001.19 \\
.9800-001.20\end{array}$ & $\begin{array}{l}\text { EOL MASS } \\
.5200-041 \\
.6375-026 \\
.1700-031 \\
1.0000\end{array}$ & $\begin{array}{l}\text { ISO TOP I C } \\
\text { KATIO } \\
.150-05) \\
.315-04) \\
.250-05)\end{array}$ & $\begin{array}{l}\text { INN } \\
\text { EO } \\
.005 \\
.633 \\
.016 \\
9.344\end{array}$ & $\begin{array}{l}V E N T O R Y \\
V(A N 0) \\
2(.0001) \\
3(.0030) \\
9(.0002) \\
6(.0399)\end{array}$ & $\begin{array}{l}.496 \\
.611 \\
.16 \\
.97 \\
.97\end{array}$ & $\begin{array}{l}O L(G M) \\
=04(.146-05) \\
=02(.305-04) \\
=03(.243-05) \\
=00(.431-03) \\
=00(.200-03)\end{array}$ & $\begin{array}{l}\text { NET PRODUCTIOI } \\
-.5273-051.15 \\
-.8573-031.31 \\
.1637-031.24 \\
-.1739-026.38 \\
-.2434-026.38\end{array}$ \\
\hline $\begin{array}{l}\text { ISOTOPE } \\
\text { PU230 } \\
\text { rU239 } \\
\text { PU240 } \\
\text { PU241 } \\
\text { PU242 } \\
\text { TOTALS }\end{array}$ & $\begin{array}{c}\delta 0 L(A / 0) \\
.0000(.0000) \\
91.1282(.0370) \\
8.0856(.0309) \\
.7450(.0005) \\
.0410(.0009)\end{array}$ & $\begin{array}{l}\text { EOL MASS RATIO } \\
.4920-03(.100-04) \\
1.0000 \\
.1703-00(.900-03) \\
.2855-01(.140-03) \\
.2031-02(.240-04)\end{array}$ & $\begin{array}{r}\text { EOL }(A / 0) \\
.04091 .0008 \\
83.12151 .063 \\
14.15561 .0642 \\
2.51321 .0128 \\
.16881 .002\end{array}$ & & $\begin{array}{c}E O L(G M) \\
.7550-03(.200-06 \\
.1541-011.260-0 \\
.2635-021.466-0 \\
.4698-031.830-05 \\
.3169-041.654-06 \\
.1855-011.314-0\end{array}$ & $\begin{array}{l}031 \\
313 \\
513 \\
031\end{array}$ & $\begin{array}{c}\mathrm{BOL}(\mathrm{GM}) \\
.0000(.000 \\
.1822-01(.182-03) \\
.1623-02(.178-04) \\
.1502-03(.199-05) \\
.8301-05(.200-06) \\
.2000-01(.200-03)\end{array}$ & $\begin{array}{l}\text { NET PRODUCT } \\
.7550-051 . \\
-.2809-021 . \\
.1012-021 . \\
.3196-031 . \\
.2339-041 . \\
-.1446-021 .\end{array}$ \\
\hline $\begin{array}{l}\text { ISOTOPE } \\
\text { U-23. } \\
\text { U-230 } \\
\text { PU239 } \\
\text { PU241 } \\
\text { TUTALS }\end{array}$ & $\begin{array}{l}\text { FISS } \\
.6921-1 \\
.2028- \\
.2933- \\
.6381- \\
.3891-1\end{array}$ & 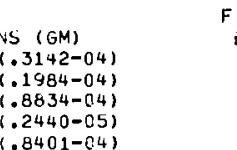 & $\begin{array}{c}\text { I S S I } I \text { O N S } \\
\text { BURNUP (MWD/MTM) } \\
663.4 \\
190.4 \\
2875.1 \\
63.0 \\
3799.8\end{array}$ & BY & $\begin{array}{l}\text { ND } 148 \\
\text { FISSION YIELD } \\
.0169(.0001) \\
.0193(.0010) \\
.0170(.0003) \\
.0189(.0005)\end{array}$ & & $\begin{array}{c}\text { DEL } \\
.0491(.0049) \\
.0563(.0056) \\
.0604(.0060)\end{array}$ & $\begin{array}{l}\text { MEV/FISSION } \\
201.7 \\
208.5 \\
210.7 \\
213.8\end{array}$ \\
\hline
\end{tabular}

U235/U23bBOL $=.077(.004)$ ALPHA235 $=.2387(.0117)$ U238 INTO PU239=.1542-02(.38-03) GM U236 INTO NP237=.220-05GM

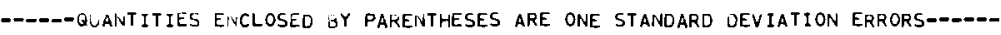

ELEMENT L06o KOD FO79( O-ROD FIING) BATCH CORE THE SAMPLE WAS CUT 47.3 INCHES FROM THE TOP OF THE FUEL

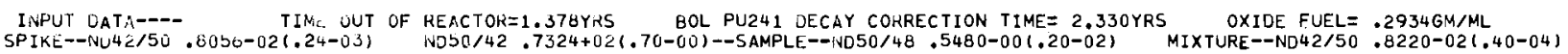

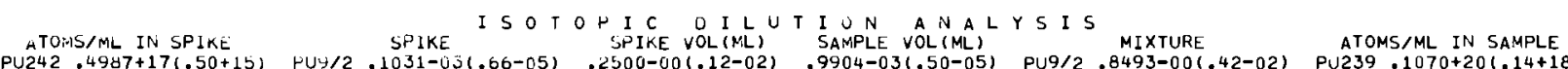

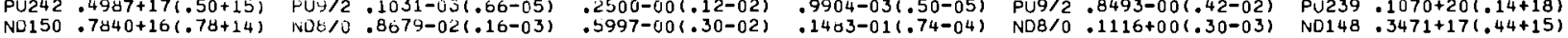
BOL U+PU $=.2580(.0026) \mathrm{GM} / \mathrm{ML}$ AS CALCULATED BY FUEL WEIGHT AND SOLUTION VOLUME

\begin{tabular}{|c|c|c|c|c|c|c|c|c|}
\hline $\begin{array}{l}\text { ISUTOPE } \\
U-234 \\
U=233 \\
U-230 \\
U-23 \dot{ } \\
\text { TOTAL'S }\end{array}$ & $\begin{array}{c}\text { DUL }(G N) \\
.5493-04(.487 \\
.0971-026.691 \\
.0000 \quad 1.000 \\
.9730-001.199 \\
.9800-001.200\end{array}$ & $\begin{array}{l}\text { EOL MASS } \\
.5100-041 \\
.0587-021 \\
.1340-031 \\
1.0000\end{array}$ & 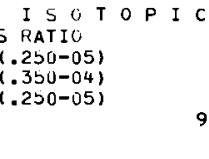 & $\begin{array}{r}\text { I I } \\
\text { ED } \\
.005 \\
.654 \\
.013 \\
99.327\end{array}$ & $\begin{array}{l}V E N T O R Y \\
(A / 0) \\
1(.0002) \\
3(.0034) \\
3(.0002) \\
4(.0399)\end{array}$ & & $\begin{array}{l}O L(G M) \\
-04(.243-05) \\
-02(.339-04) \\
=03(.243-05) \\
-00(.434-03) \\
-00(.199-03)\end{array}$ & $\begin{array}{l}\text { NET PRODUCTIO } \\
-.6238-051.24 \\
-.6552-031.34 \\
.1290-031.24 \\
-.1924-021.38 \\
-.2452-021.38\end{array}$ \\
\hline $\begin{array}{l}\text { ISOTOPE } \\
\text { PU235 } \\
\text { PU239 } \\
\text { PU240 } \\
\text { PU241 } \\
\text { PU242 } \\
\text { TOTALS }\end{array}$ & $\begin{array}{c}0 O L(A / O) \\
.0000(.0000) \\
91.1282(.0370) \\
8.0858(.0369) \\
.7450(.0065) \\
.0410(.0009)\end{array}$ & $\begin{array}{l}\text { EUL MASS RATIO } \\
.3950-03(.800-05) \\
1.0000 \\
.1517-00(.800-03) \\
.2328-01(.130-03) \\
.1470-02(.170-04)\end{array}$ & $\begin{array}{r}\text { EOL }(A / 0) \\
.0335(.000 \\
84.8587(.058 \\
12.87311 .059 \\
2.11001 .012 \\
.12471 .001\end{array}$ & $\begin{array}{l}1 \\
071 \\
861 \\
921 \\
221 \\
141\end{array}$ & $\begin{array}{c}\text { EOL }(\mathrm{GM}) \\
.6461-051.169- \\
.1642-011.273- \\
.2502-021.436- \\
.4118-031.725- \\
.2445-041.495- \\
.1937-011.322-\end{array}$ & $\begin{array}{l}061 \\
031 \\
041 \\
051 \\
061 \\
031\end{array}$ & $\begin{array}{l}{ }^{B O L}(G M) \\
.0000^{2}(.000 \\
.1822-01(.182-03) \\
.1623-02(.178-04) \\
.1502-03(.199-05) \\
.8301-05(.200-06) \\
.2000-01(.200-03)\end{array}$ & $\begin{array}{l}\text { NET PRODUCT } \\
.6461-051 . \\
-.1793-021 . \\
.8788-031 \\
.2617-031 \\
.1615-041 . \\
-.6304-031 .\end{array}$ \\
\hline $\begin{array}{l}\text { ISUTUPE } \\
\text { U-235 } \\
\text { U-230 } \\
\text { PU239 } \\
\text { PU24I } \\
\text { TOTAL'S }\end{array}$ & $\begin{array}{l}\text { FISSI } \\
.3254-0 \\
.2121-0 \\
.2312-0 \\
.4405-0 \\
.3094-0\end{array}$ & $\begin{array}{l}.5(G M) \\
(.3471-04) \\
(.2051-04) \\
.7363-04) \\
(.1617-05) \\
(.6549-04)\end{array}$ & $\begin{array}{c}\text { ISSS I ON N S } \\
\text { BURNUP (N,WD/MTM) } \\
503.6 \\
207.5 \\
2260.7 \\
43.5 \\
3021.3\end{array}$ & BY & $\begin{array}{l}\text { ND } 14{ }^{8} \\
\text { FISSION YIEL } \\
.0169(.0001) \\
.0193(.0010) \\
.0170(.0003) \\
.0189(.0005)\end{array}$ & & $\begin{array}{c}\text { DEL } \\
.0609(.0061) \\
.0766(.0077) \\
.0799(.0080)\end{array}$ & $\begin{array}{l}\text { MEV/FISSION } \\
201.7 \\
208.5 \\
210.7 \\
213.8\end{array}$ \\
\hline
\end{tabular}

U235/U235BOL $=.900(.005)$ ALPHA235=.2470(.0173) U23B INTO PU239=.1719-02(.39-03)GM U236 INTO NP237=.129-05GM 
ELEMENT 6066 ROD FE77(CENTRAL ROD) BATCH CORE THE SAMPLE WAS CUT 6.5 INCHES FROM THE TOP OF THE FUEL INPUT DATA---: TIME OUT OF REACTOR=1.378YRS BOL PU241 DECAY CORRECTION TIME= 2.330YRS OXIDE FUEL= .1614GM/ML TIME OUT OF REACTOR=1.378YRS
SPIKE--NOL $42 / 50.8056-021.24-03)$
NOSO PU2

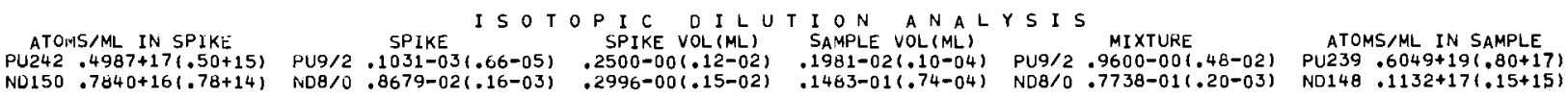
BOL U+PU $=.1423(.0014) \mathrm{GM} / \mathrm{ML}$ AS CALCULATED BY FUEL WEIGHT ANO SOLUTION VOLUME .34\% SAMPLE---ND CONTAMINATION---MIXTURE . $44 \%$

\begin{tabular}{|c|c|c|c|c|c|c|c|c|}
\hline $\begin{array}{l}\text { ISOTOPE } \\
U-234 \\
U-235 \\
U-236 \\
U-234 \\
\text { TOTALS }\end{array}$ & \multicolumn{5}{|c|}{ 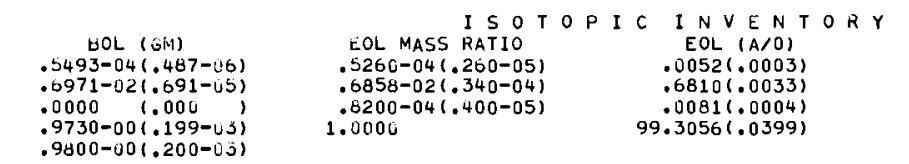 } & \multicolumn{2}{|c|}{$\begin{array}{l}E O L(G M) \\
.5027-04(.253-05) \\
.6582-02(.329-04) \\
.7904-04(.389-05) \\
.9721-00(.431-03) \\
.9788-00(.200-03)\end{array}$} & $\begin{array}{l}\text { NET PRODUCTIOI } \\
-.4658-051.25 \\
-.3885-031.33 \\
.7904-041.38 \\
-.9151-031.38 \\
-.1225-021.38\end{array}$ \\
\hline $\begin{array}{l}\text { ISOTOPE } \\
\text { PU230 } \\
\text { PU239 } \\
\text { PU240 } \\
\text { PU241 } \\
\text { PU242 } \\
\text { TOTALS }\end{array}$ & $\begin{array}{r}\text { BOL }(A / 0) \\
.0263(.0006) \\
90.5182(.0375) \\
8.2281(.0374) \\
1.1478(.0070) \\
.0796(.0018)\end{array}$ & $\begin{array}{l}\text { EOL IMASS RATIO } \\
.4690-03(.110-04) \\
1.0000 \\
.1254-00(.600-03) \\
.2001-01(.100-03) \\
.1476-02(.230-04)\end{array}$ & $\begin{array}{r}E O L(A / 0) \\
.04081 .0010 \\
87.05361 .0463 \\
10.91651 .0465 \\
1.86061 .0098 \\
.12851 .002\end{array}$ & & $\begin{array}{l}E O L(G M) \\
.7882-051.227-0 \\
.1688-011.280-0 \\
.2125-021.367-0 \\
.3637-031.635-0 \\
.2522-04(.575-0 \\
.1940-011.322-0\end{array}$ & & $\begin{array}{c}\text { BOL (GM) } \\
.5244-05(.137-06) \\
.1810-01(.181-03) \\
.1652-02(.181-04) \\
.2314-03(.271-05) \\
.1611-04(.396-06) \\
.2000-01(.200-03)\end{array}$ & $\begin{array}{l}\text { NET PRODUCT } \\
.2638-051 . \\
-.1219-021 . \\
.4734-031 . \\
.1323-031 . \\
.9116-051 . \\
-.6020-031 .\end{array}$ \\
\hline $\begin{array}{l}\text { ISOTOPE } \\
\text { U-235 } \\
\text { U-238 } \\
\text { PU239 } \\
\text { PU241 } \\
\text { TOTALS }\end{array}$ & $\begin{array}{r}\text { FISSI } \\
.3094-0 \\
.1343-0 \\
.1365-0 \\
.2487-0 \\
.1833-0\end{array}$ & $\begin{array}{l}\text { Y }(G M) \\
.3389-04) \\
(1312-C 4) \\
(5148-C 4) \\
(2001-05) \\
3695-04)\end{array}$ & $\begin{array}{c}\text { I SSS I O NS } \\
\text { BURNUF (MWD/MTM) } \\
296.5 \\
131.4 \\
1337.8 \\
24.5 \\
1790.3\end{array}$ & $B Y$ & $\begin{array}{l}\text { ND } 148 \\
\text { FISSION YIELD } \\
.0169(.0001) \\
.0193(.0010) \\
.0170(.0003) \\
.0189(.0005)\end{array}$ & & $\begin{array}{c}\text { DEL } \\
.0636(.0064) \\
.0826(.0083) \\
.0855(.0085)\end{array}$ & $\begin{array}{c}\text { MEV/FISSI ON } \\
201.7 \\
208.5 \\
210.7 \\
213.8\end{array}$ \\
\hline
\end{tabular}

U235/U235BOL $=.944(.005)$ ALPHA235 $=.25591 .0320)$ U238 INTO PU239=.7840-03(.38-03) GM U236 INTO NP237=.458-06GM ------QuaniIIIES ENCLOSED BY PARENTHESES ARE ONE STANDARD DEVIATION ERRORS------

ELENENT $6060^{\circ}$ ROD FE77(CENTRAL RUD) BATCH CORE THE SAMPLE WAS CUT 30.3 INCHES FROM THE TOP OF THE FUEL

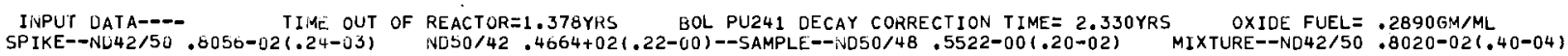
I SOTOPIC DILUT ION A N A L Y S IS ATOMS/ML IN SPIKE SPIKE ISO TSE VOL (ML) SAMPLE VOL (ML)

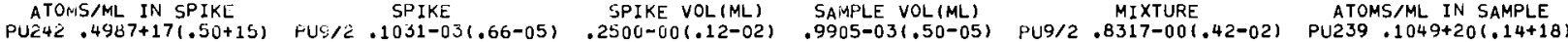

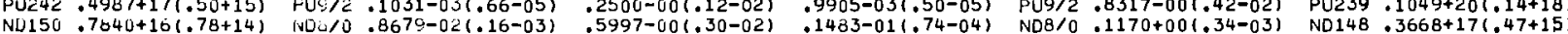
BOL U+PU $=.2546(.0025) \mathrm{GM} / \mathrm{ML}$ AS CALCULATED GY FUEL WEIGHT AND SOLUTION VOLUME

\begin{tabular}{|c|c|c|c|c|c|c|c|c|}
\hline $\begin{array}{l}\text { ISOTOPE } \\
U-234 \\
U-235 \\
U-230 \\
U-235 \\
\text { TOTALS }\end{array}$ & $\begin{array}{c}00 L(0 M) \\
.0493-04(.487-00) \\
.0971-02(.691-05) \\
.0000(.000) \\
.9730-00(.199-03) \\
.9800-00(.200-03)\end{array}$ & \multicolumn{2}{|c|}{$\begin{array}{l}\text { ISOT } \\
\text { COL MASS RATIC } \\
.540 G-04(.250-05) \\
.6542-02(.300-04) \\
.1540=03(.200-05) \\
1.0000\end{array}$} & \multicolumn{2}{|c|}{$\begin{array}{c}\text { C IN } N \text { N } N T \\
E O L(A / 0) \\
.0054(.0002) \\
.6498(.0029) \\
.0153(.0002) \\
99.3295(.0398)\end{array}$} & \multicolumn{2}{|c|}{$\begin{array}{c}\text { EOL }(G M) \\
.5154-04(.243-05) \\
.6271-02(.290-04) \\
.1482-03(.195-05) \\
.9708-00(.436-03) \\
.9772-00(.199-03)\end{array}$} & $\begin{array}{l}\text { NET PRODUCTI I } \\
-.3388-051.248 \\
-.7002-031.299 \\
.1482-031.195 \\
-.2204-021.388 \\
-.2757-021.387\end{array}$ \\
\hline $\begin{array}{l}\text { ISUTOFE } \\
\text { PU230 } \\
\text { PU23\% } \\
\text { PU24U } \\
\text { PU241 } \\
\text { PU24L } \\
\text { TOTALS }\end{array}$ & $\begin{array}{c}\text { dOL }(A / 0) \\
.0253(.0006) \\
90.0182(.0375) \\
8.2281(.0374) \\
1.1478(.0070) \\
.0790(.0018)\end{array}$ & $\begin{array}{l}\text { EJL MaSS RATIO } \\
.0530-03(.130-04) \\
1.0000 \\
.1555-00(.800-03) \\
.2928-01(.150-03) \\
.2322-02(.230-04)\end{array}$ & $\begin{array}{r}\text { EOL }(A / 0) \\
.05491 .001 \\
64.05141 .057 \\
13.07001 .058 \\
2.62861 .014 \\
.19521 .001\end{array}$ & & $\begin{array}{c}\text { EOL (GM) } \\
.1062-041.276-c \\
.1634-011.272- \\
.2551-021.444- \\
.5153-031.902- \\
.3842-041.744- \\
.1945-011.324-\end{array}$ & $\begin{array}{l}06) \\
03) \\
043 \\
05) \\
06) \\
03)\end{array}$ & $\begin{array}{c}\text { BOL (GM) } \\
.5244-05(.137-06) \\
.1810-01(.181-03) \\
.1652-02(.181-04) \\
.2314-03(.271-05) \\
.1611-04(.396-06) \\
.2000-01(.200-03)\end{array}$ & $\begin{array}{l}\text { NET PRODUCTI } \\
.5381-051.3 \\
-.1757-021.3 \\
.8995-031.4 \\
.2839-031.0 \\
.2231-041.8 \\
-.5456-031.3\end{array}$ \\
\hline $\begin{array}{l}\text { ISOTOPE } \\
\text { U-235 } \\
\text { U-230 } \\
\text { PU239 } \\
\text { PU241 } \\
\text { TOTALS }\end{array}$ & $\begin{array}{l}\text { FYSS } \\
.5510- \\
.2431- \\
.2400- \\
.6087- \\
.3315-\end{array}$ & $\begin{array}{l}b(G M) \\
.2998-04) \\
.2333-04) \\
7004-04) \\
.2746-05) \\
.7024-041\end{array}$ & $\begin{array}{c}\text { F I } 55 \text { I ON } 5 \\
\text { DUENNUP (RiWD/MTM) } \\
528.1 \\
237.8 \\
2412.2 \\
60.1 \\
3238.2\end{array}$ & B & $\begin{array}{l}\text { ND } 148 \\
\text { FISSION YIELL } \\
.0169(.0001) \\
.0193(.0010) \\
.0170(.0003) \\
.0189(.0005)\end{array}$ & & $\begin{array}{c}\text { DEL } \\
.0636(.0064) \\
.0826(.0083) \\
.0855(.0085)\end{array}$ & $\begin{array}{c}\text { MEV/FISSION } \\
201.7 \\
208.5 \\
210.7 \\
213.8\end{array}$ \\
\hline
\end{tabular}

U235/U2350L $=.900(.004)$ ALFHA235 $=.27061 .0154)$ U238 INTO PU239=.1969-021.39-03)GM U236 INTO NP237=.160-05GM 


\section{A-10}

ELEMENT OU6Ó ROD FE77(CËNTRAL ROD) BATCH CORE THE SAMPLE WAS CUT 47.6 INCHES FROM THE TOP OF THE FUEL

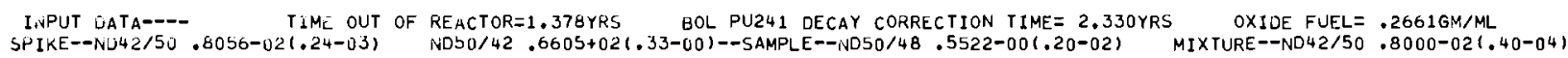
I S O T OP I C D I L U T I ON A N A L Y S I S

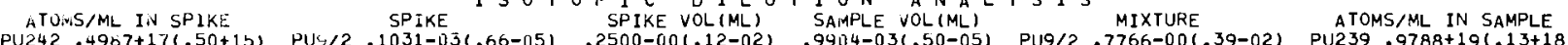

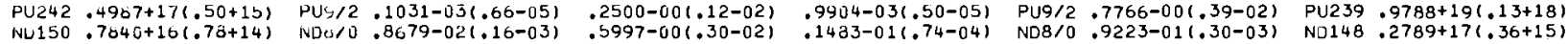
BÜL U+PUE $=.2346(.0023) \mathrm{GM} / \mathrm{ML}$ AS CALCULATED BY FUEL WEIGHT AND SOLUTION VOLUME

.18\% SAMPLE---iND CONTAMINATION---MIXTURE .08\%

\begin{tabular}{|c|c|c|c|c|c|c|c|c|}
\hline $\begin{array}{l}\text { ISUTOHE } \\
U-234 \\
U-230 \\
\text { J-230 } \\
\text { U-23. } \\
\text { TUTALS }\end{array}$ & 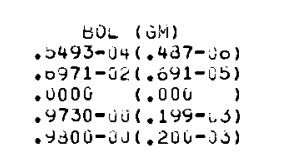 & $\begin{array}{l}\text { EOL MASS } \\
.5300-041 \\
.6709-021 \\
.1250-031 \\
1.0000\end{array}$ & $\begin{array}{l}\text { ISO T O P I C } \\
\text { SATIO } \\
(.250-U 5) \\
(.350-04) \\
(.200-05)\end{array}$ & \multicolumn{2}{|c|}{$\begin{array}{c}\text { INVENT } \\
\text { EOL (A/O) } \\
.0053(.0002) \\
.6663(.0034) \\
.0124(.0002) \\
99.3160(.0399)\end{array}$} & \multicolumn{2}{|c|}{$\begin{array}{c}\text { EOL (GM) } \\
.5061-04(.243-05) \\
.6434-02(.339-04) \\
.1204-03(.195-05) \\
.9712-00(.434-03) \\
.9778-00(.200-03)\end{array}$} & $\begin{array}{l}\text { NET PRODUCTION (GM) } \\
-.4319-05(.248-05) \\
-.5371-03(.346-04) \\
.1204-03(.195-05) \\
-.1753-02(.386-03) \\
-.2170-02(.385-03)\end{array}$ \\
\hline $\begin{array}{l}\text { ISOTOFE } \\
\text { PU230 } \\
\text { PU239 } \\
\text { PU24U } \\
\text { PU241 } \\
\text { PU24L } \\
\text { TOTALS }\end{array}$ & $\begin{array}{c}\text { UUL }(\mathrm{A} / 0) \\
.0263(.0006) \\
90.0182(.0375) \\
6.2281(.0374) \\
1.1478(.0070) \\
.0790(.0018)\end{array}$ & $\begin{array}{l}\text { EOL MASS RATIO } \\
.0030-03(.140-04) \\
1.0000 \\
.1432-00(.700-03) \\
.2547-01(.130-03) \\
.1939-02(.190-04)\end{array}$ & $\begin{array}{r}\text { EOL }(\mathrm{A} / 0) \\
.05141 .001 \\
\mathrm{d5} .25541 .052 \\
12.20861 .052 \\
2.31931 .012 \\
.16531 .001\end{array}$ & $\begin{array}{l}12) \\
\text { 20) } \\
24) \\
24) \\
16)\end{array}$ & $\begin{array}{c}E O L(G M) \\
.9946-05(.284-0 \\
.1056-01(.275-0 \\
.2382-02(.413-0 \\
.4544-031.794-0 \\
.3252-041.628-0 \\
.1944-011.323-0\end{array}$ & $\begin{array}{l}061 \\
031 \\
041 \\
05) \\
06) \\
03)\end{array}$ & $\begin{array}{c}30 L(6 M) \\
.5244-05(.137-06) \\
.1810-01(.181-03) \\
.1652-02(.181-04) \\
.2314-03(.271-05) \\
.1611-04(.396-06) \\
.2000-01(.200-03)\end{array}$ & $\begin{array}{l}\text { NET PRODUCTION (GM) } \\
.4702-05(.315-06) \\
-.1532-02(.330-03) \\
.7301-03(.451-04) \\
.2230-03(.839-05) \\
.1641-04(.742-06) \\
-.5576-03(.380-03)\end{array}$ \\
\hline $\begin{array}{l}\text { ISOTOPE } \\
\text { U-230 } \\
\text { U-23U } \\
\text { PU23S } \\
\text { PUS241 } \\
\text { TCTALS }\end{array}$ & $\begin{aligned} & \text { FISS } \\
& .+162-c \\
& .2014-0 \\
& .2075-6 \\
& .4479-0 \\
& .2738-\end{aligned}$ & 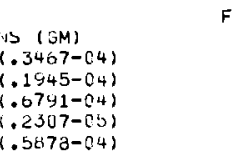 & $\begin{array}{c}\text { I S S I ONS } \\
\text { DURIUP (M/WD/MTM) } \\
399.0 \\
197.0 \\
2035.3 \\
44.2 \\
2675.6\end{array}$ & B Y & $\begin{array}{l}\text { ND } 148 \\
\text { FISSION YIELD } \\
.0169(.0001) \\
.0193(.0010) \\
.0170(.0003) \\
.0189(.0005)\end{array}$ & & $\begin{array}{c}\text { DEL } \\
.0636(.0064) \\
.0826(.0083) \\
.0855(.0085)\end{array}$ & $\begin{array}{l}M E V / F I S S I O N \\
201.7 \\
208.5 \\
210.7 \\
213.8\end{array}$ \\
\hline
\end{tabular}

U235/U2358OL $=.923(.005)$ ALPHA235 $=.2903(.0249)$ U238 INTO PU239 $=.1558-02(.39-03)$ GM U236 INTO NP237=.978-06GM

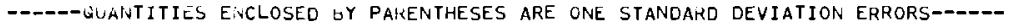

ELEHENT UOLS ROC FSQ1(12-RCU RIIVG) BATCH CORE THE SAMPLL WAS CUT 28.9 INCHES FROM THE TOP OF THE FUEL

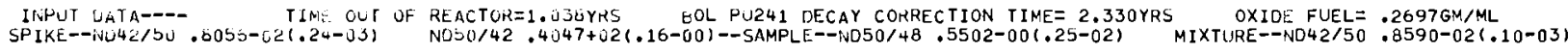

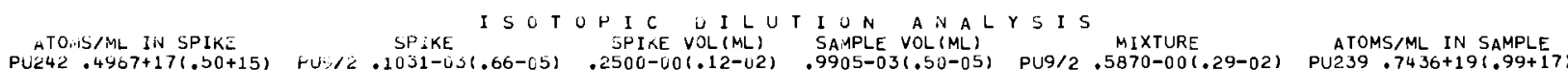

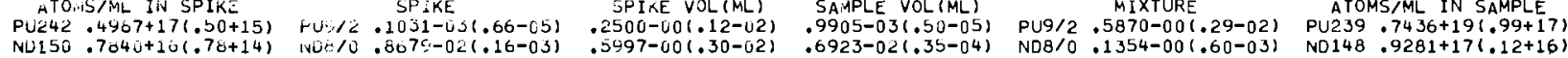
BUL U+PU $=.2377(.0024) \Theta M / M L$ AS CALCLLLATEU SY FUEL WEIGHT AIVO SOLUTIUN VOLUME. .29\% SAMPLE---IND CONTAMINATION---MIXTURE $.18 \%$

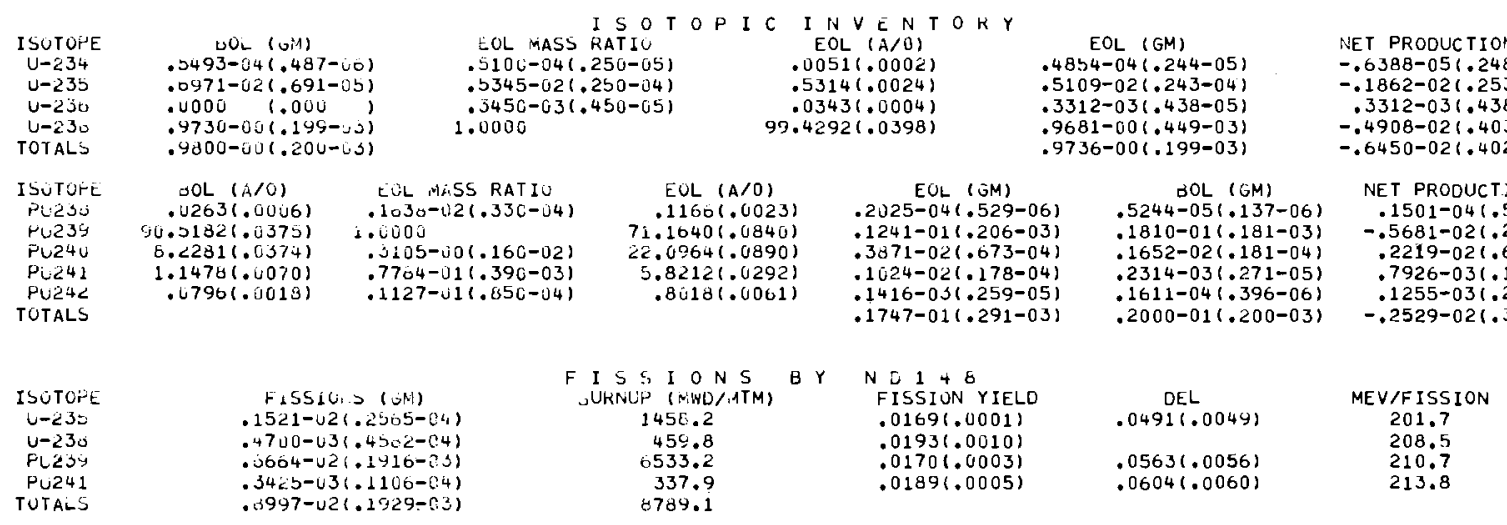

U2Jb/U2350OL=.73S1.0U4) ALIHA235=.22391.00D1) U238 INTO PU239=.44b7-02(.40-03)GM U236 INTO NP237=.108-04GM 


\section{A-11}

ELEMENT 6067 ROD FS23(12-ROD RING) BATCH CORE THE SAMPLE WAS CUT 6.5 INCHES FROM THE TOP OF THE FUEL INPUT DATA-D-- TIME OUT OF REACTOR=1.038YRS BOL PU24I DECAY CORRECTION TIME= 2.33OYRS OXIDE FUEL= .2591GM/ML SPIKE--NO42/50.8056-02(.24-03) NC50/42 .3746+02(.37-00)--SAMPLE--ND50/48 .5502-00(.20-02) MIXTURE- ND42/50 .7900-02(.40-04)

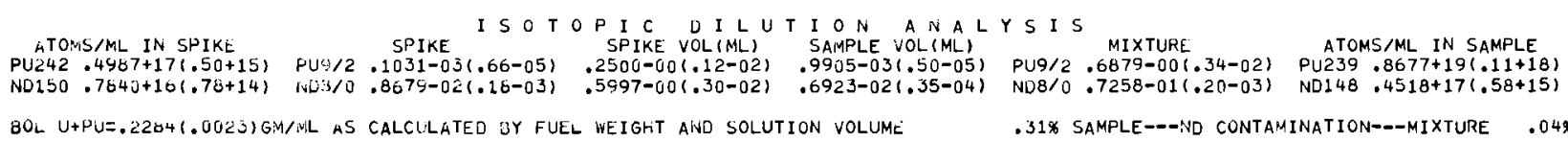

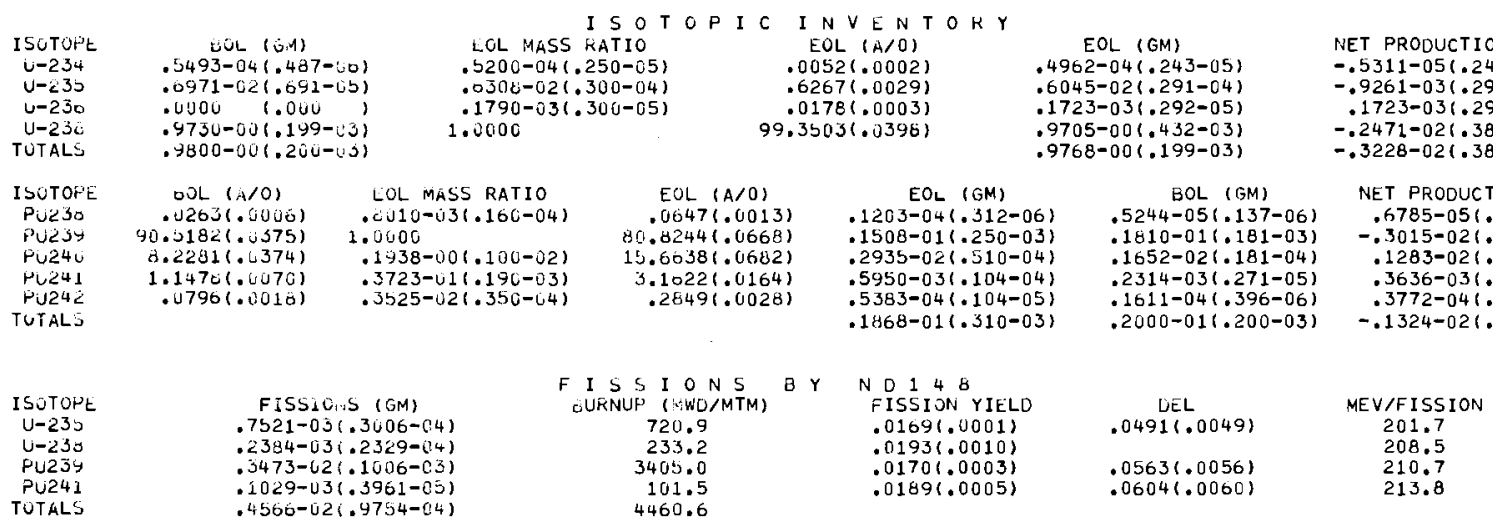

U2SS/U2350OL $=.867(.004)$ ALPHA235 $=.2314(.0104)$ U238 INTO PU239=.2242-02(.38-03)GM U236 INTO NP237=.251-05GM

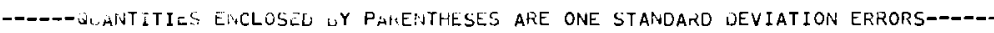

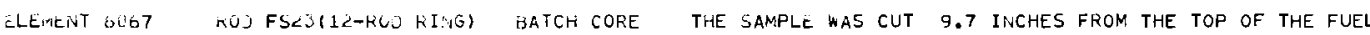

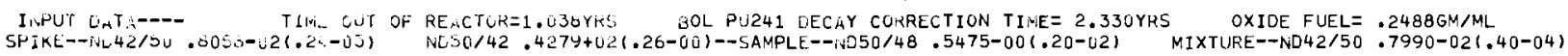
I 5 OTOPIC UILUT ION ANALYSIS $M T O M S / M L I N S P I K C$
$P U \angle 42.4407+17(.550+15)$ BUL U+PU $=.2190(.002$ C) GIM/ML AS IALCULATEO GY FLEL NEIGHT AND SOLUTION VOLUME

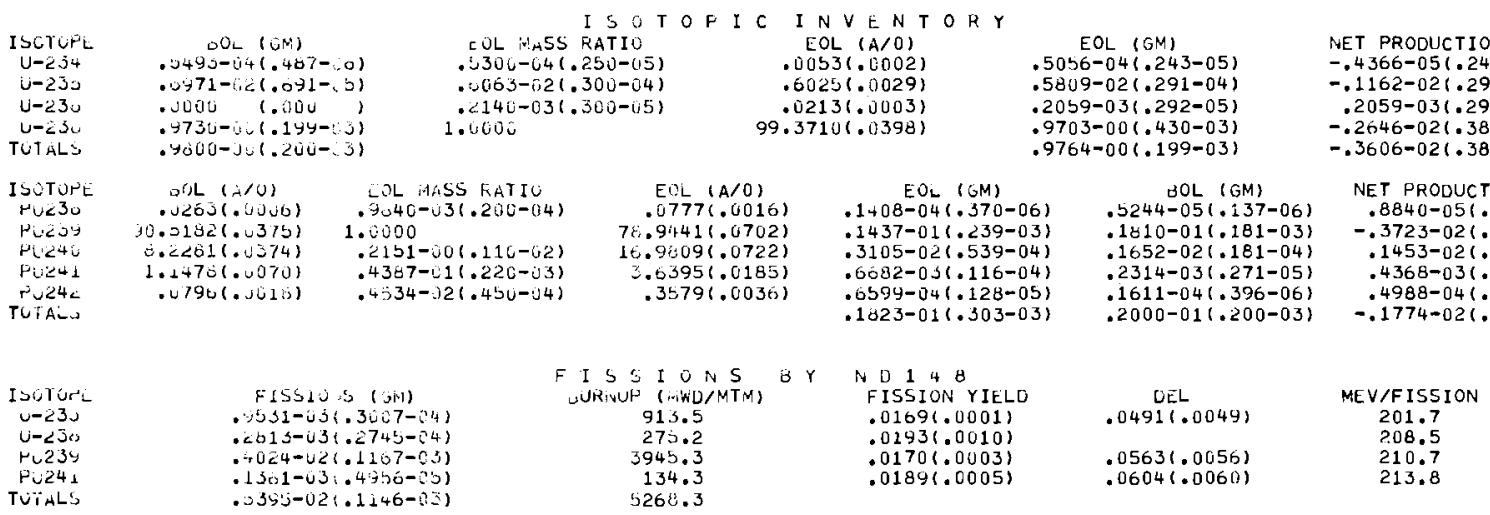




\section{A-12}

ELEMENT 6067 ROD FS23(12-ROD RING) BATCH CORE THE 5AMPLE WAS CUT 29.1 INCHES FROM THE TOP OF THE FUEL IINPUT DATA-D-D TIME. OUT OF REACTOR=1.038YRS BOL PU241 DECAY CORRECTION TIME $2.330 Y$ YRS OXIDE FUEL= .2713GM/ML SPIKE--NU42/50.8050-02(.24-03) OF NDS0/42.2735+02(.20-00)--SAMPLE--ND50/48.5508-006.30-02) MIXTURE--ND42/50 :8280-021.40-04)

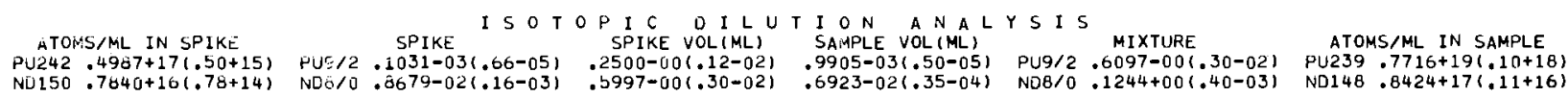
BOL U+PUE $=.2392(.0024) \mathrm{GM} / \mathrm{ML}$ AS CALCULATED BY FUEL WEIGHT AND SOLUTION VOLUME

.43\% SAMPLE---ND CONTAMINATION---MIXTURE .13\%

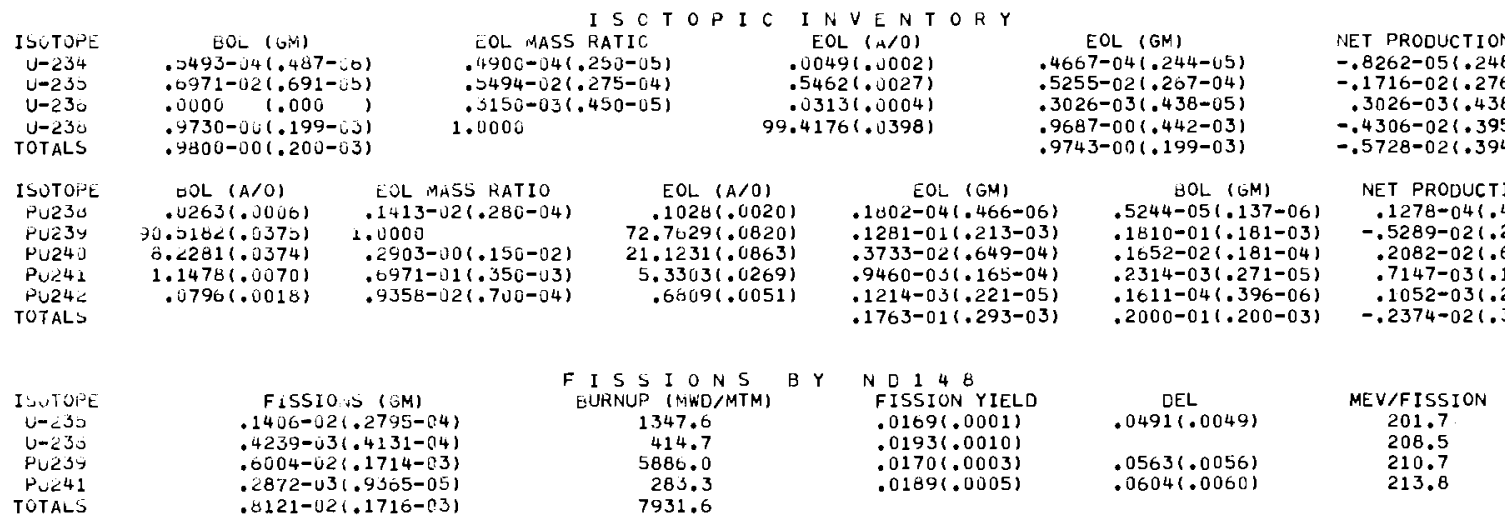

ULSS/U235JOL $=.734(.004)$ ALIHA235 $=.22061 .0058)$ U238 INTO PU239=.3898-02(.39-03)GM U236 INTO NP237=.895-05GM

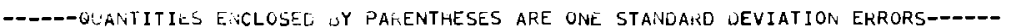

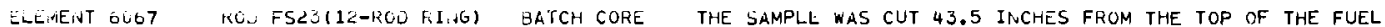

INPUT UATA---- TLMZ UUT OF REACTOR=1.26OYKS BOL PU241 DECAY COKRECTION TIME= 2.330YRS OXIDE FUEL= .2833GM/ML

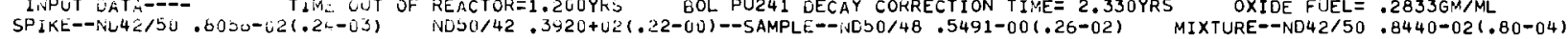
I S O T O F F I C D ILU U T I O N A IN A L Y S I 5

ATOHS/ML IN SPIKL SPIKE SPIKE VOL (ML) SAMPLE VOL(ML)

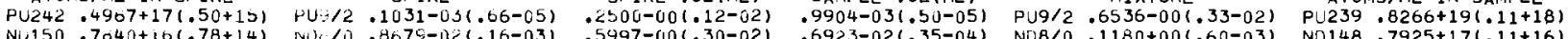
BOL J+PU $=.2497(.0025) G, M / M, L$ AS CALCULATED BY FUEL IEEIGMT AIND SOLUTION VOLUME

.30\% SAMPLE---ND CONTAMINATION---MIXTURE

$.17 \%$

\begin{tabular}{|c|c|c|c|c|c|c|c|c|}
\hline $\begin{array}{l}\text { ISJTOPE } \\
U-234 \\
U-230 \\
U-230 \\
U=230 \\
\text { TOTALS }\end{array}$ & $\begin{array}{c}\mathrm{DOL}(0 \mathrm{U}) \\
.0495-04(.487-j 0) \\
.0971-02(.691-53) \\
.0000(.000) \\
.9730-00(.199-00) \\
.9800-01(.200-03)\end{array}$ & \multicolumn{2}{|c|}{$\begin{array}{l}\text { ISO T O } \\
\text { ECL MASS TRATIO } \\
.2100-04(.150-05) \\
.5671-02(.265-04) \\
.2080-03(.450-05) \\
1.0000\end{array}$} & \multicolumn{2}{|c|}{$\begin{array}{c}C I N V E N T \\
\text { EOL }(A / 0) \\
.0051(.0001) \\
.5637(.0026) \\
.0286(.0004) \\
99.4026(.0398)\end{array}$} & \multicolumn{2}{|c|}{$\begin{array}{c}\text { EOL (GM) } \\
.4860-04(.146-05) \\
.5428-02(.257-04) \\
.2708-03(.438-05) \\
.9693-00(.438-03) \\
.9751-00(.199-03)\end{array}$} & $\begin{array}{l}\text { NET PRODUCT ION } \\
-.6324-051.154 \\
-.1543-021.267 \\
-.2768-031.438 \\
-.3633-021.390 \\
-.4907-021.38\end{array}$ \\
\hline $\begin{array}{l}\text { ISOTOAE } \\
\text { PU230 } \\
\text { PU239 } \\
\text { PU24U } \\
\text { PU24i } \\
\text { PU24L } \\
\text { TOTAL'S }\end{array}$ & $\begin{array}{c}\text { bül }(a / 0) \\
.0263(.0006) \\
30.5182(.0375) \\
0.2281(.0374) \\
1.1470(.0070) \\
.0796(.0013)\end{array}$ & $\begin{array}{l}\text { EUL NASS RATIU } \\
.1434-U 2(.240-04) \\
1.0000 \\
.2639-10(.130-02) \\
.0179-01(.300-03) \\
.7451-02(.750-04)\end{array}$ & $\begin{array}{r}E C L(A / 0) \\
.10721 .001 \\
74.72601 .075 \\
19.72021 .078 \\
4.83991 .024 \\
.55681 .0056\end{array}$ & $\begin{array}{l}\text { (a) } \\
\text { 31) } \\
\text { 32) } \\
\text { (2) } \\
\text { 56) }\end{array}$ & $\begin{array}{c}\text { EOL (GM) } \\
.1876-041.443-0 \\
.1314-011.219-0 \\
.3482-021.004-0 \\
.8069-031.151-0 \\
.9912-0+1.193-0 \\
.1760-011.293-0\end{array}$ & $\begin{array}{l}61 \\
31 \\
41 \\
51 \\
31\end{array}$ & $\begin{array}{c}\text { BOL (GM) } \\
.5244-05(.137-06) \\
.1810-01(.181-03) \\
.1652-02(.181-04) \\
.2314-03(.271-05) \\
.1611-04(.396-06) \\
.2000-01(.200-03)\end{array}$ & $\begin{array}{l}\text { NET PRODUCT } \\
.1352-041.2 \\
-.4958-021.2 \\
.1830-021 . \\
.6356-031 . \\
.3302-041 . \\
-.2396-021 .\end{array}$ \\
\hline $\begin{array}{l}\text { ISLTORE } \\
\text { U-230 } \\
\text { U-230 } \\
\text { PLE3Y } \\
\text { PUE41 } \\
\text { TOTALS }\end{array}$ & $\begin{aligned} F+S S 1 \\
.1200- \\
.38<0-0 \\
.2450-0 \\
.2205-0 \\
.7319-0\end{aligned}$ & $\begin{array}{l}(5(0) 1) \\
(.2702-84) \\
.3726-04) \\
1582-83) \\
.7751-05) \\
.1586-(3)\end{array}$ & 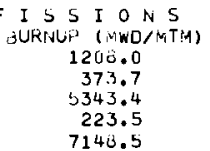 & BY & $\begin{array}{l}\text { ND } 148 \\
\text { FISSIUN YIELD } \\
.0169(.0001) \\
.0193(.0010) \\
.0170(.0003) \\
.0189(.0005)\end{array}$ & & $\begin{array}{c}\text { DEL } \\
.0491(.0049)\end{array}$ & $\begin{array}{l}\text { MEV/FISSION } \\
201.7 \\
208.5 \\
210.7 \\
213.8\end{array}$ \\
\hline
\end{tabular}

U235/U2350UL $=.779(.004)$ ALFHA235 $=.22441 .0064)$ U238 INTO PU239=.3265-021.39-03) GM U236 INTO NP237=.721-05GM 


\section{A-13}

ELEMENT 0067 HOD FS23(12-RUD RING) BATCH CORE THE SAMPLE WAS CUT 48.5 INCHES FROM THE TOP OF THE FUEL

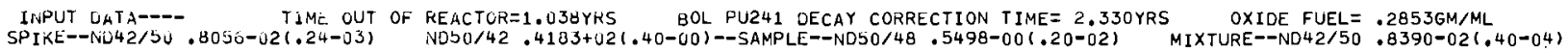
I S O T O P I C D I L U T I ON A N A L Y S I S

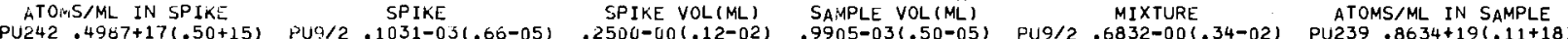

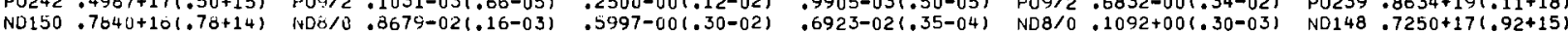
BOL U+PUE $=.2513(.0023)$ GHIML AS CALCULATED BY FUEL WEIGHT ANO SOLUTION VOLUME

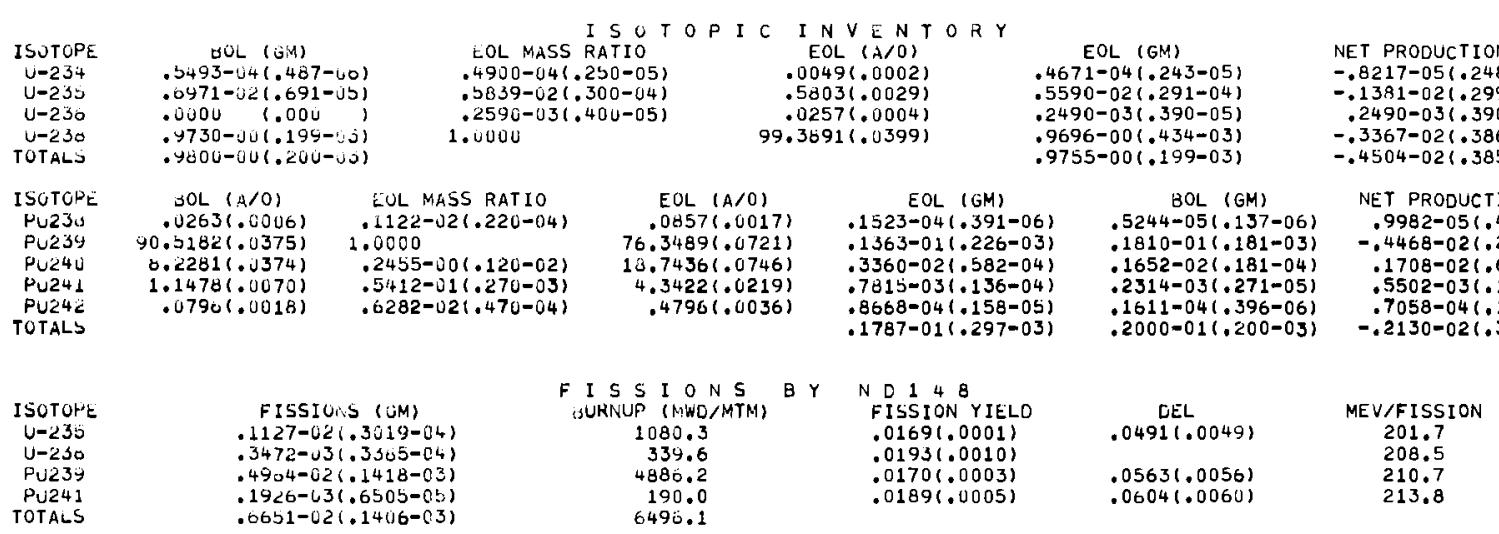

U235/UCS5EGL $=.8026 .004)$ ALPHA235=.22301.0073) U238 IIVTO PU239=.3033-02(.39-03)GM U236 INTO NP237=.570-05GM

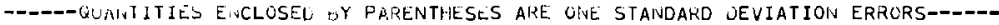

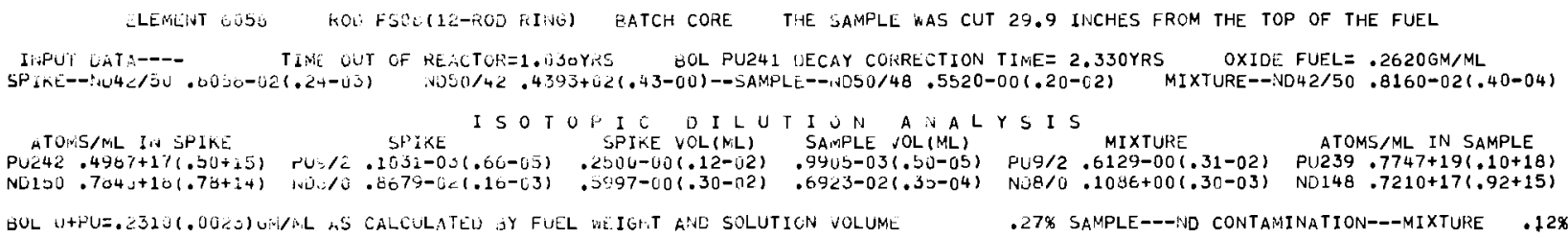

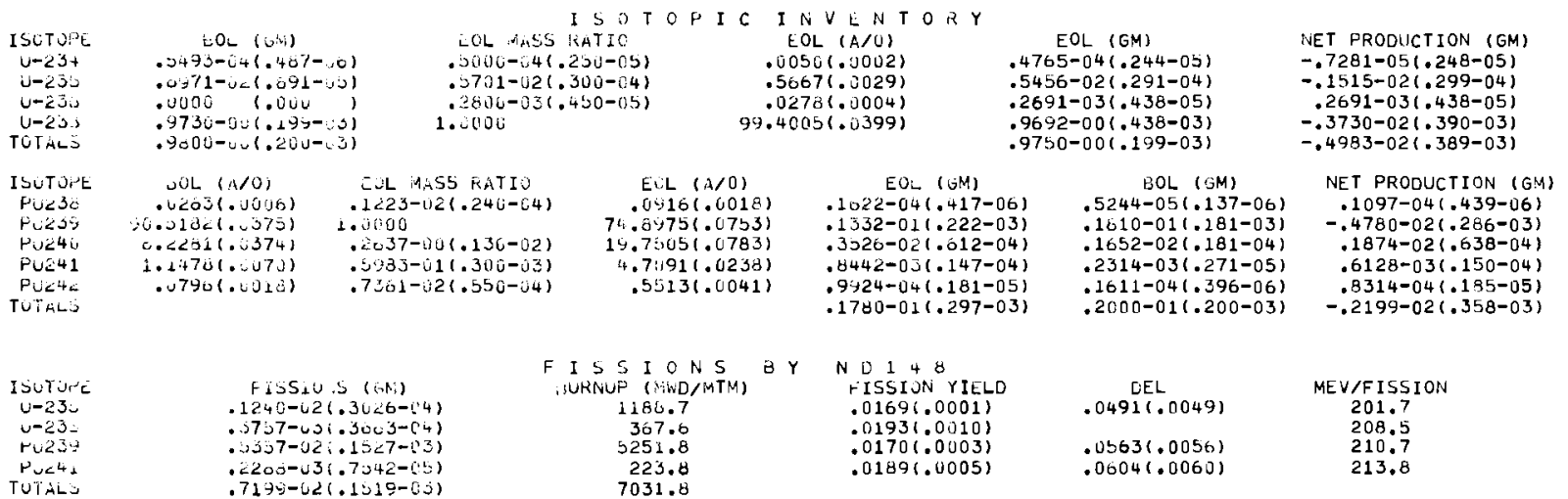

ULSb/UC20SULL $=.7 .01 .004)$ ALFtiA23b $=.2210(.0009)$ U238 INTO PU239=.3368-02(.39-03) GM U236 INTO NP237=.686-05GM 


\section{A-14}

ELEMENT 6516 ROD FR64(12-ROD RING) BATCH CORE THE SAMPLE WAS CUT 30.5 INCHES FROM THE TOP OF THE FUEL INPUT DATA--- TIME OUT OF REACTOR=1.200YRS BOL PU241 DECAY CORRECTION TIME=-2.100YRS OXIDE FUEL= .2987GM/ML SPIKE--NU42/50.8056-02(.24-03) ND50/42.4526+02(.80-00)--SAMPLE--ND50/48.5521-00(.20-02) MIXTURE--ND42/50:8120-02(.40-04)

I SOTOPIC DILUT ION A N A L Y S IS

ATOMS/ML IN SPIKE SPIKE SPIKE VOL (ML) SAMPLE VOL (ML)

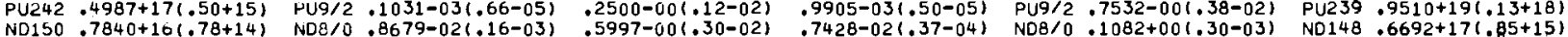
BOL U+PU $=.2633(.0026) \mathrm{GM} / \mathrm{ML}$ AS CALCULATED BY FUEL WEIGHT AND SOLUTION VOLUME

$.26 \%$ SAMPLE---ND CONTAMINATION---MIXTURE . .11X

\begin{tabular}{|c|c|c|c|c|c|c|c|c|}
\hline $\begin{array}{l}\text { ISOTOPE } \\
U-234 \\
U-235 \\
U-230 \\
U-236 \\
\text { TOTALS }\end{array}$ & \multicolumn{5}{|c|}{$\begin{array}{l}\text { I SOT } \\
\text { EOL MASS RATIO } \\
.5000-04(.250-05) \\
.5950-02(.300-04) \\
.2300-03(.400-05) \\
1.0000\end{array}$} & \multicolumn{2}{|c|}{$\begin{array}{c}\text { EOL (GM) } \\
.4767-04(.243-05) \\
.5698-02(.291-04) \\
.2212-03(.390-05) \\
.9698-00(.435-03) \\
.9758-00(.199-03)\end{array}$} & $\begin{array}{l}\text { NET PRODUCT IO } \\
-.7252-051.24 \\
-.1273-021.29 \\
.2212-031.39 \\
-.3141-021.38 \\
-.4197-021.38\end{array}$ \\
\hline $\begin{array}{l}\text { PTOPE } \\
-\cup 230 \\
\text { PU239 } \\
\text { PU240 } \\
\text { PU241 } \\
\text { PU242 } \\
\text { TUTALS }\end{array}$ & $\begin{array}{c}\text { BOL }(A / O) \\
.0000(.0000) \\
91.0504(.0381) \\
6.0865(.0377) \\
.8111(.0084) \\
.0520(.0013)\end{array}$ & $\begin{array}{l}\text { EOL MASS RATIO } \\
.7330-03(.150-04) \\
1.0000 \\
.2252-00(.110-02) \\
.4558-01(.230-03) \\
.4378-02(.440-04)\end{array}$ & $\begin{array}{r}\text { EOL }(\mathrm{A} / 0) \\
.05731 .0012 \\
78.21161 .0691 \\
17.61331 .0710 \\
3.77541 .0195 \\
.34241 .0034\end{array}$ & & $\begin{array}{c}\text { EOL (GM) } \\
.1046-04(.276-0 \\
.1434-011.238-0 \\
.3242-02(.562-0 \\
.6978-031.122-0 \\
.6355-04(.124=0 \\
.1835-01(.306-0\end{array}$ & & $\begin{array}{c}\text { BOL (GM) } \\
.0000 \quad(.000 \\
.1820-01(.182-03) \\
.1623-02(.179-04) \\
.1635-03(.234-05) \\
.1054-04(.276-06) \\
.2000-01(.200-03)\end{array}$ & $\begin{array}{l}\text { NET PRODUCT } \\
.1046-041 . \\
-.3867-021 . \\
.1618-021 . \\
.5343-031 . \\
.5301-041 . \\
-.1651-021 .\end{array}$ \\
\hline $\begin{array}{l}\text { ISOTOPE } \\
\text { U-235 } \\
\text { U-230 } \\
\text { PU239 } \\
\text { PU24I } \\
\text { TOTALS }\end{array}$ & $\begin{array}{r}\text { FISSI } \\
.1048-0 \\
.3057-0 \\
.4366-0 \\
.1446-0 \\
.5865=0\end{array}$ & $\begin{array}{l}\text { NS (GM) } \\
(.3019-04) \\
(.2982-04) \\
(.1254-03) \\
.4966-05) \\
(.1236-03)\end{array}$ & 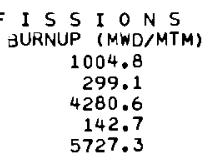 & $B Y$ & $\begin{array}{l}\text { NDI } 188 \\
\text { FISSION YIELO } \\
.0169(.0001) \\
.0193(.0010) \\
.0170(.0003) \\
.0189(.0005)\end{array}$ & & $\begin{array}{c}\text { DEL } \\
.04911 .0049)\end{array}$ & $\begin{array}{c}\text { MEV/FISSION } \\
201.7 \\
208.5 \\
210.7 \\
213.8\end{array}$ \\
\hline
\end{tabular}

U235/U235BOL $=.827(.004)$ ALPHA235 $=.2145(.0076)$ U238 INTO PU239 $=.2847-02(.39-03)$ GM U236 INTO NP237=.461-05GM

-----OUANTITIES ENCLOSED BY PARENTHESES ARE ONE STANDARD DEVIATION ERRORS------

ELEMENT 6\$20 KOL FR7E(12-ROD RING) BATCH CORE THE SAMPLE WAS CUT 30.4 INCHES FROM THE TOP OF THE FUEL INPUT OATA--- TIMC OUT OF REACTOR=1.700YRS BOL PU241 OECAY CORRECTION TIME=-2.100YRS OXIDE FUEL= .2252GM/ML SPINE-- $+042 / 50.6050-021.24-03) \quad$ NOSO $142.4298+01(.20-01)--S A M P L E--N D 50 / 48.5588-00(.20-02)$ MIXTURE--ND42/50 .11B0-01(.20-03)

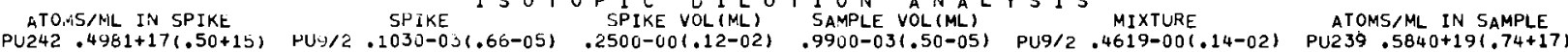
SPIKE I 5 O T O P I C D I L U T I ON A A N A L Y S I S

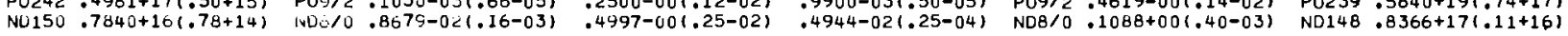
BOL U+PU $=.19831 .0020) \mathrm{GM} / \mathrm{ML}$ AS CALCULATED BY FUEL WEIGHT AND SOLUTION VOLUME

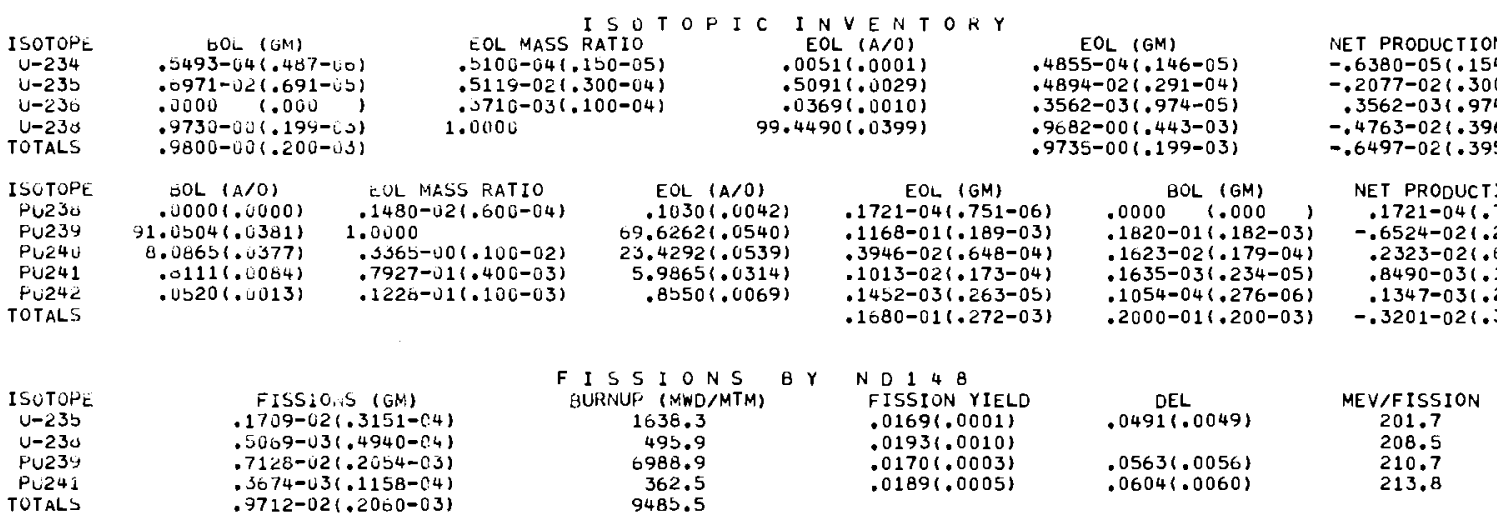

U235/U233OUUL=.7U2(.004) ALFHA235=.2153(.0079) U238 INTO PU239=.4274-02(.39-03)GM U236 INTO NP237=.133-04GM 
ELEMENT 6065 KOD FS07(12-ROD RING) BATCH CORE THE SAMPLE WAS CUT 30.4 INCHES FROM THE TOP OF THE FUEL TIME OUT OF REACTOR=1.700YRS BOL PU241 DECAY CORRECTION TIME= 2.330YRS PATA----
SPIKE--NO42/50.8056-02(.24-03) OXIDE FUEL= .2729GM/ML
ND50/42.3109+01(.15-01) I SO TOP I C D ILU T ION A N A L Y S I S

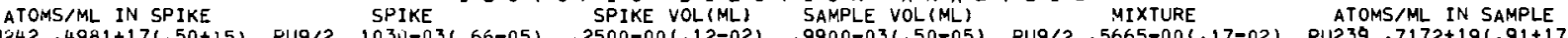

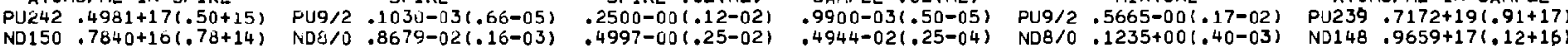
BOL U+PU $=.2400(.0024) \mathrm{GM} / M L$ AS CALCULATED BY FUEL KEIGHT AND SOLUTION VOLUME

\begin{tabular}{|c|c|c|c|c|c|c|c|c|}
\hline \multirow{2}{*}{$\begin{array}{l}\text { ISOTOPE } \\
\text { U-234 } \\
\text { U-235 } \\
\text { U-236 } \\
\text { U-238 } \\
\text { TOTALS } \\
\text { ISOTOPE } \\
\text { PU238 } \\
\text { PU239 } \\
\text { PU240 } \\
\text { PU241 } \\
\text { PU242 } \\
\text { TOTALS }\end{array}$} & \multicolumn{5}{|c|}{ 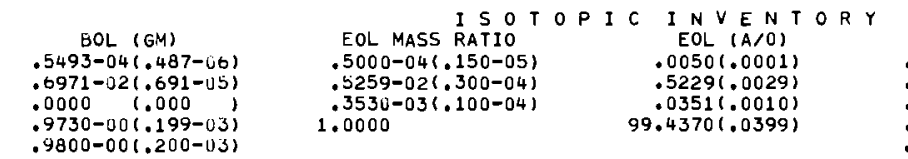 } & \multicolumn{2}{|c|}{$\begin{array}{c}\text { EOL }(G M) \\
.4761-04(.146-05) \\
.5029-02(.291-04) \\
.3390-03(.974-05) \\
.9685-00(.439-03) \\
.9739-00(.199-03)\end{array}$} & $\begin{array}{l}\text { NET PRODUCTI ON } \\
-.7318-051.154 \\
-.1942-021.300 \\
.3390-031.974 \\
-.4482-021.392 \\
-. .6097-021.390\end{array}$ \\
\hline & $\begin{array}{r}\text { BOL }(1 / 0) \\
.0263(.0006) \\
90.5182(.0375) \\
8.2281(.0374) \\
1.1478(.0070) \\
.0796(.0018)\end{array}$ & $\begin{array}{l}\text { EOL MASS RATIO } \\
.1840-02(.700-04) \\
1.0000 \\
.3235-00(.100-02) \\
.7815-01(.400-03) \\
.1218-01(.100-03)\end{array}$ & $\begin{array}{r}\text { EOL }(A / O) \\
.1294(.004 \\
70.3093(.055) \\
22.7451(.054 \\
5.9598(.031 \\
.8564(.007\end{array}$ & & $\begin{array}{r}E O L(G M) \\
.2168-04(.896-06 \\
.1183-01(.191-0) \\
.3844-02(.632-04 \\
.1012-02(.173-04 \\
.1460-03(.265-05 \\
.1686-01(.272-0)\end{array}$ & $\begin{array}{l}\text { 6) } \\
\text { 3) } \\
\text { 4) } \\
\text { 5) } \\
\text { 3) }\end{array}$ & $\begin{array}{l}\mathrm{BOL}(\mathrm{GM}) \\
.5244-05(.137-06) \\
.1810-01(.181-03) \\
.1652-02(.181-04) \\
.2314-03(.271-05) \\
.1611-04(.396-06) \\
.2000-01(.200-03)\end{array}$ & $\begin{array}{l}\text { NET PRODUCT: } \\
.1644-041 . \\
-.6261-021 . \\
.2193-021 . \\
.7802-031 \\
.1298-031 . \\
-.3142-021 .\end{array}$ \\
\hline $\begin{array}{l}\text { ISOTOPE } \\
\text { U-235 } \\
\text { U-236 } \\
\text { PU239 } \\
\text { PU241 } \\
\text { TOTALS }\end{array}$ & $\begin{array}{r}\text { FISSI } \\
.1593-0 \\
.4832-0 \\
.6824-0 \\
.3543-0 \\
.9254-0\end{array}$ & $\begin{array}{l}(S)(G M) \\
(.3150-04) \\
(.4709-04) \\
(.1953-03) \\
(.1138-04) \\
(.1955-03)\end{array}$ & $\begin{array}{c}\text { ISS S I O N S } \\
\text { OURNUP (MWD/MTM) } \\
1526.6 \\
472.7 \\
6690.0 \\
349.5 \\
9038.8\end{array}$ & $B Y$ & $\begin{array}{l}\text { ND } 148 \\
\text { FISSION YIELD } \\
.0169(.0001) \\
.0193(.0010) \\
.0170(.0003) \\
.0189(.0005)\end{array}$ & & $\begin{array}{c}\text { DEL } \\
.0491(.0049)\end{array}$ & $\begin{array}{c}\text { MEV/FISSI ON } \\
201.7 \\
208.5 \\
210.7 \\
213.8\end{array}$ \\
\hline
\end{tabular}

U235/U235BOL $=.721(.004)$ ALPHA235 $=.2192(.0085)$ U238 INTO PU239 $=.4016-02(.39-03)$ GM U236 INTO NP237=.117-04GM

------QuANTITIES ENCLOSED GY PARENTHESES ARE ONE STANOARD DEVIATION ERRORS------

ELEMENT O065 ROD FO17( O-RGD FING) BATCH CORE THE SAMPLE WAS CUT 10.0 INCHES FROM THE TOP OF THE FUEL INPUT DATA---- TIME OUT OF REACTOR=1.666YFS BOL PU241 DECAY CORRECTION TIME= 2.330YRS OXIDE FUEL= .2532GM/ML SPIKE--NU42/50.6056-02(.24-03) NDSO/42.4344+02(.30-00)--SAMPLE--ND50/48.5513-00(.20-02) MIXTURE--ND42/50.8020-02(.40-04)

IS OT OPIC DILUTION ANALYSIS

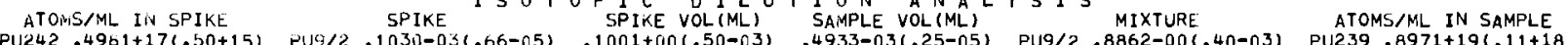

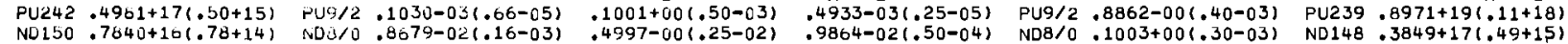
BOL U+PU $=.2232(.0022) \mathrm{GM} / \mathrm{ML}$ AS CALCULATEO BY FUEL WEIGHT AND SOLUTION VOLUME $.27 \%$ SAMPLE---ND CONTAMINATION---MIXTURE .09\%

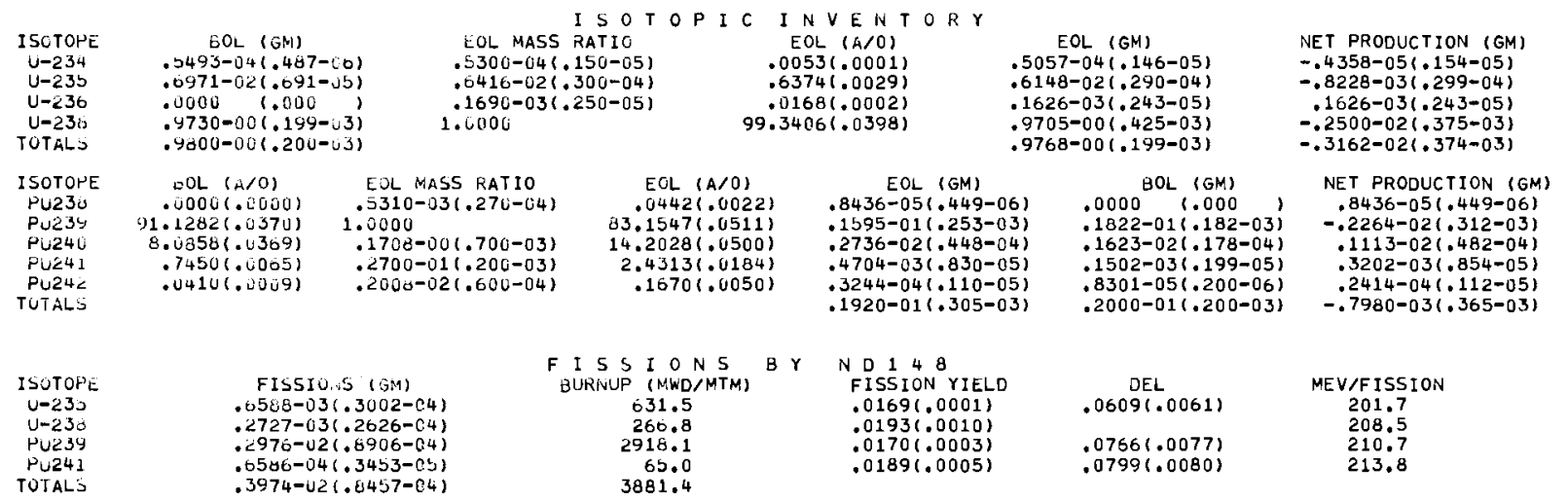




\section{A-16}

ELEMENT 6065 ROD FO17( G-ROD RING) BATCH CORE THE SAMPLE WAS CUT 30.4 INCHES FROM THE TOP OF THE FUEL

INPUT DATA---- TIME OUT OF REACTOR=1.666YRS BOL PU241 DECAY CORRECTION TIME= 2.330YRS OXIDE FUEL= .2622GM/ML SPIKE--ND42/50.8056-02(.24-03) ND50/42 .4052+02(.75-00)--SAMPLE--ND50/48 .5544-00(.20-02) MIXTURE--ND42/50 .8290-02(.30-04)

$$
\text { I } 50 T O P I C \text { T ILU T ION A NALYSIS }
$$

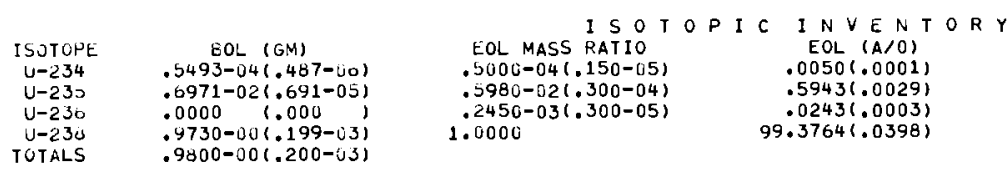

I $\triangle$ UTOPE
PU230
PU239
PU240
PU241
PU242

SOL $(A / O)$ $.0000(.0000$ $91.1282(.0370)$ $8.0850(.0309)$ .74501.0065i

$$
\begin{aligned}
& \text { EOL MASS RAT 10 } \\
& .7680-03(.380-04) \\
& 1.0000 \\
& .2156-00(.800-03) \\
& .3936-01(.28 \mathrm{G}-03) \\
& .3615-02(.900-04)
\end{aligned}
$$
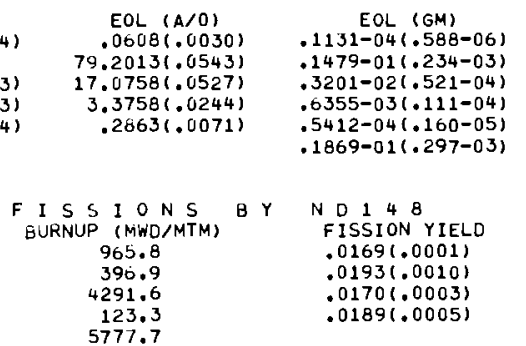
FISSIONS (GM) $.4057-031.3896-04$ $.4377-02(.1275-03)$ $1250-03(.5374-05)$

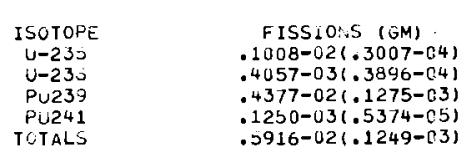

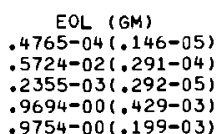

$.9694-00(.429-03)$
NET PRODUCTION (GM) -.7273-05(.154-05) -.1247-02(.299-04) - $3571-021.380-031$ $-.4587-02(.379-03)$

U235/U23500L $=.821(.004)$ ALPHA235 $=.2375(.0079)$ U238 INTO PU239=.3178-02(.38-03) GM U236 INTO NP237=.479-05GM

ELENENT OOG SO ROD FE74(CENTRAL ROD) BATCH CORE THE SAMPLE WAS CUT 10.0 INCHES FROM THE TOP OF THE FUEL

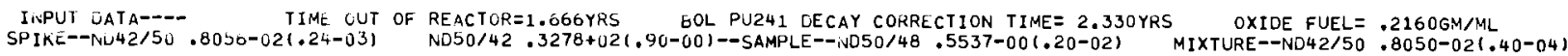
I S O T O P I C D D I L U T I O N A N A L YSIS ATONS M IN SPIKE

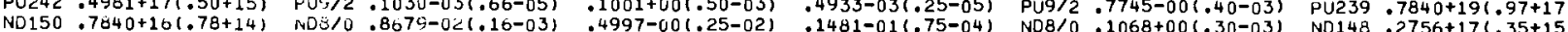
BOL UTPU $=.1904(.0019)$ GMIML AS CALCLLATED BY FUEL WEIGHT AND SOLUTION VOLUME

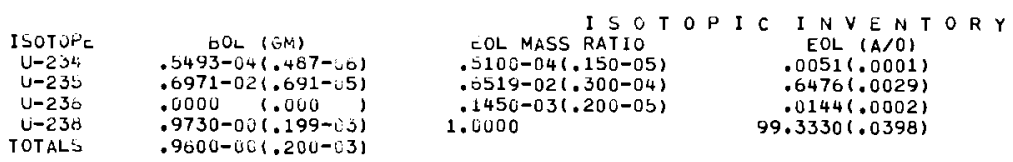

ISOTOFE

Pu236

Pu24

PU240

PU24C
TOTALS

$$
\begin{array}{r}
.00 L(A / 0) \\
.0263(.0006) \\
90.5182(.0375) \\
8.2281(.0374) \\
1.1478(.0070)
\end{array}
$$

EOL MASS RATIO

. 6380-03(.340-04)

1.0000
$.1545-10(.500-03)$

$.1545-40(.500-03)$
$.2685-01(.900-04)$

$.0211-02(.66(j-04)$

- EOL (A/O)

$84.0580(.0029)$

13.02181 .0367

$13.0218(.0367)$
$2.4506(.0096)$

$.4506(.0096)$

EOL (GM)

$.1120-04(.581-06)$

$.1634-01(.259-031$

$.2536-02(.411-04)$

$.4792-03(.784-05)$

$.1941-01(.308-03)$

$\begin{array}{ll}\text { F I S S I ONS N B Y NDI } 48 \\ \text { OURNUP (MWD/MTM) } \\ 557.2 & \text { FISSION YIELD } \\ 238.6 & .0169(.0001) \\ 2404.2 & .0193(.0010) \\ 55.1 & .0170(.0003) \\ 3255.1 & .0189(.0005)\end{array}$

1SOTOPE

U-235

U-23o
PL239

PU: 41

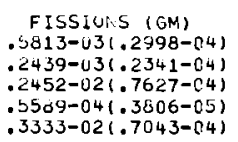

3255.1
EOL (GM)

.4868-04(.146-05) $.6249-02(.290-04)$ $.1396-03(.195-05)$ $9708-00(.424=03$ 9773-00(.199-03)

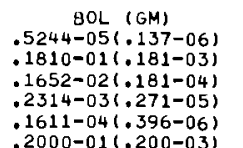

$.1611-04(.396-06)$

$\begin{array}{cc}\text { DEL } & \text { MEV/FISSION } \\ .0636(.0064) & 201.7 \\ & 208.5 \\ .0826(.0083) & 210.7 \\ .0855(.0085) & 213.8\end{array}$

$\begin{array}{lc}\text { NDI } 148 & \\ \text { FISSION YIELD } & \text { DEL } \\ .0169(.0001) & .0636(.0064) \\ .0193(.0010) & \\ .0170(.0003) & .0826(.0083) \\ .0189(.0005) & .0855(.0085)\end{array}$

NET PRODUCTION (GM) $-.6249-05(.154-05)$
$-.7218-03(.299-04)$ .1396-03(.195-05) $-.2144-02(.375-03)$ $-.2727-02(.374-03)$

NET PRODUCTION .5953-05 (.597-06) $.1752-02(.316-03)$ $.8839-03(.449-04)$
$2478-03(.829-05)$ $.2478-03(.829-05)$ $.2048-04(.130-05)$

213.8 


\section{A-17}

ELEMENT 6065 ROD FE74(CENTRAL ROD) BATCH CORE THE SAMPLE WAS CUT 30.4 INCHES FROM THE TOP OF THE FUEL INPUT DATA---- TIME OUT OF REACTOR=1.666YRS BOL PU241 DECAY CORRECTION TIME= 2.330YRS OXIDE FUEL= .31B2GM/ML SPIKE--NO42/50.8056-02(.24-03) NDb0/42 .5745+02(.25-00)--SAMPLE--ND50/48 .5531-00(.20-02) MIXTURE--ND42/50 .8210-02(.40-04)

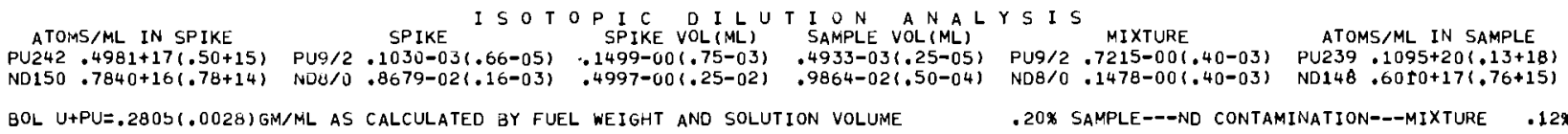

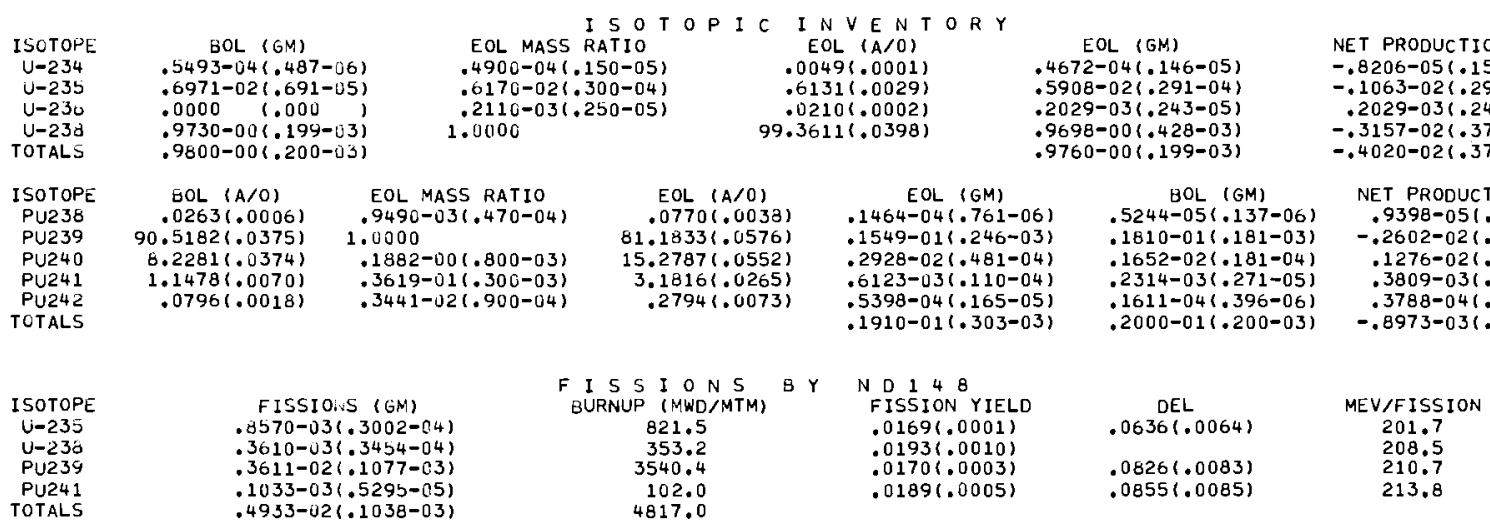

U235/U235BOL $=.848(.004)$ ALPHA235 $=.2398(.0091)$ U238 INTO PU239=.2807-02(.38-03)GM U236 INTO NP237=.345-05GM

------QLANTITIES ENCLOSED BY PARENTHESES ARE ONE STANDARD DEVIATION ERRORS------

ELEMENT $6 \$ 19$ ROD FNOG( O-ROD RING) BATCH CORE THE SAMPLE WAS CUT 7.8 INCHES FROM THE TOP OF THE FUEL INPUT JATA---- TIME OUT OF REACTOR=1.666YHS BOL PU241 DECAY CORRECTION TIME=-2.100YRS OXIDE FUEL= .2403GM/ML

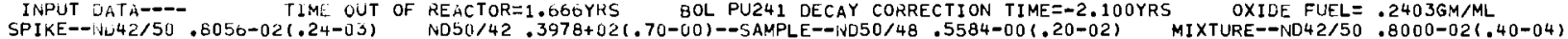
ISOTOPIC DILUTION ANALYSIS ATOMS/ML IN SPIKE SPIKE SPIKE VOL(ML) SAMPLE VOL(ML) MIXTURE PU242 .4981+17(.50+15) PU9/2 .1031)-03(.66-05) .1001+00(.50-03) .4933-03(.25-05) PU9/2 .9004-00(.50-03) PU239.9109+19(.11+18)

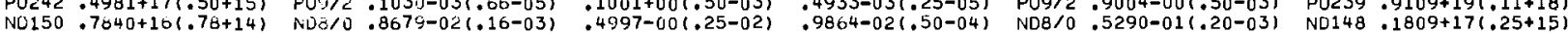
BOL U+PLi $=.2118(.0021) \mathrm{GM} / \mathrm{ML}$ AS CALCLLATED BY FUEL WEIGHT AND SOLUTION VOLUME .30\% SAMPLE---ND CONTAMINATION---MIXTURE .07\%

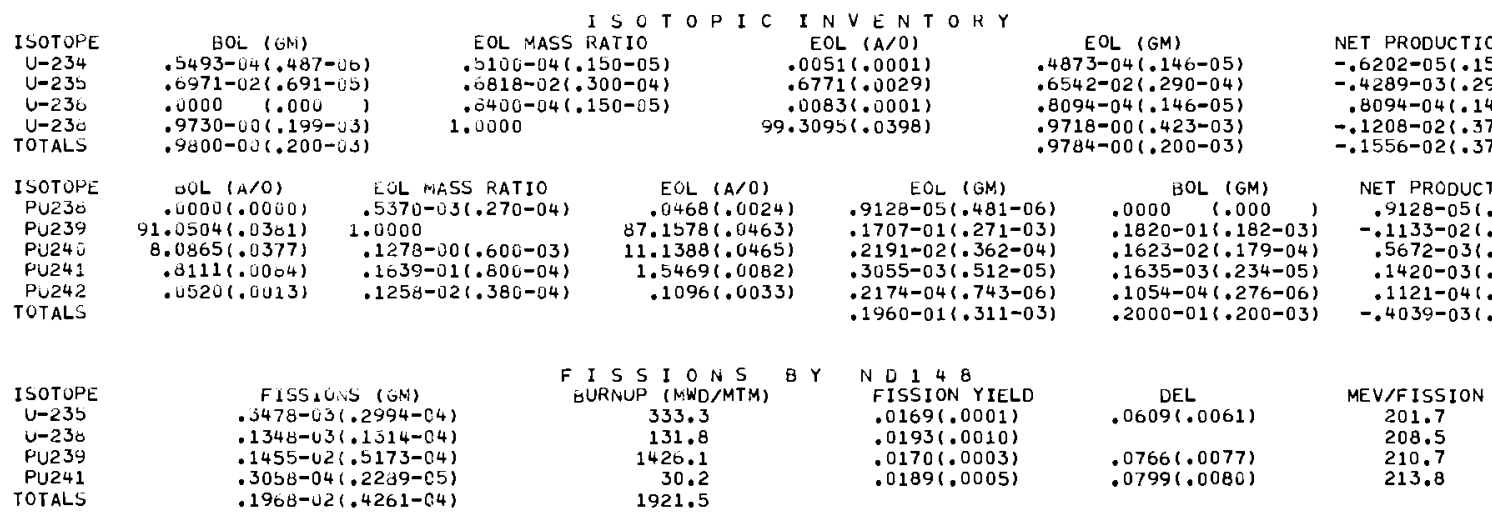

U235/U235BOL $=.930(.004)$ ALPHA235 $=.23331 .0207)$ U238 INTO PU239 $=.1077-02(.37-03)$ GM U236 INTO NP237=.519-06GM 


\section{A-18}

ELEMENT 6519 ROO FN86( 6-ROD RING) BATCH CORE THE SAMPLE WAS CUT 30.4 INCHES FROM THE TOP OF THE FUEL INPUT DATA--- TIME OUT OF REACTOR=1.666YRS BOL PU241 DECAY CORRECTION TIME=-2.10OYR5 OXIDE FUEL= .3082GM/ML SPIKE--ND 42/50.8056-02(.24-03) ND5U/42.5430+02(.30-00)--SAMPLE--ND50/48.5524-00(.20-02) MIXTURE--ND42/50.8140-02(.40-04.

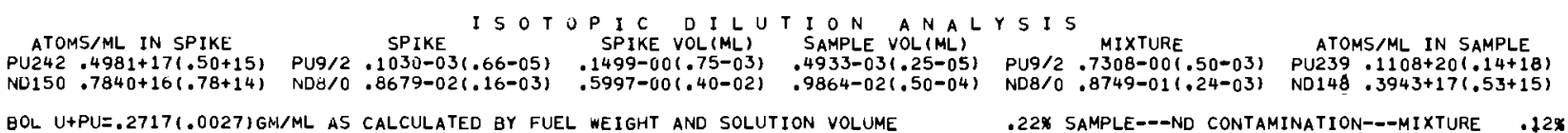

\begin{tabular}{|c|c|c|c|c|c|c|c|c|}
\hline $\begin{array}{l}\text { ISOTOPE } \\
\text { U-234 } \\
U-235 \\
U-236 \\
U-238 \\
\text { TOTALS }\end{array}$ & $\begin{array}{c}B O L(G M) \\
.0493-04(.487-00) \\
.6971-02(.691-05) \\
.0000 \quad(.000 \\
.9730-00(.199-03) \\
.9800-00(.200-03)\end{array}$ & \multicolumn{4}{|c|}{$\begin{array}{l}\text { I SOT } \\
\text { EOL MASS RATIO } \\
.5200-04(.150-05) \\
.6521-02(.300-04) \\
.144(0-03(.200-05) \\
1.0000\end{array}$} & \multicolumn{2}{|c|}{$\begin{array}{c}\text { EOL }(G M) \\
.4964-04(.146-05) \\
.6252-02(.290-04) \\
.1387-03(.195-05) \\
.9711-00(.422-03) \\
.9775-00(.199-03)\end{array}$} & $\begin{array}{l}\text { NET PRODUCTIC } \\
-.5283-051.15 \\
-.7185-031.29 \\
.1387-031.19 \\
-.1923-021.37 \\
-.2505-021.37\end{array}$ \\
\hline $\begin{array}{l}\text { ISOTOPE } \\
\text { PU238 } \\
\text { PU239 } \\
\text { PU240 } \\
\text { PU241 } \\
\text { PU242 } \\
\text { TOTALS }\end{array}$ & $\begin{array}{r}\text { HOL }(A / O) \\
.0000(.0060) \\
91.0504(.0381) \\
8.0865(.0377) \\
.8111(.0084) \\
.0520(.0013)\end{array}$ & $\begin{array}{l}\text { EOL IMASS RAT10 } \\
.4460-03(.220-04) \\
1.0000 \\
.1562-00(.600-03) \\
.2341-01(.100-03) \\
.1751-02(.530-04)\end{array}$ & $\begin{array}{r}\text { EOL }(A / 0) \\
.0377(.001 \\
84.4775(.043 \\
13.1954(.044 \\
2.14161 .010 \\
.1479(.004\end{array}$ & $\begin{array}{l}191 \\
\text { 39) } \\
40) \\
01) \\
45)\end{array}$ & $\begin{array}{c}\text { EOL }(\mathrm{GM}) \\
.7187-051.372-0 \\
.1618-011.257-0 \\
.2538-021.415-0 \\
.4137-031.686-0 \\
.2869-041.982-0 \\
.1917-011.304-0\end{array}$ & & $\begin{array}{c}\text { BOL (GM) } \\
.0000(.000 \\
.1820-01(.182-03) \\
.1623-02(.179-04) \\
.1635-03(.234-05) \\
.1054-04(.276-06) \\
.2000-01(.200-03)\end{array}$ & $\begin{array}{l}\text { NET PRODUCT } \\
.7187-051 . \\
-.2019-021 \\
.9150-031 \\
.2502-031 \\
.1816-041 \\
-.8290-031\end{array}$ \\
\hline $\begin{array}{l}\text { ISOTOPE } \\
\text { U-235 } \\
\text { U-236 } \\
\text { PU239 } \\
\text { PU241 } \\
\text { TOTALS }\end{array}$ & $\begin{aligned} & \text { FISS } \\
& .5749- \\
& .2292- \\
& .2487- \\
& .4954- \\
& .3345-\end{aligned}$ & $\begin{array}{l}.5(G M) \\
.2998-04) \\
.2212-(04) \\
.7770-(04) \\
.3038-05) \\
.7245-04)\end{array}$ & $\begin{array}{c}\text { F I S } 5 \text { I O N } 5 \\
\text { BURNUP (MWD } / M T M) \\
554.9 \\
224.9 \\
2438.4 \\
48.9 \\
3266.4\end{array}$ & BY & $\begin{array}{l}\text { ND } 148 \\
\text { FISSION YIELD } \\
.0169(.0001) \\
.0193(.0010) \\
.0170(.0003) \\
.0189(.0005)\end{array}$ & & $\begin{array}{c}\text { DEL } \\
.0609(.0061) \\
\\
.0766(.0077) \\
.0799(.0080)\end{array}$ & $\begin{array}{c}\text { MEV/FISSION } \\
201.7 \\
208.5 \\
210.7 \\
213.8\end{array}$ \\
\hline
\end{tabular}

U235/U235BOL $=.897(.004)$ ALPHA235 $=.2412(.0131)$ U238 INTO PU239 $=.1701-02(.37-03)$ GM U236 INTO NP237 $=.154-05 G M$

-----GUANTITIES ENCLOSED GY PARENTHESES ARE ONE STANDARD DEVIATION ERRORS------

ÉLEMENT ÓS19 ROU FE69(CENTRAL ROD) BATCH CORE THE SAMPLE WAS CUT 7.8 INCHES FROM THE TOP OF THE FUEL INPUT DATA---- TIML UUT OF REACTOR=1.666YRS BOL PU241 DECAY CORRECTION TIME =-2.100YRS OXIDE FUEL= .2496GM/ML SPIKE--NU42/50.8056-02(.24-03) ND50/42.3102+02(.12+00)--SAMPLE-ND50/48 .5573-00(.20-02) MIXTURE--ND42/50 .7930-02(.30-04)

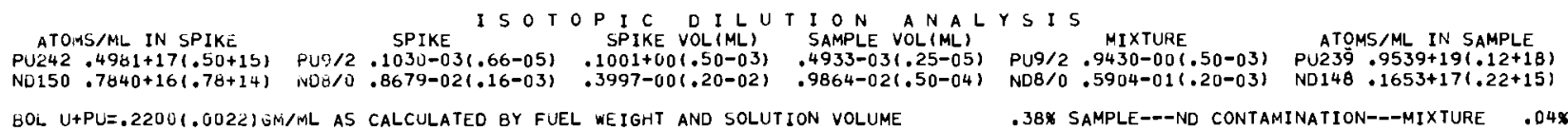

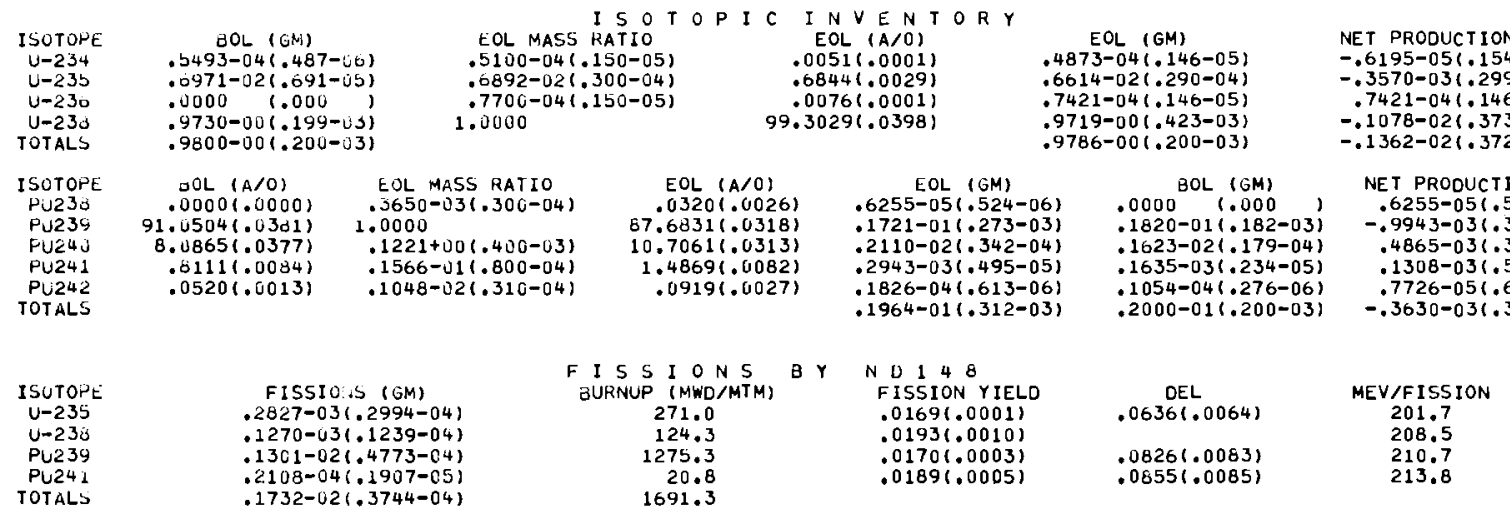

U235/U235HOL $=.949(.004)$ ALFHA235 $=.2628(.0285)$ U238 INTO PU239 $=.9554-03(.37-03)$ GM U236 INTO NP237=.394-06GM 


\section{A-19}

ELEMENT 6519 ROD FE69(CENTRAL ROD) BATCH CORE THE SAMPLE WAS CUT 30.4 INCHES FROM THE TOP OF THE FUEL

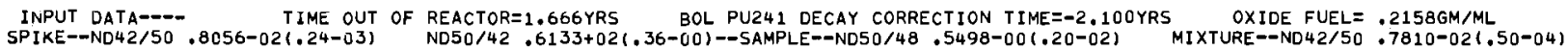
SPIKE ISOTOPIC DILUTION A NALYSIS

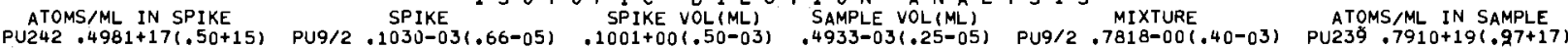
ND150 $.7840+16(.78+14) \quad$ ND8/0 $.8679-02(.16-03) \quad .4997-00(.25-02) \quad .1481-01(.75-04)$ ND8/0.9534-01(.40-03) ND148.2418+17(.32+15) BOL U+PU: $=.19021 .0019)$ GM/ML AS CALCULATED BY FUEL WEIGHT AND SOLUTION VOLUME

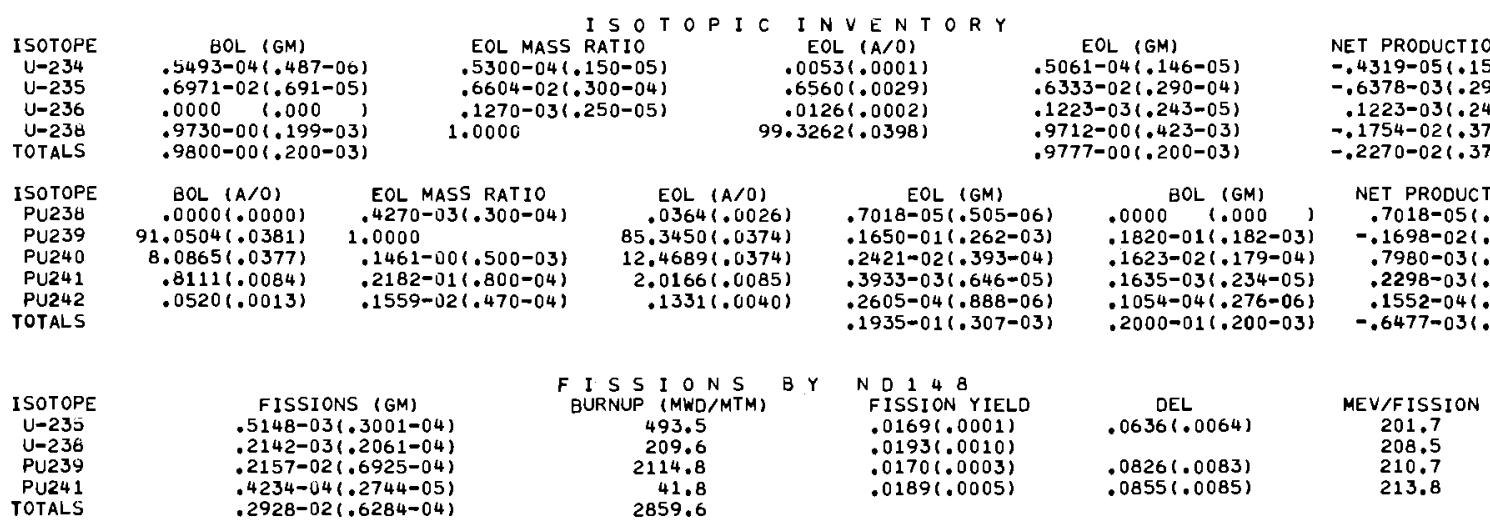

U235/U235BOL $=.909(.004)$ ALPHA235 $=.2389(.0151)$ U238 INTO PU239=.1546-02(.37-03) GM U236 INTO NP237=.119-05GM

------QUANTITIES ENCLOSED BY PARENTHESES ARE ONE STANDARD DEVIATION ERRORS------

ELEMENT 6519 ROD 4 (12-ROD RING) BATCH CORE THE SAMPLE WAS CUT 30.0 INCHES FROM THE TOP OF THE FUEL INPUT DATA---- TIME OUT OF REACTOR=1.879YRS BOL PU241 DECAY CORRECTION TIME=-2.100YRS SPIKE--ND42/50,8050-02(.24-03) ND50/42.7599+02(.20-00)--SAMPLE--N050/48.5496-00(.10-02) MIXTURE--ND42/50 .9950-02(.50-04) ISPTE I T OPIC DILUT ION A NALYSIS

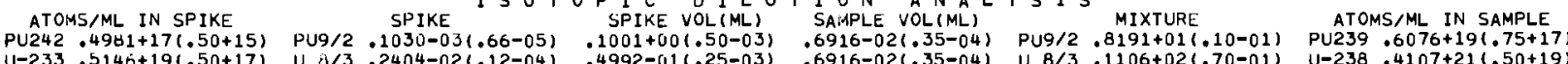

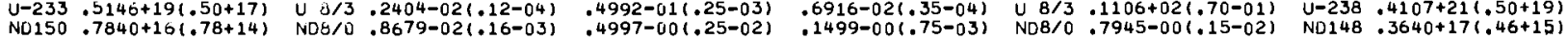
BOL U+PU $=.1672(.0020) \mathrm{GM} / M L$ AS CALCULATED BY ISOTOPIC DILUTION $.15 \%$ SAMPLE---ND CONTAMINATION---MIXTURE . .14\%

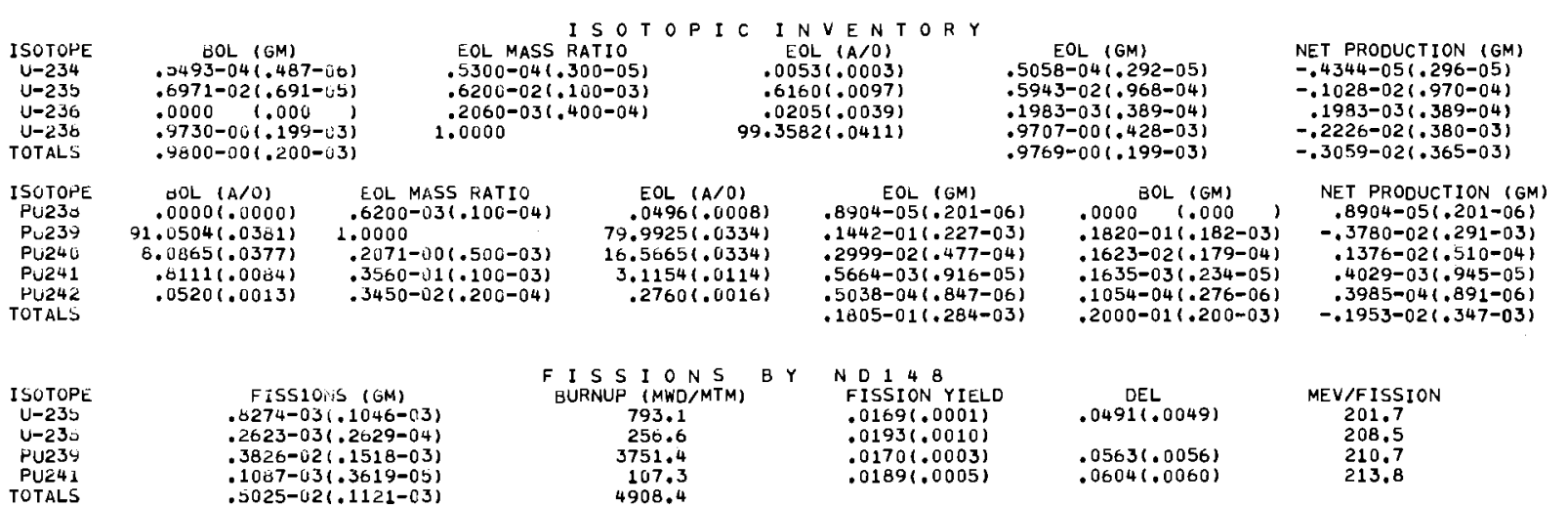

U235/U235BOL $=.853(.014)$ ALPHA235 $=.2425(.0650)$ U238 INTO PU239 $=.1972-02(.38-03)$ GM U236 INTO NP237=.325-05GM 
-

.

.

. 
APPENDIX B 
APPENDIX B

ANALYSIS OF FERTF FUEL ASSEMBLY 9035 BY GAMMA SCANNING

INTRODUCTION

Test fuels have been irradiated in the Fuel Element Rupture Test Facility (FERTF) of the PRTR. To assist in researching the behavior of these fuels*, gamma activities have been measured to obtain the relative power generation profile around the circumference of the FERTF fuel assembly as part of the post-irradiation analysis. The rods from FERTF assembly 9035 have been scanned on three different occasions using different procedures to determine the power profile and to establish the reliability of the measured relative power generation values. A brief summary of the results was included in a previous quarterly report. The detailed results are presented here along with a description of assembly 9035 and the procedures used to measure the circumferential power profile. IRRADIATION HISTORY

Fuel assembly 9035 was an 8-rod test assembly containing vibrationally compacted $\mathrm{UO}_{2}-2 \mathrm{wt} \% \mathrm{PuO}_{2}$ fuel and the configuration of the assembly is illustrated in Figure B-1. The assembly was irradiated in the FERTF location (process channe1 1550) between June 29 and Ju1y 14, 1968, accumulating a total exposure of 4.08 MWd (324.1 MWd/MTM). After its removal from the reactor, the assembly was disassembled so that each rod could be analyzed separately. A punch mark was put on the top end cap of each rod so that the orientation of the rod in the assembly would be known.

* Conducted by the Fuels Research Section of the Materials Department 


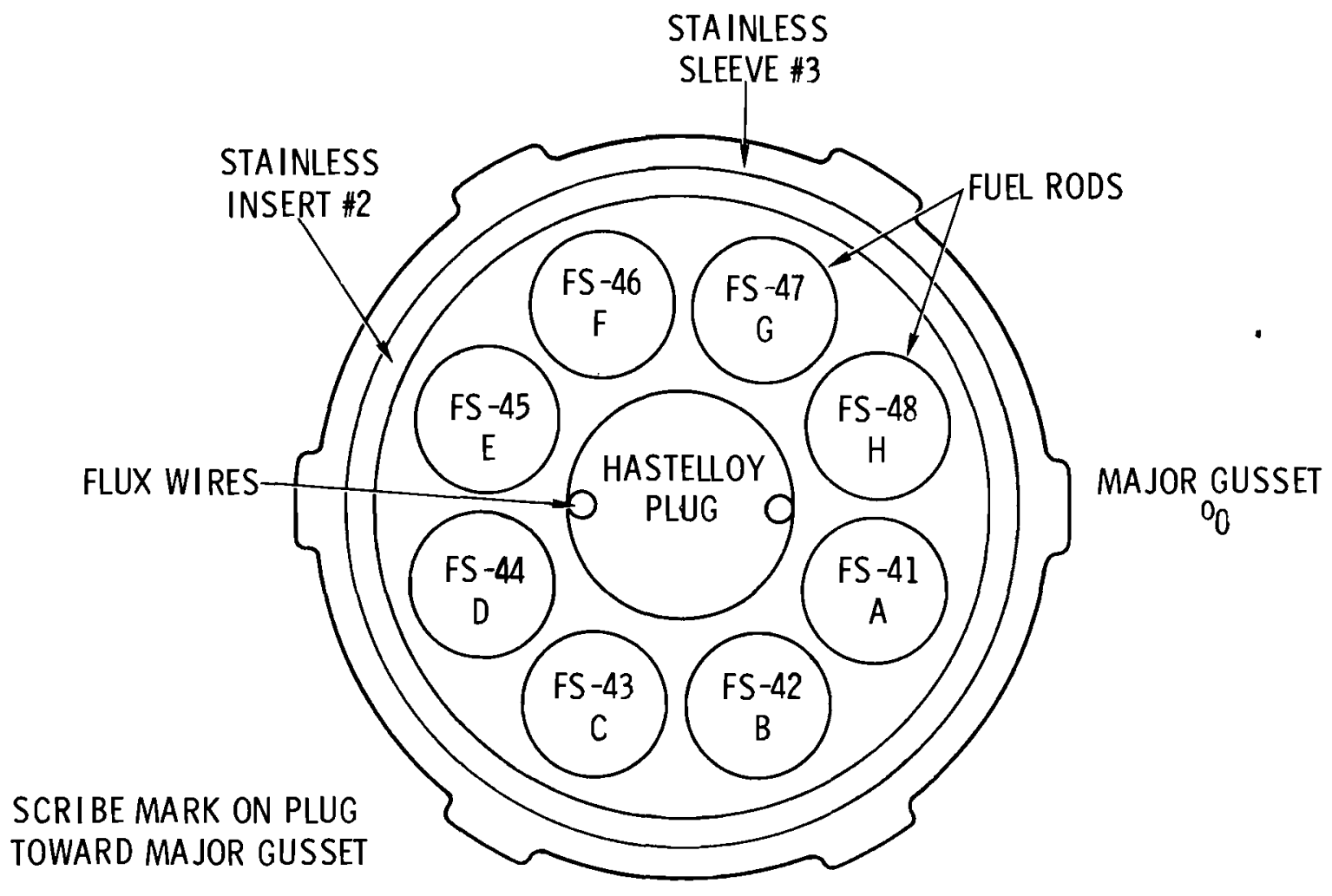

FIGURE B-1. FUEL ASSEMBLY 9035 
The rods were then scanned after cooling times of 29 days, 232 days, and 240 days. The scans were made using the PNL Underwater Gamma Scan Facility.

\section{SCANNING PROCEDURES}

The first measurements were made after 29 days cooling time, using an automated continuous scan drive over a 6 in. length on each rod in the region of maximum power generation (26 7/16 in. to $327 / 16$ in. from the bottom tip of the rod). The ${ }^{95} \mathrm{Zr} /{ }^{95} \mathrm{Nb}$ gamma rays were monitored by setting a single channel analyzer (SCA) to accept the pulses from a $\mathrm{NaI}(\mathrm{Tl})$ detector in the energy range from $660 \mathrm{KeV}$ to $880 \mathrm{KeV}$. The activity profile over the 6 in. span was recorded using a linear ratemeter and a strip-chart recorder. Also, the area beneath the activity profile was integrated by means of a scaler in parallel with the ratemeter. The activity plots could then be compared as well as the integrated counts. Two scans were made in opposite directions over the 6 in. length to check the reproducibility of the data. A timer was used with the scaler to indicate the total elapsed time of each scan. The integrated counts were recorded as counts per second. The scan rate was 1 in. per 3 minutes (i.e., 18 min to scan the 6 in. length). A ${ }^{137}$ Cs source was used after each scan for calibration. No corrections were made for background since it was constant and less than $1 \%$ of the total counts. All the data for this set of measurements were taken on the same day over a 9 hour period and no corrections for decay were made. Although an $815 \mathrm{KeV}$ gamma ray from 12.8 day half-1 ife 
$140_{\mathrm{Ba} /}{ }^{140} \mathrm{La}$ was present in the energy range counted, it was not the major contributor to the measured activity and decayed only $2 \%$ during the measurement interval.

After 232 days cooling time, 6 of the 8 rods were rescanned using a Ge(Li) detector (2 rods had been destructively analyzed). Continuous scans were made over the total length of the $\mathrm{UO}_{2}-\mathrm{PuO}_{2}$ fuel in each rod. Due to the long cooling time and the low efficiency of the detector, the single channel analyzer was set to accept all gamma rays of energies greater than $30 \mathrm{KeV}$ in order to operate the ratemeter in a range that would give statistical significance to variations in the power generation profile of a rod. The area beneath the profile was again integrated by use of a scaler and timer so that, in this case, the total relative power generated by each rod could be compared. The scan rate was approximately $1.5 \mathrm{in}$. per minute. Five of the six rods were scanned on the same day and the sixth, rod FS-43, was scanned on the following day. The values measured for FS-43 appeared low and may have resulted from an electronic gain shift caused by a temperature change of $60 \mathrm{~F}$ which occurred.

The third set of measurements scanned the same $6 \mathrm{in}$. of fuel as the first measurements and were done after 240 days cooling. The $\mathrm{Ge}(\mathrm{Li})$ detector was used to take this set of data, on the same day, including two repeat measurements. Again, the single channel analyzer was set to accept all gamma rays of energy greater than $30 \mathrm{KeV}$ and a scaler was used to integrate the area beneath the activity profiles. The scan rate was approximately 1 in. per 5 minutes. 


\section{$\underline{\text { RESULTS }}$}

The activity profiles for rod FS-43 by each method are shown in Figures B-2. These results are typical of the results obtained from a11 the rods. The profiles for the $6 \mathrm{in}$. (from $267 / 16$ to $323 / 16$ inches) show very nearly the same details. Three points of low activity are discernible in each trace. The integrated activities in counts per second are presented in Table B-I for al1 measurements. In addition, each set of data has been averaged and the average value used to normalize each count rate of the set. The normalized values as a function of rod position in element 9035 are shown in Figure B-3.

It is concluded from Table B-I and Figure B-3 that the relative power generation profile around the circumference of element 9035 in the region of peak power was constant to within $\pm 1.0 \%$. Each of the three measurement procedures gave this result, independent of the other two sets of data. Measurement method number two where the entire fuel length was scanned appeared the most favorable since the total power generation profile of each rod was determined.

\section{REFERENCE}

1. D. G. Carter, T. B. Burley and M. D. Freshley, "PRTR Fuel Evaluations," Plutonium Utilization Program Technical Activities Quarterly Report September, October, November 1969, BNWL-1289, p. 3.1 (January 1970). 


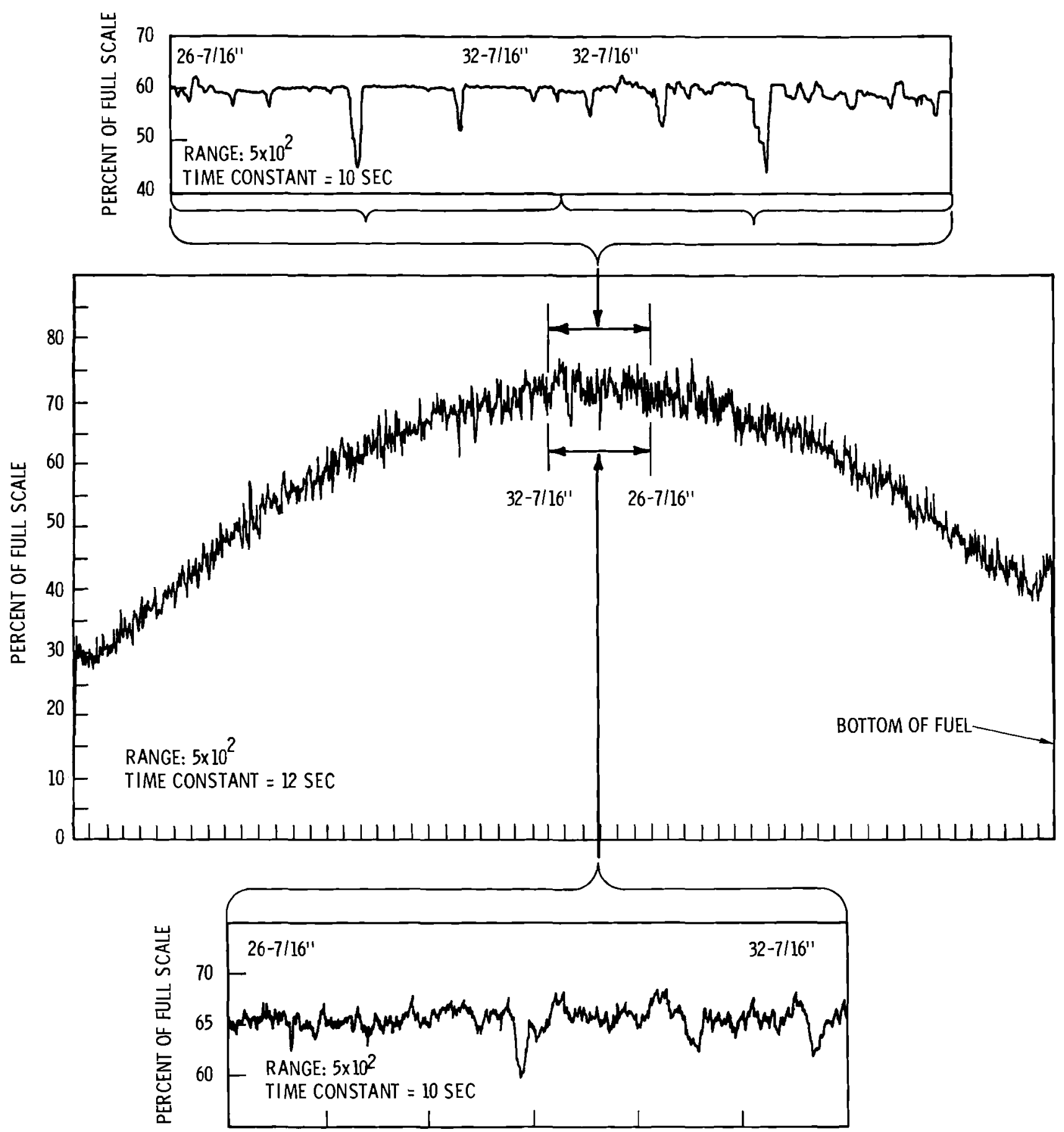

FIGURE B-2. GAMMA-RAY ACTIVITY PROFILES FOR ROD FS43 A) OVER 6-INCH LENGTH AFTER 29 DAYS COOLING, B) OVER TOTAL LENGTH AFTER 233 DAYS COOLING AND CI OVER 6 INCH LENGTH AFTER 240 DAYS COOLING. THE 6 INCH LENGTH IS IDENTIFIED WITH REFERENCE TO THE BOTTOM TIP OF THE ROD 


\section{TABLE B-I}

INTEGRATED ACTIVITY EXPRESSED AS COUNTS PER SECOND

FROM CONTINUOUS SCANS OF FERTF RODS

MEASURED AFTER 29, 232, AND 240 DAYS COOLING TIME

Set I: Over 6 in. Length

Toward Top of Rod Toward Bottom of Rod

FS 41

FS 42

FS 43

FS 44

FS. 45

FS 46

FS 47

FS 48

Averages
$678.5(1.0012)$

$680.9(1.0048)$

$676.6(0.9984)$

$682.8 \quad(1.0075)$

$680.7(1.0045)$

$680.9(1.0048)$

$684.7(1.0104)$

$684.1 \quad(10094)$

$675.8 \quad(0.9972)$

$675.9(0.9973)$

$674.3 \quad(0.9951)$

$674.0 \quad(0.9946)$

$673.6 \quad(0.9941)$

$673.3(0.9936)$

$673.3(0.9936)$

$673.0 \quad(0.9932)$

677.7

$\frac{\text { Set II: Over }}{\text { Entire Fuel Length }}$

$309.0 \quad(1.0006)$

$308.8 \quad(1.0001)$

$305.1 \quad(0.9880)$

$309.2 \quad(1.0012)$

$310.6 \quad(1.0059)$

$310.1 \quad(1.0041)$

308.8
Set III: Over 6 in. Length

Toward Bottom of Rod Repeat

$\begin{array}{llll}359.2 & (1.0093) & 356.9 & (1.0028)\end{array}$

$356.5(1.0045)$

$354.4 \quad(0.9958) \quad 356.1 \quad(1.0006)$

$354.9(0.9972)$

$352.3(0.9899)$

$355.6(0.9992)$

355.9

355.9

Note: Numbers in parentheses () are the integrated activity of the $i$ th rod in the assembly divided by the integrated activity averaged over all rods in the assembly. 


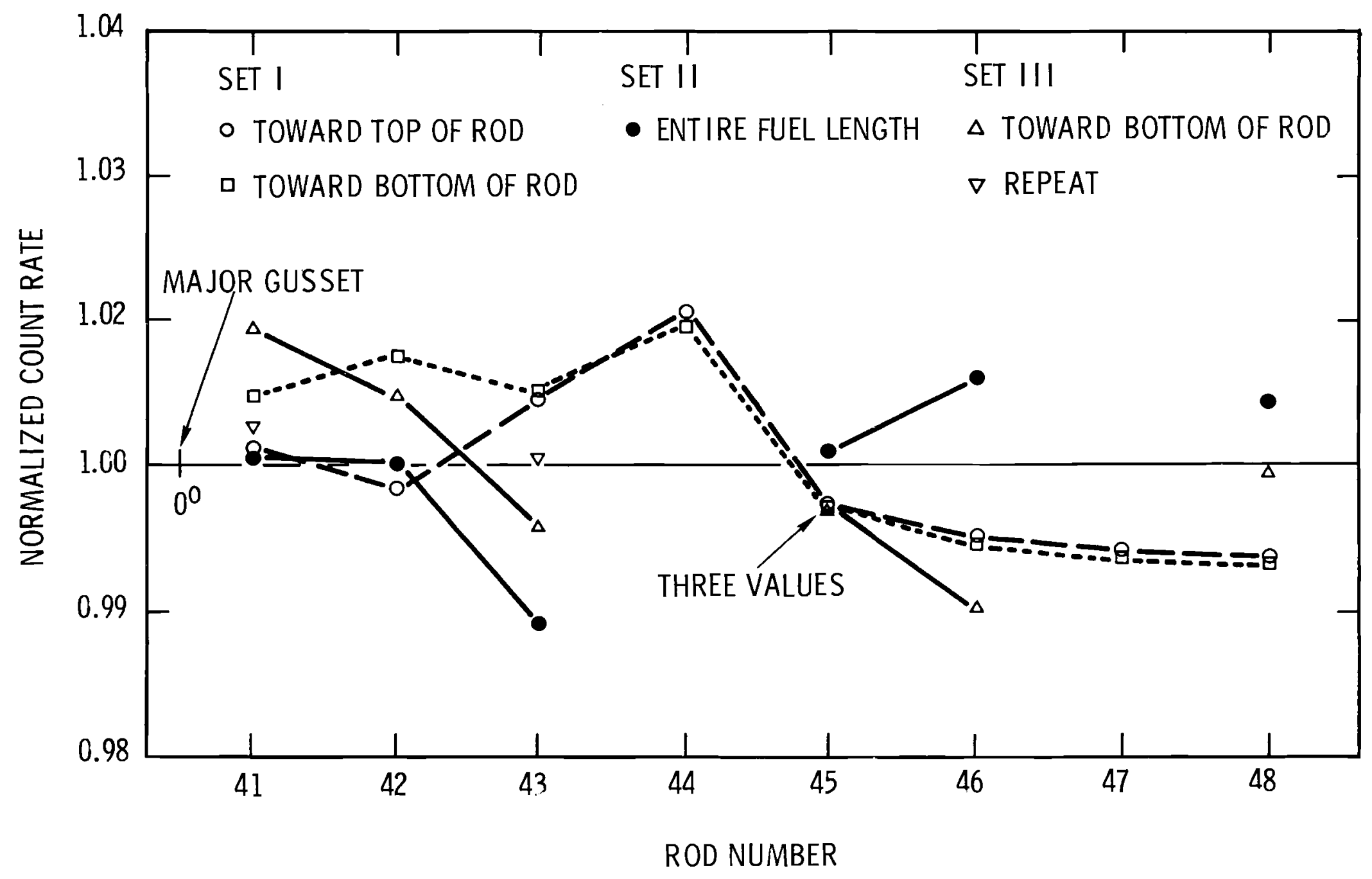

嗢

FIGURE B-3. NORMALIZED ACTIVITY OF EIGHT RODS FROM FUEL ASSEIMBLY 9035 AS A FUNCTION OF ROD POSITION. THE VALUES SHOWN ARE LISTED IN TABLE B-1. 


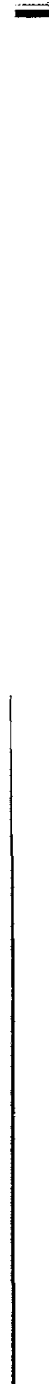


C-1

$\underline{\text { APPENDIX C }}$

GAMMA-RAY SCANNING OF INTACT FUEL ASSEMBLIES

PROCEDURE

During the scanning of intact 19-rod fuel assemblies using a $\mathrm{NaI}(\mathrm{T} \ell)$ detector, gamma-ray spectra were accumulated on a 512 multichannel analyzer (MCA). Simultaneously, single channel analyzer (SCA) data were also taken. The ${ }^{140} \mathrm{Ba}-{ }^{140} \mathrm{La}$ and ${ }^{95} \mathrm{Zr}-{ }^{95} \mathrm{Nb}$ gammas were monitored with the SCA using energy ranges from 1480 to $1700 \mathrm{KeV}$ and from 662 to 878 KeV, respectively. The procedure was to accumulate a 10 minute live time count on the MCA at the same time taking two four minute counts with the SCA and a scaler, one in each energy range mentioned above. After a spectrum was accumulated, the spectrum was stored in the first 256 channels of the MCA and the procedure was repeated with the aperature closed to collect a background spectrum in the second 256 channels and to determine SCA backgound counts. The background spectrum was then subtracted from the first spectrum while both spectra were stored in the MCA. The resulting background corrected spectrum was then typed out. Using this procedure, a total of 23 spectra were obtained and recorded along with SCA data from eight assemblies.

The eight assemblies represented one assembly each from core ring positions $1,2,4,5,6$ and 7 and two assemblies from core ring position 3 of the Batch Core. Measurements were made at the axial peak power position of each assembly. A 1/16 inch diameter aperture positioned at 
27-9/16 inches from the top of the fuel was used while scanning. Since the orientation of each assembly in its process tube was fixed (see Figure 5 in the body of the report), measurements were made on the inside (major gusset up) and outside (major gusset down) edge rods of the assemblies relative to the center of the core. The assemblies were positioned horizontally during scanning.

The purpose of the measurements was to infer the shape of the core radial power profile. The MCA and SCA data were taken concurrently to give more credence to the results from either set of measurements and to expand upon the results obtained solely from SCA measurements. $\underline{\text { RESULTS }}$

The assemblies scanned are identified in Table C-I. As indicated, the data were collected February 21 to 23,1968 , which was three weeks after reactor shutdown, January 28,1968 . Spectra obtained using the $\mathrm{NaI}(\mathrm{Tl})$ detector and the MCA are shown in Figure $\mathrm{C}-1$ where a spectrum plus background, a background spectrum and a spectrum minus background are illustrated. Also illustrated are the SCA window settings used to monitor the ${ }^{140} \mathrm{Ba}-{ }^{140}$ La gamma ray at $1600 \mathrm{KeV}$ and the ${ }^{95} \mathrm{Zr}-{ }^{95} \mathrm{Nb}$ gamma rays.

Due to the short cooling time of the fuel and the half-life of $140 \mathrm{Ba}-{ }^{140} \mathrm{La}$ ( 12.8 days), the peak at $1600 \mathrm{KeV}$ was contributed to primarily by ${ }^{140} \mathrm{Ba}-{ }^{140} \mathrm{La}$ and would thus indicate the power profile during the last month of reactor operation. Although other fission products contributed gamma-ray activity within the energy setting of the SCA, the relative contributions were small compared to ${ }^{140} \mathrm{Ba}-{ }^{140} \mathrm{La}$ and no corrections were made to account for them. 


\section{TABLE C-I}

GAMMA-RAY ACTIVITY MEASUREMENTS FROM INTACT FUEL ASSEMBLIES

\begin{tabular}{|c|c|c|c|c|c|c|c|c|c|c|c|}
\hline \multicolumn{6}{|c|}{ ASSEMBLY AND MEASUREMENT DATA } & \multicolumn{6}{|c|}{ GAMMA-RAY ACTIVITY RESULTS } \\
\hline & Core & & & & Distance** & ${ }^{140} \mathrm{~L}$ & $40_{\mathrm{BaC}}$ & $1600 \mathrm{KeV}$ & ${ }^{95} \mathrm{Zr}$ & $\mathrm{Nb}: \mathbb{a} \sim 7$ & $\mathrm{KeV}$ \\
\hline $\begin{array}{l}\text { Assembly } \\
\text { No. }\end{array}$ & $\begin{array}{c}\text { Ring } \\
\text { Position }\end{array}$ & Orientation* & Date & Time & $\begin{array}{l}\text { From } \\
\text { Core Center }\end{array}$ & $\begin{array}{l}\mathrm{SCA} \\
\mathrm{C} / \mathrm{M}\end{array}$ & $\begin{array}{l}M C A \\
C / M\end{array}$ & $\frac{M C A}{S C A}$ & $\begin{array}{l}\text { SCA } \\
C / M\end{array}$ & $\begin{array}{l}M C A \\
C / M\end{array}$ & $\frac{M C A}{S C A}$ \\
\hline \multirow[t]{2}{*}{6055} & \multirow[t]{2}{*}{1} & Up & \multirow[t]{5}{*}{$2-22-68$} & $11: 15$ & 17.16 & 7739 & 6591 & 0.852 & 36214 & 22801 & 0.630 \\
\hline & & Down & & $11: 55$ & 23.48 & 7586 & 6540 & 0.862 & 36550 & 23265 & 0.636 \\
\hline \multirow[t]{7}{*}{6051} & \multirow[t]{7}{*}{2} & Up & & $9: 35$ & 32.03 & 9836 & 8617 & 0.876 & 40999 & 26410 & 0.644 \\
\hline & & Down & & $10: 23$ & 38.36 & 7051 & 6067 & 0.860 & 34260 & 21648 & 0.632 \\
\hline & & Up & & $13: 32$ & 32.03 & 8424 & 7245 & 0.860 & 40025 & 24070 & 0.638 \\
\hline & & Up & \multirow[t]{4}{*}{$2-23-68$} & $8: 15$ & 32.03 & 9257 & 8127 & 0.878 & 39142 & 24932 & 0.637 \\
\hline & & Up & & $11: 05$ & 32.03 & 9260 & 7970 & 0.861 & 38410 & 24256 & 0.631 \\
\hline & & Up & & $11: 27$ & 32.03 & 8584 & 7424 & 0.865 & 37377 & 23877 & 0.639 \\
\hline & & Up & & $11: 50$ & 32.03 & 8940 & 7740 & 0.866 & 37748 & 24239 & 0.642 \\
\hline \multirow[t]{2}{*}{6066} & \multirow[t]{2}{*}{3} & Up & \multirow[t]{2}{*}{$2-22-68$} & $14: 30$ & 37.48 & 7358 & 6327 & 0.860 & 33900 & 21059 & 0.621 \\
\hline & & Down & & 15.10 & 43.80 & 6975 & 5951 & 0.853 & 33111 & 20663 & 0.624 \\
\hline \multirow[t]{3}{*}{6067} & \multirow[t]{3}{*}{3} & Up & \multirow[t]{2}{*}{$2-21-68$} & $16: 00$ & 37.48 & 7043 & 5935 & 0.843 & 34279 & 21003 & 0.613 \\
\hline & & Down & & $15: 10$ & 43.80 & 6638 & 5637 & 0.849 & 31374 & 19041 & 0.607 \\
\hline & & Up & $2-22-68$ & $8: 20$ & 37.48 & 6994 & 6003 & 0.858 & 33573 & 20739 & 0.618 \\
\hline \multirow[t]{2}{*}{6047} & \multirow[t]{2}{*}{4} & Up & \multirow[t]{9}{*}{$2 \div 21-68$} & $8: 17$ & 53.14 & 6335 & 5397 & 0.852 & 32186 & 20217 & 0.628 \\
\hline & & Down & & $8: 50$ & 59.46 & 5870 & 5094 & 0.868 & 26950 & 16665 & 0.618 \\
\hline \multirow[t]{2}{*}{6035} & \multirow[t]{2}{*}{5} & Up & & $13: 37$ & 57.80 & 5962 & 5115 & 0.858 & 29271 & 18142 & 0.620 \\
\hline & & Down & & $14: 19$ & 64.12 & 5528 & 4711 & 0.852 & 24342 & 14391 & 0.591 \\
\hline \multirow[t]{3}{*}{6031} & \multirow[t]{3}{*}{6} & Up & & $11: 37$ & 67.23 & 5879 & 5152 & 0.876 & 20741 & 17247 & 0.638 \\
\hline & & Down & & $12: 20$ & 73.55 & 4753 & 5057 & 0.854 & 21120 & 12526 & 0.593 \\
\hline & & Up & & $12: 43$ & 67.23 & 5258 & 4480 & 0.852 & 25973 & 16300 & 0.628 \\
\hline \multirow[t]{2}{*}{6019} & \multirow[t]{2}{*}{7} & Up & & $10: 00$ & 70.10 & 4965 & 4308 & 0.867 & 22286 & 136171 & 0.611 \\
\hline & & Down & & $10: 45$ & 76.43 & 4397 & 3821 & 0.869 & 19494 & 11588 & 0.594 \\
\hline
\end{tabular}

* Major gusset up or down. $\quad$ ** Distance in $\mathrm{cm}$. 
C-4

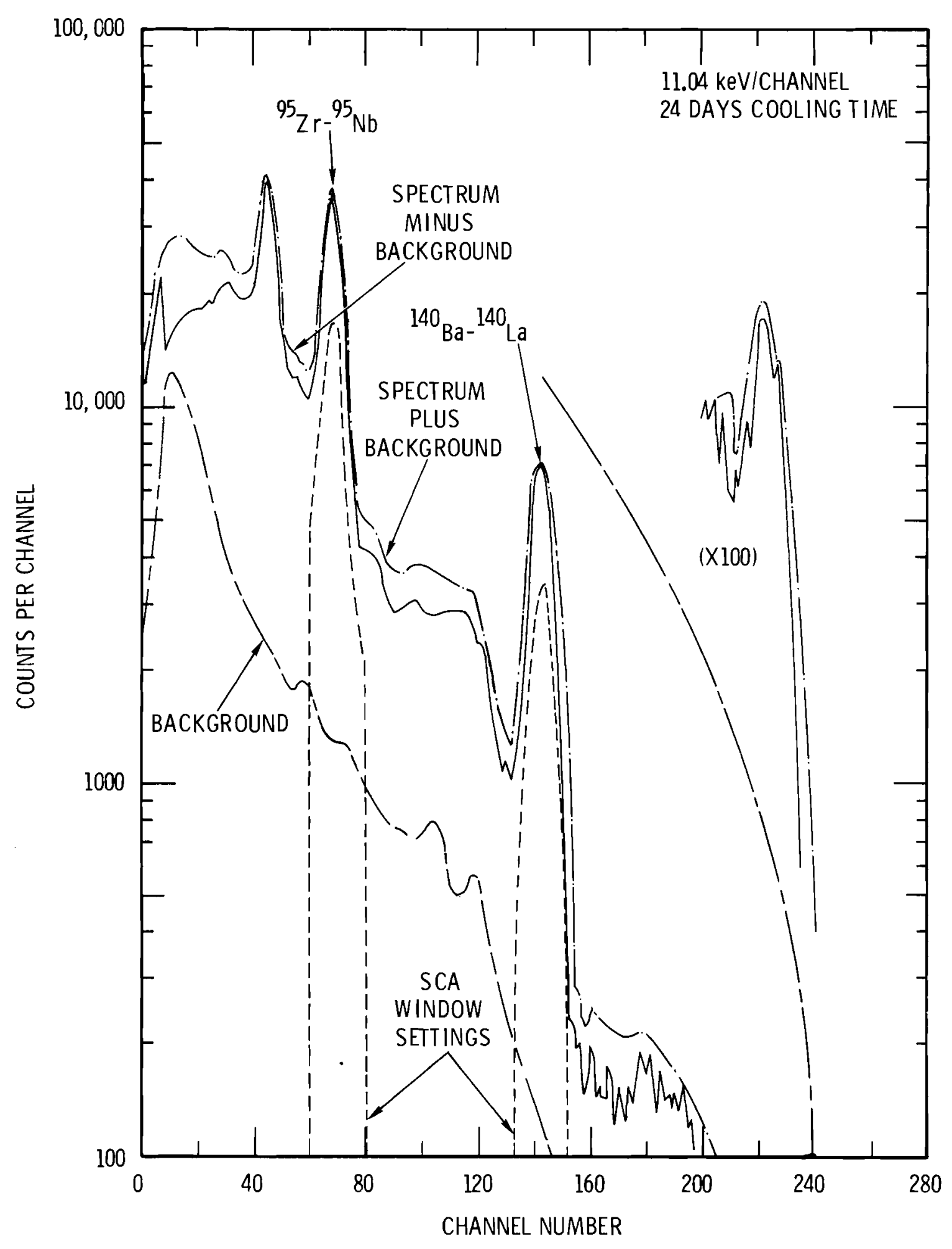

FIGURE C-1. TYPICAL GAMMA RAY SPECTRA OBTA INED WITH NaI(TL) DETECTOR AND MCA 
The ${ }^{140} \mathrm{Ba}-{ }^{140} \mathrm{La}$ SCA results are given in Table C-I where the numbers in counts per minute, corrected for background and decay, are listed. The zero time reference point was chosen as 8:17 A.M. on February 21,1968 , and the largest decay correction was $12 \%$. The ${ }^{140} \mathrm{Ba}-{ }^{140} \mathrm{La}$ MCA results are also shown in Table C-I and were obtained by integrating the peak plus Compton and then subtracting the Compton values. The MCA values were also decay corrected. The SCA data correspond directly to the MCA integrated peak plus Compton values and the ratio of peak plus Compton integrated values to SCA values (both decay corrected) was $1.087 \pm 0.008$ or $\pm 0.7 \%$. The MCA integrated values and the SCA values thus provided the same information to within one percent. The ratio of the peak minus Compton values from the MCA spectra which are given in Table C-I to the SCA values was $0.860 \pm 0.009$ or $\pm 1.1 \%$ indicating that peak minus Compton values and the SCA data also provided the same information. The latter ratio values for each set of measurements are listed in Table C-I.

The ${ }^{140} \mathrm{Ba}-{ }^{140}$ La results (at $1600 \mathrm{KeV}$ ) for both the SCA and MCA measurements are shown in Figure $\mathrm{C}-2$ as a function of assembly core position. Except for two measurements, from assemblies 6051 , ring position 2, and 6031 , ring position 6 , the results indicated a cosine power distribution consistent with measured tube power data. The results also provided quantitative information about the power tilt across an assembly at various radial positions in the core. The high

* The value represents the average ratio from all measurements and the plus and minus value is the one standard deviation of the average Al1 comparison values used in this appendix are given in this same format. 


\section{C-6}

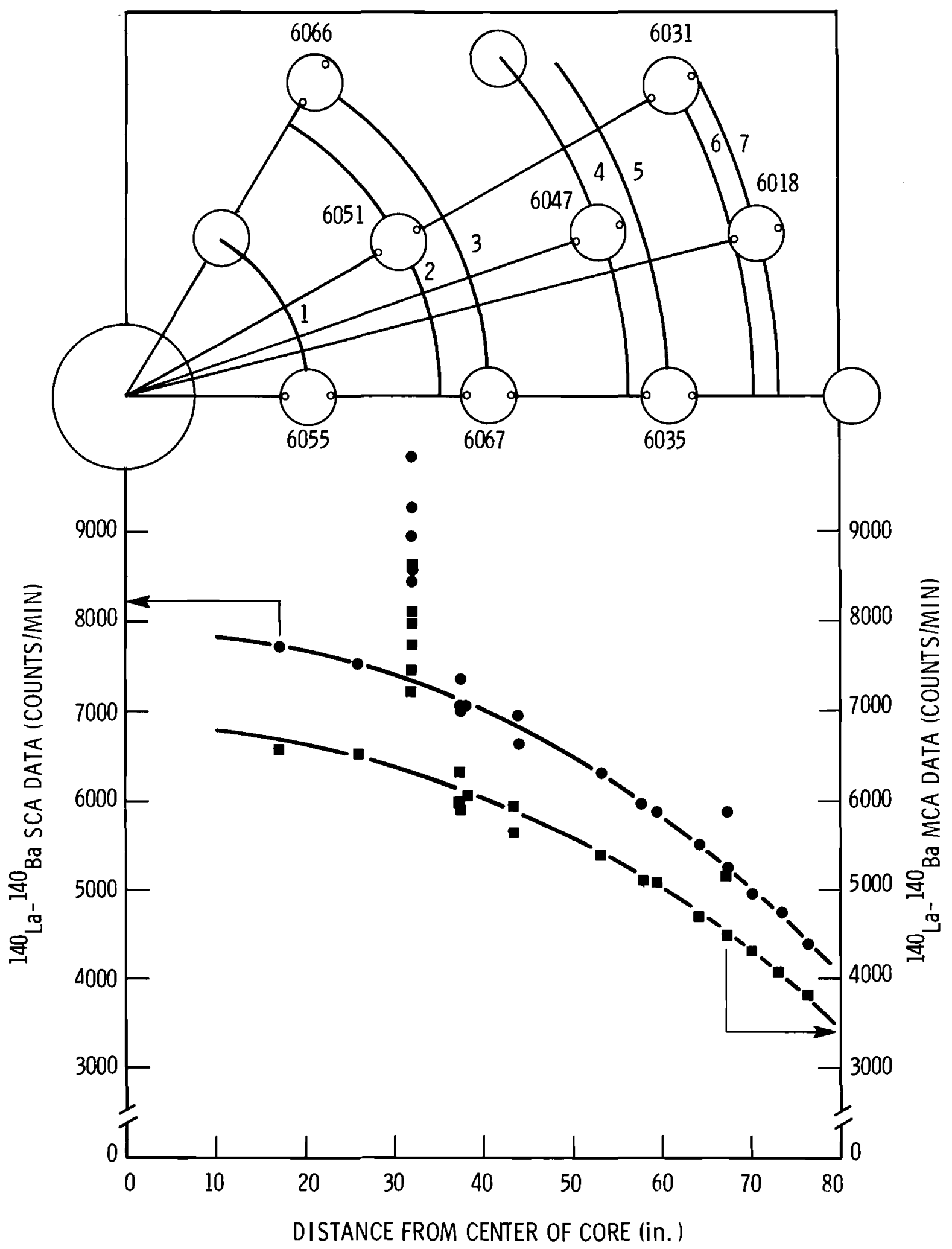

FIGURE C-2. RADIAL POWER DISTRI BUTION FOR BATCH CORE PRIOR TO SHUTDOWN ON JANUARY 28, 1968 
values from assembly 6051 (up) as seen in Figure C-2 and Table C-I was determined to be due to a localized accumulation of fission products similar to those indicated by the gamma-ray activity profiles of several rods given in the body of the report. The high values did not represent a peak in the power distribution. Additional axial measurements over a 17 -inch span using SCA readings only indicated an activity of 7350 counts per minute on either side of the point of localized accumulation of fission products which was consistent with other measurements given. In the case of assembly 6031, a remeasurement gave results consistent with the rest of the data.

Due to the low resolution of a $\mathrm{NaI}(\mathrm{Tl})$ detector, the ${ }^{95} \mathrm{Zr}-{ }^{95} \mathrm{Nb}$ data collected were not as readily interpreted as the ${ }^{140} \mathrm{Ba}-{ }^{140} \mathrm{La}$ data (1600 KeV) were. In addition to the ${ }^{95} \mathrm{Zr}-{ }^{95} \mathrm{Nb}$ gamma rays at 724,756 and $764 \mathrm{KeV},{ }^{137} \mathrm{Cs}(662 \mathrm{KeV}){ }^{144} \mathrm{Ce}-{ }^{144} \mathrm{Pr}(696 \mathrm{KeV}),{ }^{134} \mathrm{Cs}$ (796 and $801 \mathrm{KeV}),{ }^{140} \mathrm{Ba}-{ }^{140} \mathrm{La}(815 \mathrm{KeV})$ and ${ }^{106} \mathrm{Ru}-{ }^{106} \mathrm{Rh}$ (874 KeV) contributed gamma rays in the energy range indicated by the single gross gamma activity peak at approximately $750 \mathrm{KeV}$ discernable in Figure C-1. Furthermore, the peak was superimposed upon the Compton contributions from the $1600 \mathrm{KeV}{ }^{140} \mathrm{Ba}-{ }^{140}$ La gammas. The largest single contributor to $\mathrm{SCA}$ readings other than ${ }^{95} \mathrm{Zr}-{ }^{95} \mathrm{Nb}$ was the Compton contribution. The 65 day half-life of ${ }^{95} \mathrm{Zr}$ indicates that ${ }^{95} \mathrm{Zr}-{ }^{95} \mathrm{Nb}$ data would provide an indication of the average radial power distirbution over a period of reactor operation of approximately 180 days or since the start of the Batch Core. However, the SCA data given in Table C-I 
included major contributions from ${ }^{140} \mathrm{Ba}-{ }^{140}$ La which affects the interpretation of what the true average distribution should be. Using the MCA data, it is possible to approximate the true distribution more closely.

To illustrate, first, the ratio of SCA values and the integrated peak plus Compton values was $1.090 \pm 0.010$ or $\pm 0.9 \%$, indicating both the SCA and MCA systems were providing the same gross information. In this case, the Compton contributions from ${ }^{140} \mathrm{Ba}-{ }^{140} \mathrm{La}$ amounted to approximately $43 \%$ of the total SCA counts as indicated by the MCA results. The ratio of Compton to integrated peak plus Compton was $0.429 \pm 0.016$ or $\pm 3.8 \%$. The $3.8 \%$ variation was due in part to the fact that the Compton decreased with a 12.8 day half-life while the peak was decaying with essentially a 65 day half-life. Subtracting away the Compton, as was done for the MCA values given in Table C-I, values more representative of the average radial power distribution for the first 180 days were determined. Also, because the Compton contributions were subtracted for the MCA data, the variation of the ratio of MCA to SCA values was larger. The ratio for each set of measurements are also listed in Table C-I and the average value was $0.623 \pm 0.016$ or $\pm 2.5 \%$.

The ${ }^{140} \mathrm{Ba}-{ }^{140}$ La contributions from the $815 \mathrm{KeV}$ gamma ray are still present in the MCA ${ }^{95} \mathrm{Zr}-{ }^{95} \mathrm{Nb}$ data 1 isted in Table $\mathrm{C}-\mathrm{I}$. The contribution was not as large as the Compton contribution, however, and no attempt was made to subtract the activity from the ${ }^{95} \mathrm{Zr}-{ }^{95} \mathrm{Nb}$ activity. All other contributions from other gamma-ray sources were 
also small compared to the ${ }^{95} \mathrm{Zr}-{ }^{95} \mathrm{Nb}$ activity and no corrections were made. Decay corrections were not made either since these corrections were less than $2 \%$ using $8: 17$ A.M. on February 21 as the zero time reference.

It is seen from the ${ }^{95} \mathrm{Zr}-{ }^{95} \mathrm{Nb}$ values for assembly 6051 (up) in Table C-I that they were also high as were the ${ }^{140} \mathrm{Ba}-{ }^{140} \mathrm{La}$ values. A possible explanation for the high values may be that ${ }^{95} \mathrm{Nb}$ may have concentrated as did the ${ }^{140} \mathrm{Ba}-{ }^{140} \mathrm{La}$ as part of the localized concentration of fission products. Additional data are needed, high resolution gamma-ray spectra for example, to determine the exact composition of fission products present at the point. The additional SCA data taken on each side of the localized concentration indicated that a ${ }^{95} \mathrm{Zr}-{ }^{95} \mathrm{Nb}$ value of 35800 counts/min was more representative of the activity for assembly 6051 (up).

Since the power distribution changes with increasing exposure, it was expected that the distribution from ${ }^{140} \mathrm{Ba}-{ }^{140}$ La activity data would be different from the distribution derived from the ${ }^{95} \mathrm{Zr}-{ }^{95} \mathrm{Nb}$ activity data. This was the case as the ratio of the activity of assembly 6055 (up) to the activity of assembly 6019 (down) was 1.74 (average of SCA and MCA values) for ${ }^{140} \mathrm{Ba}-{ }^{140} \mathrm{La}$ (see Table C-I for the activity values) while the same ratio for ${ }^{95} \mathrm{Zr}-{ }^{95} \mathrm{Nb}$ was 1.97 (from MCA data only). The power distribution thus flattened as the exposure increased, as was expected. 


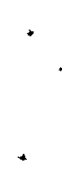


APPENDIX D 


\section{D-1}

APPENDIX D

\section{TABULATION OF GAMMA-RAY SPECTRA}

The spectra obtained from gamma-ray measurements are tabulated in Table D-I on the pages that follow. The order of the spectra is the same as that given in Table XII of the body of the report. 

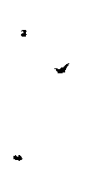

• 
$\underline{\text { TABLE D-I }}$

GAMMA-RAY SPECTRA COLLECTED FROM BATCH CORE FUEL RODS

pp. $D-2$ to $D-34$ 
GAMMA RAY SPECTRUM DATA

PRTR ROD FS-16 SPECTRUM NO, 14 DATA TAKEN 3/02/69 LIVE TIME 733.MIN. POSITION 9.IN, ENERGY(KEV) $=1.112 *$ CHAN =8.5 CHAN COUNT COUNT COUNT COUNT COUNT COUNT COUNT COUNT COUNT COUNT

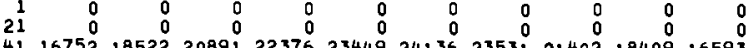

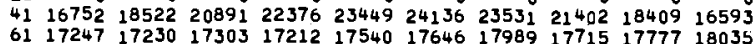
$\begin{array}{lllllllllllll}61 & 17247 & 17230 & 17303 & 17212 & 17540 & 17646 & 17989 & 17715 & 17777 & 18035 \\ 8 & 18189 & 18591 & 18545 & 18878 & 18888 & 18979 & 18642 & 18715 & 18646 & 18890\end{array}$ $\begin{array}{rrlllllllll}81 & 18189 & 18591 & 18545 & 18878 & 18888 & 18979 & 18642 & 18715 & 18646 & 18890\end{array}$ $121 \quad 1790017805 \quad 17644 \quad 17747 \begin{array}{llllllllll}17855 & 18293 & 19009 & 20362 & 19779 & 18855\end{array}$ $16116831171611694316983170961731817643 \quad 17444178317046$ $\begin{array}{lllllllllllll}181 & 17431 & 17932 & 17466 & 17787 & 17523 & 17515 & 17409 & 17203 & 17354 & 17274\end{array}$

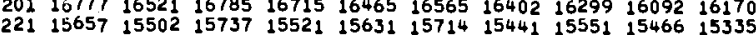
$\begin{array}{lllllllllll}241 & 15481 & 15121 & 15383 & 15382 & 15111 & 15098 & 15158 & 15201 & 15082 & 15166\end{array}$

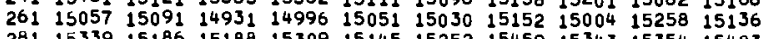
$\begin{array}{lllllllllll}281 & 15339 & 15186 & 15188 & 15309 & 15145 & 15252 & 15459 & 15343 & 15354 & 15403\end{array}$

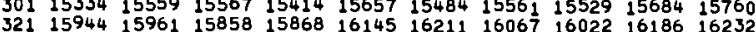
$341 \quad 1666416425 \quad 16871 \quad 16759 \begin{array}{llllllllll}16914 & 16860 & 160648 & 16022 & 16186 & 16232\end{array}$ $\begin{array}{llllllllllll}361 & 16651 & 16532 & 16645 & 16442 & 16218 & 16453 & 16394 & 16509 & 16356 & 16263\end{array}$ $\begin{array}{llllllllllll}381 & 10091 & 15821 & 15833 & 15590 & 15603 & 15314 & 14947 & 14673 & 14210 & 13702\end{array}$

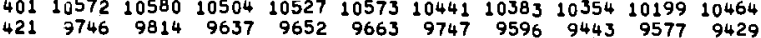
$\begin{array}{lllllllllll}441 & 8970 & 8963 & 8835 & 8934 & 8853 & 8752 & 8876 & 8813 & 8813 & 8678\end{array}$ $\begin{array}{lllllllllll}461 & 3580 & 8469 & 8636 & 8760 & 9238 & 9769 & 10830 & 11227 & 10856 & 9496\end{array}$ $\begin{array}{llllllllllll}501 & 9174 & 8620 & 8576 & 8646 & 8628 & 8569 & 8725 & 8812 & 8827 & 8626\end{array}$

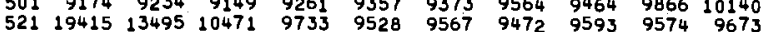
$\begin{array}{lllllllllll}541 & 9825 & 10075 & 9828 & 9863 & 10143 & 10250 & 12103 & 17670 & 32158 & 58335\end{array}$ $\begin{array}{lllllllllll}561 & 4867 & 4870 & 4801 & 4813 & 4900 & 5224 & 5160 & 4957 & 4741 & 4406\end{array}$ $\begin{array}{lllllllllll}581 & 4073 & 4026 & 3908 & 3948 & 3954 & 3933 & 3939 & 3882 & 3956 & 3742 \\ 601 & 4539 & 5233 & 5367 & 4830 & 3940 & 3449 & 3241 & 3264 & 3209 & 3096\end{array}$ $\begin{array}{lllllllllll}621 & 2771 & 2677 & 2705 & 2599 & 2591 & 2566 & 2519 & 2501 & 2497 & 2548\end{array}$

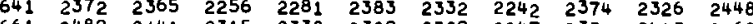
$\begin{array}{lllllllllll}661 & 2489 & 2341 & 2315 & 2338 & 2309 & 2329 & 2247 & 2331 & 2413 & 2268\end{array}$ $\begin{array}{lllllllllll}701 & 2559 & 2547 & 2390 & 2455 & 2551 & 2693 & 2819 & 3018 & 2945 & 2811 \\ & 254 & 2625 & 2638 & 2630 & 2691 & 2741 & 2688\end{array}$ $\begin{array}{lllllllllll}721 & 22350 & 35747 & 46897 & 45112 & 30394 & 14409 & 7382 & 6504 & 6765 & 5782\end{array}$ $\begin{array}{lllllllllll}741 & 2088 & 2223 & 2152 & 2298 & 2207 & 2235 & 2247 & 2267 & 2186 & 2196\end{array}$

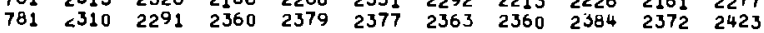

$\begin{array}{lllllllllll}801 & 2416 & 2516 & 2531 & 2440 & 2518 & 2498 & 2484 & 2536 & 2507 & 2501\end{array}$ $\begin{array}{lllllllllll}821 & 2597 & 2622 & 2671 & 2623 & 2616 & 2634 & 2639 & 2654 & 2634 & 2674 \\ 841 & 2767 & 2777 & 2713 & 2750 & 2699 & 2749 & 2811 & 2777 & 2856 & 2782\end{array}$ $\begin{array}{llllllllllll}861 & 2844 & 2850 & 2739 & 2728 & 2785 & 2708 & 2719 & 2741 & 2645 & 2589\end{array}$

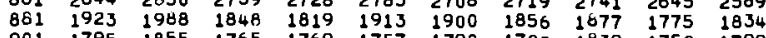
$\begin{array}{lllllllllll}901 & 1795 & 1855 & 1765 & 1769 & 1757 & 1790 & 1702 & 1839 & 1750 & 1799\end{array}$ $\begin{array}{lllllllllll}921 & 1782 & 1769 & 1811 & 1715 & 1741 & 1759 & 1742 & 1739 & 1771 & 1706 \\ 941 & 2034 & 2061 & 1969 & 1838 & 1769 & 1738 & 1736 & 1705 & 1688 & 1667\end{array}$ $\begin{array}{lllllllllll}961 & 1635 & 1639 & 1594 & 1737 & 1672 & 1727 & 1645 & 1612 & 1627 & 1668\end{array}$

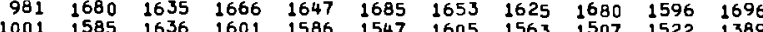

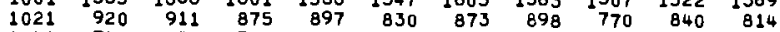
$\begin{array}{lllllllllll}1041 & 742 & 751 & 714 & 727 & 693 & 742 & 694 & 716 & 710 & 723\end{array}$ $\begin{array}{lllllllllll}1061 & 6921 & 8602 & 9048 & 7441 & 4301 & 1811 & 762 & 489 & 460 & 463\end{array}$

$\begin{array}{lllllllllll}1101 & 202 & 189 & 210 & 199 & 232 & 340 & 301 & 299 & 342 & 300\end{array}$

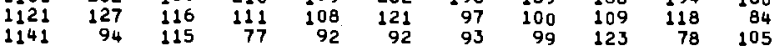

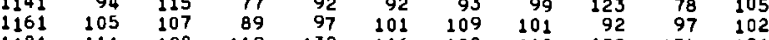

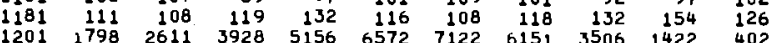

$\begin{array}{rrrrrrrrrrr}1201 & 1798 & 2611 & 3928 & 5156 & 6572 & 7122 & 6151 & 3506 & 1422 & 402 \\ 1221 & 24 & 23 & 22 & 21 & 24 & 22 & 36 & 69 & 117 & 172 \\ 1241 & 16 & 14 & 9 & 9 & 12 & 11 & 16 & 8 & 16 & 7\end{array}$

128

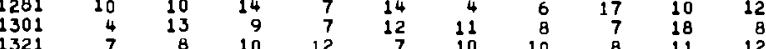

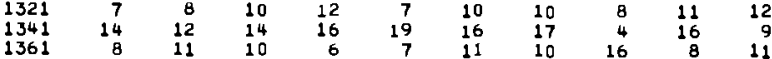

1381

142

144

1481

154

1561

160

1621

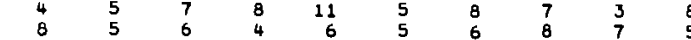

168

1701
1721

1741
1761

$\begin{array}{rrrrrrrrrrr}1781 & 5 & 5 & 2 & 5 & 5 & 9 & 1 & 1 & 5 & 7 \\ 1801 & 3 & 3 & 3 & 4 & 7 & 5 & 3 & 3 & 5 & 6 \\ 1821 & 7 & 4 & 4 & 7 & 3 & 4 & 4 & 5 & 7 & 6 \\ 1841 & 3 & 2 & 4 & 3 & 3 & 0 & 2 & 2 & 2 & 2 \\ 1861 & 4 & 4 & 2 & 4 & 4 & 2 & 1 & 7 & 2 & 6 \\ 1881 & 2 & 4 & 2 & 1 & 4 & 4 & 7 & 5 & 3 & 9 \\ 1901 & 6 & 3 & 3 & 6 & 2 & 1 & 3 & 2 & 7 & 4 \\ 1921 & 1 & 4 & 6 & 5 & 2 & 3 & 5 & 3 & 6 & 3 \\ 1941 & 3 & 2 & 5 & 3 & 5 & 7 & 3 & 2 & 3 & 4 \\ 1961 & 2 & 6 & 5 & 5 & 9 & 10 & 9 & 9 & 11 & 15 \\ 1941 & 1 & 3 & 1 & 4 & 1 & 3 & 3 & 3 & 6 & 1 \\ 2001 & 4 & 1 & 1 & 5 & 2 & 8 & 7 & 1 & 1 & 2 \\ 2021 & 1 & 4 & 3 & 3 & 1 & 5 & 4 & 1 & 6 & 1 \\ 2041 & 4 & 3 & 1 & 2 & 2 & 2 & 3 & 0 & & \end{array}$

COUNT COUNT COUNT COUNT COUNT COUNT COUNT COUNT COUNT COUNT $\begin{array}{rrrrrrrrrr}0 & 0 & 0 & 0 & 0 & 0 & 0 & 0 & 0 & 0 \\ 0 & 0 & 0 & 0 & 1 & 1 & 65 & 594 & 7004 & 15508\end{array}$ $\begin{array}{llllllllll}16433 & 16115 & 16108 & 16500 & 16539 & 16548 & 16891 & 16801 & 16845 & 16766\end{array}$ $\begin{array}{lllllllllll}17956 & 18177 & 18540 & 19194 & 19511 & 19623 & 18911 & 18460 & 18543 & 18332\end{array}$

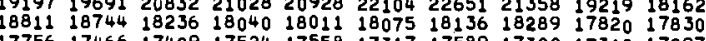
$\begin{array}{llllllllll}17756 & 17466 & 17409 & 17524 & 17558 & 17317 & 17589 & 17300 & 17318 & 17297\end{array}$ $\begin{array}{llllllllllll}17875 & 17851 & 17638 & 17781 & 17777 & 17563 & 17289 & 17499 & 17203 & 17445\end{array}$ $\begin{array}{llllllllll}16914 & 16984 & 17091 & 16715 & 16751 & 16968 & 16843 & 16695 & 16740 & 16748\end{array}$ $156615306 \quad 15324152531534915162 \quad 1543815317 \quad 552759$ $1497814934 \quad 15173 \quad 1517015253 \quad 15098 \quad 1492315104 \quad 1482815024$

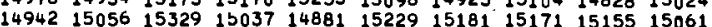
$\begin{array}{llllllllllll}15013 & 15187 & 15277 & 15280 & 15167 & 15439 & 15494 & 15490 & 15193 & 15494\end{array}$ $1572015834 \quad 15602 \quad 1589915922 \quad 15897 \quad 15778 \quad 15883 \quad 1554015909$ $\begin{array}{llllllllllllll}16446 & 16063 & 16470 & 16420 & 16476 & 16367 & 16451 & 16726 & 16814 & 16638\end{array}$ $\begin{array}{lllllllllll}17263 & 17067 & 16854 & 17061 & 16739 & 16838 & 16644 & 16822 & 16909 & 16714\end{array}$ $\begin{array}{lllllllllll}16497 & 16334 & 16250 & 16424 & 16183 & 16301 & 16348 & 16225 & 16135 & 16306\end{array}$

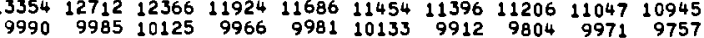

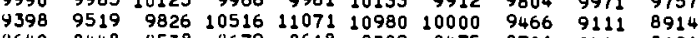
$\begin{array}{lllllllllll}8640 & 8448 & 8538 & 8679 & 8618 & 8389 & 8475 & 8701 & 6411 & 8621 \\ 8773 & 8583 & 8545 & 8623 & 8389 & 8558 & 8574 & 8339 & 8608 & 8374\end{array}$

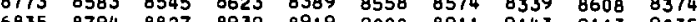

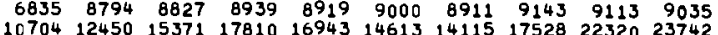
$\begin{array}{llllllllll}9660 & 9728 & 9604 & 9644 & 9805 & 9767 & 9912 & 9830 & 9894 & 9818\end{array}$

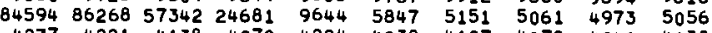
$\begin{array}{llllllllll}4277 & 4221 & 4138 & 4270 & 4224 & 4038 & 4127 & 4072 & 4973 & 5056 \\ 4138\end{array}$ $\begin{array}{llllllllll}4277 & 4221 & 4138 & 4270 & 4224 & 4038 & 4127 & 4072 & 4046 & 4138 \\ 3819 & 3757 & 3660 & 3669 & 3616 & 3582 & 3611 & 3643 & 3805 & 4071\end{array}$ $\begin{array}{llllllllll}3148 & 3086 & 3109 & 3022 & 2916 & 2880 & 2884 & 2809 & 2822 & 2779 \\ 2430 & 2485 & 2541 & 2564 & 2499 & 2413 & 2480 & 2428 & 2360 & 2308\end{array}$ $\begin{array}{llllllllll}2362 & 2306 & 2330 & 2262 & 2314 & 2413 & 2480 & 2428 & 2360 & 2308 \\ 2571 & 2579 & 2798 & 2842 & 2680\end{array}$ $\begin{array}{llllllllll}2363 & 2385 & 2389 & 2398 & 2418 & 2410 & 2462 & 2330 & 2324 & 2451 \\ 2671 & 2786 & 2929 & 3495 & 4058 & 4635 & 4571 & 3784 & 2935 & 2638\end{array}$ $\begin{array}{rrrrrrrrrr}2671 & 2786 & 2929 & 3495 & 4058 & 4635 & 4571 & 3784 & 2935 & 2638 \\ 2820 & 2716 & 2843 & 2909 & 2970 & 3156 & 3346 & 4263 & 6802 & 12497\end{array}$ $\begin{array}{llllllllll}3906 & 2736 & 2412 & 2671 & 3102 & 3658 & 3720 & 3410 & 2794 & 2318\end{array}$ $\begin{array}{llllllllll}2268 & 2128 & 231 & 2288 & 2288 & 2298 & 2372 & 2347 & 2405 & 2260 \\ 2252 & 2263 & 2264 & 2231 & 2206 & 2238 & 2361 & 2293 & 2358 & 2266\end{array}$ $\begin{array}{llllllllll}2431 & 2421 & 2426 & 2488 & 2399 & 2458 & 2414 & 2482 & 2471 & 2435\end{array}$ $\begin{array}{llllllllll}2742 & 2570 & 2747 & 2718 & 2619 & 2739 & 2630 & 2654 & 2739 & 2672\end{array}$ $\begin{array}{llllllllll}2854 & 2836 & 2712 & 2896 & 2784 & 2759 & 2757 & 2727 & 2695 & 2666\end{array}$

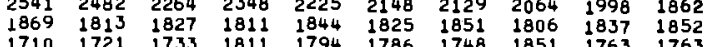
$\begin{array}{llllllllll}1710 & 1721 & 1733 & 1811 & 1794 & 1786 & 1748 & 1851 & 1763 & 1763\end{array}$ $\begin{array}{llllllllll}1830 & 1783 & 1670 & 1807 & 1796 & 1804 & 1768 & 1842 & 1936 & 2073\end{array}$ $\begin{array}{llllllllll}1725 & 1770 & 1731 & 1731 & 1678 & 1703 & 1683 & 1582 & 1715 & 1633 \\ 1669 & 1737 & 1639 & 1680 & 1661 & 1694 & 1720 & 1691 & 1645 & 1670\end{array}$ $\begin{array}{lllllllllll}1669 & 1737 & 1639 & 1680 & 1661 & 1694 & 1720 & 1691 & 1645 & 1670 \\ 1727 & 1673 & 1634 & 1637 & 1681 & 1633 & 1612 & 1667 & 1678 & 1707\end{array}$ $\begin{array}{rrrrrrrrrr}1727 & 1673 & 1634 & 1637 & 1681 & 1633 & 1612 & 1667 & 1678 & 1707 \\ 1359 & 1373 & 1304 & 1231 & 1156 & 1140 & 1037 & 1020 & 945 & 938\end{array}$ $\begin{array}{llllllllll}795 & 822 & 801 & 828 & 777 & 762 & 768 & 745 & 734 & 738\end{array}$ $\begin{array}{rrrrrrrrrr}438 & 421 & 431 & 932 & 1122 & 1391 & 1966 & 2682 & 3893 & 5093 \\ 433 & 407 & 433 & 426 & 373 & 396 & 400 & 380\end{array}$ $\begin{array}{llllllllll}257 & 293 & 303 & 255 & 251 & 261 & 228 & 233 & 224 & 209\end{array}$ $\begin{array}{rrrrrrrrrr}161 & 166 & 174 & 178 & 184 & 166 & 166 & 147 & 125 & 113 \\ 96 & 89 & 90 & 105 & 90 & 101 & 85 & 94 & 100 & 89\end{array}$ $\begin{array}{rrrrrrrrrr}88 & 84 & 93 & 89 & 88 & 105 & 101 & 94 & 107 & 98 \\ 108 & 90 & 112 & 110 & 96 & 111 & 116 & 116 & 117 & 125 \\ 141 & 152 & 156 & 165 & 165 & 199 & 268 & 405 & 707 & 1152\end{array}$ $\begin{array}{rrrrrrrrrr}141 & 152 & 156 & 165 & 165 & 199 & 268 & 405 & 707 & 1152 \\ 83 & 22 & 17 & 16 & 25 & 20 & 21 & 23 & 20 & 18\end{array}$ $\begin{array}{rrrrrrrrrr}261 & 325 & 456 & 611 & 653 & 579 & 432 & 187 & 68 & 22 \\ 13 & 17 & 10 & 11 & 11 & 7 & 6 & 10 & 8 & 17 \\ 12 & 10 & 13 & 7 & 7 & 10 & 6 & 9 & 12 & 10\end{array}$ 12 11

$$
\begin{array}{rr}
11 & 6 \\
7 & 7 \\
9 & 7 \\
6 & 14 \\
6 & 5 \\
8 & 8 \\
8 & 5 \\
6 & 9 \\
6 & 8 \\
6 & 7 \\
9 & 9 \\
6 & 8 \\
7 & 11 \\
9 & 6 \\
9 & 11 \\
3 & 5 \\
7 & 11 \\
7 & 6 \\
5 & 9 \\
11 & 6 \\
9 & 1 \\
6 & 6
\end{array}
$$

9
8
5
4
9
8
11
8
8
7
13
11
5
7
6
9
6
10
6
8
5
5
6
6
6

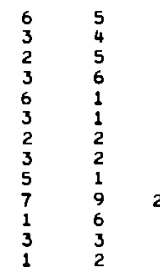

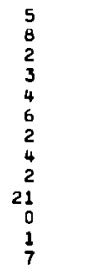

5
5
2
5
7
4
3
6
2
10
3
3
6

$\begin{array}{ll}4 & 6 \\ 4 & 4 \\ 6 & 4 \\ 5 & 3 \\ 2 & 5 \\ 4 & 5 \\ 5 & 2 \\ 2 & 3 \\ 3 & 5 \\ 3 & 3 \\ 4 & 3 \\ 5 & 9 \\ 3 & 3 \\ & \end{array}$

$\begin{array}{lll}3 & 7 & 5 \\ 8 & 7 & 6 \\ 7 & 4 & 8 \\ 3 & 4 & 2 \\ 3 & 3 & 9 \\ 8 & 3 & 6 \\ 4 & 3 & 2 \\ 3 & 3 & 8 \\ 4 & 4 & 5 \\ 7 & 4 & 5 \\ 3 & 2 & 5 \\ 1 & 0 & 1 \\ 0 & 2 & 3\end{array}$


D-3

GAMMA RAY SPECTRUM DATA

PATR RUO FS-16 SPECTRUM NO. 15 DATA TAKEN 3/02769 LIVE TIME G00.MIN. POSITION O.IN, ENERGY(KEV)= 1.112*CHAN -B.4 CHAN COUNT COUNT COUNT COUNT COUNT GOUNT COUNT COUNT COUNT COUNT

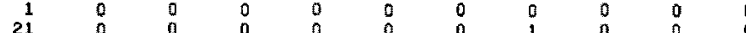
$\begin{array}{rrrrrrrrrrr}41 & 6165 & 6503 & 7407 & 8015 & 8491 & 8859 & 8653 & 7970 & 6869 & 5955 \\ 61 & 6226 & 6178 & 6243 & 6111 & 6279 & 6325 & 6359 & 6342 & 6571 & 6467\end{array}$ $\begin{array}{rrrrrrrrrrr}81 & 7157 & 7267 & 7343 & 7568 & 7683 & 8098 & 7986 & 8005 & 8111 & 8352 \\ 101 & 7510 & 7410 & 7476 & 7491 & 7968 & 9034 & 1925 & 15227 & 15127 & 12087\end{array}$ $\begin{array}{rrrrrrrrrrr}101 & 7518 & 7410 & 7476 & 7491 & 7968 & 9034 & 11925 & 15227 & 15127 & 12087 \\ 121 & 6254 & 6151 & 6376 & 6200 & 6178 & 6474 & 7362 & 9120 & 9292 & 7796\end{array}$ $\begin{array}{lllllllllll}121 & 6254 & 6151 & 6376 & 6200 & 6178 & 6474 & 7382 & 9120 & 9292 & 7796 \\ 141 & 5662 & 5782 & 5613 & 5693 & 5671 & 5823 & 5681 & 5380 & 5530 & 5504\end{array}$ $\begin{array}{lllllllllll}141 & 5662 & 5782 & 5613 & 5693 & 5671 & 5823 & 5681 & 5380 & 5530 & \mathbf{5 5 0 4} \\ 161 & 5263 & \mathbf{5 2 8 1} & \mathbf{5 4 4 7} & \mathbf{5 4 2 5} & \mathbf{5 3 9 8} & \mathbf{5 3 0 5} & \mathbf{5 3 1 8} & \mathbf{5 2 7 3} & 5179 & \mathbf{5 2 4 1}\end{array}$ $\begin{array}{lllllllllll}181 & 5163 & 5226 & 5054 & 5048 & 4999 & 5009 & 4881 & 4852 & 4997 & 4889\end{array}$

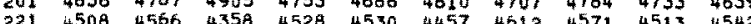
$241 \quad 4412 \quad 4366 \quad 4401 \quad 4317 \quad 4346 \quad 4237 \quad 4306 \quad 4321 \quad 4262$ $261418242844124 \quad 42534308 \quad 4233 \quad 4216 \quad 4306 \quad 4244 \quad 4011$

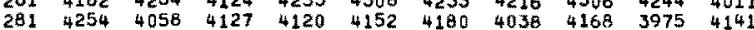

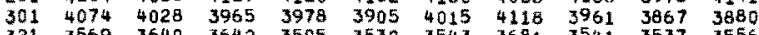

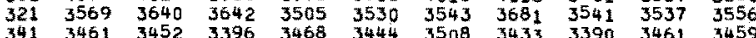
$\begin{array}{lllllllllll}341 & 3461 & 3452 & 3396 & 3468 & 3444 & 3508 & 3433 & 3390 & 3461 & 3459\end{array}$ $\begin{array}{lllllllllll}381 & 3173 & 3212 & 3204 & 3306 & 3132 & 3239 & 3197 & 3207 & 3229 & 3179\end{array}$

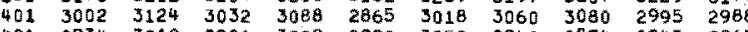
$\begin{array}{lllllllllll}421 & 2934 & 3012 & 2901 & 3009 & 2990 & 3050 & 2942 & 2874 & 2943 & 2967 \\ 441 & 2676 & 2559 & 2589 & 2588 & 2582 & 2629 & 2608 & 2579 & 2521 & 2429\end{array}$ $\begin{array}{lllllllllll}441 & 2676 & 2559 & 2589 & 2588 & 2582 & 2629 & 2608 & 2579 & 2521 & 2429 \\ 461 & 2545 & 2611 & 2559 & 2733 & 3220 & 4191 & 5161 & 7696 & 7143 & 4977\end{array}$ $\begin{array}{lllllllllll}481 & 2386 & 2331 & 2400 & 2479 & 2282 & 2322 & 2237 & 2294 & 2281 & 2301\end{array}$

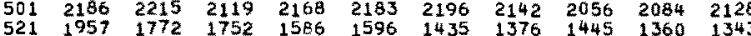

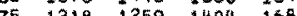

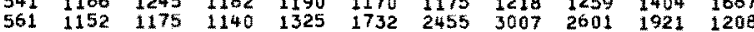
$\begin{array}{lllllllllll}581 & 847 & 796 & 738 & 756 & 793 & 723 & 757 & 740 & 737 & 725\end{array}$

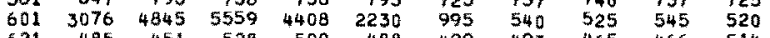

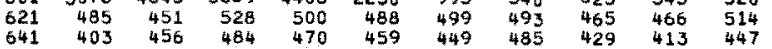
$\begin{array}{rrrrrrrrrrr}661 & 1156 & 697 & 509 & 433 & 382 & 443 & 407 & 444 & 433 & 437\end{array}$ $\begin{array}{rrrrrrrrrrr}681 & 463 & 462 & 554 & 646 & 923 & 1343 & 1954 & 2574 & 2404 & 1691 \\ 701 & 367 & 301 & 330 & 334 & 321 & 322 & 297 & 303 & 332 & 320\end{array}$

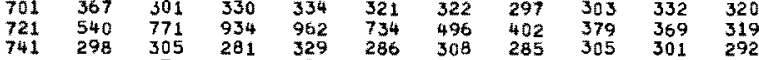
$\begin{array}{lllllllllll}761 & 324 & 376 & 281 & 329 & 286 & 308 & 285 & 305 & 301 & 292 \\ 781 & 278 & 282 & 303 & 292 & 282 & 309 & 283 & 277 & 264 & 278 \\ & & & & & & & & & & \end{array}$ COUNT COUNT COUNT COUNT COUNT COUNT COUNT COUNT COUNT COUNT $\begin{array}{rrrrrrrrrr}0 & 0 & 0 & 0 & 1 & 0 & 0 & 0 & 0 & 0 \\ 0 & 0 & 0 & 0 & 0 & 0 & 1 & 74 & 1045 & 5527 \\ 5794 & 5732 & 5789 & 5761 & 5789 & 5928 & 5774 & 5756 & 6096 & 6164\end{array}$

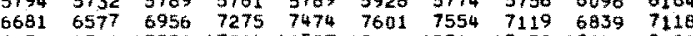

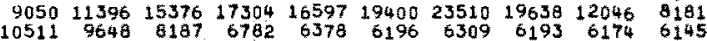
$\begin{array}{rlllllllll}6378 & 5934 & 5850 & 5788 & 5810 & 5922 & 5776 & 5696 & 5890 & 5946\end{array}$ $\begin{array}{llllllllll}5651 & 5513 & 5547 & 5436 & \mathbf{5 5 2 6} & \mathbf{5 5 6 4} & \mathbf{5 3 9 8} & \mathbf{5 4 0 6} & 5487 & 5348\end{array}$ $\begin{array}{llllllllll}4805 & 4964 & 4930 & 4867 & 4778 & 5029 & 4998 & 4870 & 4735 & 4790\end{array}$

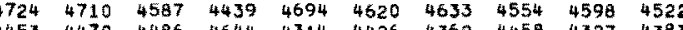
$4274 \quad 4371 \quad 4305 \quad 4187 \quad 4188 \quad 4289 \quad 4294 \quad 4298 \quad 4213 \quad 4126$ $4093 \quad 4141 \quad 4105 \quad 4111 \quad 4245 \quad 4286 \quad 4175 \quad 4144 \quad 41304095$ $\begin{array}{llllllllll}4177 & 4004 & 4079 & 4019 & 4077 & 4085 & 4067 & 4049 & 4060 & 4158\end{array}$ $\begin{array}{llllllllll}3943 & 3843 & 3763 & 3630 & 3777 & 3791 & 3667 & 3714 & 3686 & 3559\end{array}$ $\begin{array}{llllllllll}3542 & 3448 & 3417 & 3469 & 3455 & 3499 & 3542 & 3453 & 3335 & 3496 \\ 3409 & 3461 & 3376 & 3329 & 3414 & 3379 & 3373 & 3297 & 3385 & 3336\end{array}$

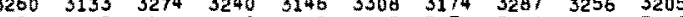

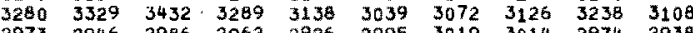
$\begin{array}{llllllllll}2973 & 2946 & 2986 & 2962 & 2926 & 2995 & 3019 & 3014 & 2974 & 2938\end{array}$ $\begin{array}{llllllllll}2839 & 2948 & 2865 & 2917 & 2875 & 2798 & 2797 & 2680 & 2647 & 2724\end{array}$

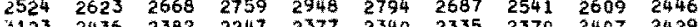
$\begin{array}{llllllllll}216 & 2258 & 2228 & 2255 & 2196 & 2188 & 2172 & 2256 & 2222 & 2163\end{array}$

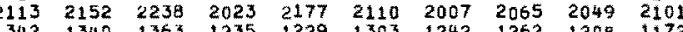
$\begin{array}{llllllllll}2038 & 2213 & 1794 & 1380 & 1065 & 1049 & 1044 & 989 & 1021 & 1053\end{array}$

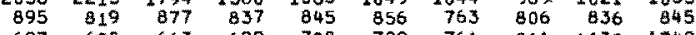
$\begin{array}{llllllllll}697 & 698 & 663 & 699 & 708 & 729 & 761 & 811 & 1130 & 1749\end{array}$ $\begin{array}{llllllllll}505 & 532 & 486 & 461 & 502 & 472 & 502 & 490 & 483 & 466\end{array}$

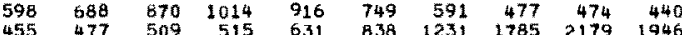

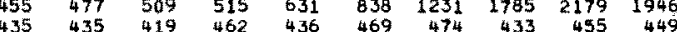
$\begin{array}{llllllllll}1061 & 1065 & 1969 & 3650 & 5945 & 7906 & 7876 & 5321 & 2231 & 740\end{array}$ $\begin{array}{llllllllll}329 & 342 & 327 & 281 & 307 & 307 & 339 & 309 & 346 & 439 \\ 329 & 296 & 319 & 327 & 312 & 333 & 340 & 298 & 301\end{array}$ $\begin{array}{llllllllll}281 & 275 & 266 & 245 & 250 & 255 & 291 & 264 & 268 & 262\end{array}$

\begin{tabular}{|c|c|c|c|c|c|c|c|c|c|c|c|c|c|c|c|c|c|c|c|}
\hline $\begin{array}{l}801 \\
821\end{array}$ & $\begin{array}{l}272 \\
237\end{array}$ & $\begin{array}{l}255 \\
294\end{array}$ & $\begin{array}{l}244 \\
254\end{array}$ & $\begin{array}{l}245 \\
256\end{array}$ & $\begin{array}{l}248 \\
290\end{array}$ & $\begin{array}{l}301 \\
272\end{array}$ & $\begin{array}{l}280 \\
244\end{array}$ & 253 & 310 & $\begin{array}{l}290 \\
279\end{array}$ & 265 & $\begin{array}{l}256 \\
293\end{array}$ & $2^{48}$ & 278 & 231 & $\begin{array}{r}245 \\
256\end{array}$ & $\begin{array}{r}291 \\
284\end{array}$ & 298 & $\begin{array}{r}276 \\
273\end{array}$ \\
\hline 841 & 249 & 218 & 232 & 245 & 283 & $\begin{array}{l}247 \\
247\end{array}$ & 267 & 282 & 250 & 277 & $\begin{array}{r}260 \\
298\end{array}$ & $\begin{array}{r}283 \\
245\end{array}$ & $\begin{array}{l}242 \\
284\end{array}$ & 284 & $\begin{array}{l}251 \\
290\end{array}$ & $\begin{array}{l}250 \\
296\end{array}$ & $\begin{array}{l}284 \\
252\end{array}$ & $\begin{array}{l}211 \\
265\end{array}$ & $\begin{array}{l}263 \\
265\end{array}$ \\
\hline 255 & 258 & 257 & 277 & 267 & 249 & 259 & 242 & 219 & 245 & 229 & 219 & 245 & 252 & 238 & 241 & 221 & 211 & 222 & 206 \\
\hline 199 & $1 \in 8$ & 191 & 176 & 201 & 176 & 204 & 108 & 200 & 102 & 188 & 213 & 201 & 184 & 158 & 186 & 182 & 184 & 179 & 164 \\
\hline 200 & 185 & 183 & 170 & 182 & 184 & 201 & 169 & 177 & 167 & 167. & 190 & 193 & 167 & 185 & 154 & 188 & 163 & 176 & 197 \\
\hline 191 & 172 & 184 & 162 & 164 & 145 & 159 & 157 & 188 & 197 & 157 & 172 & 177 & 178 & 171 & 180 & 177 & 162 & 175 & 156 \\
\hline 186 & 166 & 151 & 179 & 180 & 170 & 190 & 190 & 213 & 259 & 266 & 310 & 334 & 292 & 204 & $\begin{array}{l}100 \\
147\end{array}$ & 180 & $\begin{array}{l}182 \\
182\end{array}$ & $\begin{array}{l}175 \\
139\end{array}$ & $\begin{array}{l}150 \\
156\end{array}$ \\
\hline 145 & 169 & 173 & 148 & 171 & 164 & 189 & 142 & 187 & $10_{1}$ & 157 & 137 & 131 & 182 & 137 & 159 & $\begin{array}{l}148 \\
\end{array}$ & $\begin{array}{l}147 \\
\end{array}$ & 144 & 152 \\
\hline 156 & 144 & 155 & 145 & 177 & 163 & 140 & 157 & 145 & 160 & 146 & 159 & 152 & 149 & 172 & 131 & 160 & 145 & 154 & 175 \\
\hline 153 & 170 & 151 & 140 & 145 & 157 & 136 & 166 & 168 & 188 & 156 & 199 & 136 & 100 & 126 & 110 & 108 & 121 & 129 & 117 \\
\hline 125 & 143 & 121 & 132 & 100 & 93 & 82 & 93 & 99 & 88 & 86 & 91 & 87 & 84 & 92 & 97 & 89 & 97 & 78 & 81 \\
\hline 92 & 87 & 95 & 98 & 80 & $7 B$ & 89 & 75 & 85 & 108 & 108 & 133 & 139 & 157 & 146 & 163 & 157 & 146 & 228 & 248 \\
\hline 368 & 520 & 613 & 516 & 299 & 148 & 73 & 71 & 64 & 69 & 63 & 69 & 63 & 71 & 57 & 59 & 52 & 60 & 72 & 49 \\
\hline 63 & 67 & 74 & 72 & 63 & 57 & 56 & 56 & 58 & 53 & 55 & 52 & 49 & 43 & 68 & 50 & 47 & 55 & 58 & 43 \\
\hline 55 & 63 & 51 & 44 & 53 & 54 & 50 & 53 & 42 & 47 & 41 & 53 & 54 & 39 & 51 & 47 & 37 & 41 & 42 & 35 \\
\hline $4 B$ & 50 & 52 & 49 & 49 & 40 & 40 & 39 & 53 & 41 & 41 & 37 & 45 & 31 & 55 & 44 & 60 & 42 & 31 & 43 \\
\hline 42 & so & 51 & 36 & 41 & 61 & $\$ 5$ & 37 & 48 & 41 & 42 & 48 & 39 & 33 & 29 & 46 & 47 & 28 & 44 & 35 \\
\hline 42 & 33 & 47 & 32 & 35 & 38 & 48 & 46 & 39 & 27 & 30 & 37 & 36 & 37 & 33 & $\begin{array}{r}74 \\
44\end{array}$ & 35 & 40 & 39 & 37 \\
\hline 25 & 28 & 41 & 39 & 27 & 41 & 38 & 27 & 44 & 45 & 34 & 32 & 37 & 33 & 43 & 58 & 63 & 58 & 69 & 84 \\
\hline 201 & 143 & 216 & 310 & 413 & 476 & 402 & 218 & 95 & 55 & 27 & 32 & 25 & 16 & 17 & 21 & 21 & 32 & 26 & 30 \\
\hline 28 & 19 & 38 & 32 & 28 & 25 & 23 & 18 & 22 & 30 & 20 & 24 & 20 & 29 & 43 & 47 & 29 & 23 & 29 & 16 \\
\hline 22 & 29 & 28 & 27 & 27 & 18 & 27 & 16 & 26 & 26 & 21 & 28 & 16 & 27 & 20 & 21 & 36 & 19 & 19 & 21 \\
\hline 21 & 20 & 21 & 27 & 20 & 29 & 17 & 21 & 15 & 24 & 22 & 26 & 24 & 17 & 14 & 22 & 20 & 11 & 17 & 19 \\
\hline 23 & 19 & 28 & 27 & 24 & 17 & 22 & 11 & 20 & 22 & 23 & 31 & 22 & 22 & 14 & 23 & 21 & 24 & 27 & 27 \\
\hline 25 & 19 & 20 & 3.3 & 25 & 24 & 25 & 28 & 14 & 15 & 17 & 16 & 21 & 26 & 19 & 21 & 18 & 14 & 22 & 35 \\
\hline 11 & 16 & 25 & 22 & 27 & 27 & 18 & 19 & 29 & 18 & 25 & 19 & 16 & 20 & 29 & 21 & 21 & 16 & 31 & 33 \\
\hline 29 & 29 & 39 & 44 & 48 & 51 & 54 & 52 & 32 & 25 & 22 & 17 & 19 & 27 & 27 & $\begin{array}{l}19 \\
19\end{array}$ & $\begin{array}{l}1 \\
16\end{array}$ & 13 & 19 & 17 \\
\hline 9 & 27 & 26 & 17 & 9 & 17 & 11 & 11 & 22 & 25 & 20 & 23 & 13 & 16 & 21 & 15 & 13 & 21 & 19 & 17 \\
\hline 21 & 20 & 10 & 18 & 18 & 17 & 22 & 19 & 18 & 11 & 18 & 23 & 15 & 16 & 16 & 21 & 13 & 16 & 21 & 21 \\
\hline 9 & 16 & 16 & 17 & 26 & 21 & 28 & 24 & 25 & 26 & 31 & 27 & 27 & 31 & 19 & 17 & 24 & 9 & 8 & 14 \\
\hline 24 & 16 & 19 & 22 & 15 & 14 & 17 & 14 & 22 & 16 & 16 & 13 & 20 & 13 & $\begin{array}{l}17 \\
16\end{array}$ & 17 & 17 & 15 & $\begin{array}{r}8 \\
16\end{array}$ & 17 \\
\hline 11 & 17 & 15 & 12 & 15 & 18 & 13 & 12 & i1 & 16 & $\begin{array}{l}16 \\
16\end{array}$ & 22 & 11 & 16 & 21 & 19 & 9 & 19 & 17 & 11 \\
\hline 8 & 17 & 21 & 14 & 17 & 24 & 17 & 15 & 14 & 14 & 13 & 13 & 11 & 10 & 11 & 14 & 21 & 19 & 16 & 10 \\
\hline 25 & 18 & 16 & 17 & 18 & 12 & 10 & 23 & 19 & 12 & 17 & 26 & 22 & 16 & 13 & 13 & 11 & 12 & 13 & 15 \\
\hline 13 & 9 & 17 & 16 & 11 & 18 & 14 & 13 & 15 & 27 & 11 & 21 & 10 & 19 & 20 & 23 & 12 & 13 & 25 & 24 \\
\hline 16 & 11 & 14 & 10 & 21 & 17 & $\theta$ & 22 & 12 & 15 & $\begin{array}{l}15 \\
15\end{array}$ & 11 & 22 & 21 & 12 & 12 & 18 & 50 & 22 & 14 \\
\hline 15 & 16 & 24 & 12 & 18 & 9 & 19 & 16 & 18 & 16 & 20 & 11 & 22 & 18 & $\begin{array}{l}17 \\
17\end{array}$ & 21 & 11 & 25 & $\begin{array}{r}4.2 \\
9\end{array}$ & 13 \\
\hline 22 & 13 & 16 & 23 & 16 & 24 & 23 & 12 & 16 & 12 & 15 & 17 & 21 & 12 & 22 & 23 & 17 & 16 & 19 & 18 \\
\hline 9 & 18 & 12 & 15 & 14 & 12 & 12 & 20 & 11 & 10 & 9 & 16 & 15 & 18 & 16 & 12 & $2 i$ & 16 & 12 & 17 \\
\hline 13 & 14 & 16 & 16 & 11 & 14 & 23 & 16 & 15 & 20 & 14 & 18 & 17 & 27 & 19 & 17 & 17 & 16 & 16 & 22 \\
\hline 14 & 15 & 22 & 16 & 18 & 19 & 18 & 36 & 24 & 11 & 13 & 13 & 14 & 23 & 12 & 13 & 15 & 15 & 21 & 14 \\
\hline $\begin{array}{l}18 \\
18\end{array}$ & 13 & 17 & 17 & $\begin{array}{l}10 \\
14\end{array}$ & 18 & $\begin{array}{l}10 \\
20\end{array}$ & 21 & $\begin{array}{l}24 \\
15\end{array}$ & $\begin{array}{l}11 \\
12\end{array}$ & 17 & $\begin{array}{l}13 \\
24\end{array}$ & $\begin{array}{l}17 \\
17\end{array}$ & 19 & 23 & $\begin{array}{l}13 \\
17\end{array}$ & 14 & $\begin{array}{l}120 \\
14\end{array}$ & $\begin{array}{l}15 \\
15\end{array}$ & $\begin{array}{r}14 \\
9\end{array}$ \\
\hline 16 & 20 & 18 & 12 & 22 & 19 & 16 & 27 & 15 & 18 & 13 & 12 & 22 & 18 & 16 & 16 & 18 & 16 & 20 & 15 \\
\hline 28 & 24 & 26 & 17 & 20 & 22 & 19 & 21 & 21 & 15 & 16 & 15 & 15 & 14 & 19 & 19 & 17 & 18 & 22 & 17 \\
\hline 15 & 13 & 13 & 12 & 17 & 20 & 14 & 21 & 18 & 15 & 15 & 14 & 23 & 13 & 26 & 15 & 21 & 18 & 25 & 23 \\
\hline 26 & 17 & 18 & 23 & 25 & 21 & 26 & $\begin{array}{l}18 \\
16\end{array}$ & $\begin{array}{r}10 \\
9\end{array}$ & 10 & 15 & 16 & 22 & 17 & 18 & 11 & $\begin{array}{l}17 \\
\end{array}$ & $\begin{array}{l}10 \\
25\end{array}$ & 29 & 22 \\
\hline 20 & 19 & 17 & 14 & 20 & 22 & 14 & 18 & 21 & 24 & 19 & 27 & 21 & 18 & 16 & 19 & 20 & 14 & 26 & 14 \\
\hline 23 & 20 & 14 & 16 & 14 & 13 & 10 & 14 & 14 & 7 & 14 & 15 & 15 & 15 & 11 & 9 & 12 & 11 & 13 & 7 \\
\hline
\end{tabular}

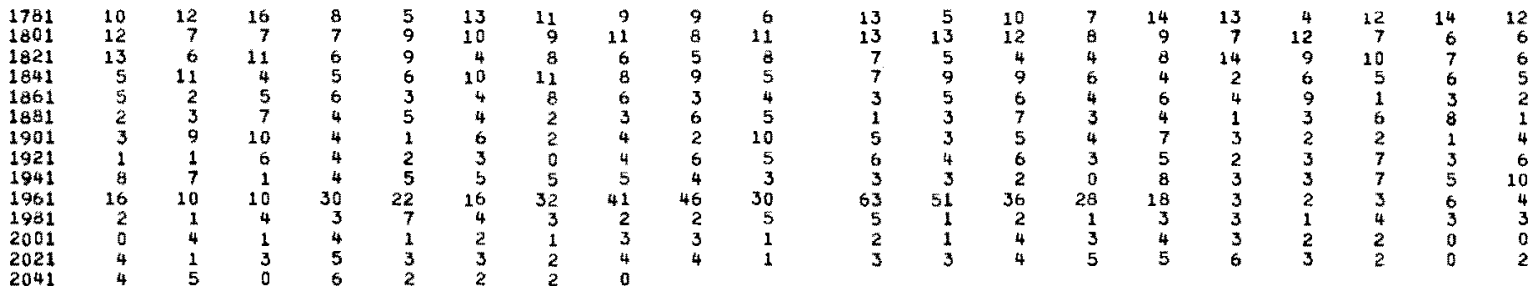




\section{D-4}

GAMMA RAY SPECTRUM DATA

PRTR ROD FS-16 SPECTRUM NO. 13 DATA TAKEN 3/01/69 LIVE TIME 700.MIN. POSITION 1.IN. ENERGY(KEV) 1 1.111*CHAN -8.1 CHAN COUNT COUNT COUNT COUNT COUNT COUNT COUNT COUNT COUNT COUNT

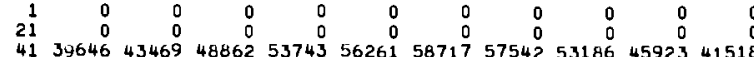
$61 \quad 42295 \quad 42772 \quad 43142 \quad 4361944003 \quad 44261 \quad 44699 \quad 44730 \quad 44597 \quad 45669$

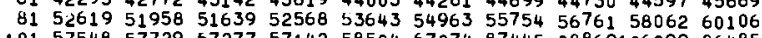
$1015754857729572775714258504 \quad 67074 \quad 87445,108869106099 \quad 86485$

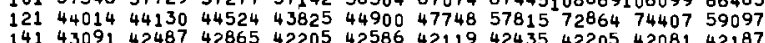
$16142196 \quad 42099 \quad 42236 \quad 42073 \quad 41983 \quad 4193142178 \quad 42041 \quad 41754 \quad 41408$ $\begin{array}{llllllllllll}181 & 41560 & 40972 & 40920 & 40697 & 40574 & 40712 & 40449 & 40433 & 40525 & 3995\end{array}$ $201399663930639882388903925439017389593906739133 \quad 39292$ $\begin{array}{lllllllllll}221 & 38403 & 38618 & 38233 & 38343 & 38276 & 38327 & 38868 & 38085 & 38639 & 38582 \\ 241 & 34384 & 38222 & 38147 & 37911 & 37913 & 37960 & 37725 & 37967 & 38270 & 37545\end{array}$ $\begin{array}{lllllllllll}241 & 34384 & 38222 & 38147 & 37911 & 37913 & 37960 & 37725 & 37967 & 38270 & 37545 \\ 261 & 37559 & 37596 & 37939 & 37590 & 37816 & 37606 & 37507 & 37715 & 37405 & 37293\end{array}$ $2813808837560 \quad 37773 \quad 378043757037828 \quad 3806437667378603818$

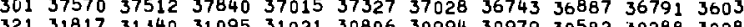

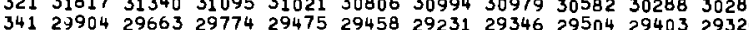
$\begin{array}{lllllllllll}361 & 29011 & 28936 & 28754 & 28779 & 28981 & 28610 & 28834 & 28810 & 28467 & 28515\end{array}$ $\begin{array}{lllllllllll}381 & 28693 & 28533 & 28624 & 28457 & 28529 & 28489 & 28373 & 27930 & 28440 & 28589\end{array}$

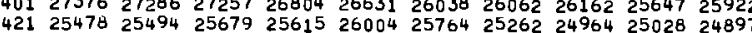

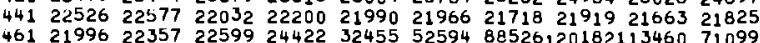

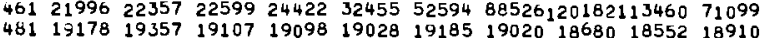
$\begin{array}{llllllllllll}481 & 19178 & 19357 & 19107 & 19098 & 19028 & 19185 & 19020 & 18680 & 18552 & 18910\end{array}$ $\begin{array}{llllllllllll}501 & 18184 & 17940 & 17810 & 17821 & 17946 & 17759 & 17775 & 17648 & 17469 & 17337 \\ 521 & 15364 & 14520 & 13665 & 12910 & 12569 & 11953 & 11741 & 11220 & 10925 & 10576\end{array}$

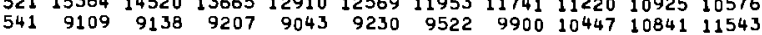
$\begin{array}{llllllllllll}561 & 10422 & 11576 & 12343 & 14772 & 22597 & 35998 & 47534 & 44638 & 28624 & 14288\end{array}$ $\begin{array}{lllllllllll}581 & 6554 & 6035 & 5808 & 5714 & 5596 & 5692 & 5520 & 5508 & 5437 & 5360\end{array}$ $\begin{array}{llllllll} & \end{array}$ $\begin{array}{lllllllllll}641 & 3193 & 3567 & 3257 & 3405 & 3381 & 3306 & 3283 & 3485 & 3449 & 3623\end{array}$ $\begin{array}{lllllllllll}661 & 10849 & 5523 & 3477 & 3134 & 2962 & 2956 & 3070 & 3023 & 3138 & 3104\end{array}$ $\begin{array}{lllllllllll}681 & 3335 & 3519 & 3894 & 4738 & 6819 & 10708 & 16148 & 20970 & 20885 & 14726\end{array}$ $\begin{array}{lllllllllll}701 & 2822 & 2038 & 2059 & 2004 & 2058 & 1924 & 1937 & 1903 & 1967 & 1932\end{array}$ $\begin{array}{lllllllllll}721 & 3015 & 3911 & 4682 & 4977 & 4145 & 2933 & 2299 & 2131 & 2134 & 2058\end{array}$ $\begin{array}{lllllllllll}741 & 1904 & 1749 & 1818 & 1918 & 1815 & 1801 & 1794 & 1833 & 1845 & 1804 \\ 761 & 1800 & 1784 & 1660 & 1722 & 1660 & 1661 & 1611 & 1703 & 1583 & 1499\end{array}$ $\begin{array}{lllllllllll}761 & 1800 & 1784 & 1660 & 1722 & 1660 & 1661 & 1611 & 1703 & 1583 & 1499 \\ 781 & 1434 & 1465 & 1409 & 1412 & 1410 & 1360 & 1439 & 1460 & 1489 & 1637\end{array}$

\begin{tabular}{|c|c|c|c|c|c|c|c|c|c|c|}
\hline 801 & 1378 & 1921 & 1327 & 1380 & 1326 & 1292 & 1250 & 1297 & 1307 & \\
\hline & 0 & 266 & 239 & 1259 & 1312 & 1181 & 1223 & $\begin{array}{l}1174 \\
\text { S }\end{array}$ & 1223 & \\
\hline 841 & 1080 & 1136 & 1057 & 1119 & 1063 & 1092 & 1096 & 1092 & $\begin{array}{l}1037 \\
\end{array}$ & $\begin{array}{l}1078 \\
\text {. }\end{array}$ \\
\hline 861 & 1046 & 976 & 993 & 974 & 1049 & 961 & 1021 & 961 & 950 & 958 \\
\hline 881 & 897 & 873 & 885 & 926 & 869 & 837 & 944 & 681 & 896 & 846 \\
\hline 901 & 890 & 777 & 844 & 863 & 812 & 793 & 818 & 801 & 819 & 78 \\
\hline 921 & 776 & 749 & 715 & 743 & 733 & 757 & 804 & 763 & 726 & 774 \\
\hline 941 & 760 & 778 & 757 & 742 & 762 & 841 & 998 & 1269 & 1666 & 2322 \\
\hline 961 & 687 & 777 & 719 & 706 & 694 & 639 & 664 & 679 & 675 & \\
\hline 981 & 627 & 611 & 659 & 588 & 595 & 621 & 631 & 650 & 700 & 65. \\
\hline 001 & 623 & 636 & 608 & 613 & 628 & 633 & 604 & & & 63 \\
\hline 021 & 1135 & 1257 & 1195 & 1003 & 735 & 586 & 546 & 518 & 532 & \\
\hline 041 & 528 & 479 & 474 & 489 & 486 & 493 & 457 & 496 & 591 & 63 \\
\hline 061 & 837 & 948 & 1088 & 995 & 800 & 553 & 541 & 468 & 465 & 46 \\
\hline 081 & 508 & 562 & 539 & 494 & 464 & 406 & 453 & 447 & 444 & 44 \\
\hline 10 & 407 & 411 & 379 & 420 & 411 & 447 & 40 & 39. & 41 & \\
\hline 12 & 400 & 376 & 401 & 395 & 415 & 395 & 385 & 417 & 408 & \\
\hline 14 & 388 & 397 & 381 & 363 & 375 & 377 & 358 & 385 & 368 & 41 \\
\hline 161 & 326 & 334 & 350 & 327 & 299 & 347 & 305 & 348 & & 33 \\
\hline 18 & 321 & 349 & 318 & 328 & 313 & 283 & 297 & 297 & 30 & 27 \\
\hline 1201 & 376 & 420 & 461 & 546 & 707 & 788 & 700 & 565 & 415 & 32 \\
\hline 1221 & 280 & 275 & 247 & 246 & 235 & 272 & 217 & 278 & 237 & \\
\hline 24 & 216 & 221 & 252 & 214 & 230 & 218 & 217 & 238 & 23 & 21 \\
\hline 26 & 226 & 202 & 220 & 217 & 192 & 235 & 200 & 219 & 20 & \\
\hline 28 & 207 & 240 & 216 & 184 & 211 & 196 & 23 & 18 & & 21 \\
\hline 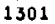 & 180 & 201 & 191 & 199 & 190 & 188 & 218 & 208 & 17 & \\
\hline 132 & 178 & 215 & 193 & 175 & 178 & 200 & 208 & 195 & 20 & 21 \\
\hline 134 & 266 & 295 & 361 & 406 & 475 & 515 & 539 & 52 & & \\
\hline 136 & 180 & 184 & 155 & 191 & 188 & 180 & 188 & 15 & & \\
\hline 138 & 145 & 186 & 182 & 154 & 167 & 17 & 17 & 17 & & 17 \\
\hline & 190 & 179 & 164 & 180 & 219 & 180 & 196 & 24 & 23 & 25 \\
\hline 142 & 148 & 175 & 159 & 165 & 165 & 151 & 154 & 13 & & 16 \\
\hline 144 & 134 & 160 & 161 & 146 & 166 & 161 & 14 & 16 & & 15 \\
\hline 146 & 165 & $\begin{array}{l}147 \\
\text {. }\end{array}$ & 138 & 147 & 156 & 16 & 17 & 15 & & \\
\hline 148 & 159 & 160 & 149 & 136 & 151 & 14 & 17 & 14 & & 1 \\
\hline & 147 & 151 & 157 & 147 & 146 & 13 & 16 & 17 & & 16 \\
\hline 15 & 161 & 152 & 159 & 160 & 146 & 161 & 159 & 163 & & 13 \\
\hline 1541 & 126 & 153 & 161 & 15 & 131 & 15 & 14 & 13 & & \\
\hline 15 & 14 & & 147 & 148 & 148 & 152 & 14 & 15 & & \\
\hline 158 & 145 & 155 & 159 & 168 & 164 & 16 & 12 & 16 & 16 & \\
\hline & 14 & 128 & 14 & 15 & 156 & 14 & 13 & 15 & & \\
\hline & 16 & 162 & 153 & 151 & 169 & 15 & 17 & 15 & & 15 \\
\hline 164. & 142 & 142 & 164 & $150^{\circ}$ & 155 & 16 & 14 & 15 & & \\
\hline 16 & 178 & 160 & 163 & 162 & 169 & & 16 & 14 & & 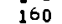 \\
\hline 168 & 158 & 161 & 181 & 178 & 17 & & 16 & 13 & 17 & 1 \\
\hline 170 & 190 & 170 & 158 & 152 & 137 & 147 & 149 & 183 & 185 & 15 \\
\hline 1723 & 166 & 165 & 177 & 168 & 176 & 184 & 16 & 14 & 16 & 17 \\
\hline 174 & 181 & 185 & 158 & 203 & 190 & 171 & 170 & 147 & 173 & 16 \\
\hline 1761 & 149 & 144 & 144 & 151 & 137 & 138 & 118 & 99 & 113 & \\
\hline 178 & 72 & 85 & 76 & 74 & 82 & 87 & 78 & 93 & 79 & \\
\hline & 7 & 72 & 67 & 60 & 87 & 66 & 81 & 70 & 67 & \\
\hline 10 & 64 & 60 & 82 & 79 & 74 & 7 & 62 & 70 & 60 & \\
\hline 18 & 6 & 6 & 54 & & & 5 & & 4 & 49 & \\
\hline & 24 & 36 & 42 & 28 & 34 & 34 & 35 & $\begin{array}{l}34 \\
34\end{array}$ & 32 & \\
\hline & 28 & 23 & 19 & 42 & 29 & 31 & 27 & 2 & 2 & \\
\hline & 35 & 24 & 40 & 32 & 46 & 3 & 40 & 4 & 38 & \\
\hline 192 & 18 & 22 & 21 & 26 & 19 & 27 & 35 & 24 & 21 & \\
\hline 19 & 19 & 16 & 24 & 27 & 18 & 22 & 36 & 20 & 19 & \\
\hline & 74 & 78 & 106 & 154 & 158 & 243 & 257 & 290 & 360 & \\
\hline 19 & 12 & 7 & 13 & 15 & 14 & 9 & 15 & 8 & 12 & \\
\hline 200 & 8 & 7 & 7 & 13 & 10 & 7 & 3 & 13 & 4 & \\
\hline 202 & 10 & 9 & 7 & 5 & 10 & 7 & 5 & 8 & 11 & \\
\hline 2041 & 11 & 4 & 12 & 8 & 6 & 6 & 7 & 0 & & \\
\hline
\end{tabular}

COUNT COUNT COUNT COUNT COUNT COUNT COUNT COUNT COUNT COUNT $\begin{array}{rrrrrrrrrr}0 & 0 & 0 & 0 & 0 & 0 & 0 & 0 & 0 & 0 \\ 39972 & 39362 & 39737 & 40129 & 40388 & 40940 & 41150 & 41429 & 13573 & 33430\end{array}$ 49834494535007251575 66575828841089791188981164081384971620871338688593461455

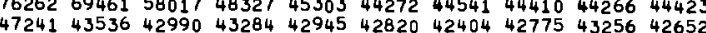

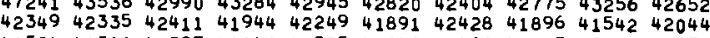
$\begin{array}{lllllllllll}41584 & 41381 & 41397 & 41246 & 41335 & 41242 & 41482 & 41605 & 41420 & 41151\end{array}$ $\begin{array}{lllllllllll}39942 & 39937 & 39797 & 40136 & 39594 & 39471 & 39940 & 39777 & 39433 & 39212 \\ 39507 & 38838 & 38720 & 38740 & 38853 & 38463 & 38587 & 38485 & 38722 & 38786\end{array}$ $\begin{array}{lllllllllllll}37889 & 37989 & 38271 & 38059 & 37999 & 38178 & 37987 & 38010 & 37703 & 38458\end{array}$ $\begin{array}{lllllllllll}37606 & 37589 & 37629 & 38218 & 37645 & 37757 & 37380 & 37780 & 37610 & 37338\end{array}$ $\begin{array}{lllllllllll}37852 & 37719 & 37550 & 37591 & 37362 & 37613 & 38133 & 37817 & 37722 & 37421 \\ 37957 & 37528 & 37508 & 38014 & 37759 & 37846 & 37755 & 37561 & 37468 & 37241\end{array}$ $\begin{array}{llllllllll}35914 & 35727 & 35312 & 34682 & 34185 & 33248 & 32975 & 32507 & 32119 & 31544\end{array}$

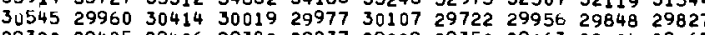

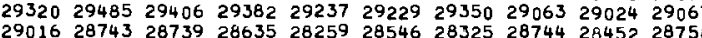

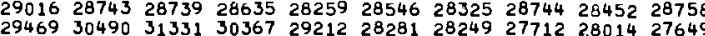
$\begin{array}{llllllllll}25416 & 25732 & 25845 & 25493 & 25317 & 25506 & 25347 & 25451 & 25469 & 25559\end{array}$ $\begin{array}{lllllllllllll}24673 & 24681 & 24756 & 24811 & 24419 & 24083 & 23750 & 23230 & 23000 & 22963\end{array}$ $\begin{array}{llllllllll}21900 & 22204 & 23814 & 24888 & 25940 & 25084 & 23604 & 22083 & 22071 & 22104 \\ 35840 & 22699 & 20062 & 19594 & 19421 & 19349 & 19469 & 19462 & 19401 & 19513\end{array}$ $\begin{array}{lllllllllll}18577 & 18331 & 18442 & 18288 & 18284 & 18215 & 18150 & 17923 & 17954 & 17890\end{array}$ $\begin{array}{rrrrrrrrrr}17271 & 17184 & 17407 & 17297 & 17287 & 16851 & 16299 & 16053 & 16005 & 15838 \\ 14384 & 10410 & 9963 & 9998 & 9704 & 9648 & 9758 & 9528 & 9347 & 9250\end{array}$ $\begin{array}{llllllllll}13050 & 13672 & 12016 & 10223 & 8927 & 8234 & 8401 & 8328 & 8570 & 9147\end{array}$ $\begin{array}{lllllllllll}8023 & 6669 & 6413 & 6393 & 6202 & 6311 & 6278 & 6423 & 6692 & 6716\end{array}$ $\begin{array}{rrrrrrrrrr}5423 & 5220 & 5321 & 5275 & 5352 & 5160 & 5533 & 6418 & 9157 & 15957 \\ 3841 & 3723 & 3712 & 3714 & 3651 & 3574 & 3501 & 3510 & 3451 & 3501\end{array}$ $\begin{array}{lllllllllll}3312 & 3296 & 3480 & 3702 & 4576 & 6547 & 10230 & 15208 & 18416 & 16836\end{array}$ $\begin{array}{rrrrrrrrrr}2991 & 3128 & 3232 & 3024 & 3130 & 3088 & 3287 & 3250 & 3342 & 3330 \\ 9132 & 8901 & 15193 & 28022 & 48867 & 68655 & 71670 & 50123 & 22822 & 7371 \\ 2009 & 1938 & 1934 & 1972 & 2029 & 1996 & 1947 & 2068 & 2169 & 2533\end{array}$ $\begin{array}{llllllllll}2046 & 1938 & 1934 & 1972 & 2029 & 1996 & 1947 & 2068 & 2169 & 2533 \\ 27836 & 1891 & 1884 & 1853 & 1825 & 1885 & 1904 & 1860 & 1860\end{array}$ $\begin{array}{llllllllll}1789 & 1854 & 1779 & 1901 & 1862 & 1838 & 1851 & 1841 & 1838 & 1747\end{array}$ $\begin{array}{llllllllll}1525 & 1452 & 1524 & 1485 & 1398 & 1450 & 1478 & 1472 & 1445 & 1440 \\ 2013 & 2304 & 2502 & 2469 & 2033 & 1666 & 1411 & 1440 & 1308 & 1396\end{array}$

\begin{tabular}{|c|c|c|c|c|c|c|c|c|c|}
\hline $\begin{array}{l}1350 \\
1170\end{array}$ & $\begin{array}{l}1301 \\
1237\end{array}$ & $\begin{array}{l}1292 \\
1265\end{array}$ & $\begin{array}{l}1277 \\
1168\end{array}$ & $\begin{array}{l}1244 \\
1126\end{array}$ & $\begin{array}{l}1256 \\
1178\end{array}$ & $\begin{array}{l}1273 \\
1177\end{array}$ & $\begin{array}{l}1256 \\
1181\end{array}$ & $\begin{array}{l}1245 \\
1108\end{array}$ & $\begin{array}{l}1230 \\
1155\end{array}$ \\
\hline & 1008 & 1066 & 1005 & 1015 & 990 & 1009 & 1009 & 1047 & 1073 \\
\hline & 914 & 977 & 938 & 933 & 966 & 921 & 865 & 920 & 830 \\
\hline 85 & 847 & 853 & & 812 & & & & & 817 \\
\hline & 843 & 773 & 865 & 839 & 782 & 789 & 771 & 781 & 781 \\
\hline & 786 & 786 & 725 & 762 & 748 & 752 & 700 & 829 & 784 \\
\hline & 3664 & 3863 & 2888 & 1750 & 99 & & 714 & 69 & 678 \\
\hline 66 & 643 & 687 & 645 & 680 & 617 & 583 & 653 & & 650 \\
\hline 59 & 593 & 651 & 64 & 615 & 582 & 653 & 624 & 662 & 639 \\
\hline 615 & 645 & 584 & 577 & 552 & 605 & 629 & 755 & & 948 \\
\hline & 512 & 494 & 495 & 526 & 461 & 537 & 503 & & 522 \\
\hline & 828 & 866 & 937 & 927 & 90 & 750 & 69 & & 672 \\
\hline 43 & 458 & 450 & 471 & 447 & 406 & 499 & 453 & 494 & 485 \\
\hline 484 & 446 & 427 & 431 & 429 & 398 & 428 & 385 & 417 & 419 \\
\hline 419 & 417 & 399 & 418 & 421 & 411 & 415 & 395 & 40 & 394 \\
\hline 43. & 422 & 404 & 392 & 443 & 40 & 39 & 40 & 4 & 367 \\
\hline & 415 & 379 & 386 & 405 & 37 & 37 & 327 & 3 & 353 \\
\hline 32 & 297 & 319 & 301 & 334 & 32 & 338 & 312 & & 330 \\
\hline 32 & 356 & 300 & 33 & 329 & 32 & 31 & & & 372 \\
\hline & 28 & 294 & & 282 & & 28 & & & 279 \\
\hline & & 254 & & 295 & & 251 & & 27 & 230 \\
\hline & & 238 & 25 & 236 & & & & & 213 \\
\hline 20 & 199 & 20 & 21 & 199 & & 20 & 2. & & 197 \\
\hline & 216 & 20 & & 181 & 2 & & & & 176 \\
\hline 19 & & 19 & & 20 & & & & & $i$ \\
\hline & 20 & 188 & & 17 & & & & & 250 \\
\hline & 20 & 212 & 21 & 200 & & 18 & & & 191 \\
\hline 16 & 180 & 190 & & 17 & 14 & & & & 15 \\
\hline & 17 & 16 & & & & & & & 190 \\
\hline 308 & 331 & 30 & 26 & 20 & $1 \theta$ & & 15 & & 171 \\
\hline & 16 & 15 & 16 & & 17 & & & & 175 \\
\hline & & 142 & 141 & 152 & 16 & 15 & & 1 & 159 \\
\hline 15 & 154 & 156 & & 163 & & & & & 141 \\
\hline & & & & & & & & & 155 \\
\hline 19 & 18 & 17 & & & 17 & 1 & & & 144 \\
\hline 15 & 13 & 15 & 14 & 164 & 13 & 13 & 13 & 1 & 148 \\
\hline 13 & 14 & 12 & 15 & 146 & & 14 & & & 142 \\
\hline 15 & 15 & & & & & & & & $14 \overline{6}$ \\
\hline 15 & 166 & 16 & & $18 E$ & 15 & 14 & 17 & 15 & 135 \\
\hline 13 & 166 & 17 & 14 & 135 & $16^{\circ}$ & $1 \epsilon$ & 15 & & \\
\hline 15 & 18 & 15 & 16 & 13 & 14 & & & & 150 \\
\hline 15 & 162 & 15 & 15 & 15 & 16 & & & & 137 \\
\hline & & & & & & & & & 170 \\
\hline & 148 & 183 & 16 & 164 & 173 & 17 & 19 & 17 & 146 \\
\hline & 158 & 180 & 15 & 166 & 18 & 15 & 17 & & 156 \\
\hline 16 & 17 & 17 & 16 & 16 & & & & & 181 \\
\hline 14. & 153 & 136 & 155 & 144 & 153 & 157 & 161 & 133 & 160 \\
\hline & 99 & 95 & 99 & 71 & 86 & 90 & 74 & 91 & \\
\hline
\end{tabular}

$\begin{array}{rrrrrrrrrr}76 & 79 & 98 & 84 & 88 & 80 & 80 & 68 & 69 & 79 \\ 67 & 76 & 61 & 58 & 50 & 65 & 77 & 69 & 65 & 63 \\ 59 & 71 & 64 & 67 & 52 & 68 & 72 & 63 & 55 & 41 \\ 41 & 42 & 36 & 34 & 50 & 45 & 40 & 41 & 41 & 46 \\ 39 & 35 & 37 & 31 & 29 & 32 & 30 & 27 & 32 & 31 \\ 30 & 24 & 19 & 24 & 33 & 31 & 33 & 35 & 29 & 32 \\ 23 & 25 & 18 & 24 & 15 & 26 & 19 & 27 & 26 & 33 \\ 26 & 18 & 14 & 23 & 14 & 22 & 25 & 31 & 28 & 36 \\ 29 & 22 & 28 & 31 & 34 & 31 & 27 & 31 & 43 & 59 \\ 454 & 410 & 380 & 269 & 170 & 74 & 39 & 17 & 20 & 17 \\ 12 & 9 & 13 & 9 & 12 & 4 & 8 & 12 & 7 & 6 \\ 7 & 5 & 14 & 8 & 10 & 8 & 5 & 6 & 15 & 12 \\ 10 & 5 & 3 & 8 & 9 & 9 & 4 & 14 & 9 & 7\end{array}$




\section{D-5}

GAMMA RAY SPECTRUM DATA

PRTR ROD FS-16 SPECTRUM NO. 9 DATA TAKEN 2/27/69 LIVE TIME 900.MIN. POSITION 6.IN. ENERGY(KEV)= $1.110 * C H A N ~-7.5$ CHAN COUNT COUNT COUNT COUNT COUNT COUNT COUNT COUNT COUNT COUNT COUNT COUNT COUNT COUNT COUNT COUNT COUNT COUNT COUNT COUNT

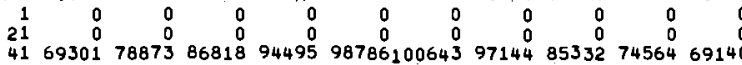

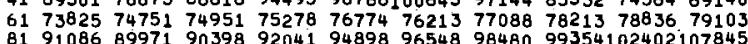
1011005901005369996799222105124127591169597194156173733143294 1217612276812768727645978404871711100921303431209989398 $141 \quad 7385673580734357355373510 \quad 73245 \quad 72607 \quad 7300173116 \quad 7276$ $\begin{array}{lllllllllll}161 & 72397 & 71940 & 72692 & 72516 & 72721 & 72652 & 72950 & 72067 & 71394 & 71190 \\ 189 & 72097 & 70766 & 70660 & 70266 & 70297 & 70404 & 69550 & 69894 & 69490 & 69394\end{array}$

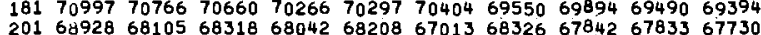

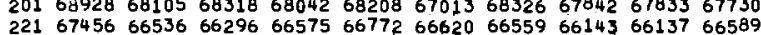
$26165315648916518164865 \quad 65048 \quad 65123 \quad 65042 \quad 65480 \quad 6503565401$ $\begin{array}{lllllllllllllll}281 & 65792 & 65561 & 65096 & 65184 & 65788 & 65725 & 65107 & 65033 & 65478 & 65410\end{array}$

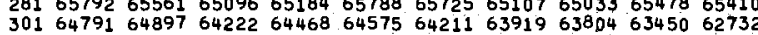

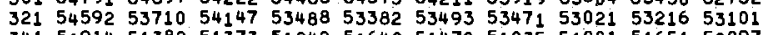

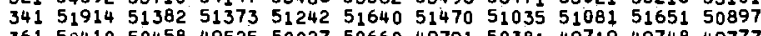
$361504105045849525500275066049791503814971949748 \quad 49777$ $\begin{array}{lllllllllll}381 & 49476 & 49812 & 49302 & 49437 & 49661 & 49465 & 49417 & 49337 & 49128 & 5022 \\ 401 & 47417 & 47007 & 46770 & 46400 & 45866 & 45563 & 44784 & 44548 & 44510 & 4433\end{array}$

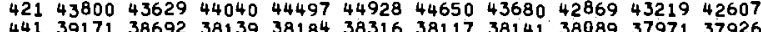
$\begin{array}{lllllllllll}441 & 39171 & 38692 & 38139 & 38184 & 38316 & 38117 & 38141 & 38089 & 37971 & 37926 \\ 461 & 38943 & 38763 & 39924 & 44467 & 58914 & 97671161685211900189540114870 \\ 481 & 33868 & 33495 & 33757 & 33604 & 33430 & 33234 & 33433 & 32993 & 32961 & 32908\end{array}$ $5013156531516 \quad 31339313683138930791 \quad 31145 \quad 31176 \quad 3081930776$ $\begin{array}{lllllllllll}521 & 27532 & 25486 & 24308 & 22863 & 21919 & 20929 & 20246 & 19653 & 19148 & 18701\end{array}$ $\begin{array}{lllllllllll}561 & 18522 & 20061 & 21249 & 26276 & 40181 & 63558 & 82408 & 75850 & 48300 & 23983\end{array}$

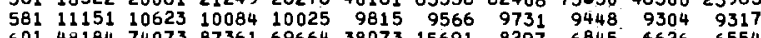
$\begin{array}{lllllllllll}621 & 5989 & 5890 & \mathbf{5 7 7 3} & \mathbf{5 7 7 1} & \mathbf{5 8 6 0} & \mathbf{5 7 3 4} & \mathbf{5 8 4 6} & \mathbf{5 7 8 8} & 5989 & 6363\end{array}$ $\begin{array}{lllllllllll}661 & 19071 & 10146 & 6239 & 5323 & 5301 & 5206 & 5338 & 5166 & 5199 & 5399\end{array}$ $\begin{array}{lllllllllll}681 & 5937 & 6082 & 6531 & 8100 & 11673 & 18576 & 27947 & 36458 & 36886 & 26695\end{array}$ $\begin{array}{lllllllllll}721 & 5936 & 7851 & 9821 & 10292 & 8279 & 5738 & 4237 & 3802 & 3883 & 378\end{array}$ $\begin{array}{lllllllllll}741 & 3157 & 3121 & 3135 & 3061 & 3114 & 3177 & 3164 & 3140 & 3113 & 3058\end{array}$ $\begin{array}{lllllllllll}761 & 3091 & 2824 & 2997 & 2962 & 2901 & 2934 & 2754 & 2738 & 2696 & 2634 \\ 781 & 2361 & 2411 & 2344 & 2427 & 2383 & 2386 & 2376 & 2490 & 2578 & 2855\end{array}$

$\begin{array}{lllllllllll}801 & 2290 & 2385 & 2274 & 2340 & 2308 & 2295 & 2152 & 2238 & 2214 & 2196\end{array}$ $\begin{array}{lllllllllll}821 & 2193 & 2054 & 2059 & 2136 & 2028 & 2146 & 2095 & 2039 & 2048 & 2018\end{array}$ $1617 \quad 1566 \quad 1593 \quad 1631 \quad 1603 \quad 1641 \quad 1690$ $\begin{array}{llllllllllll}881 & 1489 & 1446 & 1457 & 1384 & 1435 & 1403 & 1452 & 1454 & 1478 & 1377\end{array}$ $\begin{array}{lllllllllll}901 & 1374 & 1419 & 1409 & 1441 & 1396 & 1402 & 1344 & 1331 & 1390 & 1421\end{array}$ $\begin{array}{lllllllllll}941 & 1306 & 1307 & 1304 & 1343 & 1364 & 1379 & 1573 & 1977 & 2580 & 3709\end{array}$ $\begin{array}{lllllllllll}961 & 1171 & 1101 & 1181 & 1180 & 1147 & 1087 & 1119 & 1112 & 1062 & 1133 \\ 981 & 1082 & 1050 & 1037 & 1042 & 1081 & 1055 & 1065 & 1040 & 1031 & 1080\end{array}$ $\begin{array}{lllllllllll}1001 & 1062 & 1125 & 938 & 1021 & 1045 & 986 & 1039 & 1047 & 1056 & 1026\end{array}$ $\begin{array}{rrrrrrrrrrr}1021 & 1859 & 2079 & 2096 & 1851 & 1440 & 1061 & 915 & 901 & 845 & 863 \\ 1041 & 891 & 899 & 888 & 918 & 831 & 817 & 843 & 889 & 1007 & 1041\end{array}$ $\begin{array}{rrrrrrrrrrr}1061 & 1190 & 1393 & 1550 & 1508 & 1291 & 1040 & 878 & 809 & 822 & 788\end{array}$ $\begin{array}{lllllllllll}1081 & 881 & 941 & 898 & 919 & 840 & 733 & 792 & 811 & 720 & 697 \\ 1101 & 707 & 705 & 699 & 720 & 685 & 710 & 693 & 725 & 758 & 717\end{array}$ $\begin{array}{lllllllllll}1101 & 707 & 705 & 699 & 720 & 685 & 710 & 693 & 725 & 758 & 717 \\ 1121 & 710 & 752 & 717 & 717 & 732 & 716 & 701 & 699 & 645 & 692\end{array}$ $\begin{array}{lllllllllll}1141 & 693 & 685 & 679 & 669 & 678 & 654 & 664 & 640 & 643 & 638\end{array}$ $\begin{array}{lllllllllll}1161 & 561 & 591 & 565 & 582 & 583 & 599 & 579 & 539 & 575 & 555 \\ 1181 & 557 & 518 & 525 & 552 & 559 & 560 & 521 & 567 & 544 & 511\end{array}$

$\begin{array}{rrrrrrrrrrr}1201 & 588 & 682 & 678 & 771 & 972 & 1159 & 1196 & 1003 & 746 & 561 \\ 1221 & 461 & 404 & 427 & 411 & 474 & 430 & 418 & 452 & 415 & 432\end{array}$ $\begin{array}{lllllllllll}1241 & 421 & 431 & 375 & 394 & 447 & 392 & 386 & 411 & 434 & 415 \\ 1261 & 360 & 375 & 397 & 353 & 358 & 391 & 380 & 367 & 369 & 360\end{array}$ $\begin{array}{lllllllllll}\mathbf{1 2 8 1} & \mathbf{3 9 6} & \mathbf{3 7 1} & \mathbf{3 9 5} & \mathbf{3 3 4} & \mathbf{3 2 1} & \mathbf{3 5 0} & \mathbf{3 5 9} & \mathbf{3 5 3} & \mathbf{3 5 2} & \mathbf{3 4 7} \\ \mathbf{1 3 0 1} & \mathbf{3 4 2} & \mathbf{3 5 3} & \mathbf{3 3 4} & \mathbf{3 4 1} & \mathbf{3 4 9} & \mathbf{3 5 6} & \mathbf{3 2 1} & \mathbf{3 6 2} & \mathbf{3 0 5} & \mathbf{3 5 5}\end{array}$ $\begin{array}{lllllllllll}1301 & \mathbf{3 4 2} & \mathbf{3 5 3} & \mathbf{3 3 4} & \mathbf{3 4 1} & \mathbf{3 4 9} & \mathbf{3 5 6} & \mathbf{3 2 1} & \mathbf{3 6 2} & \mathbf{3 0 5} & \mathbf{3 5 5} \\ \mathbf{1 3 2 1} & \mathbf{3 5 3} & \mathbf{3 2 6} & \mathbf{3 4 0} & \mathbf{3 2 7} & \mathbf{3 0 9} & \mathbf{3 5 1} & \mathbf{3 2 5} & \mathbf{3 2 0} & \mathbf{3 3 5} & \mathbf{3 6 5}\end{array}$

\section{$\begin{array}{lllllllllll}1791 & 151 & 132 & 143 & 125 & 164 & 148 & 145 & 135 & 135 & 133\end{array}$}

$\begin{array}{rrrrrrrrrrr}1701 & 151 & 132 & 143 & 126 & 164 & 148 & 145 & 135 & 135 & 133 \\ 1801 & 116 & 119 & 141 & 138 & 121 & 126 & 120 & 115 & 123 & 101 \\ 1821 & 130 & 122 & 115 & 106 & 107 & 106 & 103 & 103 & 115 & 122 \\ 1841 & 76 & 97 & 117 & 82 & 63 & 89 & 89 & 86 & 90 & 84 \\ 1861 & 67 & 74 & 75 & 73 & 57 & 55 & 57 & 67 & 48 & 63 \\ 1881 & 51 & 39 & 52 & 44 & 54 & 44 & 47 & 47 & 46 & 45 \\ 1901 & 62 & 62 & 59 & 83 & 75 & 60 & 72 & 74 & 55 & 67 \\ 1921 & 32 & 38 & 45 & 37 & 46 & 51 & 33 & 45 & 46 & 40 \\ 1941 & 33 & 32 & 38 & 36 & 37 & 24 & 43 & 50 & 41 & 42 \\ 1961 & 55 & 100 & 133 & 171 & 216 & 290 & 347 & 475 & 500 & 596 \\ 1981 & 24 & 20 & 23 & 18 & 23 & 27 & 22 & 26 & 15 & 16 \\ 2001 & 17 & 18 & 16 & 19 & 19 & 21 & 19 & 12 & 14 & 18 \\ 2021 & 15 & 11 & 18 & 21 & 16 & 16 & 17 & 9 & 15 & 13 \\ 2041 & 15 & 15 & 8 & 10 & 17 & 15 & 10 & 0 & & \end{array}$

$\begin{array}{rrrrrrrrrr}0 & 0 & 0 & 0 & 0 & 0 & 0 & 0 & 0 & 0 \\ 0 & 0 & 0 & 0 & 1 & 5 & 295 & 3640 & 42787 & 64162 \\ 68196 & 68141 & 68462 & 69315 & 70126 & 70092 & 71166 & 71034 & 71667 & 73221\end{array}$ 79960809398284784342849968575085850862478830690082 124484163116204738206934211560267831281465201102128181102630 $\begin{array}{lllllllll}130281113766 & 92749 & 80578 & 77735 & 76271 & 76249 & 76883 & 76208 & 76569\end{array}$ $\begin{array}{lllllllllll}78949 & 74443 & 74816 & 74016 & 73554 & 74366 & 74756 & 74161 & 73718 & 73930 \\ 72646 & 72498 & 73088 & 72626 & 72591 & 72218 & 72465 & 72475 & 72069 & 72011\end{array}$ $71746 \quad 71633$
70885 $71620 \quad 71384 \quad 71345 \quad 71701 \quad 7098471467 \quad 71359$

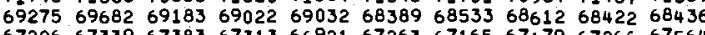
$\begin{array}{llllllllll}67226 & 67339 & 67383 & 67313 & 66921 & 67263 & 67165 & 67179 & 67266 & 67564 \\ 66033 & 66235 & 66148 & 65938 & 66219 & 66024 & 65608 & 65493 & 6576 ? & 65943\end{array}$ $\begin{array}{llllllllll}66033 & 66235 & 66148 & 65938 & 66219 & 66024 & 65608 & 65493 & 65762 & 65943 \\ 65382 & 65191 & 65605 & 65397 & 65160 & 64885 & 65271 & 65203 & 65290 & 64802\end{array}$ $6510864956 \quad 64765 \quad 65303 \quad 65386 \quad 6485965848659276535165055$ $\begin{array}{lllllllllllll}65408 & 65274 & 65427 & 65696 & 65567 & 65121 & 65423 & 65493 & 65008 & 64899\end{array}$ $62889611006064459342584765725456826 \quad 56101 \quad 5571855045$ $\begin{array}{llllllllll}52321 & 52276 & 52313 & 52397 & 52289 & 52062 & 52357 & 51815 & 51961 & 51710\end{array}$ 51039505735103350693509455071550306504165052950209 $\begin{array}{lllllllllll}49989 & 49201 & 49887 & 49067 & 49249 & 48764 & 49415 & 49988 & 49521 & 48923 \\ 5 & 332 & 53247 & 54200 & 52504 & 49904 & 48509 & 48097 & 47892 & 47801 & 47570\end{array}$ $\begin{array}{lllllllllll}4302 & 43794 & 43802 \quad 43522 & 43658 & 43568 \quad 43938 & 43573 \quad 43867 & 43265\end{array}$ $\begin{array}{llllllllll}42794 & 42345 & 42470 & 42186 & 42022 & 41213 & 40506 & 40219 & 39944 & 39327 \\ 38197 & 39151 & 41235 & 43977 & 45462 & 43626 & 40318 & 38679 & 38375 & 38333\end{array}$ $\begin{array}{llllllllllll}59015 & 38882 & 35162 & 34048 & 33734 & 33766 & 34156 & 33865 & 33574 & 33628\end{array}$ $\begin{array}{lllllllllll}32451 & 32517 & 32263 & 31686 & 32187 & 32114 & 31837 & 31643 & 31624 & 31641\end{array}$ $\begin{array}{lllllllllll}18336 & 18072 & 17913 & 17668 & 17315 & 17213 & 16958 & 16811 & 16516 & 16644\end{array}$ $\begin{array}{lllllllllll}25332 & 26305 & 22891 & 18119 & 15649 & 14573 & 14532 & 14546 & 14976 & 16409\end{array}$ $915892989098 \quad 9166 \quad 9029 \quad 89969782110981546326615$ $\begin{array}{rrrrrrrrrr}9158 & 9298 & 9098 & 9166 & 9029 & 8996 & 9782 & 11098 & 15463 & 26615 \\ 6578 & 6512 & 6484 & 6420 & 6222 & 6271 & 6185 & 6146 & 6039 & 6017\end{array}$ $\begin{array}{rrrrrrrrrr}6578 & 6512 & 6484 & 6420 & 6222 & 6271 & 6185 & 6,146 & 6039 & 6017 \\ 7279 & 9412 & 12226 & 14587 & 13875 & 10366 & 7212 & 5913 & 5440 & 5444\end{array}$ $\begin{array}{rrrrrrrrrr}7279 & 9412 & 12226 & 14587 & 13875 & 10366 & 7212 & 5913 & 5440 & 5444 \\ 5810 & 5920 & 5933 & 6468 & 8000 & 11238 & 17815 & 26555 & 32751 & 29312\end{array}$ $\begin{array}{lllllllllll}5274 & 5348 & 5308 & 5383 & 5478 & 5443 & 5587 & 5567 & 5623 & 5815\end{array}$ $\begin{array}{llllllll}16487 & 15377 & 25750 & 49315 & 84175119289126226 & 90297 & 41807 & 13835\end{array}$ $\begin{array}{llllllllll}3496 & 3205 & 3136 & 3110 & 3128 & \mathbf{3 1 1 9} & \mathbf{3 1 4 2} & \mathbf{3} 192 & \mathbf{3 2 3 0} & \mathbf{3 2 0 5} \\ \mathbf{3 0 8 7} & 3032 & 3126 & 2911 & 3108 & \mathbf{3 1 7 8} & \mathbf{3 1 0 7} & \mathbf{3} 078 & \mathbf{3 1 9 9} & \mathbf{3 2 1 3}\end{array}$ $\begin{array}{llllllllll}3087 & 3032 & 3126 & 2911 & 3108 & 3178 & 3107 & 3078 & 3199 & 3213\end{array}$ $\begin{array}{llllllllll}2682 & 2542 & 2496 & 2508 & 2504 & 2442 & 2421 & 2563 & 2463 & 2456 \\ 3232 & 3719 & 4395 & 4289 & 3782 & 2880 & 2555 & 2279 & 2242 & 2375\end{array}$

$\begin{array}{llllllllll}2186 & 2122 & 2221 & 2149 & 2097 & 2161 & 2238 & 2100 & 2125 & 2173\end{array}$ $\begin{array}{lllllllllll}2027 & 2024 & 2034 & 1875 & 1874 & 1897 & 1899 & 1911 & 1889 & 1744\end{array}$ $\begin{array}{llllllllll}1612 & 1587 & 1607 & 1578 & 1574 & 1482 & 1532 & 1515 & 1528 & 1481\end{array}$ $\begin{array}{llllllllll}1452 & 1409 & 1439 & 1476 & 1336 & 1316 & 1396 & 1355 & 1368 & 1388\end{array}$ $\begin{array}{llllllllll}391 & 1397 & 1334 & 1371 & 1317 & 1277 & 1290 & 1273 & 1268 & 1322\end{array}$ $\begin{array}{llllllllll}4729 & 6062 & 6456 & 5262 & 3455 & 1858 & 1357 & 1203 & 1135 & 1106\end{array}$ $\begin{array}{llllllllll}1125 & 1095 & 1029 & 1005 & 1028 & 1124 & 1100 & 1132 & 105 \mathrm{~A} & 1079\end{array}$ $\begin{array}{rrrrrrrrrr}1047 & 1078 & 997 & 1028 & 1013 & 1008 & 1027 & 1037 & 1011 & 1045 \\ 1037 & 1018 & 1022 & 1025 & 953 & 1019 & 996 & 1189 & 1339 & 1563\end{array}$ $\begin{array}{rrrrrrrrrr}1037 & 1018 & 1022 & 1025 & 953 & 1019 & 996 & 1189 & 1339 & 1563 \\ 793 & 907 & 877 & 869 & 882 & 866 & 786 & 867 & 813 & 830\end{array}$ $\begin{array}{rrrrrrrrrr}793 & 907 & 877 & 869 & 882 & 866 & 786 & 867 & 813 & 830 \\ 1183 & 1347 & 1404 & 1602 & 1685 & 1572 & 1273 & 1184 & 1113 & 1122 \\ 818 & 741 & 802 & 780 & 743 & 856 & 805 & 783 & 796 & 838\end{array}$ $\begin{array}{llllllllll}818 & 741 & 802 & 780 & 743 & 856 & 805 & 783 & 796 & 838 \\ 745 & 761 & 780 & 762 & 701 & 732 & 795 & 763 & 731 & 748\end{array}$ $\begin{array}{lllllllllll}725 & 654 & 670 & 712 & 719 & 739 & 687 & 671 & 722 & 682 \\ 720 & 720 & 679 & 705 & 709 & 652 & 662 & 654 & 673 & 685\end{array}$ $\begin{array}{llllllllll}674 & 695 & 749 & 726 & 735 & 633 & 576 & 584 & 564 & 581\end{array}$ $\begin{array}{llllllllll}585 & 496 & 548 & 558 & 570 & 554 & 533 & 536 & 557 & 551 \\ 568 & 537 & 537 & 528 & 525 & 547 & 511 & 558 & 528 & 581\end{array}$ $\begin{array}{lllllllllll}511 & 463 & 482 & 460 & 492 & 508 & 478 & 558 & 52 A & 581 \\ 493 & 488 & 523 & 480 & 515 & 536 & 544 & 473 & 477 & 456\end{array}$ $\begin{array}{llllllllll}456 & 425 & 427 & 420 & 444 & 432 & 404 & 473 & 414 & 448 \\ & 349 & 372 & 380 & 353 & 360 & 369 & 345 & 354 & 385\end{array}$ $\begin{array}{llllllllll}365 & 349 & 372 & 380 & 353 & \mathbf{3 6 0} & \mathbf{3 6 9} & \mathbf{3 4 5} & \mathbf{3 5 4} & \mathbf{3 7 4} \\ \mathbf{3 7 2} & \mathbf{3 7 0} & \mathbf{3 5 1} & \mathbf{3 4 6} & \mathbf{3 4 2} & \mathbf{3 8 0} & \mathbf{3 6 6} & \mathbf{3 6 5} & \mathbf{3 3 3} & 329\end{array}$ $\begin{array}{llllllllll}372 & 370 & \mathbf{3 5 1} & \mathbf{3 4 6} & \mathbf{3 4 2} & \mathbf{3 8 0} & \mathbf{3 6 6} & \mathbf{3 6 5} & \mathbf{3 3 3} & \mathbf{3 2 9} \\ \mathbf{3 8 0} & \mathbf{3 3 0} & \mathbf{3 3 4} & \mathbf{3 5 9} & \mathbf{3 8 7} & \mathbf{3 6 4} & \mathbf{3 6 0} & \mathbf{3 2 9} & \mathbf{3 5 9} & \mathbf{3 3 1}\end{array}$ $\begin{array}{llllllllll}317 & 313 & 356 & 314 & 355 & 342 & 370 & 326 & 389 & 402\end{array}$ $\begin{array}{llllllllll}481 & 384 & 355 & 364 & 341 & 342 & 281 & 312 & 325 & 314 \\ 269 & 295 & 308 & 281 & 315 & 308 & 305 & 304 & 303 & 283\end{array}$ $\begin{array}{llllllllll}306 & 288 & 304 & 301 & 304 & 305 & 311 & 302 & 283 & 323 \\ 529 & 581 & 552 & 540 & 429 & 360 & 302 & 278 & 251 & 277\end{array}$

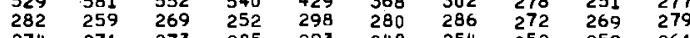
$\begin{array}{llllllllll}274 & 271 & 273 & 285 & 293 & 248 & 254 & 252 & 259 & 261 \\ 263 & 259 & 264 & 240 & 259 & 253 & 231 & 257 & 247 & 277\end{array}$ $\begin{array}{llllllllll}280 & 253 & 263 & 240 & 259 & 253 & 231 & 257 & 247 & 277 \\ & 259 & 245 & 291 & 229 & 289 & 275 & 256\end{array}$ $\begin{array}{llllllllll}305 & 328 & 312 & 348 & 335 & 312 & 287 & 258 & 244 & 255 \\ 268 & 257 & 221 & 275 & 235 & 262 & 263 & 244 & 268 & 231\end{array}$ $\begin{array}{llllllllll}268 & 257 & 221 & 275 & 235 & 262 & 263 & 244 & 269 & 231 \\ 276 & 257 & 228 & 251 & 233 & 243 & 277 & 261 & 223 & 218\end{array}$ $\begin{array}{llllllllll}253 & 244 & 233 & 265 & 290 & 276 & 248 & 252 & 271 & 255\end{array}$ $\begin{array}{llllllllll}268 & 268 & 273 & 261 & 260 & 331 & 279 & 298 & 287 & 265 \\ 273 & 251 & 251 & 296 & 229 & 264 & 291 & 294 & 265 & 277\end{array}$ $\begin{array}{llllllllll}261 & 266 & 249 & 256 & 251 & 204 & 248 & 271 & 264 & 251 \\ 257 & 270 & 271 & 261 & 241 & 262 & 248 & 275 & 244 & 258\end{array}$

$\begin{array}{llllllllll}262 & 254 & 288 & 272 & 292 & 258 & 282 & 291 & 285 & 296\end{array}$

$\begin{array}{llllllllll}283 & 289 & 273 & 282 & 268 & 283 & 297 & 275 & 297 & 278 \\ 286 & 284 & 295 & 270 & 248 & 303 & 267 & 268 & 266 & 297\end{array}$ $\begin{array}{llllllllll}267 & 288 & 294 & 306 & 288 & 277 & 295 & 320 & 302 & 282\end{array}$ $\begin{array}{llllllllll}313 & 261 & 296 & 329 & 322 & 281 & 261 & 255 & 251 & 255 \\ 165 & 178 & 175 & 172 & 153 & 151 & 153 & 147 & 143 & 168\end{array}$

$\begin{array}{rrrrrrrrrr}139 & 155 & 145 & 164 & 163 & 153 & 147 & 154 & 137 & 137 \\ 127 & 96 & 127 & 108 & 125 & 114 & 109 & 110 & 111 & 121 \\ 117 & 87 & 109 & 91 & 102 & 83 & 110 & 108 & 113 & 93 \\ 83 & 75 & 69 & 79 & 61 & 70 & 77 & 73 & 59 & 67 \\ 60 & 47 & 38 & 55 & 50 & 56 & 58 & 54 & 51 & 56 \\ 43 & 45 & 50 & 41 & 42 & 55 & 42 & 40 & 53 & 57 \\ 53 & 45 & 44 & 42 & 42 & 43 & 41 & 49 & 38 & 43 \\ 60 & 43 & 40 & 33 & 40 & 42 & 36 & 35 & 43 & 38 \\ 41 & 40 & 42 & 36 & 35 & 44 & 61 & 40 & 37 & 71 \\ 653 & 771 & 834 & 701 & 518 & 282 & 102 & 54 & 31 & 30 \\ 24 & 12 & 12 & 19 & 16 & 21 & 12 & 26 & 14 & 13 \\ 11 & 21 & 12 & 24 & 11 & 20 & 12 & 9 & 11 & 14 \\ 15 & 10 & 10 & 19 & 7 & 11 & 16 & 14 & 12 & 14\end{array}$


GAMMA RAY SPECTRUM DATA

PRTR ROD FS-16 SPECTRUM NO, 10 DATA TAKEN 2/28/69 LIVE TIME 330.MIN. POSITION 28.IN, ENERGY(KEVI= 1.110*CHAN -7.3 CHAN COUNT COUNT COUNT COUNT COUNT COUNT COUNT COUNT COUNT COUNT

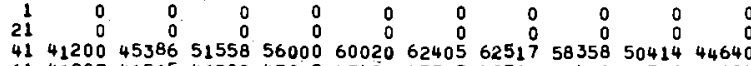
$6146207 \quad 46565 \quad 46382 \quad 47019 \quad 47480 \quad 47725 \quad 48707 \quad 48466 \quad 48716 \quad 49624$

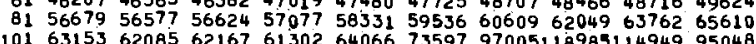

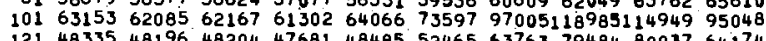
$\begin{array}{lllllllllll}121 & 48335 & 48196 & 48204 & 47681 & 48485 & 52465 & 63763 & 79484 & 80037 & 64174\end{array}$

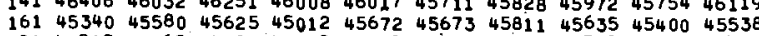

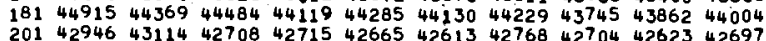
$\begin{array}{lllllllllll}201 & 42946 & 43114 & 42708 & 42715 & 42665 & 42613 & 42768 & 42704 & 42623 & 42697 \\ 221 & 41799 & 41833 & 41761 & 42337 & 42388 & 42126 & 41955 & 41658 & 41540 & 41985\end{array}$ $24141314 \quad 41193 \quad 41050 \quad 41242 \quad 41387 \quad 41201 \quad 41315 \quad 41321 \quad 4171541019$ 2814140641324407884111241456410944130940444077940908

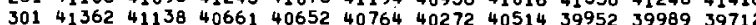
$321 \quad 34399 \quad 34523 \quad 34195 \quad 34033 \quad 337413342 \quad 3320233467399893971$

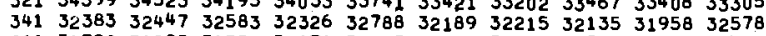
$\begin{array}{llllllllllll}361 & 31790 & 31923 & 31731 & 31171 & 31443 & 31305 & 31349 & 31259 & 31006 & 31291 \\ 381 & 31030 & 30937 & 31085 & 30913 & 30981 & 30785 & 30690 & 30985 & 30797 & 30852\end{array}$

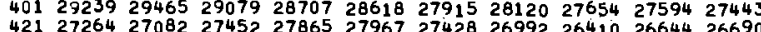

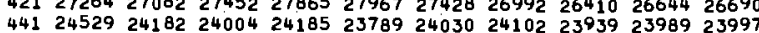

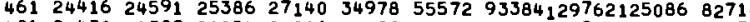

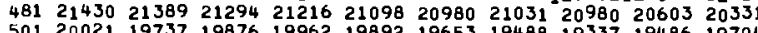

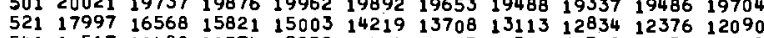
$\begin{array}{llllllllllll}541 & 10517 & 10488 & 10374 & 9959 & 10414 & 10605 & 11308 & 11782 & 13252 & 15245\end{array}$ 56111421125921350515911235653764550527 $\begin{array}{lllllllllll}581 & 7025 & 6846 & 6394 & 6264 & 6082 & 6203 & 5933 & 5910 & 5756 & 5784\end{array}$ $\begin{array}{lllllllllll}621 & 3944 & 3837 & \mathbf{3 7 0 3} & \mathbf{3 6 8 9} & 3796 & \mathbf{3 6 1 3} & 3624 & 4436 & 4356 & 4250 \\ 641 & 3549 & 3461 & 3505 & 3531 & 3595 & 3504 & 3554 & 3584 & 3598 & 4035\end{array}$ $\begin{array}{lllllllllll}641 & 3549 & 3461 & 3505 & 3531 & 3595 & 3504 & 3554 & 3584 & 3576 & 3612\end{array}$ $\begin{array}{rrrrrrrrrrr}061 & 13096 & 6918 & 4352 & 3474 & 3319 & 3242 & 3337 & 3428 & 3281 & 3303\end{array}$ $\begin{array}{lllllllllll}681 & 3648 & 3718 & 4060 & 5067 & 7072 & 11174 & 16744 & 22440 & 23171 & 17662\end{array}$ $\begin{array}{llllllllllll}701 & 3924 & 2508 & 2154 & 2170 & 2140 & 2218 & 2167 & 2230 & 2157 & 2195 \\ 721 & 4200 & 5848 & 7438 & 8177 & 6836 & 4449 & 3018 & 2633 & 2676 & 2600\end{array}$ $\begin{array}{lllllllllll}721 & 4200 & 5848 & 7438 & 8177 & 6836 & 4449 & 3018 & 2633 & 2676 & 2600 \\ 741 & 2049 & 1949 & 1978 & 1936 & 2012 & 1987 & 2031 & 1947 & 1973 & 1985\end{array}$ $\begin{array}{lllllllllll}761 & 20493 & 1949 & 1978 & 1936 & 2012 & 1987 & 2031 & 1947 & 1973 & 1985\end{array}$ $\begin{array}{lllllllllll}761 & 1893 & 1894 & 1819 & 1769 & 1750 & 1707 & 1734 & 1782 & 1650 & 1639 \\ 781 & 1542 & 1425 & 1473 & 1480 & 1477 & 1580 & 1557 & 1508 & 1628 & 1786\end{array}$

\begin{tabular}{|c|c|c|c|c|c|c|c|c|c|c|}
\hline 801 & 1467 & 1502 & 1467 & 1548 & 1507 & 1371 & 1364 & 1318 & 1397 & 1374 \\
\hline 821 & 1287 & 1299 & 1225 & 1277 & 1269 & 1302 & 1253 & 1263 & 1210 & 1258 \\
\hline 841 & 1122 & 1093 & 1144 & 1122 & 1133 & 1118 & 1080 & 1054 & 1103 & 1103 \\
\hline 861 & 1088 & 995 & 993 & 992 & 1049 & 1021 & 969 & 1023 & 1013 & 99 \\
\hline 881 & 944 & 963 & 905 & 884 & 923 & 19 & 8 & 959 & & \\
\hline 901 & 901 & 879 & 920 & 893 & 903 & 14 & 842 & 898 & & \\
\hline 921 & 828 & 842 & 760 & 748 & 781 & 83 & 808 & 730 & 790 & 758 \\
\hline 941 & 872 & 827 & 841 & 836 & 805 & 927 & 1033 & 1302 & 1699 & 2360 \\
\hline & 721 & 736 & 782 & 766 & 733 & 647 & 643 & 687 & 657 & \\
\hline 981 & 658 & 670 & 641 & 632 & 625 & 647 & 639 & 658 & 679 & \\
\hline 1001 & 624 & 619 & 612 & 648 & 634 & 616 & & 638 & & \\
\hline 1021 & 1100 & 1284 & 1330 & 1191 & 825 & 654 & & 581 & & \\
\hline 1041 & 522 & 566 & 556 & 522 & 518 & 498 & 549 & 541 & 62 & \\
\hline & 647 & 743 & 789 & 788 & 688 & & 580 & 517 & 514 & \\
\hline 1081 & 598 & 575 & 573 & 519 & 548 & 489 & 493 & & & \\
\hline 1101 & 472 & 436 & 454 & 454 & 469 & 464 & 437 & 471 & 459 & \\
\hline 1121 & 431 & 460 & 466 & 460 & 475 & 445 & 477 & 422 & 422 & \\
\hline & 414 & 443 & 454 & 438 & 42 & 419 & 426 & 448 & 452 & \\
\hline & 378 & 347 & 357 & 375 & 359 & 353 & 359 & 351 & 326 & \\
\hline 1181 & 360 & 362 & 322 & 361 & 331 & 376 & 345 & 341 & 345 & \\
\hline 1201 & 390 & 384 & 386 & 478 & 490 & 542 & 577 & 499 & 39 & \\
\hline 1221 & 257 & 305 & 290 & 296 & 29 & 271 & $28_{1}$ & 282 & & \\
\hline & 267 & 288 & 275 & 214 & 25 & & & 24 & & \\
\hline & 239 & 250 & 246 & 240 & 231 & 257 & 269 & 235 & 234 & \\
\hline 1281 & 242 & 241 & 219 & 238 & 224 & 2 & 209 & 218 & 217 & \\
\hline 1301 & 229 & 196 & 225 & 213 & 229 & & 232 & 199 & & \\
\hline 1321 & 193 & 222 & 223 & 204 & 214 & & 20 & 21 & & \\
\hline 134 & 271 & 313 & 36 & 401 & 540 & & & 63 & & \\
\hline & 207 & 217 & 219 & 204 & 187 & & 182 & 188 & 177 & \\
\hline 1381 & 195 & 202 & 200 & 178 & 199 & 18 & 189 & 197 & 183 & \\
\hline 1401 & 163 & 185 & 204 & 185 & 190 & & 231 & 271 & & \\
\hline 1421 & 149 & 172 & 167 & 162 & 13 & & & 19 & & \\
\hline 144 & 180 & 195 & & & 18 & & 19 & & & \\
\hline 1461 & 165 & 154 & 162 & 162 & 174 & 158 & 179 & 16 & & \\
\hline 481 & 168 & 165 & 156 & 177 & 169 & 16 & 17 & 171 & 162 & \\
\hline & 15 & 167 & 165 & 15 & 182 & 17 & 173 & 171 & 19 & \\
\hline 1521 & 172 & 185 & 163 & 157 & 166 & 1 & 16 & 149 & & \\
\hline 1541 & 170 & 172 & 171 & & 15 & & & 17 & & \\
\hline 156 & 172 & 151 & 161 & 166 & 187 & 181 & 154 & 164 & 183 & \\
\hline 158 & 168 & 172 & 159 & 155 & 15 & 160 & 180 & 180 & 174 & \\
\hline 1601 & 162 & 166 & 145 & 177 & 141 & & 16 & 154 & 158 & \\
\hline 162 & 203 & 165 & 21 & 19 & 204 & 18 & 181 & 156 & 15 & \\
\hline & 155 & 166 & 167 & 174 & 175 & 15 & 148 & 137 & 179 & \\
\hline & 164 & 174 & 180 & 161 & 178 & & 16 & 18 & 187 & \\
\hline & 19 & 15 & 20 & 163 & 177 & 17 & 17 & 186 & 174 & \\
\hline 1701 & 195 & 197 & 189 & 201 & 195 & 168 & 187 & 202 & 185 & \\
\hline 172 & 183 & 191 & 177 & 19 & 174 & & 184 & 173 & 229 & \\
\hline & 20 & 203 & 212 & 195 & 189 & & 172 & 179 & 184 & \\
\hline 961 & 153 & 160 & 161 & 173 & 130 & 135 & 141 & 120 & 134 & \\
\hline
\end{tabular}

COUNT COUNT COUNT COUNT COUNT COUNT COUNT COUNT COUNT COUNT $\begin{array}{rrrrrrrrrr}0 & 0 & 1 & 0 & 0 & 0 & 0 & 0 & 0 & 0 \\ 0 & 0 & 0 & 0 & 1 & 0 & 37 & 527 & 8990 & 35022 \\ 8 & 02431 & 43000 & 42918 & 43337 & 43845 & 44302 & 44356 & 44929 & 45635\end{array}$

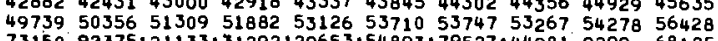

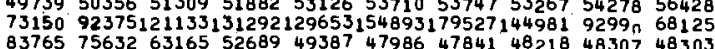
$\begin{array}{lllllllllllll}51998 & 47809 & 46342 & 46590 & 46556 & 46254 & 46404 & 46651 & 46828 & 46503\end{array}$ $\begin{array}{llllllllll}45566 & 45510 & 45853 & 45674 & 45765 & 45369 & 45251 & 45128 & 45806 & 45211\end{array}$ $\begin{array}{llllllllll}44781 & 45097 & 44918 & 44872 & 44890 & 44820 & 44745 & 44647 & 44974 & 44502 \\ 43191 & 43314 & 43442 & 43208 & 43216 & 43387 & 43277 & 42938 & 42868 & 43280\end{array}$ $\begin{array}{llllllll}42456 & 42146 & 42252 & 42331 & 42049 & 42147 & 42169 & 44215 \\ 4208 & 41839\end{array}$ $\begin{array}{llllllllll}41820 & 41278 & 41738 & 41708 & 41383 & 41548 & 41370 & 41484 & 41963 & 41144\end{array}$

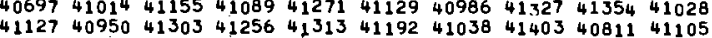
$\begin{array}{llllllllll}41288 & 41143 & 41133 & 41188 & 41560 & 41035 & 41004 & 41015 & 41215 & 40319\end{array}$ $\begin{array}{llllllllll}39743 & 39212 & 38426 & 37871 & 37074 & 36542 & 36129 & 35180 & 35104 & 34835 \\ 33031 & 33158 & 33146 & 32845 & 32741 & 32720 & 32572 & 32553 & 32694 & 32557\end{array}$

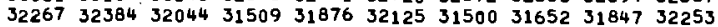
$\begin{array}{llllllllll}31521 & 30874 & 31004 & 31187 & 30798 & 30876 & 31183 & 31061 & 31096 & 31209 \\ 31299 & 32864 & 33828 & 32652 & 31205 & 30110 & 30043 & 30228 & 29929 & 29788\end{array}$

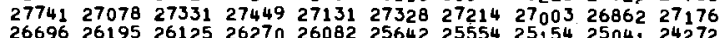
$\begin{array}{lllllllllll}24234 & 24562 & 25362 & 27177 & 28353 & 27515 & 25768 & 24606 & 24077 & 24202\end{array}$ $\begin{array}{llllllllll}42893 & 26988 & 22560 & 21408 & 21362 & 21628 & 21188 & 21384 & 21358 & 21372\end{array}$ $2069920550204272025220103202622027919934 \quad 1990520115$

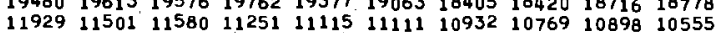

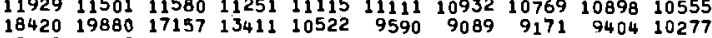
$\begin{array}{llllllllll}9818 & 7639 & 7299 & 7125 & 6873 & 6867 & 6871 & 6914 & 7142 & 7331 \\ 5902 & 5649 & 5792 & 5694 & 5705 & 5800 & 5927 & 6686 & 8887 & 14244\end{array}$ $\begin{array}{rlllllllll}4225 & 4250 & 4089 & 4035 & 4122 & 4021 & 3889 & 3989 & 3963 & 3962\end{array}$ $\begin{array}{rrrrrrrrrr}4535 & 5794 & 7587 & 9101 & 8900 & 6976 & 4867 & 3917 & 3578 & 3622 \\ 3651 & 3673 & 3764 & 4006 & 4895 & 6907 & 10679 & 15912 & 200338 & 19091\end{array}$ $\begin{array}{lllllllllll}3392 & 3427 & 3403 & 3459 & 3535 & 3468 & 3524 & 3680 & 3671 & 3623\end{array}$ $\begin{array}{lllllllllll}11255 & 9407 & 15489 & 29056 & 50245 & 72660 & 80158 & 59723 & 2972 & 10500\end{array}$ $\begin{array}{llllllllll}2149 & 2129 & 2145 & 2134 & 2176 & 2157 & 2275 & 2212 & 2561 & 3002\end{array}$ $\begin{array}{llllllllll}1885 & 1922 & 1907 & 1941 & 1885 & 1970 & 1926 & 1858 & 1940 & 1958 \\ & 1591 & 1543 & 1604 & 1561 & 1541 & 156 & 1489 & 1586 & 1511\end{array}$ $\begin{array}{llllllllll}1582 & 1591 & 1543 & 1604 & 1561 & 1541 & 1539 & 1489 & 1586 & 1511 \\ 2065 & 2439 & 2742 & 2768 & 2541 & 1823 & 1562 & 1529 & 1441 & 1340\end{array}$

\begin{tabular}{|c|c|c|c|c|c|c|c|c|c|}
\hline 1406 & 1348 & 1355 & 1339 & 1299 & 1387 & 1303 & 1273 & 1364 & 1333 \\
\hline 1246 & 1213 & 1250 & 1189 & 1205 & 1149 & 1121 & 1211 & 1099 & 1136 \\
\hline 1059 & 1154 & 1133 & 1028 & 1002 & 1053 & 1033 & 1041 & 1077 & 954 \\
\hline 968 & 980 & 987 & 948 & 946 & 958 & 912 & 943 & & 941 \\
\hline 84 & 850 & 844 & 873 & 832 & 894 & 881 & 852 & & 50 \\
\hline 934 & 955 & 924 & 848 & 811 & 839 & 877 & 826 & & \\
\hline 818 & 810 & 740 & 764 & 773 & 766 & 785 & & & 808 \\
\hline 153 & 3823 & 4121 & 3480 & 2177 & 1257 & 846 & & & \\
\hline 662 & 677 & 67 & 605 & 86 & & & & & \\
\hline 63 & & & & 626 & & 623 & & 669 & \\
\hline 641 & 631 & 635 & 610 & 614 & & 679 & 719 & & \\
\hline 565 & 506 & 558 & 565 & 534 & 497 & 615 & 49 & & \\
\hline & 859 & 90 & 993 & 1097 & 938 & 855 & 73 & & 6 \\
\hline 489 & 493 & 496 & 497 & 499 & & 483 & 500 & & \\
\hline 476 & 509 & 532 & 461 & 506 & 449 & 443 & 448 & 450 & \\
\hline 476 & 444 & 491 & 456 & 454 & 463 & 455 & 487 & 438 & 432 \\
\hline 446 & 452 & 468 & 434 & 477 & & 405 & 445 & 44 & 462 \\
\hline 46 & 480 & 499 & 57 & 529 & 49 & 411 & 38 & 407 & 87 \\
\hline 376 & 320 & & & 389 & & 354 & & 349 & 344 \\
\hline 372 & & & & 331 & & 348 & & 366 & 37 \\
\hline 335 & & 31 & 31 & 304 & 30 & 306 & & & \\
\hline 29 & 283 & 37 & 33 & 346 & & 315 & 32 & & \\
\hline 298 & 288 & 27 & 290 & 258 & 258 & 266 & 250 & & 66 \\
\hline 228 & & & 25 & 243 & & 263 & & & \\
\hline 224 & 237 & & 25 & 231 & & 229 & & & \\
\hline 210 & 189 & & 21 & 196 & 22 & 21 & & & 23 \\
\hline & 21 & & & & & & & & 26 \\
\hline 258 & 228 & & 22 & & & 22 & 16 & & 185 \\
\hline 176 & 198 & 192 & 188 & 227 & & & & & 192 \\
\hline 179 & 216 & & 17 & 199 & & & & & \\
\hline 275 & 357 & & 32 & 275 & & 17 & & & 18 \\
\hline 182 & 140 & 148 & 166 & 16 & 17 & 17 & 16 & 16 & 168 \\
\hline & & & & & 18 & 17 & & & 182 \\
\hline & & & & 177 & & 169 & 16 & & 166 \\
\hline 15 & 180 & 16 & 137 & 154 & & & & & 167 \\
\hline 22 & & & & & & & & & \\
\hline 158 & & & & & & & 17 & 16 & 163 \\
\hline 172 & 135 & & 14 & 17 & 16 & 18 & 14 & & 150 \\
\hline 16. & 15 & & 15 & 17 & & 16 & & & 160 \\
\hline 168 & 181 & 15 & 18 & 20 & & & & & 174 \\
\hline 154 & 171 & & & & & & & 17 & 184 \\
\hline 166 & 151 & 17 & 18 & 183 & 16 & 164 & 16 & 16 & 176 \\
\hline 17 & 15 & & 18 & 160 & 16 & 15 & & & 171 \\
\hline 194 & 17 & 16 & 15 & 152 & 16 & & & & 158 \\
\hline 177 & 18 & 18 & & & 19 & 16 & & & 192 \\
\hline 172 & 18 & 17 & 17 & 209 & 17 & 16 & 180 & 201 & 193 \\
\hline 196 & 184 & 20 & 16 & 192 & 18 & 20 & 18 & 18 & 186 \\
\hline 17 & 18 & 17 & 17 & 187 & & 18 & & 15 & 16 \\
\hline 102 & 111 & 105 & 108 & 96 & 102 & 116 & 104 & 104 & \\
\hline
\end{tabular}

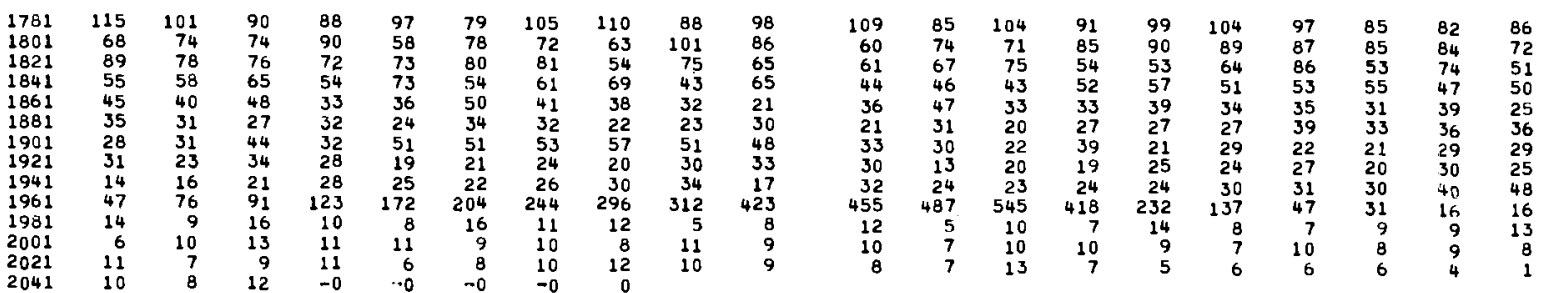




\section{D-7}

GAMMA RAY SPECTRUIA DATA

PRTR ROD FS-16 SPECTRUM NO. 11 DATA TAKEN 2/28/69 LIVE TIME O70.MIN. POSITION 48.IN. ENERGYIKEVI= 1.111*CHAN -7.8 CHAN COUNT COUNT COUNT COUNT COUNT COUNT COUNT COUNT COUNT COUNT

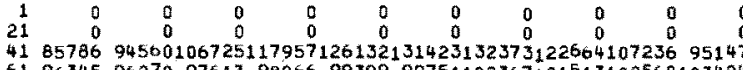
61963459627097613982669939999751100367101513102568103424 81118707118256118100118720121137124378126583128895131844136323 121100474100450100362100849102758107559127767160879169868139987 14197640968009693296595968869642296884959429629795952 16195885954439554995295955889583796631953909526394840

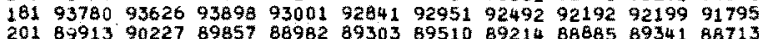
$2018991390227898578898287303 \quad 8951089214888858934188713$

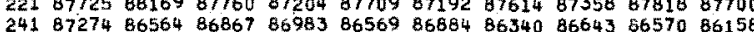
$261 \quad 8578485401 \quad 86122 \quad 86005 \quad 85936 \quad 8629485947 \quad 85852 \quad 861658609$ $281864198651986039864098649386732 \quad 8696986390862578628$

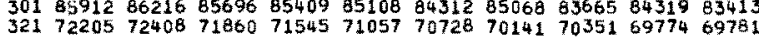

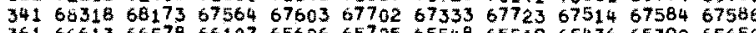
$38165526 \quad 649146467465156647876513064974648386506165165$ $401 \quad 6265761681616906058160130 \quad 59130 \quad 59438 \quad 5835458354 \quad 57733$ $421 \quad 57190 \quad 56925579515826358740 \quad 582195702756805 \quad 56497 \quad 56222$ 46150900509805080750119505415002550036501914995349943 $\begin{array}{lllllllllll}481 & 44781 & 44320 & 44075 & 44141 & 44221 & 43249 & 43839 & 43671 & 43292 & 43104\end{array}$

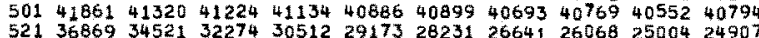

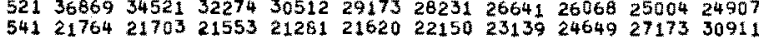

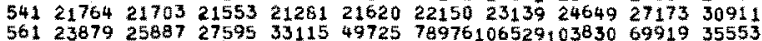

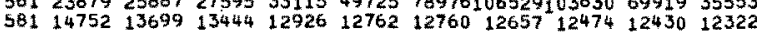

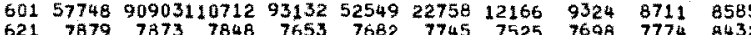
$\begin{array}{lllllllllll}641 & 7319 & 7303 & 7319 & 7270 & 7284 & 7334 & 7108 & 7156 & 7342 & 7469\end{array}$ $\begin{array}{rrrrrrrrrrr}661 & 25895 & 13944 & 8700 & 7067 & 6862 & 6804 & 6806 & 5912 & 6985 & 7085 \\ 681 & 7701 & 7859 & 8777 & 10496 & 14993 & 23572 & 35937 & 47021 & 48687 & 35461\end{array}$ $\begin{array}{lrrrrrrrrrr}701 & 7495 & 5032 & 4579 & 4572 & 4513 & 4483 & 4548 & 4449 & 4371 & 4534 \\ 721 & 8685 & 1927 & 15199 & 16085 & 12982 & 8555 & 5908 & 5345 & 5511 & 517\end{array}$ $\begin{array}{lllllllllll}741 & 4111 & 4156 & 4083 & 4133 & 4023 & 4091 & 4149 & 4147 & 4149 & 4027\end{array}$ $\begin{array}{lllllllllll}761 & 4026 & 3896 & 3853 & 3654 & 3721 & 3654 & 3538 & 3455 & 3440 & 341 \\ 781 & 3071 & 3165 & 3087 & \mathbf{3 1 7 0} & \mathbf{3 1 2 1} & \mathbf{3 1 6 6} & \mathbf{3 1 1 6} & \mathbf{3 2 6 1} & \mathbf{3 3 3 1} & \mathbf{3 7 5 1}\end{array}$

COUNT COUNT COUNT COUNT COUNT COUNT COUNT COUNT COUNT COUNT

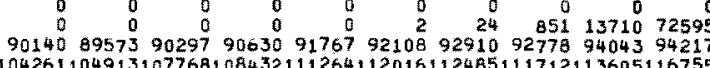
104261104913107768109432111264112016112485111712113605116755 17729516212813644411210610374110093310062410064510116146847 1118191001349789098137973289780698221981089837197189

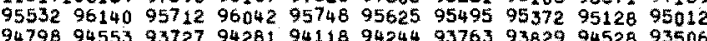
$913059121791868907999094790871911918982190628 \quad 89949$ $\begin{array}{llllllllll}8723 & 88663 & 88426 & 88310 & 89370 & 88402 & B 8761 & 88206 & 88504 & 87777 \\ 87842 & 87618 & 87112 & 87157 & 87265 & 87345 & 86585 & 86873 & 86725 & 86293\end{array}$

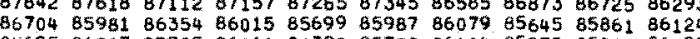
$8569586227859258601186390 \quad 85720 \quad 8616485975 \quad 8580186193$

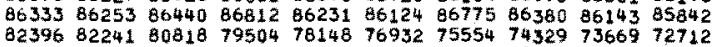

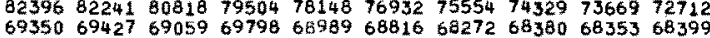

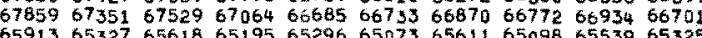
66825694307094769308651496389063376632096262762862 $58306 \quad 57986 \quad 581335788357196 \quad 5719757016 \quad 57122 \quad 5724356958$ 55583552225548154761551465441953718529195205951217 50502510055309656853593115794554116510485021450661 $908755262 \quad 46654 \quad 45069 \quad 44668 \quad 44577 \quad 44630 \quad 44468 \quad 4419444474$

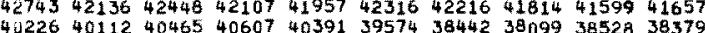
$2459123938 \quad 23900234392303722934 \quad 2247722602 \quad 22463 \quad 22067$ $3691339491 \quad 34746267082176019551 \quad 19312194071966121011$

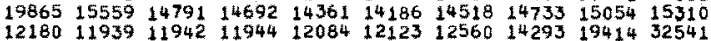
$86268696 \quad 8358 \quad 8168 \quad 8135 \quad 8152 \quad 8050 \quad 8000 \quad 01428012$ $7671 \quad 7694 \quad 7721 \quad 8487 \quad 9959 \quad 14304 \quad 22528 \quad 3365441860 \quad 39028$ $\begin{array}{llllllllll}6852 & 7075 & 7146 & 7011 & 7031 & 7230 \quad 7233 & 7401 & 7457 & 7524\end{array}$

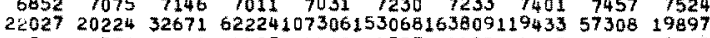
$\begin{array}{llllllllll}4500 & 4592 & 4411 & 4465 & 4420 & 4557 & 4493 & 4665 & 5146 & 6413\end{array}$ $\begin{array}{llllllllll}4041 & 4072 & 4081 & 4053 & 4184 & 4106 & 4112 & 4110 & 4211 & 4131 \\ 3338 & 3376 & 3244 & 3319 & 3296 & 3972 & 4098 & 4063 & 4150 & 4022 \\ 3 & 3927 & 3187 & 3211 & 3148 & 3093\end{array}$ $\begin{array}{llllllllll}4222 & 5008 & 5795 & 5319 & 3298 & 3227 & 3187 & 3211 & 3148 & 3093 \\ & & 4968 & 3673 & 3140 & 3032 & 3008 & 2991\end{array}$

$\begin{array}{lllllllllll}801 & 3003 & 3118 & 3175 & 3210 & 3076 & 3033 & 2964 & 2886 & 2876 & 2854\end{array}$ $\begin{array}{lllllllllll}221 & 2873 & 2214 & 2764 & 2784 & 2644 & 2610 & 2654 & 2753 & 2668 & 2620 \\ 641 & 2401 & 2373 & 2446 & 2303 & 2390 & 2304 & 2269 & 3302 & 2354 & 232\end{array}$

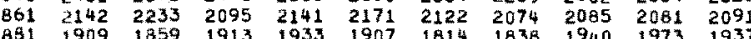
$\begin{array}{lllll} & \\ 1857 & 1838 & 1940 & 1973 & 1937\end{array}$ $\begin{array}{lllllllllll}901 & 1838 & 1860 & 1851 & 1911 & 1818 & 1857 & 1841 & 1726 & 1828 & 1825 \\ 921 & 1698 & 1662 & 1683 & 1647 & 1674 & 1651 & 1586 & 1657 & 1607 & 1616\end{array}$ $\begin{array}{lllllllllll}941 & 1751 & 1713 & 1666 & 1729 & 1782 & 1806 & 2160 & 2795 & 3778 & 4862 \\ 961 & 1498 & 1474 & 1548 & 1557 & 1568 & 1388 & 1378 & 1356 & 1413 & 1394\end{array}$

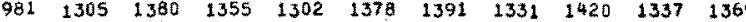
$\begin{array}{lllllllllll}1001 & 1321 & 1331 & 1362 & 1282 & 1398 & 1314 & 1308 & 1318 & 1427 & 1340 \\ 1021 & 2594 & 2781 & 2764 & 2342 & 1627 & 1344 & 1224 & 1191 & 1101 & 1085\end{array}$

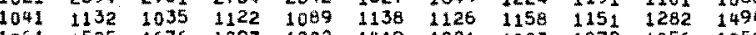

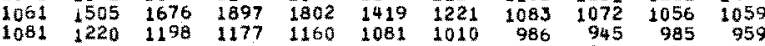
$\begin{array}{rrrrrrrrrrr}1081 & 1220 & 1198 & 1177 & 1160 & 1081 & 1010 & 986 & 945 & 985 & 959 \\ 1101 & 945 & 985 & 986 & 959 & 917 & 947 & 930 & 991 & 941 & 969\end{array}$ $\begin{array}{lllllllllll}1121 & 938 & 907 & 950 & 944 & 928 & 992 & 932 & 979 & 917 & 916 \\ 1141 & 922 & 886 & 863 & 890 & 857 & 905 & 892 & 913 & 872 & 969\end{array}$ $\begin{array}{lllllllllll}1141 & 922 & 886 & 863 & 890 & 857 & 905 & 892 & 913 & 872 & 969 \\ 1161 & 756 & 757 & 751 & 758 & 769 & 783 & 713 & 732 & 735 & 706\end{array}$ $\begin{array}{rrrrrrrrrrr}1181 & 730 & 719 & 667 & 738 & 677 & 719 & 692 & 706 & 740 & 724 \\ 1201 & 823 & 811 & 881 & 1018 & 1151 & 1353 & 1291 & 1091 & 818 & 732\end{array}$ $\begin{array}{rrrrrrrrrrr}1201 & 823 & 811 & 981 & 1018 & 1151 & 1353 & 1231 & 1051 & 818 & 732 \\ 1221 & 549 & 566 & 576 & 572 & 555 & 559 & 592 & 602 & 613 & 590 \\ 1241 & 540 & 515 & 539 & 547 & 581 & 526 & 514 & 575 & 505 & 514\end{array}$ 1261

$\begin{array}{lr}1781 & 205 \\ 1801 & 161 \\ 1821 & 139 \\ 1841 & 106 \\ 1861 & 85 \\ 1881 & 70 \\ 1901 & 79 \\ 1921 & 53 \\ 1941 & 44 \\ 1961 & 113 \\ 1981 & 17 \\ 2001 & 17 \\ 2021 & 22 \\ 2041 & 16\end{array}$

$462 \quad 5$

$\begin{array}{lll}489 & 502 & 483 \\ 459 & 423 & 465\end{array}$

$\begin{array}{lll}501 & 685 & 455 \\ 434 & 397 & 379\end{array}$

$411 \quad 378 \quad 397$

334
347

$\begin{array}{lll}353 & 547 & 320\end{array}$

313

$\begin{array}{lll}365 & 382 & 395 \\ 337 & 529 & 331\end{array}$

$\begin{array}{lll}355 & 334 & 323\end{array}$

$\begin{array}{rrr}178 & 192 & 179 \\ 157 & 176 & 164 \\ 149 & 144 & 155 \\ 143 & 134 & 192 \\ 92 & 68 & \\ 53 & 56 & \\ 97 & 83 & 99 \\ 53 & 54 & 44 \\ 54 & 45 & \\ 156 & 204 & \\ 25 & 25 & \\ 17 & 21 \\ 22 & 18 \\ 16 & 12\end{array}$

$\begin{array}{lll}384 & 397 & 409\end{array}$

$\begin{array}{lll}371 & 375 & 379 \\ 375 & 327 & 365\end{array}$

$\begin{array}{lll}361 & 339 & 352 \\ 322 & 346 & 335\end{array}$

$\begin{array}{lll}328 & 394 & 363 \\ 324 & 373 & 378\end{array}$

$\begin{array}{lll}389 & 390 & 399 \\ 332 & 350 & 305\end{array}$ $\begin{array}{lllll}527 & 538 & 510 & 462 & 488\end{array}$

$\begin{array}{lllll}488 & 493 & 466 & 471 & 457 \\ 85 & 451 & 420 & 442 & 430\end{array}$

$\begin{array}{rrrrr}926 & 428 & 386 & 431 & 446 \\ 4078 & 1171 & 1270 & 1161\end{array}$

$400 \quad 417$

$\begin{array}{lll}01 & 389 & 403\end{array}$

$\begin{array}{lll}355 & 341 & 370 \\ 351 & 336\end{array}$

$\begin{array}{ll}319 & 3 \\ 370 & 350\end{array}$

$\begin{array}{lll}344 & 348 & 326 \\ 354 & 319 & 323\end{array}$

$\begin{array}{lll}340 & 319 & 323 \\ 340 & 331 & 332 \\ 348\end{array}$

$\begin{array}{lll}340 & 331 & 348 \\ 351 & 346 & 343\end{array}$

$\begin{array}{lll}383 & 328 & 353\end{array}$

$\begin{array}{lll}360 & 357 & 364 \\ 352 & 352 & 359\end{array}$

$\begin{array}{lll}399 & 369 & 382 \\ 402 & 399 & 399\end{array}$

$\begin{array}{lllllll}402 & 398 & 378 & 368 & 354 & 397 & 376\end{array}$

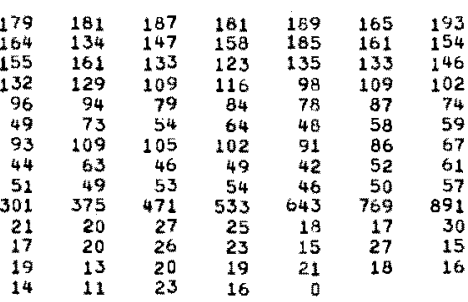

$\begin{array}{lll}358 & 341 & 440 \\ 362 & 352\end{array}$

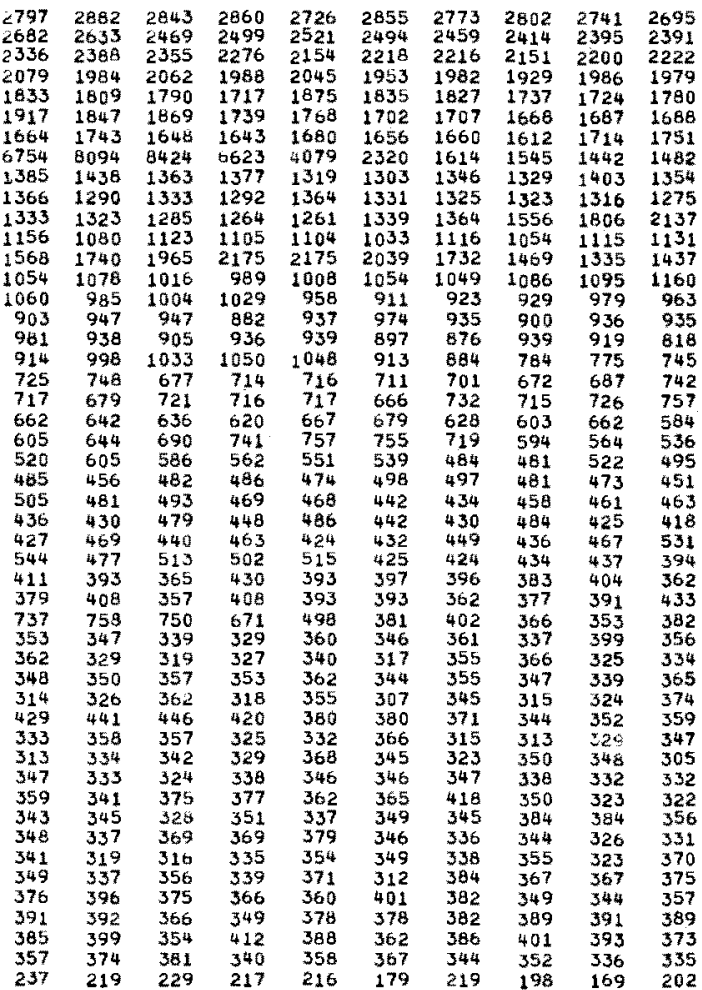

$\begin{array}{rrrrrrrrrr}196 & 194 & 206 & 224 & 218 & 209 & 208 & 200 & 180 & 160 \\ 157 & 145 & 149 & 158 & 147 & 161 & 152 & 146 & 149 & 164 \\ 138 & 151 & 121 & 133 & 140 & 120 & 129 & 125 & 97 & 118 \\ 118 & 98 & 90 & 108 & 97 & 103 & 106 & 91 & 100 & 98 \\ 77 & 85 & 57 & 57 & 76 & 60 & 73 & 58 & 60 & 61 \\ 52 & 49 & 49 & 53 & 41 & 49 & 67 & 70 & 53 & 78 \\ 45 & 59 & 49 & 60 & 65 & 46 & 38 & 48 & 50 & 61 \\ 49 & 51 & 40 & 55 & 53 & 47 & 49 & 48 & 33 & 53 \\ 53 & 45 & 48 & 63 & 52 & 71 & 62 & 68 & 78 & 105 \\ 1021 & 1113 & 927 & 676 & 367 & 142 & 55 & 30 & 24 & 30 \\ 27 & 20 & 21 & 24 & 27 & 11 & 14 & 18 & 25 & 22 \\ 16 & 22 & 25 & 20 & 13 & 17 & 22 & 14 & 25 & 23 \\ 17 & 18 & 17 & 16 & 12 & 13 & 20 & 28 & 17 & 20\end{array}$ 


\section{D-8}

GAMMA RAY SPECTRUM DATA

PRTR ROD FS-16 SPECTRUM NO. 12 DATA TAKEN 3/01/69 LIVE TIME 650.MIN. POSITION 56.IN. ENERGY(KEV)= $1.113 *$ FHAN -9.1 CHAN COUNT COUNT COUNT COUNT COUNT COUNT COUNT COUNT COUNT COUNT

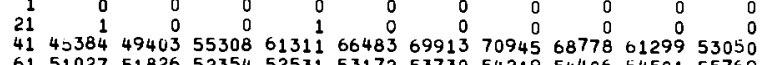

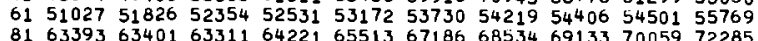

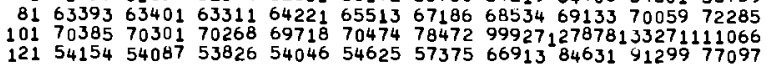

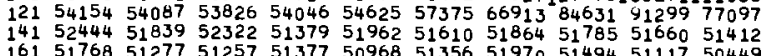
$\begin{array}{lllllllllll}181 & 49976 & 50583 & 50217 & 49969 & 50159 & 49910 & 49595 & 49411 & 49653 & 49180\end{array}$

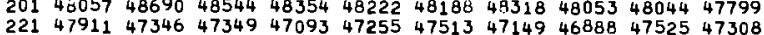
$241465494670946944 \quad 46578 \quad 46773 \quad 46395 \quad 46551 \quad 463544615746112$ $\begin{array}{lllllllllll}261 & 46406 & 46278 & 46236 & 45916 & 46448 & 46326 & 46593 & 46399 & 46439 & 46172\end{array}$ $\begin{array}{lllllllllll}281 & 46042 & 46476 & 46747 & 46053 & 46349 & 46025 & 46575 & 46193 & 46634 & 46107\end{array}$ $\begin{array}{llllllllllll}301 & 40208 & 46117 & 45767 & 45752 & 45696 & 45765 & 45755 & 45523 & 45107 & 44759\end{array}$ $\begin{array}{lllllllllll}341 & 36497 & 38809 & 38547 & 38352 & 38123 & 38170 & 37929 & 37322 & 37407 & 37209\end{array}$ $\begin{array}{lllllllllll}361 & 30062 & 35136 & 35563 & 35061 & 35277 & 35173 & 35366 & 34926 & 35394 & 35335\end{array}$ $\begin{array}{lllllllllll}381 & 34923 & 34760 & 34885 & 35227 & 34748 & 34872 & 34814 & 34799 & 35022 & 34990 \\ 401 & 33235 & 33406 & 33081 & 32992 & 32417 & 32161 & 31910 & 31533 & 31814 & 31316\end{array}$ $\begin{array}{llllllllllll}421 & 30880 & 31149 & 31133 & 31904 & 31780 & 31443 & 31040 & 30741 & 30600 & 29942 \\ 441 & 27699 & 27222 & 27453 & 27276 & 27350 & 26879 & 27278 & 26728 & 27092 & 27117\end{array}$ 46127611275362852030532400396516011041414985214062287737 $\begin{array}{lllllllllll}481 & 23868 & 23823 & 24056 & 23900 & 23894 & 23733 & 23598 & 23451 & 23740 & 23241\end{array}$

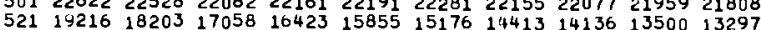
$\begin{array}{lllllllllll}561 & 12989 & 14387 & 15173 & 18528 & 28867 & 45661 & 59364 & 54699 & 33996 & 16712\end{array}$

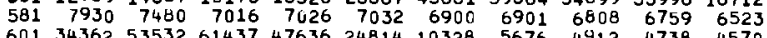
$\begin{array}{lllllllllll}601 & 34362 & 53532 & 61437 & 47636 & 248114 & 10328 & 5676 & 4912 & 4738 & 4570\end{array}$ $\begin{array}{lllllllllll}641 & 3932 & 3970 & 3976 & 4091 & 4042 & 3985 & 3988 & 3941 & 4323 & 4603 \\ \end{array}$ $\begin{array}{lllllllllll}661 & 12493 & 6505 & 4322 & 3871 & 3833 & 3767 & 3806 & 3714 & 3858 & 3711\end{array}$ $\begin{array}{rrrrrrrrrrr}681 & 4217 & 4465 & 4955 & 6041 & 8915 & 14262 & 21414 & 26948 & 25464 & 17624 \\ 701 & 3436 & 2433 & 2448 & 2322 & 2392 & 2478 & 2489 & 2352 & 2402 & 2425\end{array}$ $\begin{array}{lllllllllll}721 & 4250 & 5404 & 6460 & 6313 & 5023 & 3569 & 2880 & 2719 & 2769 & 2631\end{array}$ $\begin{array}{lllllllllll}761 & 2145 & 2106 & 2055 & 2051 & 2069 & 2011 & 1880 & 1900 & 1927 & 1980\end{array}$ $\begin{array}{lllllllllll}781 & 1739 & 1627 & 1702 & 1746 & 1761 & 1707 & 1731 & 1787 & 1872 & 2142\end{array}$

\begin{tabular}{|c|c|c|c|c|c|c|c|c|c|c|}
\hline 801 & 1676 & 1632 & 1744 & 1685 & 1611 & 1605 & 1633 & 1674 & 1612 & 1602 \\
\hline & 1539 & 1517 & 1540 & 1554 & 1507 & 1475 & 1498 & 1434 & 1427 & \\
\hline 4 & 1323 & 1361 & 1301 & 1250 & 1319 & 1340 & 1302 & 1315 & 1268 & 1286 \\
\hline 861 & 1215 & 1185 & 1199 & 1134 & 1223 & 1170 & 1143 & 1146 & 1140 & 1156 \\
\hline 881 & 1056 & 1113 & 1045 & 1100 & 1019 & 1043 & 1044 & 1124 & 1050 & \\
\hline 901 & 977 & 1040 & 1013 & 1013 & 995 & 979 & 998 & 945 & 986 & \\
\hline 21 & 940 & 919 & 1008 & 949 & 897 & 972 & 926 & 889 & 980 & \\
\hline & 941 & 920 & 970 & 876 & 989 & 1049 & 1328 & 1641 & 2320 & \\
\hline 961 & 794 & 875 & 872 & 877 & 85 & 805 & 772 & 789 & 797 & \\
\hline 981 & 817 & 764 & 796 & 781 & 727 & 747 & B11 & 767 & 735 & \\
\hline 1001 & 751 & 708 & 799 & 799 & 762 & 746 & 784 & 737 & 798 & \\
\hline 021 & 1444 & 1601 & 1405 & 1141 & 813 & 676 & 653 & 573 & 641 & \\
\hline 04 & 626 & 626 & 620 & 627 & 59 & 641 & 650 & 665 & 739 & \\
\hline 1061 & 967 & 1113 & 1131 & 1024 & 744 & 694 & 582 & ל57 & 609 & \\
\hline 1081 & 627 & 675 & 637 & 590 & 519 & 561 & 537 & 596 & 602 & \\
\hline 1101 & 537 & 491 & 480 & 540 & 506 & 486 & 522 & 487 & 510 & \\
\hline 12 & 527 & 473 & 566 & 540 & 510 & 479 & 531 & 476 & 503 & \\
\hline 1141 & 500 & 462 & 468 & 494 & 467 & 469 & 479 & 462 & 455 & \\
\hline 116 & 433 & 432 & 428 & 454 & 421 & 378 & 429 & 413 & 409 & \\
\hline 1181 & 391 & 419 & 386 & 418 & 411 & 392 & 385 & 415 & 400 & \\
\hline 1201 & 443 & 511 & 576 & 702 & 857 & 796 & 715 & 570 & 426 & \\
\hline 1221 & 342 & 303 & 333 & 335 & 377 & 301 & 30 & & 325 & \\
\hline 124 & 310 & $\$ 13$ & 295 & 273 & 320 & 281 & 272 & 298 & 307 & \\
\hline & 26 & 264 & 30 & 306 & 275 & 283 & 267 & 265 & 246 & \\
\hline 1281 & 247 & 269 & 272 & 258 & 281 & 277 & 287 & 258 & 240 & \\
\hline 130 & 241 & 260 & $27 \overline{3}$ & 234 & & 222 & & & 244 & \\
\hline 1321 & 229 & 217 & 262 & 252 & 257 & 260 & 223 & & 246 & \\
\hline 134 & 35 & 429 & 47 & 55 & 633 & 688 & 664 & 515 & 401 & \\
\hline & 232 & 216 & 22 & 208 & 206 & 228 & 232 & 249 & 225 & \\
\hline 133 & 235 & 212 & $21 \epsilon$ & 199 & 201 & 228 & 20 & 180 & 206 & \\
\hline 1401 & 203 & 227 & 203 & 236 & & 257 & & & & \\
\hline 142 & 181 & & 190 & & & 194 & & & & \\
\hline 144 & 188 & 191 & 165 & 237 & 191 & 180 & 1 & & 16 & \\
\hline 146 & 198 & 180 & 187 & 196 & 194 & 171 & 18 & 18 & 162 & \\
\hline 148 & $16 t$ & 204 & 20 & 210 & 20 & 179 & 18 & 18 & 181 & \\
\hline 150 & 17 & 199 & 195 & 2 & & & & & 212 & \\
\hline 152 & 18 & & & 17 & 17 & 195 & 10 & 19 & 166 & \\
\hline 1541 & 157 & 177 & 182 & 178 & 187 & 186 & 20 & $i_{5}$ & 211 & \\
\hline 1561 & 162 & 156 & 171 & 169 & 162 & 192 & 17 & 19 & 188 & \\
\hline 158 & 18 & 190 & 187 & 100 & 185 & 176 & 175 & 1 & 211 & \\
\hline 160 & 17 & 198 & & & & 178 & 19 & & 176 & \\
\hline 1621 & 182 & & 238 & 214 & 188 & 197 & 206 & 188 & 184 & \\
\hline 1041 & $17 \overline{4}$ & 189 & 208 & 134 & 175 & 198 & 18 & 158 & 181 & \\
\hline 106 & 207 & 204 & 186 & 193 & 18 & 22 & 20 & & 1 & \\
\hline 168 & 18 & 20 & 1 & & 15 & 2 & 22 & & 211 & \\
\hline 1701 & 1 & 19 & 210 & 19 & 19. & 219 & 204 & 218 & 194 & \\
\hline 17 & 219 & 203 & 223 & 228 & 197 & 215 & 223 & 207 & 199 & \\
\hline 174 & 211 & 211 & 190 & 168 & 221 & 245 & 20 & 228 & 213 & \\
\hline 176 & 179 & 164 & 166 & 164 & 132 & 145 & 126 & 123 & 147 & \\
\hline
\end{tabular}

COUNT COUNT COUNT COUNT COUNT COUNT COUNT COUNT COUNT COUNT

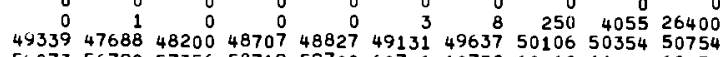

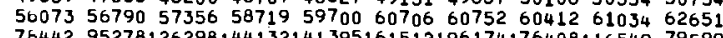
764429527812629814413214139516151219617417640811654979599 $\begin{array}{llllllllll}9506 & 87136 & 74192 & 61168 & 55590 & 54076 & 53835 & 53938 & 53700 & 53688\end{array}$

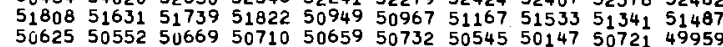
$\begin{array}{lllllllllllllll}49164 & 49079 & 49080 & 48615 & 48994 & 48911 & 48399 & 48704 & 48580 & 48184\end{array}$ $\begin{array}{llllllllll}47780 & 47852 & 47899 & 47637 & 47426 & 47635 & 47853 & 47526 & 47253 & 47267\end{array}$ $\begin{array}{lllllllllll}40413 & 46207 & 46341 & 46224 & 46199 & 46071 & 46533 & 46248 & 46395 & 46278\end{array}$ $\begin{array}{llllllllll}46132 & 46185 & 46256 & 46283 & 46508 & 46008 & 46563 & 46370 & 46156 & 46333\end{array}$

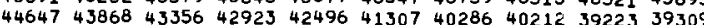
$\begin{array}{llllllllll}47647 & 43868 & 43356 & 42923 & 42496 & 41307 & 40286 & 40212 & 39223 & 39309\end{array}$ $\begin{array}{llllllllllllll}30320 & 36296 & 36150 & 36059 & 36057 & 35570 & 35620 & 35682 & 35635 & 35544\end{array}$ $\begin{array}{lllllllllll}35389 & 35023 & 35226 & 35102 & 35178 & 35151 & 35040 & 3508 \mathrm{C} & 35203 & 34623\end{array}$ $\begin{array}{lllllllllllll}36360 & 37492 & 38872 & 37861 & 35920 & 34492 & 34465 & 34188 & 34152 & 33803\end{array}$ $\begin{array}{llllllllll}30254 & 3278 & 31364 & 31158 & 30991 & 30862 & 30978 & 31073 & 30972 & 31002\end{array}$ $\begin{array}{llllllllll}27247 & 27785 & 29049 & 30974 & 32342 & 31240 & 29081 & 27613 & 27636 & 27230\end{array}$ $\begin{array}{llllllllll}44661 & 28252 & 25068 & 24474 & 24199 & 24268 & 24073 & 24070 & 24260 & 24243\end{array}$ $\begin{array}{llllllllll}23169 & 23021 & 23111 & 22830 & 22936 & 22445 & 22440 & 22542 & 22679 & 22542 \\ 21689 & 21353 & 21849 & 21743 & 21709 & 20908 & 20776 & 20282 & 20198 & 19820\end{array}$ $\begin{array}{lllllllllll}13096 & 12768 & 12677 & 12343 & 12493 & 12205 & 12116 & 11775 & 11767 & 11661\end{array}$ $\begin{array}{lllllllllll}10951 & 17643 & 15726 & 12732 & 10954 & 10503 & 10259 & 10289 & 10631 & 11720\end{array}$ $\begin{array}{llllllllll}9792 & 8279 & 7983 & 7859 & 7794 & 7697 & 8032 & 7990 & 8077 & 8231 \\ 6370 & 6536 & 6556 & 6445 & 6399 & 6531 & 6976 & 7910 & 11176 & 19684\end{array}$ $\begin{array}{llllllllllll}4716 & 4726 & 4526 & 4623 & 4605 & 4537 & 4366 & 4277 & 4280 & 4344\end{array}$ $\begin{array}{rrrrrrrrrr}4542 & 6865 & 9128 & 10723 & 9608 & 7104 & 4860 & 4192 & 3960 & 3856\end{array}$ $\begin{array}{llllllllll}4078 & 4090 & 4221 & 4642 & 5896 & 8655 & 13558 & 19913 & 23895 & 20318\end{array}$ $\begin{array}{rrrrrrrrrr}3814 & 3807 & 3907 & 3942 & 3883 & 3947 & 4109 & 4101 & 4067 & 4192 \\ 14957 & 11506 & 20455 & 39149 & 65615 & 89097 & 88252 & 58291 & 24615 & 7871\end{array}$ 1471150620455391496561589097882525829124615787 $\begin{array}{llllllllll}2420 & 2377 & 2471 & 2475 & 2458 & 2454 & 2480 & 2590 & 2811 & 3314 \\ 2520 & 2378 & 2238 & 2277 & 2271 & 2396 & 2207 & 2293 & 2279 & 2250\end{array}$ $\begin{array}{llllllllll}2253 & 2238 & 2358 & 2229 & 2274 & 2245 & 2243 & 2336 & 2281 & 2213\end{array}$ $\begin{array}{llllllllll}2484 & 2921 & 3146 & 2984 & 2547 & 1938 & 1771 & 1762 & 1626 & 1711\end{array}$

$\begin{array}{llllllllll}1607 & 1545 & 1573 & 1567 & 1587 & 1614 & 1533 & 1605 & 1593 & 1545\end{array}$ $\begin{array}{lllllllllll}1456 & 1473 & 1471 & 1423 & 1446 & 1402 & 1370 & 1364 & 1401 & 1400\end{array}$ $\begin{array}{llllllllll}1374 & 1291 & 1225 & 1267 & 1272 & 1213 & 1240 & 1177 & 1117 & 1151\end{array}$

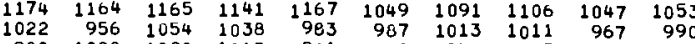
$\begin{array}{rrrrrrrrrr}980 & 1020 & 1020 & 1013 & 961 & 960 & 949 & 955 & 980 & 970\end{array}$ $\begin{array}{rrrrrrrrrr}939 & 967 & 907 & 869 & 947 & 880 & 902 & 942 & 859 & 993 \\ 4050 & 4750 & 4469 & 3098 & 1783 & 1102 & 899 & 790 & 866 & 860\end{array}$ $\begin{array}{rrrrrrrrrr}4050 & 4750 & 4469 & 3098 & 1783 & 1102 & 899 & 790 & 866 & 860 \\ 782 & 769 & 815 & 784 & 793 & 792 & 722 & 763 & 780 & 754\end{array}$ $\begin{array}{llllllllll}\mathbf{7 8 2} & 769 & \mathbf{8 1 5} & \mathbf{7 8 4} & \mathbf{7 9 3} & \mathbf{7 9 2} & \mathbf{7 2 2} & \mathbf{7 6 3} & \mathbf{7 8 0} & \mathbf{7 5 4} \\ \mathbf{7 4 2} & \mathbf{7 3 7} & \mathbf{7 7 2} & \mathbf{7 4 0} & \mathbf{7 5 5} & \mathbf{7 0 8} & \mathbf{7 4 2} & \mathbf{7 6 9} & \mathbf{7 6 1} & \mathbf{7 1 6}\end{array}$ $\begin{array}{rrllllllll}779 & 727 & 742 & 702 & 731 & 773 & 742 & 769 & 761 & 716 \\ 661 & 621 & 576 & 625 & 607 & 647 & 628 & 925 & 1083 & 1224\end{array}$ $\begin{array}{rrrrrrrrrr}661 & 621 & 576 & 625 & 607 & 647 & 628 & 618 & 647 & 637 \\ 869 & 973 & 1072 & 1191 & 1170 & 1001 & 844 & 728 & 782 & 852\end{array}$ $\begin{array}{lrrrrrrrrr}581 & 575 & 512 & 560 & 595 & 544 & 573 & 572 & 603 & 619 \\ 546 & 587 & 500 & 500 & 523 & 500 & 514 & 549 & 490 & 530\end{array}$ $\begin{array}{llllllllll}536 & 476 & 500 & 515 & 517 & 400 & 514 & 549 & 490 & 530 \\ 484 & 461 & 517 & 518 & 500 & 474 & 472 & 550 & 503 & 520\end{array}$ $\begin{array}{llllllllll}484 & 461 & 517 & 518 & 500 & 474 & 472 & 483 & 539 & 518 \\ 471 & 560 & 507 & 537 & 489 & 477 & 423 & 445 & 375 & 435\end{array}$ $\begin{array}{llllllllll}431 & 428 & 409 & 408 & 414 & 390 & 430 & 383 & 430 & 375 \\ 362 & 421 & 366 & 392 & 391 & 417 & 409 & 371 & 412 & 397\end{array}$ $\begin{array}{llllllllll}344 & 355 & 368 & 336 & 375 & 370 & 365 & 359 & 307 & 304 \\ 343 & 343 & 353 & 381 & 375 & 392 & 329 & 309 & 296 & 317\end{array}$ $\begin{array}{llllllllll}314 & 314 & 311 & 271 & 280 & 314 & 309 & 309 & 296 & 317\end{array}$ $\begin{array}{llllllllll}314 & 314 & 111 & 271 & 280 & 314 & 309 & 274 & 293 & 257 \\ 260 & 263 & 229 & 305 & 251 & 239 & 264 & 265 & 254 & 266\end{array}$ 
GAMMA RAY SPECTRUM DATA

PRTR ROD FO-79 SPECTRUM NO. 8 DATA TAKEN 2/27/69 LIVE TIME 310.MIN. POSITION 30.IN. ENERGY(KEV) $=1.112 *$ CHAN -O.3 CHAN COUNT COUNT COUNT COUNT COUNT COUNT COUNT COUNT COUNT COUNT

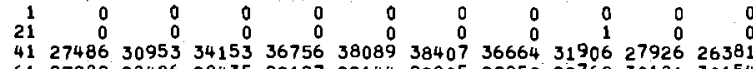
$\begin{array}{lllllllllll}61 & 27982 & 28406 & 28435 & 29197 & 29144 & 29805 & 29852 & 29762 & 30181 & 30154\end{array}$

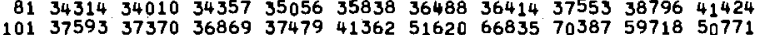

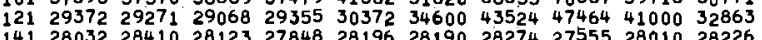

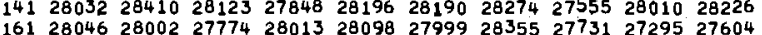
$\begin{array}{lllllllllll}161 & 28046 & 28002 & 27774 & 28013 & 28098 & 27999 & 28355 & 27731 & 27295 & 27604 \\ 181 & 27467 & 27531 & 27139 & 27531 & 26974 & 27139 & 26639 & 26827 & 26955 & 26822\end{array}$ $\begin{array}{llllllllllll}181 & 27467 & 27531 & 27139 & 27531 & 26974 & 27139 & 26639 & 26827 & 26955 & 26822 \\ 201 & 26519 & 26362 & 26578 & 26443 & 26265 & 26402 & 26410 & 26131 & 26234 & 26031\end{array}$

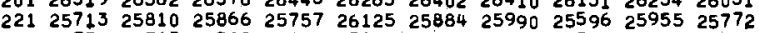

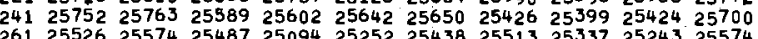
$\begin{array}{llllllllllll}281 & 25561 & 25360 & 25541 & 25432 & 25482 & 25622 & 25571 & 25664 & 25438 & 25542\end{array}$

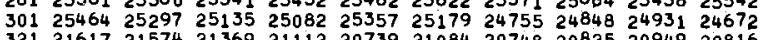
32121617215742136921112207392108420748208252094920816

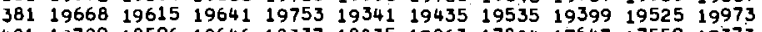
$\begin{array}{llllllllllll}401 & 18729 & 18596 & 18646 & 18337 & 18035 & 17863 & 17804 & 17647 & 17559 & 17373\end{array}$ $\begin{array}{llllllllllll}421 & 17563 & 17456 & 17597 & 17592 & 17538 & 17731 & 16967 & 17047 & 17071 & 16875 \\ 441 & 15505 & 15327 & 15374 & 15175 & 15412 & 14953 & 14983 & 15247 & 15034 & 14943\end{array}$ $4611564015600 \quad 1639418737 \quad 27557 \quad 46475 \quad 71741 \quad 80758 \quad 61494 \quad 33642$ $\begin{array}{lllllllllll}481 & 13360 & 13242 & 13453 & 13364 & 13322 & 13491 & 13223 & 13109 & 13074 & 13086 \\ 501 & 12815 & 12478 & 12516 & 12484 & 12504 & 12239 & 12539 & 12150 & 12253 & 12275\end{array}$

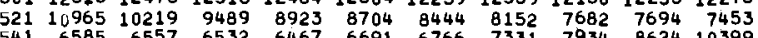
$\begin{array}{lllllllllll}561 & 7518 & 8142 & 8899 & 12061 & 19186 & 28248 & 31950 & 25382 & 14168 & 7214\end{array}$ $\begin{array}{lllllllllll}581 & 4462 & 4133 & 3917 & 3938 & 3913 & 3851 & 3815 & 3805 & 3690 & 3677\end{array}$ $\begin{array}{lllllllllll}621 & 2419 & 2414 & 2347 & 2344 & 2270 & 2348 & 2315 & 2334 & 2399 & 2763\end{array}$ $\begin{array}{rrrrrrrrrrr}661 & 5574 & 3125 & 2315 & 2102 & 2041 & 2064 & 2082 & 2005 & 2159 & 2117 \\ 681 & 2334 & 2494 & 2837 & 3778 & 5709 & 8896 & 12749 & 14842 & 13048 & 8400\end{array}$ $\begin{array}{lllllllllll}701 & 1712 & 1409 & 1384 & 1341 & 1361 & 1383 & 1440 & 1327 & 1322 & 1374\end{array}$ $\begin{array}{lllllllllll}721 & 3101 & 4161 & 5061 & 4634 & 3258 & 2071 & 1635 & 1673 & 1571 & 1522 \\ 741 & 1234 & 1250 & 1189 & 1228 & 1250 & 1215 & 1227 & 1242 & 1235 & 1184\end{array}$ $\begin{array}{lllllllllll}741 & 1234 & 1250 & 1189 & 1228 & 1250 & 1215 & 1227 & 1242 & 1235 & 1184 \\ 761 & 1181 & 1196 & 1181 & 1117 & 1142 & 1110 & 1043 & 1093 & 1053 & 1038\end{array}$

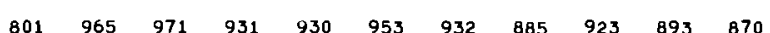

\begin{tabular}{|c|c|c|c|c|c|c|c|c|c|c|}
\hline 801 & 965 & 971 & 931 & 930 & 953 & 932 & 885 & 923 & 893 & 870 \\
\hline & & 158 & 385 & 806 & & 821 & 825 & 880 & 796 & 776 \\
\hline 841 & 30 & 735 & 724 & 674 & 713 & 715 & 670 & 690 & 720 & 731 \\
\hline 861 & 648 & 695 & 604 & 644 & 686 & 689 & 582 & 673 & 655 & 647 \\
\hline 881 & 86 & 561 & 638 & 566 & 585 & & 584 & 572 & 576 & 583 \\
\hline 901 & 557 & 566 & 600 & 602 & 578 & & 522 & 546 & 579 & 592 \\
\hline 921 & 488 & 498 & 523 & 514 & 508 & 508 & 494 & 500 & 542 & 496 \\
\hline 941 & 544 & 553 & 522 & 534 & 54 & 55 & 7 & .941 & 1214 & 1674 \\
\hline 961 & 97 & 464 & 471 & 452 & 476 & 448 & 410 & 450 & 425 & 399 \\
\hline 981 & 883 & 415 & 430 & 425 & 445 & 431 & 4 & 408 & 447 & 442 \\
\hline 001 & 423 & 351 & 414 & 394 & 393 & 431 & 40 & 393 & 448 & 396 \\
\hline & 66 & 805 & 747 & 641 & 649 & & & & 352 & 345 \\
\hline 104 & 334 & 318 & 342 & 305 & 321 & & & & 444 & 439 \\
\hline 061 & 494 & 556 & 619 & 580 & 427 & 383 & 354 & 297 & 333 & 325 \\
\hline 081 & 329 & 28 & 341 & 329 & 299 & 314 & 296 & 277 & 282 & 320 \\
\hline 1101 & 272 & 287 & 278 & 289 & 303 & & & & 275 & 281 \\
\hline 12 & 269 & 312 & 269 & 272 & 285 & & & & 296 & 281 \\
\hline 1141 & 264 & 271 & 249 & 279 & 269 & 260 & 264 & 245 & 282 & 257 \\
\hline 116 & 220 & $25 \overline{6}$ & 215 & 230 & 207 & 250 & 2 & 2 & 244 & 228 \\
\hline 181 & 212 & 221 & 196 & 229 & 188 & 19 & 20 & 28 & 222 & 227 \\
\hline 120 & 242 & 256 & 292 & 363 & 39 & 43 & 3 & 3 & 252 & 220 \\
\hline 1221 & 177 & 182 & 167 & 173 & 174 & 19 & 164 & 195 & 178 & 183 \\
\hline 124 & 177 & 169 & 162 & 165 & 160 & & & 159 & 177 & 176 \\
\hline 126 & 160 & 149 & 121 & 168 & 16 & & & & 163 & 160 \\
\hline 12 & 154 & 126 & 154 & 126 & 1 & & & & 137 & 145 \\
\hline & 144 & 134 & 141 & 138 & 13 & 14 & 1 & 14 & 125 & 139 \\
\hline 13 & 152 & 127 & 13 & 113 & 13 & 14 & 12 & 135 & 129 & 137 \\
\hline $\begin{array}{l}1341 \\
\end{array}$ & 195 & 237 & 246 & 278 & 33 & $\frac{1}{3}$ & & 326 & 245 & $\begin{array}{l}101 \\
192\end{array}$ \\
\hline 136 & 122 & 127 & 130 & 122 & 12 & & & 95 & & 109 \\
\hline 138 & 130 & 125 & 126 & 98 & 11 & 1 & & & 12 & 110 \\
\hline 14 & 99 & 106 & 117 & 122 & 13 & $1=$ & 12 & 14 & 190 & 200 \\
\hline 1421 & 112 & 96 & 114 & 93 & 10 & 11 & 10 & 98 & 96 & 114 \\
\hline 1441 & 107 & 105 & 91 & 105 & 12 & & & & & 118 \\
\hline 146 & 103 & 95 & 115 & 99 & 10 & & 11 & 104 & 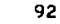 & 105 \\
\hline 14 & 100 & 115 & 105 & 108 & 97 & 111 & 10 & & & 95 \\
\hline 150 & 102 & 94 & 96 & 103 & 130 & & 9 & 1 & 128 & 128 \\
\hline 152 & 113 & 99 & 93 & 89 & 93 & 10 & 11 & $i$ & 111 & 100 \\
\hline 15 & 110 & 85 & 95 & 107 & 109 & & & 1 & 83 & 105 \\
\hline 15 & 115 & 93 & 108 & 100 & 9 & 10 & 10 & & 92 & 83 \\
\hline 158 & 109 & 101 & 87 & 95 & 111 & 109 & 108 & 1 & 96 & 101 \\
\hline 160 & 91 & 70 & 98 & 110 & 10 & 95 & 8 & 113 & 90 & 118 \\
\hline 16 & 98 & 128 & 118 & 111 & 10 & 9 & 10 & 11 & 93 & 90 \\
\hline 1641 & 96 & 107 & 98 & 111 & 10 & 100 & 11 & 1 & 97 & 102 \\
\hline 166 & 110 & 105 & 106 & 109 & 122 & 110 & 104 & 107 & 92 & 99 \\
\hline 168 & 114 & 112 & 107 & 93 & 114 & 86 & 103 & 102 & 93 & 109 \\
\hline 170 & 101 & 106 & 115 & 92 & 10 & 79 & 114 & 131 & 116 & 125 \\
\hline 172 & 119 & 10 & 132 & 123 & 11 & 95 & & 10 & 108 & 108 \\
\hline 174 & 117 & 130 & 142 & 92 & 143 & 109 & 115 & 103 & 114 & 122 \\
\hline 1761 & 100 & 97 & 95 & 72 & 91 & 71 & 69 & 63 & 82 & 70 \\
\hline
\end{tabular}

COUNT COUNT COUNT COUNT COUNT COUNT COUNT COUNT COUNT COUNT

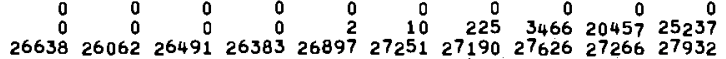

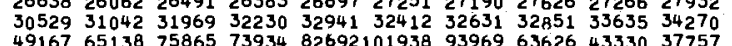

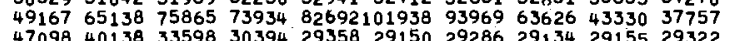

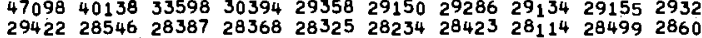
$\begin{array}{llllllllll}28115 & 27511 & 27933 & 28082 & 28220 & 27679 & 28029 & 27949 & 27715 & 2808\end{array}$ $\begin{array}{llllllllll}27219 & 27639 & 27566 & 27519 & 27439 & 27346 & 27608 & 27471 & 27624 & 27623 \\ 27029 & 26620 & 26571 & 26715 & 26654 & 26295 & 26650 & 26478 & 26212 & 26453\end{array}$ $\begin{array}{llllllllll}27029 & 26620 & 26571 & 26715 & 26654 & 26295 & 26650 & 26478 & 26212 & 26453 \\ 26253 & 26024 & 26498 & 25805 & 25751 & 25810 & 25969 & 26104 & 25904 & 25855\end{array}$ $\begin{array}{lllllllllll}26253 & 26024 & 26498 & 25805 & 25751 & 25810 & 25969 & 26104 & 25904 & 25855 \\ 25829 & 25797 & 25758 & 25832 & 25847 & 25796 & 25695 & 25492 & 25823 & 25555\end{array}$

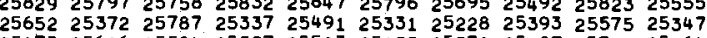
(25584 25397 25503 25233 25370 254872571325464 $\begin{array}{llllllll}25756 & 25524 & 25653 & 25420 & 25790 & 25572 & 25449 & 25578 \\ 25605 & 25496\end{array}$

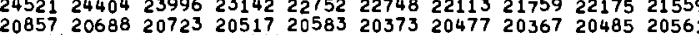
$20067200412050920151 \quad 1987220010199302005620131 \quad 19924$ $\begin{array}{lllllllllll}19656 & 19833 & 19607 & 19603 & 19669 & 19729 & 19356 & 19377 & 19563 & 19528\end{array}$

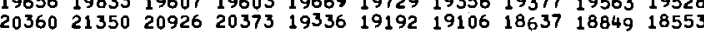
$\begin{array}{llllllllll}17338 & 17355 & 17195 & 17486 & 17294 & 17181 & 17314 & 17178 & 17183 & 17298\end{array}$ $\begin{array}{lllllllllll}17094 & 16858 & 16620 & 16691 & 16229 & 16335 & 16157 & 15775 & 15715 & 15497 \\ 15351 & 15688 & 16731 & 17685 & 17806 & 16745 & 15491 & 15329 & 15167 & 15368\end{array}$ $\begin{array}{lllllllllll}18680 & 14409 & 13685 & 13641 & 134 & 12 & 13412 & 13498 & 13379 & 13695 & 13350\end{array}$ $\begin{array}{llllllllll}12951 & 12733 & 12784 & 12615 & 12899 & 12773 & 12680 & 12800 & 12542 & 12672\end{array}$ $\begin{array}{rrrrrrrrrr}12122 & 12008 & 12240 & 12388 & 12032 & 11724 & 11520 & 11603 & 11764 & 11356 \\ 7396 & 7302 & 7113 & 7211 & 6950 & 6793 & 6767 & 6679 & 6777 & 6776\end{array}$ $\begin{array}{rrrrrrrrrr}7396 & 7302 & 7113 & 7211 & 6950 & 6793 & 6767 & 6679 & 6777 & 6776 \\ 11911 & 11284 & 8956 & 6916 & 6221 & 5721 & 5885 & 5983 & 6182 & 6827\end{array}$ $\begin{array}{lllllllllll}5030 & 4640 & 4471 & 4346 & 4411 & 4391 & 4453 & 4540 & 4589 & 4597\end{array}$ $\begin{array}{llllllllll}3739 & 3562 & 3561 & 3707 & 3698 & 3884 & 4034 & 5080 & 7614 & 13575 \\ 2614 & 2616 & 2540 & 2532 & 2570 & 2396 & 2445 & 2375 & 2433 & 2405\end{array}$ $\begin{array}{llllllllll}3181 & 4124 & 5208 & 5723 & 4898 & 3312 & 2536 & 2181 & 2113 & 2180 \\ 2297 & 2404 & 2426 & 2711 & 3607 & 5445 & 8522 & 11899 & 2140 & 9930\end{array}$ $\begin{array}{lllllllll}2166 & 2206 & 2202 & 2115 & 2218 & 2232 & 2213 & 2261 & 2355\end{array}$ $\begin{array}{lllllllllll}5733 & 7380 & 13761 & 25543 & 40177 & 50296 & 44447 & 26279 & 10094 & 3308\end{array}$ $\begin{array}{llllllllll}1347 & 1318 & 1360 & 1331 & 1397 & 1353 & 1406 & 1548 & 1693 & 2280 \\ 1474 & 1240 & 1232 & 1260 & 1304 & 1205 & 1244 & 1260 & 1231 & 1249\end{array}$ $\begin{array}{lllllllllll}1474 & 1240 & 1232 & 1260 & 1304 & 1205 & 1244 & 1260 & 1231 & 1249 \\ 1132 & 1204 & 1169 & 1196 & 1235 & 1227 & 1205 & 1209 & 1321 & 1158\end{array}$ $\begin{array}{rrrrrrrrrr}1020 & 1004 & 1057 & 949 & 992 & 979 & 963 & 1020 & 977 & 972 \\ 1383 & 1640 & 1779 & 1689 & 1366 & 1010 & 942 & 898 & 935 & 863\end{array}$

\begin{tabular}{|c|c|c|c|c|c|c|c|c|c|}
\hline $\begin{array}{l}873 \\
800 \\
760 \\
638 \\
603 \\
632 \\
492\end{array}$ & $\begin{array}{l}831 \\
813 \\
659 \\
658 \\
613 \\
574 \\
492\end{array}$ & $\begin{array}{l}911 \\
818 \\
679 \\
582 \\
561 \\
550 \\
503\end{array}$ & $\begin{array}{l}877 \\
778 \\
718 \\
596 \\
561 \\
555 \\
495\end{array}$ & $\begin{array}{l}880 \\
746 \\
653 \\
669 \\
549 \\
497 \\
477\end{array}$ & $\begin{array}{l}836 \\
760 \\
649 \\
634 \\
579 \\
504 \\
529\end{array}$ & $\begin{array}{l}820 \\
741 \\
644 \\
627 \\
529 \\
502 \\
501\end{array}$ & $\begin{array}{l}871 \\
778 \\
662 \\
636 \\
562 \\
525 \\
517\end{array}$ & $\begin{array}{l}843 \\
744 \\
674 \\
600 \\
543 \\
546 \\
526\end{array}$ & $\begin{array}{l}844 \\
769 \\
646 \\
590 \\
551 \\
534 \\
480\end{array}$ \\
\hline 2255 & 2517 & 2419 & 1633 & 964 & 619 & 496 & 465 & 440 & 447 \\
\hline $\begin{array}{l}434 \\
423\end{array}$ & $\begin{array}{l}421 \\
398\end{array}$ & & & & & $\begin{array}{l}441 \\
414\end{array}$ & $\begin{array}{l}377 \\
443\end{array}$ & & $\begin{array}{l}429 \\
416\end{array}$ \\
\hline 404 & 372 & 387 & 418 & 378 & 414 & 426 & 473 & & 662 \\
\hline $\begin{array}{l}357 \\
501\end{array}$ & $\begin{array}{l}342 \\
581\end{array}$ & $\begin{array}{l}350 \\
607\end{array}$ & $\begin{array}{l}344 \\
680\end{array}$ & & & & $\begin{array}{r}372 \\
454\end{array}$ & & $\begin{array}{l}343 \\
460\end{array}$ \\
\hline 287 & 353 & 319 & 315 & 296 & & & 33 & & 341 \\
\hline 289 & 300 & 29 & 33 & 308 & & 28 & 28 & & \\
\hline & 27 & & & & & & 25 & & \\
\hline 264 & 274 & 27 & 25 & 28 & 18 & & 273 & & 300 \\
\hline 295 & 333 & 28 & 29 & 29 & & 24 & 21 & & 231 \\
\hline 220 & 203 & 21 & 21 & 21 & & 21 & 22 & & 212 \\
\hline 198 & 19 & 2 & 1 & & & & 22 & 24 & 233 \\
\hline 190 & 188 & 206 & 18 & 18. & 2 & 22 & 174 & 19 & 169 \\
\hline 20 & 163 & 214 & 20 & 215 & & 22 & 17 & 19 & 161 \\
\hline 17 & 155 & 175 & 175 & 168 & 186 & 15 & 16 & 16 & 131 \\
\hline 17 & 133 & 12 & & 14 & & & 14 & & \\
\hline & 144 & & & & & & & & 142 \\
\hline 131 & 133 & 15 & 147 & 15 & 14 & 12 & 12 & 14 & 154 \\
\hline 153 & 105 & 13 & 137 & 13 & & 13 & 16 & & 153 \\
\hline 138 & 164 & 14 & 14 & 13 & & & 13 & & 141 \\
\hline 113 & 142 & 10 & 11 & 11 & 9 & & & & 127 \\
\hline & 102 & ic & 11 & & & & 12 & 12 & 123 \\
\hline 228 & 224 & 20 & 18 & 16 & 10 & 11 & 10 & 12 & 114 \\
\hline 97 & 99 & & 91 & 10 & 10 & & 9 & & 85 \\
\hline 10 & 93 & & & & & & 11 & & 124 \\
\hline 109 & 85 & & 9 & 101 & & & 9 & & 97 \\
\hline 97 & 100 & & 10 & 93 & 11 & & 9 & & 99 \\
\hline 127 & 122 & & & & & & & & 114 \\
\hline 9 & 9 & & 9 & & & & & & 102 \\
\hline 8 & 98 & 10 & 9 & 10 & 12 & & 10 & & 111 \\
\hline 89 & 102 & & & & 10 & & & & 105 \\
\hline 129 & $\begin{array}{l}107 \\
\end{array}$ & 109 & 124 & 114 & 11 & 12 & 12 & 11 & 84 \\
\hline 108 & 91 & 88 & 121 & 98 & 10 & 14 & 11 & 12 & 108 \\
\hline & & & & & & & 10 & & 122 \\
\hline 110 & 114 & 9 & 89 & & 10 & 10 & 9 & & 122 \\
\hline 111 & 97 & 110 & 115 & & & & 8 & & 116 \\
\hline 93 & 107 & & & & & & & & 112 \\
\hline 97 & & & & & & & 10 & & 125 \\
\hline 110 & 126 & 10 & 128 & 131 & 122 & 132 & 104 & 130 & 128 \\
\hline 122 & 111 & 125 & 114 & 124 & 97 & 107 & 111 & 119 & 105 \\
\hline 71 & 52 & 66 & 66 & 64 & 54 & 60 & 81 & 58 & \\
\hline
\end{tabular}

\begin{tabular}{|c|c|c|c|c|c|c|c|c|c|c|c|c|c|c|c|c|c|c|c|c|}
\hline 1781 & 64 & 54 & 65 & 63 & 49 & 52 & 59 & 46 & 54 & 62 & 55 & 61 & 66 & 61 & 76 & 50 & 64 & 73 & 56 & 45 \\
\hline 1801 & 48 & 44 & 57 & 59 & 44 & 48 & 56 & 35 & 38 & 48 & 49 & 55 & 51 & 40 & 60 & 38 & 34 & 49 & 47 & 64 \\
\hline 1821 & 41 & 51 & 41 & 51 & 49 & 34 & 36 & 40 & 41 & 45 & 47 & 39 & 47 & 49 & 36 & 36 & 36 & 39 & 28 & 24 \\
\hline 1841 & 40 & 47 & 33 & 39 & 35 & 32 & 42 & 37 & 39 & 34 & 33 & 26 & 32 & 30 & 41 & 31 & 20 & 28 & 24 & 21 \\
\hline 1861 & 17 & 27 & 22 & 32 & 29 & 25 & 22 & 20 & 25 & 16 & 28 & 22 & 22 & 17 & 19 & 21 & 24 & 23 & 14 & 22 \\
\hline 1881 & 18 & 21 & 17 & 18 & 15 & 13 & 17 & 12 & 19 & 16 & 17 & 20 & 6 & 12 & 15 & 19 & 20 & 21 & 26 & 25 \\
\hline 1901 & 18 & 24 & 33 & 19 & 31 & 34 & 28 & 36 & 27 & 19 & 18 & 31 & 5 & 10 & 11 & 22 & 17 & 20 & 14 & 10 \\
\hline 1921 & 12 & 10 & 21 & 10 & 15 & 20 & 15 & 15 & 14 & 11 & 20 & 14 & 18 & 18 & 15 & 14 & 18 & 18 & 22 & 13 \\
\hline 1941 & 19 & 12 & 11 & 10 & 18 & 15 & 17 & 13 & 11 & 11 & 18 & 13 & 23 & 15 & 17 & 19 & 26 & 21 & 19 & 23 \\
\hline 1961 & 35 & 47 & 63 & 86 & 105 & 146 & 187 & 213 & 206 & 290 & 316 & 317 & 338 & 215 & 131 & 40 & 25 & 14 & 11 & 13 \\
\hline 1981 & 11 & 4 & 6 & 6 & 6 & 6 & 10 & 4 & 7 & 14 & 8 & 8 & 14 & 8 & 5 & 4 & 5 & 8 & 7 & 4 \\
\hline 2001 & 5 & 5 & 8 & 4 & 9 & 7 & 5 & 6 & 5 & 10 & 3 & 3 & 6 & 6 & 9 & 3 & 5 & 5 & 8 & 9 \\
\hline 2021 & 4 & 6 & 4 & 4 & 3 & 7 & 5 & 4 & 2 & 8 & 6 & 8 & 4 & 5 & 5 & 3 & 4 & 6 & 5 & 5 \\
\hline 2041 & 4 & 6 & 6 & 5 & 6 & 4 & 7 & 0 & & & & & & & & & & & & \\
\hline
\end{tabular}




\section{D-10}

GAMMA RAY SPECTRUM DATA

PRTR ROO FE-77 SPECTRUM NO, 7 DATA TAKEN 2/27/69 LIVE TIME 960.MIN. POSITION 30.IN. ENERGY(KEVI= 1.110*AHAN -7.3

CHAN COUNT COUNT COUNT COUNT COUNT COUNT COUNT COUNT COUNT COUNT

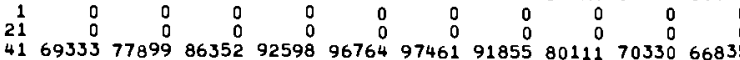

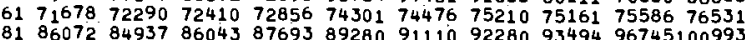
10193858938579286793084991331204651579889349496745100993 $12173568 \quad 736567336574203 \quad 751428301010167411589010565985502$ $14171343710917055270816706547002470708 \quad 704667096270456$ $\begin{array}{llllllllllll}161 & 70275 & 70032 & 69859 & 69714 & 70970 & 70949 & 70580 & 70251 & 69586 & 69588\end{array}$

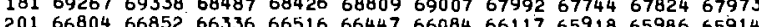

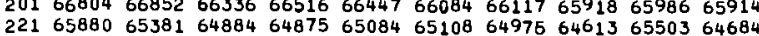
24164294653816488464875650846510864975646136550364684 $2616428164445 \quad 64470 \quad 6440464096 \quad 644516427863916 \quad 6428964327$ 28164880643746459264489647186436164161644036469465132 $32154540 \quad 54361 \quad 54518 \quad 5409453892 \quad 53641 \quad 53370 \quad 529935342953180$

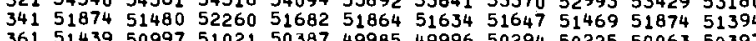
$\begin{array}{lllllllllll}361 & 51439 & 50997 & 51021 & 50387 & 49985 & 49996 & 50294 & 50225 & 50063 & 50397\end{array}$ $\begin{array}{lllllllllll}381 & 50116 & 49688 & 49991 & 49693 & 49855 & 50115 & 49937 & 49219 & 49659 & 50272\end{array}$ $\begin{array}{llllllllllll}401 & 47741 & 47490 & 47407 & 46179 & 46218 & 45792 & 45582 & 44897 & 45501 & 44849 \\ 421 & 44237 & 44863 & 45165 & 45224 & 45542 & 44913 & 44233 & 43764 & 43682 & 43602\end{array}$

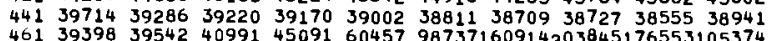
$\begin{array}{llllllllllll}481 & 34702 & 33975 & 34521 & 34445 & 34296 & 34035 & 34027 & 33882 & 33665 & 33448 \\ 501 & 32677 & 32205 & 32123 & 32232 & 32095 & 31933 & 31714 & 31483 & 31464 & 31075\end{array}$ $\begin{array}{lllllllllll}501 & 32677 & 32205 & 32123 & 32232 & 32095 & 31933 & 31714 & 31483 & 31464 & 31075 \\ 521 & 27859 & 26144 & 25041 & 23616 & 2288 & 21733 & 21100 & 20441 & 19513 & 19397\end{array}$

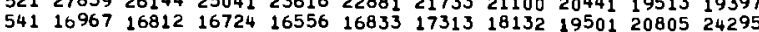
$\begin{array}{lllllllllll}561 & 18496 & 20136 & 21604 & 26507 & 40628 & 63117 & 80447 & 72453 & 45726 & 22672\end{array}$

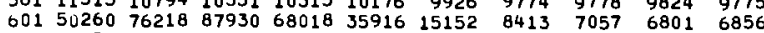
$\begin{array}{llllllllllll}621 & 6235 & 6146 & 6061 & 6017 & 6129 & 6149 & 5999 & 6018 & 6115 & 6612\end{array}$ $\begin{array}{lllllllllll}1 / 1 & 5873 & 5680 & 5621 & 5586 & 5731 & 5683 & 5822 & 5706 & 5670 & 5962\end{array}$ $\begin{array}{llllllllllll}661 & 18738 & 10041 & 6292 & 5532 & 5432 & 5357 & 5358 & 5400 & 5543 & 5378\end{array}$ $\begin{array}{lllllllllll}681 & 6191 & 6286 & 6740 & 6375 & 12298 & 19289 & 29172 & 37466 & 36795 & 26293\end{array}$ $\begin{array}{lllllllllll}701 & 5280 & 3677 & 3548 & 3514 & 3425 & 3419 & 3474 & 3471 & 3451 & 3440\end{array}$ $\begin{array}{llllllllllll}721 & 6501 & 8974 & 11688 & 11923 & 9564 & 6348 & 4467 & 4078 & 4226 & 4002 \\ 741 & 3185 & 3197 & 3272 & 3311 & 3281 & 3225 & 3188 & 3132 & 3187 & 3153\end{array}$ $\begin{array}{lllllllllll}761 & 3087 & 2971 & 2957 & 2982 & 2879 & 2826 & 2818 & 2788 & 2742 & 2742 \\ 781 & 2527 & 2411 & 2510 & 2582 & 2492 & 2522 & 2411 & 2516 & 2703 & 2985\end{array}$

\begin{tabular}{|c|c|c|c|c|c|c|c|c|c|c|}
\hline 801 & 2365 & 2455 & 2416 & 2445 & 2456 & 2299 & 2281 & 2330 & 2313 & 2227 \\
\hline & & 219 & & 199 & 2209 & 2162 & 2269 & 2053 & 2133 & 2112 \\
\hline 841 & 1890 & 1862 & 1855 & 1863 & 1919 & 1835 & 1810 & 1864 & 1879 & \\
\hline 861 & 1824 & 1720 & 1713 & 1699 & 1747 & 1799 & 1795 & 1682 & 1646 & 1654 \\
\hline 881 & 1549 & 1452 & 1518 & 1561 & 1535 & 1554 & 1513 & 1502 & 1543 & \\
\hline & 1465 & 1449 & 1503 & 1485 & 1508 & 1498 & 1446 & $\begin{array}{l}1479 \\
\end{array}$ & 1453 & 1478 \\
\hline 921 & 1330 & 1359 & 1316 & 1331 & 1292 & 1247 & 1306 & 1300 & 1352 & \\
\hline & 1407 & 1410 & 1320 & 1349 & 1365 & 1485 & 1696 & 1952 & 2793 & \\
\hline & 1168 & 1209 & 1254 & 1171 & 1158 & 1162 & 1145 & 1115 & 1161 & \\
\hline & 1046 & 1129 & 1090 & 1159 & 1097 & 1088 & 1099 & 1153 & $\begin{array}{l}1089 \\
1089\end{array}$ & \\
\hline & 1023 & 1067 & 1065 & 1073 & 1090 & 1092 & 1043 & 1090 & $\begin{array}{l}1151 \\
\text {. }\end{array}$ & 114 \\
\hline & 1864 & 2087 & 2192 & & 1411 & & 977 & & 942 & \\
\hline & 857 & 936 & 830 & 867 & 860 & 872 & & 859 & 972 & \\
\hline & 1300 & 1479 & 1637 & 1624 & 1326 & 1011 & 884 & 864 & 846 & \\
\hline & 955 & 927 & 948 & 863 & 866 & & & & & \\
\hline & 77 & 73 & 744 & & & & 75 & 70 & 723 & \\
\hline & 735 & 730 & 719 & 743 & 757 & 695 & 737 & 702 & 659 & \\
\hline 41 & 680 & 706 & 735 & 659 & 683 & 643 & 682 & 669 & 658 & \\
\hline & & 56 & 542 & & & & & & 601 & \\
\hline & 571 & 544 & 516 & 576 & 599 & 544 & 566 & 582 & 541 & \\
\hline 20 & 659 & 629 & 743 & 900 & 1011 & 1203 & 1203 & 1055 & 761 & \\
\hline & 447 & 413 & 488 & 484 & & 449 & & & & \\
\hline 4 & 435 & 404 & 431 & 394 & 390 & 410 & 396 & 393 & 452 & \\
\hline & 37 & 407 & 409 & 42 & & 359 & & & & \\
\hline & 33 & 35 & 35 & & & 40 & 35 & & & \\
\hline & 375 & 332 & 359 & 367 & 344 & 349 & 356 & & 35 & \\
\hline 1321 & 353 & 362 & 319 & 34 & 36 & 337 & 362 & 315 & 337 & \\
\hline & 438 & & & & & & 1003 & & & \\
\hline & 34 & & & & & & 31 & & & \\
\hline & 325 & 29 & 2 & & 33 & 29 & 28 & & 302 & \\
\hline .402 & 287 & 30 & 25 & 322 & 319 & 32 & & & 43 & \\
\hline 1421 & 284 & & & & & & & & & \\
\hline & 270 & & & & & & & & & \\
\hline & 298 & & & & & & & & & \\
\hline & 24 & 29 & & 26 & & 22 & 26 & & 25 & \\
\hline 50 & $26^{\circ}$ & 30 & 2 & & & & 2 & & 30 & \\
\hline 52 & 28 & 27 & 2 & & & & & & & \\
\hline 154 & 28 & & & & & & & & & \\
\hline & 268 & 258 & 288 & 249 & 25 & 2 & 25 & 3 & 28 & \\
\hline & 27 & & & & & & & & & \\
\hline 60 & 245 & & & & & & & & & \\
\hline 1621 & 30 & & & & & & & & & \\
\hline 60 & 280 & 265 & 275 & 260 & & 265 & 28 & 25 & 251 & \\
\hline & 257 & 30 & 262 & 295 & & 2 & & & & \\
\hline & 281 & 28 & 28 & 28 & 2 & & & & & \\
\hline 01 & 302 & 299 & 30 & 305 & 303 & 270 & 28 & 30 & 286 & \\
\hline 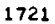 & 289 & 294 & 287 & 292 & 269 & 281 & 294 & 300 & 310 & \\
\hline & 330 & 279 & 300 & 308 & & & 27 & & 337 & \\
\hline & $=7$ & & & & & & & & & \\
\hline
\end{tabular}

$\begin{array}{rrrrrrrrrrr}1781 & 128 & 154 & 138 & 137 & 135 & 160 & 145 & 142 & 138 & 137 \\ 1801 & 125 & 132 & 100 & 115 & 120 & 134 & 132 & 92 & 132 & 121 \\ 1821 & 107 & 108 & 126 & 94 & 115 & 111 & 120 & 106 & 137 & 98 \\ 1841 & 93 & 93 & 115 & 71 & 99 & 104 & 94 & 91 & 72 & 85 \\ 1861 & 71 & 70 & 60 & 58 & 54 & 75 & 77 & 56 & 50 & 69 \\ 1881 & 42 & 56 & 45 & 43 & 45 & 54 & 45 & 35 & 49 & 49 \\ 1901 & 58 & 63 & 59 & 68 & 75 & 79 & 88 & 82 & 74 & 49 \\ 1921 & 42 & 45 & 40 & 45 & 40 & 37 & 36 & 41 & 35 & 25 \\ 1941 & 32 & 38 & 46 & 27 & 62 & 38 & 34 & 46 & 46 & 34 \\ 1961 & 89 & 94 & 147 & 157 & 237 & 295 & 365 & 425 & 523 & 640 \\ 1981 & 20 & 24 & 21 & 20 & 23 & 18 & 19 & 15 & 22 & 16 \\ 2001 & 16 & 11 & 15 & 12 & 20 & 23 & 16 & 7 & 9 & 18 \\ 2021 & 16 & 19 & 22 & 20 & 12 & 20 & 15 & 12 & 16 & 21 \\ 2041 & 12 & 10 & 19 & 11 & 13 & 19 & 13 & 0 & & \end{array}$
COUNT COUNT COUNT COUNT COUNT COUNT COUNT COUNT COUNT COUNT $\begin{array}{rrrrrrrrrr}0 & 0 & 0 & 0 & 0 & 0 & 0 & 0 & 0 & 0 \\ 65907 & 66628 & 674177 & 67461 & 68224 & 25 & 574 & 9080 & 52049 & 62598 \\ 6596 & 69122 & 69314 & 69922 & 70549\end{array}$ $\begin{array}{ll}77113151037195856155148194136242941243874172928 & 8337185599\end{array}$ 1188601045198645377303741557344873655732507392073775 74489715737162971441715217115271069714127196771320 $703937061770536705157029170124 \quad 70098 \quad 70234 \quad 6972269727$ $\begin{array}{llllllllll}69720 & 69428 & 69215 & 69337 & 68946 & 69395 & 69005 & 69629 & 69624 & 68837 \\ 67281 & 67486 & 67091 & 67295 & 67394 & 66470 & 66967 & 66385 & 66035 & 66781\end{array}$

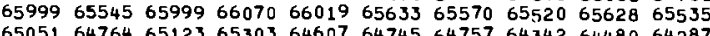
65051647646512365303646076474564757643426448064287 $6476563885642606451164152643046451363826 \quad 6368363986$

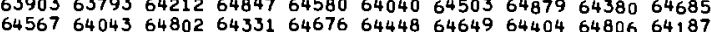

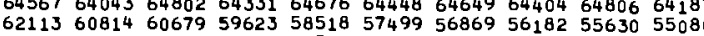

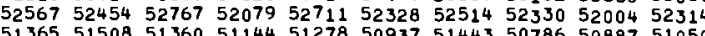
$\begin{array}{lllllllllll}51365 & 51508 & 51360 & 51144 & 51278 & 50937 & 51443 & 50786 & 50887 & 51050 \\ 50512 & 49986 & 50112 & 50513 & 50144 & 49834 & 49768 & 49933 & 50137 & 49683\end{array}$ $51695 \quad 53835 \quad 542985249750561 \quad 49242 \quad 48663 \quad 48702 \quad 47931 \quad 48252$ $\begin{array}{llllllllll}44629 & 44602 & 44644 & 44589 & 44350 & 43875 & 44493 & 44254 & 43930 & 44422 \\ 43747 & 43245 & 43290 & 42486 & 42738 & 41930 & 41584 & 40649 & 40328 & 39434\end{array}$ $\begin{array}{lllllllllll}39182 & 39569 & 41979 & 44817 & 46097 & 44065 & 40927 & 38930 & 39154 & 38488 \\ 55797 & 38762 & 35477 & 34859 & 35176 & 34340 & 34599 & 34604 & 34810 & 34678\end{array}$

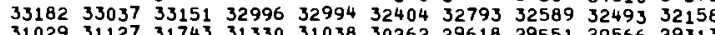

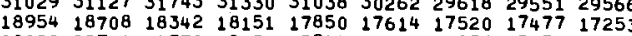
$\begin{array}{lllllllllll}28022 & 28721 & 24779 & 19054 & 15944 & 15118 & 14876 & 15054 & 15412 & 16555\end{array}$

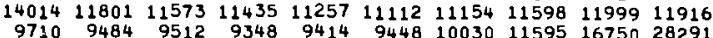
$\begin{array}{lllllllllrr}6730 & 6764 & 6659 & 6478 & 6516 & 6231 & 6317 & 6207 & 6374 & 6040\end{array}$ $\begin{array}{rrrrrrrrrrr}7689 & 9625 & 12644 & 14915 & 13748 & 10412 & 7123 & 5993 & 5748 & 5660 \\ 5890 & 5979 & 6327 & 6723 & 8267 & 12016 & 18697 & 27362 & 33025 & 29515\end{array}$ $\begin{array}{rrrrrrrrrr}5367 & 5470 & 5319 & 5687 & 5752 & 5823 & 5853 & 5829 & 5715 & 5821 \\ 16429 & 15775 & 26983 & 51251 & 87897122220126929 & 89025 & 40178 & 13067\end{array}$ $\begin{array}{llllllllll}3416 & 3499 & 3530 & 3399 & 3448 & 3496 & 3490 & 3684 & 4185 & 4871\end{array}$ $\begin{array}{llllllllll}3130 & 3143 & 3130 & 3157 & 3190 & 3233 & 3155 & 3188 & 3125 & 3199\end{array}$ $\begin{array}{llllllllll}2689 & 2651 & 2576 & 2594 & 2471 & 2533 & 2505 & 2562 & 2544 & 2592 \\ 3299 & 3898 & 4429 & 4387 & 3856 & 3021 & 2580 & 2410 & 2314 & 2431\end{array}$

\begin{tabular}{|c|c|c|c|c|c|c|c|c|c|}
\hline 2243 & 2283 & 2238 & 2275 & 2241 & 2154 & 2229 & 2230 & 2240 & 2217 \\
\hline 2024 & 2170 & 2047 & 1960 & 2094 & 2048 & 1965 & 1881 & 1985 & 1887 \\
\hline 878 & 1857 & 1839 & 1789 & 1778 & 1775 & 1709 & 1772 & 1756 & \\
\hline 51 & 1684 & 1659 & 1667 & 1563 & $\begin{array}{l}1650 \\
\end{array}$ & 1612 & 1566 & $\begin{array}{l}1756 \\
1641\end{array}$ & \\
\hline 571 & 1393 & 1450 & 451 & 1458 & 1373 & 1344 & & & \\
\hline 470 & 1492 & 1457 & 1458 & 1393 & 136 & 1364 & 345 & 35 & \\
\hline 334 & 1285 & 133 & 1255 & 1307 & & 5 & & & \\
\hline 01 & 6235 & 658 & 5313 & 3317 & & 1363 & & $\begin{array}{l}157 \\
\end{array}$ & 17 \\
\hline 120 & 1121 & 1078 & 1064 & 1159 & 1050 & 1139 & 1180 & & \\
\hline 086 & 1145 & 1122 & 1089 & 1109 & 10 & 1070 & 1119 & & \\
\hline 134 & 1104 & 1035 & 1049 & 971 & 109 & 11 & 118 & & \\
\hline 873 & 932 & 90 & 905 & 910 & & 905 & & & \\
\hline 216 & 1298 & 1514 & 1709 & 1751 & 1745 & 1428 & 1250 & & 1 \\
\hline 846 & 817 & 825 & 819 & 835 & 803 & 791 & & & \\
\hline 74 & & & & & & & & & \\
\hline 73 & 689 & 73 & 765 & 694 & 74 & & & & \\
\hline 708 & 703 & 716 & 712 & 710 & 73 & 697 & 69 & 14 & \\
\hline 726 & 719 & 78 & 835 & 80 & 71 & 698 & 59 & & \\
\hline 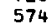 & & & & 52 & & & & 56 & \\
\hline 564 & 572 & 54 & 557 & 554 & 53 & 53 & 59 & 61 & \\
\hline 510 & 499 & 476 & 488 & 461 & 52 & 51 & 46 & & \\
\hline 480 & 543 & 492 & 547 & 571 & 58 & 56 & $\begin{array}{l}40 \\
50\end{array}$ & $\begin{array}{l}45 \\
45\end{array}$ & \\
\hline 439 & 450 & 461 & 474 & 440 & 415 & 43 & 38 & 35 & \\
\hline & 366 & 36 & & & & & & & \\
\hline & 37 & 396 & & & & & & & \\
\hline 31 & 323 & 34 & & 34 & & 35 & 33 & & \\
\hline 31 & & & & & & & & & \\
\hline 37 & & 40 & & & & 35 & & & \\
\hline 30 & & & & 28 & & 33 & & & \\
\hline 30 & 30 & & & 29 & & 31 & 29 & 31 & 2 \\
\hline 53 & 58 & 5 & & 474 & & & & 27 & \\
\hline 24 & & & & & & & & & \\
\hline 290 & 250 & & & & & & & & \\
\hline 27 & 266 & & & 24 & & & 25 & & 25 \\
\hline 275 & & & & & & 26 & 306 & & \\
\hline 333 & 330 & & 332 & 318 & & 27 & 290 & 27 & \\
\hline 25 & 22 & & & & & & & & \\
\hline 25 & & & & & 26 & & & 27 & \\
\hline 256 & 239 & 24 & & 23 & 27 & 25 & 28 & 27 & \\
\hline 248 & 278 & & & & & & & & \\
\hline 25 & 26 & & & & & & & & \\
\hline 283 & & & & & & & 27 & & \\
\hline & 283 & 249 & 233 & 278 & 282 & 261 & 23 & 269 & \\
\hline & 26 & & & 28 & 27 & & & & \\
\hline 31 & 26 & $2 E$ & & & & & & & \\
\hline 27 & 279 & & & & 28 & & 8 & 313 & \\
\hline 29 & 27 & 28 & 286 & 32 & 34 & 338 & 298 & 298 & \\
\hline & & & & & & & & 28 & \\
\hline 17 & & & & & & 3 & 5 & $\begin{array}{l}202 \\
142\end{array}$ & 5 \\
\hline
\end{tabular}

$\begin{array}{rrrrrrrrrr}160 & 137 & 144 & 152 & 162 & 159 & 150 & 163 & 137 & 116 \\ 135 & 133 & 130 & 121 & 129 & 143 & 136 & 126 & 128 & 95 \\ 88 & 103 & 96 & 101 & 85 & 107 & 110 & 84 & 93 & 83 \\ 90 & 85 & 86 & 77 & 80 & 87 & 80 & 71 & 58 & 80 \\ 51 & 58 & 51 & 57 & 41 & 51 & 66 & 63 & 47 & 46 \\ 56 & 31 & 54 & 44 & 54 & 50 & 65 & 51 & 43 & 44 \\ 45 & 47 & 39 & 39 & 42 & 54 & 36 & 41 & 30 & 52 \\ 45 & 38 & 47 & 36 & 32 & 37 & 32 & 41 & 33 & 49 \\ 44 & 34 & 43 & 44 & 39 & 46 & 58 & 47 & 69 & 53 \\ 716 & 799 & 902 & 799 & 520 & 275 & 109 & 50 & 19 & 25 \\ 13 & 15 & 28 & 13 & 14 & 13 & 12 & 12 & 27 & 17 \\ 16 & 16 & 12 & 9 & 17 & 13 & 20 & 17 & 16 & 11 \\ 14 & 20 & 17 & 10 & 20 & 20 & 8 & 18 & 8 & 16\end{array}$




\section{D-11}

GAMMA RAY SPECTRUM DATA

PRTR ROD FS-01 SPECTRUM NO. 25 DATA TAKEN 3/07/69 LIVE TIME 1127.MIN. POSITION 27.IN. ENERGY(KEV)= 1.112*CHAN -7.7 CHAN COUNT COUNT COUNT COUIUT COUNT COUNT COUNT COUNT COUNT COUNT

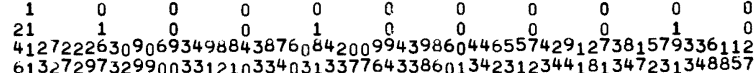
61327297329900331210334031337764338601342312344181347231348857
81393268392456393162395757404211410202417989424217433636449214 813932683924563931623957574042114102024179894242174333636449214
10144795743236542709542584043791748832861669475356764639662436 121345029344856344971345126349458365901422586501466518676449275 1413386443352803319783314913321263317923323 161328978328847329249328176329919331405331767329670328924327194
181327045327215325783323952324047323014322167319851319277319493 $2013137903135953118763114 \pi 73_{13} 3251312370311801311243311570311043$ 22131056430877330813730628030709330669930744330586430777630674 26130520530451130491230374530513230442330423030452930495030398 2813,613830484330513930397630576530562930566530551430642930526 301303089301286300827298859299075298216296177295489294084293112 34125188525212025142525086225102125018524997124893025021325008 361247353246461245668245923245300244699244865244664245766244252 401234367233396233354231967230341228884227526226811225546225268 441214610213420213043213478213930213918215148213765215430216901 461219378220022227743251627329792493416706782796374657081428917 $4012 \mathrm{j} 6602207516206890206681206821205485204064203639202393201520$ 501197879197391197285195330195063195227194968193664194041192270 54193083916119061590714914189338896955105404122175146502

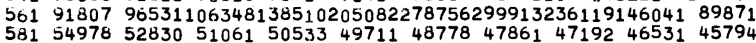
$601205878262284245783164763 \quad 8813149743 \quad 365573299531971 \quad 31640$ $\begin{array}{lllllllllll}641 & 26874 & 26903 & 27030 & 26989 & 26667 & 26881 & 27194 & 27453 & 27641 & 27979\end{array}$ 68131301354954437467106112026176250238510252007197349126031 $70121897 \quad 14559132261260112575 \quad 12476 \quad 12327 \quad 126301229512309$

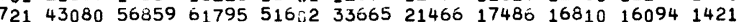

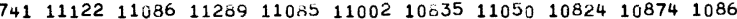

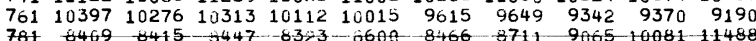
COUNT COUNT COUNT COUNT COUNT COUNT COUNT COUNT COUNT COUNT

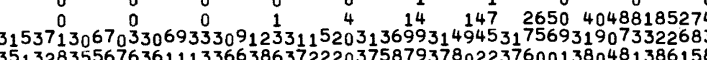
487124590590753796843289851521979758129445994086702508512293 381457348366340940335821335831334373337087339974346580344446 330338330316330001329331329799328529328639327981327962328216 325209325389326768325490325734325383326357326009325387325396 30957331047830977430999030975730877530920130894531018930991 306260305750306432305797306074304643305189305577304548305068 30417230357130498930373130391130374130282430349330534630333 30401230472530546830560030436530434630491830499430566730536 306207304463306547305154305511303671304757304501303542302482 255972253579255002254189253684252923252821252526252412251454 2491482490412488062482412486222473282474472471582479 245025243820244917243678243836244024244446244610244207244137
250173255229255899251027243788238853238166237541237681235186 224870224282223986223642224305224097224182225599224382224391 223401242391278675318391324337289037248161226232220314217134 281929226458211418207665206282206183207052207015207421206491 192246192386193337192271188467183193180691180839179999175377 10641310420210265510067499254982989727395532950389378 $1643201542901244179854986727 \quad 83616 \quad 817328092081526 \quad 85843$ $\begin{array}{rllllllllll}68189 & 61938 & 60533 & 59166 & 58354 & 57761 & 57871 & 57686 & 58030 & 56626 \\ 45565 & 44341 & 44267 & 43274 & 43439 & 43869 & 47617 & 57272 & 82481132859\end{array}$

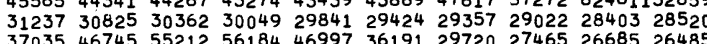
2823729304321754116562230103190161458210610207064146924

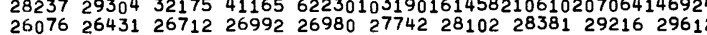
10437815996730578253622777987286147867162135936814040749140 $\begin{array}{lllllllllll}12430 & 12574 & 12660 & 12650 & 12895 & 13061 & 13608 & 15760 & 20277 & 29489\end{array}$ $\begin{array}{llllllllllll}12595 & 11589 & 11451 & 11210 & 11156 & 11258 & 11306 & 11359 & 11381 & 11135\end{array}$ $\begin{array}{llllllllllll}10746 & 10694 & 10878 & 10877 & 10849 & 10647 & 10940 & 10790 & 10827 & 10594\end{array}$

$\begin{array}{lllllllllll}801 & 8849 & 9244 & 9191 & 8788 & 8255 & 7692 & 7576 & 7709 & 7625 & 7668\end{array}$ $\begin{array}{llllllllllll}821 & 7403 & 7319 & 7120 & 7014 & 7067 & 7015 & 7029 & 7056 & 7257 & 7106\end{array}$ $\begin{array}{lllllllllll}841 & 6378 & 6259 & 6140 & 6273 & 6289 & 6226 & 6038 & 6545 & 6605 & 6656 \\ 861 & 5708 & 5733 & 5451 & 5441 & 5646 & 5578 & 5552 & 5536 & 5376 & 5456\end{array}$ $\begin{array}{llllllllllll}881 & 5153 & 5097 & 5140 & 4920 & 4894 & 5022 & 4966 & 5054 & 5062 & 4947\end{array}$ $\begin{array}{lllllllllll}901 & 5144 & 5080 & 5072 & 516.7 & 4903 & 5017 & 4895 & 5075 & 5144 & 5411 \\ 921 & 4516 & 4416 & 4429 & 4509 & 4534 & 4464 & 4476 & 4385 & 4567 & 4360\end{array}$ $\begin{array}{llllllllllll}941 & 4908 & 4836 & 4677 & 4820 & 5157 & 6184 & 7975 & 10533 & 14318 & 18813\end{array}$ $\begin{array}{rllllllllll}941 & 4908 & 4836 & 4677 & 4820 & 5157 & 6184 & 7975 & 10533 & 14318 & 18813 \\ 961 & 4161 & 4120 & 4099 & 3858 & 3714 & 3770 & 3686 & 3767 & 3668 & 3644 \\ 981 & 3687 & 3512 & 3646 & 3536 & 3597 & 3601 & 3665 & 3630 & 3600 & 3582\end{array}$ $\begin{array}{lllllllllll}101 & 3687 & 3512 & 3646 & 3536 & 3597 & 3601 & 3665 & 3630 & 3600 & 3582 \\ 1001 & 3407 & 3525 & 3619 & 3498 & 3546 & 3518 & 3511 & 3647 & 3562 & 3612\end{array}$ $\begin{array}{llllllllllll}1021 & 7244 & 7065 & 5657 & 4308 & 3487 & 3286 & 3134 & 3196 & 3102 & 3051 \\ 1041 & 2990 & 3018 & 3073 & 2930 & 3084 & 3090 & 3214 & 3560 & 3981 & 4473\end{array}$ $\begin{array}{lllllllllll}1061 & 3720 & 3887 & 3073 & 2930 & 3084 & 3090 & 3214 & 3560 & 3981 & 4473 \\ & 3698 & 3277 & 2932 & 2931 & 2898 & 2850 & 3009 & 281\end{array}$ $\begin{array}{lllllllllll}1081 & 3133 & 3132 & 2975 & 2760 & 2717 & 2792 & 2793 & 2825 & 2807 & 2852 \\ 1101 & 2514 & 2568 & 2597 & 2594 & 2600 & 2602 & 2578 & 2536 & 2587 & 2545\end{array}$ $\begin{array}{lllllllllll}1121 & 2429 & 2472 & 2509 & 2514 & 2548 & 2503 & 2521 & 2436 & 2429 & 2474 \\ 1141 & 2408 & 2461 & 2410 & 2336 & 2363 & 2373 & 2491 & 2502 & 2617 & 2888\end{array}$ $\begin{array}{lllllllllll}1161 & 2022 & 2059 & 1939 & 2034 & 2070 & 2052 & 1957 & 2018 & 2063 & 1910\end{array}$ $\begin{array}{llllllllllll}1181 & 1949 & 1901 & 1867 & 1721 & 1948 & 1879 & 1932 & 1918 & 1967 & 1943\end{array}$ $\begin{array}{lllllllllll}1201 & 2085 & 2171 & 2361 & 2470 & 2628 & 2501 & 2112 & 1984 & 1803 & 1708\end{array}$ $\begin{array}{lllllllllll}1241 & 1501 & 1454 & 1364 & 1423 & 1378 & 1516 & 1513 & 1595 & 1607 & 1722 \\ 1261 & 1376 & 1393 & 1340 & 1280 & 1328 & 1389 & 1264 & 1352 & 1323 & 1344\end{array}$ $\begin{array}{lllllllllll}1281 & 1252 & 1327 & 1252 & 1256 & 1201 & 1264 & 1267 & 1213 & 1287 & 1234\end{array}$ $\begin{array}{lllllllllll}13_{11} & 1101 & 1244 & 1197 & 1216 & 1247 & 1212 & 1262 & 1200 & 1150 & 1226 \\ 1321 & 1202 & 1192 & 1172 & 1269 & 1141 & 1177 & 1161 & 1223 & 1185 & 1251\end{array}$ $\begin{array}{lllllllllll}1341 & 2104 & 2382 & 2844 & 3105 & 3218 & 3198 & 2461 & 1903 & 1443 & 1353 \\ 1351 & 1123 & 1113 & 1101 & 1106 & 1052 & 1054 & 1052 & 1155 & 1022 & 1029\end{array}$ $\begin{array}{lllllllllll}1361 & 1123 & 1113 & 1101 & 1106 & 1052 & 1054 & 1052 & 1155 & 1022 & 1029 \\ 1301 & 1030 & 1028 & 1002 & 985 & 1074 & 1008 & 1010 & 1006 & 1017 & 1065\end{array}$ $\begin{array}{rrrrrrrrrrr}1401 & 1032 & 1091 & 1075 & 1179 & 1262 & 1463 & 1557 & 1618 & 1881 & 2004 \\ 1421 & 949 & 967 & 995 & 1006 & 941 & 934 & 915 & 933 & 947 & 904\end{array}$ $\begin{array}{lllllllllll}1441 & 996 & 944 & 988 & 952 & 932 & 990 & 942 & 943 & 907 & 945 \\ 1461 & 889 & 917 & 899 & 936 & 913 & 892 & 907 & 939 & 874 & 911\end{array}$ $\begin{array}{rrrrrrrrrrr}1481 & 903 & 909 & 916 & 907 & 870 & 939 & 959 & 895 & 885 & 925\end{array}$

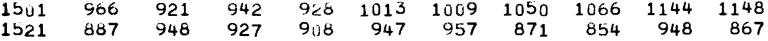

$\begin{array}{lllllllllll}1541 & 924 & 848 & 837 & 870 & 888 & 921 & 923 & 858 & 860 & 854 \\ 1561 & 969 & 371 & 878 & 892 & 852 & 881 & 869 & 880 & 880 & 906\end{array}$ $\begin{array}{lllllllllll}1581 & 904 & 929 & 891 & 874 & 894 & 918 & 986 & 893 & 940 & 940 \\ 1601 & 898 & 870 & 920 & 866 & 858 & 909 & 938 & 922 & 919 & 892\end{array}$ $\begin{array}{rrrrrrrrrrr}1601 & 898 & 870 & 920 & 866 & 858 & 909 & 938 & 922 & 919 & 892 \\ 1621 & 1041 & 1002 & 1043 & 942 & 933 & 935 & 848 & 896 & 881 & 905\end{array}$ $\begin{array}{rrrrrrrrrrr}1661 & 937 & 947 & 931 & 934 & 921 & 948 & 963 & 986 & 944 & 928\end{array}$ $\begin{array}{lllllllllll}1721 & 997 & 1049 & 1044 & 1013 & 1043 & 1026 & 960 & 1054 & 1055 & 1030\end{array}$

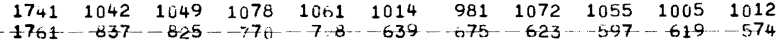

$\begin{array}{lllllllllll}7367 & 7546 & 7467 & 7363 & 7433 & 7291 & 7243 & 7399 & 7416 & 7348\end{array}$ $\begin{array}{llllllllll}6941 & 6828 & 6832 & 6807 & 6725 & 6271 & 6468 & 6286 & 6299 & 6300\end{array}$ $\begin{array}{lllllllllll}5385 & 5458 & 5455 & 5374 & 5232 & 5231 & 5334 & 5262 & 5158 & 5004\end{array}$ $\begin{array}{lllllllll}4993 & 4722 & 4884 & 4815 & 4720 & 4832 & 4831 & 4900 & 496\end{array}$ $\begin{array}{llllllllll}5396 & 5156 & 4807 & 4609 & 4589 & 4642 & 4642 & 4664 & 4664 & 4562 \\ 4467 & 4354 & 4280 & 4440 & 4550 & 4404 & 4506 & 4648 & 4660 & 4875\end{array}$ $\begin{array}{lllllllllll}21656 & 21051 & 10072 & 5974 & 4500 & 3964 & 3974 & 3935 & 3950 & 3893\end{array}$ $\begin{array}{lllllllllll}3610 & 36339 & 3702 & 3658 & 3664 & 3650 & 3575 & 3649 & 3599 & 349 \\ 3531 & 3495 & 3610 & 3351 & 3499 & 3476 & 3394 & 3392 & 3441 & 3422\end{array}$ $\begin{array}{lllllllllll}3461 & 3452 & 3326 & 3529 & 3742 & 3883 & 4492 & 5129 & 5969 & 6833\end{array}$ $\begin{array}{llllllllll}4835 & 5453 & 5947 & 5857 & 5202 & 4336 & 3946 & 3687 & 3726 & 3476\end{array}$ $\begin{array}{llllllllll}2779 & 2773 & 2805 & 2630 & 2911 & 2830 & 3006 & 2963 & 3089 & 3171 \\ 2763 & 2776 & 2635 & 2470 & 2689 & 2595 & 2658 & 2633 & 2613 & 2702\end{array}$ $\begin{array}{llllllllll}2511 & 2393 & 2442 & 2423 & 2417 & 2424 & 2403 & 2444 & 2533 & 2444\end{array}$ $\begin{array}{llllllllll}1934 & 2027 & 1977 & 1898 & 1927 & 1901 & 2004 & 1968 & 1876 & 1956\end{array}$ $\begin{array}{llllllllll}1856 & 1836 & 1847 & 1844 & 1889 & 1862 & 1884 & 1835 & 1967 & 2070\end{array}$ $\begin{array}{lllllllllll}1753 & 1798 & 1701 & 1779 & 1714 & 1746 & 1716 & 1624 & 1586 & 1583 \\ 2008 & 2148 & 2214 & 2254 & 2226 & 1873 & 1624 & 1563 & 1436 & 1524\end{array}$ $\begin{array}{llllllllll}2084 & 1720 & 1586 & 1476 & 1466 & 1443 & 1340 & 1344 & 1404 & 1311 \\ 1315 & 1249 & 1265 & 1262 & 1287 & 1337 & 1291 & 1341 & 1301 & 1255\end{array}$ $\begin{array}{llllllllll}1221 & 1283 & 1289 & 1262 & 1206 & 1271 & 1238 & 1227 & 1243 & 1174\end{array}$ $\begin{array}{llllllllll}1264 & 1250 & 1172 & 1191 & 1147 & 1149 & 1178 & 1186 & 1159 & 1193 \\ 1236 & 1196 & 1185 & 1235 & 1278 & 1234 & 1303 & 1328 & 1640 & 1778\end{array}$ $\begin{array}{llllllllll}1318 & 1272 & 1266 & 1223 & 1179 & 1215 & 1168 & 1225 & 1213 & 1211 \\ 1053 & 1083 & 1085 & 1046 & 1076 & 1011 & 1054 & 1085 & 1100 & 1049\end{array}$ $\begin{array}{rrrrrrrrrr}1053 & 1083 & 1085 & 1046 & 1076 & 1011 & 1054 & 1085 & 1100 & 1049 \\ 983 & 1080 & 1085 & 1019 & 1087 & 1039 & 1084 & 1061 & 1075 & 1033\end{array}$ $\begin{array}{rrrrrrrrrr}983 & 1080 & 1085 & 1019 & 1087 & 1039 & 1084 & 1061 & 1075 & 1033 \\ 1962 & 1681 & 1355 & 1196 & 1024 & 977 & 885 & 954 & 956 & 992\end{array}$ $\begin{array}{llllllllll}970 & 952 & 918 & 969 & 946 & 936 & 960 & 984 & 953 & 943 \\ 925 & 903 & 871 & 879 & 928 & 905 & 895 & 898 & 962 & 922\end{array}$ $\begin{array}{rrrrrrrrrr}918 & 941 & 911 & 910 & 914 & 836 & 921 & 904 & 862 & 957 \\ 895 & 878 & 934 & 858 & 927 & 893 & 909 & 928 & 889 & 934\end{array}$ $\begin{array}{rrrrrrrrrr}895 & 878 & 934 & 858 & 927 & 893 & 909 & 928 & 889 & 934 \\ 1123 & 1212 & 1111 & 1018 & 929 & 901 & 913 & 909 & 887 & 925 \\ 941 & 877 & 869 & 874 & 859 & 883 & 838 & 858 & 912 & 911\end{array}$ $\begin{array}{llllllllll}891 & 868 & 868 & 923 & 899 & 914 & 932 & 858 & 912 & 911 \\ 8 & 940 & 874 & 898 & 919 & 946 & 966 & 886 & 921 & 903\end{array}$ $\begin{array}{rrrrrrrrrr}891 & 940 & 874 & 898 & 919 & 946 & 866 & 886 & 921 & 903 \\ 955 & 1010 & 982 & 1007 & 1048 & 980 & 917 & 873 & 864 & 855\end{array}$ $\begin{array}{rrrrrrrrrr}885 & 932 & 935 & 1007 & 1048 & 980 & 917 & 873 & 864 & 855 \\ 927 & 914 & 899 & 849 & 927 & 934 & 997 & 988 & 1075 & 993\end{array}$ $\begin{array}{llllllllll}927 & 914 & 899 & 849 & 904 & 865 & 919 & 850 & 891 & 928 \\ 892 & 942 & 950 & 911 & 917 & 909 & 965 & 919 & 885 & 922\end{array}$ $\begin{array}{rrrrrrrrrr}998 & 1007 & 955 & 950 & 981 & 1022 & 968 & 1001 & 969 & 993 \\ 990 & 993 & 995 & 1039 & 988 & 920 & 981 & 981 & 967 & 1007\end{array}$ $\begin{array}{rrrrrrrrrr}965 & 1025 & 982 & 1017 & 968 & 1010 & 1034 & 997 & 1105 & 1021\end{array}$ $\begin{array}{rrrrrrrrrr}1040 & 1044 & 1072 & 992 & 1111 & 1090 & 1064 & 1142 & 1055 & 1121 \\ 1021 & 978 & 1034 & 977 & 981 & 993 & 910 & 887 & 913 & 850\end{array}$ $\begin{array}{rrrrrrrrrrr}1781 & 538 & 504 & 477 & 5: 4 & 507 & 481 & 521 & 523 & 511 & 528 \\ 1801 & 417 & 413 & 452 & 4 j 6 & 385 & 431 & 422 & 461 & 422 & 425 \\ 1821 & 390 & 359 & 377 & 342 & 397 & 366 & 339 & 368 & 336 & 388 \\ 1841 & 310 & 321 & 321 & 315 & 319 & 285 & 269 & 272 & 255 & 252 \\ 1801 & 207 & 210 & 235 & 232 & 223 & 203 & 185 & 212 & 181 & 196 \\ 1881 & 147 & 174 & 166 & 171 & 152 & 149 & 157 & 153 & 126 & 165 \\ 1901 & 237 & 259 & 248 & 286 & 274 & 235 & 163 & 17100 & 142 & 127 \\ 1921 & 139 & 132 & 145 & 1>7 & 125 & 120 & 126 & 116 & 140 & 137 \\ 1941 & 130 & 136 & 148 & 112 & 143 & 128 & 134 & 136 & 134 & 134 \\ 1901 & 700 & 889 & 1055 & 1331 & 1676 & 1894 & 2242 & 2549 & 2845 & 2650 \\ 1981 & 58 & 57 & 59 & 64 & 42 & 57 & 68 & 66 & 43 & 58 \\ 2001 & 43 & 44 & 51 & 50 & 49 & 54 & 62 & 41 & 50 & 63 \\ 2021 & 42 & 51 & 55 & 43 & 36 & 44 & 40 & 57 & 42 & 41 \\ 2041 & 33 & 47 & 40 & 43 & 43 & 49 & 41 & 0 & & \end{array}$

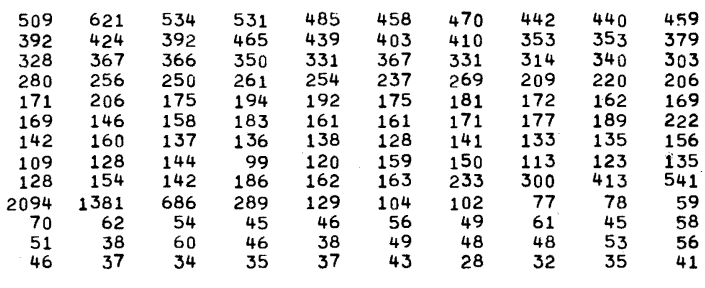


GAMMA RAY SPECTRUM DATA

PHTR HOD FS-01 SPECTRUM NO. 26 OATA TAKEN 3/09/69 LIVE TIME 1192.MIN. POSITION 27.IN. ENERGY(KEVIE 1.109\%CHAN -8.1

CHAN COUNT COUNI COUNT COUNT COUNT COUNT COUNT COUNT COUNT COUNT COUNT COUNT COUNT COUNT COUNT COURT COUNT COUNT COUNT COUNT

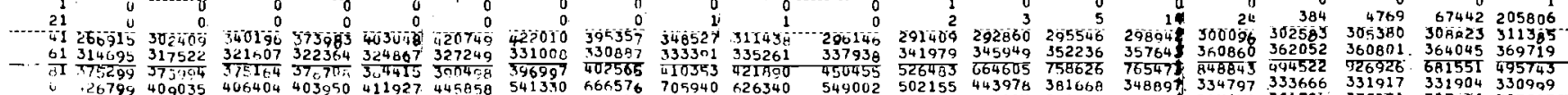

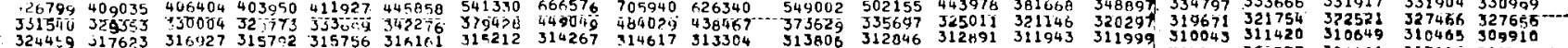

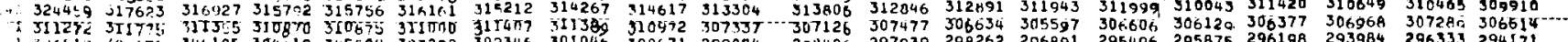

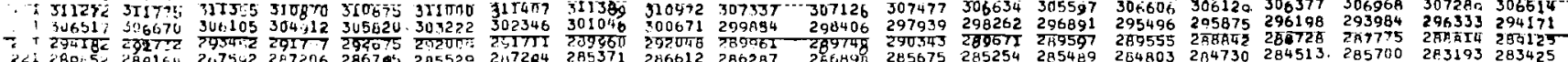

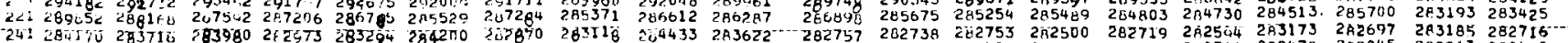

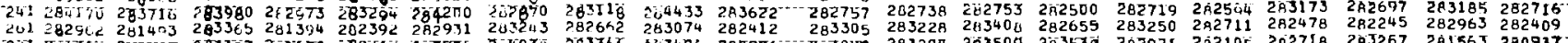

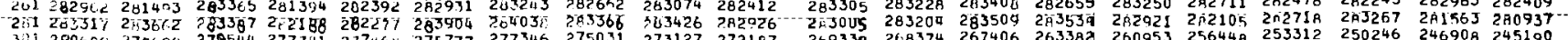

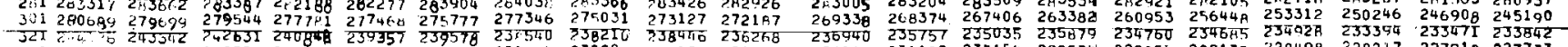

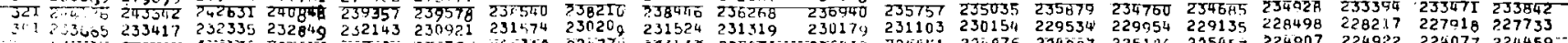

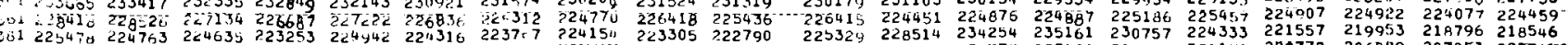

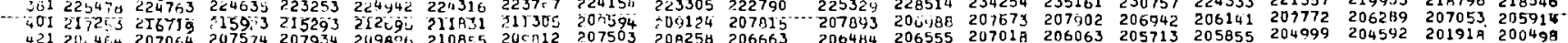

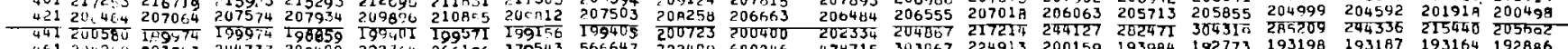

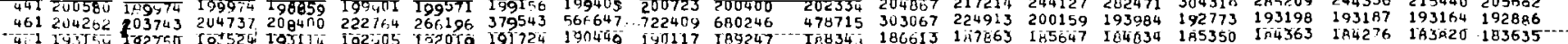

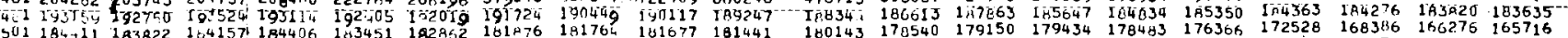

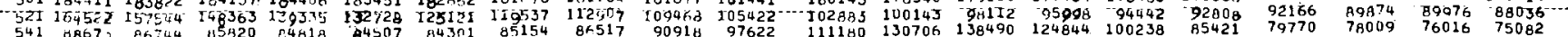

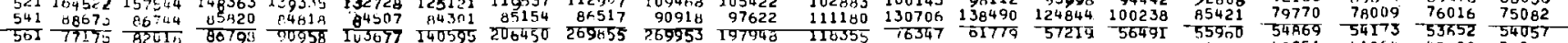

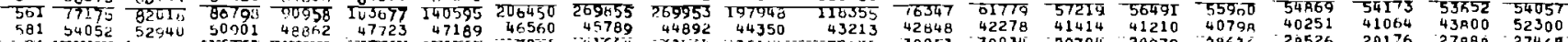

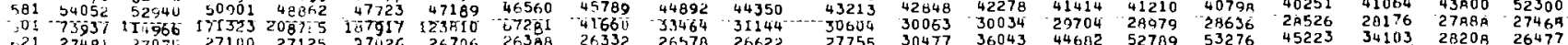

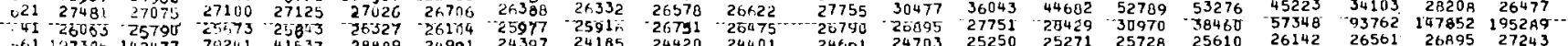

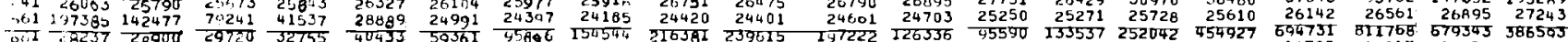

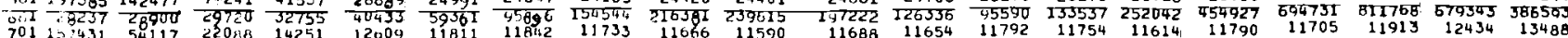

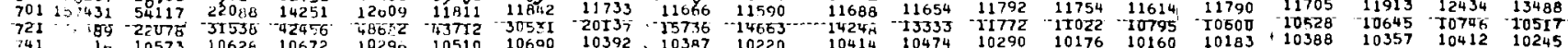

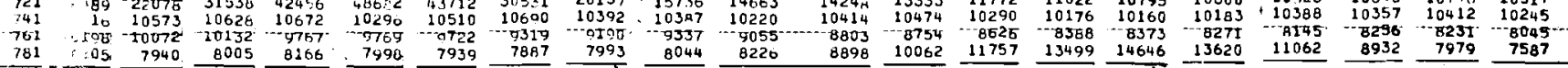

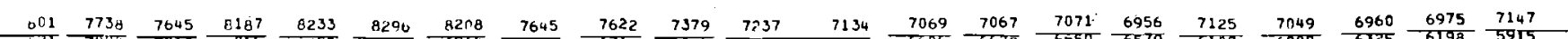

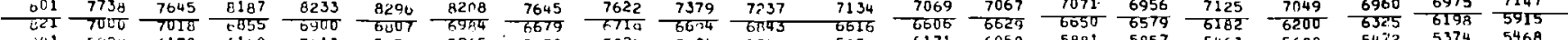

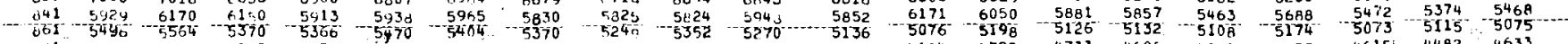

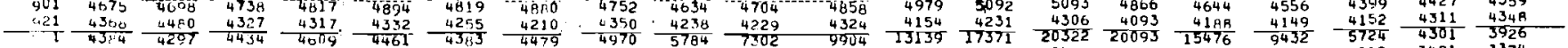

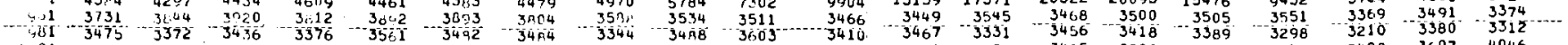

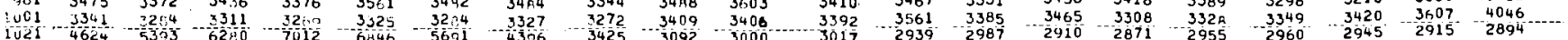

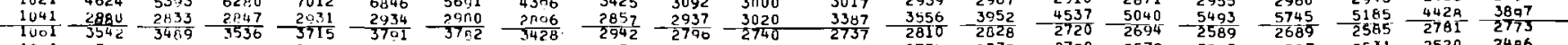

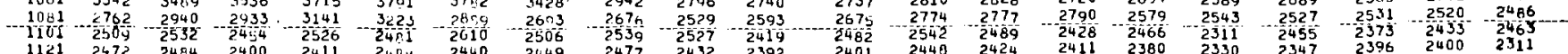

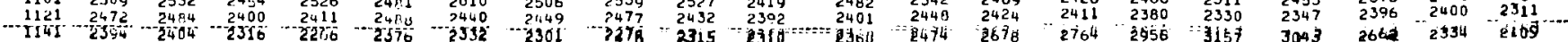
$\frac{1101}{1141} \frac{2010}{1651} \frac{1997}{1875} \frac{1974}{1872} \frac{1946}{1822} \frac{1456}{1561} \frac{1964}{1759} \frac{1908}{1823}$

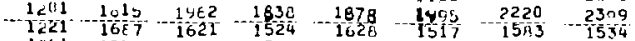

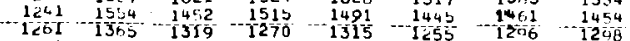
$\frac{122: 1}{1301} \frac{1259}{1172} \frac{1319}{1224} \frac{1210}{1169} \frac{1264}{1191} \frac{1170}{1152} \frac{1279}{1136} \frac{1255}{1157}$ -

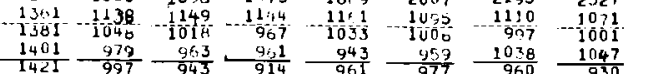

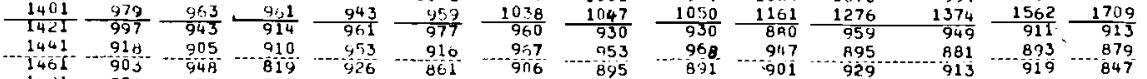

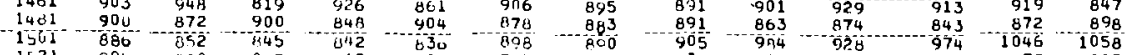

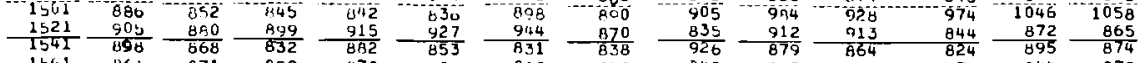

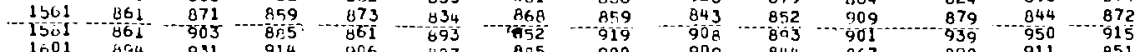

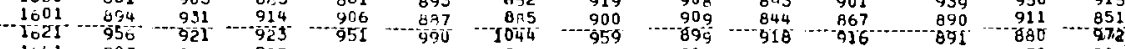

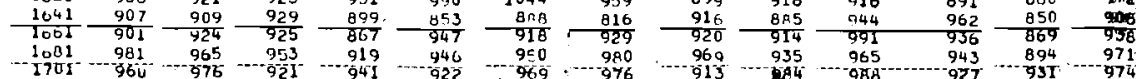

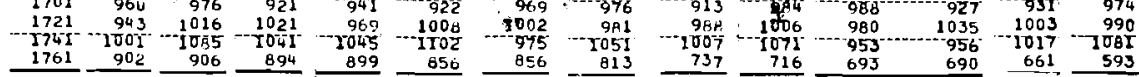

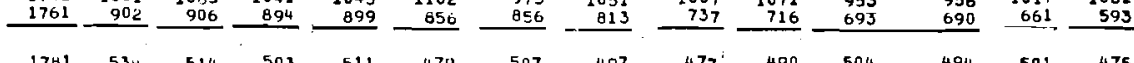

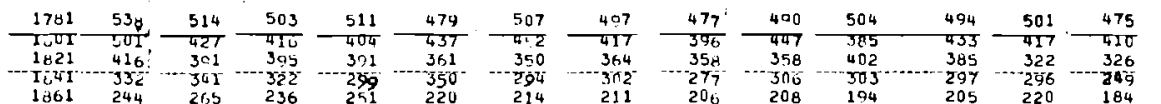

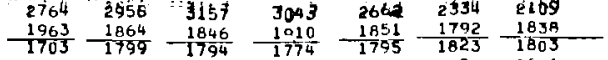

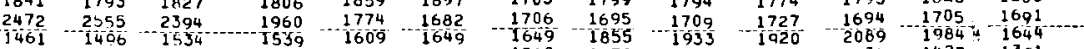

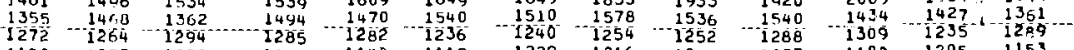
$\frac{1190}{1190}-\frac{1273}{1295}-\frac{1220}{1173}-1192-\frac{1148}{1150}-\frac{1119}{1235}-\frac{1229}{1191}-\frac{1216}{1193}-\frac{1246}{1132}-\frac{1227}{1159}-\frac{1182}{1125} \frac{1205}{1154}-\frac{1153}{1176}$

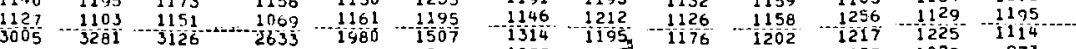

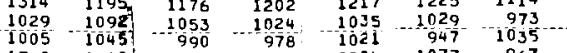
$\frac{1798}{966}-\frac{1913:}{924}-\frac{1836}{878}-\frac{1659}{952}-\frac{1234}{879}-\frac{1077}{932} \frac{967}{889}$

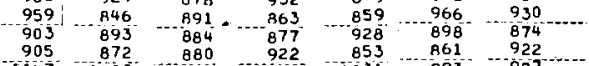

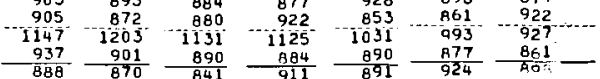

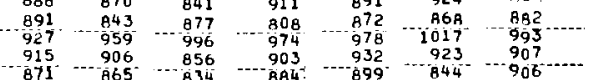

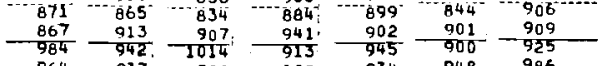

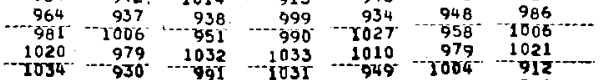

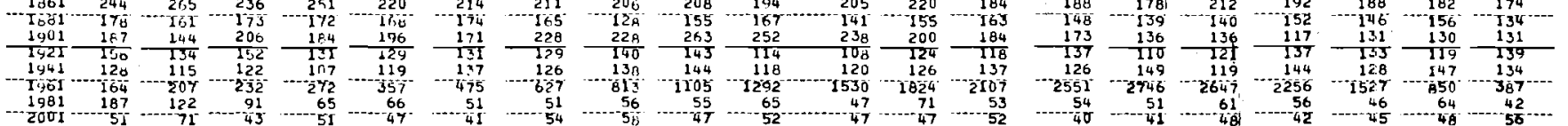

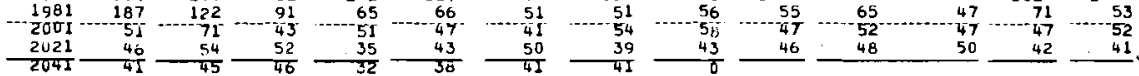

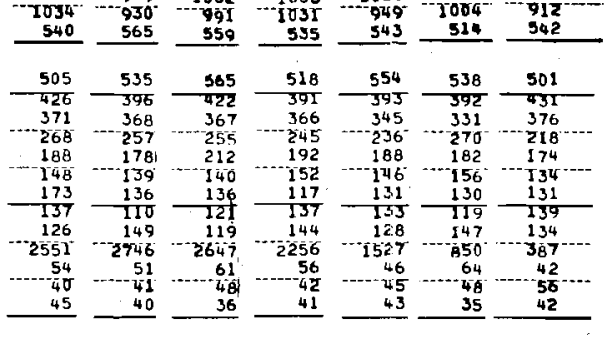




\section{D-13}

GAMMA RAY SPECTRUM DATA

PRTR ROO FS-01 SPECTRUM NO. 23 DATA TAKEN 3/07/69 LIVE TIME 210.MIN. POSITION 27.IN. ENERGY(KEV)= 1.112*CHAN -7.7

CHAN COUNT COUNT COUNT COUNT COUNT COUNT COUNT COUNT COUNT COUNT

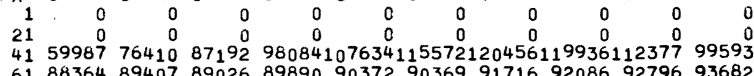

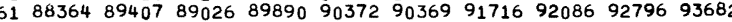
$8110173610^{2} 022103240102131104082105002106586107836109819112021$
101118545111564110395109015110535116253135202163384177737162992 121934139331893078928159344295632102708117317125864118198 14192944918569129890213908029129290945909189059291090 16190835908739074190972908339120690800907449087990223 1819045589386897229006889949893148893288300888528888 $\begin{array}{lllllllllll}201 & 87503 & 87104 & 87261 & 86898 & 87437 & 87198 & 86952 & 87230 & 87443 & 86841 \\ 221 & 87429 & 87067 & 87055 & 86904 & 87334 & 87076 & 86977 & 86651 & 87555 & 87239\end{array}$ $\begin{array}{llllllllllll}241 & 87724 & 87193 & 87499 & 87551 & 87313 & 87742 & 87378 & 87750 & 88044 & 87114\end{array}$ $\begin{array}{lllllllllll}261 & 89064 & 88872 & 88814 & 89053 & 89202 & 89367 & 89448 & 89277 & 89711 & 89382 \\ 281 & 91307 & 90964 & 91563 & 91234 & 91410 & 91721 & 91444 & 91510 & 91351 & 91955\end{array}$

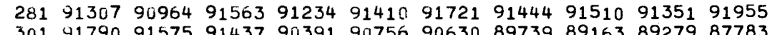

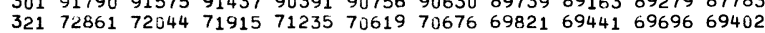
341 67180 6742966872 67265 666336715266417658746641366003 $\begin{array}{lllllllllll}361 & 64851 & 65272 & 65160 & 64913 & 64466 & 64491 & 64318 & 63712 & 64212 & 6427\end{array}$ $\begin{array}{lllllllllll}401 & 59506 & 58550 & 57520 & 56905 & 56187 & 55172 & 54823 & 53959 & 53335 & 52822 \\ 421 & 51719 & 51918 & 51900 & 52144 & 52662 & 52285 & 51832 & 51200 & 51031 & 51151\end{array}$ 44150192501365018249295498465014349833502705001850545 $461518785304455575 \quad 66412100666179630291990356548308426191027$ $\begin{array}{lllllllllll}4 b 1 & 44425 & 43858 & 44108 & 43875 & 43753 & 43578 & 43262 & 43273 & 43306 & 42738\end{array}$ $\begin{array}{lllllllllll}501 & +1812 & 41670 & 41591 & 41527 & 41528 & 41602 & 41353 & 41021 & 41019 & 40941\end{array}$ $\begin{array}{lllllllllll}b_{21} 1 & 35685 & 34079 & 31918 & 30416 & 29393 & 27651 & 26912 & 26392 & 25636 & 24944 \\ 541 & 21778 & 21740 & 21461 & 21118 & 21420 & 21775 & 22313 & 23070 & 24951 & 27792\end{array}$ 56120942326853749654156862921304681472141199777156137340

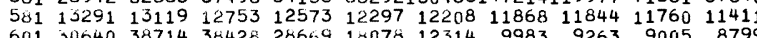
$\begin{array}{llrlllllllll}621 & 8108 & 8060 & 0148 & 8044 & 7942 & 8160 & 7937 & 7946 & 8129 & 8579\end{array}$ $\begin{array}{lrrrrrrrrrrr}641 & 7550 & 7788 & 7814 & 7992 & 7911 & 7717 & 7835 & 7954 & 7927 & 7811 \\ 661 & 19797 & 2025 & 8945 & 7694 & 7551 & 7364 & 7287 & 7278 & 7524 & 7515\end{array}$ $\begin{array}{llllllllllll}681 & 8624 & 9218 & 10832 & 14779 & 22887 & 34649 & 46652 & 50683 & 41772 & 28855\end{array}$ $\begin{array}{llllllllllll}701 & 8014 & 5728 & 5067 & 4936 & 4857 & 4747 & 4849 & 4771 & 4813 & 4745\end{array}$ $\begin{array}{lllllllllll}721 & 8485 & 10196 & 11197 & 9688 & 7682 & 6168 & 5476 & 5349 & 5437 & 5090\end{array}$ $\begin{array}{lllllllllll}741 & 4610 & 4713 & 4552 & 4660 & 4505 & 4761 & 4782 & 4536 & 4685 & 4589\end{array}$ $\begin{array}{rllllllllll}761 & 4569 & 4444 & 4282 & 4271 & 4147 & 4024 & 3895 & 3745 & 3845 & 3610 \\ -781 & 3267 & 3246 & 3326 & 3245 & 3325 & 3244 & 3387 & 3565 & 4199 & 4921\end{array}$ COUNT COUNT COUNT COUNT COUNT COUNT COINT COUNT COUNT COUNT $\begin{array}{rrrrrrrrrr}0 & 0 & 0 & 0 & 0 & 0 & 7 & 115 & 1379 & 22171 \\ 89823 & 84824 & 84421 & 83335 & 84701 & 84850 & 85507 & 85699 & 86094 & 86791\end{array}$ 年 $117013_{130} 371159655184676189231203265237063234378185842139445$

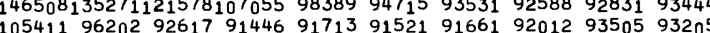
90810909209089090758905919091090593904569044690774 $\begin{array}{lllllllllll}89863 & 89844 & 90170 & 90120 & 89834 & 89926 & 89842 & 89665 & 90404 & 89914 \\ 88077 & 88413 & 88352 & 88763 & 88067 & 87905 & 87914 & 87486 & 87950 & 87447\end{array}$ $\begin{array}{llllllll}87466 & 87129 & 87339 & 87060 & 87074 & 86552 \quad 86998 \quad 87046 \quad 86973 \quad 86592\end{array}$ $\begin{array}{lllllll}8675687311 & 8706486840 \quad 86907 & 86450 & 87461 & 87141 & 87122 & 87153\end{array}$ $\begin{array}{lllllllllll}87852 & 87724 & 88892 & 88046 & 88409 & 87642 & 89161 & 87919 & 88705 & 87814 \\ 89542 & 90086 & 90767 & 90313 & 90249 & 90663 & 91095 & 90782 & 90813 & 90519\end{array}$

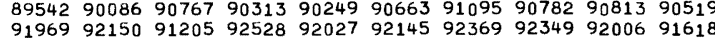
$\begin{array}{llllllllll}7327 & 85955 & 84288 & 83269 & 81106 & 78461 & 77740 & 75808 & 74495 & 7415\end{array}$ $\begin{array}{lllllllllll}68958 & 68599 & 68110 & 68111 & 68215 & 68178 & 67866 & 67675 & 67616 & 67019 & \end{array}$ $\begin{array}{lllllllllll}63969 & 63161 & 63167 & 62917 & 63388 & 63662 & 63512 & 63163 & 63205 & 62414\end{array}$ $\begin{array}{lllllllllll}52698 & 52281 & 52439 & 52460 & 51528 & 51870 & 51941 & 51710 & 51696 & 51574 \\ 51056 & 50781 & 51202 & 50703 & 51100 & 50665 & 50396 & 50101 & 50116 & 49813\end{array}$ $\begin{array}{rlllllllll}51985 & 55737 & 63531 & 74187 & 77593 & 71155 & 61541 & 54440 & 52683 & 51771 \\ 1032200 & 63339 & 49460 & 45833 & 44777 & 44076 & 43610 & 44033 & 43887 & 44076\end{array}$ $\begin{array}{rlllllllllll}42864 & 42066 & 42140 & 42010 & 42518 & 42122 & 41939 & 41718 & 41741 & 41639\end{array}$ $\begin{array}{llllllllllll}40989 & 40467 & 40707 & 40228 & 40144 & 38770 & 38445 & 38270 & 37574 & 369 \\ 24513 & 23769 & 23503 & 23470 & 22852 & 22671 & 227688 & 22703 & 22054 & 22007\end{array}$ $\begin{array}{lllllllllll}30535 & 29998 & 26506 & 23085 & 21359 & 21139 & 21288 & 21469 & 22729 & 25525\end{array}$ $\begin{array}{lllllllllll}21610 & 16543 & 14898 & 14385 & 14146 & 14187 & 14178 & 14017 & 13717 & 13803\end{array}$ $\begin{array}{llllllllll}8999 & 8815 & 8665 & 8679 & 8522 & 8509 & 8344 & 8515 & 8272 & 8171\end{array}$ $\begin{array}{llllllllll}9466 & 11173 & 13019 & 13321 & 11919 & 9794 & 8482 & 7831 & 7780 & 7674\end{array}$ $\begin{array}{rrrrrrrrrr}7935 & 8334 & 8672 & 10328 & 13958 & 21171 & 32226 & 41829 & 42959 & 3239 \\ 7512 & 7496 & 7417 & 7671 & 7748 & 7667 & 7907 & 8034 & 8223 & 8285\end{array}$ 23628324555809399836146542165977136533800913604215370 $\begin{array}{lllllllllll}4888 & 4809 & 4861 & 4813 & 4749 & 4869 & 5010 & 5001 & 5689 & 6851\end{array}$ $\begin{array}{lllllllllll}4972 & 4730 & 4764 & 4589 & 4610 & 4643 & 4629 & 4639 & 4619 & 4719\end{array}$ $\begin{array}{lllllllllll}3594 & 3539 & 3486 & 3517 & 3419 & 3406 & 3469 & 3380 & 3339 & 3356 \\ 6090 & 6998 & 7095 & 5947 & 4724 & 3655 & 3223 & 3075 & 3136 & 3063\end{array}$

$\begin{array}{lllllllllll}801 & 3065 & 3129 & 3156 & 3129 & 3068 & 2985 & 2951 & 2914 & 2871 & 2815\end{array}$ $\begin{array}{lllllllllll}821 & 2742 & 2881 & 2807 & 2817 & 2703 & 2679 & 2724 & 2570 & 2651 & 2566 \\ 841 & 2316 & 2246 & 2186 & 2203 & 2207 & 2142 & 2163 & 2176 & 2158 & 2214\end{array}$ $\begin{array}{lllllllllll}601 & 1921 & 1920 & 1925 & 1893 & 1939 & 1556 & 1890 & 1826 & 1896 & 1849\end{array}$ $\begin{array}{llllllllllll}881 & 1709 & 1718 & 1684 & 1738 & 1782 & 1754 & 1689 & 1741 & 1747 & 1723 \\ 901 & 1689 & 1727 & 1642 & 1668 & 1646 & 1553 & 1641 & 1608 & 1677 & 1716\end{array}$

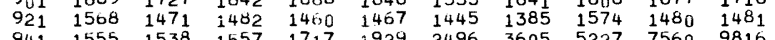
$\begin{array}{lllllllllll}961 & 1308 & 1372 & 136 i & 1314 & 1210 & 1211 & 1216 & 1114 & 1124 & 1122 \\ 981 & 1119 & 1175 & 1178 & 1181 & 1159 & 1224 & 1159 & 1163 & 1198 & 1133\end{array}$ $\begin{array}{rrrrrrrrrrr}1001 & 1100 & 1068 & 1165 & 1129 & 1140 & 1117 & 1222 & 1169 & 1169 & 1147 \\ 1021 & 3451 & 3195 & 2409 & 1681 & 1261 & 1090 & 1018 & 996 & 914 & 941\end{array}$ $\begin{array}{rrrrrrrrrrr}1021 & 3451 & 3195 & 2409 & 1681 & 1261 & 1090 & 1018 & 996 & 914 & 941 \\ 1041 & 1000 & 950 & 960 & 977 & 945 & 972 & 1047 & 1096 & 1153 & 1266\end{array}$ $\begin{array}{rrrrrrrrrrr}1061 & 1118 & 1064 & 1052 & 1022 & 999 & 989 & 980 & 947 & 958 & 885 \\ 1081 & 1244 & 1133 & 1000 & 8 \times 1 & 916 & 827 & 842 & 823 & 929 & 890\end{array}$ $\begin{array}{rrrrrrrrrrr}1081 & 1244 & 1133 & 1000 & 841 & 916 & 827 & 842 & 823 & 929 & 890 \\ 1101 & 848 & 838 & 817 & 854 & 909 & 845 & 882 & 821 & 801 & 795\end{array}$ $\begin{array}{lllllllllll}1101 & 848 & 838 & 817 & 854 & 909 & 845 & 882 & 821 & 801 & 795 \\ 1121 & 770 & 812 & 856 & 774 & 810 & 812 & 832 & 819 & 816 & 724\end{array}$ $\begin{array}{lllllllllll}1141 & 795 & 765 & 824 & 758 & 751 & 785 & 816 & 827 & 884 & 877 \\ 1161 & 723 & 703 & 749 & 731 & 695 & 712 & 670 & 718 & 715 & 742\end{array}$ $\begin{array}{llllllllll}1181 & 723 & 756 & 569 & 755 & 728 & 630 & 706 & 626 & 635\end{array}$ $\begin{array}{lllllllllll}1181 & 722 & 656 & 569 & 705 & 728 & 630 & 706 & 626 & 635 & 685 \\ 1201 & 730 & 714 & 759 & 764 & 766 & 765 & 707 & 691 & 581 & 542\end{array}$ $\begin{array}{lllllllllll}1221 & 542 & 524 & 526 & 539 & 534 & 515 & 572 & 489 & 546 & 565\end{array}$ $\begin{array}{lllllllllll}1241 & 506 & 513 & 501 & 432 & 503 & 502 & 506 & 538 & 530 & 513 \\ 12 n 1 & 479 & 477 & 449 & 464 & 443 & 438 & 482 & 431 & 467 & 403\end{array}$

$\begin{array}{lllllllllll}1231 & 456 & 446 & 424 & 412 & 402 & 461 & 443 & 467 & 425 & 402 \\ 13.1 & 362 & 389 & 368 & 403 & 412 & 416 & 433 & 392 & 378 & 387\end{array}$

$\begin{array}{lllllllllll}1321 & 391 & 402 & 410 & 350 & 418 & 389 & 347 & 365 & 385 & 381 \\ 1341 & 572 & 615 & 711 & 779 & 780 & 751 & 608 & 550 & 474 & 427\end{array}$

$\begin{array}{lllllllllll}1361 & 384 & 353 & 362 & 340 & 329 & 365 & 346 & 372 & 355 & 377\end{array}$

$\begin{array}{lllllllllll}1401 & 347 & 356 & 331 & 322 & 360 & 324 & 337 & 320 & 306 & 333 \\ 1411 & 374 & 368 & 435 & 448 & 478 & 568 & 636 & 724 & 801 & 874\end{array}$

$\begin{array}{lllllllllll}1421 & 335 & 324 & 272 & 3.34 & 279 & 281 & 304 & 286 & 284 & 282 \\ 1441 & 293 & 283 & 311 & 316 & 284 & 288 & 274 & 277 & 298 & 301\end{array}$

$\begin{array}{lllllllllll}1461 & 284 & 313 & 296 & 272 & 264 & 272 & 298 & 273 & 301 & 254\end{array}$

$\begin{array}{lllllllllll}1401 & 296 & 262 & 266 & 285 & 277 & 274 & 261 & 263 & 258 & 262 \\ 1501 & 295 & 266 & 289 & 261 & 274 & 300 & 298 & 327 & 321 & 289\end{array}$

$\begin{array}{lllllllllll}1521 & 280 & 278 & 286 & 266 & 276 & 296 & 273 & 247 & 262 & 264\end{array}$

$\begin{array}{lllllllllll}1541 & 248 & 254 & 264 & 248 & 266 & 252 & 264 & 257 & 264 & 270 \\ 1561 & 258 & 255 & 237 & 247 & 276 & 233 & 264 & 208 & 256 & 258\end{array}$

$\begin{array}{lllllllllll}1501 & 221 & 247 & 227 & 269 & 271 & 238 & 260 & 266 & 306 & 287 \\ 1601 & 225 & 242 & 267 & 227 & 255 & 236 & 215 & 224 & 229 & 273\end{array}$

$\begin{array}{lllllllllll}1601 & 225 & 242 & 267 & 227 & 255 & 236 & 215 & 224 & 229 & 273 \\ 1621 & 293 & 291 & 320 & 246 & 260 & 254 & 270 & 259 & 232 & 235\end{array}$

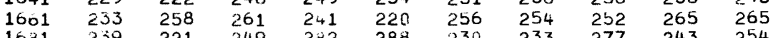

$\begin{array}{llll}282 & 288 & 230 & 233\end{array}$

$\begin{array}{lllllllllll}1701 & 230 & 243 & 249 & 259 & 225 & 217 & 227 & 226 & 201 & 229 \\ 1721 & 246 & 226 & 247 & 244 & 244 & 257 & 229 & 233 & 222 & 239 \\ 1741 & 264 & 228 & 239 & 233 & 246 & 261 & 255 & 236 & 255 & 249\end{array}$

$17 n 1-220-193-194-1=1-174-594-148-159-161-458$

$\begin{array}{rrrrrrrrrrr}1701 & 135 & 140 & 152 & 142 & 157 & 155 & 154 & 152 & 169 & 180 \\ 1801 & 127 & 118 & 127 & 109 & 125 & 103 & 125 & 118 & 125 & 112 \\ 1021 & 102 & 119 & 90 & 119 & 109 & 112 & 95 & 1199 & 99 & 124 \\ 1841 & 94 & 101 & 105 & 94 & 117 & 77 & 79 & 88 & 86 & 73 \\ 1861 & 77 & 70 & 73 & 76 & 94 & 82 & 76 & 75 & 88 & 81 \\ 1881 & 53 & 60 & 61 & 53 & 50 & 48 & 63 & 63 & 59 & 64 \\ 1961 & 117 & 145 & 108 & 135 & 116 & 96 & 79 & 51 & 60 & 53 \\ 1921 & 51 & 46 & 39 & 51 & 64 & 52 & 57 & 45 & 55 & 30 \\ 1941 & 55 & 40 & 46 & 40 & 46 & 56 & 61 & 62 & 59 & 45 \\ 1961 & 156 & 198 & 227 & 327 & 398 & 432 & 490 & 541 & 576 & 474 \\ 1981 & 25 & 30 & 20 & 20 & 21 & 24 & 30 & 25 & 23 & 19 \\ 2001 & 16 & 34 & 24 & 29 & 26 & 21 & 29 & 28 & 24 & 26 \\ 2021 & 29 & 25 & 30 & 21 & 20 & 15 & 23 & 13 & 24 & 15 \\ 2041 & 16 & 10 & 19 & 19 & 17 & 23 & 21 & 0 & & \end{array}$ $\begin{array}{llllllllll}2947 & 2932 & 2869 & 2868 & 2954 & 2902 & 2851 & 2815 & 2751 & 2766\end{array}$ $\begin{array}{llllllllll}2622 & 2491 & 2508 & 2458 & 2353 & 2392 & 2341 & 2337 & 2327 & 2202 \\ 2143 & 2097 & 2027 & 2018 & 1985 & 2059 & 1956 & 2010 & 1947 & 2071\end{array}$ $\begin{array}{llllllll}1933 & 1859 & 1846 & 1922 & 1820 & 1867 & 1791\end{array}$ $\begin{array}{lllllllllll}1666 & 1658 & 1686 & 1657 & 1634 & 1671 & 1661 & 1677 & 1697 & 1621 \\ 1601 & 1686 & 1644 & 1535 & 1526 & 1610 & 1544 & 1505 & 1466 & 1531\end{array}$ $\begin{array}{rrrrrrrrrr}1420 & 1454 & 1565 & 1524 & 1444 & 1456 & 1453 & 1479 & 1512 & 1457 \\ 11408 & 10939 & 8382 & 4717 & 2743 & 1697 & 1417 & 1275 & 1390 & 1363\end{array}$ $\begin{array}{rrrllllllll}1183 & 1171 & 1123 & 1134 & 1234 & 1156 & 1215 & 1140 & 1112 & 1107 \\ 1074 & 1094 & 1067 & 1131 & 1156 & 1097 & 1004 & 1137 & 1034 & 1112\end{array}$ $\begin{array}{rrrrrrrrrr}1155 & 1100 & 1127 & 1101 & 1247 & 1453 & 1738 & 2200 & 2658 & 3211 \\ 987 & 895 & 983 & 980 & 1048 & 927 & 1020 & 985 & 946 & 939\end{array}$ $\begin{array}{rrrrrrrrrr}987 & 895 & 983 & 980 & 1048 & 927 & 1020 & 985 & 946 & 939 \\ 1305 & 1452 & 1534 & 1507 & 1320 & 1151 & 1124 & 1081 & 1016 & 1011\end{array}$ $\begin{array}{rrrrrrrrrr}1305 & 1452 & 1534 & 1507 & 1320 & 1151 & 1124 & 1081 & 1016 & 1011 \\ 889 & 906 & 830 & 908 & 931 & 956 & 1010 & 989 & 1105 & 1151\end{array}$ $\begin{array}{llllllllll}863 & 878 & 863 & 881 & 870 & 837 & 863 & 885 & 849 & 849 \\ 896 & 837 & 821 & 792 & 848 & 805 & 851 & 820 & 828 & 795\end{array}$ $\begin{array}{llllllllll}896 & 837 & 821 & 792 & 848 & 805 & 851 & 820 & 828 & 795 \\ 763 & 804 & 826 & 850 & 800 & 830 & 800 & 837 & 824 & 769\end{array}$ $\begin{array}{llllllllll}909 & 930 & 911 & 888 & 821 & 773 & 722 & 743 & 763 & 693 \\ 722 & 696 & 647 & 611 & 684 & 705 & 675 & 675 & 670 & 642\end{array}$ $\begin{array}{llllllllll}660 & 685 & 654 & 624 & 655 & 649 & 589 & 690 & 713 & 659\end{array}$ $\begin{array}{llllllllll}621 & 600 & 640 & 617 & 622 & 648 & 645 & 562 & 570 & 563 \\ 613 & 602 & 637 & 571 & 571 & 570 & 524 & 500 & 533 & 506\end{array}$ $\begin{array}{llllllllll}508 & 512 & 496 & 482 & 497 & 489 & 489 & 470 & 457 & 468\end{array}$ $\begin{array}{llllllllll}434 & 440 & 442 & 416 & 451 & 429 & 439 & 462 & 469 & 411\end{array}$

$\begin{array}{llllllllll}403 & 415 & 383 & 413 & 411 & 442 & 421 & 419 & 394 & 402 \\ 373 & 389 & 383 & 382 & 415 & 413 & 384 & 403 & 400 & 406\end{array}$ $\begin{array}{llllllllll}373 & 365 & 357 & 370 & 425 & 366 & 379 & 412 & 460 & 512 \\ 521 & 436 & 443 & 394 & 413 & 429 & 375 & 368 & 315 & 376\end{array}$ $\begin{array}{llllllllll}349 & 397 & 341 & 358 & 357 & 331 & 339 & 318 & 335 & 316 \\ 336 & 329 & 305 & 337 & 334 & 349 & 374 & 326 & 305 & 349\end{array}$ $\begin{array}{llllllllll}836 & 741 & 580 & 429 & 337 & 327 & 308 & 297 & 296 & 297 \\ 327 & 322 & 315 & 269 & 290 & 272 & 272 & 282 & 296 & 322\end{array}$ $\begin{array}{lllllll}289 & 295 & 265 & 242 & 279 & 267 & 27 \\ 299 & 269 & 254 & 271 & 276 & 260 & 306\end{array}$

$\begin{array}{llllllllll}265 & 262 & 293 & 262 & 259 & 281 & 267 & 280 & 267 & 257 \\ 317 & 297 & 285 & 292 & 284 & 268 & 274 & 280 & 289 & 297\end{array}$

$\begin{array}{llllllllll}225 & 268 & 229 & 248 & 258 & 252 & 253 & 240 & 257 & 267\end{array}$ $\begin{array}{llllllllll}232 & 250 & 272 & 267 & 290 & 250 & 243 & 251 & 255 & 254 \\ 265 & 241 & 236 & 256 & 246 & 240 & 262 & 259 & 253 & 217\end{array}$

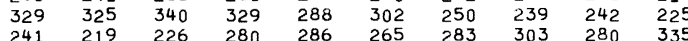
$\begin{array}{llllllllll}262 & 219 & 226 & 280 & 286 & 265 & 283 & 303 & 280 & 335 \\ 267 & 227 & 236 & 224 & 259 & 243 & 266 & 257 & 250\end{array}$ $\begin{array}{llllllllll}265 & 254 & 276 & 253 & 248 & 234 & 229 & 255 & 252 & 239\end{array}$

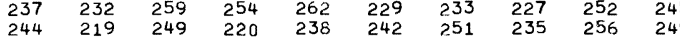
$\begin{array}{llllllllll}251 & 255 & 261 & 249 & 256 & 260 & 300 & 296 & 261 & 265\end{array}$ $\begin{array}{rrrrrrrrrr}239 & 222 & 235 & 229 & 239 & 254 & 220 & 247 & 210 & 208 \\ -+46 & -144 & 146 & 136 & -138 & 152 & -43 & 130 & 162 & 137\end{array}$

$\begin{array}{rrrrrrrrrr}197 & 191 & 170 & 169 & 163 & 142 & 129 & 132 & 145 & 114 \\ 108 & 98 & 123 & 107 & 118 & 110 & 112 & 114 & 98 & 101 \\ 88 & 98 & 111 & 112 & 105 & 80 & 98 & 96 & 101 & 101 \\ 104 & 95 & 83 & 95 & 86 & 68 & 71 & 91 & 67 & 88 \\ 70 & 73 & 80 & 78 & 80 & 74 & 61 & 67 & 72 & 58 \\ 82 & 57 & 57 & 71 & 82 & 71 & 95 & 97 & 98 & 91 \\ 46 & 58 & 56 & 51 & 61 & 49 & 48 & 66 & 48 & 55 \\ 41 & 52 & 59 & 56 & 39 & 52 & 49 & 47 & 63 & 46 \\ 47 & 55 & 49 & 69 & 66 & 54 & 74 & 77 & 110 & 133 \\ 347 & 205 & 130 & 88 & 52 & 37 & 37 & 41 & 37 & 32 \\ 25 & 28 & 19 & 26 & 20 & 22 & 25 & 27 & 23 & 20 \\ 20 & 23 & 28 & 21 & 18 & 24 & 26 & 29 & 23 & 24 \\ 18 & 19 & 11 & 19 & 13 & 16 & 21 & 15 & 20 & 20\end{array}$




\section{$D-14$}

GAMMA RAY SPECTRUM DATA

PRTR ROE FS-01 SPECTRUM NO. 24 DATA TAKEN 3/07/69 LIVE TIME 350.MIN. POSITION 29. IN. ENERGY(KEV) $=1.114 *$ CHAN -8.5

CHaN COUNT COUNT COUNT COU T COUNT COUNT COUNT COUNT COUNT COUNT

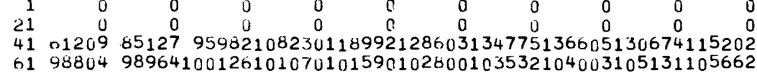
10114423413368113173113008122088124362125739127040130726133738 1211,5104105378104785105124106044107538117552140773160956150878 1411,4438102238101751101174101193101877101532101356100708100686 $\begin{array}{lll}1 b 11,01841002091004531003 n 0100417100156100448100927100080 & 99484 \\ 161 & 99602994579806699112990979812197993971919711196889\end{array}$

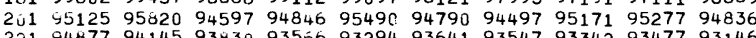
24192961920729281892422929959271692395924679240392568 26192606926269225192399926109241592487925499235191995 28192953928139273292759935189283192713922859293892807

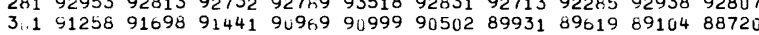

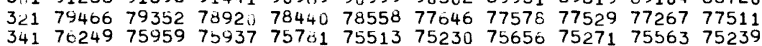

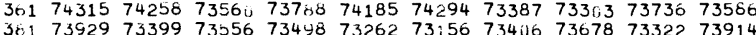
$40170830 \quad 70627 \quad 7,449569773 \quad 695536939768503685326833368337$

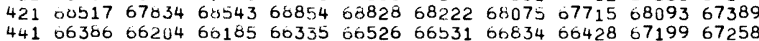

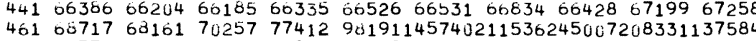

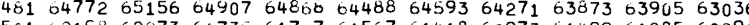
$\begin{array}{lllllllllll}501 & 62198 & 62273 & 61735 & 61707 & 61567 & 61418 & 60973 & 61499 & 61285 & 60291 \\ 521 & 51052 & 48036 & 45250 & 42946 & 40364 & 38675 & 37397 & 35589 & 34403 & 33340\end{array}$ $\begin{array}{lllllllllll}541 & 28455 & 27874 & 27978 & 27556 & 27970 & 28349 & 29211 & 30783 & 34199 & 39557\end{array}$ bo1 $2036829935 \quad 32753 \quad 4287163916 \quad 86101 \quad 9157470955 \quad 44130 \quad 26950$ $\begin{array}{lllllllllll}601 & 49277 & 60958 & 55972 & 37060 & 21081 & 13088 & 10699 & 10031 & 9947 & 9905\end{array}$

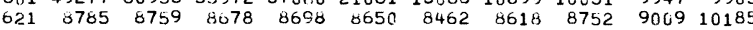
$\begin{array}{lrrrrrrrrrr}641 & 0319 & 8490 & 0514 & 0594 & 8262 & 8556 & 8672 & 8590 & 8476 & 8954 \\ 661 & 22880 & 12424 & 9481 & 7965 & 7927 & 7778 & 7927 & 8001 & 8041 & 8015\end{array}$ $\begin{array}{lllllllllll}661 & 22880 & 12424 & 9481 & 7985 & 7927 & 7778 & 7927 & 8001 & 8041 & 8015 \\ 681 & 10170 & 11333 & 1504 & 23447 & 30944 & 60509 & 79600 & 79398 & 58515 & 36526\end{array}$ $\begin{array}{rrrrrrrrrrr}681 & 10170 & 11333 & 15049 & 23447 & 30944 & 60505 & 79600 & 79398 & 5815 & 36526 \\ 71,1 & 5887 & 4362 & 4032 & 3861 & 3807 & 3854 & 3915 & 3799 & 3701 & 3725 \\ 721 & 11078 & 13601 & 14020 & 10937 & 7426 & 5407 & 4782 & 4636 & 4382 & 3990\end{array}$ $\begin{array}{rrrrrrrrrrr}721 & 11078 & 13601 & 14020 & 10937 & 7426 & 5407 & 4782 & 4636 & 4382 & 3990 \\ 741 & 3465 & 3516 & 3547 & 34.2 & 3442 & 3275 & 3394 & 3343 & 3495 & 3352\end{array}$ $\begin{array}{lllllllllll}741 & 3465 & 3516 & 3547 & 34,2 & 3442 & 3275 & 3394 & 3343 & 3495 & 3352 \\ 761 & 3152 & 3218 & 3168 & 3037 & 3005 & 3,34 & 2906 & 2443 & 2962 & 2815 \\ 701 & 2049 & 2075 & 4474 & 2506 & 5593 & 2069 & 2654 & 2769 & 3270 & 3753\end{array}$

$\begin{array}{lllllllllll}001 & 2599 & 2675 & 2708 & 2496 & 2387 & 2392 & 2277 & 2332 & 2364 & 2383\end{array}$ $\begin{array}{lllllllllll}821 & 2214 & 2154 & 2160 & 232 b & 2175 & 2235 & 2191 & 2137 & 2165 & 2205 \\ 841 & 2017 & 1914 & 1910 & 1894 & 1879 & 1926 & 1922 & 1934 & 1948 & 1941\end{array}$ $\begin{array}{lllllllllll}861 & 1815 & 1676 & 1766 & 1636 & 1735 & 1006 & 1650 & 1713 & 1708 & 1683\end{array}$ $\begin{array}{lllllllllll}801 & 1609 & 1570 & 1560 & 15 c 7 & 1613 & 1539 & 1550 & 1545 & 1505 & 1508\end{array}$ $\begin{array}{lllllllllll}901 & 1555 & 1578 & 1630 & 1527 & 1545 & 1485 & 1539 & 1603 & 1721 & 1772 \\ 921 & 1409 & 1391 & 1455 & 1366 & 1313 & 1300 & 1324 & 1368 & 1307 & 1391\end{array}$ $\begin{array}{lllllllllll}941 & 1498 & 1373 & 1433 & 1424 & 1770 & 2111 & 2849 & 3800 & 5216 & 6389 \\ 9.1 & 1292 & 1270 & 1203 & 1176 & 1128 & 1161 & 1154 & 1117 & 1111 & 1190\end{array}$ $\begin{array}{lllllllllll}9.11 & 1099 & 1133 & 1134 & 1004 & 1110 & 1112 & 1172 & 1116 & 1116 & 1114\end{array}$ $\begin{array}{rrrrrrrrrrr}1001 & 1124 & 1097 & 1080 & 1015 & 1127 & 1091 & 1115 & 1038 & 1060 & 1077 \\ 1021 & 2249 & 1044 & 1475 & 11 \text { fio } & 1032 & 943 & 1019 & 894 & 942 & 924\end{array}$ $\begin{array}{rrrrrrrrrrr}1021 & 2249 & 1044 & 1475 & 1166 & 1032 & 943 & 1019 & 894 & 942 & 924 \\ 1041 & 975 & 968 & 952 & 990 & 950 & 947 & 1020 & 1217 & 1333 & 1456\end{array}$ $\begin{array}{rrrrrrrrrrr}1061 & 1204 & 1116 & 1076 & 9 n 4 & 887 & 930 & 906 & 926 & 881 & 866 \\ 1061 & 1019 & 939 & 522 & 871 & 881 & 551 & 932 & 846 & 897 & 897\end{array}$ $\begin{array}{rrrrrrrrrrr}1001 & 1419 & 939 & 522 & 871 & 881 & 051 & 932 & 846 & 897 & 897 \\ 1141 & 815 & 814 & 836 & 810 & 820 & 824 & 783 & 866 & 734 & 786\end{array}$ $\begin{array}{lllllllllll}1121 & 778 & 783 & 629 & 792 & 788 & 791 & 773 & 787 & 719 & 802 \\ 1141 & 753 & 752 & 749 & 758 & 752 & 761 & 823 & 892 & 903 & 996\end{array}$ $\begin{array}{lllllllllll}1141 & 753 & 752 & 749 & 758 & 752 & 761 & 823 & 892 & 903 & 996 \\ 1161 & 071 & 063 & 037 & 641 & 587 & 596 & 594 & 658 & 600 & 595 \\ 11 & 651 & 580 & 001 & 551 & 615 & 591 & 609 & 541 & 593 & 605\end{array}$ $\begin{array}{lllllllllll}1101 & 651 & 580 & 001 & 551 & 615 & 591 & 609 & 541 & 593 & 605\end{array}$ $\begin{array}{lllllllllll}1201 & 705 & 750 & 777 & 790 & 745 & 069 & 633 & 562 & 590 & 552\end{array}$ $\begin{array}{lllllllllll}1221 & 526 & 463 & 481 & 409 & 495 & 481 & 535 & 561 & 591 & 548 \\ 1241 & 452 & 461 & 453 & 401 & 480 & 504 & 501 & 493 & 487 & 528\end{array}$

$\begin{array}{lllllllllll}1<n 1 & 433 & 422 & 440 & 404 & 435 & 405 & 381 & 446 & 406 & 406 \\ 1201 & 441 & 414 & 39 i & 400 & 400 & 574 & 392 & 349 & 381 & 374\end{array}$

$\begin{array}{lllllllllll}1231 & 347 & 414 & 390 & 400 & 400 & 374 & 392 & 349 & 381 & 374 \\ 13: 1 & 377 & 346 & 380 & 355 & 308 & 406 & 371 & 367 & 378 & 416\end{array}$

$\begin{array}{lllllllllll}1341 & 767 & 884 & 1431 & 1041 & 1019 & 761 & 583 & 458 & 448 & 375\end{array}$

$\begin{array}{lllllllllll}13 r 1 & 331 & 303 & 377 & 327 & 337 & 323 & 340 & 360 & 328 & 353 \\ 13 r 1 & 316 & 330 & 343 & 250 & 314 & 318 & 332 & 325 & 329 & 356\end{array}$

$\begin{array}{lllllllllll}1401 & 314 & 315 & 371 & 309 & 437 & 477 & 539 & 579 & 636 & 610 \\ 1421 & 304 & 314 & 260 & 313 & 320 & 296 & 283 & 286 & 277 & 283\end{array}$

$\begin{array}{lllllllllll}1421 & 304 & 314 & 268 & 313 & 320 & 296 & 283 & 286 & 277 & 283 \\ 1441 & 313 & 315 & 313 & 2 \% 7 & 268 & 302 & 312 & 284 & 283 & 294\end{array}$

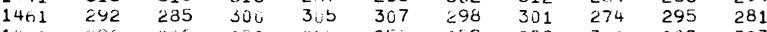

$\begin{array}{lllllllllll}1401 & 296 & 266 & 282 & 250 & 250 & 259 & 289 & 311 & 289 & 303 \\ 1501 & 254 & 293 & 290 & 254 & 330 & 329 & 324 & 372 & 357 & 387\end{array}$

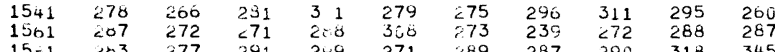

$\begin{array}{lllllllllll}1501 & 203 & 277 & 291 & 299 & 271 & 289 & 237 & 290 & 318 & 345 \\ 1641 & 204 & 280 & 285 & 241 & 273 & 280 & 204 & 297 & 307 & 291\end{array}$

$\begin{array}{lllllllllll}1621 & 315 & 292 & 309 & 2.2 & 270 & 578 & 299 & 302 & 306 & 271 \\ 1641 & 282 & 323 & 306 & 241 & 262 & 300 & 293 & 265 & 301 & 305\end{array}$

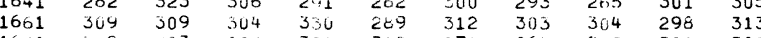

$\begin{array}{lllllllllll}1661 & 309 & 323 & 506 & 321 & 312 & 271 & 264 & 312 & 300 & 300 \\ 1701 & 210 & 300 & 281 & 3.0 & 310 & 300 & 319 & 325 & 316 & 346\end{array}$

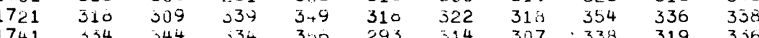

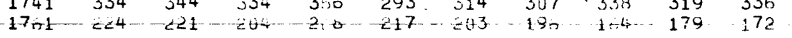

$\begin{array}{lllllllllll}1701 & 147 & 107 & 130 & 1 \% 4 & 162 & 169 & 159 & 166 & 177 & 172\end{array}$

$\begin{array}{rrrrrrrrrrr}1701 & 147 & 107 & 130 & 174 & 162 & 169 & 159 & 166 & 177 & 172 \\ 1001 & 131 & 120 & 131 & 137 & 136 & 152 & 135 & 137 & 137 & 153 \\ 1021 & 91 & 134 & 130 & 139 & 122 & 107 & 131 & 163 & 94 & 113 \\ 1801 & 91 & 77 & 102 & 105 & 109 & 95 & 84 & 89 & 78 & 83 \\ 1801 & 55 & 62 & 70 & 64 & 60 & 60 & 54 & 52 & 48 & 60 \\ 1001 & 44 & 52 & 45 & 42 & 50 & 55 & 57 & 58 & 48 & 53 \\ 1941 & 69 & 04 & 84 & 69 & 59 & 38 & 35 & 41 & 27 & 38 \\ 1901 & 56 & 35 & 29 & 43 & 55 & 40 & 50 & 40 & 37 & 33 \\ 1941 & 42 & 33 & 42 & 44 & 55 & 35 & 52 & 43 & 34 & 51 \\ 1961 & 310 & 407 & 511 & 607 & 734 & 849 & 941 & 937 & 757 & 486 \\ 1901 & 10 & 13 & 13 & 17 & 12 & 19 & 13 & 14 & 16 & 20 \\ 2001 & 14 & 9 & 12 & 10 & 21 & 10 & 14 & 18 & 21 & 17 \\ 2021 & 14 & 10 & 20 & 13 & 14 & 7 & 13 & 13 & 18 & 20 \\ 2041 & 10 & 10 & 21 & 10 & 9 & 6 & 15 & 0 & & \end{array}$

COUNT COUNT COUNT COUNT COUNT COUNT COINT COUNT COUNT COUNT

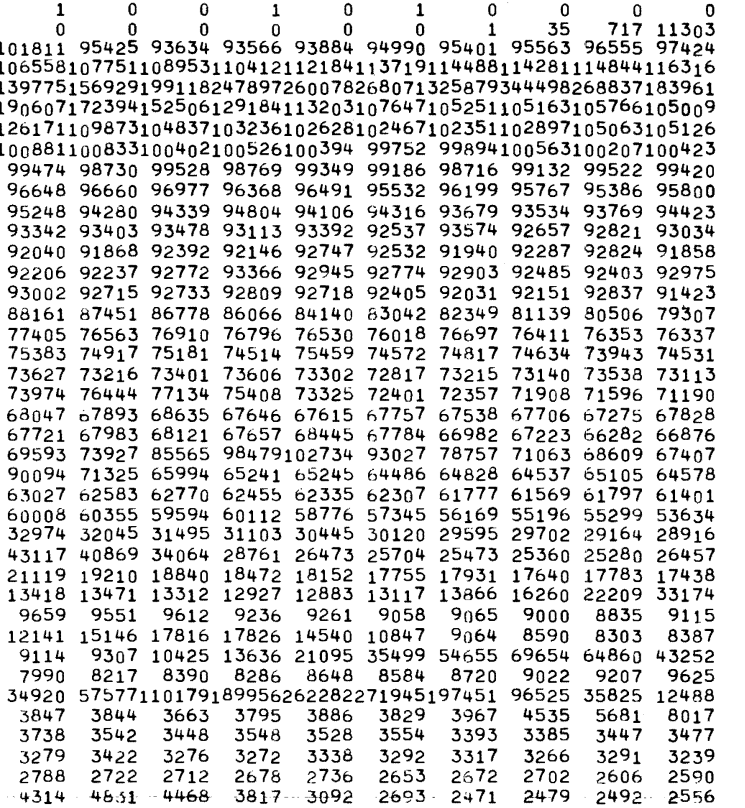

$\begin{array}{lllllllll}2309 & 2260 \quad 2377 \quad 2320 & 2330 & 2350 & 2296 & 2281 & 2256 & 2290\end{array}$ $\begin{array}{llllllllll}2309 & 2268 & 2377 & 2320 & 2330 & 2358 & 2296 & 2281 & 2256 & 2290 \\ 2167 & 2138 & 2060 & 2061 & 2084 & 1904 & 1954 & 1923 & 1985 & 1944\end{array}$

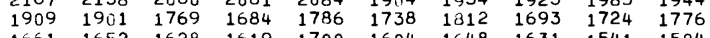
$\begin{array}{llllllllll}1661 & 1652 & 1628 & 1619 & 1700 & 1604 & 1648 & 1631 & 1541 & 1584 \\ 1631 & 1540 & 1526 & 1527 & 1547 & 1506 & 1469 & 1441 & 1593 & 1587\end{array}$ $\begin{array}{lllllllllll}1677 & 1566 & 1491 & 1407 & 1448 & 1419 & 1451 & 1342 & 1354 & 1382 \\ 1380 & 1338 & 1349 & 1364 & 1365 & 1384 & 1387 & 1358 & 1436 & 1475\end{array}$ $\begin{array}{llllllllll}1380 & 1338 & 1349 & 1364 & 1365 & 1384 & 1387 & 1358 & 1436 & 1475 \\ 1813 & 5749 & 3853 & 2272 & 1518 & 1235 & 1250 & 1194 & 1264 & 1237\end{array}$ $\begin{array}{llllllllll}6813 & 5749 & 3853 & 2272 & 1518 & 1235 & 1250 & 1194 & 1264 & 1237 \\ 1065 & 1155 & 1055 & 1061 & 1123 & 1076 & 1156 & 1103 & 1097 & 1162 \\ 1058 & 1073 & 1084 & 1044 & 1017 & 1076 & 1092 & 1073 & 1052 & 1090\end{array}$ $\begin{array}{llllllllll}1058 & 1073 & 1084 & 1044 & 1017 & 1076 & 1092 & 1073 & 1052 & 1090 \\ 1033 & 1065 & 1084 & 1066 & 1155 & 1275 & 1494 & 1724 & 2054 & 2223\end{array}$ $\begin{array}{rrrrrrrrrr}1033 & 1065 & 1084 & 1066 & 1155 & 1275 & 1494 & 1724 & 2054 & 2223 \\ 930 & 964 & 1028 & 948 & 893 & 979 & 943 & 979 & 943 & 985\end{array}$

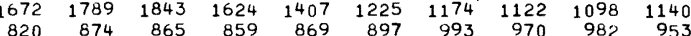
$\begin{array}{lllllllllll}820 & 874 & 865 & 859 & 869 & 897 & 993 & 970 & 982 & 953 \\ 870 & 842 & 867 & 789 & 773 & 850 & 784 & 830 & 783 & 846 \\ 761 & 813 & 810 & 824 & 839 & 834 & 763 & 775 & 781 & 775\end{array}$ $\begin{array}{lllllllllll}761 & 813 & 810 & 824 & 839 & 834 & 763 & 775 & 781 & 775 \\ 765 & 736 & 767 & 742 & 783 & 765 & 775 & 735 & 725 & 763\end{array}$ $\begin{array}{rrrrrrrrrr}984 & 1009 & 1016 & 843 & 735 & 619 & 661 & 642 & 633 & 590 \\ 581 & 628 & 599 & 628 & 575 & 604 & 668 & 586 & 600 & 598\end{array}$ $\begin{array}{lrrrrrrrrr}581 & 628 & 599 & 628 & 575 & 604 & 668 & 586 & 600 & 598 \\ 637 & 603 & 581 & 587 & 540 & 593 & 574 & 591 & 617 & 621\end{array}$ $\begin{array}{llllllllll}545 & 533 & 534 & 519 & 549 & 487 & 513 & 526 & 483 & 539 \\ 600 & 637 & 652 & 614 & 534 & 515 & 524 & 432 & 454 & 417\end{array}$ $\begin{array}{llllllllll}600 & 637 & 652 & 614 & 534 & 515 & 524 & 432 & 454 & 417 \\ 508 & 512 & 486 & 466 & 463 & 462 & 419 & 442 & 423 & 466\end{array}$

$\begin{array}{llllllllll}385 & 423 & 395 & 405 & 409 & 404 & 373 & 409 & 366 & 383 \\ 382 & 406 & 411 & 389 & 416 & 379 & 368 & 383 & 402 & 403\end{array}$

$\begin{array}{llllllllll}382 & 383 & 378 & 374 & 409 & 384 & 374 & 372 & 410 & 401 \\ 353 & 359 & 383 & 407 & 397 & 447 & 474 & 524 & 573 & 743\end{array}$

$\begin{array}{llllllllll}420 & 367 & 384 & 372 & 389 & 368 & 349 & 367 & 360 & 344\end{array}$

$\begin{array}{llllllllll}347 & 329 & 395 & 350 & 303 & 342 & 329 & 310 & 308 & 336 \\ 328 & 312 & 346 & 339 & 302 & 345 & 318 & 341 & 306 & 325\end{array}$

$\begin{array}{llllllllll}559 & 426 & 342 & 313 & 289 & 317 & 3109 & 282 & 320 & 309 \\ 293 & 314 & 293 & 284 & 343 & 308 & 301 & 309 & 311 & 293\end{array}$

$\begin{array}{llllllllll}289 & 314 & 293 & 284 & 343 & 308 & 301 & 309 & 311 & 293 \\ 273 & 302 & 313 & 283 & 319 & 289 & 295 & 294 & 284 & 295\end{array}$

$\begin{array}{llllllllll}273 & 305 & 278 & 281 & 272 & 294 & 326 & 263 & 292 & 289 \\ 323 & 239 & 264 & 291 & 299 & 290 & 283 & 285 & 323 & 294\end{array}$

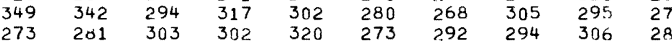

$\begin{array}{llllllllll}284 & 277 & 307 & 258 & 300 & 311 & 293 & 294 & 263 & 291 \\ 297 & 310 & 287 & 254 & 312 & 282 & 254 & 281 & 265 & 303\end{array}$

$\begin{array}{llllllllll}337 & 365 & 339 & 354 & 309 & 277 & 292 & 299 & 291 & 279\end{array}$

$\begin{array}{llllllllll}269 & 285 & 321 & 317 & 318 & 319 & 303 & 361 & 345 & 320 \\ 301 & 305 & 283 & 298 & 290 & 285 & 306 & 301 & 299 & 308\end{array}$

$\begin{array}{llllllllll}299 & 298 & 334 & 304 & 310 & 315 & 304 & 269 & 286 & 266\end{array}$

$\begin{array}{llllllllll}280 & 284 & 292 & 303 & 289 & 289 & 307 & 274 & 323 & 298 \\ 317 & 353 & 328 & 320 & 299 & 302 & 330 & 323 & 302 & 326\end{array}$

$\begin{array}{llllllllll}314 & 341 & 326 & 293 & 336 & 344 & 322 & 316 & 351 & 314\end{array}$

$\begin{array}{llllllllll}372 & 373 & 323 & 3.35 & 352 & 358 & 338 & 330 & 334 & 355 \\ 320 & 302 & 299 & 303 & 292 & 314 & 273 & 268 & 249 & 250\end{array}$

$\begin{array}{rrrrrrrrrr}158 & 181 & 148 & 151 & 157 & 176 & 132 & 156 & 166 & 137 \\ 119 & 113 & 129 & 149 & 128 & 135 & 113 & 123 & 125 & 131 \\ 109 & 112 & 115 & 111 & 124 & 92 & 107 & 88 & 119 & 96 \\ 87 & 79 & 83 & 68 & 85 & 76 & 85 & 79 & 73 & 57 \\ 58 & 62 & 57 & 50 & 61 & 60 & 52 & 68 & 45 & 47 \\ 41 & 71 & 44 & 59 & 51 & 68 & 77 & 77 & 58 & 69 \\ 41 & 56 & 42 & 40 & 43 & 50 & 44 & 41 & 34 & 37 \\ 38 & 40 & 34 & 37 & 47 & 32 & 51 & 40 & 42 & 34 \\ 52 & 26 & 50 & 55 & 65 & 90 & 120 & 171 & 205 & 295 \\ 245 & 99 & 53 & 30 & 24 & 28 & 21 & 12 & 19 & 15 \\ 13 & 20 & 11 & 24 & 10 & 13 & 21 & 13 & 17 & 16 \\ 16 & 20 & 15 & 12 & 18 & 18 & 15 & 10 & 13 & 18 \\ 14 & 11 & 8 & 13 & 15 & 14 & 8 & 19 & 17 & 10\end{array}$ 


\section{D-15}

GAMMA RAY SPECTRUM DATA

PRTR ROD FS-OI SPECTRUM NO. 28 DATA TAKEN $3 / 10 / 69$ LIVE IIME 90.MIN. ROSITION 55.IN. ENERGYIKEVIL 1.112*CHAN -8.2 CHAN COUNT COUNT COUNT COUNT COUNT COUNT COUNT COUNT COUNT COUNT COUNT COUNT COUNT COUNT COUNT COUNT COUNT COUNT COUNT COINT

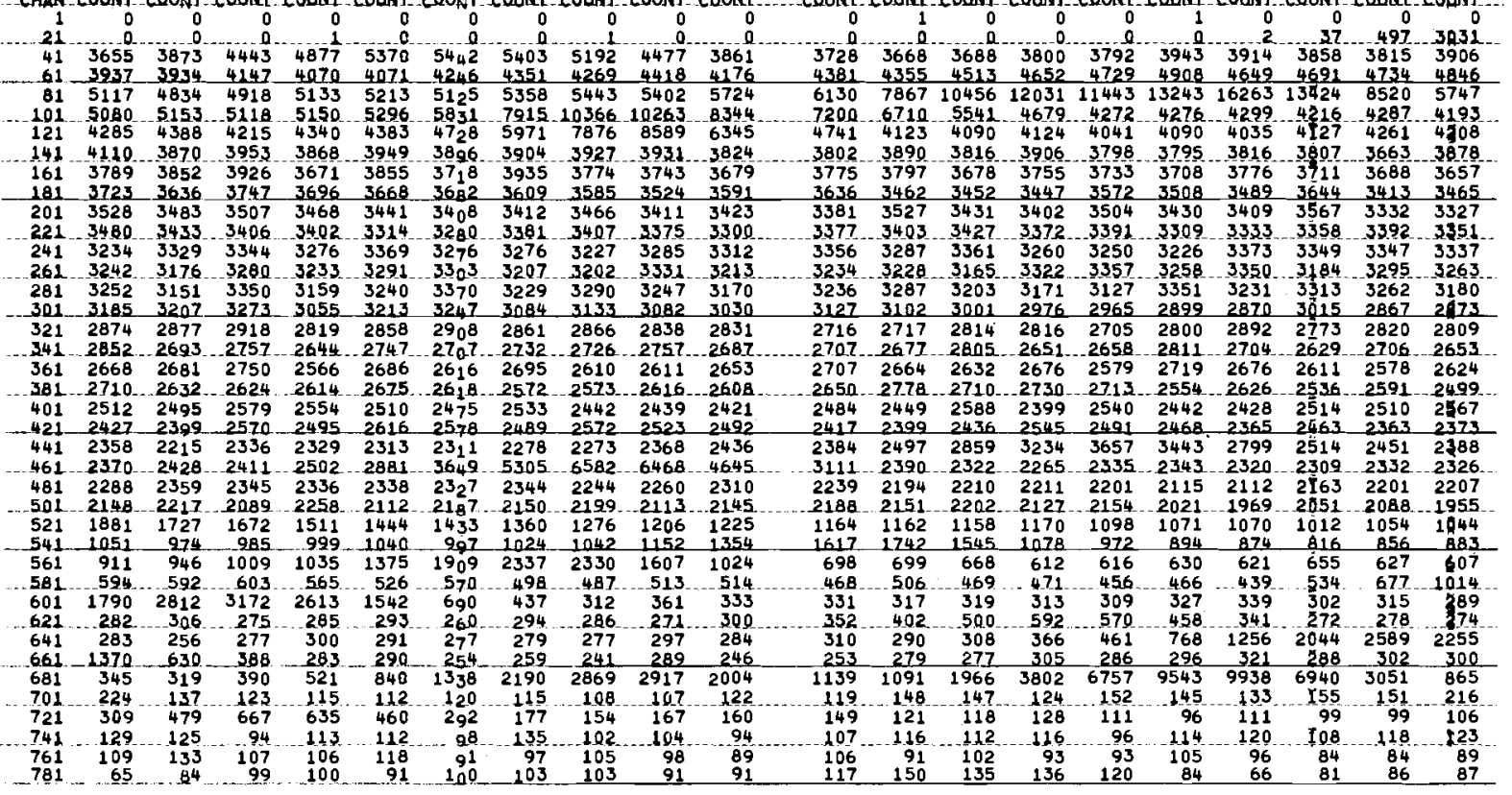

\begin{tabular}{|c|c|c|c|c|c|c|c|c|c|c|c|c|c|c|c|c|c|c|c|c|}
\hline 801 & 91 & 105 & 81 & 100 & 85 & 85 & 100 & 67 & 92 & 85 & 89 & -77 & 83 & 94 & 78 & 74 & 78 & 87 & 86 & 93 \\
\hline 821 & 76 & 83 & 95 & 95 & 90 & 82 & 61 & 78 & 96 & 100 & 76 & 77 & 77 & 72 & $\begin{array}{l}63 \\
84\end{array}$ & $\begin{array}{l}67 \\
68\end{array}$ & $\begin{array}{l}60 \\
68\end{array}$ & 70 & $\begin{array}{l}72 \\
48\end{array}$ & $\begin{array}{l}81 \\
87\end{array}$ \\
\hline $\begin{array}{l}841 \\
861 \\
881\end{array}$ & $\begin{array}{l}60 \\
81 \\
69\end{array}$ & $\begin{array}{r}80 \\
78 \\
68\end{array}$ & $\begin{array}{l}75 \\
64 \\
59\end{array}$ & $\begin{array}{l}86 \\
73 \\
65\end{array}$ & $\begin{array}{r}78 \\
72 \\
70\end{array}$ & $\begin{array}{r}72 \\
72 \\
\end{array}$ & $\begin{array}{r}73 \\
80 \\
61\end{array}$ & $\begin{array}{l}77 \\
78 \\
55\end{array}$ & $\begin{array}{l}93 \\
70 \\
63\end{array}$ & $\begin{array}{c}70 \\
-\quad 70 \\
51 \\
53\end{array}$ & $\begin{array}{r}77 \\
-59 \\
69\end{array}$ & $\begin{array}{r}-88 \\
-70 \\
53\end{array}$ & $\begin{array}{l}65 \\
82 \\
69\end{array}$ & $\begin{array}{r}70 \\
47 \\
60\end{array}$ & $\begin{array}{l}84 \\
66 \\
46\end{array}$ & $\begin{array}{l}68 \\
66 \\
60\end{array}$ & $\begin{array}{l}68 \\
52 \\
50\end{array}$ & $\begin{array}{l}70 \\
73 \\
63\end{array}$ & $\begin{array}{r}48 \\
70 \\
45\end{array}$ & $\begin{array}{l}87 \\
67 \\
61\end{array}$ \\
\hline 901 & 59 & 62 & 50 & 70 & 72 & 50 & 54 & 46 & 59 & 45 & 46 & 63 & 54 & 53 & 51 & 48 & 58 & 54 & 54 & 58 \\
\hline 921 & 37 & 45 & 53 & -46 & -50 & 48 & 57 & 51 & -44 & 59 & 59 & -43 & 43 & 51 & 57 & 58 & 47 & 59 & 55 & 51 \\
\hline 941 & $\begin{array}{l}65 \\
48\end{array}$ & $\begin{array}{l}55 \\
31\end{array}$ & $\begin{array}{l}52 \\
53\end{array}$ & $\begin{array}{l}51 \\
54\end{array}$ & $\begin{array}{l}38 \\
49\end{array}$ & 46 & $\begin{array}{l}70 \\
49\end{array}$ & 65 & $\begin{array}{l}81 \\
45\end{array}$ & $\begin{array}{r}121 \\
46\end{array}$ & $\begin{array}{r}148 \\
46\end{array}$ & $\begin{array}{r}185 \\
37\end{array}$ & $\begin{array}{r}160 \\
48\end{array}$ & $\begin{array}{r}139 \\
52\end{array}$ & $\begin{array}{l}91 \\
47\end{array}$ & $\begin{array}{l}64 \\
33\end{array}$ & $\begin{array}{l}47 \\
39\end{array}$ & $\begin{array}{l}53 \\
44\end{array}$ & $\begin{array}{l}42 \\
37\end{array}$ & $\begin{array}{l}43 \\
35\end{array}$ \\
\hline 981 & 35 & 39 & 46 & 47 & 49 & -46 & 56 & 44 & 52 & 48 & 49 & 45 & 46 & 43 & 40 & 45 & 47 & $40^{\circ}$ & 44 & 37 \\
\hline 1001 & 41 & 39 & 48 & 36 & 49 & $\begin{array}{l}47 \\
47\end{array}$ & 53 & 44 & 26 & 48 & 37 & 39 & 29 & 44 & 46 & 33 & 39 & 38 & 45 & 53 \\
\hline 1021 & 63 & 67 & 63 & 44 & 44 & 36 & 35 & 31 & 31 & 31 & 35 & 29 & 34 & 35 & 32 & 37 & 30 & 34 & 44 & 28 \\
\hline 1041 & 34 & 28 & 32 & 36 & 33 & 39 & -43 & 43 & 43 & 35 & 41 & 33 & 74 & 68 & 66 & 70 & 65 & 54 & 45 & 61 \\
\hline 1061 & 69 & 80 & 119 & 88 & 62 & 50 & 30 & 30 & 24 & 28 & 28 & 25 & 25 & 18 & 33 & 19 & 23 & 26 & 36 & 38 \\
\hline 1081 & 33 & 34 & $\begin{array}{r}39 \\
27\end{array}$ & 27 & 27 & 32 & $\begin{array}{r}31 \\
30\end{array}$ & 30 & -19 & 31 & -35 & 30 & 27 & 26 & 17 & 20 & $\begin{array}{l}27 \\
27\end{array}$ & $-\frac{22}{20}$ & 27 & 26 \\
\hline $\begin{array}{l}1101 \\
1121\end{array}$ & 30 & $\begin{array}{l}20 \\
13\end{array}$ & $\begin{array}{l}27 \\
17\end{array}$ & $\begin{array}{l}20 \\
20\end{array}$ & $\begin{array}{l}20 \\
30\end{array}$ & $\begin{array}{l}10 \\
21\end{array}$ & 25 & $\begin{array}{l}14 \\
17\end{array}$ & $\begin{array}{l}10 \\
20\end{array}$ & 18 & 34 & $\begin{array}{l}24 \\
16\end{array}$ & $\begin{array}{l}18 \\
22\end{array}$ & $\begin{array}{l}20 \\
19\end{array}$ & $\begin{array}{l}25 \\
25\end{array}$ & 22 & $\begin{array}{l}27 \\
26\end{array}$ & $\begin{array}{l}29 \\
22\end{array}$ & $\begin{array}{l}29 \\
17\end{array}$ & 20 \\
\hline 1141 & 20 & 18 & 18 & 21 & 16 & 17 & 17 & 21 & 20 & 24 & 22 & 23 & 26 & 18 & 24 & 21 & 18 & 27 & 23 & 16 \\
\hline 1161 & 15 & 20 & 16 & 18 & 18 & 19 & 21 & 24 & 13 & 22 & 12 & 17 & 23 & 11 & 19 & 17 & 23 & 12 & 17 & 18 \\
\hline 1181 & 18 & 19 & 21 & 13 & 18 & 16 & 20 & 13 & 25 & 20 & 16 & 21 & 15 & 10 & 16 & 23 & 13 & 19 & 27 & 21 \\
\hline 1201 & 32 & 41 & 40 & 57 & 62 & 79 & 58 & 42 & 37 & 24 & 19 & 14 & 17 & 10 & 13 & 14 & 16 & 12 & 16 & 6 \\
\hline $\begin{array}{l}1221 \\
1241\end{array}$ & $\begin{array}{r}13 \\
7\end{array}$ & 11 & 11 & 11 & $\begin{array}{l}7 \\
8\end{array}$ & $\begin{array}{l}16 \\
16\end{array}$ & $\begin{array}{l}17 \\
12\end{array}$ & $\begin{array}{r}15 \\
9\end{array}$ & 13 & $\begin{array}{l}12 \\
13\end{array}$ & $\begin{array}{l}11 \\
11\end{array}$ & $\begin{array}{l}23 \\
10\end{array}$ & $\begin{array}{l}19 \\
13\end{array}$ & $\begin{array}{l}20 \\
14\end{array}$ & $\begin{array}{l}22 \\
16\end{array}$ & $\begin{array}{l}26 \\
10\end{array}$ & $\begin{array}{l}11 \\
10\end{array}$ & $\begin{array}{l}23 \\
11\end{array}$ & 20 & $\begin{array}{r}6 \\
11\end{array}$ \\
\hline 1261 & 9 & 14 & 9 & 12 & 7 & 14 & 6 & 12 & 9 & 7 & 11 & 8 & 8 & 12 & 8 & 7 & 8 & 7 & 10 & 12 \\
\hline 1281 & 14 & 8 & 9 & 5 & 8 & 7 & $B$ & 13 & 14 & 10 & 10 & 6 & 10 & 8 & 8 & 16 & 8 & 3 & 12 & 12 \\
\hline 1301 & 11 & 11 & 10 & 10 & 13 & 11 & 15 & 13 & $-\frac{17}{5}$ & 8 & 8 & $\frac{8}{6}$ & 19 & 9 & 9 & 9 & 22 & 9 & 12 & 17 \\
\hline 1321 & 11 & 15 & 16 & 11 & 10 & 7 & 8 & 14 & 7 & 14 & 10 & 10 & 7 & 11 & 8 & 15 & 11 & 17 & 12 & 12 \\
\hline 1341 & 15 & 11 & 31 & 28 & 24 & 33 & 31 & 28 & 15 & 18 & 10 & 14 & 9 & 10 & 17 & 11 & 12 & 16 & 11 & 9 \\
\hline 1361 & 11 & 10 & 8 & 16 & 8 & 11 & 11 & 12 & 7 & 17 & 8 & 10 & 9 & 12 & 8 & 9 & 7 & 8 & 12 & 6 \\
\hline 1381 & 11 & 12 & 7 & 12 & 13 & 13 & 16 & 7 & 8 & 7 & 9 & 8 & 6 & 13 & 8 & 11 & 7 & 3 & 7 & 11 \\
\hline 1401 & 9. & 7 & 6 & 7 & 10 & 8 & 7 & 15 & 15 & 12 & 10 & 17 & 16 & 9 & 13 & 10 & 6 & 9 & 7 & 15 \\
\hline 1421 & 7 & 12 & 7 & 4 & 0 & 10 & 7 & 13 & 5 & 8 & 4 & 9 & 9 & 7 & 8 & $B$ & 8 & 8 & 5 & 8 \\
\hline $\begin{array}{l}1441\end{array}$ & 12 & 7 & 11. & 9 & & $\begin{array}{r}10 \\
-10\end{array}$ & 10 & & 7 & -13 & 10 & 8 & 12 & 6 & 8 & 7 & 9 & 8 & 7 & 5 \\
\hline 1461 & 8 & 7 & 13 & 9 & 4 & $\frac{6}{6}$ & 8 & 5 & 7 & 4 & 7 & 6 & 7 & 14 & 6 & 8 & 11 & 5 & 9 & 5 \\
\hline 1481 & 2 & 9 & 8 & 10 & 5 & 8 & 7 & 13 & 9 & 8 & 8 & 10 & 8 & 6. & 13 & 12 & 5 & 8 & 6 & \\
\hline $\begin{array}{l}1501 \\
1521\end{array}$ & 9 & 5 & 9 & $?$ & 8 & 8 & 9 & 11 & 12 & 7 & 15 & 5 & 8 & 12 & 15 & 7 & 7 & 11 & 12 & 9 \\
\hline $\begin{array}{l}1521 \\
1541\end{array}$ & 7 & $\begin{array}{r}14 \\
8\end{array}$ & $12^{9}$ & 8 & 9 & 10 & 5 & $\frac{8}{3}$ & $\begin{array}{l}4 \\
8\end{array}$ & 6 & $\frac{4}{13}$ & 20 & 9 & 11 & 11 & 7 & 10 & $-\frac{6}{7}$ & 11 & -0 \\
\hline 1561 & 9 & 13 & 12 & 7 & 15 & 14 & 8 & 7 & 4 & 8 & 5 & 10 & 11 & 8 & 6 & 10 & 3 & 7 & 10 & 8 \\
\hline 1581 & 9 & 10 & 9 & 13 & 8 & 10 & 9 & 12 & 6 & 9 & 7 & 11 & 8 & 13 & 11 & 11 & 9 & 10 & 14 & 6 \\
\hline 1601 & 10 & 9 & $\vec{g}$ & 9 & 9 & 10 & 6 & 5 & 9 & 12 & 4 & 11 & 11 & 7 & 13 & 9 & 10 & 11 & 3 & 7 \\
\hline 1621 & 13 & 11 & 10 & 12 & 10 & 12 & 3 & 10 & 12 & 11 & 9 & 7 & 9 & 7 & 13 & 7 & 8 & 11 & 10 & 7 \\
\hline 1641 & 8 & 5 & 9 & 14 & 9 & 0 & 10 & 6 & 7 & 7 & 12 & 8 & 6 & 8 & 10 & 5 & 6 & 10 & 5 & $B$ \\
\hline 1661 & 5 & 11 & 5 & 9 & 7 & 9 & 9 & 8 & 7 & 9 & 6 & 11 & 14 & 8 & 9 & 11 & 11 & 7 & 6 & 9 \\
\hline 1681 & 8 & 14 & 7 & 8 & 7 & 4 & 10 & 7 & 8 & 12 & 10 & 15 & 6 & 12 & 9 & 12 & 12 & 4 & 13 & $B$ \\
\hline 1701 & 8 & 10 & 13 & 6 & 5 & 15 & 12 & 13 & 11 & 12 & $B$ & 9 & 8 & 7 & 10 & 7 & 11 & 14 & 13 & 6 \\
\hline 1721 & 10 & i1 & 10 & 5 & 10 & 8 & 9 & 5 & 11 & 8 & 16 & 11 & 8 & 11 & 8 & 7 & 10 & 10 & 7 & 6 \\
\hline 1741 & 15 & 6 & 13 & 12 & 10 & 14 & 4 & 5 & 7 & 9 & 13 & 12 & 16 & 7 & 11 & 5 & 11 & 10 & 11 & 9 \\
\hline 1761 & 6 & 11 & 6 & 7 & 8 & 7 & 6 & 7 & 5 & 5 & 5 & 9 & 4 & 4 & 6 & 7 & 5 & 5 & 8 & 3 \\
\hline
\end{tabular}

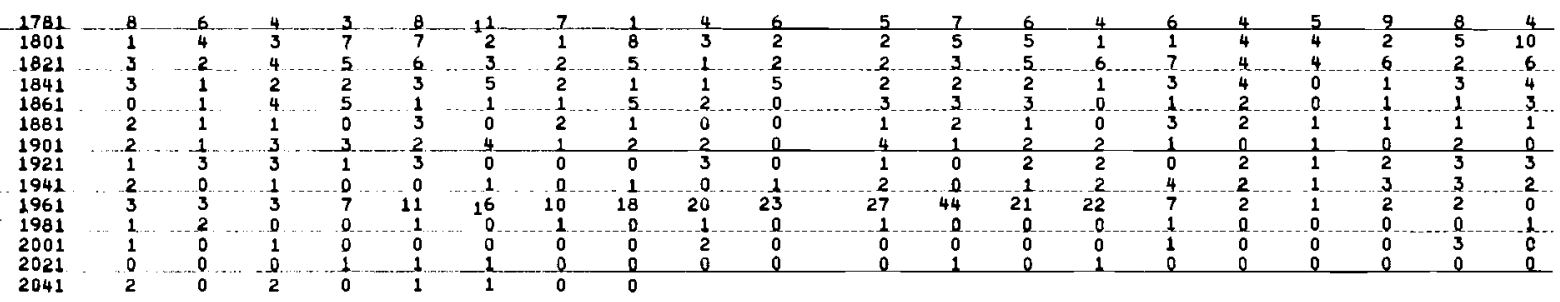


GAMMA RAY SPECTRUM DATA

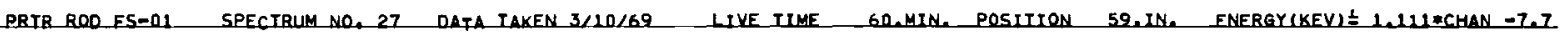

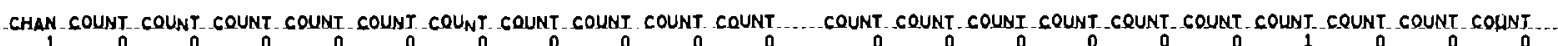

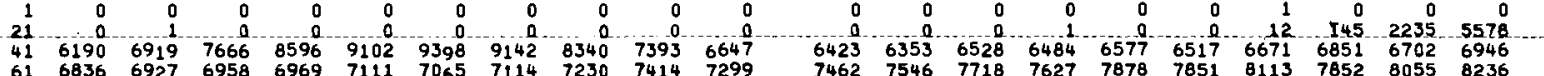

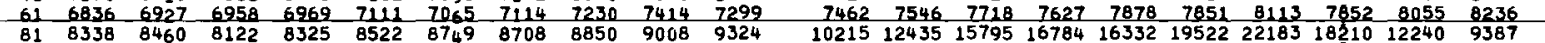

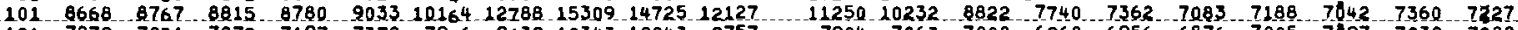

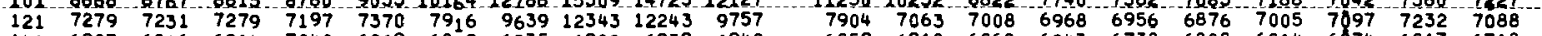

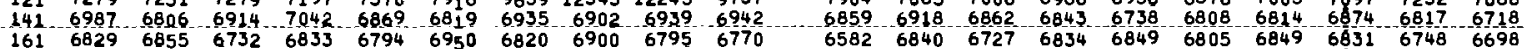

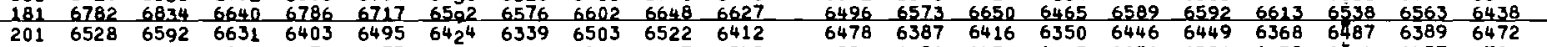

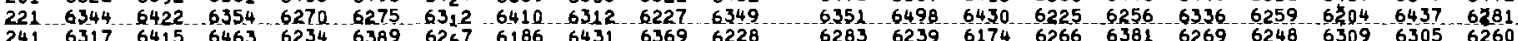

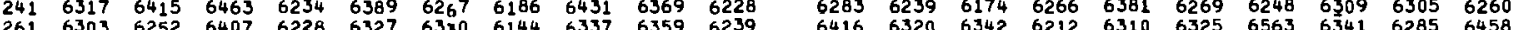
\begin{tabular}{llllllllllllllllll}
261 & 6303 & 6252 & 6407 & 6228 & 6327 & 6330 & 6144 & 6337 & 6359 & 6239 & 6416 & 6320 & 6342 & 6212 & 6310 & 6325 & 6563 \\
281 & 6339 & 6315 & 6223 & 6224 & 6332 & 6379 & 6283 & 6233 & 6464 & 6240 & 6341 & 6285 & 6458 \\
\hline
\end{tabular}

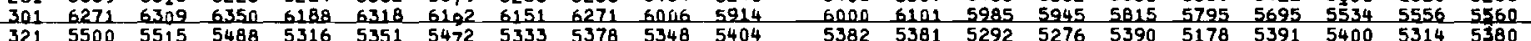

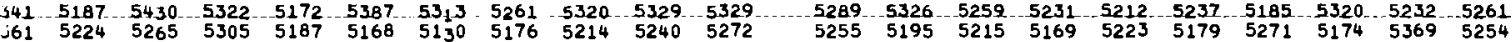

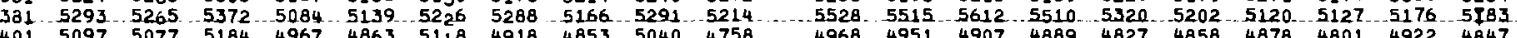
$\begin{array}{llllllllll}4968 & 4951 & 4907 & 4889 & 4827 & 4858 & 4878 & 4801 & 4922 & 4847\end{array}$

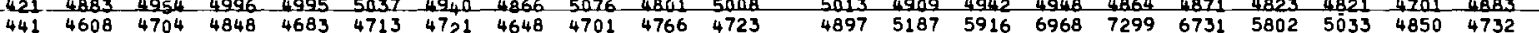

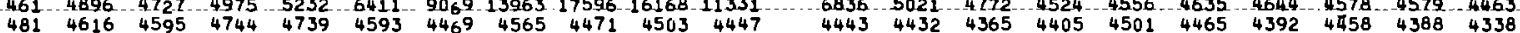

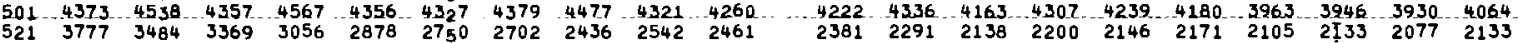

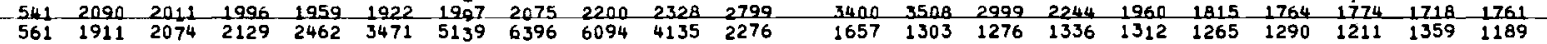

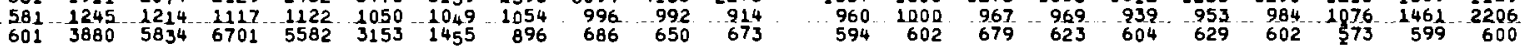

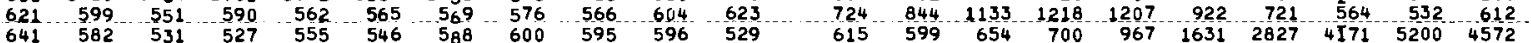

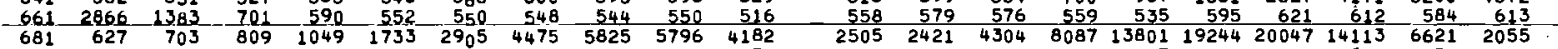

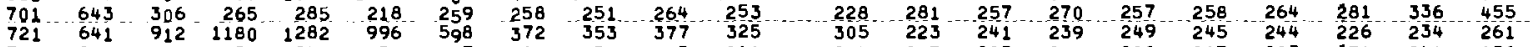

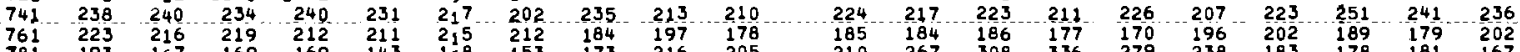
$\begin{array}{llllllllllllllllllllll}781 & 193 & 167 & 169 & 169 & 143 & 168 & 153 & 173 & 216 & 205 & 210 & 267 & 308 & 336 & 279 & 238 & 183 & 178 & 181 & 167\end{array}$

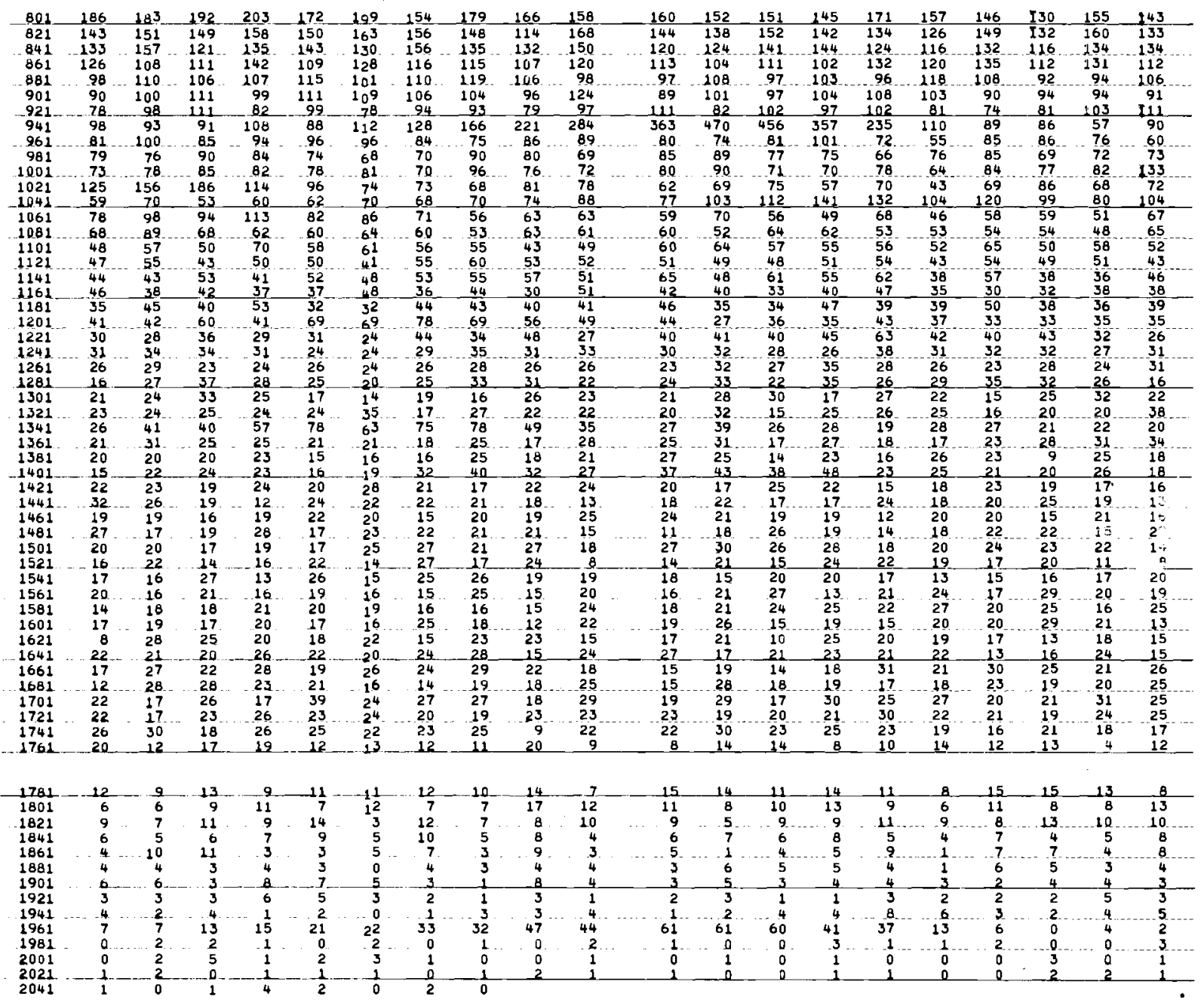




\section{D-17}

GAMMA RAY SPECTRUM DATA

PRTR ROU FS-23 SPECTRUM NO. 22 DATA TAKEN 3/06/69 LIVE TIME 870.MIN. POSITION 6.IN. ENERGY(KEV)= 1.112*CHAN -8.8

CHUN COUNT COUNT COUNT COU, T COUNT COUNT COUNT COUNT COUNT COUNT 21 41120688133615150386167003179934187957191279182632159079139547 61139072138945140784141958142797143795145467145803147662147927
61166379166080166115168151171081174147177754178740183388189080 B1 166379166080166115168151171081174147177754178740183388189080 121144838146080146280145296147536151981172324209624224413194138 141144544142222141562140597140705140549141062140402140774139940 (1) 181138859138739138647137966137168137036136671136066134877135022 201133462132459133076132856132473131944132111132048131982132387 221131602130573130465130237130871130053130229130090129885129295 24112980812905012969112906012928512845612865812916312898512910 281129868129001129608129291129847130195129273129974129637130232 321111805111147110460109833109394109356109112109423108833108586
341106636106649106542105832106356106180105871105192106451105719 361104047103567104372104032104252103407103495102959104430103341 $3 \mathrm{~B} 11 \cup 3129102838103208103361103953102816103464102879103451103731$
4011,049710088910011999357990179828397819973299740396580 4219682997118973939892198907981859775397290969519697

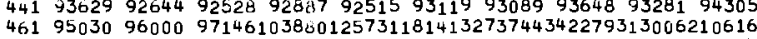

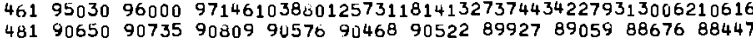

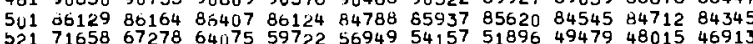

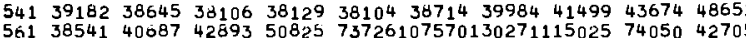

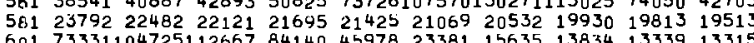

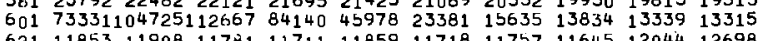
$\begin{array}{llllllllllll}621 & 11853 & 11908 & 11781 & 11711 & 11859 & 11718 & 11757 & 11645 & 12044 & 12698\end{array}$ $\begin{array}{llllllllllll}641 & 11297 & 11421 & 11342 & 11566 & 11360 & 11437 & 11574 & 11570 & 11728 & 11961 \\ 661 & 46399 & 22197 & 13321 & 11161 & 10680 & 10863 & 10768 & 10797 & 10753 & 10980 \\ 681 & 33375 & 14286 & 16886 & 23875 & 34823 & 64686 & 97599118063104801 & 68427\end{array}$ $\begin{array}{rrrrrrrrrrr}681 & 13375 & 14286 & 16886 & 23875 & 38823 & 64686 & 97599118063104801 & 68427 \\ 701 & 9654 & 5926 & 5261 & 5205 & 5095 & 5087 & 4877 & 5144 & 5014 & 4999\end{array}$ $\begin{array}{lllllllllll}721 & 10985 & 14396 & 17482 & 16277 & 11930 & 7931 & 6148 & 5908 & 6014 & 5624\end{array}$

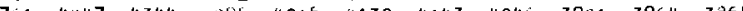
$\begin{array}{ccccccccccc}761 & 4447 & 4344 & 4295 & 4215 & 4132 & 4143 & 4046 & 3921 & 3964 & 3868 \\ -701 & -3530-\cdots 3563 & 3527- & 3599 & -3536 & 3544 & 3529 & 3669 & 3864 & 4280\end{array}$

$\begin{array}{lllllllllll}801 & 3392 & 3579 & 3624 & 3497 & 3389 & 3278 & 3193 & 3201 & 3232 & 3085\end{array}$ $\begin{array}{lllllllllll}821 & 3066 & 3067 & 3027 & 2995 & 2952 & 3045 & 2935 & 2931 & 2934 & 2932\end{array}$ $\begin{array}{lllllllllll}841 & 2663 & 2617 & 2671 & 2544 & 2610 & 2589 & 2547 & 2644 & 2594 & 2630 \\ 861 & 2339 & 2466 & 2391 & 2510 & 2342 & 2276 & 2314 & 2304 & 2304 & 2310\end{array}$ $\begin{array}{lllllllllll}881 & 2172 & 2079 & 2172 & 2112 & 2137 & 2154 & 2102 & 2208 & 2055 & 2107 \\ 901 & 2074 & 2061 & 2147 & 2010 & 2008 & 1918 & 1981 & 1987 & 2012 & 204\end{array}$ $\begin{array}{lllllllllll}901 & 2074 & 2061 & 2147 & 2010 & 2008 & 1918 & 1981 & 1987 & 2012 & 2041 \\ 921 & 1855 & 1889 & 1932 & 1812 & 1869 & 1836 & 1884 & 1817 & 1842 & 1847\end{array}$ $\begin{array}{lllllllllll}921 & 1855 & 1889 & 1932 & 1812 & 1869 & 1836 & 1884 & 1817 & 1842 & 1847 \\ 941 & 1925 & 1945 & 1905 & 1959 & 1979 & 2159 & 2779 & 3681 & 4980 & 6626\end{array}$ $\begin{array}{lllllllllll}961 & 1729 & 1710 & 1794 & 1670 & 1616 & 1613 & 1548 & 1558 & 1512 & 1521 \\ 981 & 1466 & 1544 & 1450 & 1533 & 1502 & 1511 & 1435 & 1559 & 1515 & 1516\end{array}$ $\begin{array}{rrrllllllll}981 & 1466 & 1544 & 1450 & 1533 & 1502 & 1511 & 1435 & 1559 & 1515 & 1516 \\ 1001 & 1462 & 1514 & 1440 & 1455 & 1466 & 1519 & 1527 & 1520 & 1481 & 1487 \\ 1021 & 2949 & 3069 & 2763 & 2144 & 1575 & 1378 & 1362 & 1305 & 1291 & 1281\end{array}$ $\begin{array}{llllllllllll}1041 & 1217 & 1247 & 1267 & 1201 & 1348 & 1286 & 1249 & 1397 & 1570 & 1701\end{array}$ $\begin{array}{llllllllllll}1061 & 1771 & 1983 & 1857 & 1691 & 1491 & 1272 & 1164 & 1268 & 1237 & 1198\end{array}$

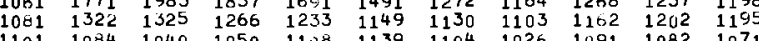
$\begin{array}{rllllllllll}1101 & 1084 & 1040 & 1050 & 1108 & 1139 & 1104 & 1026 & 1091 & 1082 & 1071\end{array}$ $\begin{array}{lllllllllll}1121 & 1065 & 1036 & 1021 & 976 & 1033 & 1015 & 1044 & 1039 & 1027 & 955\end{array}$ $\begin{array}{rrrrrrrrrrr}1141 & 1011 & 1017 & 1029 & 994 & 1075 & 986 & 1063 & 984 & 1068 & 1114 \\ 1161 & 874 & 822 & 815 & 847 & 873 & 805 & 802 & 867 & 813 & 828\end{array}$ $\begin{array}{lllllllllll}1181 & 839 & 822 & 804 & 828 & 800 & 782 & 769 & 803 & 768 & 818\end{array}$ $\begin{array}{rrrrrrrrrrr}12,11 & 874 & 971 & 1153 & 1257 & 1409 & 1307 & 1149 & 977 & 857 & 687\end{array}$ $\begin{array}{lllllllllll}1221 & 669 & 629 & 667 & 664 & 667 & 654 & 663 & 715 & 661 & 686 \\ 1241 & 611 & 642 & 616 & 589 & 553 & 578 & 602 & 592 & 670 & 670\end{array}$ $\begin{array}{lllllllllll}1241 & 611 & 642 & 616 & 589 & 553 & 578 & 602 & 592 & 670 & 670 \\ 1201 & 530 & 564 & 575 & 5 n 3 & 555 & 568 & 568 & 578 & 600 & 566\end{array}$ $\begin{array}{lllllllllll}1281 & 545 & 536 & 572 & 547 & 551 & 513 & 526 & 520 & 570 & 506\end{array}$ $\begin{array}{rrrrrrrrrrr}1301 & 504 & 518 & 530 & 451 & 536 & 491 & 495 & 526 & 484 & 510 \\ 1321 & 473 & 470 & 497 & 450 & 526 & 519 & 516 & 508 & 515 & 532\end{array}$ $\begin{array}{rrrrrrrrrrr}1341 & 778 & 384 & 1072 & 1142 & 1377 & 1497 & 1344 & 1047 & 707 & 564 \\ 13 \mathrm{r} 1 & 470 & 448 & 483 & 434 & 456 & 485 & 446 & 425 & 459 & 432\end{array}$ $\begin{array}{lllllllllll}13 n 1 & 470 & 448 & 483 & 434 & 456 & 485 & 446 & 425 & 459 & 432 \\ 1301 & 402 & 406 & 433 & 445 & 462 & 434 & 417 & 428 & 447 & 422 \\ 14 i 1 & 425 & 421 & 445 & 475 & 465 & 505 & 625 & 635 & 679 & 822\end{array}$ $\begin{array}{lllllllllll}14 i 11 & 425 & 421 & 445 & 475 & 465 & 505 & 625 & 635 & 679 & 822 \\ 1421 & 374 & 403 & 440 & 409 & 381 & 392 & 396 & 401 & 397 & 382\end{array}$ $\begin{array}{lllllllllll}1441 & 396 & 424 & 426 & 440 & 443 & 401 & 396 & 422 & 383 & 423 \\ 14 n 1 & 334 & 370 & 385 & 372 & 361 & 387 & 365 & 388 & 413 & 405\end{array}$ $\begin{array}{lllllllllll}1401 & 380 & 382 & 373 & 398 & 364 & 372 & 394 & 372 & 407 & 346 \\ 1501 & 394 & 370 & 407 & 409 & 390 & 403 & 409 & 487 & 444 & 474\end{array}$

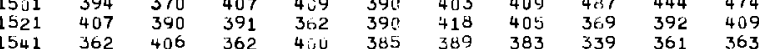
$\begin{array}{lllllllllll}1541 & 362 & 406 & 362 & 4 i, & 385 & 389 & 383 & 339 & 361 & 363 \\ 1501 & 362 & 403 & 377 & 460 & 361 & 410 & 426 & 341 & 380 & 380\end{array}$ $\begin{array}{lllllllllll}1561 & 360 & 474 & 348 & 379 & 361 & 410 & 426 & 381 & 380 & 380 \\ 1611 & 367 & 376 & 399 & 374 & 375 & 376 & 391 & 389 & 408 & 426\end{array}$

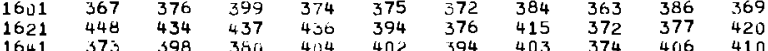
$\begin{array}{lllllllllll}1641 & 373 & 398 & 380 & 404 & 402 & 394 & 403 & 374 & 406 & 410 \\ 1601 & 395 & 361 & 387 & 367 & 370 & 406 & 422 & 412 & 417 & 396\end{array}$ $\begin{array}{lllllllllll}1681 & 406 & 401 & 389 & 306 & 433 & 430 & 386 & 411 & 394 & 393\end{array}$ $\begin{array}{lllllllllll}1701 & 437 & 398 & 415 & 451 & 435 & 391 & 400 & 413 & 423 & 427 \\ 1721 & 451 & 400 & 447 & 460 & 458 & 458 & 454 & 432 & 475 & 475\end{array}$

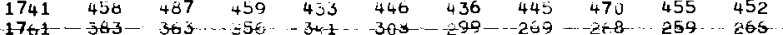

$\begin{array}{rrrrrrrrrrr}1701 & 193 & 195 & 205 & 210 & 229 & 202 & 225 & 195 & 213 & 236 \\ 1801 & 100 & 197 & 171 & 1100 & 170 & 188 & 175 & 162 & 192 & 144 \\ 1021 & 156 & 174 & 144 & 110 & 156 & 157 & 171 & 142 & 168 & 161 \\ 1041 & 131 & 121 & 141 & 131 & 135 & 131 & 130 & 127 & 113 & 106 \\ 1601 & 92 & 104 & 97 & 62 & 90 & 93 & 87 & 81 & 71 & 86 \\ 1581 & 73 & 77 & 62 & 199 & 73 & 70 & 65 & 64 & 68 & 65 \\ 1901 & 100 & 100 & 114 & 120 & 116 & 105 & 110 & 73 & 75 & 85 \\ 1921 & 79 & 49 & 59 & 65 & 59 & 54 & 60 & 55 & 53 & 59 \\ 1941 & 62 & 59 & 56 & 51 & 66 & 62 & 79 & 59 & 42 & 60 \\ 1951 & 200 & 274 & 300 & 432 & 580 & 696 & 813 & 1002 & 1108 & 1221 \\ 1901 & 20 & 20 & 29 & 25 & 19 & 28 & 20 & 15 & 29 & 28 \\ 2001 & 16 & 21 & 19 & 27 & 19 & 23 & 23 & 24 & 21 & 26 \\ 2021 & 30 & 23 & 15 & 19 & 22 & 25 & 24 & 23 & 25 & 18 \\ 2041 & 15 & 22 & 15 & 10 & 16 & 24 & 19 & 0 & & \end{array}$
COHNT COUNT COUNT COUNT COUNT COUNT COUNT COUNT COUNT COUNT

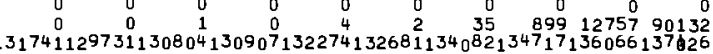
(1) 202602242372317048364620356347401599488686443404302536212265
246967227205195719165831150716146663145661145588145925145231 61647147048143798142072142370141661143157144423147047146427 140214140101140797140186139860139889139527140031139669139105

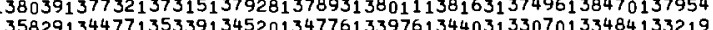
等 31991131669131267131393132064130972130866131522131566131643
129878129753130317129692130125128566130327128609129288130177 129586128530128752128814128805127989128583128724128983128703 $1297621291461296791296331298091283_{1} 4128828129534129196128954$ 130012128963130024129563129424128953129881129075129352128229 123668122386121016119413117846115765115429114049112804112318 $10802210^{8147107519107834107781106544107477107459107086106590}$ 103188103316103389104133103842102888103294102811103496103203 $10581510^{8164109716107781105008102557102540101889101726101281}$ $\begin{array}{llllllllll}97169 & 95843 & 96637 & 96868 & 96039 & 96791 & 96528 & 96592 & 96506 & 97165 \\ 96217 & 96635 & 96054 & 96239 & 96926 & 95950 & 94940 & 94597 & 93345 & 94084\end{array}$

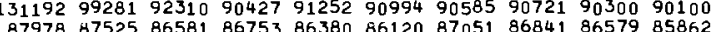
$\begin{array}{llllllllll}83343 & 83679 & 82532 & 82993 & 81965 & 79239 & 78094 & 76771 & 76347 & 74434\end{array}$

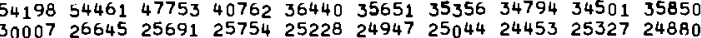
$\begin{array}{lllllllllll}18998 & 18713 & 18659 & 18423 & 18309 & 18222 & 19010 & 21209 & 28726 & 44996\end{array}$ $\begin{array}{lllllllllll}13182 & 13198 & 12893 & 12743 & 12467 & 12387 & 12386 & 12250 & 12251 & 12019\end{array}$ $\begin{array}{llllllllllll}14754 & 17976 & 22363 & 24808 & 22298 & 16925 & 13375 & 11765 & 11292 & 11352\end{array}$ $\begin{array}{llllllllll}12429 & 12427 & 13025 & 15556 & 22276 & 36330 & 60544 & 88652100641 & 80656 \\ 11157 & 1092 & 11278 & 11366 & 11720 & 11737 & 12216 & 12161 & 12208 & 12631\end{array}$ 44108524471002001888763082713963883641932242129133127987 $\begin{array}{llllllllll}4995 & 4987 & 5023 & 5062 & 5005 & 4935 & 5226 & 5465 & 6260 & 7980 \\ 5115 & 4762 & 4789 & 4545 & 4625 & 4714 & 4658 & 4634 & 4604 & 4616\end{array}$ $\begin{array}{lllllllllll}5115 & 4762 & 4789 & 4545 & 4625 & 4714 & 4658 & 4634 & 4604 & 4616 \\ 4542 & 4506 & 4625 & 4413 & 4536 & 4550 & 4502 & 4631 & 4450 & 4543\end{array}$ $\begin{array}{llllllllll}3858 & 3727 & 3674 & 3653 & 3633 & 3521 & 3686 & 3661 & 3583 & 3554 \\ 5174 & 5886 & 6541 & 5865 & 4775 & 3869 & 3386 & 3368 & 3352 & 3365\end{array}$

$\begin{array}{llllllllll}3152 & 3120 & 3124 & 3157 & 3050 & 3050 & 3218 & 3015 & 3084 & 3023\end{array}$ $\begin{array}{lllllllllll}2993 & 2945 & 2812 & 2744 & 2804 & 2752 & 2695 & 2768 & 2669 & 2669 \\ 2542 & 2628 & 2503 & 2521 & 2436 & 2452 & 2510 & 2529 & 2463 & 2310\end{array}$ $\begin{array}{llllllllll}2359 & 2205 & 2200 & 2252 & 2196 & 2148 & 2213 & 2233 & 2217 & 2150\end{array}$ $\begin{array}{llllllllll}2048 & 2183 & 2040 & 1942 & 1936 & 2042 & 2055 & 2031 & 2034 & 2049\end{array}$ $\begin{array}{llllllllll}2081 & 2018 & 1996 & 1947 & 1932 & 1931 & 1963 & 1880 & 1973 & 1832\end{array}$ $\begin{array}{llllllllll}1822 & 1799 & 1822 & 1831 & 1862 & 1876 & 1886 & 1889 & 1893 & 1919\end{array}$ $\begin{array}{llllllllll}8415 & 9310 & 8467 & 5620 & 3233 & 2044 & 1705 & 1726 & 1611 & 1678\end{array}$ $\begin{array}{llllllllll}510 & 1460 & 1468 & 1447 & 1499 & 1476 & 1410 & 1516 & 1445 & 1432\end{array}$ $\begin{array}{llllllllll}1502 & 1356 & 1442 & 1396 & 1395 & 1524 & 1655 & 1909 & 2193 & 2638 \\ 1319 & 1341 & 1231 & 1307 & 1210 & 1293 & 1212 & 1266 & 1283 & 1240\end{array}$ $\begin{array}{llllllllll}1894 & 2060 & 2339 & 2489 & 2410 & 2052 & 1733 & 1506 & 1555 & 1624\end{array}$ $\begin{array}{llllllllll}1242 & 1106 & 1071 & 1095 & 1165 & 1210 & 1216 & 1249 & 1248 & 1327 \\ 1243 & 1150 & 1148 & 1125 & 1093 & 1053 & 1009 & 1084 & 1108 & 1094\end{array}$ $\begin{array}{llllllllll}1077 & 1064 & 1057 & 1003 & 1059 & 1052 & 1085 & 1034 & 1071 & 1036\end{array}$

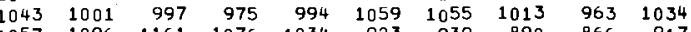
$\begin{array}{rrrrrrrrrr}1057 & 1096 & 1161 & 1076 & 1034 & 923 & 939 & 890 & 866 & 917 \\ 841 & 807 & 836 & 811 & 814 & 835 & 824 & 768 & 810 & 777\end{array}$ $\begin{array}{lllllllllll}790 & 831 & 842 & 753 & 815 & 793 & 751 & 811 & 906 & 876 \\ 689 & 767 & 725 & 781 & 773 & 698 & 675 & 693 & 689 & 729\end{array}$ $\begin{array}{llllllllll}689 & 767 & 725 & 781 & 773 & 698 & 675 & 693 & 689 & 729 \\ 749 & 787 & 845 & 832 & 824 & 742 & 727 & 650 & 583 & 630\end{array}$

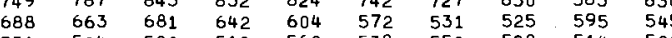
$\begin{array}{llllllllll}531 & 504 & 520 & 510 & 562 & 538 & 550 & 528 & 514 & 520 \\ 532 & 562 & 562 & 566 & 487 & 551 & 504 & 518 & 545 & 513\end{array}$ $\begin{array}{llllllllll}459 & 501 & 488 & 506 & 479 & 486 & 508 & 494 & 476 & 461\end{array}$ $\begin{array}{llllllllll}521 & 538 & 544 & 501 & 466 & 514 & 552 & 584 & 584 & 696 \\ 553 & 551 & 525 & 531 & 519 & 527 & 465 & 484 & 470 & 452\end{array}$ $\begin{array}{llllllllll}476 & 461 & 474 & 445 & 498 & 473 & 460 & 445 & 434 & 459\end{array}$ $\begin{array}{llllllllll}400 & 412 & 405 & 439 & 434 & 440 & 442 & 392 & 441 & 439 \\ 831 & 807 & 717 & 641 & 499 & 463 & 425 & 385 & 436 & 407 \\ 362 & 401 & 396 & 427 & 416 & 414 & 400 & 414 & 420 & 416\end{array}$ $\begin{array}{llllllllll}362 & 401 & 396 & 427 & 416 & 414 & 400 & 414 & 420 & 416 \\ 427 & 384 & 439 & 398 & 372 & 417 & 422 & 397 & 406 & 402\end{array}$ $\begin{array}{llllllllll}403 & 305 & 370 & 410 & 404 & 411 & 389 & 377 & 399 & 413 \\ 391 & 399 & 409 & 375 & 365 & 387 & 394 & 410 & 388 & 380\end{array}$ $\begin{array}{llllllllll}391 & 399 & 409 & 375 & 365 & 387 & 394 & 410 & 388 & 380 \\ 516 & 513 & 492 & 436 & 446 & 378 & 400 & 364 & 374 & 393\end{array}$ $\begin{array}{lllll}359 & 395 & 375 & 405 & 392\end{array}$ $\begin{array}{llllllllll}394 & 381 & 394 & 420 & 356 & 390 & 384 & 389 & 366 & 396 \\ 4 & 490 & 418 & 461 & 439 & 427 & 387 & 402 & 367 & 381\end{array}$ $\begin{array}{llllllllll}405 & 439 & 418 & 461 & 439 & 427 & 387 & 402 & 367 & 381 \\ 381 & 379 & 392 & 376 & 395 & 362 & 429 & 426 & 407 & 384\end{array}$ $\begin{array}{llllllllll}403 & 365 & 383 & 375 & 379 & 391 & 367 & 376 & 375 & 400 \\ 367 & 401 & 408 & 449 & 379 & 366 & 396 & 409 & 362 & 398\end{array}$ $\begin{array}{llllllllll}445 & 374 & 398 & 434 & 430 & 433 & 407 & 441 & 400 & 397\end{array}$ $\begin{array}{llllllllll}405 & 410 & 409 & 444 & 387 & 443 & 404 & 427 & 421 & 426 \\ 431 & 401 & 454 & 407 & 426 & 407 & 454 & 400 & 440 & 434\end{array}$ $\begin{array}{llllllllll}455 & 430 & 477 & 457 & 426 & 407 & 454 & 400 & 440 & 434 \\ 435 & 435 & 434 & 388 & 456 & 473 & 463 & 423 & 510 & 438 \\ & 441 & 411 & 393 & 411 & 375\end{array}$

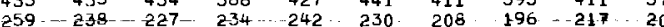

$\begin{array}{rrrrrrrrrr}223 & 224 & 230 & 212 & 232 & 236 & 197 & 162 & 187 & 182 \\ 191 & 179 & 171 & 158 & 156 & 192 & 176 & 160 & 186 & 174 \\ 149 & 163 & 133 & 139 & 143 & 135 & 135 & 136 & 157 & 126 \\ 116 & 144 & 103 & 101 & 117 & 96 & 111 & 109 & 83 & 83 \\ 91 & 77 & 83 & 86 & 75 & 85 & 92 & 59 & 73 & 86 \\ 67 & 63 & 62 & 71 & 76 & 73 & 64 & 64 & 91 & 86 \\ 48 & 49 & 57 & 52 & 62 & 72 & 57 & 58 & 51 & 54 \\ 59 & 42 & 59 & 72 & 46 & 58 & 53 & 52 & 57 & 62 \\ 55 & 63 & 55 & 69 & 65 & 56 & 73 & 102 & 131 & 176 \\ 1291 & 1043 & 576 & 301 & 120 & 50 & 40 & 31 & 33 & 25 \\ 25 & 35 & 24 & 28 & 22 & 26 & 22 & 20 & 22 & 30 \\ 20 & 25 & 23 & 31 & 32 & 16 & 23 & 24 & 22 & 21 \\ 22 & 13 & 22 & 19 & 11 & 12 & 28 & 19 & 22 & 23\end{array}$




\section{D-18}

GAMMA RAY SPECTRUM DATA

PRTR ROD FS-23 SPECTRUM NO. 21 DATA TAKEN 3/06/69 LIVE TIME 330.MIN. POSITION 9.IN. ENERGY(KEV) 1.111 . 1 CHAN -8.1

CHAN COUNT COUNT COUNT COUINT COUNT COUNT COUNT COUNT COUNT COUNT

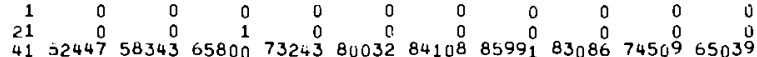

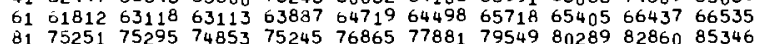

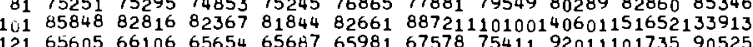
$\begin{array}{lllllllllll}141 & 65628 & 64383 & 63809 & 63649 & 63593 & 63135 & 63594 & 63207 & 63222 & 63026\end{array}$ $\begin{array}{llllllllllll}141 & 65628 & 6458 & 62748 & 63019 & 62719 & 62785 & 62463 & 63470 & 62894 & 62363 & 62369\end{array}$

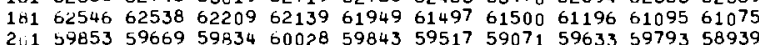
$\begin{array}{lllllllllll}2 \text { Li } 1 & 59853 & 59669 & 59834 & 60028 & 59843 & 59517 & 59071 & 59633 & 59793 & 58939 \\ 221 & 59165 & 59081 & 58798 & 58640 & 59137 & 58791 & 58931 & 58605 & 58498 & 58846\end{array}$ $241588125811458265580145859758746 \quad 58448 \quad 580945874058002$ $\begin{array}{lllllllllll}201 & 58050 & 58005 & 57860 & 57772 & 58234 & 57918 & 58405 & 57987 & 58516 & 58116\end{array}$ $\begin{array}{lllllllllll}281 & 28356 & 57990 & 57959 & 58006 & 58397 & 58486 & 58454 & 58740 & 58609 & 58504 \\ 31,1 & 57666 & 57504 & 57457 & 57183 & 57336 & 56947 & 56501 & 56463 & 56239 & 56149\end{array}$ $32150402501244973649711 \quad 49674 \quad 4925449092486944903949006$ $\begin{array}{lllllllllll}341 & 47783 & 47859 & 47996 & 47888 & 47946 & 47378 & 47727 & 47362 & 47313 & 47643 \\ 301 & 47044 & 47047 & 47149 & 40553 & 46569 & 46543 & 46659 & 46549 & 46862 & 46695\end{array}$ $\begin{array}{lllllllllllll}301 & 46099 & 46347 & 46680 & 46290 & 46427 & 46311 & 46343 & 46656 & 46827 & 46512\end{array}$

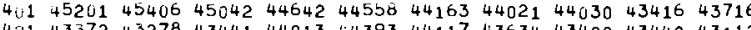
$\begin{array}{llllllllllll}421 & 43372 & 43278 & 43441 & 44013 & 44393 & 44117 & 43634 & 43400 & 43440 & 43112\end{array}$

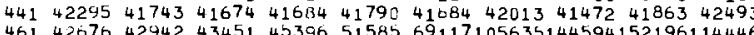
$4 a 140483 \quad 40035 \quad 41185 \quad 40594 \quad 40717 \quad 40766 \quad 40346 \quad 40366 \quad 4069539934$ $521 \quad 331193152729483278392619024903 \quad 23966 \quad 22824 \quad 21956 \quad 21320$

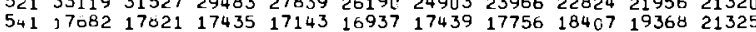
$\begin{array}{llllllllllll}5 \text { tr } & 10842 & 18060 & 18540 & 20558 & 27718 & 41038 & 54771 & 56572 & 42591 & 25378\end{array}$ $\begin{array}{rrrrrrrrrrr}581 & 14893 & 10507 & 10143 & 9751 & 9658 & 9622 & 9348 & 9139 & 9168 & 8765 \\ 6.01 & 25151 & 38526 & 48255 & 43449 & 28179 & 14534 & 8392 & 6715 & 6196 & 6133\end{array}$ $\begin{array}{lllll}534 & 8392 & 6715 & 6196 & 6133\end{array}$

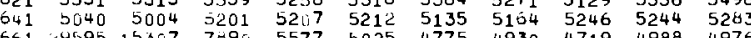

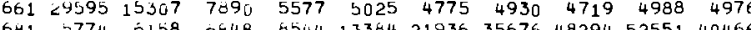
$\begin{array}{lllllllllll}641 & 5774 & 0158 & 0848 & 8544 & 13384 & 21936 & 35676 & 48294 & 52551 & 40466\end{array}$ $\begin{array}{lllllllllll}701 & 8404 & 3502 & 2566 & 2442 & 2370 & 2260 & 2310 & 2268 & 2263 & 2190 \\ 721 & 4219 & 5699 & 7742 & 8340 & 7260 & 4953 & 3326 & 2775 & 2737 & 2750\end{array}$ $\begin{array}{lllllllllll}741 & 2095 & 2071 & 2033 & 1945 & 2172 & 2133 & 2021 & 2057 & 2086 & 2027\end{array}$

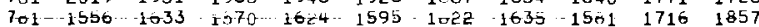

COINT COUNT COUNT COUNT COUNT COUNT COUNT COUNT COUNT COUNT

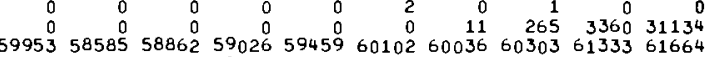

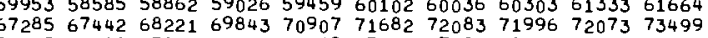
$903251056881378001633444163069_{177863217783208438149338102886}$ $\begin{array}{lllllllll}14086104564 & 90814 & 76792 & 69024 & 67059 & 65659 & 65834 & 65561 & 65231\end{array}$ $\begin{array}{llllllllll}62798 & 63336 & 62942 & 63004 & 62828 & 62673 & 62847 & 62795 & 62627 & 62514\end{array}$ $\begin{array}{llllllllll}62499 & 62001 & 62763 & 62286 & 62296 & 62033 & 62493 & 62568 & 62396 & 62315\end{array}$ $\begin{array}{llllllllll}60649 & 60578 & 60343 & 60734 & 60645 & 59953 & 60310 & 60057 & 59882 & 59839 \\ 59697 & 59299 & 59318 & 59335 & 59087 & 58740 & 59135 & 58965 & 58976 & 59198\end{array}$

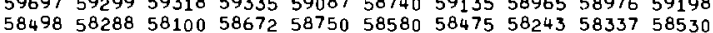
$\begin{array}{llllllllll}58498 & 58288 & 58100 & 58672 & 58750 & 58580 & 58475 & 58243 & 58337 & 58530 \\ 58023 & 57898 & 58290 & 57721 & 57578 & 57999 & 57614 & 58052 & 58404 & 57871\end{array}$ $\begin{array}{llllllllll}58023 & 57898 & 58290 & 57721 & 57578 & 57999 & 57614 & 58052 & 58404 & 57871 \\ 58267 & 57741 & 58370 & 58754 & 58812 & 58040 & 58183 & 58043 & 57972 & 58403\end{array}$ $\begin{array}{llllllllll}58825 & 58076 & 58175 & 58634 & 58351 & 58182 & 58270 & 57666 & 58132 & 57563 \\ 55900 & 55256 & 55045 & 53896 & 53207 & 52554 & 52087 & 51079 & 50709 & 50599\end{array}$ $\begin{array}{llllllllll}48358 & 48594 & 48852 & 48531 & 48570 & 48236 & 48048 & 48348 & 48443 & 47907\end{array}$ $\begin{array}{llllllllllll}48081 & 47699 & 47646 & 47329 & 47113 & 47058 & 46998 & 47236 & 47192 & 46843\end{array}$ $\begin{array}{llllllllllll}46637 & 46274 & 46576 & 46556 & 46302 & 46738 & 46437 & 46467 & 46504 & 46250\end{array}$ $\begin{array}{llllllllll}47291 & 48105 & 49099 & 48961 & 47602 & 46087 & 45980 & 45979 & 45618 & 45217 \\ 43667 & 42718 & 43258 & 43194 & 43265 & 43021 & 43078 & 43361 & 43358 & 43331\end{array}$

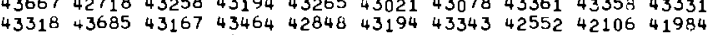
$\begin{array}{llllllllll}42795 & 44644 & 49447 & 58076 & 65237 & 636446 & 54970 & 46700 & 43617 & 42814 \\ 71819 & 49453 & 42751 & 40926 & 40815 & 40389 & 41118 & 40964 & 40658 & 40695\end{array}$

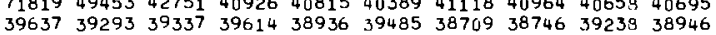
$\begin{array}{llllllllll}39637 & 39293 & 39337 & 39614 & 38936 & 39485 & 38709 & 38746 & 39238 & 38946 \\ 37986 & 37422 & 37703 & 37671 & 36911 & 36517 & 35265 & 34828 & 34757 & 34235\end{array}$

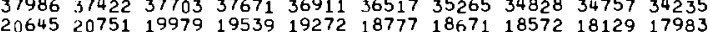
$\begin{array}{lllllllllll}20645 & 20751 & 19979 & 19539 & 19272 & 18777 & 18671 & 18572 & 18129 & 17983 \\ 24288 & 26056 & 24207 & 20225 & 17477 & 16312 & 16149 & 15874 & 15696 & 15644\end{array}$

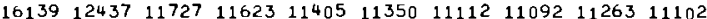
$\begin{array}{rrrrrrrrrr}8674 & 8737 & 8417 & 8388 & 8304 & 8135 & 8298 & 8736 & 10683 & 15490 \\ 6109 & 6085 & 5931 & 5778 & 5777 & 5606 & 5638 & 5513 & 5508 & 5286\end{array}$

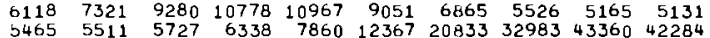
$\begin{array}{rrrrrrrrrr}5465 & 5511 & 5727 & 6338 & 7860 & 12367 & 20833 & 32983 & 43360 & 42284 \\ 4942 & 4869 & 5073 & 5057 & 5219 & 5241 & 5314 & 5399 & 5597 & 5605\end{array}$ $2512019791 \quad 3079360530107045159098178703139613 \quad 72792 \quad 26715$ $\begin{array}{llllllllll}2278 & 2314 & 2342 & 2248 & 2311 & 2341 & 2372 & 2428 & 2686 & 3115 \\ 2549 & 2312 & 2141 & 2098 & 2156 & 2115 & 2079 & 2119 & 2094 & 2101\end{array}$ $\begin{array}{llllllllll}2026 & 2040 & 2073 & 2007 & 2040 & 1969 & 2097 & 1952 & 1996 & 1951 \\ 1662 & 1781 & 1771 & 1680 & 1658 & 1600 & 1633 & 1609 & 1675 & 1598\end{array}$

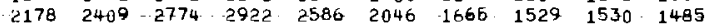

\begin{tabular}{|c|c|c|c|c|c|c|c|c|c|c|c|c|c|c|c|c|c|c|c|}
\hline 1543 & 1526 & 1012 & $16 b 2$ & 1573 & 1489 & 1465 & 1445 & 1460 & 1418 & 1418 & 1387 & 1451 & 1430 & 1440 & 1308 & 1407 & 1410 & 1376 & 1378 \\
\hline 1401 & 1428 & 1382 & 1328 & 1378 & 1379 & 1307 & 1320 & 1343 & 1329 & 1349 & 1296 & 1293 & 1211 & 1286 & 1265 & 1263 & 1193 & 1146 & 1156 \\
\hline 1196 & 1198 & 1207 & 1190 & 1129 & 1152 & $117_{0}$ & 1190 & 1122 & 1161 & 1245 & 1154 & 1178 & 1106 & 1137 & 1077 & 1073 & 1065 & 1079 & 1085 \\
\hline 1077 & 1011 & 1110 & 1084 & 1080 & 1053 & 1017 & 1039 & 1015 & 1024 & 1041 & 1051 & 991 & 1021 & 1005 & 986 & 1060 & 994 & 999 & 940 \\
\hline 956 & 947 & 948 & $10 \cup 1$ & 945 & 931 & 938 & 934 & 989 & 909 & 960 & 838 & 953 & 876 & 866 & 907 & 907 & 897 & $89_{0}$ & 899 \\
\hline 901 & 928 & 968 & 900 & 939 & 924 & 884 & 887 & 965 & 956 & 928 & 1048 & 974 & 912 & 889 & 859 & 829 & 859 & 822 & 859 \\
\hline 806 & 830 & 812 & 835 & 820 & 827 & 841 & 872 & 797 & 828 & 847 & $B_{0} 8$ & 800 & 797 & 851 & 842 & 824 & 853 & 848 & 846 \\
\hline 841 & 872 & 894 & 832 & 882 & 926 & $1010^{\circ}$ & 1326 & 1811 & 2475 & 3265 & 3998 & 4150 & 3333 & 2213 & 1365 & 882 & 769 & 755 & 722 \\
\hline 753 & 765 & 806 & 709 & 687 & 580 & 743 & 713 & 645 & 738 & 679 & 686 & 658 & 672 & 670 & 664 & 661 & 691 & 644 & $7_{13}$ \\
\hline 654 & 691 & 721 & 700 & 705 & 702 & 686 & 709 & 664 & 714 & 685 & 652 & 627 & 659 & 643 & 635 & 639 & 627 & 667 & 725 \\
\hline 645 & 058 & 645 & 7.1 & 679 & 707 & 706 & 655 & 666 & 677 & 656 & 687 & 645 & 673 & 634 & 675 & 738 & 814 & 935 & 1026 \\
\hline 1157 & 1394 & 1395 & 1101 & 889 & 708 & 598 & 570 & 568 & 578 & 554 & 605 & 589 & 586 & 589 & 596 & 574 & 566 & 552 & 550 \\
\hline 582 & $\begin{array}{r}541 \\
\end{array}$ & $\begin{array}{r}569 \\
\end{array}$ & 526 & 546 & 611 & 568 & 627 & 629 & 724 & 798 & 852 & 921 & 1141 & 1103 & 1035 & 857 & 715 & 698 & 693 \\
\hline 001 & 780 & $\therefore 03$ & 751 & 713 & 590 & 534 & 511 & 547 & 522 & 521 & 509 & 541 & 464 & 539 & 489 & 508 & 526 & 619 & 608 \\
\hline 620 & 600 & 651 & $5 \vdash 4$ & 542 & 521 & 533 & 507 & 510 & 575 & 535 & 549 & 527 & 535 & 526 & 484 & 488 & 497 & 483 & 511 \\
\hline 405 & 495 & 485 & $4 \varepsilon 5$ & 506 & 480 & 526 & 442 & 508 & 460 & 440 & 513 & 486 & 465 & 472 & 493 & 434 & 446 & 489 & 449 \\
\hline 556 & 475 & 500 & 500 & 475 & 490 & 462 & 516 & 439 & 475 & 484 & 501 & 458 & 438 & 428 & 454 & 470 & 465 & 454 & 450 \\
\hline 463 & 454 & 459 & $4 \angle 0$ & 456 & 432 & 473 & 475 & 470 & 510 & 479 & 509 & 537 & 575 & 514 & 513 & 445 & 383 & 375 & 416 \\
\hline 375 & 386 & 402 & 416 & 377 & 383 & 363 & 386 & 386 & 386 & 412 & 373 & 360 & 362 & 408 & 395 & 351 & 409 & 367 & 356 \\
\hline 393 & 366 & 367 & $3 ; 7$ & 382 & 369 & 338 & 357 & 371 & 339 & 355 & 394 & 386 & 349 & 377 & 326 & 388 & 367 & 334 & 369 \\
\hline 359 & 356 & 419 & 477 & 509 & 556 & 537 & 482 & 417 & 379 & 324 & 320 & 321 & 339 & 325 & 318 & 348 & 322 & 345 & $3 \geq 9$ \\
\hline 310 & 310 & 309 & 294 & 280 & 289 & 311 & 338 & 318 & 329 & 338 & 356 & 361 & 382 & $\begin{array}{l}20 \\
408\end{array}$ & $\begin{array}{l}310 \\
\end{array}$ & 358 & 345 & 306 & 200 \\
\hline 294 & 291 & $28 \mathrm{~B}$ & 270 & 267 & 260 & 293 & 283 & 293 & 302 & 272 & 322 & 279 & 309 & 282 & 313 & 284 & 259 & 236 & 227 \\
\hline $25 b$ & 259 & 268 & 244 & 221 & 272 & 248 & 256 & 272 & 270 & 242 & 273 & 294 & 259 & 233 & 257 & 245 & 246 & 245 & 240 \\
\hline 270 & $2 b 0$ & 239 & לS & 252 & 260 & 257 & 241 & $25 \overline{3}$ & 230 & 256 & 259 & 220 & 255 & 233 & 235 & 251 & 222 & 248 & 212 \\
\hline 225 & 254 & 209 & 253 & 232 & 233 & 231 & 209 & 251 & 222 & 209 & 203 & 252 & 232 & 228 & 251 & 211 & 222 & 223 & 234 \\
\hline 244 & 229 & 190 & 225 & 210 & 232 & 240 & 236 & 236 & $\begin{array}{l}2228 \\
228\end{array}$ & 273 & 215 & 237 & 229 & 241 & 210 & 267 & 229 & 255 & 281 \\
\hline 316 & 321 & 376 & 468 & 606 & 664 & 701 & 589 & 491 & 336 & 276 & 245 & 259 & 254 & 277 & 239 & 236 & 234 & 234 & 231 \\
\hline 222 & 229 & 224 & 229 & 211 & 200 & 241 & 200 & 221 & 222 & 208 & 223 & 195 & 192 & 180 & 208 & 222 & 196 & 213 & 201 \\
\hline 103 & 209 & 193 & 208 & 230 & 207 & 196 & 184 & 195 & 181 & 187 & 198 & 199 & 226 & 197 & 201 & 194 & 173 & 213 & 201 \\
\hline 209 & 207 & 231 & 229 & 226 & 218 & 263 & 262 & 293 & 265 & 344 & 359 & 415 & 303 & 264 & 223 & 181 & 164 & 182 & 213 \\
\hline 194 & 170 & 181 & 174 & 180 & 187 & 204 & 174 & 182 & 181 & 172 & 173 & 200 & 190 & 163 & 172 & $\begin{array}{l}181\end{array}$ & 144 & 167 & 176 \\
\hline 191 & 192 & $\begin{array}{r}0 \\
i 79\end{array}$ & 179 & 177 & 201 & $\begin{array}{l}194 \\
\end{array}$ & $\begin{array}{l}192 \\
\end{array}$ & 150 & $\begin{array}{l}181 \\
182\end{array}$ & $\begin{array}{l}196 \\
19\end{array}$ & $\begin{array}{l}182 \\
\text { S }\end{array}$ & 186 & 188 & $\begin{array}{l}103 \\
194\end{array}$ & 169 & $\begin{array}{l}101 \\
193\end{array}$ & $\begin{array}{l}144 \\
158\end{array}$ & $\begin{array}{l}107 \\
155\end{array}$ & $\begin{array}{l}179 \\
189\end{array}$ \\
\hline 183 & 171 & 158 & 1 ino & 163 & 175 & 193 & 208 & 181 & 204 & 197 & 175 & 198 & 187 & 199 & 166 & 178 & 154 & 196 & 172 \\
\hline 193 & 107 & 104 & 170 & 181 & 198 & 180 & 168 & 179 & 174 & 203 & 199 & 193 & 160 & 171 & 190 & 199 & 167 & 173 & 172 \\
\hline 165 & $2 \cup 1$ & 202 & 163 & 190 & 172 & 163 & 188 & 227 & 202 & 204 & 202 & 236 & 224 & 234 & 193 & 208 & 191 & 178 & 181 \\
\hline 171 & 171 & 186 & $\operatorname{lin} 1$ & 100 & 187 & 175 & 179 & 201 & 166 & 148 & 130 & 181 & 182 & 208 & 151 & 156 & 160 & 164 & 161 \\
\hline 188 & 193 & 160 & 176 & 147 & 153 & 161 & 157 & 179 & 173 & 159 & 102 & 181 & 201 & 186 & 161 & 175 & 156 & 179 & 172 \\
\hline 165 & 159 & 191 & 176 & 187 & 180 & 178 & 200 & 164 & 179 & 160 & 169 & 164 & 162 & 192 & 181 & 177 & 171 & 204 & 177 \\
\hline 175 & 155 & 157 & $1 \cap 1$ & 178 & 170 & 171 & 164 & 164 & 166 & 195 & 178 & 189 & 159 & 209 & 201 & 176 & 178 & 195 & 174 \\
\hline 171 & 171 & 102 & 197 & 162 & 166 & 171 & 153 & 173 & 172 & 185 & 150 & 181 & 197 & 170 & 162 & 191 & 177 & 191 & 186 \\
\hline 202 & 200 & 203 & 144 & 167 & 191 & 184 & 177 & 180 & 175 & 174 & $\begin{array}{l}169 \\
169\end{array}$ & 198 & 159 & 179 & 167 & 185 & 180 & 159 & $\begin{array}{l}172 \\
17\end{array}$ \\
\hline 176 & 170 & 176 & 192 & 177 & 170 & 169 & 170 & 193 & 171 & 183 & 163 & 208 & 184 & 182 & 156 & 175 & 194 & 177 & 189 \\
\hline 177 & 180 & 189 & 107 & 172 & 182 & 187 & 197 & 187 & 205 & 175 & 201 & 179 & 175 & 185 & 182 & 147 & 191 & 162 & 190 \\
\hline 174 & 183 & 200 & 215 & 197 & 226 & 165 & 168 & 190 & 15 & 160 & 186 & 183 & 206 & 177 & 184 & 178 & 202 & 209 & 193 \\
\hline 206 & 188 & $19 \mathrm{~b}$ & 1 n 4 & 178 & 191 & 177 & 195 & 205 & 170 & 190 & 195 & 203 & 199 & 201 & 189 & 199 & 160 & $\begin{array}{l}194 \\
\end{array}$ & 212 \\
\hline 109 & 193 & 193 & 197 & 175 & 212 & 174 & 197 & 192 & 191 & 184 & 201 & 191 & 200 & 178 & 215 & 217 & 213 & 192 & 181 \\
\hline$<03$ & 190 & 192 & 212 & 199 & 205 & 201 & 211 & 211 & 198 & 199 & 176 & 176 & 181 & 201 & 208 & 172 & 184 & 176 & 174 \\
\hline 196 & 171 & 173 & 179 & 150 & 39 & 120 & 120 & 160 & 111 & 104 & 105 & 126 & 105 & 93 & 96 & 118 & 114 & 103 & 103 \\
\hline 90 & 100 & م11 & $\therefore 2$ & 101 & 107 & 89 & 98 & 93 & 90 & 101 & 85 & 106 & 110 & 99 & 96 & 97 & 94 & 68 & 83 \\
\hline 73 & 83 & 84 & 99 & 09 & 76 & 80 & 76 & 77 & 72 & 79 & 72 & 89 & 80 & 78 & 84 & 69 & 79 & 81 & 71 \\
\hline 75 & 80 & 74 & 70 & 60 & 61 & 76 & 66 & 68 & 64 & 65 & 72 & 76 & 69 & 78 & 75 & 65 & 58 & 66 & 74 \\
\hline 79 & 71 & 59 & 57 & 51 & 57 & 67 & 55 & 67 & 55 & 54 & 46 & 54 & 48 & 56 & 50 & $\begin{array}{r}43 \\
42\end{array}$ & 47 & $\begin{array}{l}80 \\
46\end{array}$ & 52 \\
\hline 40 & 47 & 30 & $\$ 1$ & 49 & 32 & 45 & 36 & 52 & 44 & 34 & 30 & 44 & 33 & 40 & 30 & 38 & 34 & 37 & 34 \\
\hline 35 & 27 & 26 & 27 & 33 & 20 & 28 & 28 & 27 & 25 & 3 & 24 & 35 & 35 & 24 & 31 & 36 & 41 & 34 & 30 \\
\hline 35 & 37 & 35 & 40 & 45 & 44 & 44 & 41 & 30 & 28 & 25 & 27 & 27 & 33 & 33 & 29 & 27 & 28 & 38 & 26 \\
\hline 26 & 26 & 28 & 33 & 22 & 24 & 28 & 30 & 20 & 25 & 25 & 30 & 28 & 28 & 19 & 23 & 22 & 29 & 28 & 31 \\
\hline 31 & 24 & 24 & D & $\begin{array}{r}22 \\
23\end{array}$ & 28 & $\begin{array}{l}20 \\
26\end{array}$ & 25 & 26 & 21 & 30 & $\begin{array}{l}27 \\
\end{array}$ & $\begin{array}{l}20 \\
26\end{array}$ & $\begin{array}{l}20 \\
26\end{array}$ & 28 & 29 & 32 & 37 & $\begin{array}{l}28 \\
47\end{array}$ & 48 \\
\hline 70 & 100 & 104 & 135 & 180 & 232 & 304 & 357 & 460 & 489 & 563 & 562 & 524 & 410 & 197 & 94 & 32 & 27 & 14 & 19 \\
\hline 13 & 19 & 13 & 35 & 1 & 8 & 8 & 11 & 10 & 1 & 9 & 10 & 9 & 12 & 11 & 7 & 6 & 9 & 10 & 9 \\
\hline 10 & 12 & 13 & 9 & 13 & 9 & 16 & 8 & 10 & 10 & 9 & 5 & 4 & 6 & 8 & 7 & 6 & 8 & 17 & 13 \\
\hline a & 10 & 6 & 10 & 19 & 10 & 12 & 15 & 8 & 8 & 7 & 8 & 12 & 12 & 7 & 10 & 9 & 5 & 15 & 6 \\
\hline & & 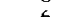 & 5 & 1 & & & 15 & & & & & & & & & & & & \\
\hline
\end{tabular}


GAMMA RAY SPECTRUM DATA

PRTR ROO F5-23 SPECTRUM NO. 18 DATA TAKEN 3105,69 LIVE TIME 300.MIN. POSITION 29.IN, ENERGY(KEVI= 1.115*CHANM10.4 CMAN CUUNT COUNT COLNT COUNT COUNT COUNT COUNT COUNT COUNT COUNT

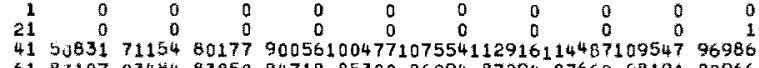
$6183107834448385084718 \quad 85300 \quad 862948729487662 \quad 8819188966$ 101123268112909110039100571102452103818106054107847109991112117 $12158212883778011287704 \quad 887258975396201115182133854128289$

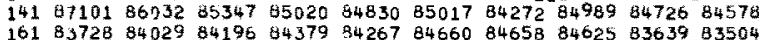

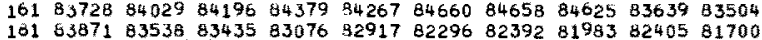
20160415801238030480369798407980279585793347998079555

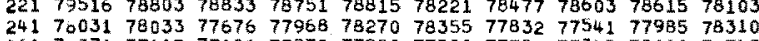
$2817 d 408782907845477690780507837078130783327768378391$ $30177337772417636776711 \quad 7680376367 \quad 76031 \quad 756497538574871$ (1)

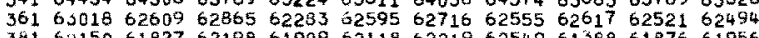
$30162150 \quad 6182762198619096211862219625496136861876 \quad 61956$

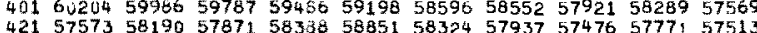
$4415757358190 \quad 578715833858851 \quad 58324 \quad 57937 \quad 57476 \quad 57771 \quad 57513$ $401517025741850774 \quad 63016 \quad 76764109891162983201416164713129215$ $481 \quad 55083 \quad 55118 \quad 5485654742 \quad 5483354566 \quad 5381354381 \quad 53732 \quad 53318$

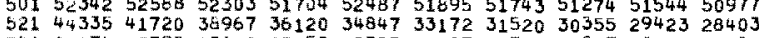
$\begin{array}{lllllllllllllll}541 & 24174 & 23798 & 23619 & 23258 & 23593 & 23587 & 24733 & 25647 & 28460 & 32980\end{array}$ $\begin{array}{lllllllllll}561 & 23453 & 24671 & 26420 & 32909 & 47538 & 67011 & 76738 & 55634 & 42885 & 261 \mathrm{BJ}\end{array}$ $\begin{array}{lllllllllll}501 & 14422 & 13901 & 13156 & 13170 & 12874 & 12889 & 12671 & 12223 & 12009 & 11765\end{array}$

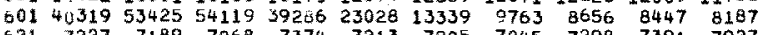
$\begin{array}{lllllllllll}621 & 7227 & 7189 & 7268 & 7374 & 7213 & 7205 & 7045 & 7298 & 7391 & 7927 \\ 641 & 5993 & 7128 & 7075 & 7100 & 7023 & 7091 & 7117 & 7180 & 7250 & 7362\end{array}$ $\begin{array}{rrrrrrrrrrr}561 & 24953 & 13015 & 8409 & 6954 & 6651 & 6722 & 6515 & 6568 & 6618 & 6652 \\ 661 & 5314 & 9069 & 11206 & 16945 & 27687 & 44416 & 62381 & 68696 & 56300 & 36926\end{array}$ $\begin{array}{rrrrrrrrrrr}701 & 6675 & 4013 & 3437 & 3143 & 3258 & 3212 & 3193 & 3063 & 3100 & 3193 \\ 721 & 007 & 11364 & 12829 & 11005 & 7714 & 5079 & 4168 & 4070 & 3892 & 3487\end{array}$

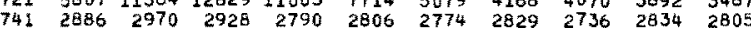
$\begin{array}{lllllllllll}761 & 703 & 2655 & 2595 & 2516 & 2591 & 2448 & 2388 & 2450 & 2369 & 2261 \\ 761 & 2182 & 2181 & 2201 & 2105 & 2117 & 2178 & 2158 & 2325 & 2593 & 2925\end{array}$

\begin{tabular}{|c|c|c|c|c|c|c|c|c|c|c|}
\hline 801 & 2114 & 2299 & 2221 & 2057 & 2130 & 1956 & 1980 & 1875 & 1863 & 1936 \\
\hline 021 & 1865 & 1070 & 1765 & 1857 & 1799 & 1915 & 171 & 1789 & 1036 & 1741 \\
\hline 841 & 1581 & 1556 & 1524 & 1602 & 1616 & 1528 & 1551 & 1636 & 1568 & 1616 \\
\hline 861 & 1421 & 1431 & 1393 & 1400 & 1476 & 1455 & 1395 & 13 . & 1441 & 1388 \\
\hline 831 & 1324 & 1288 & 1323 & 1212 & 1257 & 1307 & 1281 & 1318 & 1330 & 1329 \\
\hline 901 & 1290 & 1349 & 1333 & 1270 & 1203 & 1295 & 1250 & 1221 & 1320 & 1314 \\
\hline 921 & 1182 & 1120 & 1105 & 1140 & 1143 & 1097 & 1126 & 1148 & 1124 & 1043 \\
\hline 941 & 1257 & 1182 & 1150 & 1204 & 1343 & 1576 & 2049 & 2640 & 3728 & 4875 \\
\hline 961 & 1051 & 1093 & 1044 & 966 & 919 & 925 & 973 & 934 & 937 & 872 \\
\hline 981 & 919 & 9118 & 912 & 969 & 859 & 900 & 920 & 954 & 900 & 864 \\
\hline 301 & 855 & 875 & 909 & 902 & 839 & 898 & 897 & 900 & 914 & 899 \\
\hline 1021 & 1853 & 1649 & 1311 & 1106 & 840 & 856 & 794 & 751 & 780 & 774 \\
\hline 1041 & 790 & 758 & 825 & $8+2$ & 780 & B29 & 876 & 975 & 1061 & 1115 \\
\hline 61 & 989 & 935 & 956 & 541 & 780 & 770 & 690 & 741 & 763 & 738 \\
\hline 21 & 794 & 254 & 746 & 764 & 677 & 687 & & 714 & 726 & 730 \\
\hline 101 & 668 & 601 & 037 & 681 & 655 & 566 & 60 & 645 & 685 & 672 \\
\hline 121 & 636 & 625 & 594 & 648 & 669 & 660 & 6.88 & 650 & 640 & 615 \\
\hline 141 & 654 & 624 & 667 & 606 & 617 & 625 & 626 & 678 & 662 & 741 \\
\hline 161 & 540 & 536 & 519 & 530 & 496 & 556 & 467 & 495 & 525 & 477 \\
\hline 81 & 508 & 497 & 502 & 497 & 511 & 494 & 460 & 487 & 473 & 479 \\
\hline 01 & 590 & 576 & $6+24$ & 694 & 653 & 627 & 511 & 480 & 461 & 470 \\
\hline 221 & 405 & $42 B$ & 417 & 400 & 409 & 403 & 414 & 438 & 435 & 481 \\
\hline 241 & 380 & 380 & 384 & 357 & 355 & 385 & 376 & 420 & 417 & 426 \\
\hline 261 & 360 & 351 & 335 & 375 & 392 & 356 & 317 & 335 & 365 & 311 \\
\hline$B$ & 364 & 322 & 335 & 356 & 337 & 316 & 297 & 304 & 327 & 315 \\
\hline 1301 & 345 & 325 & 301 & 297 & 301 & 346 & 342 & 321 & 306 & 325 \\
\hline 1321 & 289 & 32.3 & 345 & 310 & 312 & 312 & 306 & 335 & 312 & 321 \\
\hline 341 & 581 & 606 & 754 & $\$ 95$ & 854 & 744 & 612 & 437 & 378 & 331 \\
\hline 01 & 294 & 291 & 269 & 259 & 311 & 265 & & 267 & & 257 \\
\hline 1 & 269 & 261 & 269 & 26 & 29 & 264 & & 241 & & 240 \\
\hline 1401 & 269 & 285 & 303 & 298 & 338 & 387 & 390 & 451 & 466 & 481 \\
\hline 1421 & 251 & 237 & 258 & 251 & 230 & 218 & 238 & 231 & 249 & 254 \\
\hline 1441 & 263 & 243 & 243 & 38 & 224 & 235 & 248 & 243 & 238 & 255 \\
\hline 1461 & 254 & 240 & 247 & 222 & 228 & 209 & 24 & 258 & 252 & 244 \\
\hline 41 & 241 & 244 & 221 & & & & & 247 & & 230 \\
\hline & 250 & $23 \mathrm{E}$ & 23 & 24 & 28 & 26 & & 30 & 288 & 333 \\
\hline 1521 & 241 & 274 & 219 & 231 & 25 & 235 & 21 & 214 & 237 & 236 \\
\hline 1541 & 219 & 217 & & & & & & 22 & 23 & 225 \\
\hline 150 & 241 & 22 & 26 & 22 & & & & 24 & 232 & 253 \\
\hline 8 & 216 & 24 & 24 & & $2 E$ & & & 24 & 287 & 259 \\
\hline 0 & 218 & 218 & 21 & 25 & 21 & 23 & & 23 & & 240 \\
\hline 1621 & 263 & 264 & 25 & 26 & 26 & 220 & 22 & 22 & 228 & 221 \\
\hline 1641 & 265 & 208 & 238 & 249 & 237 & 24 & & 236 & 244 & 246 \\
\hline 1601 & 239 & 235 & & & & & & 23 & & 237 \\
\hline 160 & 235 & 248 & 256 & 26 & 240 & 24 & 25 & 23 & 232 & 228 \\
\hline 170 & 252 & 221 & 26 & 27 & & 228 & 25 & 25 & 259 & 269 \\
\hline 1721 & 279 & 240 & 287 & 28 & 274 & & & 29 & 278 & 269 \\
\hline 1741 & 267 & 293 & 259 & 298 & 269 & 269 & 253 & 254 & 264 & 282 \\
\hline 1761 & 213 & 220 & 202 & 193 & 164 & 164 & 157 & 158 & 155 & 134 \\
\hline 1 & 115 & 124 & & 125 & & & & & & 139 \\
\hline 180 & 102 & 110 & 113 & 39 & 11 & 10 & i3? & 100 & $\begin{array}{l}181 \\
99\end{array}$ & 11.1 \\
\hline 132 & 109 & 114 & 87 & 102 & 11 & 90 & 93 & 8 & 87 & 85 \\
\hline & 79 & $8 B$ & 7 & 31 & & 100 & 6 & 6 & 64 & 68 \\
\hline & 50 & 44 & & & 5 & & 6 & 57 & 48 & 59 \\
\hline 081 & 45 & 42 & 44 & 36 & 53 & 33 & 60 & 36 & 50 & 40 \\
\hline & 79 & 61 & 62 & 56 & 60 & 39 & 54 & 43 & 37 & 39 \\
\hline & 35 & 44 & 37 & 34 & 2 & 44 & 38 & 29 & 34 & 25 \\
\hline & 26 & 24 & 29 & 28 & 24 & 39 & 25 & 34 & 35 & 39 \\
\hline & 240 & 287 & 331 & 427 & 520 & 556 & 694 & 747 & 633 & 527 \\
\hline 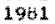 & 11 & 20 & 11 & 16 & 17 & 20 & 21 & 16 & 11 & 12 \\
\hline & 10 & 12 & 12 & 10 & 18 & 14 & 16 & 13 & 11 & 13 \\
\hline 2021 & 6 & 10 & 7 & 10 & 16 & 15 & 11 & 8 & 24 & B \\
\hline & 15 & 15 & 10 & 10 & 15 & 9 & 15 & 0 & & \\
\hline
\end{tabular}

COUNT COUNT COUNT COUNT COUNT COUNT COUNT COUNT COUNT COUNT

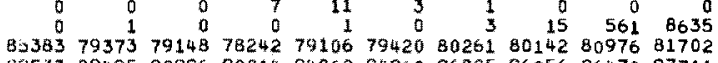
$89533 \quad 894959099692814940629496096825962569647097711$ 117947130270166750209185220577225101273535296033234905159685

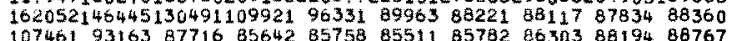

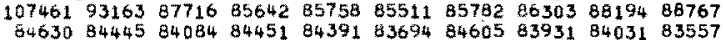
$\begin{array}{llllllllll}83413 & 82767 & 83124 & 83070 & 82825 & 83367 & 22809 & 33241 & 83420 & 83358\end{array}$

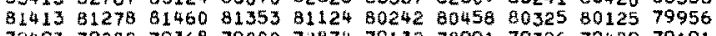

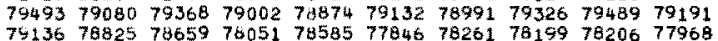

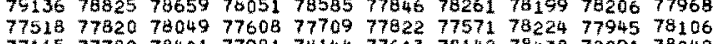
77936780967320077963776317813478096784887601277834 7793678096782207796377631770479096784687601277834

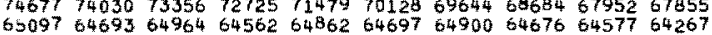
$6356063046 \quad 63626 \quad 63037 \quad 63264 \quad 6316963444063092 \quad 63063 \quad 63411$ $62590 \quad 62283 \quad 6249362207 \quad 6222962442 \quad 6229862415 \quad 62113 \quad 62182$

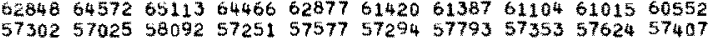
$5776157630 \quad 57521 \quad 5715457572 \quad 56836 \quad 5703256391 \quad 56167 \quad 56225$

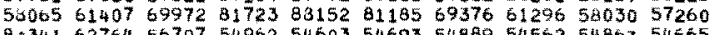
Q $53416276456707549625460354693 \quad 54869545625486354665$ $5340252991 \quad 5283352957519965171952617524155218052086$

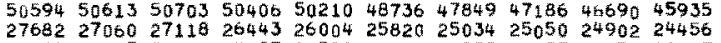

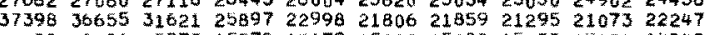

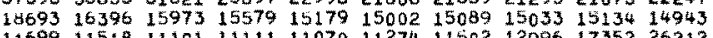

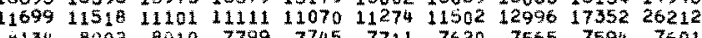

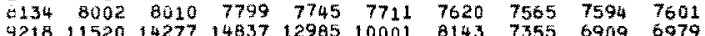

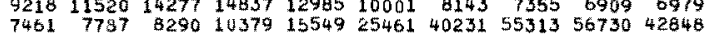

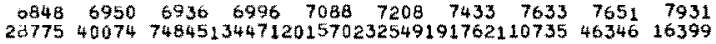
$\begin{array}{llllllllll}3161 & 3098 & 3159 & 3134 & 3148 & 3334 & 3233 & 3741 & 4606 & 6059\end{array}$ $\begin{array}{llllllllll}3231 & 2946 & 2931 & 2869 & 2859 & 2924 & 2817 & 2846 & 2851 & 2903 \\ 2805 & 2848 & 2719 & 2821 & 2773 & 2785 & 2738 & 2764 & 2757 & 2679\end{array}$ $\begin{array}{llllllllll}2288 & 2303 & 2298 & 2303 & 2290 & 2170 & 2202 & 2166 & 2245 & 2218 \\ 3348 & 3902 & 3837 & 3341 & 2765 & 2285 & 2081 & 2028 & 2042 & 2190\end{array}$

\begin{tabular}{|c|c|c|c|c|c|c|c|c|c|}
\hline 1801 & 1927 & 1935 & 1919 & 1653 & 1896 & 1802 & 1914 & 1891 & 1822 \\
\hline 1812 & 1031 & 1680 & 1699 & 1703 & 1677 & 1648 & 1626 & 1674 & 1601 \\
\hline 1646 & 1575 & 1545 & 1510 & 1499 & 1423 & 1487 & 1423 & 1487 & 1505 \\
\hline $\begin{array}{l}1366 \\
1366\end{array}$ & 1320 & 1336 & 1339 & 1366 & $\begin{array}{r}1384 \\
13\end{array}$ & $\begin{array}{r}1324 \\
\end{array}$ & 1318 & 1374 & $\begin{array}{l}129 \mathrm{~B} \\
\end{array}$ \\
\hline $\begin{array}{l}1214 \\
121\end{array}$ & 1231 & 1237 & 1207 & 1181 & 1261 & 1205 & 1257 & 1226 & 1274 \\
\hline 1297 & 1285 & 1223 & 1176 & 1150 & 1167 & 1127 & 1172 & 1244 & 1212 \\
\hline 1123 & 1102 & 1163 & 1153 & 1102 & Ii11 & 1088 & 1122 & 1153 & 1224 \\
\hline 5526 & 5161 & 3967 & 2547 & 1607 & 1204 & 1036 & 1032 & 1022 & 1007 \\
\hline $\begin{array}{r}883 \\
8\end{array}$ & 952 & 939 & 914 & 917 & 92 & 886 & $\begin{array}{r}054 \\
954\end{array}$ & 1014 & 903 \\
\hline 871 & 915 & 912 & 383 & 670 & 672 & 924 & 848 & 891 & 823 \\
\hline 850 & 835 & 901 & 806 & 918 & 1032 & 1123 & 1368 & $\begin{aligned} 8712 \\
1512\end{aligned}$ & $\begin{array}{l}1752 \\
\end{array}$ \\
\hline 767 & 781 & 623 & 797 & 745 & 784 & 810 & 772 & $78_{0}$ & 825 \\
\hline 1324 & 1322 & 1536 & 1449 & 1214 & 1141 & 992 & 907 & 898 & 960 \\
\hline $\begin{array}{r}684 \\
684\end{array}$ & 746 & $\begin{array}{r}695 \\
\end{array}$ & 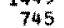 & 722 & $71^{4}$ & 706 & 740 & 801 & 803 \\
\hline 715 & 666 & 870 & 668 & 634 & 684 & 680 & 630 & 675 & 662 \\
\hline 673 & 718 & 602 & 695 & 668 & 655 & 654 & 678 & 670 & 639 \\
\hline 645 & 617 & 010 & 625 & 647 & 597 & 600 & 648 & 652 & 649 \\
\hline 829 & 806 & 751 & 749 & 595 & 561 & 565 & $5 \mathrm{Ba}$ & 507 & 560 \\
\hline 526 & 476 & 543 & 533 & 309 & 507 & 485 & 520 & 51.4 & 455 \\
\hline 530 & 490 & 506 & 485 & 481 & 501 & 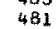 & 493 & $\begin{array}{r}474 \\
474\end{array}$ & 537 \\
\hline 419 & 470 & 427 & 426 & 476 & 447 & 429 & 446 & 412 & 392 \\
\hline $\begin{array}{r}447 \\
\end{array}$ & $5_{11}$ & 527 & 515 & 564 & 470 & $\begin{array}{r}407 \\
403\end{array}$ & 391 & 395 & 408 \\
\hline 464 & 405 & 424 & 410 & 390 & 392 & 351 & 376 & 321 & 330 \\
\hline 331 & 332 & 329 & 353 & 294 & 301 & 351 & 351 & 361 & 315 \\
\hline 327 & $x_{18}$ & 300 & 311 & 303 & 296 & 315 & 312 & 321 & 302 \\
\hline 302 & 323 & 279 & 327 & 305 & 301 & 308 & 256 & 302 & 303 \\
\hline 327 & 297 & 313 & 290 & 331 & $\begin{array}{l}301 \\
3.54\end{array}$ & $\begin{array}{r}378 \\
345\end{array}$ & $\begin{array}{l}230 \\
382\end{array}$ & $\begin{array}{r}302 \\
420\end{array}$ & 472 \\
\hline 308 & 341 & 333 & 330 & 323 & 313 & 283 & 276 & 324 & 273 \\
\hline 273 & 259 & 255 & 263 & 231 & 265 & 235 & 275 & 261 & 272 \\
\hline 266 & 269 & 252 & 229 & 249 & 287 & 267 & 262 & 247 & 285 \\
\hline 509 & 404 & 341 & 287 & 248 & 234 & 230 & 273 & 241 & 270 \\
\hline 245 & 226 & 233 & 263 & 263 & 236 & 272 & 239 & 287 & 250 \\
\hline 222 & $\begin{array}{l}220 \\
230\end{array}$ & 240 & $\begin{array}{l}205 \\
259\end{array}$ & 262 & 241 & $\begin{array}{l}27 \\
247\end{array}$ & 256 & $\begin{array}{l}207 \\
254\end{array}$ & 215 \\
\hline 246 & 239 & 228 & 245 & $22^{9}$ & 242 & 247 & 236 & 252 & 230 \\
\hline 227 & 215 & 245 & 211 & 247 & 242 & 245 & 214 & $2 \geqslant 0$ & 235 \\
\hline 301 & 207 & 276 & 278 & 251 & $2 \times 5$ & 229 & 243 & 23. & 273 \\
\hline 221 & 217 & 205 & 247 & 240 & 238 & 223 & 235 & 246 & 227 \\
\hline 225 & 233 & 243 & 248 & 235 & 225 & 200 & 224 & 234 & 259 \\
\hline 249 & $\begin{array}{r}233 \\
223\end{array}$ & 228 & 247 & 236 & 235 & 233 & $\begin{array}{l}2 \times 4 \\
249\end{array}$ & 221 & 225 \\
\hline 247 & 292 & 293 & 255 & 226 & 259 & 250 & 242 & 228 & 221 \\
\hline 237 & $\begin{array}{r}32 \\
238\end{array}$ & 257 & 262 & 238 & 246 & 273 & 204 & 251 & 258 \\
\hline 227 & 241 & 248 & $\begin{array}{r}202 \\
249\end{array}$ & $\begin{array}{r}230 \\
277\end{array}$ & $\begin{array}{r}280 \\
234\end{array}$ & 229 & $\begin{array}{r}204 \\
252\end{array}$ & 237 & 230 \\
\hline 235 & 250 & 229 & 247 & 236 & 253 & 231 & 245 & 222 & 24. \\
\hline 251 & 233 & 287 & 236 & 241 & 211 & 251 & 257 & $\begin{array}{l}258 \\
251\end{array}$ & 267 \\
\hline 267 & 255 & 305 & 269 & 266 & $\begin{array}{l}276 \\
\end{array}$ & 255 & $2^{4} 48$ & 260 & 277 \\
\hline $\begin{array}{l}201 \\
250\end{array}$ & 261 & 267 & 214 & 256 & $\begin{array}{l}279 \\
\end{array}$ & 27 & $\begin{array}{r}251 \\
\end{array}$ & $\begin{array}{l}250 \\
258\end{array}$ & 258 \\
\hline 269 & 292 & 293 & 273 & 258 & 279 & 288 & 207 & 270 & 233 \\
\hline 263 & 245 & 251 & 257 & 246 & 262 & 245 & 230 & 214 & 229 \\
\hline $\begin{array}{l}147 \\
\end{array}$ & 143 & 157 & 134 & 130 & 141 & 132 & 130 & 134 & 152 \\
\hline
\end{tabular}

$\begin{array}{rrrrrrrrrr}152 & 149 & 120 & 121 & 124 & 118 & 113 & 107 & 101 & 107 \\ 120 & 92 & 99 & 99 & 106 & 129 & 123 & 102 & 119 & 98 \\ 93 & 88 & 96 & 92 & 54 & 78 & 86 & 77 & 90 & 74 \\ 62 & 66 & 56 & 59 & 64 & 65 & 69 & 59 & 56 & 54 \\ 55 & 54 & 52 & 46 & 46 & 44 & 56 & 42 & 49 & 37 \\ 49 & 24 & 49 & 42 & 55 & 44 & 47 & 48 & 55 & 52 \\ 37 & 37 & 37 & 28 & 35 & 44 & 31 & 41 & 40 & 40 \\ 32 & 33 & 32 & 41 & 23 & 25 & 33 & 31 & 29 & 33 \\ 45 & 35 & 52 & 54 & 60 & 68 & 83 & 105 & 136 & 153 \\ 360 & 207 & 108 & 55 & 46 & 21 & 24 & 21 & 15 & 18 \\ 10 & 13 & 11 & 6 & 12 & 17 & 8 & 13 & 15 & 15 \\ 15 & 12 & 6 & 8 & 14 & 14 & 14 & 20 & 13 & 13 \\ 13 & 9 & 7 & 12 & 9 & 10 & 14 & 11 & 11 & 9\end{array}$


GAMMA RAY SPECTRUM DATA

PRTR RUD F5-23 SPECTRUM NO. 19 DATA TAKEN 3/05/69 LIVE TIME 310.MIN. POSITION 43. IN. ENERGYIKEVI= 1.112*CHAN -8.8 CHLIV COUNT COUNT COUNT CUU GOUNT COUNT COUNT COUNT COUNT COUNT

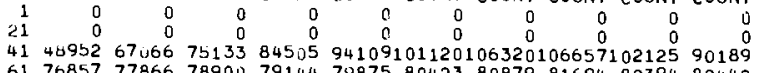
$\begin{array}{lll}6176857 & 77866789007914479875 & 8042380879816948239482442\end{array}$ 10111524110488610271910254410210910623812305115747818823104731 12181686819048240382286827328342789286104673122979121037

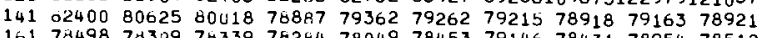

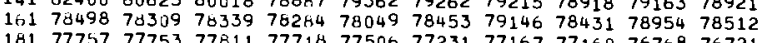

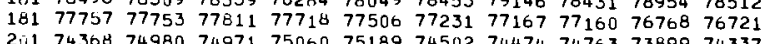

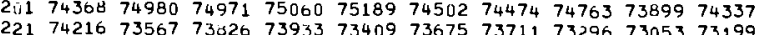

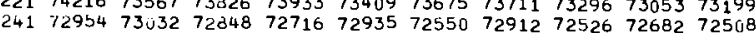

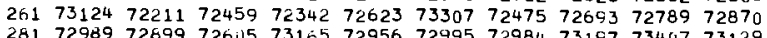
$\begin{array}{lllllllllll}281 & 72989 & 72899 & 72645 & 73165 & 72956 & 72995 & 72984 & 73197 & 73407 & 73129\end{array}$

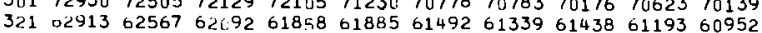
$34159962 \quad 601346016559519596345980859504593985924659333$

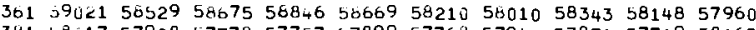
$\begin{array}{lllllllllll}381 & 8017 & 57908 & 57778 & 57757 & 57899 & 57768 & 57941 & 57876 & 57719 & 58065\end{array}$ $\begin{array}{lllllllllll}401 & 50679 & 50236 & 55956 & 55383 & 54758 & 54762 & 54551 & 54546 & 54341 & 53905 \\ 421 & 3631 & 53475 & 54684 & 54473 & 55054 & 54620 & 54368 & 53826 & 53622 & 53766\end{array}$

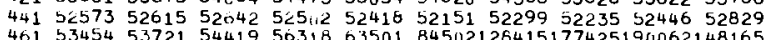
47115113450940512255083513825101550432507344997850073

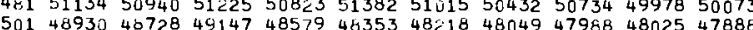

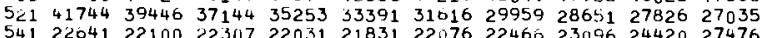
$\begin{array}{lllllllllll}\operatorname{Sn} 1 & 21205 & 22333 & 236448 & 204 \dot{ } 5 & 35609 & 52396 & 69333 & 71268 & 52732 & 31465\end{array}$ $\begin{array}{lllllllllll}5.1 & 13626 & 12914 & 12791 & 124 \mathrm{hs} & 12223 & 11715 & 11895 & 11431 & 11180 & 11010\end{array}$ $\begin{array}{lllllllllll}6 \text { (i1 } & 29229 & 43529 & 52361 & 466,4 & 31182 & 16143 & 10218 & 8354 & 7837 & 7717\end{array}$ $\begin{array}{lllllllllll}621 & 6832 & 6768 & 6825 & 6741 & 6692 & 6650 & 6789 & 6666 & 6746 & 7167 \\ 641 & 6358 & 0677 & 6090 & 6611 & 6468 & 6570 & 6605 & 6629 & 6657 & 6631\end{array}$

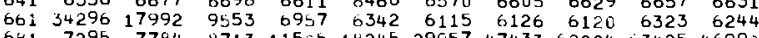
$\begin{array}{rrrrrrrrrrr}681 & 7295 & 7784 & 4713 & 11505 & 18245 & 29557 & 47433 & 62224 & 63425 & 46990 \\ 7 \cup 1 & 9004 & 4679 & 3345 & 3039 & 2931 & 2969 & 2959 & 2922 & 2936 & 2854 \\ 721 & 0817 & 8524 & 10548 & 10977 & 8947 & 5901 & 4186 & 3658 & 3626 & 3419\end{array}$ $\begin{array}{lllllllllll}741 & 2553 & 2717 & 272 b & 2570 & 2671 & 2041 & 2717 & 2606 & 2591 & 2642\end{array}$ $\begin{array}{lllllllllll}761 & 2637 & 1991 & 1964 & 1901 & 2064 & 21.26 & 2072 & 2074 & 2172 & 2420\end{array}$

COINT COUNT COUNT COUNT COUNT COUNT COLNT COUNT COUNT COUNT

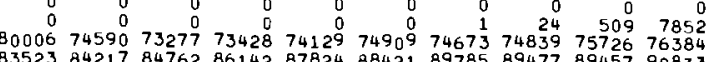
$\begin{array}{lll}83523842178476286142 \quad 87824 & 88421897858947789457 \quad 90833\end{array}$ 109459120608151682192071205666206930249735276912223752151232
153090136988123140104033904138436083066825158194082077 101601877538237280856806527994680202807768259382843 $\begin{array}{llllllllllll}78657 & 78649 & 78714 & 79086 & 78627 & 78893 & 78529 & 79142 & 78457 & 78598\end{array}$ $\begin{array}{llllllllll}7958 & 77963 & 77556 & 77813 & 77466 & 77696 & 77720 & 77527 & 77989 & 77587\end{array}$ $\begin{array}{llllllllll}74622 & 73933 & 74169 & 74252 & 73999 & 73790 & 73698 & 73785 & 74000 & 73839\end{array}$ $\begin{array}{lllllllllll}73417 & 73158 & 72888 & 72868 & 73100 & 73290 & 73110 & 72813 & 73398 & 72774\end{array}$ $\begin{array}{llllllllll}72831 & 72517 & 72934 & 73276 & 72268 & 72414 & 72558 & 72170 & 72972 & 72329\end{array}$

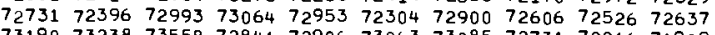
$731907323873558 \quad 7284172906 \quad 73003 \quad 730857273172216$
61909 $\begin{array}{llllllllll}69628 & 68580 & 68644 & 67704 & 66775 & 65936 & 65054 & 64026 & 64278 & 63225 \\ 60535 & 60819 & 60744 & 60081 & 60401 & 60282 & 60586 & 60721 & 60903 & 59828\end{array}$ $59505 \quad 58914587335919059328 \quad 5903158782588895892958914$ $\begin{array}{llllllllll}58066 & 58066 & 58174 & 58320 & 57900 & 58241 & 58081 & 58012 & 57754 & 57982\end{array}$ $\begin{array}{lllllllllll}58417 & 59927 & 60977 & 60729 & 59116 & 57669 & 57066 & 56834 & 56744 & 56566\end{array}$

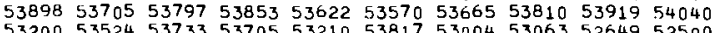
$53605 \quad 554986115471650 \quad 80949804977003859727 \quad 5493953434$ 49858495794943348871487004886949144488424878248836 $\begin{array}{llllllllll}49858 & 49579 & 49433 & 48871 & 48700 & 48869 & 49144 & 48842 & 48782 & 48836 \\ 47294 & 47520 & 47700 & 47502 & 46513 & 45607 & 44698 & 43953 & 43568 & 42800\end{array}$ $\begin{array}{llllllllll}47294 & 47520 & 47700 & 47502 & 46513 & 45607 & 44698 & 43953 & 43568 & 42800 \\ 26118 & 26086 & 24912 & 24967 & 24202 & 24089 & 23539 & 23298 & 22748 & 22664\end{array}$ $\begin{array}{llllllllll}26118 & 26086 & 24912 & 24967 & 24202 & 24089 & 23539 & 23298 & 22748 & 22664 \\ 31548 & 34118 & 31503 & 25806 & 22186 & 20611 & 20652 & 19948 & 19461 & 19805\end{array}$ $\begin{array}{lllllllllll}19959 & 16019 & 15111 & 14896 & 14301 & 13979 & 14025 & 14182 & 13843 & 14076 \\ 11084 & 10824 & 10427 & 10306 & 10199 & 10085 & 10343 & 11143 & 13366 & 18894\end{array}$ $\begin{array}{llllllllll}7523 & 7495 & 7393 & 7229 & 7431 & 7161 & 7185 & 7136 & 6931 & 6820\end{array}$ $\begin{array}{rrrrrrrrrr}7834 & 9462 & 11964 & 13551 & 13649 & 10974 & 8403 & 7024 & 6434 & 6398 \\ 6812 & 7032 & 7344 & 8229 & 10706 & 16906 & 28016 & 43037 & 54675 & 50577\end{array}$ $\begin{array}{rrrrrrrrrr}6812 & 7032 & 7344 & 8229 & 10706 & 16906 & 28016 & 43037 & 54675 & 50577 \\ 6307 & 6502 & 6480 & 6647 & 6550 & 6797 & 6847 & 6987 & 6947 & 7307\end{array}$ $29861264264357384134145200205095218704160590 \quad 8022329197$ $\begin{array}{llllllllll}3005 & 2915 & 2972 & 2827 & 2941 & 3001 & 2991 & 3184 & 3516 & 4474 \\ 3143 & 2855 & 2741 & 2635 & 2673 & 2785 & 2656 & 2735 & 2740 & 2721\end{array}$ $\begin{array}{llllllllll}2572 & 2635 & 2581 & 2600 & 2513 & 2602 & 2703 & 2583 & 2580 & 2573\end{array}$ $\begin{array}{llllllllll}2142 & 2165 & 2172 & 2094 & 1985 & 2098 & 2013 & 2062 & 2055 & 2010 \\ 2883 & 3242 & 3693 & 3484 & 3027 & 2414 & 2037 & 1865 & 1901 & 1943\end{array}$

\begin{tabular}{|c|c|c|c|c|c|c|c|c|c|c|}
\hline $8 \cup 1$ & 1881 & 2003 & 1975 & 20 b & 1975 & 1948 & 1815 & 1861 & 1753 & 179 \\
\hline $8 \angle 1$ & 1746 & 1736 & 1733 & $17 i, 0$ & 1682 & 1620 & 1644 & 1706 & 1656 & \\
\hline 841 & 1608 & 1472 & 1514 & 1457 & 1425 & 1465 & 1464 & 1466 & 1438 & 2. \\
\hline 861 & 1333 & 1325 & 1340 & 1327 & 1364 & 1321 & 1293 & 1263 & 1297 & 31 \\
\hline 881 & 1241 & 1101 & 1262 & 1136 & 1220 & 1220 & 1209 & 1156 & 1203 & \\
\hline 901 & 1204 & $\begin{array}{l}1157 \\
11\end{array}$ & 1186 & 1200 & 1217 & 1186 & $\begin{array}{l}1157 \\
1157\end{array}$ & 1177 & 1198 & \\
\hline 921 & 1061 & 1103 & 1022 & 1026 & 1118 & 1068 & 1031 & 1080 & 1064 & 101 \\
\hline 941 & 1009 & 1048 & 1164 & 1131 & 1131 & 1281 & 1500 & 1987 & 2639 & \\
\hline 961 & 894 & 996 & 971 & 9,67 & 945 & 918 & 828 & 885 & 846 & \\
\hline 901 & 860 & 629 & 873 & 817 & 847 & 841 & 866 & 855 & 895 & \\
\hline 1001 & 772 & 763 & 806 & 820 & 785 & 845 & 890 & 642 & 869 & \\
\hline 1021 & 1610 & 1746 & 1626 & 13400 & 949 & 637 & 793 & 7 & 740 & \\
\hline 1641 & 751 & 739 & 650 & 600 & 620 & 706 & 706 & $7 \notin 1$ & 873 & \\
\hline 1 Un 1 & 804 & 886 & 694 & 857 & 779 & 767 & 727 & 711 & 670 & \\
\hline 1081 & 763 & 748 & 733 & 710 & 687 & 080 & 653 & 654 & 718 & \\
\hline $11 \cup 1$ & 636 & 637 & 607 & 630 & 638 & 603 & 592 & 670 & 582 & \\
\hline 1121 & 565 & 594 & 565 & $5 \varsigma_{1} 1$ & 593 & 565 & 623 & $5 t 0$ & 585 & \\
\hline 1141 & 617 & 617 & 549 & 550 & 572 & 535 & 596 & 583 & 566 & \\
\hline 1161 & 502 & 491 & 469 & 5,6 & 527 & 459 & 494 & $4 \otimes 1$ & 448 & \\
\hline 1181 & 471 & 429 & 430 & $4 e^{2}$ & 483 & 433 & 479 & 432 & 469 & \\
\hline $2 \pm 1$ & 494 & 509 & 532 & 6.6 & 62 & 652 & 61 & 494 & 455 & \\
\hline 1221 & 386 & 385 & $35 \overline{3}$ & 368 & 396 & 382 & 379 & 373 & 389 & \\
\hline 1241 & 337 & 350 & 310 & 330 & 350 & 345 & 351 & 359 & 352 & \\
\hline 1261 & 347 & 340 & 326 & 318 & 318 & 349 & 339 & 301 & 331 & \\
\hline $12+1$ & 313 & 324 & 312 & 290 & 330 & 320 & 321 & 305 & 304 & \\
\hline 1301 & 306 & 290 & 316 & 273 & 282 & 288 & 314 & 285 & 260 & \\
\hline 1321 & 254 & $<97$ & 207 & 273 & 289 & 309 & 278 & 278 & 280 & \\
\hline 1341 & $3 E .5$ & 521 & 557 & 664 & 743 & 831 & 782 & 60 & 463 & \\
\hline 1301 & 271 & 256 & 265 & 259 & 261 & 271 & 205 & 27 & 252 & \\
\hline $13+1$ & 236 & 242 & 237 & 2,8 & 259 & 251 & 25 & 25 & 231 & \\
\hline $14 \mathrm{EI}$ & 253 & 243 & 202 & 2133 & 255 & 300 & 330 & 349 & 385 & \\
\hline $142 i$ & 237 & 243 & 210 & $2 ? 0$ & 252 & 223 & 229 & 209 & 246 & \\
\hline 1441 & 243 & 226 & & 237 & 236 & $\angle 1$ & 234 & & 222 & \\
\hline $14 \mathrm{EI}$ & 222 & 225 & 207 & & 19 & $\div 4$ & 21 & & 234 & \\
\hline $14 \curvearrowleft 1$ & 211 & 229 & 232 & 2 & 230 & 19 & 23 & $2 \hat{x}$ & 208 & \\
\hline 15,1 & 225 & 235 & 223 & 216 & 224 & 21 & 23 & & 263 & \\
\hline 1521 & 235 & 229 & 224 & $2 \times 4$ & 225 & $<69$ & 196 & 197 & 200 & \\
\hline 1541 & $<33$ & 200 & 197 & & 207 & & & & 192 & \\
\hline 1 bo 1 & 200 & $<35$ & 27, & 215 & 221 & 23 & 19 & 224 & 207 & \\
\hline 1501 & 242 & $\angle 10^{\circ}$ & alon & 23s & 220 & 223 & 23 & $<30$ & 225 & \\
\hline $10_{i j 1}$ & $22 \hat{c}$ & 190 & 202 & $2 i 0$ & 202 & $\because 17$ & $22 t$ & 201 & 230 & \\
\hline 1021 & 244 & 260 & $\leqslant 26$ & & 229 & $\pm b$ & 224 & & 223 & \\
\hline 1641 & 203 & $<09$ & 255 & 2.35 & 220 & 222 & 24 & $2>5$ & 212 & \\
\hline $16+1$ & 247 & $<24$ & 243 & 2.3 & 264 & 432 & 224 & 225 & 236 & \\
\hline $1 \mathrm{~b} \downarrow 1$ & $\approx 45$ & 225 & 25 & 211 & 235 & 244 & 259 & $<19$ & 212 & \\
\hline $17 \cup 1$ & 243 & $\angle 86$ & $\angle 36$ & 227 & 239 & 239 & $26 \mathrm{~J}$ & 223 & 219 & \\
\hline 1721 & $<\rightarrow y$ & 234 & 242 & $2, y$ & 240 & 234 & 219 & 2nd & 256 & \\
\hline 174 & 267 & $<44$ & 274 & $2 \div 20$ & 240 & 36 & 241 & 245 & 246 & \\
\hline$z=1$ & 200 & 100 & $\therefore$ & $1 . \cdots$ & $=64$ & +65 & 14 & $1+-5$ & 141 & \\
\hline
\end{tabular}

\begin{tabular}{|c|c|c|c|c|c|c|c|c|c|}
\hline 1772 & 1854 & 1824 & 1726 & 1835 & 1665 & 1681 & 1767 & 1837 & 1735 \\
\hline 1700 & 1617 & 1647 & 1591 & 1533 & 1579 & 1538 & 1533 & 1557 & 1505 \\
\hline 1562 & 1473 & 1527 & 1373 & 1351 & 1366 & 1336 & 1398 & 1351 & 1383 \\
\hline 1237 & 1294 & 1329 & 1283 & 1260 & 1249 & 1206 & 1287 & 1254 & 1217 \\
\hline 1102 & 1191 & 1172 & 1177 & 1189 & 1134 & 1132 & 1150 & 1183 & 1190 \\
\hline 1218 & 1243 & 1212 & 1089 & 1074 & 1074 & 1144 & 1116 & 1101 & 1120 \\
\hline 1034 & 1020 & 1056 & 999 & 1037 & 999 & 1065 & 1006 & 1019 & 1076 \\
\hline 4517 & 5323 & 5129 & 3739 & 2248 & 1326 & 1049 & 1001 & 931 & 872 \\
\hline 840 & 823 & 875 & 839 & 862 & 900 & 373 & & 890 & 896 \\
\hline 843 & 846 & 795 & 847 & 810 & 825 & 846 & 835 & 792 & 783 \\
\hline 816 & 857 & 791 & 825 & 785 & 846 & 383 & 1049 & 1193 & 1434 \\
\hline 721 & 702 & 721 & 697 & 749 & 670 & 736 & 715 & 701 & 698 \\
\hline 1014 & 1203 & 1279 & 1415 & 1293 & 1166 & 975 & 922 & 824 & 860 \\
\hline 634 & 682 & 636 & 639 & 650 & 677 & 688 & 681 & 697 & \\
\hline 714 & 695 & & 573 & & 609 & 614 & & 611 & 579 \\
\hline 609 & 637 & 576 & 560 & 623 & 575 & 597 & 668 & 583 & 593 \\
\hline 586 & 594 & 502 & 609 & 573 & 593 & 606 & 608 & 572 & 607 \\
\hline 695 & 655 & 714 & 739 & 630 & 578 & 527 & 523 & 508 & 462 \\
\hline 484 & 479 & 474 & 486 & 492 & 464 & 458 & 472 & 449 & 437 \\
\hline 440 & 460 & 439 & 442 & 454 & 474 & 482 & 466 & 500 & 477 \\
\hline 445 & 421 & 424 & 414 & 433 & 415 & 405 & 388 & 407 & 411 \\
\hline 384 & 449 & 455 & 503 & 473 & 464 & 420 & 389 & 381 & 371 \\
\hline 374 & 401 & 388 & 365 & 371 & 344 & 352 & 360 & $33 \mathrm{~B}$ & 347 \\
\hline 301 & 313 & 311 & 331 & 319 & 311 & & 30 & 317 & 329 \\
\hline 306 & 287 & 324 & 300 & 277 & 315 & 274 & 296 & 283 & 260 \\
\hline 283 & 279 & 294 & 272 & 286 & 297 & 277 & 284 & 288 & 302 \\
\hline 253 & 290 & 323 & 290 & 297 & 328 & 287 & 278 & 350 & 38 \\
\hline 326 & 316 & 290 & 312 & 279 & 304 & 299 & 263 & 294 & 284 \\
\hline 276 & 255 & 27 & 242 & & & & 25 & 254 & 233 \\
\hline 266 & 226 & 244 & 287 & 258 & 214 & 261 & 274 & 255 & 200 \\
\hline 440 & 500 & 397 & 355 & 297 & 280 & 229 & 246 & 260 & 231 \\
\hline 245 & 203 & 211 & 211 & 237 & 229 & 230 & 230 & 223 & 230 \\
\hline 237 & 226 & 197 & 202 & 199 & 22 & & 21 & 227 & 211 \\
\hline 233 & 220 & 23 & 214 & 2 & & 23 & 21 & 222 & 202 \\
\hline 190 & 207 & 218 & 22 & 21 & 219 & 212 & 21 & 201 & 224 \\
\hline 259 & 278 & & 263 & 254 & & & 23 & 206 & 215 \\
\hline 223 & 201 & 223 & & & & & 208 & 44 & 195 \\
\hline 223 & 226 & 21 & 214 & & 2 & 20 & 22 & 235 & 185 \\
\hline 225 & 204 & 19 & 210 & 2 & 21 & & 20 & 21 & 200 \\
\hline 243 & 23 & 23 & 25 & 27 & 22 & 21 & 20 & 195 & 254 \\
\hline 219 & 240 & 213 & 22 & 22 & 22 & 217 & 250 & 250 & 259 \\
\hline 232 & 19 & & & & & & 23 & 23 & \\
\hline 232 & 22 & 20 & 196 & 24 & & 243 & 243 & 213 & 206 \\
\hline 241 & 21 & 228 & 24 & 25 & 2 & 21 & 22 & 25 & 242 \\
\hline 242 & 238 & 21 & 23 & 25 & 238 & 24 & 25 & 250 & 259 \\
\hline 252 & 24 & & 242 & 27 & 23 & 2 & 25 & 243 & 238 \\
\hline 252 & 203 & 265 & 231 & 28 & 24 & 22 & 284 & 269 & 240 \\
\hline 256 & 243 & & 242 & 22 & 21 & 20 & 205 & 238 & 208 \\
\hline+33 & -149 & 129 & 127 & 145 & 119 & $\$ 19$ & 125 & 130 & 118 \\
\hline 126 & 1 bo & 162 & 167 & 125 & 127 & 121 & 12 & 126 & 10 \\
\hline 104 & 108 & 92 & 103 & 81 & 10 & 10 & 107 & 78 & 9 \\
\hline 94 & 91 & 8 & & 7 & 87 & 88 & 78 & 75 & 92 \\
\hline 59 & 66 & 59 & 56 & 60 & 70 & 54 & 65 & 54 & 58 \\
\hline 46 & 40 & 35 & 57 & & $2^{8}$ & & 51 & 48 & 42 \\
\hline 45 & 39 & 50 & 49 & 49 & 37 & 43 & 48 & 42 & \\
\hline 32 & 32 & 32 & 39 & 31 & 40 & 39 & 35 & 32 & 37 \\
\hline 28 & 36 & 28 & 34 & & 30 & 2 & 30 & 30 & $2^{8}$ \\
\hline 42 & 40 & 24 & 33 & & & & & 60 & \\
\hline 710 & 601 & 376 & 154 & 6 & & & 17 & 9 & 5 \\
\hline 15 & 11 & 16 & 8 & 11 & 11 & 12 & 5 & 8 & \\
\hline 10 & 12 & 12 & 16 & 10 & 15 & 11 & 11 & 12 & 14 \\
\hline 10 & 5 & 10 & 16 & 13 & 9 & 6 & 7 & 12 & 11 \\
\hline
\end{tabular}

\begin{tabular}{|c|c|c|c|c|c|c|c|c|c|c|c|c|c|c|c|c|c|c|c|c|}
\hline $17 \delta 1$ & 142 & 116 & 91 & 115 & 136 & 115 & 131 & 143 & 141 & 135 & 126 & 1 bo & 162 & 167 & 125 & 127 & 121 & 122 & 126 & 105 \\
\hline 1601 & 101 & 100 & o's & cio & 100 & 107 & 101 & 03 & 98 & 96 & 104 & 108 & 92 & 103 & 81 & 100 & 102 & 107 & 78 & 99 \\
\hline $102 i$ & 94 & 104 & 82 & 107 & 87 & 81 & 85 & 94 & 66 & 86 & 94 & 91 & 85 & 84 & 73 & 87 & 88 & 78 & 75 & 92 \\
\hline 1841 & 80 & 76 & 69 & isi & 60 & 78 & 80 & 75 & 42 & 61 & 59 & 66 & 59 & 56 & 60 & 70 & 54 & 65 & 54 & 58 \\
\hline 1801 & 33 & 65 & jo & is 4 & 53 & 55 & 37 & 44 & 42 & 54 & 46 & 40 & 35 & 57 & 44 & 28 & 50 & 51 & 48 & 42 \\
\hline 1081 & 34 & 31 & 39 & +3 & 30 & 33 & 31 & 38 & 42 & 29 & 45 & 39 & 50 & 49 & 49 & 37 & 43 & 48 & 42 & 45 \\
\hline $19_{i j 1}$ & 44 & 46 & 59 & 1 & 55 & 65 & 65 & 45 & 35 & 39 & 32 & 32 & 32 & 39 & 31 & 40 & 39 & 35 & 32 & 37 \\
\hline 1921 & 35 & 31 & $4 i$ & 35 & 32 & 38 & 31 & 44 & 40 & 30 & 28 & 36 & 28 & 34 & 26 & 30 & 28 & 30 & 30 & \\
\hline 1941 & 27 & 32 & 35 & 33 & 36 & 31 & 30 & 29 & 29 & 34 & 42 & 40 & 24 & 33 & 35 & 41 & 43 & 45 & 60 & 90 \\
\hline 1901 & 110 & 100 & 225 & 273 & 355 & 368 & 490 & $5 \div 4$ & 602 & 716 & 710 & 601 & 376 & 154 & 67 & 49 & 26 & 17 & 9 & 15 \\
\hline 1901 & 17 & 19 & 11 & 5 & 12 & 7 & 12 & 10 & 19 & 17 & 15 & 11 & 16 & 8 & 11 & 11 & 12 & 5 & 8 & 8 \\
\hline 2 viv 1 & 5 & 11 & 10 & 0 & 10 & 7 & 9 & 9 & 15 & 16 & 10 & 12 & 12 & 16 & 10 & 15 & 11 & 11 & 12 & 14 \\
\hline 2421 & 11 & 10 & 16 & 15 & in & 9 & 9 & 8 & 7 & 7 & 10 & 5 & 10 & 16 & 13 & 9 & 6 & 7 & 12 & 11 \\
\hline
\end{tabular}




\section{D-21}

GAMMA RAY SPECTRUM DATA

PRTR KUL FS-23 SPECTRUM NO. 20 DATA TAKEN 3/05/69 LIVE TIME 530.MIN. POSITION 48. IN. ENERGY(KEV)= 1.111*CHAN -8.1 CHAN COUNT COUNT COUNT COUNT COUNT COUNT COUNT COUNT COUNT COUNT

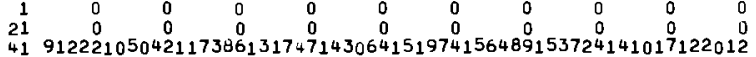
61113107114081115466115591116655118742119128119524120607121288
81130618137330136839137205139703142616144240146772149788153454 81136818137330136839137205139703142616144240146772149788153454
101161680151736150156148450149855158017187794242500277922253632 12112067119012050120147121089123061134126160076183230171203 141119762117201166451170511748311580115765115494115693116264 1411449814537113570114209113394113455112576112614112251111768 (2) 2211.8574108463107698107804108047107340107694107591107714107566 24117218106915107152107202106930106956106553106871107011106569 261106908106054106102106332106580106463106423105610106941106830 $2 \times 11_{106586106956106810106543106812106389106648107277107258106820}$ $3011,563 d 105949105235105616105563104001104556104055103513102494$ $\begin{array}{lllllllllll}321 & 92388 & 91774 & 91720 & 90830 & 91164 & 90720 & 89980 & 89608 & 89882 & 89699 \\ 341 & 88479 & 88177 & 83372 & 87644 & 88207 & 87710 & 87376 & 87417 & 87740 & 87198\end{array}$

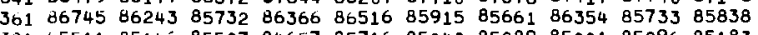
$\begin{array}{llllllllllll}381 & 85511 & 85146 & 85507 & 84657 & 85716 & 85040 & 85089 & 85001 & 85296 & 85183 \\ 401 & 133953 & 83030 & 82183 & 81898 & 81378 & 80695 & 80506 & 80430 & 80068 & 80037\end{array}$

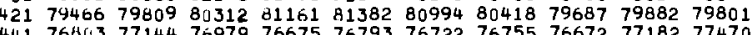

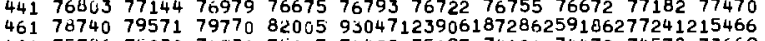
$50171775 \quad 72003 \quad 7235071773 \quad 71113 \quad 70877 \quad 7077070571 \quad 70765 \quad 70003$ $\begin{array}{llllllllllll}541 & 33345 & 33063 & 32507 & 32034 & 31945 & 32092 & 32886 & 34356 & 35886 & 39674\end{array}$ $\begin{array}{lllllllllll}561 & 31252 & 32765 & 34162 & 38036 & 50253 & 74191100199103997 & 78335 & 47171\end{array}$ $\begin{array}{llllllllllll}561 & 20120 & 19<57 & 18631 & 18319 & 17777 & 17433 & 17315 & 16954 & 16534 & 16182 \\ 6 & 44214 & 67431 & 84468 & 76835 & 50325 & 26469 & 15549 & 12431 & 11410 & 11326\end{array}$ $\begin{array}{lllllllllll}621 & 10133 & 9810 & 10040 & 9795 & 9689 & 9766 & 9665 & 9519 & 9908 & 10296\end{array}$ $\begin{array}{llllllllllll}641 & 9262 & 9579 & 9383 & 9514 & 9567 & 9666 & 9584 & 9767 & 9698 & 9938\end{array}$

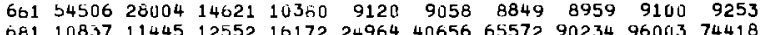
$\begin{array}{rrrrrrrrrrr}\dot{b} 81 & 10837 & 11445 & 12552 & 16172 & 24964 & 40656 & 65572 & 90234 & 96003 & 74418 \\ 701 & 15641 & 6711 & 4661 & 4375 & 4280 & 4232 & 4270 & 4174 & 4223 & 4223\end{array}$ $\begin{array}{lllllllllll}721 & 8276 & 11586 & 15043 & 16441 & 13954 & 9280 & 6223 & 5330 & 5218 & 5056\end{array}$ $\begin{array}{lllllllllll}741 & 3843 & 3864 & 36446 & 3822 & 3962 & 3831 & 3859 & 3736 & 3766 & 3698 \\ 761 & 3630 & 3660 & 3536 & 3508 & 3447 & 3395 & 3498 & 3321 & 3224 & 3229\end{array}$

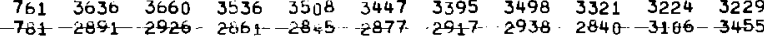

$\begin{array}{lllllllllll}801 & 2804 & 2955 & 2913 & 2994 & 2831 & 2786 & 2637 & 2607 & 2599 & 2590\end{array}$ $\begin{array}{lllllllllll}821 & 2524 & 2581 & 2497 & 2519 & 2455 & 2400 & 2486 & 2444 & 2395 & 2477\end{array}$ $\begin{array}{lllllllllll}861 & 2074 & 1958 & 1929 & 1869 & 2039 & 2052 & 2137 & 2090 & 2149 & 2153 \\ & 1934 & 2010 & 1873 & 1952 & 1881 & 1843\end{array}$ $\begin{array}{lllllllllll}861 & 1832 & 1732 & 1792 & 1768 & 1739 & 1793 & 1751 & 1781 & 1725 & 1747 \\ \mathbf{9}_{i 11} & 1642 & 1725 & 1790 & 174 & 1713 & 1671 & 1662 & 1696 & 1748 & 1685\end{array}$ $\begin{array}{lllllllllll}921 & 1542 & 1582 & 1502 & 1560 & 1500 & 1511 & 1584 & 1585 & 1528 & 1566 \\ 941 & 1605 & 1036 & 1057 & 1596 & 1613 & 1724 & 2031 & 2615 & 3436 & 4711\end{array}$ $\begin{array}{lllllllllll}941 & 1605 & 1036 & 1057 & 1596 & 1613 & 1724 & 2031 & 2615 & 3436 & 4711 \\ 961 & 1375 & 1432 & 1500 & 1364 & 1301 & 1295 & 1274 & 1295 & 1292 & 1264\end{array}$

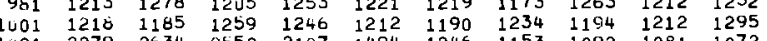

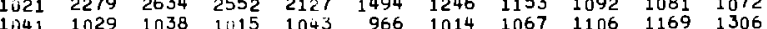
$\begin{array}{rrrrrrrrrrr}1061 & 1216 & 1446 & 1433 & 1403 & 1271 & 1092 & 1061 & 1045 & 972 & 965 \\ 1081 & 1109 & 1147 & 1107 & 1013 & 1036 & 909 & 933 & 941 & 960 & 1027\end{array}$

$\begin{array}{rrrrrrrrrrr}1101 & 866 & 873 & 921 & 907 & 907 & 891 & 826 & 882 & 873 & 829\end{array}$

$\begin{array}{lllllllllll}1121 & 383 & 851 & 882 & 884 & 877 & 855 & 883 & 837 & 849 & 851 \\ 1141 & 636 & 849 & 075 & 816 & 790 & 871 & 806 & 639 & 872 & 869\end{array}$

$\begin{array}{lllllllllll}1141 & 636 & 849 & 675 & 810 & 790 & 871 & 806 & 639 & 872 & 869 \\ 1161 & 678 & 099 & 742 & 720 & 736 & 715 & 729 & 722 & 731 & 676\end{array}$

$\begin{array}{rrrrrrrrrrr}1181 & 665 & 070 & 618 & 664 & 693 & 705 & 635 & 583 & 735 & 698 \\ 12 i 1 & 688 & 747 & 734 & 797 & 967 & 1033 & 1013 & 840 & 698 & 649\end{array}$

$\begin{array}{llllllrrrrr}1221 & 523 & 598 & 575 & 5 \times 7 & 533 & 563 & 574 & 489 & 569 & 579\end{array}$

$\begin{array}{lllllllllll}1241 & 553 & 500 & 516 & 537 & 473 & 498 & 554 & 482 & 515 & 560 \\ 1261 & 473 & 438 & 522 & 478 & 459 & 476 & 464 & 497 & 472 & 448\end{array}$

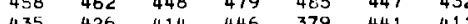

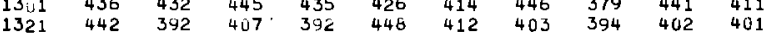

$\begin{array}{lllllllllll}1341 & 579 & 680 & 786 & 951 & 1065 & 1153 & 1228 & 1052 & 732 & 572\end{array}$

$\begin{array}{lllllllllll}1361 & 417 & 417 & 365 & 357 & 408 & 413 & 363 & 339 & 359 & 342 \\ 13 \mathrm{ri1} & 400 & 402 & 381 & 370 & 385 & 352 & 339 & 380 & 340 & 340\end{array}$

$\begin{array}{lllllllllll}1401 & 376 & 474 & 381 & 376 & 385 & 352 & 339 & 380 & 340 & 340 \\ 1421 & 356 & 306 & 316 & 375 & 380 & 411 & 452 & 476 & 526 & 588 \\ 1421 & 320 & 370 & 326 & 323 & 322 & 326 & 339 & 319 & 322 & 322\end{array}$

$\begin{array}{lllllllllll}1441 & 320 & 370 & 326 & 323 & 336 & 307 & 365 & 344 & 351 & 335 \\ 1461 & 329 & 344 & 312 & 365 & 364 & 323 & 310 & 339 & 348 & 317\end{array}$

$\begin{array}{llllllllllll}14,1 & 310 & 335 & 34, & 339 & 305 & 336 & 326 & 303 & 321 & 348 \\ 1501 & 326 & 326 & 317 & 328 & 319 & 349 & 332 & 333 & 396 & 378\end{array}$

$\begin{array}{lllllllllll}1521 & 312 & 326 & 317 & 328 & 319 & 349 & 332 & 333 & 396 & 378 \\ 1521 & 312 & 325 & 321 & 327 & 309 & 320 & 340 & 330 & 310 & 321\end{array}$

$\begin{array}{lllllllllll}1541 & 319 & 348 & 297 & 3.16 & 294 & 297 & 319 & 320 & 344 & 326 \\ 1561 & 293 & 331 & 309 & 3.19 & 312 & 295 & 339 & 312 & 328 & 299\end{array}$

$\begin{array}{lllllllllll}1581 & 300 & 206 & 336 & 340 & 314 & 313 & 325 & 301 & 346 & 340\end{array}$

$\begin{array}{lllllllllll}1601 & 340 & 301 & 320 & 296 & 232 & 295 & 339 & 332 & 290 & 295 \\ 1621 & 372 & 371 & 375 & 400 & 323 & 320 & 317 & 294 & 321 & 329\end{array}$

$\begin{array}{lllllllllll}1641 & 342 & 308 & 339 & 321 & 314 & 345 & 343 & 332 & 291 & 316 \\ 1601 & 313 & 349 & 304 & 327 & 320 & 343 & 313 & 358 & 346 & 351\end{array}$

$\begin{array}{lllllllllll}1661 & 357 & 318 & 355 & 31.7 & 331 & 356 & 347 & 333 & 323 & 338\end{array}$

$\begin{array}{lllllllllll}1701 & 329 & 346 & 333 & 357 & 340 & 347 & 349 & 329 & 374 & 307 \\ 1721 & 351 & 352 & 371 & 342 & 373 & 372 & 372 & 356 & 369 & 379 \\ 1741 & 357 & 392 & 367 & 370 & 361 & 377 & 347 & 361 & 361 & 355\end{array}$

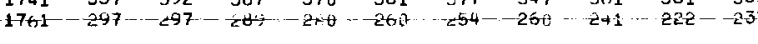

$\begin{array}{rrrrrrrrrrr}17 \circ 1 & 182 & 189 & 197 & 163 & 170 & 170 & 180 & 172 & 165 & 180 \\ 1801 & 157 & 158 & 149 & 150 & 142 & 163 & 157 & 146 & 144 & 131 \\ 1021 & 130 & 124 & 157 & 104 & 132 & 113 & 132 & 110 & 148 & 136 \\ 1841 & 80 & 93 & 100 & 109 & 116 & 106 & 94 & 109 & 89 & 100 \\ 18,11 & 82 & 64 & 73 & 72 & 79 & 76 & 72 & 62 & 65 & 67 \\ 1861 & 64 & 57 & 55 & 54 & 46 & 43 & 55 & 40 & 61 & 60 \\ 19.11 & 67 & 79 & 81 & 110 & 86 & 80 & 87 & 93 & 59 & 62 \\ 1921 & 47 & 42 & 46 & 50 & 43 & 46 & 45 & 49 & 53 & 48 \\ 1941 & 49 & 51 & 47 & 55 & 43 & 51 & 53 & 53 & 56 & 47 \\ 1901 & 101 & 175 & 257 & 330 & 364 & 491 & 561 & 681 & 771 & 985 \\ 1991 & 19 & 19 & 23 & 23 & 21 & 22 & 19 & 27 & 20 & 20 \\ 2001 & 15 & 22 & 13 & 31 & 19 & 22 & 19 & 22 & 19 & 15 \\ 2421 & 16 & 14 & 21 & 15 & 14 & 20 & 17 & 22 & 11 & 15 \\ 2441 & 19 & 20 & 11 & 10 & 11 & 21 & 15 & 0 & & \end{array}$

$\begin{array}{llllllllll}2687 & 2593 & 2595 & 2604 & 2622 & 2650 & 2656 & 2575\end{array}$

$\begin{array}{llllllllll}2415 & 2420 & 2377 & 2421 & 2320 & 2232 & 2236 & 2182 & 2225 & 2135 \\ & 2152 & 2111 & 2063 & 2080 & 1976 & 1965 & 2075 & 2025 & 1980\end{array}$

$\begin{array}{llllllllll}2141 & 2152 & 2111 & 2063 & 2080 & 1976 & 1965 & 2075 & 2025 & 1980 \\ 1967 & 1917 & 1897 & 1875 & 1910 & 1821 & 1855 & 1748 & 1791 & 1802\end{array}$

$\begin{array}{llllllllll}1634 & 1687 & 1697 & 1699 & 1635 & 1638 & 1679 & 1597 & 1622 & 1632 \\ 1837 & 1775 & 1748 & 1665 & 1596 & 1628 & 1615 & 1632 & 1565 & 1688\end{array}$

$\begin{array}{llllllllll}1485 & 1491 & 1484 & 1557 & 1544 & 1518 & 1508 & 1596 & 1515 & 1547 \\ 6144 & 7456 & 7714 & 5993 & 3779 & 2166 & 1534 & 1327 & 1373 & 1382\end{array}$

$\begin{array}{llllllllll}1300 & 1269 & 1353 & 1196 & 1322 & 1233 & 1200 & 1247 & 1300 & 1230 \\ 1291 & 1237 & 1220 & 1247 & 1233 & 1178 & 1205 & 1252 & 1175 & 1158\end{array}$

$\begin{array}{llllllllll}1183 & 1252 & 1166 & 1133 & 1176 & 1211 & 1285 & 1425 & 1593 & 1994\end{array}$

$\begin{array}{llllllllll}1074 & 1077 & 1023 & 969 & 1030 & 1078 & 1064 & 1073 & 1055 & 1015 \\ 1544 & 1625 & 1869 & 2043 & 2075 & 1857 & 1504 & 1363 & 1302 & 1231\end{array}$

$\begin{array}{rrrrrrrrrr}964 & 943 & 928 & 977 & 964 & 948 & 973 & 979 & 1060 & 1100 \\ 1020 & 1029 & 940 & 916 & 983 & 857 & 947 & 937 & 917 & 874\end{array}$

$\begin{array}{llllllllll}848 & 891 & 936 & 926 & 818 & 873 & 886 & 862 & 895 & 866\end{array}$

$\begin{array}{lllrlllllll}856 & 825 & 869 & 872 & 896 & 837 & 844 & 865 & 842 & 820 \\ 899 & 955 & 978 & 1016 & 977 & 845 & 746 & 693 & 729 & 789\end{array}$

$\begin{array}{rrrrrrrrrr}699 & 955 & 978 & 1016 & 977 & 845 & 746 & 693 & 729 & 789 \\ 690 & 682 & 726 & 684 & 700 & 684 & 654 & 692 & 695 & 685\end{array}$

$\begin{array}{llllllllll}666 & 670 & 670 & 690 & 715 & 619 & 646 & 642 & 732 & 640 \\ 633 & 563 & 586 & 613 & 586 & 622 & 599 & 544 & 621 & 567\end{array}$

$\begin{array}{llllllllll}607 & 655 & 607 & 714 & 658 & 755 & 654 & 587 & 528 & 530\end{array}$

$\begin{array}{llllllllll}572 & 580 & 544 & 530 & 514 & 508 & 502 & 501 & 457 & 489 \\ 431 & 485 & 462 & 460 & 469 & 450 & 469 & 432 & 439 & 435\end{array}$

$\begin{array}{llllllllll}405 & 405 & 448 & 419 & 426 & 394 & 398 & 421 & 404 & 378\end{array}$

$\begin{array}{llllllllll}399 & 421 & 434 & 410 & 421 & 417 & 449 & 441 & 504 & 504\end{array}$

$\begin{array}{llllllllll}452 & 473 & 450 & 479 & 454 & 410 & 404 & 417 & 368 & 432 \\ 353 & 375 & 400 & 359 & 379 & 401 & 368 & 388 & 372 & 357\end{array}$

$\begin{array}{llllllllll}353 & 375 & 400 & 359 & 379 & 401 & 368 & 388 & 372 & 357 \\ 388 & 344 & 367 & 362 & 408 & 367 & 353 & 394 & 353 & 329 \\ 697 & 762 & 654 & 554 & 475 & 345 & 365 & 371 & 326 & 320\end{array}$

$\begin{array}{llllllllll}\mathbf{3 1 8} & 320 & \mathbf{3 3 9} & \mathbf{3 2 1} & \mathbf{3 1 4} & \mathbf{3 2 8} & 329 & \mathbf{3 5 3} & 326 & 325 \\ \mathbf{3 2 4} & 366 & 297 & \mathbf{3 1 5} & \mathbf{3 3 6} & \mathbf{3 2 5} & \mathbf{3 4 7} & 339 & 314 & 314\end{array}$

$\begin{array}{llllllllll}343 & 335 & 310 & 315 & 336 & 325 & 347 & 339 & 314 & 31 \\ 3112 & 311 & 306 & 282 & 295 & 315 & 28\end{array}$

$\begin{array}{llllllllll}318 & 283 & 306 & 332 & 360 & 343 & 312 & 281 & 317 & 321 \\ 432 & 378 & 451 & 399 & 388 & 336 & 313 & 364 & 351 & 316\end{array}$

$\begin{array}{lll}324 & 289 & 320\end{array}$

$\begin{array}{lll}324 & 289 & 307 \\ 376 & 352 & 326\end{array}$

$\begin{array}{lll}302 & 317 & 298 \\ 311 & 308 & 305\end{array}$

$\begin{array}{lll}352 & 314 & 342 \\ 347 & 330 & 358\end{array}$

$\begin{array}{lll}347 & 330 & 358 \\ 333 & 336 & 343\end{array}$

$\begin{array}{lll}318 & 328 & 354 \\ 328 & 367 & 348\end{array}$

322
297

$\begin{array}{lllllll}304 & 323 & 354 & 348 & 338 & 318 & 343 \\ 315 & 297 & 318 & 334 & 321 & 305 & 332\end{array}$

$\begin{array}{lllllll}3158 & 297 & 318 & 319 & 309 & 330 & 347 \\ 355 & 354 & 315 & 320 & 364 & 350 & 340\end{array}$

$\begin{array}{lllllll}355 & 315 & 353 & 343 & 361 & 325 & 316\end{array}$

$\begin{array}{lllllll}379 & 367 & 365 & 408 & 360 & 397 & 378 \\ 375 & 324 & 338 & 338 & 336 & 334 & 308\end{array}$

PLOT CaLLE) FOHI 
GAMMA RAY SPECTRUM DATA

PRTR ROD FS-08 SPECTRUM NO. 16 DATA TAKEN 3/02/69 LIVE TIME 240.MIN. POSITION 29.IN. ENERGY(KEV)= 1.111*CHAN -8.1

CHAN COUNT COUNT COUNT COUNT COUNT COUNT COUNT COUNT COUNT COUNT

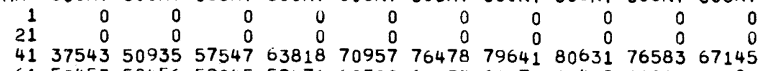

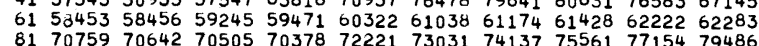

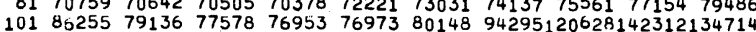
$1216194461787 \quad 61531 \quad 617516212163134 \quad 67830802509369990427$ $\begin{array}{llllllllllll}141 & 61867 & 60693 & 60228 & 59920 & 59993 & 59543 & 59660 & 59261 & 59562 & 59560 \\ 161 & 58978 & 59091 & 59337 & 58793 & 59106 & 59103 & 58976 & 59616 & 59294 & 58700\end{array}$

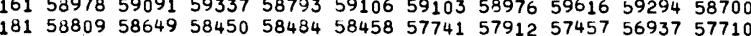
$201563455607556149562375619556090 \quad 55838 \quad 5556955599 \quad 55852$ $\begin{array}{llllllllllll}221 & 55887 & 55277 & 55383 & 55395 & 55150 & 54879 & 55412 & 55418 & 54767 & 54612 \\ 241 & 54722 & 54723 & 54988 & 54576 & 55266 & 54844 & 54845 & 55082 & 54878 & 54682\end{array}$ $261546415476654616545475472754526 \quad 54656 \quad 54590 \quad 54895 \quad 54601$ $\begin{array}{lllllllllll}281 & 55324 & 54660 & 54764 & 54926 & 55361 & 54975 & 55182 & 54633 & 54845 & 55140 \\ 301 & 54662 & 54526 & 54518 & 53702 & 53719 & 53778 & 53236 & 52958 & 52822 & 52123\end{array}$

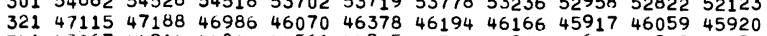
$34145267 \quad 44914 \quad 44814 \quad 44566 \quad 44945 \quad 44521 \quad 44969 \quad 44610 \quad 44908 \quad 44480$ $\begin{array}{llllllllllll}361 & 44267 & 44108 & 43656 & 43906 & 44080 & 43465 & 43611 & 43744 & 43308 & 43683\end{array}$ $4014240542392 \quad 422864155143250745324353435404325942936$ $4214045740278 \quad 40724 \quad 40800 \quad 41118 \quad 41399 \quad 40901 \quad 40314 \quad 40447 \quad 40243$

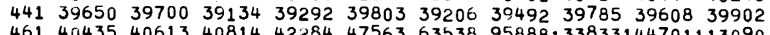
$48130793 \quad 39373 \quad 39443 \quad 38846 \quad 38564 \quad 38821 \quad 38712 \quad 38143 \quad 38119 \quad 37800$ $\begin{array}{llllllllllll}501 & 37479 & 36831 & 37133 & 37459 & 36842 & 36867 & 36735 & 36561 & 36774 & 36533\end{array}$ $\begin{array}{lllllllllll}521 & 31894 & 29992 & 28128 & 26716 & 24991 & 23878 & 22611 & 21879 & 21011 & 20620 \\ 541 & 10852 & 16893 & 16508 & 16485 & 16683 & 16525 & 16819 & 17329 & 18386 & 19776\end{array}$ $\begin{array}{llllllllllll}541 & 10852 & 16893 & 16508 & 16485 & 16683 & 16525 & 16819 & 17329 & 18386 & 19776 \\ 561 & 10056 & 16963 & 17822 & 19568 & 26043 & 38748 & 51649 & 53910 & 41057 & 24819\end{array}$ $\begin{array}{lllllllllll}581 & 10280 & 9968 & 9501 & 9376 & 9194 & 8880 & 8977 & 8606 & 8645 & 8426\end{array}$

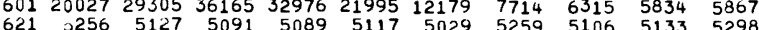
$\begin{array}{lllllllllll}641 & 4887 & 4763 & 4953 & 4995 & 4907 & 5105 & 5096 & 5041 & 5159 & 5144\end{array}$ $\begin{array}{lllllllllll}601 & 27586 & 14525 & 7494 & 5365 & 4917 & 4528 & 4594 & 4694 & 4708 & 4667\end{array}$ $\begin{array}{lllllllllll}681 & 5644 & 5829 & 6539 & 8418 & 13011 & 21758 & 34606 & 46654 & 49579 & 37758\end{array}$ $\begin{array}{lllllllllll}701 & 0130 & 3588 & 2610 & 2326 & 2184 & 2122 & 2108 & 2226 & 2212 & 2088 \\ 721 & 3975 & 5578 & 7104 & 7368 & 6289 & 4367 & 2968 & 2618 & 2607 & 2581\end{array}$ $\begin{array}{lllllllllll}7+1 & 2016 & 2028 & 2042 & 2057 & 2014 & 1952 & 2036 & 1964 & 1939 & 1906 \\ 761 & 1849 & 1786 & 1826 & 1782 & 1839 & 1758 & 1669 & 1715 & 1641 & 1665\end{array}$ $\begin{array}{lllllllllll}781 & 1548 & 1459 & 1461 & 1482 & 1480 & 1505 & 1548 & 1521 & 1632 & 1703\end{array}$

\begin{tabular}{|c|c|c|c|c|c|c|c|c|c|c|}
\hline 801 & 1454 & 1466 & 1651 & $15 \overline{2} 9$ & $146 \overline{2}$ & 1388 & 1459 & 1363 & $13 \overline{9}$ & $1=3$ \\
\hline 821 & 1375 & 1256 & 1243 & 1254 & 1288 & 1290 & 1342 & 1228 & 1291 & 1287 \\
\hline 841 & 1149 & 1138 & 1136 & 1043 & 1083 & 1165 & 1075 & 1149 & 1128 & 1128 \\
\hline 801 & 989 & 948 & 945 & .030 & 1026 & & 1001 & 958 & 1006 & 1000 \\
\hline & 938 & 928 & 912 & 937 & 909 & 970 & 925 & 903 & 916 & 947 \\
\hline 901 & 880 & 865 & 903 & 871 & 929 & 887 & & 82 & & 943 \\
\hline 921 & 833 & $\$ 29$ & 793 & 030 & 836 & 800 & 785 & 746 & 793 & 802 \\
\hline 911 & 833 & 859 & 793 & 803 & 873 & 892 & 1035 & $13_{11}$ & 1847 & 2496 \\
\hline 951 & 707 & 685 & 720 & 677 & 728 & 692 & 620 & 655 & 652 & 636 \\
\hline 981 & 641 & 631 & 627 & 597 & 65 & 64 & & & & 648 \\
\hline 001 & 631 & 626 & 610 & 621 & 617 & 62 & 58 & 628 & 639 & 631 \\
\hline 21 & 1269 & 1306 & 1287 & 1040 & 833 & 612 & 557 & 326 & 548 & 570 \\
\hline & 504 & 541 & 528 & 544 & & & & & & 722 \\
\hline & 63 & & 724 & 68 & & 55 & & 52 & 51 & 52 \\
\hline 381 & 584 & 560 & 589 & 525 & 497 & 502 & 473 & 484 & 486 & 45 \\
\hline 01 & 457 & 476 & 452 & 439 & 473 & 436 & 451 & 432 & 463 & 418 \\
\hline & 491 & 445 & 453 & 498 & 467 & $\begin{array}{r}450 \\
457\end{array}$ & 435 & 468 & 493 & 470 \\
\hline & 434 & 434 & 451 & 429 & 40 & 45 & & 44 & 48 & 480 \\
\hline & 39 & 36 & 319 & 374 & 378 & 38 & 357 & 329 & & 358 \\
\hline 18 & 357 & 348 & 343 & 380 & 327 & & 341 & 369 & & \\
\hline 0 & 376 & 411 & 410 & 404 & 52 & 52 & 48 & 42 & & 11 \\
\hline & 313 & 28 & 29 & 26 & & & & & & \\
\hline & & 27 & 23 & 25 & & & & & & 293 \\
\hline & 24 & 244 & 24 & 251 & 224 & 23 & 236 & 27 & 22 & 255 \\
\hline 28 & 23 & 247 & 21 & 257 & 217 & & 24 & & & \\
\hline 0 & & & & 23 & & & & & & \\
\hline & 248 & 238 & 23 & 21 & & & & & & \\
\hline & & & 45 & & 58 & & & & & \\
\hline & & & 19 & 20 & 18 & 16 & 18 & 17 & & \\
\hline 138 & 16 & 198 & & & & & & & & \\
\hline & & & & & & & & & & \\
\hline & & & & & & & & & & \\
\hline+6 & 189 & 161 & 18 & 18 & 16 & 16 & 1 & & & \\
\hline & 15 & & 17 & 16 & 17 & 19 & 18 & 18 & 19 & \\
\hline 146 & 17 & & 19 & 17 & 16 & & & & & \\
\hline & & & & & & & & & & \\
\hline & 16 & & & 15 & 15 & 17 & 17 & 17 & & \\
\hline & 165 & 172 & 164 & 17 & 15 & 18 & 16 & 16 & & 15 \\
\hline 156 & 17 & 16 & 15 & 1 a & 17 & 17 & & 15 & & \\
\hline 15 & & 17 & & & & & & & & \\
\hline & & & & & & & & & 17 & \\
\hline 1621 & 19 & 203 & 177 & 134 & 17 & 150 & 17 & 17 & 16 & 17 \\
\hline & 19 & 18 & 17 & 18 & 17 & & & 15 & & \\
\hline & 18 & & 1 & & & & & & & \\
\hline & 157 & & 17 & & 17 & 18 & 17 & 16 & 18 & \\
\hline & 197 & 20 & 165 & 186 & 18 & & 17 & 19 & 179 & 17 \\
\hline & 161 & 187 & 19 & 18 & 22 & & 1 & 10 & & \\
\hline 174 & 18 & 21 & 20 & 21 & 19 & & & & & \\
\hline 177 & 171 & 159 & 163 & 146 & 150 & 135 & 117 & 119 & 113 & \\
\hline
\end{tabular}

$\begin{array}{rrrrrrrrrrr}1701 & 101 & 72 & 97 & 98 & 100 & 76 & 92 & 106 & 81 & 94 \\ 1801 & 90 & 80 & 95 & 31 & 53 & 91 & 75 & 73 & 67 & 84 \\ 1821 & 69 & 66 & 76 & 60 & 74 & 70 & 50 & 63 & 77 & 82 \\ 1841 & 68 & 58 & 62 & 47 & 48 & 58 & 47 & 53 & 59 & 51 \\ 1861 & 38 & 40 & 37 & 40 & 55 & 35 & 40 & 38 & 36 & 47 \\ 1881 & 40 & 42 & 26 & 29 & 37 & 33 & 22 & 27 & 31 & 28 \\ 1901 & 29 & 36 & 34 & 50 & 46 & 53 & 44 & 26 & 31 & 28 \\ 1921 & 23 & 28 & 30 & 17 & 22 & 30 & 28 & 25 & 23 & 32 \\ 1941 & 28 & 34 & 24 & 24 & 23 & 22 & 26 & 23 & 22 & 27 \\ 1961 & 73 & 112 & 126 & 197 & 228 & 265 & 339 & 369 & 441 & 446 \\ 1981 & 11 & 10 & 13 & 9 & 6 & 12 & 5 & 10 & 11 & 5 \\ 2001 & 8 & 7 & 10 & 9 & 7 & 4 & 9 & 6 & 6 & 8 \\ 2021 & 10 & 14 & 15 & 10 & 7 & 4 & 11 & 14 & 11 & 7 \\ 2041 & 10 & 4 & 7 & 4 & 14 & 11 & 5 & 0 & & 7\end{array}$

CUUNT COUNT COUNT COUNT COUNT COUNT COUNT COUNT COUNT COUNT

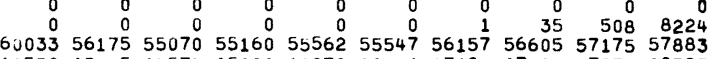

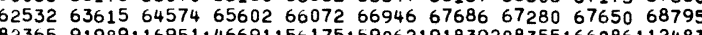
1147661028879236078017681576324261902619176193261474 $\begin{array}{lllllllll}7571165216 & 61465 & 60236 & 60239 & 60589 & 60586 & 60910 & 61880 & 62275\end{array}$ \begin{tabular}{llllllllll}
59452 & 59053 & 59099 & 59580 & 59236 & 59015 & 59304 & 58980 & 58924 & 59283 \\
\hline
\end{tabular} $\begin{array}{lllllllllll}59452 & 59053 & 59099 & 59580 & 59236 & 59015 & 59304 & 58980 & 58924 & 59283 \\ 50574 & 58263 & 58525 & 58156 & 58317 & 58002 & 57914 & 58629 & 58437 & 58548\end{array}$ $\begin{array}{lllllllllll}56796 & 57291 & 56975 & 56948 & 56788 & 56368 & 57046 & 56936 & 56726 & 56272\end{array}$ $\begin{array}{llllllllll}50419 & 55437 & 55666 & 55700 & 55731 & 55081 & 55690 & 55744 & 55105 & 55884\end{array}$ $\begin{array}{llllllllll}54486 & 54387 & 54952 & 55020 & 54812 & 54482 & 54822 & 54904 & 54866 & 55195 \\ 54797 & 54575 & 55024\end{array}$ $\begin{array}{llllllllll}54373 & 54991 & 54799 & 54909 & 55060 & 54764 & 55236 & 54819 & 54831 & 54680\end{array}$ $5481054744 \quad 5532555105549895499354823544415514454394$ $\begin{array}{llllllllll}52207 & 52222 & 51375 & 50690 & 50522 & 49620 & 49473 & 48179 & 47613 & 47051\end{array}$ $\begin{array}{llllllllll}45462 & 46115 & 45328 & 45877 & 45242 & 45175 & 44996 & 45211 & 45311 & 45198 \\ 44546 & 44594 & 44250 & 44232 & 44184 & 44350 & 44512 & 44364 & 43939 & 44035\end{array}$

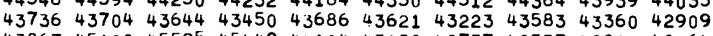

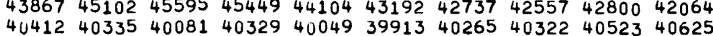
$\begin{array}{llllllllll}40412 & 40335 & 40081 & 40329 & 40049 & 39913 & 40265 & 40322 & 40523 & 40625 \\ 40076 & 40454 & 40326 & 40279 & 40406 & 40186 & 40079 & 40472 & 39577 & 39405\end{array}$ $\begin{array}{llllllllllll}400259 & 41747 & 46318 & 53729 & 61555 & 61553 & 53042 & 45083 & 41702 & 40219 \\ 717588 & 48726 & 41317 & 39153 & 38964 & 39033 & 38692 & 38830 & 38453 & 38738\end{array}$

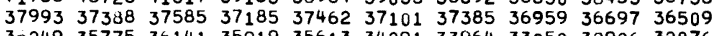
$\begin{array}{llllllllll}30249 & 35775 & 36141 & 35919 & 35613 & 34291 & 33964 & 33250 & 32926 & 32876 \\ 19933 & 19533 & 18965 & 18459 & 18390 & 17833 & 18005 & 17449 & 17469 & 17072\end{array}$

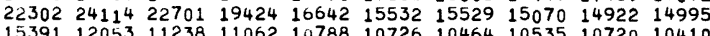
$\begin{array}{lllllllllll}15391 & 12053 & 11238 & 11062 & 10788 & 10726 & 10464 & 10535 & 10720 & 10410\end{array}$ $\begin{array}{rrrrrrrrrr}8204 & 7825 & 7883 & 7904 & 7837 & 7787 & 7667 & 8100 & 9531 & 12806 \\ 5778 & 5719 & 5569 & 5599 & 5443 & 5403 & 5338 & 5219 & 5273 & 5270\end{array}$ $\begin{array}{rllllllllll}5635 & 7011 & 8657 & 55083 & 10238 & 8661 & 6569 & 5359 & 5033 & 4762\end{array}$ $4784 \quad 4789 \quad 4869 \quad 4890 \quad 4930 \quad 5113 \quad 5327 \quad 5225 \quad 526350270$

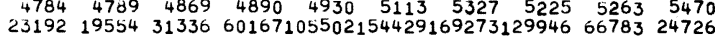
$\begin{array}{llllllllll}2240 & 2165 & 2118 & 2188 & 2097 & 2048 & 2162 & 2250 & 2485 & 2960\end{array}$ $\begin{array}{llllllllll}2326 & 2106 & 2030 & 2114 & 2032 & 1996 & 2027 & 2005 & 2031 & 2060\end{array}$ $\begin{array}{llllllllll}1965 & 1952 & 1950 & 1930 & 1872 & 1932 & 2001 & 1943 & 2037 & 1972 \\ 1626 & 1579 & 1660 & 1671 & 1513 & 1578 & 1485 & 1532 & 1575 & 1488\end{array}$ $\begin{array}{llllllllll}2066 & 2400 & 2740 & 2750 & 2329 & 1915 & 1577 & 1484 & 1449 & 1449\end{array}$

$\begin{array}{llllllllll}1375 & 1316 & 1386 & 1311 & 1304 & 1331 & 1319 & 1368 & 1316 & 1301 \\ 1296 & 1228 & 1264 & 1153 & 1201 & 1174 & 1225 & 1204 & 1112 & 1120\end{array}$

$\begin{array}{rrrrrrrrrr}1156 & 1099 & 1109 & 1049 & 1078 & 1057 & 1071 & 1042 & 1034 & 1015 \\ 1004 & 1002 & 966 & 918 & 983 & 972 & 928 & 939 & 971 & 926\end{array}$

$\begin{array}{llllllllll}948 & 912 & 847 & 846 & 836 & 865 & 928 & 939 & 971 & 926 \\ 903 & 959 & 917 & 869 & 847 & 846 & 816 & 817 & 864 & 884\end{array}$

$\begin{array}{llllllllll}793 & 758 & 779 & 803 & 769 & 769 & 732 & 803 & 833 & 754\end{array}$

$\begin{array}{lllllllllll}3265 & 3892 & 3922 & 2945 & 1806 & 1094 & 826 & 696 & 754 & 698\end{array}$

$\begin{array}{llllllllll}633 & 619 & 720 & 618 & 650 & 643 & 649 & 654 & 629 & 655\end{array}$

$\begin{array}{rrrrrrrrrr}684 & 626 & 643 & 631 & 662 & 632 & 615 & 659 & 632 & 621\end{array}$

$\begin{array}{rrrrrrrrrr}581 & 540 & 524 & 542 & 533 & 567 & 538 & 569 & 565 & 1042 \\ & 867 & 921 & 1037 & 1093 & 891 & 811 & 704 & 607 & 558\end{array}$

$\begin{array}{rrrrr}807 & 867 & 921 & 1037 & 1093 \\ 505 & 554 & 517 & 482 & 477\end{array}$

$\begin{array}{lll}566 & 538 & 474\end{array}$

$\begin{array}{lll}450 & 456 & 446 \\ 458 & 411 & 438\end{array}$

$\begin{array}{lll}458 & 411 & 438 \\ 514 & 542 & 590\end{array}$

$\begin{array}{lll}352 & 415 & 325 \\ 302 & 390 & 365\end{array}$

$\begin{array}{lll}302 & 390 & 365 \\ 334 & 318 & 303\end{array}$

$\begin{array}{lll}287 & 344 & 345 \\ 313 & 272 & 301\end{array}$

$\begin{array}{lll}313 & 272 & 301 \\ 223 & 254 & 220\end{array}$

$\begin{array}{lll}202 & 221 & 235 \\ 242 & 232 & 197\end{array}$

$\begin{array}{lll}214 & 221 & 212 \\ 256 & 204 & 229\end{array}$

$\begin{array}{lll}200 & 197 & 217 \\ 201 & 188 & 188\end{array}$

$\begin{array}{lll}201 & 188 & 188 \\ 173 & 348 & 327\end{array}$

$\begin{array}{lll}181 & 191 & 186 \\ 184 & 188 & 184\end{array}$

$\begin{array}{lll}184 & 188 & 184 \\ 178 & 158 & 158\end{array}$

$\begin{array}{lll}202 & 199 & 223 \\ 159 & 134 & 162\end{array}$

$\begin{array}{lll}176 & 152 & 152 \\ 148 & 177 & 156 \\ 194 & 192 & 174\end{array}$

$\begin{array}{lll}194 & 192 & 174 \\ 172 & 173 & 167 \\ 166 & 162 & 170\end{array}$

$\begin{array}{lll}166 & 162 & 176 \\ 167 & 169 & 185\end{array}$

$\begin{array}{lll}162 & 180 & 180 \\ 183 & 188 & 163\end{array}$

$\begin{array}{llll}183 & 188 & 163 & 1 \\ 195 & 199 & 203 & 1\end{array}$

$\begin{array}{rrr}191 & 174 & 207 \\ 196 & 176 & 186 \\ 102 & 91 & 133\end{array}$

$\begin{array}{ll}499 & 461 \\ 448 & 437\end{array}$

$441 \quad 464$

$\begin{array}{ll}605 & 486 \\ 396 & 329\end{array}$

$\begin{array}{ll}290 & 303 \\ 340 & 346\end{array}$

$300 \quad 246$

$\begin{array}{ll}246 & 227 \\ 226 & 235\end{array}$

$212 \quad 180 \quad 198$

$\begin{array}{lll}327 & 171 & 194 \\ 165 & 147 & 201\end{array}$

$170 \quad 180$

$\begin{array}{ll}182 & 168 \\ 162 & 180\end{array}$

$\begin{array}{ll}201 & 172 \\ 190 & 169\end{array}$

$\begin{array}{ll}138 & 150 \\ 152 & 183\end{array}$

191206

$\begin{array}{lll}163 & 180 & 175\end{array}$

$\begin{array}{lll}197 & 190 & 177 \\ 173 & 175 & 165\end{array}$

$\begin{array}{lll}161 & 183 & 193 \\ 192 & 182 & 207\end{array}$

$\begin{array}{lll}192 & 182 & 207 \\ 157 & 187 & 191\end{array}$

$\begin{array}{rrrrrrrrrr}96 & 124 & 110 & 110 & 105 & 103 & 108 & 73 & 88 & 69 \\ 67 & 84 & 85 & 91 & 86 & 02 & 98 & 73 & 78 & 65 \\ 60 & 65 & 57 & 59 & 52 & 60 & 53 & 53 & 64 & 45 \\ 52 & 51 & 50 & 47 & 43 & 40 & 50 & 46 & 44 & 35 \\ 38 & 34 & 29 & 32 & 33 & 22 & 34 & 29 & 30 & 20 \\ 30 & 27 & 30 & 27 & 24 & 24 & 29 & 33 & 26 & 35 \\ 25 & 21 & 26 & 23 & 27 & 27 & 37 & 30 & 18 & 23 \\ 20 & 31 & 22 & 16 & 27 & 29 & 30 & 27 & 17 & 16 \\ 26 & 25 & 26 & 20 & 32 & 27 & 32 & 40 & 54 & 69 \\ 532 & 452 & 345 & 191 & 76 & 35 & 23 & 13 & 20 & 9 \\ 11 & 10 & 10 & 17 & 12 & 11 & 10 & 12 & 13 & 10 \\ 8 & 9 & 6 & 10 & 12 & 12 & 10 & 6 & 7 & 15 \\ 8 & 9 & 7 & 9 & 10 & 9 & 15 & 6 & 5 & 12\end{array}$


GAMMA RAY SPECTRUM DATA

PRTPR ROD FR-78 SPECTRUM NO. 15 DATA TAKEN 9/17/69 LIVE TIME 180.MIN. POSITION 30,IN, ENERGY(KEV) $1,113 * C H A N=9.0$ CHAN COUNT COUNT COUNT COUNT COUNT COUNT COUNT COUNT COUNT COUNT

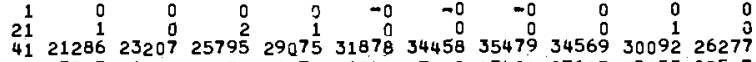
$\begin{array}{llllllllllllll}61 & 25845 & 26366 & 26331 & 26470 & 26944 & 27009 & 27421 & 27607 & 28053 & 28517\end{array}$ $\begin{array}{rllllllllll}81 & 32923 & 32584 & 32501 & 32819 & 33240 & 34111 & 34408 & 35859 & 37548 & 40448 \\ 101 & 39989 & 37922 & 37810 & 38276 & 41375 & 48979 & 60396 & 68880 & 68812 & 61168\end{array}$ $\begin{array}{lllllllllll}101 & 39989 & 37922 & 37810 & 38276 & 41375 & 48979 & 60396 & 68880 & 68812 & 61168 \\ 121 & 28820 & 28397 & 28376 & 28824 & 30404 & 34422 & 40385 & 45858 & 44963 & 39309\end{array}$ $\begin{array}{lllllllllll}121 & 28820 & 28397 & 28376 & 28824 & 30404 & 34422 & 40385 & 45858 & 4963 & 39309 \\ 141 & 27789 & 27328 & 27524 & 27629 & 27553 & 27249 & 27538 & 26927 & 27505 & 27235\end{array}$

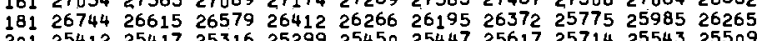
$\begin{array}{lllllllllll}221 & 25289 & 25294 & 25047 & 25142 & 25276 & 25154 & 25251 & 25083 & 25309 & 25359\end{array}$ $\begin{array}{lllllllllll}241 & 24675 & 24522 & 24794 & 24862 & 24962 & 24741 & 24791 & 24721 & 24782 & 24803\end{array}$ $\begin{array}{lllllllllll}261 & 24664 & 24687 & 24771 & 24813 & 24726 & 24794 & 24724 & 24517 & 24935 & 24935\end{array}$

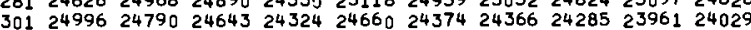
$\begin{array}{lllllllllll}321 & 20266 & 19946 & 19889 & 19717 & 19851 & 19586 & 19529 & 19519 & 19558 & 19580 \\ 341 & 19042 & 19246 & 19056 & 18994 & 18808 & 19176 & 18597 & 18871 & 18996 & 18630\end{array}$ $\begin{array}{lllllllllll}341 & 19042 & 19246 & 19056 & 18994 & 18808 & 19176 & 18597 & 18871 & 18996 & 18630 \\ 361 & 18255 & 18421 & 18247 & 18097 & 18528 & 18306 & 18352 & 18152 & 18216 & 17969\end{array}$ $\begin{array}{lllllllllll}361 & 18255 & 18421 & 18247 & 18097 & 18528 & 18306 & 18352 & 18152 & 18216 & 17969 \\ 381 & 17809 & 17628 & 18006 & 17745 & 17718 & 17639 & 18158 & 17653 & 17786 & 18178\end{array}$ $\begin{array}{lllllllllllll}401 & 16687 & 16623 & 16412 & 15987 & 16048 & 15794 & 15487 & 15245 & 15137 & 15166\end{array}$

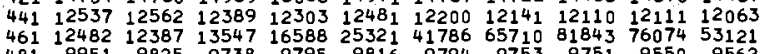
$5019014 \quad 9062 \quad 8982917791319039 \quad 9065$ $\begin{array}{llllllllllll}521 & 9161 & 8408 & 7798 & 7360 & 7154 & 6749 & 6605 & 6458 & 6392 & 6263\end{array}$ $\begin{array}{lllllllllll}541 & 5787 & 5790 & 5748 & 5683 & 5842 & 6065 & 6687 & 7567 & 9236 & 11910\end{array}$ $\begin{array}{lllllllllll}561 & 7038 & 7881 & 9332 & 13051 & 20439 & 29083 & 33698 & 29872 & 20308 & 11478\end{array}$ $\begin{array}{lllllllllll}601 & 22911 & 30141 & 30965 & 23486 & 13946 & 7279 & 4040 & 2928 & 2582 & 2477\end{array}$ $\begin{array}{lllllllllll}621 & 2345 & 2178 & 2265 & 2259 & 2196 & 2197 & 2253 & 2301 & 2397 & 2564\end{array}$

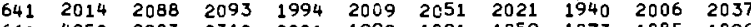
$\begin{array}{lllllllllll}661 & 4250 & 2903 & 2312 & 2001 & 1999 & 1891 & 1859 & 1873 & 1885 & 1896 \\ 681 & 2029 & 2209 & 2484 & 3140 & 4471 & 6430 & 8029 & 8525 & 7599 & 5809\end{array}$ $\begin{array}{lllllllllll}701 & 2176 & 1629 & 1556 & 1549 & 1523 & 1490 & 1588 & 1482 & 1502 & 1601\end{array}$ $\begin{array}{llllllllllll}721 & 5236 & 6710 & 7307 & 6115 & 4465 & 3077 & 2412 & 2177 & 1978 & 1813\end{array}$ $\begin{array}{lllllllllll}741 & 1428 & 1364 & 1426 & 1356 & 1345 & 1357 & 1358 & 1279 & 1371 & 1328 \\ 761 & 1286 & 1227 & 1255 & 1271 & 1182 & 1200 & 1210 & 1201 & 1096 & 1130\end{array}$ $\begin{array}{lllllllllll}781 & 1085 & 1021 & 1058 & 1014 & 1052 & 1071 & 1091 & 1168 & 1221 & 1480\end{array}$

COUNT COUNT GOUNT COUNT COUNT GOUNT COUNT COUNT COUNT COUNT $\begin{array}{rrrrrrrrrr}0 & 0 & 0 & 0 & 0 & 0 & 0 & 0 & 0 & 0 \\ 1 & 0 & 0 & 1 & 0 & 1 & 5 & 13 & 830 & 11666 \\ 24189 & 23384 & 23831 & 24134 & 24616 & 24564 & 25094 & 25064 & 25357 & 25328\end{array}$ $\begin{array}{llllllllll}28604 & 29030 & 29439 & 30201 & 30669 & 30754 & 30898 & 31249 & 31906 & 32363 \\ 47163 & 59122 & 72349 & 81064 & 88736 & 98907102125 & 87708 & 65367 & 47562\end{array}$ $\begin{array}{lllllllllll}47163 & 59122 & 72349 & 81064 & 88736 & 98907102125 & 87708 & 65367 & 47562 \\ 53001 & 45856 & 38991 & 33421 & 30273 & 28811 & 28506 & 28631 & 28424 & 28618\end{array}$ $\begin{array}{llllllllll}53001 & 45856 & 38991 & 33421 & 30273 & 28811 & 28506 & 28631 & 28424 & 28618 \\ 32973 & 29514 & 2 \varepsilon 291 & 27676 & 27589 & 27472 & 27561 & 27457 & 27809 & 27359\end{array}$

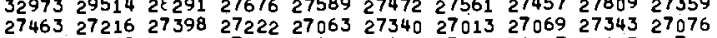

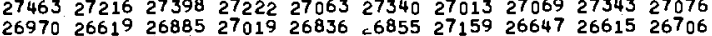

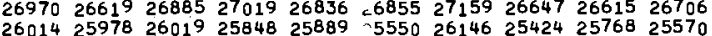
$\begin{array}{llllllllll}26014 & 25978 & 26019 & 258448 & 25889 & 5550 & 26146 & 25424 & 25768 & 25570 \\ 25276 & 25075 & 25216 & 25221 & 25295 & 25191 & 25409 & 25096 & 25333 & 25104\end{array}$

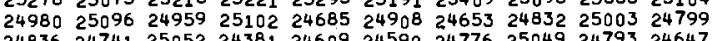
$\begin{array}{lllllllllll}24836 & 24741 & 25052 & 2438 & 24609 & 24590 & 24776 & 25049 & 24793 & 24647\end{array}$

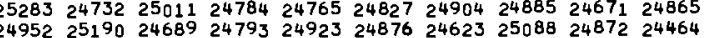
$2369123363230072245921785215602128821018 \quad 2058820368$

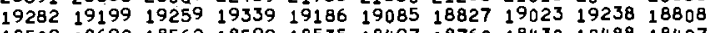
$\begin{array}{llllllllll}18509 & 18692 & 18562 & 18599 & 18535 & 18497 & 18760 & 18430 & 18488 & 18427 \\ 17796 & 17846 & 17907 & 17868 & 18220 & 17940 & 17910 & 17611 & 17701 & 178699\end{array}$ $\begin{array}{llllllllll}17796 & 17846 & 17907 & 17868 & 18220 & 17940 & 17910 & 17611 & 17701 & 17869 \\ 18667 & 19115 & 19354 & 18611 & 18208 & 17277 & 17083 & 16842 & 16880 & 16825\end{array}$ $\begin{array}{llllllllll}15063 & 14931 & 15169 & 14789 & 14776 & 15014 & 14925 & 14712 & 14521 & 14642 \\ 14091 & 14105 & 14213 & 14056 & 13930 & 13719 & 13367 & 13153 & 12895 & 12633\end{array}$ $\begin{array}{llllllllll}12157 & 12053 & 12622 & 12742 & 13200 & 128_{11} & 12578 & 123_{15} & 12261 & 12190\end{array}$

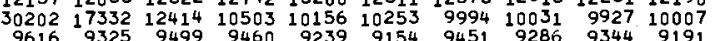
\begin{tabular}{llllllllll}
9190 & 9311 & 9417 & 9503 & 9473 & 8998 & 8944 & 9235 & 9456 & 9493 \\
\hline
\end{tabular} $\begin{array}{lllllllllll}14071 & 13956 & 11758 & 8506 & 6426 & 5329 & 5090 & 5194 & 5408 & 6222\end{array}$ $\begin{array}{llllllllll}6514 & 4460 & 3933 & 3736 & 3745 & 3706 & 3879 & 3916 & 4002 & 4044 \\ 3359 & 3462 & 3432 & 3409 & 3410 & 3533 & 4013 & 5256 & 8273 & 14341\end{array}$ $\begin{array}{rlllllllll}3359 & 3462 & 3432 & 3409 & 3410 & 3533 & 4013 & 5256 & 8273 & 14341 \\ 2558 & 2483 & 2458 & 2464 & 2411 & 2394 & 2282 & 2262 & 2310 & 2311\end{array}$ $\begin{array}{llllllllll}2558 & 2483 & 2458 & 2464 & 2411 & 2394 & 2282 & 2262 & 2310 & 2311 \\ 3299 & 4368 & 5432 & 5612 & 4959 & 3725 & 2822 & 2267 & 2059 & 1996\end{array}$ $\begin{array}{llllllllll}2073 & 2137 & 2149 & 2407 & 3122 & 432 B & 6125 & 7686 & 7596 & 6312 \\ 1870 & 1956 & 1973 & 2000 & 2007 & 1999 & 1937 & 2103 & 1950 & 2009\end{array}$ $\begin{array}{rrrrrrrrrr}1870 & 1956 & 1973 & 2000 & 2007 & 1999 & 1937 & 2103 & 1950 & 2009 \\ 5044 & 6241 & 10685 & 17847 & 25074 & 27326 & 23346 & 14923 & 7884 & 3805\end{array}$ $\begin{array}{llllllrrrr}1512 & 1513 & 1545 & 1537 & 1563 & 1525 & 1682 & 1966 & 2454 & 3681 \\ 1560 & 1474 & 1491 & 1375 & 1401 & 1383 & 1352 & 1360 & 1420 & 1438\end{array}$ $\begin{array}{llllllllll}1365 & 1314 & 1327 & 1322 & 1316 & 1314 & 1298 & 1350 & 1318 & 1283 \\ 1098 & 1143 & 1085 & 1053 & 1084 & 1059 & 1060 & 1062 & 1083 & 1039\end{array}$ $\begin{array}{rrrrrrrrrr}1791 & 2085 & 1950 & 1690 & 1473 & 1146 & 1028 & 978 & 999 & 987\end{array}$

\begin{tabular}{|c|c|c|c|c|c|c|c|c|c|}
\hline 972 & 952 & 951 & 940 & 922 & 933 & 887 & 882 & 954 & 894 \\
\hline 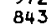 & 840 & 781 & 859 & 800 & 785 & 817 & 840 & 764 & 756 \\
\hline 764 & 24 & 15 & 749 & & 691 & 747 & 683 & 660 & 688 \\
\hline 674 & 684 & 690 & 673 & 670 & 664 & 645 & 646 & 633 & 625 \\
\hline 610 & 651 & 650 & 590 & 614 & 584 & 646 & 590 & 582 & 631 \\
\hline 764 & 653 & 639 & 600 & 589 & 549 & 598 & & 18 & 527 \\
\hline 534 & 540 & 547 & 520 & 549 & 569 & 599 & 568 & 502 & 569 \\
\hline 2817 & 2733 & 2205 & 1411 & 942 & 678 & 496 & 507 & 501 & 522 \\
\hline 453 & 446 & 444 & 492 & 457 & 426 & 449 & 479 & 449 & 417 \\
\hline 415 & 380 & 382 & 451 & 464 & 424 & 449 & 413 & 414 & 443 \\
\hline 424 & 414 & 396 & 444 & 446 & 496 & 501 & 624 & 803 & 908 \\
\hline 419 & 367 & 401 & 405 & 361 & 399 & 408 & & 365 & 355 \\
\hline 598 & 743 & 745 & 747 & 633 & 599 & & & 687 & 459 \\
\hline 347 & 342 & 331 & 339 & 335 & 373 & & 38 & & 463 \\
\hline 340 & 370 & 332 & 323 & 323 & & & & & 307 \\
\hline 298 & 318 & 316 & $=$ & 285 & 334 & & & 0 & 291 \\
\hline 316 & 313 & 331 & 338 & 311 & 305 & & 30 & 305 & 272 \\
\hline 483 & 484 & 480 & 402 & 366 & 315 & 273 & 252 & 237 & 237 \\
\hline 249 & 234 & 239 & & 228 & 246 & & & & 250 \\
\hline 22 & 231 & 219 & 235 & 232 & 217 & 21 & $2^{4}$ & 253 & 246 \\
\hline 20 & 196 & 21 & 223 & 239 & 219 & 21 & 19 & 18 & 193 \\
\hline 252 & 265 & 298 & 304 & 246 & 219 & 21 & 22 & 1 & 171 \\
\hline 182 & 215 & 196 & & 174 & 18 & & & & 176 \\
\hline 162 & $\begin{array}{l}142 \\
142\end{array}$ & 161 & 13 & 161 & 18 & & & & 140 \\
\hline 158 & 130 & 200 & 12 & 147 & 148 & & & & 146 \\
\hline 142 & 136 & 161 & 128 & 153 & 158 & 12 & 144 & 17 & 134 \\
\hline 146 & 133 & 166 & & 128 & & & & & 174 \\
\hline 164 & & 162 & & 17 & & & & & 139 \\
\hline 13 & 149 & 118 & 14 & 11 & 129 & & & & 125 \\
\hline 13 & 109 & 98 & 139 & 139 & 125 & & & & 145 \\
\hline 27 & 235 & 229 & 20 & 150 & 139 & & & & 139 \\
\hline 104 & 110 & 114 & 104 & 119 & 102 & 11 & & 9 & 111 \\
\hline 135 & 108 & 10 & & 91 & 148 & & & 1 & 104 \\
\hline 11 & 121 & 113 & & 95 & & & & & 89 \\
\hline 11 & 100 & 113 & 10 & 107 & 124 & & & 13 & 120 \\
\hline 14 & 15 & 150 & 124 & 116 & 126 & 12 & & & 107 \\
\hline & 11 & 106 & & 106 & 102 & & & & 105 \\
\hline & & 95 & & 11 & & & & & 117 \\
\hline 118 & & 99 & & 12 & & & & & 107 \\
\hline 109 & & 147 & & 12 & & & & & 123 \\
\hline 92 & 10 & 108 & 11 & 10 & 10 & $1 \mathrm{c}$ & & & 135 \\
\hline 118 & 107 & 99 & 11 & 115 & 10 & 1 & & & 111 \\
\hline 118 & & 142 & & 13 & & & & & 127 \\
\hline 121 & 1 & 12 & 11 & 11 & 11 & & & & 122 \\
\hline 11 & 11 & 115 & 12 & 11 & 10 & 13 & & 12 & 106 \\
\hline 147 & 120 & 123 & 10 & 111 & & 13 & & 55 & 126 \\
\hline 131 & 121 & 121 & 121 & 132 & 12 & 1 & 1 & & 128 \\
\hline 135 & 114 & 118 & 111 & 108 & 110 & 107 & 119 & 101 & 104 \\
\hline 85 & 65 & 56 & 56 & 73 & 62 & 60 & 59 & 58 & 66 \\
\hline
\end{tabular}

$\begin{array}{rrrrrrrrrrr}1781 & 50 & 61 & 60 & 62 & 62 & 60 & 58 & 72 & 58 & 66 \\ 1801 & 68 & 63 & 52 & 55 & 60 & 67 & 54 & 60 & 56 & 61 \\ 1821 & 45 & 55 & 62 & 47 & 46 & 52 & 53 & 41 & 42 & 46 \\ 1841 & 42 & 46 & 36 & 37 & 48 & 35 & 30 & 35 & 26 & 44 \\ 1861 & 36 & 42 & 30 & 26 & 27 & 33 & 36 & 22 & 23 & 33 \\ 1881 & 28 & 28 & 25 & 23 & 27 & 32 & 24 & 20 & 25 & 16 \\ 1901 & 29 & 33 & 47 & 36 & 35 & 40 & 36 & 27 & 22 & 31 \\ 1921 & 18 & 17 & 15 & 15 & 11 & 8 & 16 & 14 & 17 & 16 \\ 1941 & 15 & 12 & 21 & 14 & 25 & 20 & 24 & 12 & 11 & 17 \\ 1961 & 24 & 41 & 43 & 61 & 89 & 111 & 109 & 185 & 250 & 301 \\ 1981 & 13 & 11 & 8 & 11 & 8 & 7 & 10 & 10 & 9 & 8 \\ 2001 & 6 & 3 & 6 & 5 & 7 & 6 & 5 & 11 & 7 & 6 \\ 2021 & 5 & 6 & 11 & 13 & 5 & 7 & 9 & 4 & 7 & 8 \\ 2041 & 4 & 4 & 10 & 6 & 4 & 3 & 4 & 0 & & \end{array}$

$\begin{array}{rrrrrrrrrr}54 & 52 & 60 & 89 & 54 & 84 & 65 & 63 & 54 & 64 \\ 54 & 54 & 65 & 44 & 46 & 55 & 53 & 69 & 54 & 63 \\ 41 & 43 & 42 & 45 & 48 & 45 & 63 & 39 & 38 & 49 \\ 46 & 28 & 41 & 28 & 32 & 43 & 43 & 35 & 27 & 33 \\ 15 & 16 & 28 & 20 & 23 & 30 & 25 & 24 & 22 & 25 \\ 21 & 16 & 18 & 10 & 17 & 30 & 8 & 27 & 19 & 17 \\ 23 & 14 & 9 & 13 & 13 & 17 & 13 & 13 & 17 & 15 \\ 15 & 18 & 14 & 17 & 24 & 19 & 22 & 5 & 21 & 19 \\ 16 & 19 & 16 & 18 & 12 & 18 & 20 & 24 & 20 & 21 \\ 371 & 392 & 341 & 309 & 277 & 164 & 114 & 62 & 30 & 26 \\ 8 & 7 & 7 & 9 & 4 & 8 & 3 & 7 & 8 & 9 \\ 4 & 5 & 8 & 7 & 9 & 5 & 8 & 11 & 5 & 6 \\ 5 & 7 & 4 & 2 & 7 & 1 & 5 & 5 & 6 & 4\end{array}$




\section{D-24}

GAMMA RAY SPECTRUM DATA

PRTR ROD FS-07 SPECTRUM NO. 2 DATA TAKEN 9/08/69 LIVE TIME 860.MIN. POSITION 30.RN. ENERGY(KEVI= 1.111*CHAN -6.8 CHAN COUNT COUNT COUNT COUNT COUNT COUNT COUNT COUNT COUNT COUNT

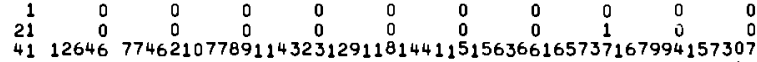
61119778121317122634124494126191126953127668129618131420132814 81154407157118157518157583160678163706167226171776178061183628 101234959193247181070178240182002191980222233273514318514322845
121136163135231135228135505137251141821157307184692213301215537 141130075129407130283129445130011129334129417128425128542128383 161128353127972128211127907128474129000129885129766128964127179
181125548125652125380124606124456123980124669124040124158123322 201121361120820120929120787120697119895120303120015119766119997 221118983118262118957117762118774118141118273117853117066118047 241116965116661116677116822116974117172117042116342116931116922 281116702116609116867116331117142116314116969116888117077116850 321971109678195866559047779394894549939829313593015 32197110967819586695180947779394894549939829313593015 $\begin{array}{lllllllllll}361 & 88655 & 88023 & 87018 & 87200 & 87583 & 87231 & 87247 & 87129 & 86984 & 86490\end{array}$ $\begin{array}{lllllllllll}381 & 85695 & 85657 & 85520 & 85402 & 85183 & 84920 & 84406 & 84684 & 85212 & 86514\end{array}$

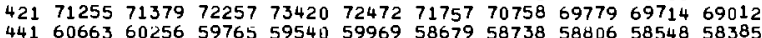
$461000106175267156 \quad 84875129324212705314875367906324563217703$ $\begin{array}{llllllllllll}481 & 48134 & 47773 & 47798 & 47512 & 48123 & 47831 & 47182 & 47078 & 46882 & 46303\end{array}$ $\begin{array}{lllllllllll}501 & 44493 & 44355 & 44383 & 44135 & 43634 & 44223 & 43699 & 43432 & 43647 & 43611\end{array}$ 52143007394673695434895335753246331549309283057129898 $\begin{array}{lllllllllll}541 & 27820 & 27563 & 27218 & 27851 & 28265 & 30668 & 33704 & 39306 & 49144 & 61561\end{array}$ $\begin{array}{lllllllllll}581 & 18580 & 17350 & 17181 & 16706 & 16491 & 16520 & 16179 & 16193 & 16305 & 16113\end{array}$ $60112113715077614444210387558592 \quad 29618 \quad 16848 \quad 1286611898 \quad 11849$

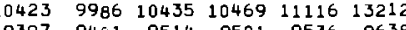

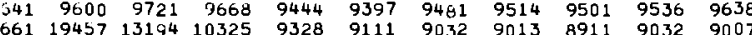

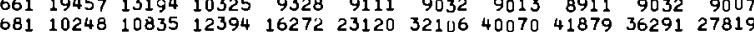
$\begin{array}{lllllllllll}701 & 9426 & 7550 & 7100 & 6877 & 6877 & 6899 & 6832 & 6953 & 6818 & 7140\end{array}$ $\begin{array}{llllllllllll}721 & 25526 & 32582 & 35100 & 29702 & 21080 & 14235 & 10730 & 9708 & 9206 & 8273\end{array}$ $\begin{array}{lllllllllll}741 & 6449 & 6254 & 6178 & 6211 & 6203 & 6024 & 5963 & 5974 & 6117 & 6107\end{array}$ $\begin{array}{lllllllllll}761 & 5869 & 5664 & 5714 & 5615 & 5442 & 5404 & 5380 & 5320 & 5178 & 5103 \\ 781 & 4769 & 4797 & 4745 & 4818 & 4754 & 4798 & 4904 & 5203 & 5806 & 6715\end{array}$

\begin{tabular}{|c|c|c|c|c|c|c|c|c|c|c|}
\hline $\begin{array}{l}801 \\
821\end{array}$ & $\begin{array}{l}4872 \\
4134\end{array}$ & $\begin{array}{l}5186 \\
3988\end{array}$ & $\begin{array}{r}4968 \\
4036\end{array}$ & $\begin{array}{l}4841 \\
4040\end{array}$ & $\begin{array}{l}4612 \\
4070\end{array}$ & $\begin{array}{r}4593 \\
4134\end{array}$ & $\begin{array}{l}4356 \\
3960\end{array}$ & $\begin{array}{r}4279 \\
3904\end{array}$ & $\begin{array}{r}4230 \\
400\end{array}$ & $\begin{array}{l}4141 \\
3872\end{array}$ \\
\hline 841 & 3484 & 3351 & 3548 & 3513 & 3492 & 3618 & 3527 & 3612 & 3609 & 3561 \\
\hline 861 & 308 & 3145 & 3106 & 3142 & & & & & & \\
\hline & 282 & & 278 & & & & & & & \\
\hline 90 & 3047 & 3051 & 3146 & 2969 & 2878 & & 3 & & & 32 \\
\hline 92 & 2457 & 2 & 2617 & & & 24 & & & & 243 \\
\hline 941 & 2725 & 2822 & 277 & 3 & & & & & 8203 & 1097 \\
\hline 901 & 27 & 30 & 234 & & & & & & & \\
\hline 981 & 1988 & 205 & 202 & 20 & 20 & & 5 & & & \\
\hline & 1901 & 19 & 19 & & & & & & & \\
\hline & 4285 & 4094 & & & & & & & & \\
\hline 14 & 1703 & 1734 & 1628 & 173 & 1632 & 17 & 1751 & & & 231 \\
\hline & 2254 & 222 & 218 & 19 & 172 & & 1629 & & & \\
\hline & 186 & 169 & 166 & $16:$ & 15 & & & & & \\
\hline 101 & 1458 & 148 & 142 & & & & & & & \\
\hline 121 & 1451 & $13 E$ & 140 & & & & & & & \\
\hline 141 & 1344 & 13 & 1277 & 130 & 1379 & 01 & 77 & & 58 & 1767 \\
\hline & & & & & & & & & & \\
\hline 181 & 1053 & 102 & 106 & 10 & 104 & & & & & \\
\hline 120 & 1141 & & & & & & & & & \\
\hline 122 & & & & & & & & & & \\
\hline 24 & 798 & & & & & 854 & & & 853 & 98 \\
\hline & & & & & & & & & & \\
\hline 128 & 72 & 7 & & & 719 & 679 & 70 & 68 & 69 & \\
\hline 30 & 66 & 70 & 64 & & & 657 & & & 6 & \\
\hline 32 & 672 & 65 & & & & 697 & 634 & & & \\
\hline & 1034 & 126 & 14 & & 1864 & 1838 & & & & \\
\hline & & & & & & 596 & & & & \\
\hline 138 & 540 & bs & 5 & 50 & & 541 & 55 & & 584 & 585 \\
\hline 40 & & & & & & $69^{7}$ & & & 1015 & 114 \\
\hline 4 & & & & & & & & & & \\
\hline & & & & & & 54 & & & 51 & 4 \\
\hline & & & & & & & & 48 & & \\
\hline & & & & & & & & & 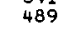 & \\
\hline 150 & 473 & 49 & & & & 52 & & & 594 & 63 \\
\hline & & & & & & & & & 48 & \\
\hline & & & & & & 494 & & 49 & 46 & 45 \\
\hline & 53 & & & & & 48 & & & & \\
\hline & 5 & & & & & & & & & \\
\hline & & & & & & & & & & \\
\hline & & & & & & 58 & & & 51 & \\
\hline & 489 & 51 & & & & & & & & 5 \\
\hline & & & & & & & & & & \\
\hline & & & & & & & & & & \\
\hline 17 & 553 & & & & & 55 & & & 569 & 5 \\
\hline & 618 & 53 & 5 & 5 & & & & 57 & 541 & 51 \\
\hline & & & & & & & & & 54 & \\
\hline & 2 & n & & & & 18 & 391 & 73 & 380 & \\
\hline
\end{tabular}

COUNT COUNT COUNT COUNT COUNT COUNT COUNT COUNT COUNT COMNT

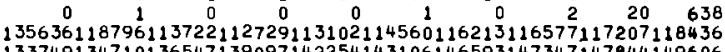
(1) (1) 290549252589221639187620161206143537137417135283135687135712
187927157725140225132714131139129887130657130102130932129394 $128764129119129147128064128238127873_{128602127694127681128121}$ 126867126873126962126609126220126409126436126111125993124587 120285119059119521119481119144119049118715118907118395118920 118393118892117880117578117831117345116899117132117730117067 116607115544116736116374116431117166116674116409116490115623 117054116559116363116667116698117041116819115607116677116475 93310922289258291812917969157591478910449136091387

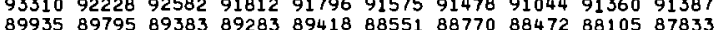
$8686086548 \quad 86308 \quad 867458666486202 \quad 86734 \quad 861728579985709$ $\begin{array}{llllllllll}86860 & 86548 & 86308 & 86745 & 86664 & 86202 & 86734 & 86172 & 85799 & 85709 \\ 89189 & 91569 & 92848 & 90612 & 86877 & 83118 & 82138 & 81541 & 80640 & 79768\end{array}$ $7252572142 \quad 72469717837100571182 \quad 70501 \quad 7103971140 \quad 71363$ $\begin{array}{llllllllll}68620 & 68217 & 67849 & 67627 & 67539 & 66021 & 64863 & 63523 & 62603 & 61016 \\ 58754 & 59123 & 61559 & 62920 & 63828 & 62558 & 60568 & 59743 & 58996 & 59059\end{array}$ $123517736165547050606 \quad 4929648646 \quad 48593 \quad 486794837948308$ $\begin{array}{llllllllll}46525 & 45953 & 45723 & 45627 & 45196 & 44869 & 44358 & 44731 & 44902 & 44651 \\ 43725 & 44497 & 45641 & 46145 & 45098 & 44050 & 44072 & 44892 & 46461 & 45378\end{array}$

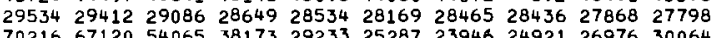
$\begin{array}{llllllllll}70216 & 67120 & 54065 & 38173 & 29233 & 25287 & 23946 & 24921 & 26976 & 30064 \\ 26593 & 20139 & 18589 & 18003 & 18107 & 18045 & 18468 & 19132 & 19348 & 19381\end{array}$ $\begin{array}{llllllllll}16176 & 159111 & 15946 & 16107 & 16806 & 17843 & 21203 & 29529 & 47124 & 79125\end{array}$ $\begin{array}{llllllllll}11706 & 11576 & 11405 & 11315 & 11062 & 10875 & 10875 & 10666 & 10691 & 10647 \\ 16174 & 20809 & 24775 & 24852 & 21303 & 16141 & 12170 & 10393 & 9632 & 9474\end{array}$

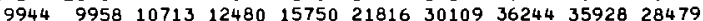
$\begin{array}{llllllllll}9027 & 8857 & 9095 & 9001 & 9294 & 9324 & 9539 & 9535 & 9507 & 9738\end{array}$ $2562233483561018946912264313321411112969626 \quad 35458 \quad 16446$ $\begin{array}{llllllllll}7111 & 7188 & 6964 & 7158 & 7197 & 7424 & 8150 & 9477 & 12494 & 17783\end{array}$ $\begin{array}{llllllllll}7307 & 6609 & 6320 & 6144 & 6197 & 6242 & 6273 & 6288 & 6279 & 6241 \\ 6078 & 6090 & 6063 & 6069 & 6234 & 5977 & 6097 & 5993 & 6054 & 5996\end{array}$ $\begin{array}{llllllllll}5147 & 5054 & 4891 & 5034 & 4788 & 4884 & 4879 & 4727 & 4756 & 4 \$ 22 \\ 7960 & 8679 & 8708 & 7812 & 6114 & 5219 & 4696 & 4618 & 4605 & 4590\end{array}$

$\begin{array}{llllllllll}4164 & 4172 & 4165 & 4229 & 4140 & 4049 & 4115 & 4231 & 4225 & 4983 \\ 3895 & 3957 & 3776 & 3698 & 3729 & 3629 & 3603 & 3647 & 3494 & 3563\end{array}$ $\begin{array}{llllllllll}3578 & 3326 & 3295 & 3355 & 3274 & 3131 & 3197 & 3149 & 3250 & 3181\end{array}$ $\begin{array}{llllllllll}2821 & 2721 & 2772 & 2765 & 2721 & 2673 & 2750 & 2725 & 2769 & 2956\end{array}$

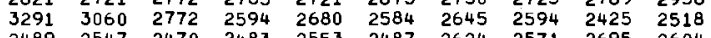

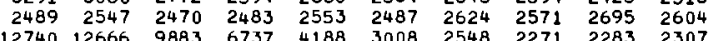
$\begin{array}{llllllllll}12740 & 12666 & 9883 & 6737 & 4188 & 3008 & 2548 & 2271 & 2283 & 2307 \\ 2041 & 2076 & 2015 & 2034 & 1957 & 2017 & 2036 & 2048 & 2022 & 1956\end{array}$ $\begin{array}{llllllllll}2051 & 2028 & 1982 & 1945 & 1946 & 1988 & 1945 & 2038 & 1958 & 1941 \\ 1964 & 1899 & 2018 & 1954 & 2054 & 2205 & 2548 & 2886 & 3433 & 3894\end{array}$ $\begin{array}{llllllllll}1711 & 1693 & 1708 & 1711 & 1698 & 1753 & 1679 & 1668 & 1714 & 1693 \\ 2615 & 2961 & 3357 & 3239 & 2961 & 2640 & 2393 & 2199 & 2141 & 2176\end{array}$ $\begin{array}{llllllllll}1582 & 1625 & 1518 & 1531 & 1544 & 1661 & 1545 & 1635 & 1755 & 1857 \\ 1489 & 1403 & 1433 & 1467 & 1474 & 1432 & 1413 & 1502 & 1451 & 1452\end{array}$

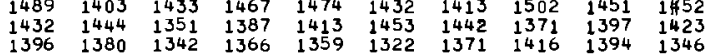
$\begin{array}{llllllllll}1964 & 2107 & 2165 & 1945 & 1674 & 1371 & 1264 & 1183 & 1136 & 1115 \\ 1147 & 1094 & 1123 & 1148 & 1118 & 1040 & 1089 & 119 & 1092 & 1066\end{array}$ $\begin{array}{llllllllll}1147 & 1094 & 1123 & 1148 & 1118 & 1040 & 1089 & 1119 & 1092 & 1086 \\ 1021 & 1047 & 1028 & 1037 & 1076 & 1039 & 1080 & 1097 & 1085 & 1085\end{array}$ $\begin{array}{rrrrrrrrrrr}1003 & 984 & 976 & 984 & 972 & 914 & 941 & 929 & 828 & 902 \\ 1101 & 1230 & 1259 & 1318 & 1315 & 1174 & 1019 & 871 & 842 & 799\end{array}$ $\begin{array}{lllllllllll}958 & 921 & 899 & 856 & 842 & 748 & 762 & 739 & 713 & 789 \\ 757 & 733 & 774 & 775 & 729 & 733 & 680 & 713 & 694 & 714\end{array}$ $\begin{array}{llllllllll}652 & 704 & 682 & 629 & 673 & 734 & 726 & 679 & 623 & 632\end{array}$ $\begin{array}{lllllllllll}680 & 647 & 751 & 724 & 663 & 741 & 684 & 676 & 693 & 640 \\ 660 & 700 & 710 & 684 & 703 & 709 & 759 & 765 & 818 & 955\end{array}$

$\begin{array}{lllllllllll}840 & 746 & 708 & 682 & 700 & 680 & 661 & 601 & 637 & 529 \\ 601 & 610 & 597 & 568 & 577 & 587 & 571 & 606 & 577 & 579\end{array}$

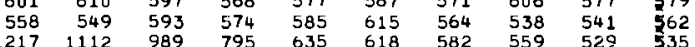

$\begin{array}{llllllllll}523 & 548 & 514 & 566 & 584 & 508 & 524 & 540 & 534 & 586 \\ 534 & 525 & 517 & 535 & 501 & 531 & 502 & 518 & 525 & 493\end{array}$

$\begin{array}{llllllllll}534 & 582 & 536 & 5507 & 524 & 466 & 525 & 535 & 493 & 523 \\ 529 & 505 & 455 & 480 & 463 & 488 & 517 & 504 & 489 & 518\end{array}$

$\begin{array}{llllllllll}631 & 636 & 650 & 608 & 604 & 525 & 525 & 549 & 473 & 508 \\ 472 & 513 & 516 & 477 & 480 & 514 & 482 & 513 & 46.4 & 451\end{array}$

$\begin{array}{llllllllll}497 & 473 & 460 & 486 & 522 & 470 & 443 & 497 & 503 & 482\end{array}$

$\begin{array}{llllllllll}473 & 506 & 518 & 486 & 494 & 515 & 475 & 489 & 479 & 989 \\ 476 & 567 & 580 & 586 & 612 & 523 & 558 & 515 & 487 & 494\end{array}$

$\begin{array}{llllllllll}530 & 541 & 518 & 489 & 480 & 539 & 572 & 515 & 489 & 53\end{array}$

$\begin{array}{llllllllll}546 & 538 & 470 & 490 & 492 & 509 & 529 & 488 & 511 & 715 \\ 545 & 537 & 550 & 573 & 544 & 522 & 545 & 527 & 510 & 551\end{array}$

$\begin{array}{llllllllll}514 & 525 & 543 & 517 & 529 & 544 & 553 & 545 & 555 & 560 \\ 588 & 520 & 546 & 584 & 575 & 531 & 516 & 577 & 571 & 576\end{array}$

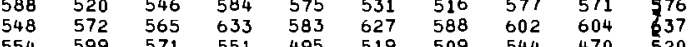

$\begin{array}{llllllllll}554 & 599 & 571 & 551 & 495 & 519 & 509 & 544 & 470 & 520 \\ 337 & 317 & 299 & 324 & 316 & 323 & 300 & 275 & 258 & 313\end{array}$

$\begin{array}{rrrrrrrrrrr}1781 & 319 & 272 & 296 & 265 & 272 & 347 & 282 & 278 & 294 & 277 \\ 1801 & 265 & 255 & 251 & 260 & 269 & 255 & 249 & 237 & 249 & 249 \\ 1821 & 206 & 227 & 200 & 216 & 208 & 208 & 196 & 205 & 200 & 203 \\ 1841 & 191 & 186 & 182 & 178 & 202 & 167 & 155 & 143 & 158 & 169 \\ 1861 & 128 & 149 & 125 & 126 & 130 & 125 & 120 & 102 & 119 & 111 \\ 1881 & 96 & 107 & 97 & 90 & 83 & 99 & 107 & 90 & 82 & 115 \\ 1901 & 127 & 114 & 136 & 151 & 133 & 138 & 143 & 148 & 141 & 102 \\ 1921 & 78 & 68 & 70 & 74 & 82 & 69 & 59 & 87 & 61 & 88 \\ 1941 & 65 & 62 & 74 & 80 & 77 & 76 & 75 & 72 & 72 & 65 \\ 1961 & 198 & 219 & 284 & 359 & 448 & 569 & 675 & 912 & 1018 & 1340 \\ 1981 & 53 & 51 & 36 & 49 & 37 & 38 & 24 & 42 & 22 & 36 \\ 2001 & 23 & 37 & 30 & 32 & 23 & 26 & 27 & 29 & 27 & 36 \\ 2021 & 21 & 34 & 41 & 42 & 20 & 23 & 24 & 23 & 23 & 25 \\ 2041 & 21 & 13 & 22 & 25 & 21 & 17 & 22 & 0 & & \end{array}$

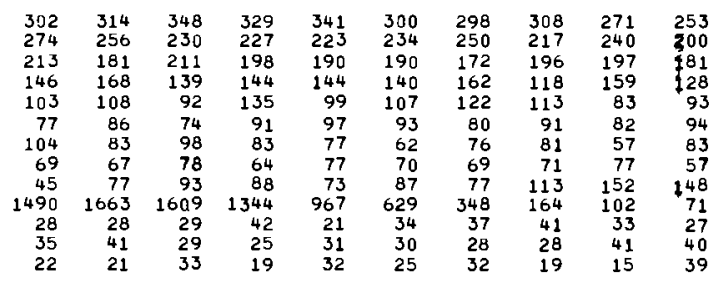


GAMMA RAY SPECTRUM DATA

PRTR ROD FS-07 SPECTRUM NO, 7 DATA TAKEN 9/10/69 LIVE TIME 120.MIN. POSITION 39.IN. ENERGY(KEV)= $1,107 \star C H A N ~-4.6$ CHAN COUNT COUNT COUNT COUNT COUNT COUNT COUNT COUNT COUNT COUNT

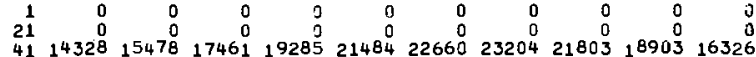
$\begin{array}{lllllllllllll}61 & 17055 & 17097 & 17372 & 17569 & 17713 & 17681 & 18049 & 18096 & 18403 & 18784\end{array}$ $\begin{array}{rlllllllllll}81 & 21387 & 21872 & 22009 & 22192 & 2288 & 23280 & 23758 & 24657 & 25710 & 28021 \\ 101 & 25656 & 24574 & 24992 & 25211 & 27617 & 33037 & 40110 & 44412 & 43183 & 37707\end{array}$ $\begin{array}{llllllllllll}101 & 25656 & 24574 & 24992 & 25211 & 27617 & 33037 & 40110 & 44412 & 43183 & 37707 \\ 121 & 18406 & 18600 & 18642 & 18989 & 20081 & 22656 & 26593 & 29222 & 28046 & 24327\end{array}$ $\begin{array}{lllllllllll}121 & 18406 & 18600 & 18642 & 18989 & 2008 & 22656 & 26593 & 29222 & 28046 & 24327 \\ 141 & 17746 & 17899 & 17708 & 17862 & 17603 & 17929 & 17995 & 17576 & 17802 & 17583\end{array}$ $\begin{array}{llllllllllll}161 & 17752 & 17395 & 17715 & 17693 & 17810 & 17642 & 18070 & 17678 & 17554 & 17424\end{array}$ $\begin{array}{llllllllllll}161 & 17752 & 17395 & 17715 & 17693 & 17810 & 17642 & 18070 & 17678 & 17554 & 17424 \\ 181 & 17328 & 17226 & 16909 & 17169 & 17287 & 17100 & 17351 & 16832 & 17015 & 17056\end{array}$ $\begin{array}{lllllllllllll}201 & 16682 & 16449 & 16635 & 16555 & 16648 & 16482 & 16585 & 16464 & 16545 & 16465\end{array}$ $\begin{array}{lllllllllllll}221 & 16451 & 16120 & 16415 & 16226 & 16100 & 16076 & 16227 & 16351 & 16285 & 16472\end{array}$ $\begin{array}{llllllllllll}261 & 16262 & 16120 & 16069 & 16198 & 16144 & 16142 & 16056 & 16199 & 16030 & 15857\end{array}$ $\begin{array}{llllllllllll}281 & 15969 & 15940 & 15729 & 16049 & 16092 & 16122 & 16067 & 15944 & 15853 & 16053\end{array}$ $\begin{array}{lllllllllll}301 & 16184 & 15889 & 15778 & 15712 & 15645 & 15754 & 15655 & 15423 & 15507\end{array}$ $\begin{array}{lllllllllll}321 & 13328 & 13265 & 13341 & 13137 & 13055 & 13117 & 13042 & 12993 & 13014 & 12848 \\ 341 & 2697 & 12700 & 12668 & 12535 & 12618 & 12585 & 12528 & 12643 & 12518 & 12577\end{array}$ $\begin{array}{lllllllllll}361 & 12303 & 12172 & 12102 & 12288 & 12066 & 12212 & 12285 & 12129 & 12149 & 12209\end{array}$ $\begin{array}{lllllllllll}381 & 12034 & 12008 & 11933 & 11868 & 11701 & 11743 & 11678 & 12037 & 12192 & 12280 \\ 401 & 11231 & 10923 & 10753 & 10619 & 10647 & 10722 & 10236 & 10333 & 10530 & 10379\end{array}$ $\begin{array}{rrrrrrrrrrr}401 & 11231 & 10923 & 10753 & 10619 & 10647 & 10722 & 10236 & 10333 & 10530 & 10379 \\ 421 & 10152 & 10236 & 10322 & 10437 & 1025 & 10179 & 9802 & 9887 & 9760 & 9798\end{array}$ $\begin{array}{lllllllllll}441 & 8501 & 8393 & 8379 & 8321 & 8403 & 8380 & 8180 & 8246 & 8163 & 8206\end{array}$ $481675868296601 \quad 6672 \quad 65946593 \quad 6614 \quad 65806558 \quad 6554$ $\begin{array}{lllllllllll}481 & 6758 & 6829 & 6601 & 6672 & 6594 & 6593 & 6614 & 6580 & 6558 & 6554\end{array}$ $\begin{array}{llllllllllll}521 & 5871 & 5393 & 5010 & 4836 & 4612 & 4455 & 4468 & 4303 & 4200 & 4295\end{array}$ $\begin{array}{rrrrrrrrrrr}541 & 3869 & 3745 & 3765 & 3781 & 4171 & 4464 & 5198 & 6202 & 7842 & 9692 \\ 561 & 4816 & 5662 & 7353 & 11047 & 5663 & 19188 & 18482 & 13782 & 8290 & 4666\end{array}$ $\begin{array}{lrrrrrrrrrr}581 & 2498 & 2463 & 2315 & 2316 & 2214 & 2312 & 2270 & 2201 & 2333 & 2196 \\ 601 & 19707 & 22838 & 19873 & 13204 & 7112 & 3513 & 2202 & 1714 & 1619 & 1608\end{array}$ $\begin{array}{llllllllll} & 1340\end{array}$ 641128113091289127 $\begin{array}{lllllllllll}661 & 2724 & 1761 & 1381 & 1245 & 1266 & 1158 & 1233 & 1202 & 1216 & 1214\end{array}$ $\begin{array}{rrrrrrrrrrr}681 & 1313 & 1467 & 1695 & 2145 & 2903 & 4117 & 5311 & 5806 & 5297 & 4134 \\ 701 & 1535 & 1043 & 989 & 938 & 951 & 913 & 941 & 917 & 949 & 923\end{array}$ $\begin{array}{llllllllllll}721 & 3072 & 4313 & 5086 & 4696 & 3584 & 2504 & 1691 & 1441 & 1372 & 1240\end{array}$ $\begin{array}{lrrrrrrrrrr}741 & 881 & 878 & 828 & 837 & 808 & 811 & 805 & 843 & 842 & 782 \\ 761 & 818 & 769 & 808 & 772 & 766 & 754 & 706 & 743 & 764 & 690\end{array}$

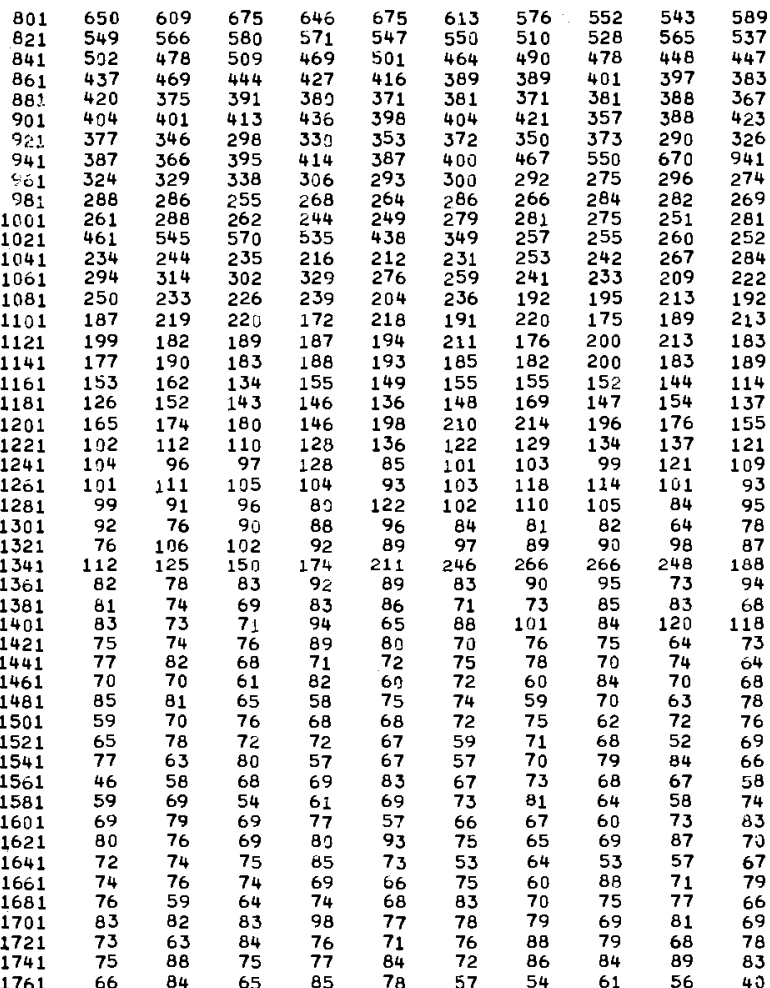
COUNT COUNT COUNT COUNT COUNT COUNT COUNT COUNT COUNT COUNT $\begin{array}{rrrrrrrrrr}0 & 0 & 0 & 0 & 0 & 0 & 0 & 0 & 0 & 0 \\ 0 & 0 & 0 & 0 & 0 & 1 & 3 & 30 & 1119 & 10624 \\ 15471 & 15661 & 15487 & 15916 & 15896 & 16047 & 16212 & 16231 & 16465 & 16599\end{array}$ $\begin{array}{lllllllllll}18652 & 18971 & 19140 & 19690 & 20264 & 20029 & 20632 & 20330 & 21053 & 21260\end{array}$ $\begin{array}{llllllllll}32932 & 40712 & 48452 & 53052 & 57637 & 63559 & 63352 & 52806 & 38817 & 29231 \\ 33118 & 28439 & 24261 & 21278 & 19398 & 18560 & 18486 & 18579 & 18673 & 18777\end{array}$ $\begin{array}{lllllllllll}20582 & 18811 & 17954 & 18052 & 17970 & 17670 & 18110 & 17981 & 17992 & 17844\end{array}$ $\begin{array}{llllllllllll}17711 & 17664 & 17513 & 17511 & 17467 & 17481 & 17333 & 17551 & 17555 & 17700\end{array}$ $\begin{array}{lllllllllll}17454 & 17377 & 17441 & 17063 & 17241 & 17211 & 17304 & 17316 & 17356 & 17372 \\ 16897 & 16847 & 16752 & 16655 & 16652 & 16824 & 16846 & 16764 & 16692 & 16697\end{array}$ $\begin{array}{llllllllll}16897 & 16847 & 16752 & 16655 & 16652 & 16824 & 16846 & 16764 & 16692 & 16697\end{array}$ $\begin{array}{llllllllll}16235 & 16315 & 16603 & 16397 & 16445 & 16529 & 16246 & 16469 & 16373 & 16259 \\ 16239 & 16416 & 16186 & 16259 & 16197 & 16210 & 16049 & 16255 & 16025 & 16135\end{array}$ $\begin{array}{lllllllllll}16215 & 15985 & 15794 & 15945 & 16051 & 15961 & 15956 & 15870 & 16093 & 15927\end{array}$ $\begin{array}{lllllllllll}16095 & 16092 & 16154 & 1607 & 16201 & 16167 & 16060 & 16138 & 16059 & 15994\end{array}$ $\begin{array}{llllllllllll}16093 & 15936 & 16208 & 16233 & 16173 & 16094 & 15891 & 15779 & 15952 & 16024 \\ 15382 & 14965 & 14977 & 14553 & 14287 & 14066 & 13807 & 13825 & 13572 & 13617\end{array}$ $\begin{array}{llllllllllll}12747 & 13058 & 12968 & 12724 & 12771 & 12760 & 12726 & 12722 & 12732 & 12523\end{array}$ $\begin{array}{lllllllllll}12573 & 12378 & 12423 & 1263 & 12391 & 12344 & 12359 & 12361 & 12339 & 12449\end{array}$

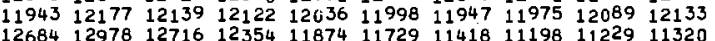
$\begin{array}{llllllllll}12684 & 12978 & 12716 & 12354 & 11874 & 11729 & 11418 & 11198 & 11229 & 11320 \\ 10317 & 10110 & 13315 & 10182 & 10151 & 10035 & 10332 & 10087 & 10178 & 10051\end{array}$

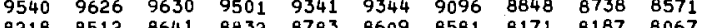
$\begin{array}{rlllllllll}8218 & 8512 & 8641 & 8832 & 8783 & 8609 & 8581 & 8171 & 8187 & 8067 \\ 12639 & 8506 & 7147 & 6778 & 6954 & 6760 & 6655 & 6683 & 6634 & 6785\end{array}$ \begin{tabular}{llllllllll}
6448 & 6374 & 6530 & 6403 & 6368 & 6232 & 6291 & 6292 & 6397 & 6173 \\
\hline
\end{tabular} $\begin{array}{llllllllll}6189 & 6293 & 6524 & 6589 & 6383 & 6221 & 6261 & 6656 & 6552 & 6336 \\ 4084 & 3935 & 4022 & 4055 & 3924 & 3919 & 3900 & 3991 & 3971 & 3827\end{array}$ $\begin{array}{rlllllllll}10249 & 8892 & 6553 & 4732 & 3750 & 3419 & 3412 & 3601 & 3865 & 4352 \\ 3089 & 2655 & 2509 & 2537 & 2522 & 2586 & 2648 & 2645 & 2717 & 2501\end{array}$ $\begin{array}{lllllllllll}2226 & 2141 & 2204 & 2256 & 2350 & 2653 & 3245 & 5077 & 8629 & 13934\end{array}$ $\begin{array}{llllllllll}1552 & 1611 & 1519 & 1589 & 1493 & 1529 & 1463 & 1426 & 1500 & 1413 \\ 2309 & 2970 & 3507 & 3375 & 2844 & 2122 & 1625 & 1351 & 1384 & 1283\end{array}$ $\begin{array}{llllllllll}2309 & 2970 & 3507 & 3375 & 2844 & 2122 & 1625 & 1351 & 1384 & 1283 \\ 1321 & 1350 & 1484 & 1652 & 2236 & 3073 & 4243 & 5010 & 4927 & 3872\end{array}$

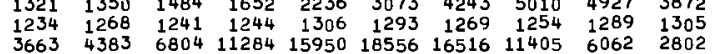
$\begin{array}{rrrrrrrrrr}3663 & 4383 & 6804 & 11284 & 15950 & 18556 & 16516 & 11405 & 6062 & 2802 \\ 967 & 912 & 942 & 1050 & 949 & 1021 & 1043 & 1206 & 1541 & 2163\end{array}$ $\begin{array}{rlllllllll}1004 & 939 & 890 & 838 & 840 & 825 & 887 & 848 & 855 & 808 \\ 816 & 806 & 834 & 787 & 839 & 827 & 854 & 783 & 809 & 807\end{array}$ $\begin{array}{rrrrrrrrrr}816 & 806 & 834 & 787 & 839 & 827 & 854 & 783 & 809 & 807 \\ 693 & 669 & 675 & 668 & 666 & 688 & 659 & 618 & 665 & 688 \\ 932 & 1071 & 1131 & 1089 & 1007 & 874 & 726 & 639 & 554 & 610\end{array}$

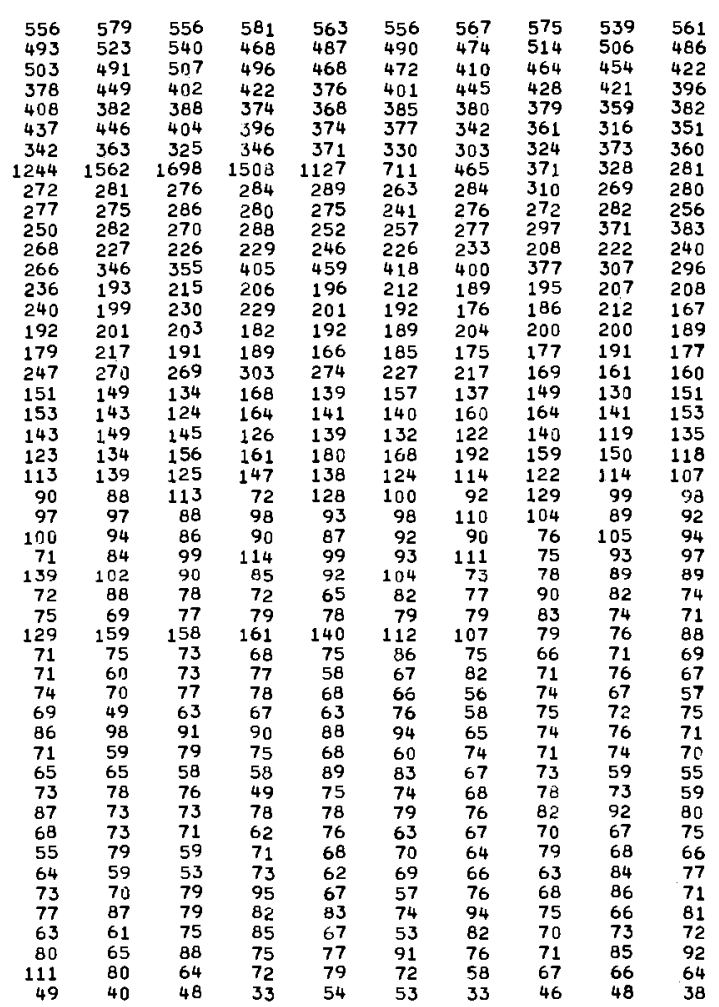

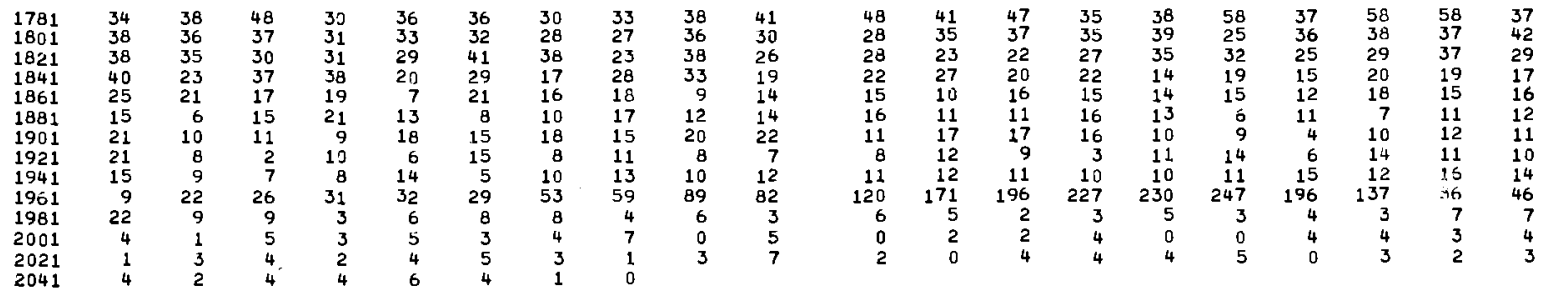


GAMMA RAY SPECTRUM DATA

PRTR ROD FS-07 SPECTRUM NO. 3 DATA TAKEN 9/09/69 LIVE TIME 180.MIN. POSITION 39. IN. ENERGY(KEVI= $1.109 \%$ CHAN -6.5

CHAN COUNT COUNT COUNT COUNT COUNT COUNT COUNT COUNT COUNT COUNT

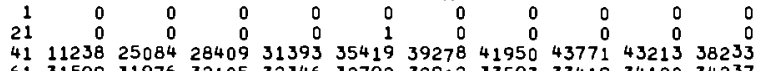

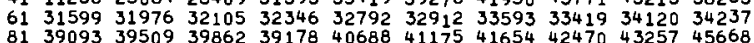
$\begin{array}{llllllllllll}101 & 52492 & 46333 & 44304 & 43654 & 44915 & 47602 & 55702 & 66284 & 74166 & 73173 \\ 121 & 35320 & 34928 & 35153 & 35022 & 35458 & 36720 & 40097 & 45951 & 50748 & 50058\end{array}$ $\begin{array}{lllllllllll}141 & 34329 & 33995 & 34178 & 33825 & 34169 & 34122 & 34163 & 34010 & 33862 & 33803\end{array}$ $\begin{array}{lllllllllll}161 & 33758 & 34145 & 34017 & 33909 & 34121 & 34191 & 33952 & 33888 & 33896 & 33655\end{array}$ $\begin{array}{lllllllllllll}181 & 32937 & 33134 & 33321 & 33370 & 33012 & 33184 & 32856 & 33124 & 32490 & 33140 \\ 201 & 32574 & 32524 & 32111 & 32544 & 32175 & 32338 & 32041 & 31859 & 32485 & 32093\end{array}$ $\begin{array}{lllllllllll}201 & 32574 & 32524 & 32111 & 32544 & 32175 & 32338 & 32041 & 31859 & 32485 & 32093 \\ 221 & 32485 & 32038 & 31881 & 31984 & 31963 & 32248 & 31843 & 32077 & 31847 & 32210\end{array}$ $\begin{array}{lllllllllllll}221 & 32485 & 32038 & 31881 & 31984 & 31963 & 32248 & 31843 & 32077 & 31847 & 32210 \\ 241 & 32058 & 31856 & 32094 & 31989 & 32087 & 32133 & 31593 & 32225 & 31854 & 32096\end{array}$ $\begin{array}{lllllllllll}261 & 32046 & 32329 & 31927 & 32172 & 32248 & 32560 & 32316 & 32642 & 32368 & 32682\end{array}$

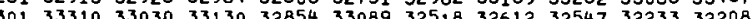
$\begin{array}{lllllllllll}301 & 33310 & 33030 & 33130 & 32854 & 33089 & 32518 & 32612 & 32547 & 32233 & 32208 \\ 321 & 26297 & 26235 & 26175 & 25478 & 25551 & 25535 & 25394 & 25280 & 24895 & 24917\end{array}$

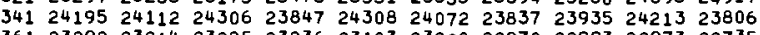

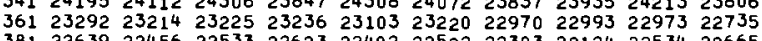

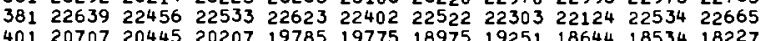
$40120707204452020719785 \quad 19775 \quad 189751925118644 \quad 18534 \quad 18227$

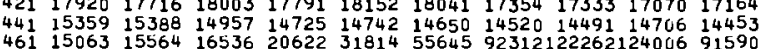
$48111067112241133311112 \quad 11151 \quad 10904 \quad 109731089310671 \quad 10892$ $\begin{array}{rrrrrrrrrrr}501 & 10373 & 10186 & 10238 & 10389 & 10124 & 10303 & 10073 & 9947 & 9812 & 9969 \\ 521 & 10318 & 9716 & 8932 & 8523 & 8171 & 79 & 7743 & 7560 & 7591 & 7389\end{array}$ $\begin{array}{rrrrrrrrrrr}521 & 10318 & 9716 & 8932 & 8523 & 8171 & 7908 & 7743 & 7560 & 7591 & 7389 \\ 541 & 6725 & 6822 & 6817 & 6885 & 6880 & 7309 & 7913 & 8794 & 10411 & 13024\end{array}$ $\begin{array}{rrrrrrrrrrr}581 & 4655 & 4506 & 4241 & 4199 & 4103 & 4119 & 4142 & 4078 & 4098 & 4065 \\ 601 & 21471 & 30899 & 36234 & 31962 & 21580 & 11806 & 6210 & 4030 & 3349 & 3200\end{array}$ $\begin{array}{llllllllllll}621 & 2886 & 2809 & 2774 & 2837 & 2779 & 2718 & 2789 & 2824 & 2769 & 2937\end{array}$ $\begin{array}{lllllllllll}641 & 2622 & 2589 & 2652 & 2554 & 2580 & 2597 & 2520 & 2522 & 2469 & 2541\end{array}$ $\begin{array}{lllllllllll}661 & 6506 & 4822 & 3364 & 2803 & 2542 & 2451 & 2466 & 2471 & 2502 & 2400\end{array}$ $\begin{array}{llllllllllll}681 & 2509 & 2622 & 2690 & 2930 & 3460 & 4668 & 6368 & 8195 & 9273 & 8550 \\ 701 & 5116 & 2882 & 2296 & 2085 & 2010 & 2007 & 1952 & 1998 & 1993 & 1993\end{array}$ $\begin{array}{lllllllllll}721 & 3587 & 5121 & 6667 & 8060 & 7955 & 6410 & 4366 & 3157 & 2812 & 2580 \\ 741 & 1881 & 1038 & 1816 & 1864 & 1847 & 1803 & 1844 & 1853 & 1815 & 1852\end{array}$ $\begin{array}{lllllllllll}761 & 1771 & 1774 & 1781 & 1753 & 1694 & 1723 & 1696 & 1644 & 1595 & 1576 \\ 781 & 1367 & 1428 & 1262 & 1345 & 1372 & 1366 & 1423 & 1402 & 1417 & 1531\end{array}$

\begin{tabular}{|c|c|c|c|c|c|c|c|c|c|c|}
\hline $\begin{array}{l}801 \\
821\end{array}$ & $\begin{array}{l}1302 \\
1203\end{array}$ & $\begin{array}{l}1255 \\
1130\end{array}$ & $\begin{array}{l}1364 \\
1170\end{array}$ & $\begin{array}{l}1365 \\
1152\end{array}$ & $\begin{array}{l}1324 \\
1186\end{array}$ & $\begin{array}{l}1308 \\
1170\end{array}$ & $\begin{array}{l}1263 \\
1111\end{array}$ & $\begin{array}{l}1256 \\
1150\end{array}$ & $\begin{array}{l}1172 \\
1123\end{array}$ & $\begin{array}{l}1234 \\
1158\end{array}$ \\
\hline 841 & 974 & 1008 & 991 & 973 & 930 & 949 & 908 & 923 & 918 & 975 \\
\hline 861 & 839 & & 869 & & & & & & & 834 \\
\hline & 757 & & 774 & 785 & 769 & 794 & 78 & 716 & 749 & $75 \mathrm{~s}$ \\
\hline 90 & 723 & 764 & 804 & 778 & 775 & 8010 & 778 & & 710 & 790 \\
\hline 92 & 697 & 704 & 640 & 657 & 663 & & & & 659 & 637 \\
\hline 941 & 739 & 728 & 758 & 725 & 712 & $77_{14}$ & & 953 & 1285 & 1735 \\
\hline 61 & 632 & 614 & 620 & 628 & 639 & 634 & 547 & & 481 & 546 \\
\hline 98 & 551 & 522 & 500 & 487 & 53 & 49 & 50 & & & 53 \\
\hline 00 & 527 & 553 & 535 & 508 & 519 & 480 & & & 537 & 517 \\
\hline & 1050 & 1200 & 1320 & 1354 & 1170 & 899 & 696 & 548 & 496 & 470 \\
\hline & 429 & 476 & 446 & 442 & 446 & 453 & 441 & 448 & 474 & 498 \\
\hline & 544 & 541 & 537 & 531 & 56 & 48 & 47 & & 41 & 45 \\
\hline & 454 & 482 & 505 & 477 & 471 & 421 & 39 & & & 390 \\
\hline & 381 & 369 & 373 & 374 & 386 & 390 & 39 & 3 & 367 & 336 \\
\hline & 362 & 370 & 358 & 350 & 36 & & 37 & & & 39 \\
\hline & 340 & & 34 & 32 & 33 & 34 & 36 & & & \\
\hline & 331 & 286 & 304 & 302 & 309 & 317 & 29 & & 313 & 313 \\
\hline 18 & 292 & 308 & 304 & 306 & 304 & 300 & 29 & 298 & 244 & 285 \\
\hline & 291 & 315 & 317 & & & & & & 329 & 347 \\
\hline & 261 & 227 & 230 & 250 & 23 & 25 & & & & \\
\hline & & & & & & & & & 185 & \\
\hline & 209 & 234 & 210 & 195 & 18 & 193 & & & 195 & 188 \\
\hline & 190 & 212 & & & & & & & & \\
\hline & 191 & 171 & 17 & 16 & 18 & 19 & & & 14 & \\
\hline & 167 & 174 & 17 & 19 & 14 & $1 t$ & & & & \\
\hline & 20 & 20 & 24 & 270 & 3 & 37 & 3 & & & \\
\hline & 159 & 159 & 18 & 137 & 15 & 179 & 17 & & 157 & 152 \\
\hline & & 167 & & 138 & & & & & & 180 \\
\hline & 14 & 151 & & & & & & & & 248 \\
\hline & $15 \mathrm{~b}$ & 14 & & & & & 1 & & & 1 \\
\hline & 15 & 14 & & & & & & & & 1 \\
\hline & 140 & 131 & & & & & & & & 15 \\
\hline & 11 & 133 & & & & & & & & 133 \\
\hline & 12 & 119 & 14 & 13 & & & & & & 110 \\
\hline & 12 & 13 & 12 & 12 & 12 & & & & 1 & \\
\hline & 11 & 12 & & 11 & & & & & & 121 \\
\hline & 1 & 12 & & & & & & & 1 & 117 \\
\hline & & & & & & & & & & \\
\hline & 123 & 117 & 110 & 11 & 10 & 108 & 12 & & 141 & 123 \\
\hline & 12 & & 14 & & & 15 & & & 129 & \\
\hline & & & & & & & & & & 122 \\
\hline & 125 & 132 & & & 11 & 13 & & & 108 & 135 \\
\hline & 126 & 139 & 126 & 131 & 11 & 13 & 13 & 13 & 119 & 132 \\
\hline & 123 & & & 11 & 12 & 12 & 12 & & & 121 \\
\hline & 113 & & 136 & 11 & 11 & 13 & 12 & & 102 & 144 \\
\hline 174 & 156 & 153 & 15 & 146 & 122 & 13 & 12 & 139 & 112 & 143 \\
\hline & 138 & 123 & 104 & 110 & 106 & 124 & 103 & 91 & 92 & \\
\hline
\end{tabular}

$\begin{array}{rrrrrrrrrrr}1781 & 70 & 70 & 59 & 09 & 50 & 68 & 56 & 59 & 78 & 73 \\ 1801 & 77 & 80 & 51 & 52 & 51 & 61 & 52 & 53 & 64 & 70 \\ 1821 & 58 & 49 & 70 & 57 & 53 & 53 & 44 & 50 & 56 & 49 \\ 1841 & 52 & 43 & 52 & 46 & 33 & 46 & 52 & 41 & 43 & 42 \\ 1861 & 28 & 31 & 34 & 41 & 33 & 36 & 31 & 21 & 28 & 33 \\ 1881 & 29 & 29 & 30 & 26 & 39 & 26 & 40 & 25 & 31 & 27 \\ 1901 & 28 & 34 & 34 & 28 & 44 & 47 & 45 & 51 & 59 & 56 \\ 1921 & 20 & 29 & 20 & 20 & 28 & 26 & 23 & 23 & 19 & 21 \\ 1941 & 19 & 20 & 20 & 10 & 14 & 16 & 17 & 26 & 18 & 17 \\ 1961 & 30 & 32 & 28 & 36 & 47 & 61 & 69 & 82 & 120 & 155 \\ 1981 & 50 & 19 & 17 & 13 & 14 & 19 & 16 & 12 & 14 & 10 \\ 2001 & 16 & 10 & 10 & 6 & 11 & 16 & 13 & 8 & 9 & 13 \\ 2021 & 9 & 6 & 10 & 7 & 5 & 11 & 11 & 9 & 8 & 6 \\ 2041 & 0 & 7 & 4 & 12 & 8 & 5 & 12 & 0 & & \end{array}$
COUNT COUNT COUNT COUNT COUNT COUNT COUNT COUNT COUNT CONNT $\begin{array}{rrrrrrrrrr}0 & 0 & 1 & 0 & 0 & 0 & 0 & 1 & 0 & 0 \\ 33536 & 30060 & 29177 & 0 & 0 & 0 & 0 & 3 & 27 & 58816 \\ 29786 & 30201 & 30337 & 30412 & 30916 & 31244\end{array}$

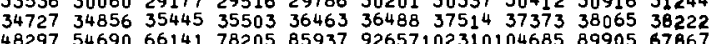
$\begin{array}{lllllllllll}65919 & 58351 & 51959 & 45189 & 39738 & 36523 & 35616 & 35474 & 35197 & 35187 \\ 44687 & 38790 & 35796 & 35032 & 33721 & 34188 & 34405 & 34442 & 34334 & 34088\end{array}$ $\begin{array}{llllllllll}34028 & 3390233928 & 34017 & 34001 & 33601 & 33684 & 33637 & 33719 & 33551\end{array}$ $\begin{array}{lllllllllll}33754 & 33376 & 33633 & 33583 & 33555 & 33372 & 33580 & 33434 & 33587 & 33395\end{array}$ $\begin{array}{llllllllll}32528 & 32772 & 32911 & 32751 & 32835 & 32392 & 32607 & 32346 & 32686 & 32372\end{array}$

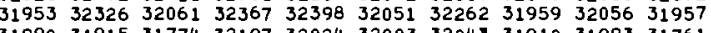
$\begin{array}{llllllllll}31990 & 31915 & 31774 & 32197 & 32024 & 32003 & 32043 & 31910 & 31993 & 31761 \\ 32069 & 31901 & 32220 & 31926 & 31923 & \mathbf{3 1 8 8 7} & 31910 & 32169 & 32119 & \mathbf{3 1 8 1 7}\end{array}$ $\begin{array}{lllllllllll}32069 & 31901 & 32220 & 31926 & 31923 & 31887 & 31910 & 32169 & 32119 & 31817 \\ 32531 & 32672 & 32716 & 32439 & 32780 & 32576 & 32747 & 32855 & 32675 & 32678\end{array}$

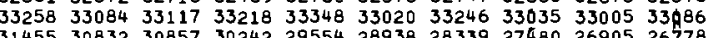
2518424934247142464024819246862454524602244692478 $23872493424742464024819246862454524602 \quad 2446924218$

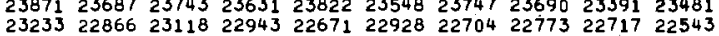

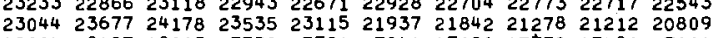
$\begin{array}{llllllllllll}18224 & 18187 & 18005 & 17738 & 17781 & 17841 & 17696 & 17772 & 17486 & 17661\end{array}$ $\begin{array}{llllllllll}14524 & 14737 & 14893 & 15491 & 16053 & 16467 & 16191 & 15842 & 15436 & 15515 \\ 15715 & 15374 & 15041 & 14736 & 14648\end{array}$

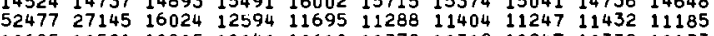
$1068510521 \quad 10805106461061010378 \quad 1031210247 \quad 1033910133$ $\begin{array}{rrrrrrrrrrr}9983 & 10178 & 10476 & 10756 & 10439 & 10324 & 10326 & 10328 & 10643 & 10594 \\ 7302 & 7137 & 7176 & 7217 & 7081 & 7006 & 6889 & 6902 & 6917 & 6828\end{array}$ $\begin{array}{lllllllllll}15569 & 16562 & 14571 & 10896 & 8116 & 6994 & 6369 & 6338 & 6958 & 8034\end{array}$ $\begin{array}{lllllllllll}4104 & 4032 & 4076 & 4027 & 4187 & 4198 & 4653 & 5670 & 8104 & 13067\end{array}$ $\begin{array}{llllllllll}3368 & 3909 & 4843 & 5723 & 5795 & 4782 & 3833 & 3112 & 2763 & 2556\end{array}$ $\begin{array}{llllllllll}2560 & 2594 & 2707 & 2726 & 3001 & 3629 & 4811 & 6610 & 7683 & 8129\end{array}$ $\begin{array}{llllllllrr}2432 & 2417 & 2383 & 2475 & 2510 & 2457 & 2504 & 2543 & 2547 & 2473\end{array}$ $\begin{array}{rrrrrrrrrr}6865 & 5592 & 6541 & 9792 & 15690 & 23309 & 27297 & 25970 & 18295 & 10469 \\ 2018 & 1952 & 2022 & 2043 & 1979 & 2057 & 2133 & 2128 & 2331 & 2769\end{array}$ $\begin{array}{llllllllll}2399 & 2221 & 1911 & 1927 & 1882 & 1895 & 1877 & 1828 & 1872 & 1994 \\ 1836 & 1835 & 1815 & 1764 & 1818 & 1859 & 1762 & 1825 & 1845 & 1811\end{array}$ $\begin{array}{llllllllll}1449 & 1502 & 1480 & 1444 & 1400 & 1473 & 1421 & 1440 & 1371 & 1407 \\ 1712 & 2124 & 2514 & 2726 & 2747 & 2457 & 1919 & 1611 & 1382 & 1302\end{array}$

\begin{tabular}{|c|c|c|c|c|c|c|c|c|c|}
\hline $\begin{array}{l}1226 \\
1118\end{array}$ & $\begin{array}{l}1186 \\
1048\end{array}$ & $\begin{array}{l}1181 \\
1045\end{array}$ & $\begin{array}{l}1218 \\
1105\end{array}$ & $\begin{array}{l}1190 \\
1026\end{array}$ & $\begin{array}{l}1156 \\
1019\end{array}$ & $\begin{array}{r}1173 \\
978\end{array}$ & $\begin{array}{l}1200 \\
1085\end{array}$ & $\begin{array}{r}1187 \\
994\end{array}$ & $\begin{array}{l}1250 \\
1059\end{array}$ \\
\hline 1021 & 999 & 909 & 968 & 915 & 882 & 934 & 876 & 892 & 853 \\
\hline 772 & 838 & 829 & & 845 & & & & & \\
\hline 772 & 777 & 747 & 764 & 701 & 729 & 732 & 763 & 780 & \\
\hline 759 & 793 & 767 & 801 & 778 & 724 & 693 & 667 & 634 & \\
\hline 660 & 640 & 653 & 680 & 699 & 677 & 690 & & & 62 \\
\hline 2372 & 3450 & 4142 & 4577 & 3871 & 2689 & 1619 & 972 & 30 & \\
\hline 537 & 529 & 543 & 515 & 472 & 513 & 547 & & 568 & \\
\hline 52 & 494 & 514 & & 48 & & 490 & 49 & 10 & 49 \\
\hline 55 & 545 & 523 & 538 & 471 & 530 & 523 & & & 53 \\
\hline 463 & 449 & 485 & 467 & 432 & 435 & 441 & 483 & 445 & 443 \\
\hline 48 & 569 & 580 & 672 & 753 & & & & 603 & \\
\hline 455 & 470 & 43 & & 425 & 422 & 393 & 427 & & \\
\hline 409 & 402 & 384 & 394 & 384 & & 390 & $\begin{array}{r}410 \\
394\end{array}$ & & 387 \\
\hline 339 & 363 & 368 & 388 & 384 & 404 & 351 & 394 & 33 & 371 \\
\hline & & & & 335 & 383 & 348 & & & \\
\hline 36 & 41 & 447 & 46 & 50 & 46 & 411 & & & \\
\hline 291 & 346 & 292 & 307 & 317 & & 304 & & & \\
\hline 306 & 291 & 277 & 284 & 272 & 282 & 292 & 258 & 281 & 398 \\
\hline 30 & 229 & 296 & 259 & 257 & 260 & 250 & & & \\
\hline $\begin{array}{l}25 \\
22\end{array}$ & $\begin{array}{l}259 \\
227\end{array}$ & & & $\begin{array}{l}303 \\
247\end{array}$ & $\begin{array}{l}321 \\
228\end{array}$ & $\begin{array}{l}316 \\
212\end{array}$ & $\begin{array}{l}316 \\
210\end{array}$ & $\begin{array}{l}282 \\
202\end{array}$ & $\begin{array}{l}244 \\
206\end{array}$ \\
\hline 21 & 182 & 177 & 191 & 201 & 166 & & & 194 & \\
\hline & & & & & & & & & \\
\hline 18 & 18 & & 19 & 16 & & & & & \\
\hline & & & & & & & & & \\
\hline 31 & 23 & 219 & 23 & 22 & 191 & 193 & & 16 & 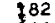 \\
\hline & 13 & & 146 & 14 & & & & & \\
\hline & & & & & & & & & \\
\hline 27 & & & & & & & & & is \\
\hline & 14 & & & 12 & & & & & \\
\hline & & & & & & & & & \\
\hline 123 & & & & & & & & & 123 \\
\hline & & & & & & & & & \\
\hline 11 & & & & & & & & & \\
\hline 14 & & & & & & 12 & & & i32 \\
\hline 12 & & & & & & & & & \\
\hline & & & & & & & & & \\
\hline & & & & & & & & & \\
\hline 11 & 120 & 117 & 109 & 120 & 106 & 109 & 110 & & i 10 \\
\hline & 12 & & & 10 & & & & & \\
\hline 1. & & & & & & & & & \\
\hline 12 & & & & & & & & 12 & 106 \\
\hline 13 & 112 & 124 & 130 & 121 & 11 & 128 & 113 & 96 & 15 \\
\hline & & & 11 & 12 & 12 & 118 & & 14 & \\
\hline 123 & 13 & & 147 & 12 & 162 & 12 & 13 & & 122 \\
\hline 154 & 118 & 137 & 118 & 104 & 13 & 133 & 130 & 124 & 131 \\
\hline 75 & 78 & 11 & 7 & 32 & & 74 & 78 & 73 & \\
\hline
\end{tabular}

$\begin{array}{rrrrrrrrrr}60 & 72 & 49 & 83 & 72 & 77 & 72 & 86 & 91 & 80 \\ 62 & 62 & 58 & 63 & 70 & 50 & 63 & 53 & 63 & 61 \\ 52 & 57 & 53 & 50 & 45 & 52 & 50 & 48 & 55 & 59 \\ 38 & 36 & 32 & 46 & 35 & 41 & 27 & 42 & 43 & 40 \\ 28 & 32 & 29 & 25 & 20 & 30 & 30 & 37 & 20 & 31 \\ 27 & 29 & 28 & 37 & 28 & 26 & 29 & 26 & 29 & 24 \\ 47 & 40 & 36 & 30 & 25 & 20 & 20 & 19 & 25 & 17 \\ 18 & 24 & 20 & 14 & 19 & 19 & 29 & 13 & 18 & 19 \\ 21 & 26 & 15 & 22 & 27 & 23 & 18 & 26 & 30 & 21 \\ 172 & 227 & 265 & 323 & 335 & 325 & 285 & 242 & 155 & 83 \\ 6 & 16 & 7 & 15 & 13 & 9 & 6 & 10 & 7 & 9 \\ 9 & 11 & 13 & 7 & 9 & 11 & 6 & 8 & 12 & 5 \\ 7 & 13 & 12 & 8 & 8 & 5 & 8 & 5 & 6 & 8\end{array}$


$D-27$

GAMMA RAY SPECTRUM DATA

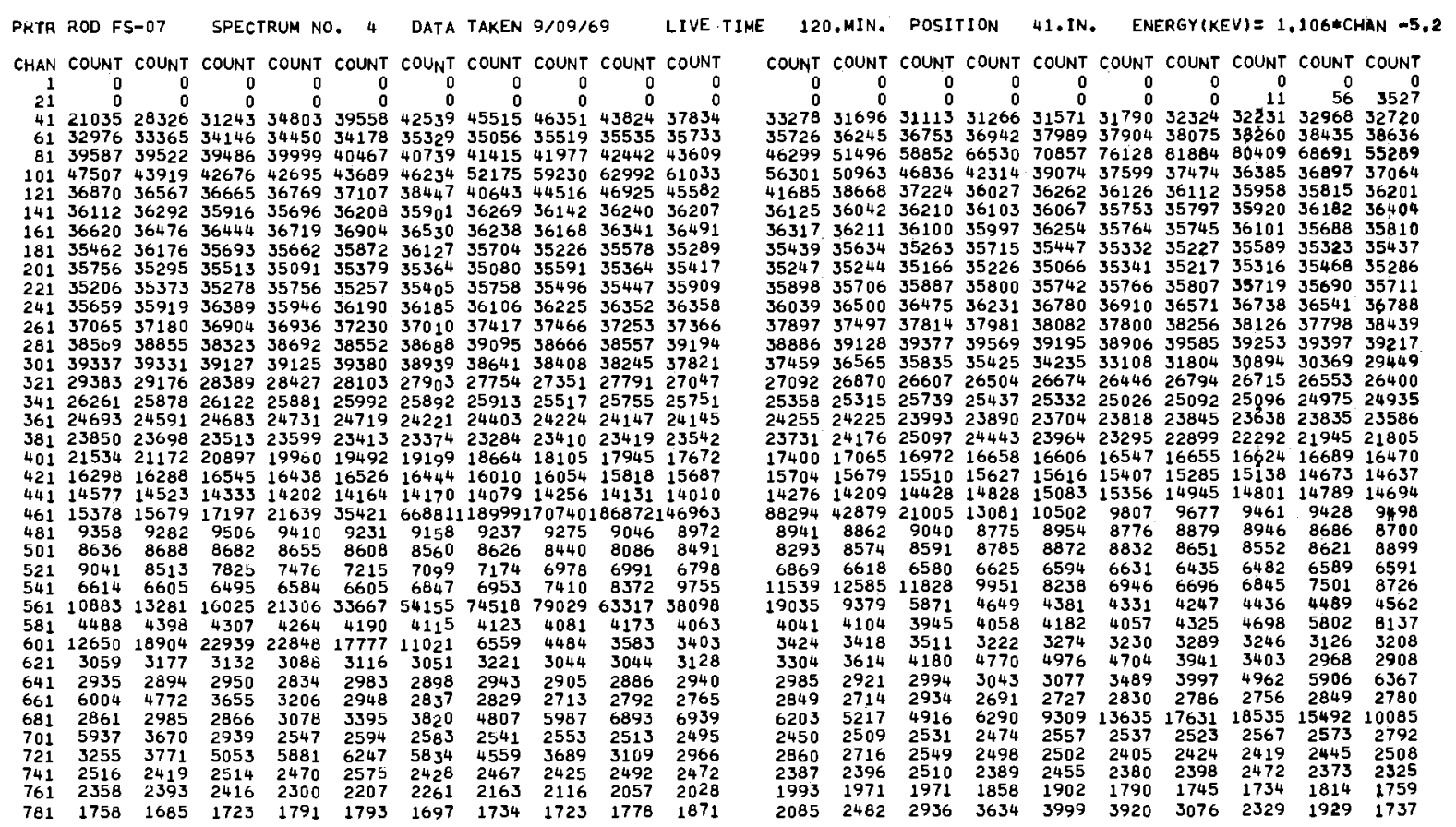

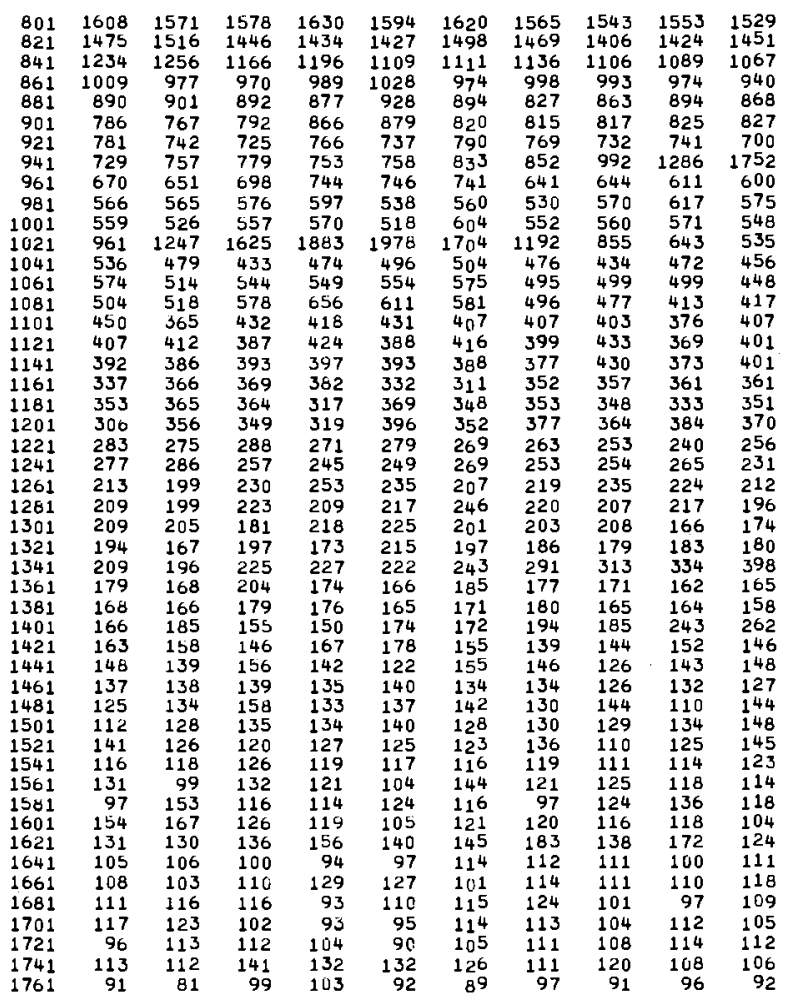

$\begin{array}{llllllllll}1562 & 1600 & 1520 & 1568 & 1528 & 1516 & 1437 & 1425 & 1484 & 1467 \\ 1379 & 1338 & 1312 & 1295 & 1290 & 1296 & 1317 & 1156 & 1207 & 1414\end{array}$

$\begin{array}{llllllllll}1379 & 1338 & 1312 & 1295 & 1290 & 1296 & 1317 & 1156 & 1207 & 1414 \\ 1101 & 1074 & 1099 & 1049 & 995 & 1053 & 1072 & 1025 & 1003 & 1914\end{array}$

$\begin{array}{rrrrrrrrrr}1101 & 1074 & 1099 & 1049 & 995 & 1053 & 1072 & 1025 & 1003 & 1914 \\ 946 & 942 & 931 & 948 & 952 & 898 & 942 & 927 & 969 & 934 \\ 840 & 854 & 882 & 797 & 814 & 792 & 824 & 823 & 820 & 819\end{array}$

$\begin{array}{rrrrrrrrrr}806 & 881 & 885 & 845 & 815 & 819 & 766 & 717 & 731 & 747 \\ 805 & 752 & 703 & 713 & 765 & 735 & 733 & 774 & 758 & 733\end{array}$

$\begin{array}{rrrrrrrrrr}805 & 752 & 703 & 713 & 765 & 735 & 733 & 774 & 758 & 73 \\ 2499 & 3771 & 5199 & 66950 & 6950 & 5956 & 3907 & 2248 & 1255 & 795 \\ 609 & 573 & 573 & 567 & 538 & 596 & 499 & 571 & 567 & 583\end{array}$

$\begin{array}{llllllllll}591 & 573 & 601 & 569 & 576 & 574 & 547 & 548 & 571 & 561\end{array}$

$\begin{array}{llllllllll}546 & 594 & 565 & 570 & 636 & 543 & 544 & 589 & 661 & 801 \\ 515 & 472 & 545 & 501 & 467 & 486 & 480 & 472 & 442 & 473\end{array}$

$\begin{array}{llllllllll}481 & 495 & 530 & 574 & 569 & 645 & 688 & 671 & 628 & 580 \\ 499 & 513 & 494 & 489 & 509 & 443 & 431 & 440 & 465 & 475\end{array}$

$\begin{array}{llllllllll}401 & 440 & 387 & 404 & 434 & 435 & 365 & 436 & 415 & 928 \\ 415 & 397 & 413 & 416 & 402 & 392 & 383 & 371 & 423 & 396\end{array}$

$\begin{array}{llllllllll}411 & 377 & 421 & \mathbf{3 8 2} & 424 & \mathbf{3 7 9} & 386 & 416 & 384 & 389 \\ \mathbf{3 8 6} & 394 & 433 & \mathbf{5 1 0} & \mathbf{5 1 3} & 442 & 452 & \mathbf{4 3 0} & 396 & 382\end{array}$

$\begin{array}{llllllllll}368 & 379 & 333 & 343 & 342 & 313 & 352 & 292 & 374 & 367\end{array}$

$\begin{array}{llllllllll}358 & \mathbf{3 4 8} & \mathbf{3 3 2} & \mathbf{3 3 8} & \mathbf{3 6 3} & \mathbf{3 1 6} & \mathbf{3 4 1} & \mathbf{3 4 2} & \mathbf{3 4 8} & \mathbf{3 1 2} \\ \mathbf{3 5 7} & \mathbf{3 1 6} & \mathbf{3 0 7} & \mathbf{3 6 2} & 295 & \mathbf{2 7 1} & \mathbf{3 1 1} & \mathbf{3 1 4} & \mathbf{3 4 6} & \mathbf{3 1 7}\end{array}$

$\begin{array}{llllllllll}249 & 307 & 280 & 273 & 280 & 300 & 299 & 354 & 311 & 312 \\ 259 & 251 & 251 & 255 & 273 & 276 & 283 & 277 & 250 & 237\end{array}$

$\begin{array}{llllllllll}231 & 231 & 218 & 212 & 218 & 227 & 215 & 235 & 228 & 213 \\ 214 & 205 & 205 & 206 & 193 & 220 & 236 & 200 & 193 & 198\end{array}$

$\begin{array}{llllllllll}214 & 205 & 205 & 206 & 193 & 220 & 236 & 200 & 193 & 198 \\ 214 & 188 & 206 & 176 & 184 & 187 & 187 & 210 & 220 & 198\end{array}$

$\begin{array}{llllllllll}180 & 192 & 179 & 181 & 163 & 206 & 176 & 205 & 181 & 183\end{array}$

$\begin{array}{llllllllll}360 & 288 & 228 & 265 & 286 & 242 & 233 & 230 & 217 & 207 \\ 157 & 186 & 178 & 158 & 164 & 173 & 168 & 197 & 175 & 175\end{array}$

$\begin{array}{llllllllll}172 & 180 & 163 & 189 & 161 & 179 & 189 & 177 & 175 & 175 \\ 298 & 342 & 386 & 491 & 567 & 536 & 436 & 341 & 148 & \$ 79\end{array}$

$\begin{array}{llllllllll}298 & 342 & 386 & 491 & 567 & 536 & 436 & 341 & 244 & 192 \\ 137 & 118 & 137 & 146 & 143 & 132 & 131 & 127 & 149 & 135\end{array}$

$\begin{array}{llllllllll}126 & 135 & 126 & 124 & 129 & 155 & 139 & 147 & 138 & 130\end{array}$

$\begin{array}{lllllllllll}126 & 134 & 126 & 124 & 129 & 155 & 139 & 147 & 138 & 130 \\ 139 & 124 & 127 & 122 & 132 & 103 & 135 & 124 & 14 & \$ 28\end{array}$

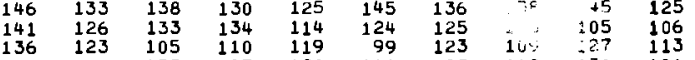

$\begin{array}{llllllllll}129 & 118 & 133 & 107 & 122 & 111 & 122 & 112 & 136 & 121\end{array}$

$\begin{array}{llllllllll}132 & 138 & 143 & 121 & 132 & 136 & 150 & 161 & 156 & \$ 66 \\ 117 & 100 & 83 & 113 & 137 & 88 & 136 & 116 & 121 & 116\end{array}$

$\begin{array}{rrrrrrrrrr}116 & 116 & 119 & 103 & 107 & 115 & 111 & 115 & 92 & 110 \\ 101 & 101 & 108 & 122 & 105 & 107 & 106 & 116 & 124 & 111\end{array}$

$\begin{array}{rrrrrrrrrr}118 & 105 & 97 & 113 & 128 & 120 & 99 & 106 & 113 & 126 \\ 107 & 100 & 127 & 93 & 105 & 104 & 91 & 112 & 127 & 114\end{array}$

$\begin{array}{rrrrrrrrrr}112 & 129 & 117 & 110 & 104 & 90 & 112 & 108 & 96 & 92 \\ 102 & 77 & 105 & 102 & 98 & 121 & 123 & 114 & 122 & 123\end{array}$

$\begin{array}{rrrrrrrrrr}102 & 115 & 105 & 102 & 98 & 121 & 123 & 114 & 122 & 123 \\ 84 & 83 & 92 & 75 & 81 & 90 & 100 & 96 & 94 & 102 \\ & & & & & & & 67 & 61 & 59\end{array}$

$\begin{array}{rrrrrrrrrrr}1781 & 62 & 67 & 60 & 60 & 67 & 71 & 67 & 61 & 63 & 57 \\ 1801 & 98 & 112 & 73 & 56 & 61 & 66 & 49 & 62 & 68 & 52 \\ 1821 & 53 & 63 & 51 & 64 & 64 & 62 & 49 & 56 & 52 & 46 \\ 1841 & 43 & 51 & 62 & 43 & 53 & 51 & 53 & 60 & 49 & 44 \\ 1861 & 34 & 49 & 49 & 36 & 39 & 45 & 39 & 43 & 38 & 41 \\ 1881 & 43 & 41 & 33 & 36 & 37 & 45 & 33 & 34 & 15 & 25 \\ 1901 & 48 & 28 & 50 & 36 & 39 & 53 & 36 & 68 & 64 & 70 \\ 1921 & 28 & 31 & 24 & 31 & 18 & 30 & 34 & 34 & 29 & 19 \\ 1941 & 26 & 32 & 21 & 23 & 19 & 26 & 23 & 24 & 28 & 25 \\ 1961 & 24 & 27 & 36 & 31 & 26 & 34 & 38 & 40 & 52 & 60 \\ 1981 & 134 & 64 & 44 & 30 & 25 & 23 & 10 & 14 & 17 & 11 \\ 2001 & 19 & 9 & 15 & 12 & 15 & 12 & 10 & 18 & 11 & 10 \\ 2021 & 15 & 6 & 13 & 12 & 14 & 9 & 12 & 18 & 16 & 9 \\ 2041 & 8 & 14 & 11 & 12 & 7 & 10 & 9 & 0 & & \end{array}$

$\begin{array}{rrrrrrrrrr}60 & 67 & 83 & 65 & 82 & 74 & 101 & 101 & 91 & 93 \\ 57 & 54 & 51 & 59 & 58 & 58 & 47 & 61 & 56 & 48 \\ 47 & 55 & 46 & 63 & 46 & 35 & 58 & 54 & 46 & 44 \\ 38 & 38 & 45 & 40 & 44 & 37 & 46 & 55 & 45 & 48 \\ 25 & 46 & 38 & 47 & 40 & 55 & 45 & 33 & 41 & 43 \\ 37 & 30 & 43 & 41 & 40 & 31 & 34 & 34 & 25 & 35 \\ 83 & 80 & 78 & 69 & 59 & 44 & 31 & 33 & 33 & 33 \\ 37 & 21 & 27 & 16 & 25 & 25 & 29 & 19 & 29 & 26 \\ 17 & 22 & 28 & 15 & 26 & 25 & 27 & 31 & 23 & 27 \\ 72 & 71 & 108 & 143 & 154 & 220 & 228 & 249 & 241 & 174 \\ 18 & 19 & 18 & 12 & 12 & 10 & 12 & 11 & 15 & 18 \\ 9 & 13 & 17 & 17 & 11 & 11 & 13 & 15 & 13 & 21 \\ 9 & 12 & 15 & 13 & 9 & 15 & 8 & 13 & 10 & 11\end{array}$


GAMMA RAY SPECTRUM DATA

\begin{tabular}{|c|c|c|c|c|c|c|c|c|c|c|c|c|c|c|c|c|c|c|c|c|}
\hline PRTR & ROD & -07 & SPEC & RUM $N$ & 6 & DATA & TAKEN & $9 / 10 / 6$ & & LIVE TIME & & MIN. & POS & TION & $44 . I N$, & ENE & ERGYI & v) $=$ & $107 *$ & IAN $-5,4$ \\
\hline CHAN & COUNT & COUNT & COUNT & OUNT & t. & $\operatorname{coU}_{N} T$ & COUN & COUN & COUNT & OUNT & & COUNT & & & & & & & $c 01 \mathrm{M}$ & COUN \\
\hline & & & & 0 & & & & 0 & 0 & & & & & & & 0 & 0 & 0 & & \\
\hline 21 & & & & & & & & & & & & 0 & & & & & & & 2728 & 46 \\
\hline 41 & 14859 & 16448 & 18356 & 20845 & 22648 & 23822 & 23903 & 22439 & 19205 & 17060 & 16169 & 16060 & 16403 & 16640 & 16586 & 16857 & 16893 & 17142 & 17248 & 174 \\
\hline 61 & 17690 & 17949 & 17679 & 18045 & 18523 & 18675 & 18597 & 18860 & 18835 & 18887 & 19331 & 19360 & 19794 & 20091 & 20330 & 20364 & 20337 & 20599 & 20977 & 21333 \\
\hline & & 21356 & 3 & 21764 & 22266 & 22298 & 22948 & & $\$ 124$ & & 29741 & 35082 & 41209 & 44468 & 47403 & 52304 & 52330 & 43350 & 33342 & 26967 \\
\hline 101 & & & 3458 & 23821 & 25873 & 29793 & 5306 & 1217 & & & 29600 & 26484 & 23514 & 21134 & 19770 & 19311 & & 19355 & & 9266 \\
\hline 121 & & 19245 & 19426 & & 20089 & 21613 & 24760 & 601 & 25905 & 22669 & 20756 & 19381 & 18984 & 18906 & 18730 & 18743 & 19003 & 9008 & 61 & 18776 \\
\hline & & 18643 & & 18893 & & & 18603 & & & & 186 & 578 & 18688 & & & & & & & 1539 \\
\hline 161 & 185 & & & & & 18907 & 3980 & & & & & 18609 & 18640 & 18567 & & 3670 & & 498 & & 25 \\
\hline 181 & & & & & & & & & & & & & & & & & & & & \\
\hline 201 & 180 & 17899 & 18126 & 18 & 17955 & 17858 & 17928 & 17884 & 17923 & 18122 & 17899 & 18037 & 18112 & 18129 & 17871 & 18008 & 17850 & 7933 & 17937 & 17947 \\
\hline 221 & 17818 & 17829 & 8053 & 1808 & 17870 & 17870 & 17961 & & 908 & & 17979 & 17783 & 18201 & 18219 & 17944 & 17995 & 180 & 17984 & 18041 & 17834 \\
\hline 241 & 18105 & 17970 & 17879 & 17915 & 18253 & 18110 & 17946 & & 18289 & 18194 & 17816 & 17974 & 18321 & 17997 & 18305 & 18055 & 182 & 18312 & & 18319 \\
\hline & & & & & & & & & & & & & & & & & & 8 & & 8574 \\
\hline & & & & & & & & & & & & & & & & & & & & \\
\hline 301 & 18989 & 18855 & 18807 & 18820 & 18822 & 18735 & 18506 & 18101 & 18177 & 18240 & 17736 & 17748 & 17083 & 16565 & 16534 & 15963 & 15575 & & & 14900 \\
\hline 321 & 14785 & 14539 & 14531 & 14527 & 14480 & 14242 & 14287 & & 3999 & & 14041 & 14127 & 13749 & 13908 & 13962 & 13814 & 14061 & 13516 & 371 & 730 \\
\hline 341 & & & 136 & 13 & 13686 & 13574 & & & & & & & 133 & & & & & & & \\
\hline & & & & & & & & & & & & & & & & & & & & \\
\hline & & & & & & & & & & & & 13527 & 13404 & 13126 & & 12117 & & & & 11584 \\
\hline 401 & 11497 & 11214 & 11196 & 10989 & 10701 & 10838 & 10385 & 10308 & 9972 & 10047 & 9896 & 9909 & 9910 & 9880 & 9853 & 9659 & 9924 & 9676 & 9780 & 9600 \\
\hline 421 & 9588 & 9810 & 9781 & 9797 & 9782 & 9723 & & 95 & 9613 & & 9283 & 9325 & & 9074 & & & & & & \\
\hline 441 & & & 8002 & & & & & & & & & & & & & & & & & \\
\hline 461 & & & 10056 & 14202 & & & & & & & & & & & & & & & & \\
\hline $\begin{array}{l}481 \\
501\end{array}$ & $\begin{array}{l}5775 \\
5278\end{array}$ & $\begin{array}{l}5758 \\
5297\end{array}$ & $\begin{array}{l}5803 \\
5218\end{array}$ & $\begin{array}{l}5744 \\
5263\end{array}$ & $\begin{array}{l}5807 \\
5342\end{array}$ & $\begin{array}{l}5677 \\
5311\end{array}$ & $\begin{array}{l}5594 \\
5227\end{array}$ & $\begin{array}{l}5581 \\
5187\end{array}$ & $\begin{array}{l}5699 \\
5183\end{array}$ & $\begin{array}{l}5608 \\
5283\end{array}$ & $\begin{array}{l}5460 \\
5237\end{array}$ & $\begin{array}{l}5657 \\
5204\end{array}$ & $\begin{array}{l}5384 \\
5551\end{array}$ & $\begin{array}{l}5528 \\
5541\end{array}$ & $\begin{array}{l}5545 \\
5582\end{array}$ & $\begin{array}{l}5457 \\
5409\end{array}$ & $\begin{array}{l}5438 \\
5208\end{array}$ & $\begin{array}{l}5305 \\
5389\end{array}$ & $\begin{array}{l}5409 \\
5584\end{array}$ & $\begin{array}{l}5284 \\
5628\end{array}$ \\
\hline 521 & 5265 & 4941 & 4513 & 4333 & 4243 & 4187 & 4062 & 3944 & 3952 & & & 3842 & & 37 & & & 37 & 3757 & & 3702 \\
\hline 541 & 3657 & & 3653 & 3769 & 3761 & 4074 & 4346 & & 5979 & & & & & & & & & & & 48 \\
\hline 561 & 639 & 6854 & 8360 & 12109 & 19618 & 28067 & 33268 & 29100 & 19068 & 10093 & 5177 & 253 & 26 & 2435 & 21 & 2491 & 59 & 485 & 672 & \\
\hline 581 & 2534 & 2357 & 2306 & & & & & & & & & 2272 & 22 & 419 & & 24 & & & & \\
\hline 601 & 13743 & 18330 & 19715 & 158 & & & & & & & & & & & & & & & & \\
\hline 621 & & & 154 & & & & & & & 174 & & & 27 & & & & & & & \\
\hline 6 & & 144 & 143 & & & & & 146 & & & & & & & & 21 & 28 & & & 78 \\
\hline 661 & 2953 & 2208 & 171 & 149 & 1318 & 140 & 140 & & & & 1375 & & & 3 & 14 & 1464 & 142 & & & 147 \\
\hline 681 & & 148 & 158 & 172 & 2082 & 26 & 3501 & 428 & 4564 & 397 & 3124 & 28 & & 553 & 8613 & 11832 & 13039 & 11347 & 75 & 4129 \\
\hline 701 & & & 127 & $\begin{array}{l}125 \\
125\end{array}$ & 1251 & $120^{3}$ & 12 & 11 & & & & & & & & & & & & \\
\hline & & & & & & & & & & & & & & & & & & & & \\
\hline 741 & 1144 & 114 & 115 & 113 & 11 & 113 & 1092 & 111 & 113 & 110 & 1117 & 1093 & 1183 & 1127 & 1184 & 1108 & 1140 & 107 & 1123 & $1 \mathrm{ic}$ \\
\hline 761 & 1145 & 1089 & 1125 & 1081 & 104 & 1040 & 997 & 978 & 1024 & 928 & 928 & 909 & 870 & 909 & 859 & 88 & 856 & 822 & 871 & 837 \\
\hline 781 & 840 & 807 & 808 & 850 & 841 & 820 & 842 & 872 & 851 & 909 & 1061 & 1320 & 1537 & 1753 & 1733 & 1467 & 1176 & 901 & 811 & 744 \\
\hline
\end{tabular}

\begin{tabular}{|c|c|c|c|c|c|c|c|c|c|c|}
\hline $\begin{array}{l}801 \\
821\end{array}$ & $\begin{array}{l}771 \\
683\end{array}$ & $\begin{array}{l}762 \\
730\end{array}$ & $\begin{array}{l}815 \\
759\end{array}$ & $\begin{array}{l}770 \\
692\end{array}$ & $\begin{array}{l}776 \\
713\end{array}$ & $\begin{array}{l}796 \\
696\end{array}$ & $\begin{array}{l}725 \\
731\end{array}$ & $\begin{array}{l}742 \\
666\end{array}$ & $\begin{array}{l}734 \\
675\end{array}$ & $\begin{array}{l}739 \\
639\end{array}$ \\
\hline 841 & & & & 596 & 515 & 517 & 574 & 562 & 536 & 543 \\
\hline 861 & 528 & 529 & 503 & 535 & 483 & 488 & 502 & $47 \overline{6}$ & 509 & 486 \\
\hline 881 & 471 & 422 & 449 & 458 & 451 & 438 & 464 & 445 & 461 & 460 \\
\hline 901 & 408 & 414 & 430 & 433 & 417 & 446 & 416 & 427 & 438 & 444 \\
\hline 921 & 359 & 389 & 355 & 403 & 358 & 396 & 386 & 387 & 390 & 384 \\
\hline 941 & 426 & 404 & 377 & 403 & 420 & 423 & 462 & 519 & 647 & 943 \\
\hline 961 & 336 & 309 & 319 & 317 & 376 & 371 & 344 & 341 & 309 & 321 \\
\hline 981 & 302 & 296 & 308 & 317 & 285 & 320 & 307 & 309 & 303 & 315 \\
\hline 1001 & 273 & 296 & 293 & 318 & 322 & 279 & 310 & 308 & 287 & 301 \\
\hline 1021 & 522 & 652 & 791 & 828 & 820 & 674 & 513 & 345 & 287 & 282 \\
\hline 1041 & 263 & 231 & 237 & 260 & 208 & 236 & 226 & 261 & 258 & 226 \\
\hline 1061 & 306 & 290 & 282 & 289 & 284 & 302 & 274 & 223 & 243 & 254 \\
\hline 1081 & 248 & 259 & 293 & 301 & 296 & 283 & 263 & 222 & 229 & 214 \\
\hline 1101 & 203 & 211 & 226 & 213 & 233 & 187 & 210 & 196 & 202 & 213 \\
\hline 1121 & 221 & 200 & 195 & 204 & 196 & 213 & 213 & 188 & 202 & 195 \\
\hline 1141 & 220 & 197 & 212 & 174 & 193 & 197 & 222 & 211 & 217 & 200 \\
\hline 1161 & 171 & 185 & 190 & 200 & 171 & 197 & 189 & 165 & 154 & 178 \\
\hline 1181 & 171 & 159 & 176 & 173 & 169 & 162 & 191 & 189 & 170 & 181 \\
\hline 1201 & 170 & 168 & 192 & 175 & 194 & 195 & 190 & 214 & 221 & 193 \\
\hline 1221 & 143 & 155 & 120 & 130 & 136 & 158 & 124 & 126 & 149 & 123 \\
\hline 1241 & 135 & 140 & 162 & 128 & 128 & 136 & 136 & 141 & 124 & 114 \\
\hline 1261 & 116 & 108 & 112 & 111 & 128 & 109 & 104 & 108 & 114 & 121 \\
\hline 1281 & 103 & 95 & 131 & 149 & 114 & 106 & 126 & 105 & 112 & 124 \\
\hline 1301 & 104 & 102 & 110 & 72 & 108 & 106 & 103 & 96 & 99 & 81 \\
\hline 1321 & 114 & 104 & 106 & 91 & 105 & 113 & 100 & 98 & & 84 \\
\hline 1341 & & 99 & 134 & 111 & 132 & 149 & 168 & 174 & 214 & 218 \\
\hline 1361 & 100 & 86 & 94 & 103 & 87 & 96 & 112 & 81 & 78 & 90 \\
\hline 1381 & 95 & 64 & 87 & 70 & 75 & 107 & 69 & 87 & 98 & 89 \\
\hline 1401 & 86 & 82 & 86 & 81 & 103 & 78 & 84 & 76 & 124 & 118 \\
\hline 1421 & 94 & 80 & 71 & 74 & 67 & 74 & 78 & 86 & 66 & 81 \\
\hline 1441 & 78 & 65 & 60 & 69 & 69 & 63 & 97 & 63 & 67 & 86 \\
\hline 1461 & 73 & 85 & 81 & 60 & 84 & 74 & 71 & 76 & 65 & 69 \\
\hline 1481 & 76 & 57 & 82 & 70 & 68 & 72 & 56 & 56 & 69 & 59 \\
\hline 1501 & 70 & 57 & 55 & 82 & 66 & 56 & 64 & 62 & 66 & 79 \\
\hline 1521 & 83 & 79 & 58 & 67 & 75 & 59 & 74 & 60 & 78 & 66 \\
\hline 1541 & 78 & 61 & 62 & 70 & 69 & 66 & 67 & 69 & 72 & 75 \\
\hline 1561 & 64 & 74 & 78 & 62 & 74 & 66 & 60 & 65 & 69 & 68 \\
\hline 1581 & 75 & 62 & 67 & 82 & 62 & 60 & 67 & 59 & 66 & 72 \\
\hline 1601 & 85 & 73 & 67 & 70 & 71 & 56 & 66 & 57 & 71 & 79 \\
\hline 1621 & 75 & 65 & 73 & 62 & 99 & 80 & 77 & 73 & 99 & 76 \\
\hline 1641 & 63 & 62 & 72 & 60 & 60 & 72 & 66 & 69 & 58 & 63 \\
\hline 1661 & 82 & 56 & 61 & 57 & 65 & 63 & 75 & 62 & 65 & 72 \\
\hline 1681 & 65 & 69 & 80 & 69 & 53 & 52 & 71 & 65 & 69 & 58 \\
\hline 1701 & 57 & 66 & $60^{\circ}$ & 69 & 52 & 61 & 62 & 60 & 46 & 51 \\
\hline 1721 & 58 & 55 & 68 & 54 & 55 & 66 & 47 & 56 & 57 & 66 \\
\hline 1741 & 73 & 50 & 82 & 79 & 67 & 62 & 74 & 65 & 63 & 78 \\
\hline 1761 & 59 & 52 & 67 & 56 & 44 & 51 & 50 & 54 & 61 & 56 \\
\hline
\end{tabular}

\begin{tabular}{|c|c|c|c|c|c|c|c|c|c|}
\hline 694 & 610 & $\begin{array}{l}686 \\
668\end{array}$ & 724 & $\begin{array}{r}729 \\
657\end{array}$ & 691 & 695 & 665 & $\begin{array}{l}751 \\
628\end{array}$ & $\begin{array}{r}739 \\
575\end{array}$ \\
\hline $\begin{array}{l}641 \\
564\end{array}$ & $\begin{array}{l}033 \\
537\end{array}$ & $\begin{array}{l}668 \\
571\end{array}$ & $\begin{array}{l}687 \\
569\end{array}$ & $\begin{array}{l}651 \\
546\end{array}$ & $\begin{array}{l}595 \\
537\end{array}$ & $\begin{array}{l}610 \\
527\end{array}$ & $\begin{array}{l}638 \\
501\end{array}$ & $\begin{array}{l}628 \\
479\end{array}$ & $\begin{array}{r}575 \\
541\end{array}$ \\
\hline 483 & 492 & 513 & 477 & 468 & 445 & 500 & 462 & 472 & \\
\hline 458 & 503 & 447 & 461 & 457 & 419 & 449 & 465 & 461 & 10 \\
\hline 436 & 464 & 452 & 458 & 419 & 395 & 427 & 397 & 428 & 89 \\
\hline 363 & 377 & 368 & 356 & 367 & 362 & 380 & 398 & 356 & \\
\hline 1287 & 1900 & 2451 & 2907 & 2759 & 2084 & 1335 & 710 & 464 & 52 \\
\hline 289 & 303 & 307 & 323 & 303 & 324 & 294 & & 308 & 100 \\
\hline 283 & 314 & 297 & 294 & 278 & 294 & 276 & 280 & 265 & \\
\hline $\begin{array}{l}200 \\
296\end{array}$ & 311 & 299 & $\begin{array}{l}294 \\
313\end{array}$ & 294 & 280 & 285 & 335 & $\begin{array}{l}200 \\
349\end{array}$ & 447 \\
\hline 235 & 258 & 267 & 259 & 292 & 233 & 241 & 273 & 266 & 222 \\
\hline 301 & 274 & 314 & 334 & 352 & 429 & 383 & 38 & 348 & \\
\hline 247 & 282 & 250 & 219 & 207 & 252 & 223 & 231 & 228 & 275 \\
\hline 246 & 216 & 223 & 223 & 207 & 211 & 202 & 199 & 197 & 210 \\
\hline 211 & 210 & 220 & 196 & 211 & 221 & 200 & 208 & 203 & 210 \\
\hline 215 & 210 & 189 & 225 & 228 & 214 & 22 & 21 & 18 & 180 \\
\hline 229 & 247 & 231 & 230 & 243 & 265 & 234 & 240 & 226 & 209 \\
\hline 166 & 179 & 165 & 179 & 196 & 180 & 180 & 183 & 172 & 175 \\
\hline $\begin{array}{l}160 \\
162\end{array}$ & $\begin{array}{l}179 \\
175\end{array}$ & 137 & 174 & $\begin{array}{l}170 \\
179\end{array}$ & 155 & $\begin{array}{l}100 \\
145\end{array}$ & 130 & 145 & $\begin{array}{l}171 \\
171\end{array}$ \\
\hline 188 & $\begin{array}{l}174 \\
\text { S }\end{array}$ & 156 & 148 & 157 & 155 & 146 & 17 & 14 & 167 \\
\hline 141 & 143 & $\begin{array}{l}159 \\
\end{array}$ & 154 & 158 & 185 & $\begin{array}{l}189 \\
\end{array}$ & 193 & 188 & $\begin{array}{l}157 \\
\end{array}$ \\
\hline $\begin{array}{l}137 \\
\end{array}$ & 107 & 121 & 138 & 133 & 136 & 148 & 159 & 122 & 125 \\
\hline 135 & 122 & 102 & 104 & 110 & 119 & $\begin{array}{l}109 \\
109\end{array}$ & 10 & 11 & 126 \\
\hline 97 & 116 & 104 & 112 & 101 & 117 & 11 & 9 & 113 & $\begin{array}{l}112 \\
112\end{array}$ \\
\hline 80 & 104 & 103 & 100 & 99 & 91 & 11 & 95 & 91 & 90 \\
\hline 90 & 93 & 106 & 98 & 97 & 99 & 11 & 90 & 90 & 104 \\
\hline 203 & 169 & 133 & 140 & 120 & 118 & 118 & 108 & 93 & 108 \\
\hline 88 & 82 & 95 & 103 & 98 & 88 & 75 & 93 & 86 & 5 \\
\hline 75 & 81 & 85 & 75 & 98 & 97 & 93 & & & 80 \\
\hline 136 & 168 & 193 & 242 & 264 & 243 & 204 & 13 & 12 & \\
\hline 74 & 75 & 80 & 85 & 82 & 69 & 75 & 7 & 66 & \\
\hline 61 & 98 & 6 & 74 & 7 & 7 & 6 & 6 & & \\
\hline 72 & 71 & 68 & 71 & 63 & 78 & 7 & 6 & 7 & \\
\hline 59 & $\begin{array}{l}68 \\
68\end{array}$ & $\begin{array}{l}60 \\
62\end{array}$ & 67 & $\begin{array}{l}03 \\
67\end{array}$ & 67 & 7 & 7 & 63 & \\
\hline 75 & 67 & $B 2$ & 77 & 95 & 92 & 91 & $B 8$ & 66 & \\
\hline 86 & 53 & 65 & 61 & 66 & 54 & 5 & 6 & 8 & \\
\hline 70 & 69 & 5 & 6 & 6 & 62 & 6 & $B$ & 70 & \\
\hline 78 & 56 & 55 & 71 & 75 & 74 & 4 & 65 & 53 & \\
\hline 58 & 65 & 75 & 58 & 75 & 91 & 8 & 8 & 108 & \\
\hline 53 & 61 & 71 & 70 & 65 & 66 & 6 & 68 & 65 & \\
\hline 64 & 58 & 73 & 62 & 6 & 6 & 5 & 6 & 7 & \\
\hline 54 & 63 & 71 & 70 & 73 & 53 & 6 & 7 & 4 & \\
\hline 55 & 63 & 65 & 75 & 62 & 66 & 72 & 57 & 62 & \\
\hline 60 & 71 & 65 & 71 & 75 & 79 & 59 & 73 & 61 & \\
\hline 69 & 68 & 45 & 47 & 85 & 60 & 67 & 76 & 71 & \\
\hline 53 & 56 & 78 & 64 & 53 & 74 & 76 & 74 & 64 & \\
\hline 58 & 62 & 70 & 66 & 52 & 65 & 63 & 63 & 63 & 5 \\
\hline 43 & 54 & 49 & 38 & 48 & 39 & 46 & 24 & 42 & \\
\hline
\end{tabular}

\begin{tabular}{|c|c|c|c|c|c|c|c|c|c|c|c|c|c|c|c|c|c|c|c|c|}
\hline $\begin{array}{l}1781 \\
1801\end{array}$ & $\begin{array}{l}42 \\
36\end{array}$ & $\begin{array}{l}39 \\
46\end{array}$ & $\begin{array}{l}34 \\
42\end{array}$ & $\begin{array}{l}40 \\
34\end{array}$ & $\begin{array}{l}30 \\
28\end{array}$ & $\begin{array}{l}37 \\
36\end{array}$ & $\begin{array}{l}31 \\
25\end{array}$ & $\begin{array}{l}35 \\
24\end{array}$ & $\begin{array}{l}45 \\
23\end{array}$ & $\begin{array}{l}39 \\
28\end{array}$ & $\begin{array}{l}35 \\
38\end{array}$ & $\begin{array}{l}44 \\
33\end{array}$ & $\begin{array}{l}38 \\
28\end{array}$ & $\begin{array}{l}39 \\
27\end{array}$ & $\begin{array}{l}42 \\
25\end{array}$ & $\begin{array}{l}47 \\
28\end{array}$ & $\begin{array}{l}42 \\
18\end{array}$ & $\begin{array}{l}49 \\
32\end{array}$ & $\begin{array}{l}46 \\
37\end{array}$ & $\begin{array}{l}45 \\
33\end{array}$ \\
\hline 1821 & 28 & 24 & 33 & 26 & 30 & 24 & 19 & 23 & 29 & 25 & 25 & 38 & 26 & 28 & 27 & 30 & 27 & 27 & 26 & 27 \\
\hline 1841 & 28 & 31 & 31 & 36 & 31 & 19 & 20 & 26 & 23 & 25 & 21 & 13 & 25 & 20 & 18 & 26 & 22 & 17 & 27 & 24 \\
\hline 1861 & 23 & 22 & 18 & 10 & 18 & 19 & 18 & 24 & 18 & 19 & 15 & 17 & 18 & 17 & 21 & 21 & 13 & 24 & 17 & 21 \\
\hline 1881 & 27 & 17 & 21 & 17 & 11 & 15 & 11 & 17 & 22 & 17 & 10 & 11 & 14 & 15 & 11 & 17 & 17 & 11 & 18 & 28 \\
\hline 1901 & 23 & 24 & 15 & 17 & 23 & 18 & 18 & 27 & 27 & $3_{1}$ & 26 & 34 & 41 & 25 & 27 & 30 & 24 & 16 & 16 & 13 \\
\hline 1921 & 12 & 18 & 12 & 14 & 17 & 16 & 13 & 9 & 11 & 12 & 10 & 17 & 11 & 13 & 10 & 9 & 17. & 15 & 19 & 4 \\
\hline 1941 & 20 & 8 & 12 & 14 & 13 & 10 & 13 & 11 & 14 & 14 & 10 & 19 & 8 & 14 & 9 & 10 & 11 & 9 & 10 & 12 \\
\hline 1961 & 9 & 9 & 14 & 16 & 15 & 14 & 32 & 23 & 27 & 50 & 44 & 60 & 84 & 88 & 97 & 132 & 192 & 173 & 181 & 116 \\
\hline 1981 & 90 & 49 & 24 & 16 & 16 & 4 & 3 & 8 & 10 & 11 & $B$ & 10 & 3 & 7 & 6 & 3 & 6 & 10 & 6 & 9 \\
\hline 2001 & 7 & 4 & 6 & 8 & 3 & 7 & 7 & 4 & 9 & 5 & 5 & 6 & 5 & 9 & 6 & 8 & 8 & 5 & 4 & 5 \\
\hline 2021 & 8 & 6 & 8 & 9 & 6 & 2 & 7 & 7 & 7 & 9 & 4 & 1 & 6 & 7 & 2 & 1 & 4 & 7 & 5 & 9 \\
\hline 2041 & 2 & 2 & 5 & 5 & 1 & $B$ & 11 & 0 & & & & & & & & & & & & \\
\hline
\end{tabular}


GAMMA RAY SPECTRUM DATA

PRTR ROD FS-07 SPECTRUM NO, 8 DATA TAKEN 9/10/69 LIVE TIME 120,MIN. POSITION 45.IN. ENERGY(KEV) $1,108 *$ CHAN -5.9

CHAN COUNT COUNT COUNT COUNT COUNT COUNT COUNT COUNT COUNT COUNT $\begin{array}{rrrrrrrrrr}1 & 0 & 0 & 0 & 0 & 0 & 0 & 0 & 0 & 0 \\ 21 & 0 & 0 & 0 & 0 & 0 & 0 & 0 & 0 & 0\end{array}$

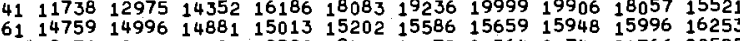

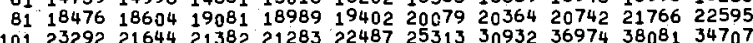
$\begin{array}{lllllllllllllll}101 & 23292 & 21644 & 21382 & 21283 & 22487 & 25313 & 30932 & 36974 & 3808_{1} & 34707\end{array}$

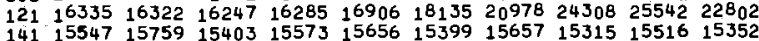
$\begin{array}{llllllllllll}161 & 15533 & 15286 & 15154 & 15431 & 15615 & 15475 & 15513 & 15590 & 15221 & 15385\end{array}$ $\begin{array}{lllllllllll}181 & 15243 & 15084 & 14900 & 15092 & 14882 & 14954 & 14944 & 14920 & 14825 & 14819\end{array}$ $\begin{array}{lllllllllll}221 & 14677 & 14544 & 14127 & 14122 & 14188 & 14309 & 14220 & 14372 & 14263 & 14217\end{array}$ $241 \quad 14194 \quad 14216 \quad 14103 \quad 1413114275 \quad 13913 \quad 14138 \quad 14041 \quad 14078 \quad 14204$ 2611422134261412314346013991410614125141611429314188 $\begin{array}{lllllllllll}281 & 14040 & 1428 & 14032 & 13746 & 14028 & 14000 & 13887 & 13785 & 14010 & 13543\end{array}$ $\begin{array}{lllllllllll}321 & 11831 & 11802 & 11746 & 11648 & 11576 & 11661 & 11648 & 11485 & 11382 & 11327\end{array}$

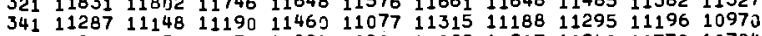
36110826107561075010884108081082910907109481077810724

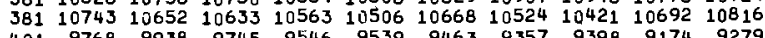
$\begin{array}{lllllllllll}401 & 9768 & 9938 & 9745 & 9546 & 9539 & 9463 & 9357 & 9398 & 9174 & 9279 \\ 4 & 8972 & 9019 & 9181 & 9332 & 9177 & 9049 & 8935 & 8794 & 8860 & 8668\end{array}$ $\begin{array}{lllllllllll}421 & 8972 & 9019 & 9181 & 9332 & 9177 & 9049 & 8935 & 8794 & 8860 & 8668\end{array}$ $\begin{array}{rrrrrrrrrrr}441 & 7437 & 7442 & 7315 & 7419 & 7277 & 7231 & 7220 & 7123 & 7252 & 7181 \\ 461 & 7149 & 7455 & 8120 & 9741 & 14653 & 23228 & 35237 & 42473 & 38674 & 26933\end{array}$ $\begin{array}{lllllllllll}481 & 5860 & 6025 & 5906 & 5835 & 5806 & 5746 & 5818 & 5684 & 5672 & 5695 \\ 501 & 5426 & 5358 & 5429 & 5419 & 5321 & 5351 & 5328 & 5281 & 5105 & 5422\end{array}$ $\begin{array}{lllllllllll}501 & 5426 & 5358 & 5429 & 5419 & 5321 & 5351 & 5328 & 5281 & 5105 & 5422 \\ 521 & 5491 & 4856 & 4544 & 4297 & 4102 & 3920 & 3986 & 3822 & 3728 & 3685\end{array}$ $\begin{array}{rrrrrrrrrrr}521 & 5491 & 4856 & 4544 & 4297 & 4102 & 3920 & 3986 & 3822 & 3728 & 368 \\ 541 & 3315 & 3292 & 3355 & 3382 & 3478 & 3696 & 4154 & 4694 & 6029 & 7516\end{array}$ $\begin{array}{lllllllllll}561 & 4102 & 4338 & 5181 & 7061 & 10623 & 14962 & 17393 & 15626 & 10849 & 6149\end{array}$ $\begin{array}{lrrrrrrrrrr}581 & 2275 & 2177 & 2188 & 1994 & 2181 & 2643 & 1939 & 2023 & 1977 & 2021 \\ 601 & 13847 & 19678 & 21551 & 17997 & 11064 & 5471 & 2761 & 1709 & 1475 & 1318\end{array}$ $\begin{array}{lllllllllll}621 & 1305 & 1231 & 1256 & 1267 & 1225 & 1220 & 1237 & 1227 & 1292 & 1442\end{array}$ $\begin{array}{lllllllllll}641 & 1199 & 1176 & 1145 & 1184 & 1194 & 1192 & 1140 & 1154 & 1103 & 1176 \\ 661 & 3262 & 2195 & 1444 & 1200 & 1110 & 1099 & 1069 & 1064 & 1077 & 1075\end{array}$ $\begin{array}{lllllllllll}681 & 1203 & 121.9 & 1269 & 1384 & 1899 & 2553 & 3576 & 4633 & 5001 & 4458\end{array}$ $\begin{array}{rrrrrrrrrrr}701 & 2306 & 1233 & 892 & 809 & 808 & 873 & 796 & 847 & 799 & 823 \\ 721 & 1920 & 2885 & 3898 & 4519 & 4322 & 3290 & 2308 & 1444 & 1192 & 1152\end{array}$ $\begin{array}{rrrrrrrrrrr}721 & 1920 & 2885 & 3898 & 4519 & 4322 & 3290 & 2308 & 1444 & 1192 & 1152 \\ 741 & 748 & 678 & 772 & 763 & 743 & 769 & 739 & 705 & 728 & 733\end{array}$ $\begin{array}{lllllllllll}741 & 748 & 678 & 772 & 763 & 743 & 769 & 739 & 705 & 728 & 733 \\ 761 & 746 & 733 & 679 & 660 & 694 & 602 & 660 & 639 & 646 & 602 \\ 781 & 545 & 631 & 554 & 560 & 594 & 590 & 587 & 575 & 551 & 617\end{array}$

$\begin{array}{lllllllllll}801 & 514 & 527 & 524 & 553 & 561 & 565 & 554 & 506 & 498 & 478\end{array}$

$\begin{array}{lllllllllll}821 & 474 & 485 & 508 & 502 & 475 & 457 & 511 & 451 & 475 & 500\end{array}$

$\begin{array}{llllllllll}861 & 408 & 376 & 407 & 381 & 361 & 382 & 384 & 355 & 379\end{array}$

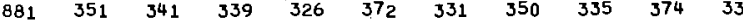

$\begin{array}{lllllllllll}901 & 281 & 339 & 336 & 410 & 370 & 328 & 344 & 383 & 346 & 308\end{array}$

$\begin{array}{llllllllllll}921 & 290 & 303 & 302 & 271 & 305 & 289 & 334 & 372 & 375 & 337 \\ 941 & 293 & 323 & 335 & 328 & 326 & 336 & 346 & 368 & 472 & 664\end{array}$

$\begin{array}{lllllllllll}961 & 285 & 265 & 267 & 283 & 307 & 274 & 243 & 275 & 248 & 253 \\ 981 & 220 & 243 & 251 & 215 & 243 & 262 & 226 & 250 & 270 & 230\end{array}$

$\begin{array}{rllllllllll}981 & 220 & 243 & 251 & 215 & 243 & 262 & 226 & 250 & 270 & 230 \\ 1001 & 256 & 239 & 221 & 237 & 218 & 242 & 252 & 264 & 233 & 270 \\ 1021 & 360 & 482 & 497 & 525 & 492 & 360 & 271 & 257 & 233 & 249\end{array}$

$\begin{array}{lllllllllll}1021 & 360 & 482 & 497 & 525 & 492 & 360 & 271 & 257 & 233 & 249 \\ 1041 & 210 & 215 & 232 & 195 & 215 & 216 & 205 & 192 & 188 & 193\end{array}$

$\begin{array}{lllllllllll}1081 & 187 & 209 & 204 & 201 & 204 & 190 & 172 & 187 & 166 & 125\end{array}$

$\begin{array}{lllllllllll}1101 & 175 & 195 & 193 & 176 & 192 & 195 & 173 & 170 & 188 & 171\end{array}$

$\begin{array}{lllllllllll}1121 & 165 & 163 & 185 & 177 & 151 & 162 & 161 & 172 & 183 & 176 \\ 1141 & 166 & 198 & 157 & 147 & 163 & 166 & 145 & 151 & 155 & 227\end{array}$

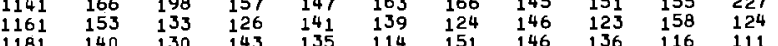

$\begin{array}{lllllllllll}1181 & 140 & 130 & 143 & 135 & 114 & 151 & 146 & 136 & 116 & 111 \\ 1201 & 128 & 140 & 148 & 147 & 174 & 182 & 189 & 198 & 191 & 165\end{array}$

$\begin{array}{rrrrrrrrrrr}1221 & 104 & 131 & 118 & 112 & 100 & 103 & 109 & 107 & 101 & 107 \\ & 105 & 106 & 102 & 93 & 71 & 107 & 95 & 107 & 89 & 110\end{array}$

$\begin{array}{llll}241 & 105 & 106 & 102\end{array}$

$\begin{array}{rrrrrrrrrrr}1281 & 100 & 88 & 93 & 95 & 87 & 95 & 103 & 78 & 78 & 91 \\ & 82 & 90 & 86 & 93 & 99 & 85 & 91 & 85 & 76 & 79\end{array}$

.

型

.

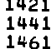

1481

1501

150

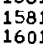

160

1641

1681

1721
1741

1741
1761

$\begin{array}{rrrrrr}1781 & 42 & 36 & 36 & 42 & 3 \\ 1801 & 46 & 29 & 31 & 30 & 3 \\ 1821 & 32 & 25 & 27 & 23 & 2 \\ 1841 & 25 & 22 & 21 & 22 & 25 \\ 1861 & 18 & 21 & 19 & 10 & 16 \\ 1881 & 12 & 14 & 12 & 15 & \\ 1901 & 11 & 11 & 11 & 13 & 1 \\ 1921 & 4 & 10 & 10 & 9 & \\ 1941 & 12 & 7 & 13 & 8 & \\ 1961 & 9 & 12 & 8 & 9 & 2 \\ 1981 & 37 & 16 & 3 & 9 & \\ 2001 & 6 & 6 & 3 & 3 & \\ 2021 & 1 & 4 & 2 & 4 & \\ 2041 & 7 & 2 & 2 & 1 & \end{array}$

COUNT COUNT COUNT COUNT COUNT COUNT COUNT COUNT COUNT COUNT

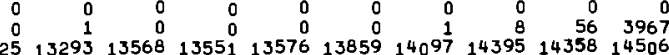
$\begin{array}{lllllllllll}14025 & 13293 & 13568 & 13551 & 13576 & 13859 & 14097 & 14395 & 14358 & 14506 \\ 16287 & 16515 & 16649 & 17144 & 17463 & 17450 & 17741 & 17807 & 17900 & 18387\end{array}$ $\begin{array}{llllllllllllll}25889 & 31091 & 38596 & 43970 & 47150 & 51813 & 55274 & 51021 & 38926 & 28461\end{array}$ $\begin{array}{llllllllll}30207 & 26450 & 23000 & 19499 & 17533 & 16451 & 16303 & 16070 & 16288 & 16449\end{array}$ $\begin{array}{lllllllllll}19422 & 16771 & 16002 & 15824 & 15895 & 15621 & 15711 & 15599 & 15796 & 15667 \\ 15451 & 15317 & 15453 & 15383 & 15391 & 15437 & 15554 & 15436 & 15382 & 15457\end{array}$ $\begin{array}{llllllllllll}15269 & 15207 & 15228 & 15098 & 15066 & 15247 & 15045 & 15471 & 15085 & 15133\end{array}$ $\begin{array}{llllllllll}14891 & 14657 & 14667 & 14534 & 14632 & 14731 & 14639 & 14556 & 14439 & 14499 \\ 14467 & 14490 & 14336 & 14260 & 14261 & 14361 & 14299 & 14504 & 14430 & 14141\end{array}$

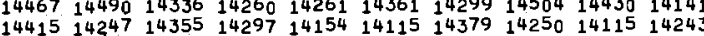
$\begin{array}{lllllllllll}14179 & 14061 & 14053 & 14100 & 14007 & 14091 & 14019 & 14150 & 14094 & 13995\end{array}$ $\begin{array}{llllllllll}14259 & 13927 & 14090 & 14190 & 14339 & 14027 & 14091 & 14059 & 14088 & 14011\end{array}$ $\begin{array}{llllllllll}14268 & 14172 & 14288 & 13977 & 14158 & 14118 & 14159 & 13879 & 14166 & 13874\end{array}$ $\begin{array}{lllllllllll}13389 & 13366 & 13261 & 13048 & 12706 & 12543 & 12239 & 12272 & 11905 & 12163\end{array}$ $\begin{array}{llllllllll}11433 & 11299 & 11320 & 1157 & 11402 & 11527 & 11274 & 11148 & 11142 & 11123\end{array}$

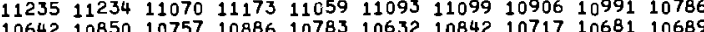
$\begin{array}{llllllllll}10642 & 10850 & 10757 & 10886 & 10783 & 10632 & 10842 & 10717 & 10681 & 10689 \\ 11078 & 11329 & 11688 & 11309 & 10788 & 10235 & 10230 & 10034 & 10157 & 9936\end{array}$

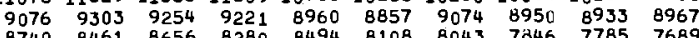
$\begin{array}{llllllllll}8740 & 8461 & 8656 & 8280 & 8494 & 8108 & 8043 & 7046 & 7785 & 7689\end{array}$ $\begin{array}{rlllllllll}7213 & 7243 & 7549 & 7573 & 7618 & 7607 & 7415 & 7302 & 7258 & 7206 \\ 15581 & 9295 & 6950 & 6207 & 6018 & 5982 & 5950 & 6058 & 5950 & 5948\end{array}$ $\begin{array}{rlllllllll}15581 & 9295 & 6950 & 6207 & 6018 & 5982 & 5950 & 6058 & 5950 & 5948 \\ 5672 & 5562 & 5526 & 5578 & 5556 & 5604 & 5412 & 5535 & 5436 & 5485\end{array}$ $\begin{array}{llllllllll}\mathbf{5 4 6 2} & \mathbf{5 3 8 4} & 5626 & 5643 & \mathbf{5 5 0 9} & \mathbf{5 4 3 3} & \mathbf{5 2 6 9} & 5470 & 5663 & 5631\end{array}$

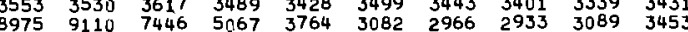
$\begin{array}{llllllllll}3486 & 2543 & 2270 & 2242 & 2147 & 2261 & 2259 & 2397 & 2261 & 2412\end{array}$ $\begin{array}{llllllllll}2013 & 2018 & 1891 & 1995 & 1967 & 2176 & 2274 & 3216 & 4994 & 8636\end{array}$ $\begin{array}{lllllllllll}1439 & 1344 & 1412 & 1351 & 1297 & 1334 & 1290 & 1296 & 1249 & 1275 \\ 1605 & 2065 & 2554 & 3053 & 2945 & 2379 & 1691 & 1391 & 1239 & 1230\end{array}$ $\begin{array}{llllllllll}1605 & 2065 & 2554 & 305 & 2945 & 2379 & 1691 & 1391 & 1239 & 1230 \\ 1162 & 1144 & 1223 & 1241 & 1460 & 1882 & 2618 & 3597 & 4345 & 4171\end{array}$ $\begin{array}{llllllllll}1031 & 1072 & 1137 & 1090 & 1063 & 1121 & 1161 & 1195 & 1142 & 1158\end{array}$ $\begin{array}{rrrrrrrrrr}3479 & 2916 & 3684 & 5892 & 9599 & 13794 & 15723 & 13981 & 9376 & 4855 \\ 821 & 826 & 820 & 840 & 880 & 886 & 907 & 915 & 1014 & 1373\end{array}$ $\begin{array}{llllllllll}1029 & 929 & 816 & 791 & 785 & 766 & 722 & 800 & 796 & 711\end{array}$ $\begin{array}{llllllllll}708 & 755 & 712 & 702 & 714 & 705 & 693 & 691 & 714 & 723\end{array}$ $\begin{array}{llllllllll}678 & 803 & 924 & 1070 & 1024 & 948 & 661 & 585 & 563 & 531\end{array}$

\begin{tabular}{|c|c|c|c|c|c|c|c|c|c|}
\hline 509 & 500 & 489 & 557 & 486 & 506 & 524 & 492 & 517 & 518 \\
\hline & 448 & 493 & 448 & 476 & 451 & 426 & 419 & 437 & 435 \\
\hline 438 & 430 & 425 & 407 & 362 & 377 & 383 & 410 & 383 & 394 \\
\hline 381 & 368 & 364 & 383 & 341 & 385 & 367 & 396 & 357 & 338 \\
\hline 348 & 316 & 326 & 343 & 311 & 322 & 286 & 314 & 337 & 349 \\
\hline 344 & 366 & 373 & 373 & 347 & 307 & 298 & 316 & 336 & 284 \\
\hline 308 & 300 & 287 & 301 & 293 & 301 & 295 & 283 & 301 & $3_{12}$ \\
\hline 840 & 1036 & 1482 & 1521 & 1346 & 948 & 610 & 396 & 274 & 283 \\
\hline 251 & 249 & 267 & 213 & 245 & 260 & 242 & 261 & 257 & 238 \\
\hline 222 & 261 & 231 & 242 & 236 & 232 & 214 & 242 & 231 & 243 \\
\hline 278 & 264 & 240 & 243 & 255 & 255 & 233 & 217 & 279 & 319 \\
\hline 213 & 224 & 202 & 191 & 205 & 215 & 196 & 196 & 222 & 190 \\
\hline 249 & 255 & 317 & $33_{1}$ & 350 & 398 & 389 & 365 & 309 & 302 \\
\hline 197 & 196 & 189 & $+7 \overline{3}$ & 165 & 182 & 171 & 191 & 159 & 205 \\
\hline 194 & 184 & 181 & 181 & 154 & 179 & $18_{1}$ & $17 \overline{7}$ & 158 & 181 \\
\hline 177 & 150 & 165 & 161 & 154 & 171 & 150 & 155 & 160 & 175 \\
\hline 180 & 174 & 169 & 150 & 173 & 153 & 159 & 145 & 154 & 145 \\
\hline 174 & 212 & 217 & 238 & 233 & 234 & 236 & 189 & 178 & 140 \\
\hline 129 & 118 & 151 & 144 & 134 & 113 & 136 & 120 & 126 & 112 \\
\hline 126 & 117 & 132 & 132 & 11.1 & 105 & 121 & 104 & 141 & 111 \\
\hline 146 & 125 & 121 & 119 & 118 & 108 & 115 & 128 & 103 & 119 \\
\hline 98 & 94 & 144 & 132 & 174 & 176 & 151 & 158 & 142 & 141 \\
\hline 98 & 117 & 95 & 101 & 108 & 110 & 96 & 82 & 93 & 104 \\
\hline 94 & 91 & 80 & 96 & 87 & 89 & 78 & 88 & 90 & 100 \\
\hline 83 & 79 & 78 & 93 & 79 & 80 & 72 & 98 & 85 & 88 \\
\hline 68 & 69 & 108 & 69 & 84 & 74 & 75 & 98 & 87 & 67 \\
\hline 99 & 79 & 63 & 85 & 74 & 80 & 88 & 88 & 95 & 84 \\
\hline 178 & 133 & 81 & 95 & 88 & 73 & 86 & 93 & 78 & 84 \\
\hline 69 & 71 & 65 & 68 & 80 & 77 & 60 & 73 & 69 & 73 \\
\hline 79 & 59 & 73 & 78 & 76 & 75 & 66 & 61 & 63 & 68 \\
\hline 107 & 108 & 116 & 127 & 151 & 102 & 109 & 78 & 69 & 73 \\
\hline 81 & 63 & 65 & 65 & 53 & 47 & 58 & 59 & 52 & 53 \\
\hline 70 & 61 & 46 & 67 & 58 & 61 & 62 & 71 & $5 \overline{9}$ & 64 \\
\hline 57 & 44 & 73 & 57 & 54 & 51 & 56 & 76 & 62 & 50 \\
\hline 46 & 64 & 63 & 71 & 47 & 58 & 48 & 56 & 61 & 59 \\
\hline 57 & 69 & 74 & 68 & 76 & 75 & 88 & 58 & 75 & 59 \\
\hline 72 & 53 & 53 & 50 & 61 & 40 & 56 & 65 & 77 & 55 \\
\hline 62 & 53 & 60 & 53 & 57 & 52 & 64 & 64 & 51 & 65 \\
\hline 59 & 61 & 50 & 70 & 38 & 54 & 75 & 53 & 63 & 56 \\
\hline 62 & 60 & 56 & 64 & 62 & 73 & 83 & 59 & 71 & 64 \\
\hline 61 & 61 & 58 & 56 & 61 & 57 & 58 & 68 & 58 & 64 \\
\hline $\begin{array}{l}63 \\
59\end{array}$ & $\begin{array}{l}62 \\
63\end{array}$ & $\begin{array}{l}57 \\
61\end{array}$ & 54 & $\begin{array}{l}58 \\
62\end{array}$ & 59 & 64 & 58 & $\begin{array}{l}66 \\
70\end{array}$ & $\begin{array}{l}51 \\
56\end{array}$ \\
\hline 65 & 51 & 69 & 68 & 72 & $\begin{array}{l}51 \\
59\end{array}$ & $\begin{array}{l}34 \\
81\end{array}$ & $\begin{array}{l}53 \\
79\end{array}$ & 63 & 59 \\
\hline 67 & 68 & 65 & 79 & 47 & 66 & 79 & 70 & 80 & 58 \\
\hline 74 & 74 & 71 & 59 & 59 & 67 & 60 & 68 & 61 & 65 \\
\hline 59 & 76 & 64 & 68 & 59 & 67 & 7 & 82 & 71 & 65 \\
\hline 64 & 83 & 62 & 75 & 83 & 75 & 67 & 81 & 57 & 64 \\
\hline 47 & 38 & 44 & 41 & 37 & 52 & 43 & 29 & 35 & 32 \\
\hline
\end{tabular}

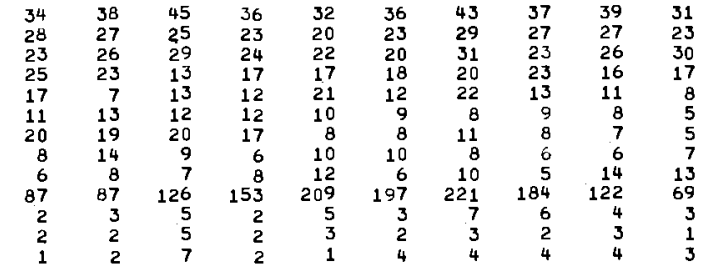


D-30

GAMMA RAY SPECTRUM DATA

PRTR ROD FS-07 SPECTRUM NO. 5 DATA TAKEN 9/09/69 LIVE TIME 915.MIN. POSITION 54.IN. ENERGY(KEV) $=1.108 * C H A N ~-7.1$ CHAN COUNT COUNT COUNT COUNT COUNT COUNT COUNT COUNT COUNT COUNT

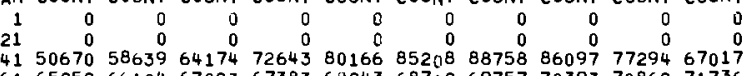
6165252661046702367383682436871269757703937086271736 8184728498544867148871291549421396824100802107180 12171831715847139972066741758002893218110392116440104223 $\begin{array}{llllllllllll}161 & 07618 & 67668 & 67920 & 67302 & 68126 & 69043 & 69151 & 68667 & 68238 & 67099\end{array}$

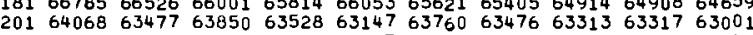
$221 \quad 618526236062112 \quad 61484 \quad 62037 \quad 617216208162016 \quad 6216961844$ $\begin{array}{llllllllllll}241 & 60417 & 61035 & 60712 & 60397 & 60590 & 60519 & 60895 & 60051 & 60171 & 60293 \\ 261 & 60129 & 60103 & 60187 & 60642 & 59951 & 60353 & 59859 & 60115 & 59697 & 59507\end{array}$ 24159768590925960159715604906068560410598056036060502 5815758534 $\begin{array}{lllllllllll}321 & 49911 & 49283 & 49827 & 48868 & 49194 & 48400 & 48480 & 48225 & 48727 & 48275\end{array}$ $\begin{array}{lllllllllllll}361 & 46266 & 46073 & 45937 & 45551 & 45801 & 45571 & 45681 & 45535 & 45426 & 45314\end{array}$ 40141991419694141041170406524028339650394963956039112 4738307382393877239159396263895038540379213761737018 $44131793 \quad 3112030860305123048230203992629902 \quad 2976239595$ $461<9796 \quad 30242 \quad 31632 \quad 3568048601 \quad 76777123292169273181837144730$ $\begin{array}{lllllllllll}481 & 24421 & 23767 & 24081 & 23970 & 23934 & 23925 & 23524 & 23536 & 23367 & 23085 \\ 501 & 22152 & 21939 & 21652 & 21657 & 22079 & 21653 & 21706 & 21360 & 21195 & 21205\end{array}$ $\begin{array}{llllllllllll}521 & 22610 & 21038 & 19132 & 17671 & 16976 & 16141 & 15681 & 15289 & 15354 & 14792 \\ 541 & 13384 & 13495 & 13387 & 13219 & 13673 & 13822 & 14794 & 16810 & 20146 & 25987\end{array}$ $\begin{array}{llllllllllll}561 & 14456 & 16306 & 18640 & 23138 & 33401 & 49591 & 65369 & 69746 & 58217 & 37624\end{array}$ $\begin{array}{rrrrrrrrrrr}581 & 9123 & 8927 & 8419 & 8326 & 8206 & 7978 & 7936 & 8101 & 8160 & 8119\end{array}$

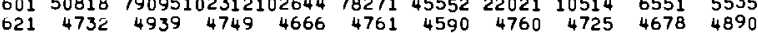
$\begin{array}{lllllllllll}641 & 4408 & 4466 & 4399 & 4244 & 4272 & 4411 & 4275 & 4349 & 4267 & 4388\end{array}$ $\begin{array}{rrrrrrrrrrr}061 & 16092 & 12120 & 8133 & 5705 & 4546 & 4090 & 4208 & 4090 & 4177 & 4209\end{array}$ $\begin{array}{lllllllllll}701 & 18800 & 8515 & 4792 & 3494 & 3344 & 3227 & 3110 & 3079 & 3240 & 3218\end{array}$ $\begin{array}{lllllllllll}721 & 5861 & 8505 & 12176 & 15912 & 17575 & 16160 & 12212 & 8389 & 5875 & 4871\end{array}$

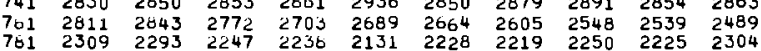

\begin{tabular}{|c|c|c|c|c|c|c|c|c|c|c|}
\hline 801 & 2110 & 2085 & 2107 & 2148 & 2109 & 2215 & 2159 & 2096 & 2108 & 2006 \\
\hline 821 & 1899 & 1965 & 1915 & 1865 & 1931 & 1891 & 1900 & 1824 & 1856 & 1819 \\
\hline 841 & 1679 & 1714 & 1710 & 1641 & 1659 & 1076 & 1622 & 1658 & 1619 & \\
\hline $\begin{array}{l}861 \\
881\end{array}$ & 1609 & 1571 & 1552 & 1567 & 1475 & 1586 & 1458 & 1531 & 1578 & \\
\hline 881 & 1346 & 1361 & 1342 & 1311 & 1365 & 1278 & 1351 & 1337 & 1305 & \\
\hline 901 & 1266 & 1280 & 1343 & 1288 & 1326 & 1370 & 1331 & 1307 & 1266 & \\
\hline 921 & 1177 & 1171 & 1235 & 1209 & 1121 & 1115 & 1176 & 1216 & 1116 & \\
\hline 941 & 1194 & 1234 & 1248 & 1201 & 1269 & 1309 & 1276 & 1429 & 1477 & \\
\hline 961 & 1136 & 1113 & 1081 & 1040 & 1120 & 1060 & 1054 & 1040 & 1081 & \\
\hline 981 & 994 & 992 & 983 & 972 & 934 & 941 & 939 & 960 & 978 & \\
\hline 001 & 998 & 974 & 943 & $9 \dot{b} \hat{c}$ & & 996 & 898 & 953 & 968 & \\
\hline 021 & 1212 & 1388 & 1469 & 1740 & 1752 & 1658 & 1495 & 1328 & 1039 & \\
\hline 041 & 759 & 774 & 793 & 792 & 804 & 775 & 740 & 739 & 740 & \\
\hline 1061 & 1331 & 1202 & 1129 & $126 \%$ & 1362 & 1192 & 1214 & 1124 & 985 & \\
\hline & 749 & 740 & & & 844 & 733 & & 768 & & \\
\hline 01 & 658 & 004 & 647 & 019 & 637 & 633 & & 611 & & \\
\hline 21 & 604 & 609 & 652 & 574 & 654 & 625 & 652 & 632 & 570 & \\
\hline 141 & 589 & 585 & 589 & 555 & 587 & 610 & & 565 & 60 & \\
\hline & 595 & 540 & 521 & 499 & 493 & 48 & & & & \\
\hline 18 & 478 & 493 & 537 & 480 & 477 & 511 & 487 & 468 & 489 & \\
\hline 20 & 500 & 494 & 531 & 555 & 603 & 729 & 797 & 822 & 901 & \\
\hline 22 & 407 & 416 & 427 & 439 & 402 & 393 & 391 & 366 & & \\
\hline 124 & 511 & 476 & $4 b 0$ & 386 & 380 & 33 & & 34 & & \\
\hline & 36 & 321 & 357 & & & 32 & & 36 & & \\
\hline & 340 & 311 & 296 & 289 & 337 & 307 & 3 & 311 & & \\
\hline & 290 & 284 & 312 & 285 & 309 & 279 & & 304 & 322 & \\
\hline & 315 & 258 & & & 295 & 317 & & & & \\
\hline 1 & 307 & 337 & 3 & 3 & 471 & 490 & & 65 & & \\
\hline & 301 & 302 & 277 & & 284 & 26 & & 26 & & \\
\hline 13 & 250 & 231 & 219 & 236 & 292 & 252 & 259 & 252 & 261 & \\
\hline 14 & 242 & & & & 2 & 247 & & & & \\
\hline & 334 & & & & & 23 & & & & \\
\hline & 218 & & & & & & & 25 & & \\
\hline & 201 & $2 b 1$ & & & 19 & 24 & & 22 & & \\
\hline & 210 & 21 & 21 & 2 & 19 & 24 & & 22 & & \\
\hline 15 & 197 & 231 & & & & 218 & & & & \\
\hline & & & & & & & & & & \\
\hline & 222 & 231 & 210 & 2 & 2 & 23 & & 222 & & \\
\hline & 210 & 201 & & & 212 & $24 \mathrm{c}$ & & 18 & & \\
\hline & 21 & 2 & & & & 23 & & 23 & & \\
\hline 16 & $2 t$ & 2 & & & 2 & 21 & & 203 & & \\
\hline 162 & 213 & & 233 & 240 & 260 & 249 & 284 & 241 & 269 & \\
\hline & 235 & 20 & 212 & & 237 & 210 & & 210 & 2 & \\
\hline & 22 & 24 & 21 & & & 22 & & 24 & & \\
\hline & & 21 & & & & 20 & & & & \\
\hline & & 244 & 233 & 200 & 240 & 242 & & & & \\
\hline & 224 & 250 & 254 & 254 & 263 & $\begin{array}{l}242 \\
235\end{array}$ & 28 & 253 & 252 & \\
\hline & 26 & 245 & 246 & 250 & 251 & 251 & 27 & 270 & 259 & \\
\hline & 23 & & & & & & 215 & 220 & 203 & \\
\hline
\end{tabular}

COUNT COUNT COUNT COUNT COUNT COUNT COUNT COUNT COUNT COUNT $\begin{array}{rrrrrrrrrr}0 & 0 & 0 & 0 & 0 & 0 & 0 & 0 & 0 & 0 \\ 61127 & 59728 & 59624 & 00595 & 61461 & 61964 & 62215 & 8262 & 3445 & 24737 \\ 72364 & 73248 & 755589 & 76468 & 78286 & 79001 & 79736 & 80109 & 63728 & 6475\end{array}$

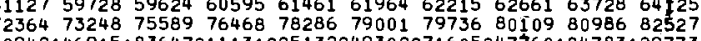
$142451124313105020 \quad 87382774397250371845716307160370854$ 85915750267048269326692596939768852691496894968348

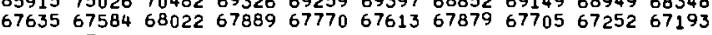
$67416 \quad 67009663546678166965669606620966513 \quad 6709966484$ $\begin{array}{llllllllll}65399 & 64761 & 64662 & 64718 & 64803 & 64103 & 64123 & 63956 & 63987 & 63988\end{array}$ $\begin{array}{lllllllllll}61750 & 61319 & 61427 & 61316 & 61080 & 61413 & 61507 & 60981 & 60836 & 60969\end{array}$ 60311602096010959882596685971760413595976031359711 $\begin{array}{lllllllllll}60064 & 59913 & 60298 & 59878 & 59925 & 59863 & 59774 & 59972 & 60280 & 59669\end{array}$

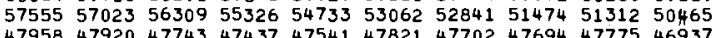
$\begin{array}{llllllllll}46981 & 46769 & 46701 & 46815 & 46594 & 46454 & 45988 & 46272 & 45698 & 46094\end{array}$ $\begin{array}{llllllllll}45234 & 45049 & 45305 & 45229 & 45324 & 45181 & 45279 & 44996 & 45078 & 44614\end{array}$ $\begin{array}{lllllllllll}46022 & 47360 & 48494 & 47776 & 45749 & 44235 & 43489 & 42840 & 42639 & 42960\end{array}$ $3742036616 \quad 36954 \quad 36007 \quad 3596435262 \quad 34532339543286732396$ $\begin{array}{lllllllllll}37420 & 36616 & 36954 & 36007 & 35964 & 35262 & 34532 & 33954 & 32867 & 32396 \\ 30065 & 30192 & 30667 & 31377 & 32255 & 31884 & 31218 & 30425 & 29787 & 29823\end{array}$ $897045008231975 \quad 2657425250 \quad 24444 \quad 24671 \quad 24239 \quad 2440424295$ $\begin{array}{llllllllll}23157 & 22858 & 22642 & 22700 & 22501 & 22463 & 22413 & 22182 & 22467 & 22103\end{array}$ $\begin{array}{lllllllllll}14603 & 14582 & 14339 & 13987 & 14033 & 13836 & 13805 & 13532 & 13676 & 13438\end{array}$ $\begin{array}{rrrrrrrrrr}32515 & 36862 & 34168 & 26561 & 18704 & 13897 & 12107 & 11695 & 11957 & 13125 \\ 21246 & 12820 & 9654 & 9029 & 8735 & 8613 & 8842 & 9065 & 9201 & 9404\end{array}$ $\begin{array}{rrrrrrrrrr}7934 & 7940 & 8072 & 8009 & 8276 & 8519 & 9465 & 11358 & 16916 & 29180\end{array}$ $\begin{array}{rrrrrrrrrr}5389 & 5370 & 5460 & 5232 & 4922 & 5065 & 4891 & 5025 & 4878 & 4944 \\ 5602 & 6777 & 8568 & 10550 & 11588 & 10856 & 8611 & 6376 & 4965 & 4514\end{array}$ $\begin{array}{rrrrrrrrrr}5602 & 6777 & 8568 & 10550 & 11588 & 10856 & 8611 & 6376 & 4965 & 4514 \\ 4387 & 4468 & 4535 & 4635 & 5053 & 6285 & 8344 & 11810 & 15502 & 17430\end{array}$ $\begin{array}{llllllllll}4029 & 4153 & 4034 & 4176 & 4046 & 4172 & 4225 & 4316 & 4319 & 4305\end{array}$ $\begin{array}{lllllllllll}3061 & 3106 & 3225 & 3258 & 3196 & 3250 & 3251 & 3526 & 3789 & 4505\end{array}$ $\begin{array}{llllllllll}3061 & 3106 & 3225 & 3258 & 3196 & 3250 & 3251 & 3526 & 3789 & 4505 \\ 4406 & 4145 & 3641 & 3168 & 3025 & 2848 & 2810 & 2855 & 2856 & 2854 \\ 2773 & 2745 & 2752 & 2709 & 2774 & 2821 & 2878 & 2877 & 2693 & 2854\end{array}$ $\begin{array}{llllllllll}2439 & 2458 & 2396 & 2298 & 2331 & 2324 & 2306 & 2256 & 2263 & 2294 \\ 2415 & 2762 & 3113 & 3652 & 3889 & 3586 & 3386 & 2834 & 2425 & 2194\end{array}$

\begin{tabular}{|c|c|c|c|c|c|c|c|c|c|}
\hline 1987 & 058 & 1981 & 2002 & 1977 & 1967 & 2027 & 1948 & 1988 & 1886 \\
\hline & 1858 & 1852 & 1780 & 1732 & & 1786 & 1748 & 1848 & 1687 \\
\hline 68 & 1629 & 1756 & 1662 & 1668 & 1670 & 1585 & 1536 & 1604 & 1529 \\
\hline $\begin{array}{l}47 \\
31\end{array}$ & $\begin{array}{l}1477 \\
1353\end{array}$ & $\begin{array}{l}1496 \\
1325\end{array}$ & $\begin{array}{r}1412 \\
1319\end{array}$ & $\begin{array}{l}1433 \\
1291\end{array}$ & $\begin{array}{l}1418 \\
1338\end{array}$ & $\begin{array}{l}1458 \\
1260\end{array}$ & $\begin{array}{l}1428 \\
1277\end{array}$ & $\begin{array}{l}1429 \\
1243\end{array}$ & $\begin{array}{l}1475 \\
1238\end{array}$ \\
\hline 30 & $\begin{array}{l}1359 \\
\end{array}$ & 1329 & 1368 & 1301 & 1359 & 1283 & 1214 & 1243 & 1152 \\
\hline 17 & 166 & 151 & 173 & 139 & 143 & 1158 & 1209 & 1142 & 1144 \\
\hline 45 & 3264 & 24 & 1909 & 5113 & 4739 & 4005 & 3028 & 2009 & 1473 \\
\hline & 971 & 009 & 986 & 979 & & & & & \\
\hline & & 969 & 100 & 100 & & & & 946 & $\$ 71$ \\
\hline 00 & & 94 & & & & 954 & 967 & 954 & 1103 \\
\hline 79 & 86 & 809 & 836 & 783 & 838 & 814 & 818 & 753 & 753 \\
\hline 827 & 872 & 979 & 1058 & 1180 & 1359 & 1335 & 1425 & 1363 & 1286 \\
\hline 812 & 768 & 711 & & 733 & 671 & 689 & 667 & 697 & 675 \\
\hline 65 & 67 & 649 & & 654 & & & & 650 & \\
\hline $\begin{array}{l}653 \\
599\end{array}$ & $\begin{array}{l}68 \\
60\end{array}$ & $\begin{array}{l}625 \\
637\end{array}$ & $\begin{array}{l}59 \\
60\end{array}$ & $\begin{array}{l}654 \\
604\end{array}$ & $\begin{array}{l}59 \\
57\end{array}$ & 60 & 62 & & 628 \\
\hline 63 & 657 & 632 & 650 & 724 & 731 & 700 & 68 & $\begin{array}{l}584 \\
588\end{array}$ & 614 \\
\hline & & & & 50 & & & & & 495 \\
\hline 47 & 508 & 491 & 501 & 48 & 45 & 52 & 44 & 448 & 481 \\
\hline 822 & 71 & 614 & 543 & 49 & $42:$ & 45 & 50 & 405 & 397 \\
\hline & 39 & & 43 & 52 & 55 & & & 50 & $\$ 02$ \\
\hline & & & & & & & & & \\
\hline & & & & & & 32 & & & 352 \\
\hline & & & & & & & & & 105 \\
\hline 28 & & & & & & & & & \\
\hline 27 & & & & & & & & & 288 \\
\hline 62 & & & & 42 & 40 & & & & \\
\hline 28 & & & & 22 & 24 & 27 & & 25 & 289 \\
\hline 26 & & 23 & 2 & 24 & & 29 & & 26 & 269 \\
\hline & & & & & & & & & \\
\hline 25 & & & & & & & & & \\
\hline & & & 22 & 20 & & & & & 226 \\
\hline 2 & 25 & 21 & & 2 & & & & & 23 \\
\hline 2 & & & & & & & & & 202 \\
\hline & & & & & & & & & 257 \\
\hline & & & & & & & & & 222 \\
\hline & & 2. & 21 & 23 & 24 & & & 2 & 18 \\
\hline & & 21 & 2 & & & & & & 192 \\
\hline & & & & & & & & & 23 \\
\hline 22 & & & & & & & & & 219 \\
\hline 23 & 23 & & 220 & 23 & 23 & 23 & 24 & 22 & 22 \\
\hline & & & & 24 & 21 & & & 2 & 23 \\
\hline 23 & & & & & & & & & \\
\hline 26 & 21 & & & & 24 & & & 26 & 225 \\
\hline 25 & 24 & & & & & & & 22 & 23 \\
\hline & & 26 & $2^{4}$ & 25 & 26 & 23 & 261 & 259 & 26 \\
\hline 25 & 25 & 265 & 256 & 267 & 255 & 25 & 2 & 241 & 24 \\
\hline & & & & & & & 143 & 150 & 15 \\
\hline
\end{tabular}

$\begin{array}{rrrrrrrrrrr}1701 & 120 & 146 & 134 & 137 & 130 & 149 & 125 & 133 & 114 & 120 \\ 1801 & 131 & 133 & 133 & 123 & 116 & 122 & 113 & 125 & 112 & 108 \\ 1821 & 104 & 117 & 128 & 103 & 118 & 99 & 129 & 102 & 107 & 97 \\ 1841 & 76 & 100 & 90 & 03 & 92 & 68 & 82 & 84 & 89 & 77 \\ 1861 & 67 & 67 & 60 & 00 & 50 & 59 & 59 & 78 & 42 & 63 \\ 1881 & 52 & 50 & 42 & 40 & 41 & 34 & 44 & 44 & 44 & 38 \\ 1901 & 44 & 41 & 41 & 44 & 48 & 40 & 41 & 50 & 69 & 69 \\ 1921 & 32 & 32 & 37 & 37 & 30 & 32 & 37 & 42 & 28 & 34 \\ 1941 & 36 & 35 & 27 & 30 & 42 & 35 & 37 & 29 & 36 & 30 \\ 1961 & 39 & 40 & 47 & 49 & 63 & 66 & 72 & 100 & 137 & 156 \\ 1981 & 416 & 411 & 354 & 277 & 226 & 141 & 97 & 50 & 32 & 19 \\ 2001 & 16 & 16 & 12 & 10 & 11 & 21 & 13 & 14 & 14 & 11 \\ 2021 & 10 & 15 & 14 & 10 & 13 & 10 & 10 & 9 & 16 & 14 \\ 2041 & 10 & 8 & 13 & 10 & 13 & 4 & 8 & 0 & & \end{array}$

$\begin{array}{rrrrrrrrrr}104 & 123 & 125 & 116 & 122 & 130 & 138 & 130 & 103 & 196 \\ 104 & 121 & 121 & 102 & 115 & 102 & 126 & 114 & 112 & 95 \\ 98 & 100 & 99 & 111 & 93 & 81 & 89 & 85 & 88 & 77 \\ 70 & 84 & 84 & 72 & 69 & 72 & 64 & 82 & 58 & 84 \\ 57 & 51 & 48 & 55 & 59 & 53 & 52 & 52 & 53 & 48 \\ 48 & 45 & 30 & 41 & 40 & 43 & 39 & 43 & 37 & 45 \\ 56 & 51 & 58 & 47 & 54 & 64 & 53 & 50 & 38 & 38 \\ 35 & 25 & 43 & 27 & 42 & 34 & 29 & 37 & 28 & 40 \\ 29 & 31 & 33 & 32 & 39 & 35 & 28 & 34 & 36 & 35 \\ 190 & 224 & 287 & 371 & 448 & 465 & 524 & 510 & 450 & 389 \\ 15 & 17 & 21 & 15 & 15 & 17 & 16 & 19 & 16 & 6 \\ 9 & 15 & 13 & 8 & 14 & 11 & 13 & 10 & 14 & 14 \\ 12 & 8 & 10 & 8 & 18 & 16 & 12 & 14 & 11 & 6\end{array}$


GAMMA RAY SPECTRUM DATA

\begin{abstract}
PRTR ROD FO-17 SPECTRUM INO, 17 DATA TAKEN 9/18/69 LIVE TIME 480.MIN. POSITION 30.IN. ENERGY(KEV)= 1.111\%CHAN -8.8 CHAN COUNT COUNT COUNT COUNT COUNT COUNT COUNT COUNT COUNT COUNT

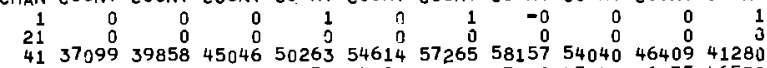

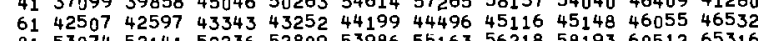

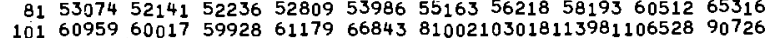
$\begin{array}{lllllllllll}101 & 60959 & 60017 & 59928 & 61179 & 66843 & 81002103018113981106528 & 90726 \\ 121 & 46691 & 46304 & 46342 & 46559 & 49107 & 55306 & 66290 & 74408 & 69604 & 57936\end{array}$ $\begin{array}{lllllllllll}121 & 46691 & 46304 & 46342 & 46559 & 49107 & 55306 & 66290 & 74408 & 69604 & 57936 \\ 141 & 44400 & 44893 & 45077 & 44816 & 44941 & 44896 & 44810 & 44914 & 44729 & 44565\end{array}$ $\begin{array}{lllllllllll}141 & 44400 & 44893 & 45077 & 44816 & 44941 & 44896 & 44810 & 44914 & 44729 & 44565 \\ 161 & 44530 & 44569 & 44650 & 44729 & 44533 & 45241 & 44724 & 44545 & 44652 & 44298 \\ 181 & 43981 & 43391 & 43571 & 43317 & 43348 & 43196 & 42701 & 42740 & 43200 & 42899\end{array}$ $\begin{array}{lllllllllllll}181 & 42189 & 42233 & 41998 & 41759 & 42193 & 41810 & 41969 & 42060 & 42232 & 41491\end{array}$ $\begin{array}{llllllllllll}201 & 42189 & 42233 & 41998 & 4179 & 42193 & 41810 & 41969 & 42060 & 42232 & 41491\end{array}$ $\begin{array}{llllllllllll}221 & 41435 & 41687 & 41486 & 41493 & 41840 & 41688 & 41588 & 41628 & 41961 & 41889 \\ 241 & 41409 & 41268 & 40925 & 40708 & 41518 & 41083 & 41054 & 40785 & 40877 & 40758\end{array}$ $\begin{array}{lllllllllll}261 & 40846 & 40938 & 40891 & 40709 & 41174 & 40921 & 40845 & 40882 & 41181 & 40920\end{array}$ $\begin{array}{llllllllllll}281 & 41601 & 41144 & 41590 & 41394 & 41356 & 41321 & 41210 & 41407 & 41328 & 41516 \\ 301 & 40992 & 40986 & 41106 & 46679 & 40925 & 40698 & 41020 & 40431 & 40320 & 3973\end{array}$ $\begin{array}{lllllllllll}321 & 34410 & 34615 & 34198 & 33638 & 33693 & 33527 & 33377 & 32940 & 33070 & 33341\end{array}$ $\begin{array}{lllllllllll}341 & 32254 & 32458 & 32242 & 32236 & 32334 & 32151 & 32266 & 31941 & 32025 & 32363\end{array}$ $\begin{array}{lllllllllllll}361 & 31621 & 31353 & 31161 & 31343 & 31517 & 31381 & 31122 & 31044 & 31274 & 30913\end{array}$

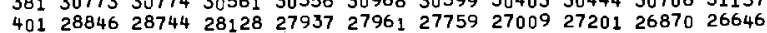
$\begin{array}{lllllllllll}421 & 26979 & 25988 & 26408 & 26559 & 26828 & 26564 & 25837 & 25842 & 25478 & 2511\end{array}$

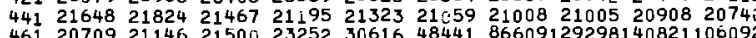
$48116977170337256 \quad 16845 \quad 16742 \quad 1696317051167911662716444$ $\begin{array}{llllllllllll}481 & 16977 & 17083 & 17256 & 16845 & 16742 & 16963 & 17051 & 1679 & 16627 & 16444\end{array}$ $\begin{array}{llllllllllll}521 & 16091 & 14847 & 13803 & 12828 & 12422 & 11844 & 11616 & 11079 & 10926 & 1098\end{array}$ $\begin{array}{lllllllllll}541 & 9823 & 9880 & 9669 & 9486 & 9698 & 9730 & 10659 & 11547 & 13047 & 16048\end{array}$ $\begin{array}{llllllllllll}561 & 10383 & 12096 & 12947 & 14287 & 20079 & 33090 & 50574 & 58492 & 48783 & 29598\end{array}$ $\begin{array}{lllllllllll}581 & 7116 & 6624 & 6381 & 6076 & 6002 & 5979 & 5830 & 5851 & 5853 & 5851 \\ 6 & 7169 & 77287 & 67831 & 70120 & 51061 & 26442 & 11976 & 6000 & 4681 & 4297\end{array}$ $\begin{array}{lllllllllll}601 & 26569 & 47287 & 67831 & 70120 & 51061 & 26442 & 11976 & 6000 & 4681 & 4297\end{array}$ $\begin{array}{lllllllllll}621 & 3779 & 3792 & 3727 & 3782 & 3739 & 3633 & 3567 & 3559 & 3630 & 3848 \\ 641 & 3334 & 3319 & 3393 & 3469 & 3339 & 3355 & 3356 & 3269 & 3362 & 3405\end{array}$ $\begin{array}{lllllllllll}661 & 11524 & 7651 & 4843 & 3683 & 3245 & 3277 & 3108 & 3192 & 3196 & 3148\end{array}$ $\begin{array}{rrrrrrrrrrr}681 & 3407 & 3455 & 3523 & 3950 & 4683 & 6345 & 9571 & 13588 & 16180 & 14118 \\ 701 & 6675 & 3521 & 2617 & 2568 & 2492 & 2540 & 2531 & 2545 & 2558 & 2509\end{array}$ $\begin{array}{lllllllllll}721 & 4768 & 7315 & 10932 & 13053 & 12357 & 8798 & 5538 & 4022 & 3558 & 353\end{array}$ $\begin{array}{llllllllllll}741 & 2391 & 2311 & 2387 & 2316 & 2271 & 2320 & 2250 & 2221 & 2218 & 2314 \\ 761 & 2234 & 2182 & 2104 & 2118 & 2129 & 2062 & 2014 & 2007 & 1916 & 1977\end{array}$ $\begin{array}{lllllllllll}781 & 1735 & 1762 & 1770 & 1799 & 1765 & 1751 & 1733 & 1724 & 1760 & 1924\end{array}$ COUNT COUNT COUNT COUNT COUNT COUNT COUNT COUNT COUNT COUNT $\begin{array}{rrrrrrrrrr}0 & 1 & 5 & 0 & 0 & 0 & 0 & 0 & 0 & 0 \\ 0 & j & 0 & 0 & 0 & 1 & 18 & 129 & 7207 & 28869 \\ 38919 & 38933 & 39271 & 39726 & 40137 & 39995 & 40661 & 41401 & 41385 & 41775\end{array}$ $\begin{array}{lllllllllll}46722 & 47301 & 47963 & 48823 & 50142 & 49854 & 50549 & 50237 & 51432 & 52322\end{array}$ 8726016165855036471524633446063461854622346043 $494584661245406452624507045578 \quad 45209449424509345002$ $\begin{array}{lllllllllll}49458 & 46612 & 45406 & 45262 & 45070 & 45578 & 45209 & 44942 & 45093 & 45002 \\ 44489 & 44492 & 45040 & 44342 & 44169 & 44336 & 44388 & 44157 & 44244 & 44491\end{array}$ $\begin{array}{llllllllll}44489 & 44492 & 45040 & 44342 & 44169 & 44336 & 44388 & 44157 & 44244 & 44491 \\ 43644 & 44113 & 44355 & 44182 & 44049 & 43967 & 43794 & 43962 & 43806 & 4374\end{array}$ $\begin{array}{llllllllll}43644 & 44113 & 44355 & 44182 & 44049 & 43967 & 43794 & 43962 & 43806 & 43741 \\ 42984 & 42334 & 42625 & 42497 & 42877 & 42436 & 42634 & 42479 & 42252 & 42282\end{array}$ $\begin{array}{llllllllll}41811 & 41648 & 41832 & 41847 & 41824 & 41591 & 41674 & 41458 & 41750 & 41622\end{array}$ $\begin{array}{llllllllll}41452 & 41568 & 41248 & 40893 & 40769 & 41078 & 41325 & 41219 & 41353 & 41076\end{array}$ $\begin{array}{lllllllllll}40808 & 40763 & 40444 & 40800 & 40861 & 40685 & 40816 & 41025 & 41158 & 40989 \\ 41163 & 41067 & 41227 & 41060 & 41378 & 41149 & 40839 & 41129 & 41415 & 41287\end{array}$ $\begin{array}{lllllllllll}41200 & 41488 & 41545 & 41453 & 41510 & 41048 & 41731 & 41349 & 41228 & 41378\end{array}$ $\begin{array}{lllllllllll}39638 & 38811 & 38408 & 37740 & 37178 & 36113 & 35908 & 35412 & 34649 & 34572 \\ 32954 & 32638 & 32785 & 33000 & 32773 & 32963 & 32506 & 32465 & 32346 & 32367\end{array}$ $\begin{array}{lllllllllllll}32052 & 32186 & 31719 & 31976 & 31928 & 31637 & 31741 & 31677 & 31668 & 31467\end{array}$ $\begin{array}{llllllllll}30855 & 30918 & 31152 & 30879 & 30689 & 30734 & 30976 & 30425 & 31173 & 30660 \\ 31769 & 33633 & 33851 & 32643 & 31325 & 30007 & 29516 & 29341 & 29019 & 29158\end{array}$

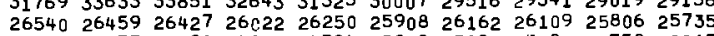
$\begin{array}{llllllllll}25303 & 24877 & 24696 & 24834 & 24584 & 23908 & 23680 & 23180 & 22758 & 22267\end{array}$ $\begin{array}{lllllllllll}20912 & 20808 & 21056 & 21969 & 22391 & 22473 & 21838 & 20924 & 20703 & 20658 \\ 59383 & 30598 & 20764 & 18170 & 17541 & 17232 & 17231 & 17072 & 17116 & 17348\end{array}$ $\begin{array}{llllllllll}59383 & 30598 & 20764 & 18170 & 17541 & 17232 & 17231 & 17072 & 17116 & 17348 \\ 16465 & 16214 & 16086 & 16023 & 16221 & 15780 & 15996 & 15771 & 15750 & 15830\end{array}$ $\begin{array}{llllllllll}16465 & 16214 & 16086 & 16023 & 16221 & 15780 & 15963 & 1571 & 15700 & 15830 \\ 15109 & 15238 & 15395 & 16060 & 16172 & 15853 & 15352 & 15013 & 15605 & 16433\end{array}$ $\begin{array}{llllllllll}10724 & 10606 & 10376 & 10228 & 9937 & 10077 & 10077 & 10108 & 9732 & 9817\end{array}$ $\begin{array}{rrrrrrrrrr}20657 & 25188 & 24284 & 18728 & 13025 & 9804 & 8787 & 8496 & 8541 & 9131 \\ 15190 & 8804 & 7115 & 6560 & 6561 & 6495 & 6431 & 6659 & 6856 & 7253\end{array}$ $\begin{array}{rrrllllllll}5923 & 5765 & 5739 & 5940 & 6561 & 6495 & 6431 & 6659 & 6856 & 7253 \\ & 5816 & 5979 & 6108 & 6943 & 8689 & 13937\end{array}$ \begin{tabular}{lllllllllll}
4292 & 4146 & 4085 & 4173 & 3996 & 4979 & 6108 & 6943 & 8689 & 13937 \\
\hline
\end{tabular} $\begin{array}{rlllllllll}4258 & 5098 & 6855 & 8996 & 9915 & 8684 & 6313 & 4579 & 3749 & 3502 \\ 3400 & 3348 & 3538 & 3489 & 3803 & 4437 & 6344 & 9617 & 13022 & 13926\end{array}$ $\begin{array}{lllllllll}3201 & 3146 & 3187 & 3192 & 3250 & 3159 & 3310 & 3295\end{array}$ $\begin{array}{lllllllllll}10293 & 7628 & 7911 & 13282 & 24769 & 40881 & 50798 & 46035 & 30378 & 14820\end{array}$ $\begin{array}{llllllllll}2496 & 2512 & 2459 & 2536 & 2539 & 2608 & 2530 & 2732 & 2878 & 3340 \\ 3158 & 2741 & 2501 & 2312 & 2085 & 2331 & 2265 & 2183 & 2226 & 2355\end{array}$ $\begin{array}{llllllllll}2371 & 2183 & 2186 & 2264 & 2248 & 2276 & 2189 & 2284 & 2243 & 2306 \\ 1842 & 1860 & 1819 & 1840 & 1785 & 1834 & 1854 & 1798 & 1784 & 1777\end{array}$ $\begin{array}{llllllllll}2192 & 2694 & 3120 & 3489 & 3166 & 2595 & 2098 & 1756 & 1693 & 1694\end{array}$
\end{abstract}

\begin{tabular}{|c|c|c|c|c|c|c|c|c|c|c|}
\hline 801 & 1666 & 1701 & 1823 & 1744 & 1865 & 1692 & 1712 & 1633 & 1610 & 1608 \\
\hline & 1514 & 1596 & 1543 & $\begin{array}{l}1553 \\
1297\end{array}$ & 1561 & $\begin{array}{l}1454 \\
1276\end{array}$ & 1525 & $\begin{array}{l}1425 \\
1424\end{array}$ & $\begin{array}{l}1473 \\
1371\end{array}$ & $\begin{array}{l}1463 \\
1371\end{array}$ \\
\hline 861 & 1212 & 1206 & 1178 & 1205 & $\begin{array}{l}1<21 \\
1152\end{array}$ & 1199 & $\begin{array}{l}1228 \\
\end{array}$ & $\begin{array}{l}1202 \\
\end{array}$ & 1110 & 1123 \\
\hline 881 & 1076 & 1076 & 1089 & 1087 & 1062 & 1049 & 1107 & 1013 & 1111 & \\
\hline 901 & 1007 & 1034 & $\begin{array}{l}1044 \\
\end{array}$ & 1119 & 1090 & 1098 & 1000 & 1033 & 1040 & \\
\hline 921 & 915 & 917 & 936 & 957 & & 962 & 952 & 952 & 880 & \\
\hline 941 & 1003 & 1015 & 970 & 981 & 980 & 961 & 1050 & 1222 & 1563 & \\
\hline 961 & 817 & 864 & 862 & $85 \overline{3}$ & 837 & 818 & 823 & 771 & 759 & \\
\hline 981 & 763 & 762 & 784 & 718 & 766 & 781 & 809 & 782 & 804 & \\
\hline 0 & 704 & 700 & 717 & 724 & 768 & 738 & 730 & 702 & 755 & \\
\hline 021 & 1274 & 1572 & 1701 & 1467 & 1286 & 916 & 731 & 647 & 656 & \\
\hline 41 & 651 & 592 & 659 & 627 & 596 & 569 & 562 & 617 & 620 & \\
\hline 061 & 872 & 876 & 970 & 986 & 867 & 755 & 712 & 616 & 608 & \\
\hline & 640 & 664 & 682 & 048 & 618 & 558 & $57 \overline{5}$ & 580 & 524 & \\
\hline & 518 & 500 & 531 & 53 & 521 & 513 & 540 & 516 & 521 & \\
\hline 121 & 517 & 505 & $47 \overline{8}$ & 510 & 500 & 491 & 515 & 471 & 513 & \\
\hline 141 & 484 & 508 & 462 & 479 & 459 & 489 & 462 & 444 & 480 & \\
\hline & 437 & 434 & 429 & 404 & 444 & 405 & 409 & 392 & 428 & \\
\hline 18 & 392 & 373 & 364 & 399 & 41 & 364 & 39 & 38 & 382 & \\
\hline 120 & 399 & 456 & 484 & 546 & 580 & 658 & 676 & 671 & $58 \overline{5}$ & \\
\hline & 338 & 378 & 360 & 327 & 340 & 319 & 331 & 311 & 358 & \\
\hline & 294 & 337 & 309 & 312 & 274 & 291 & 276 & 280 & 283 & \\
\hline 126 & 271 & 286 & 284 & 266 & 285 & $27 \overline{8}$ & 264 & 283 & 273 & \\
\hline 8 & 265 & 233 & 275 & 255 & 256 & 243 & 253 & 254 & 254 & \\
\hline 0 & 238 & 232 & 225 & 246 & 255 & 248 & 269 & 236 & 243 & \\
\hline & 219 & 267 & 22 & 21 & 237 & 241 & 255 & 249 & 203 & \\
\hline & 25 & 292 & 29 & 40 & 454 & 571 & 699 & 724 & 676 & \\
\hline & 236 & 241 & 247 & 209 & 254 & 233 & 237 & 208 & 214 & \\
\hline 1381 & 213 & 229 & 235 & 203 & 186 & 199 & 195 & 200 & 216 & \\
\hline 1401 & 221 & 201 & 178 & 195 & 204 & 221 & 238 & 238 & 244 & \\
\hline 142 & 181 & 20 & 187 & & & 212 & 200 & 233 & 207 & \\
\hline 1441 & 175 & 193 & 214 & 215 & 21 & 213 & 206 & 200 & 208 & \\
\hline 1461 & 190 & 177 & 177 & 173 & 17 & 166 & 205 & 195 & 214 & \\
\hline 1481 & 164 & 190 & 190 & & 15 & 199 & 207 & 192 & 167 & \\
\hline 1501 & 185 & 202 & 180 & 189 & 203 & 176 & 192 & 192 & 219 & \\
\hline 1521 & 188 & 195 & 195 & 181 & 20 & 188 & 194 & 18 & 182 & \\
\hline 15 & 203 & 174 & 189 & 16 & & 187 & & & 176 & \\
\hline 156 & 187 & 175 & 188 & 183 & & 166 & 169 & 177 & 197 & \\
\hline & 190 & 182 & 162 & 199 & 176 & 188 & 192 & 180 & 175 & \\
\hline 160 & 19 & 199 & & 18 & ie & 18 & 206 & 184 & 181 & \\
\hline 1621 & 190 & 164 & 198 & 210 & 19 & 253 & 182 & 193 & 191 & \\
\hline 164 & 179 & 194 & 185 & 17 & & 18 & 21 & 181 & 217 & \\
\hline & 214 & 179 & 194 & 184 & 17 & 202 & 215 & 201 & 180 & \\
\hline & 209 & 199 & 21 & 17 & 18 & 174 & 194 & 235 & 193 & \\
\hline 1701 & 224 & 209 & 214 & 20 & 187 & 198 & 195 & 209 & 190 & \\
\hline 172 & 213 & 199 & 206 & 236 & 20 & 212 & 22 & 226 & 198 & \\
\hline & 204 & 270 & 227 & 230 & 247 & 206 & 212 & 231 & 180 & \\
\hline & 198 & 188 & 189 & 186 & 193 & 170 & 173 & $16 \overline{3}$ & 154 & \\
\hline
\end{tabular}

\begin{tabular}{|c|c|c|c|c|c|c|c|c|c|}
\hline 1559 & 588 & 1587 & 1493 & 1540 & 1580 & 1615 & 1481 & 1536 & 1494 \\
\hline 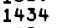 & 462 & 1454 & 1442 & 1359 & 1359 & 1439 & 1395 & 1379 & 1333 \\
\hline 280 & 332 & 344 & 327 & 1205 & 1194 & 1226 & 1270 & 1215 & 1189 \\
\hline 13 & 182 & $\begin{array}{l}1074 \\
\end{array}$ & 1107 & $\begin{array}{l}1060 \\
\end{array}$ & 1095 & 1089 & 1156 & 1058 & 1052 \\
\hline 1097 & 1067 & 1022 & 1031 & 1035 & 1012 & 1016 & 076 & & 996 \\
\hline 139 & 195 & 1192 & 1047 & 1003 & & 948 & & & 937 \\
\hline 934 & 955 & 939 & 927 & & & 895 & & & 959 \\
\hline 3253 & 4556 & 5199 & 4595 & 3436 & 2170 & 1394 & 977 & & 880 \\
\hline 808 & 761 & 803 & & & 765 & 771 & & & 713 \\
\hline 81 & 76 & 70 & 719 & 76 & 72 & 723 & 7 & & 765 \\
\hline 763 & 700 & 72 & 762 & 721 & 648 & 700 & 745 & 855 & 1020 \\
\hline 584 & 625 & 632 & 660 & 610 & 598 & 648 & 607 & 584 & 634 \\
\hline & 854 & 952 & 1112 & 1305 & 1261 & 1173 & 1028 & & 834 \\
\hline 57 & 577 & 554 & & 555 & & 567 & 5 & & 578 \\
\hline & 572 & 559 & & 591 & 578 & 542 & 525 & & 505 \\
\hline 534 & $53 \overline{6}$ & 508 & 507 & 499 & 531 & 518 & 465 & & 504 \\
\hline & 529 & 479 & & 504 & 48 & 51 & 460 & 50 & 487 \\
\hline $52 C$ & 588 & 654 & 714 & 73 & 65 & 58 & 51 & & 382 \\
\hline 405 & 420 & 417 & 414 & 395 & 387 & 407 & & & 403 \\
\hline 388 & 396 & 422 & 355 & 360 & 407 & 390 & 393 & 40 & 402 \\
\hline & 38 & & 338 & & 36 & 34 & 36 & & \\
\hline 34 & 370 & 365 & 424 & 469 & 473 & 457 & 45 & & 333 \\
\hline 300 & & & & & & 294 & & 2 & 280 \\
\hline 26 & & 287 & & & & & & & \\
\hline 22 & & & & & 25 & & & & 223 \\
\hline 231 & 24 & 25 & & & 24 & & & & 238 \\
\hline 212 & & & 2 & 2 & 23 & 24 & 28 & $2^{4}$ & 273 \\
\hline 45 & & & & & & & & & 242 \\
\hline 194 & 19 & 2 & & & & & & & \\
\hline 207 & 20 & & & & & & & & \\
\hline 31 & & & & & & & & & \\
\hline 179 & 230 & & 20 & 18 & & 20 & & & \\
\hline 193 & & & 17 & & & & 20 & 18 & 198 \\
\hline 19 & & & & & & & & & \\
\hline & & & & & & & & & \\
\hline 21 & 213 & 21 & & & & & & & \\
\hline 19 & 17 & & 19 & & & 18 & & & 1 \\
\hline 15 & 19 & & 18 & & & $1 \varepsilon$ & & & \\
\hline 18 & & & & & & & & & \\
\hline 182 & 173 & & & & & & & & \\
\hline 176 & 187 & & & & 17 & & & & \\
\hline $\begin{array}{l}16 \\
16\end{array}$ & 20 & & 17 & & 20 & 19 & 17 & 18 & 174 \\
\hline 19 & & & & & & & & & \\
\hline 165 & 1 & & & & & & 1 & 1 & 1 \\
\hline & & & & & & & & & \\
\hline 21 & 245 & & 2.0 & & 193 & 19 & 21 & 18 & 18 \\
\hline 20 & 17 & 20 & 20 & 22 & 20 & 23 & 223 & 21 & 217 \\
\hline 202 & 202 & 217 & 229 & 21 & 19 & 20 & 224 & 19 & 177 \\
\hline 143 & 125 & & 120 & & 122 & 130 & 123 & 119 & 12 \\
\hline
\end{tabular}

$\begin{array}{rrrrrrrrrrr}1781 & 124 & 105 & 97 & 123 & 108 & 118 & 102 & 118 & 91 & 117 \\ 1801 & 110 & 123 & 118 & 103 & 97 & 74 & 102 & 86 & 81 & 82 \\ 1821 & 78 & 84 & 87 & 87 & 86 & 64 & 81 & 71 & 74 & 86 \\ 1841 & 67 & 75 & 73 & 71 & 66 & 65 & 56 & 73 & 72 & 70 \\ 1861 & 54 & 59 & 45 & 50 & 50 & 54 & 55 & 43 & 45 & 42 \\ 1881 & 28 & 36 & 45 & 44 & 45 & 34 & 33 & 36 & 43 & 29 \\ 1901 & 27 & 41 & 32 & 37 & 45 & 36 & 51 & 71 & 71 & 48 \\ 1921 & 24 & 36 & 30 & 30 & 26 & 37 & 27 & 29 & 32 & 22 \\ 1941 & 20 & 24 & 24 & 22 & 31 & 31 & 26 & 24 & 17 & 22 \\ 1961 & 28 & 44 & 32 & 34 & 28 & 48 & 68 & 83 & 95 & 133 \\ 1981 & 190 & 108 & 49 & 21 & 18 & 22 & 19 & 15 & 11 & 16 \\ 2101 & 13 & 13 & 19 & 13 & 16 & 17 & 13 & 8 & 13 & 11 \\ 2021 & 14 & 5 & 12 & 8 & 13 & 4 & 13 & 9 & 13 & 9 \\ 2041 & 9 & 8 & 15 & 11 & 6 & 1 & 14 & 0 & & \end{array}$

$\begin{array}{rrrrrrrrrr}106 & 107 & 119 & 130 & 121 & 102 & 118 & 123 & 124 & 129 \\ 88 & 74 & 95 & 88 & 83 & 81 & 89 & 102 & 85 & 75 \\ 74 & 69 & 84 & 70 & 72 & 70 & 89 & 69 & 68 & 68 \\ 56 & 57 & 65 & 67 & 59 & 66 & 44 & 59 & 59 & 67 \\ 46 & 42 & 35 & 48 & 36 & 38 & 36 & 29 & 44 & 42 \\ 37 & 37 & 39 & 29 & 32 & 33 & 25 & 35 & 33 & 42 \\ 57 & 56 & 56 & 44 & 44 & 36 & 27 & 36 & 24 & 35 \\ 35 & 26 & 32 & 35 & 27 & 23 & 21 & 30 & 31 & 39 \\ 24 & 26 & 19 & 26 & 25 & 34 & 28 & 23 & 35 & 24 \\ 192 & 252 & 404 & 524 & 646 & 643 & 634 & 567 & 442 & 296 \\ 7 & 16 & 11 & 10 & 7 & 13 & 19 & 7 & 15 & 12 \\ 14 & 16 & 10 & 11 & 10 & 6 & 16 & 8 & 7 & 8 \\ 11 & 8 & 10 & 8 & 10 & 11 & 9 & 12 & 13 & 14\end{array}$




\section{D-32}

GAMMA RAY SPECTRUM DATA

PRTR ROD FE-74 SPECTRUM NO. 12 DATA TAKEN 9/15/69 LIVE TIME 840.MIN. POSITION 30.IN. ENERGY(KEV)= 1.109*CHAN -6.9

CHAN COUNT COUNT COUNT COUNT COUNT COUNT COUNT COUNT COUNT COUNT

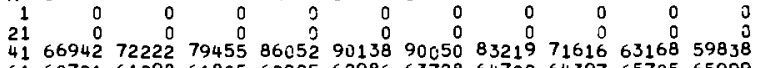

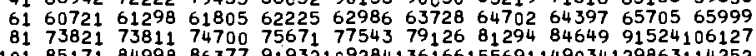
10185171 84998 8637791932109284136166155691149034129863114252 $\begin{array}{llllllllll}121 & 65717 & 65898 & 66879 & 68221 & 75833 & 88883100087 & 97788 & 83498 & 71844\end{array}$

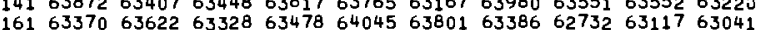
$\begin{array}{llllllllllll}181 & 62553 & 61841 & 61714 & 61280 & 61461 & 61463 & 61101 & 60773 & 61420 & 61166 \\ 201 & 60131 & 59964 & 60206 & 59414 & 59347 & 59723 & 59875 & 59725 & 59905 & 59831\end{array}$ $\begin{array}{llllllllllll}201 & 60131 & 59964 & 60206 & 59414 & 59347 & 59723 & 59875 & 59725 & 59905 & 59831\end{array}$ $\begin{array}{lllllllllll}221 & 59785 & 58969 & 59194 & 58936 & 59007 & 59016 & 59112 & 59024 & 59669 & 59167\end{array}$

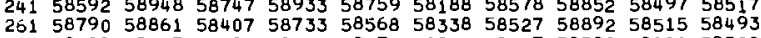
$30159065590095924058792 \quad 59116 \quad 5844158182 \quad 57609 \quad 57443 \quad 57039$

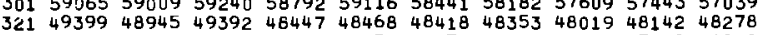
$\begin{array}{lllllllllll}341 & 47249 & 46786 & 46712 & 47005 & 46654 & 46479 & 46514 & 46507 & 47139 & 46808\end{array}$ $\begin{array}{llllllllllll}341 & 47249 & 46786 & 46712 & 47005 & 46654 & 46479 & 465114 & 46507 & 47139 & 46808 \\ 361 & 45789 & 45839 & 45536 & 45324 & 45261 & 45393 & 45392 & 45747 & 45380 & 45126\end{array}$ $\begin{array}{llllllllllll}381 & 44959 & 44702 & 44186 & 4468 & 44580 & 44684 & 44387 & 44545 & 45722 & 46645\end{array}$ $\begin{array}{lllllllllllll}401 & 42029 & 41511 & 41373 & 40419 & 40411 & 39778 & 39441 & 39238 & 38788 & 38762\end{array}$ $\begin{array}{lllllllllll}421 & 38257 & 38384 & 38958 & 39061 & 39042 & 38211 & 37600 & 37112 & 37245 & 36694 \\ 441 & 31507 & 31100 & 30906 & 30961 & 30697 & 30364 & 30216 & 29939 & 30570 & 30286\end{array}$

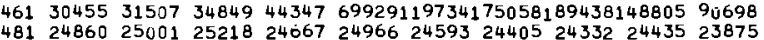
$\begin{array}{lllllllllll}501 & 23071 & 23205 & 22800 & 22743 & 22617 & 22485 & 22529 & 22397 & 22503 & 22202\end{array}$ (5)1 21891202681806518206172771691016462160791576715503 $\begin{array}{llllllllllll}541 & 14299 & 14117 & 14092 & 14099 & 14591 & 15141 & 16349 & 18571 & 21782 & 27649 \\ 561 & 16856 & 18478 & 20662 & 27197 & 42713 & 65111 & 78346 & 70696 & 49364 & 28139\end{array}$ $\begin{array}{llllllllllll}581 & 9993 & 9511 & 9184 & 8914 & 8857 & 8677 & 8558 & 8602 & 8502 & 8517\end{array}$ $\begin{array}{llllllllll}601 & 61313 & 89622100011 & 82433 & 51862 & 26608 & 13113 & 7956 & 6391 & 6201\end{array}$ $\begin{array}{lllllllllll}641 & 4750 & 4974 & 4971 & 4913 & 4905 & 4929 & 4950 & 4859 & 4939 & 5005\end{array}$ $\begin{array}{rrrrrrrrrrr}661 & 13345 & 9119 & 6470 & 5066 & 4824 & 4782 & 4729 & 4581 & 4733 & 4536\end{array}$ $\begin{array}{rrrrrrrrrrr}701 & 8543 & 5038 & 3950 & 3709 & 3648 & 3559 & 3703 & 3692 & 3634 & 3712\end{array}$ $\begin{array}{lllllllllll}721 & 8884 & 12786 & 16248 & 16447 & 14041 & 10185 & 7139 & 5567 & 5073 & 4501 \\ 741 & 3337 & 3287 & 3368 & 3369 & 3368 & 3229 & 3232 & 3399 & 3317 & 3225\end{array}$ $\begin{array}{lllllllllll}761 & 3251 & 3198 & 3092 & 2971 & 3029 & 2968 & 2973 & 2859 & 2849 & 2835 \\ 781 & 2593 & 2506 & 2499 & 2564 & 2650 & 2487 & 2548 & 2644 & 2693 & 2996\end{array}$

\begin{tabular}{|c|c|c|c|c|c|c|c|c|c|c|}
\hline 801 & 2461 & 2457 & 2546 & 2468 & 2547 & 2466 & 2381 & 2361 & 2375 & 2224 \\
\hline$B_{21}$ & 2248 & 2293 & 2172 & 2272 & 2196 & 2223 & 2229 & 2138 & 2187 & 2128 \\
\hline 841 & 2018 & 1890 & 1895 & 1837 & 1885 & 1896 & 1908 & 1867 & 1943 & 1970 \\
\hline & 1792 & 1760 & $\begin{array}{l}1742 \\
17\end{array}$ & 1768 & 1720 & 1716 & $\begin{array}{l}1735 \\
\end{array}$ & 1773 & 1708 & 1682 \\
\hline 881 & 1565 & 1585 & 1560 & 1525 & 1540 & 1541 & 1497 & 1545 & 1480 & 1512 \\
\hline & 1523 & 1578 & 1548 & 1612 & 1556 & 1555 & 1459 & 1495 & 1473 & \\
\hline 921 & 1411 & 1403 & 1374 & 1411 & 1410 & 1368 & 1318 & 1340 & 1369 & 380 \\
\hline 941 & 1404 & 1469 & 1478 & 1412 & 1505 & 1490 & 1681 & 2094 & 2729 & 4164 \\
\hline 961 & 1233 & 1191 & 1259 & 1261 & 1176 & 1176 & 1220 & 1167 & 1090 & 1057 \\
\hline 981 & 1159 & 1134 & 1107 & 1103 & 1088 & 1110 & 1204 & 1144 & 1144 & \\
\hline 1001 & 1128 & 1124 & 1094 & 1111 & 1083 & 1062 & 1104 & 1108 & 1093 & 1032 \\
\hline 021 & 1991 & 2178 & 2129 & 1995 & 1694 & 1403 & 1114 & 1053 & 1022 & \\
\hline 341 & 963 & 923 & 932 & 936 & 900 & 907 & 903 & 847 & 939 & \\
\hline & 1406 & 1449 & 1442 & 1387 & 1273 & 1062 & 1048 & 901 & 895 & \\
\hline & 961 & 965 & 964 & 904 & & 853 & & & & \\
\hline 10 & 740 & 797 & 752 & 74 & $77_{1}$ & 734 & 755 & 755 & 752 & \\
\hline 21 & 750 & 728 & 699 & 769 & 657 & 747 & 704 & 730 & 733 & \\
\hline 14 & 723 & 753 & 700 & 690 & 692 & 685 & 694 & 686 & 749 & \\
\hline & 608 & 571 & 588 & 634 & 588 & 567 & & 585 & 610 & \\
\hline 8 & 542 & 550 & 583 & 55 & 556 & 537 & 55 & 56 & 574 & \\
\hline 20 & 662 & 691 & 776 & 848 & 992 & 920 & 955 & 895 & 834 & \\
\hline 22 & 427 & 453 & 456 & 495 & 472 & 453 & 444 & 468 & 475 & \\
\hline 241 & 489 & 440 & 437 & 427 & 439 & 422 & 455 & 440 & 445 & \\
\hline & 422 & 394 & 417 & 408 & 402 & 406 & & 407 & 402 & \\
\hline & 388 & 410 & 41 & j5 & 36 & 38 & 38 & 35 & & \\
\hline 13 & 354 & 365 & 356 & 348 & 390 & 354 & 36 & 394 & 343 & \\
\hline 132 & 323 & 345 & 363 & & 356 & 339 & 362 & 354 & 349 & \\
\hline 34 & 376 & 476 & 521 & D? & 813 & 868 & 89 & 90 & 852 & \\
\hline 36 & 342 & 322 & 344 & 31 & 312 & 340 & 30 & & & \\
\hline & 34 & 318 & 31 & 26 & & & & & & \\
\hline 1401 & 277 & 346 & 312 & 28 & 299 & 31 & 32 & 36 & 387 & \\
\hline 142 & 315 & 248 & 275 & 28 & & & 2 & 24 & 263 & \\
\hline 4 & & 278 & 28 & & & & 27 & 28 & & \\
\hline 46 & 256 & 263 & 26 & 27 & 30 & 28 & 26 & 25 & 26 & \\
\hline & 27 & 25 & 26 & 24 & & & & & & \\
\hline & 27 & 299 & 252 & 28 & & & & 27 & 2 & \\
\hline 152 & 306 & 271 & 271 & 257 & 283 & 26 & 279 & 257 & 297 & \\
\hline 15 & 23 & & & & & & 23 & & & \\
\hline 15 & 24 & 257 & & & 24 & & 27 & 27 & & \\
\hline & 263 & 242 & 25 & $2 \epsilon$ & 26 & 24 & 26 & 27 & 27 & \\
\hline & 301 & 290 & 26 & & & & & & & \\
\hline 16 & 26 & 295 & 297 & 31 & 328 & 30 & 29 & 30 & 281 & \\
\hline 164 & 27 & 267 & 27 & & & & 26 & & 268 & \\
\hline & 27 & 285 & & & & 2 & 26 & & & \\
\hline & 31 & 28 & & & & & & & & 2 \\
\hline 17 & 29 & 31 & 30 & & & & & 30 & 2 & \\
\hline & 31 & & & & & & 28 & & 335 & \\
\hline 174 & 36 & 33 & 31 & 31 & & 31 & 31 & 32 & 336 & 312 \\
\hline 176 & 291 & 288 & 279 & 280 & 264 & 254 & 243 & 229 & 226 & \\
\hline 178 & & & & & & & & & & \\
\hline & $\begin{array}{l}14 \\
17\end{array}$ & 13 & & & & & 14 & & & 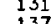 \\
\hline & 117 & 14 & & & & 14 & $\begin{array}{l}140 \\
134\end{array}$ & 11 & 134 & \\
\hline 184 & 114 & $\begin{array}{r}116 \\
97\end{array}$ & $\begin{array}{l}104 \\
104\end{array}$ & 103 & 103 & 94 & 81 & 10 & 103 & 105 \\
\hline & 78 & 8 & 68 & 74 & 82 & & 78 & 8 & 80 & 5 \\
\hline & 4 & 6 & 6 & 3 & 47 & 4 & 53 & 5 & 45 & \\
\hline & 4 & 6 & 4 & & 5 & & 64 & & 81 & 73 \\
\hline & 37 & 44 & 37 & 4 & 45 & 36 & 39 & 44 & 33 & 41 \\
\hline & 33 & 32 & 38 & & 43 & 28 & 48 & 41 & & \\
\hline & 60 & 44 & 4 & & 7 & 8 & 105 & 152 & $21 \overline{8}$ & \\
\hline & 35 & 232 & 15 & 7 & 4 & 2 & 28 & 1 & 1 & \\
\hline & 1 & 10 & 14 & 14 & 15 & 16 & 16 & 12 & 17 & 18 \\
\hline 20 & 15 & 11 & 14 & 10 & 18 & 14 & 12 & 11 & 8 & \\
\hline & 16 & 13 & 18 & 12 & 15 & 16 & 14 & 0 & & \\
\hline
\end{tabular}

COUNT COUNT COUNT. COUNT COUNT COUNT COUNT COUNT COUNT COUNT $\begin{array}{rrrrrrrrrr}0 & 0 & 0 & 0 & 0 & 0 & 0 & 0 & 0 & 0 \\ 1 & 0 & 1 & 1 & 11 & 262 & 2834 & 22534 & 55213 & 54983 \\ 58678 & 58051 & 57774 & 5771 \frac{1}{2} & 58256 & 58250 & 58783 & 59164 & 59664 & 59835\end{array}$ 67009683096978971178710917067971756723497397874206 $131049161403177285187405213560223107_{182478128933} 98023 \quad 87263$

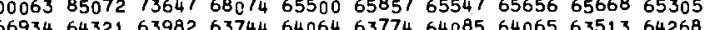
66934 6432163982 63744 64064 63774 64085 64065 63513 64268 $\begin{array}{llllllllll}63420 & 63834 & 63456 & 63651 & 63337 & 62629 & 63550 & 63246 & 62891 & 63167\end{array}$ $61150 \quad 60533 \quad 61236 \quad 604046046160531 \quad 6029560268 \quad 60432 \quad 60479$ 60211601726901059974589135911859763593275956459424 $\begin{array}{llllllllll}59014 & 59100 & 58988 & 58767 & 59153 & 59048 & 58998 & 59181 & 58557 & 59211\end{array}$ $\begin{array}{llllllllll}58545 & 58327 & 58382 & 58869 & 58173 & 58254 & 58170 & 58327 & 58404 & 58527\end{array}$ 59103591765931459159586365952859407593975907959215

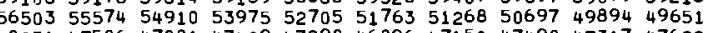
$\begin{array}{llllllllll}48031 & 47586 & 47881 & 47409 & 47298 & 46896 & 47150 & 47498 & 47317 & 47622\end{array}$ $\begin{array}{llllllllll}46532 & 46678 & 45961 & 46204 & 46178 & 45934 & 46132 & 45606 & 45394 & 45913\end{array}$ $4844549420 \quad 472474567543846 \quad 430314312542733 \quad 4264641886$ $\begin{array}{llllllllll}38900 & 38612 & 38657 & 38299 & 38313 & 38475 & 38329 & 38261 & 38306 & 37706\end{array}$ $\begin{array}{lllllllllll}35975 & 36067 & 36135 & 36051 & 35410 & 34819 & 34073 & 33511 & 32710 & 31906 \\ 30665 & 30872 & 32059 & 32593 & 32671 & 31792 & 31055 & 30629 & 30171 & 30128\end{array}$ $\begin{array}{llllllllll}50784 & 33184 & 27513 & 25979 & 25502 & 25183 & 25296 & 25576 & 25145 & 25214\end{array}$

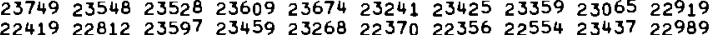
$\begin{array}{llllllllll}22419 & 22812 & 23597 & 23459 & 23268 & 22370 & 22356 & 22554 & 23437 & 22989 \\ 15335 & 15103 & 14971 & 1475 & 14529 & 14386 & 14503 & 14356 & 14442 & 13909\end{array}$ $\begin{array}{llllllllll}33066 & 33466 & 27642 & 20571 & 15604 & 13266 & 12452 & 12522 & 13131 & 14415\end{array}$ $8436 \quad 84410522 \quad 8459 \quad 8763 \quad 9053 \quad 9829122791080133801$ $\begin{array}{lllllllllll}6197 & 6086 & 5777 & 5994 & 5652 & 5725 & 5581 & 5485 & 5668 & 5580\end{array}$ $\begin{array}{lllllllllll}6903 & 8933 & 11738 & 13487 & 12700 & 10276 & 7593 & 5983 & 5221 & 4982\end{array}$ $\begin{array}{rrrrrrrrrr}6903 & 8933 & 1738 & 13487 & 12700 & 10276 & 7593 & 5983 & 5221 & 4982 \\ 5021 & 5174 & 5121 & 5328 & 6408 & 8453 & 12327 & 16750 & 19228 & 17754\end{array}$ $\begin{array}{rrrrrrrrrr}4691 & 4687 & 4628 & 4739 & 4747 & 4759 & 4836 & 4806 & 4790 & 4860 \\ 13370 & 12610 & 17613 & 30382 & 50544 & 66507 & 67174 & 53186 & 33327 & 17550\end{array}$ $\begin{array}{llllllllll}3709 & 3582 & 3540 & 3739 & 3728 & 3713 & 3829 & 4094 & 4609 & 6144 \\ 4315 & 3790 & 3523 & 3326 & 3251 & 3394 & 3255 & 3190 & 3309 & 3318\end{array}$ $\begin{array}{llllllllll}4315 & 3790 & 3523 & 3326 & 3251 & 3394 & 3255 & 3190 & 3309 & 3318 \\ 3207 & 3276 & 3296 & 3252 & 3270 & 3262 & 3307 & 3302 & 3325 & 3306\end{array}$ $\begin{array}{llllllllll}2714 & 2772 & 3296 & 3252 & 3270 & 3262 & 3307 & 3302 & 3325 & 3300 \\ 3447 & 4206 & 4559 & 4432 & 2672 & 2703 & 2572 & 2619 & 2628 & 2500 \\ 3521 & 2854 & 2619 & 2470 & 2440\end{array}$

\begin{tabular}{|c|c|c|c|c|c|c|c|c|c|}
\hline & 334 & 2268 & 2244 & 2242 & 2305 & 2285 & 2241 & 2382 & 2314 \\
\hline 2128 & 2095 & 2129 & 2057 & 2097 & 1945 & 2027 & -1959 & 2021 & 1894 \\
\hline 1974 & 1960 & 1894 & 1784 & 1852 & 1712 & 1912 & 1832 & 1824 & 1823 \\
\hline 538 & 716 & 701 & 1641 & 1674 & 1620 & 1626 & 1518 & 1528 & \\
\hline 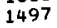 & 1 & 523 & 1431 & 1555 & 1565 & 1471 & 1466 & 1463 & 1480 \\
\hline 641 & 1595 & 623 & 1503 & 1538 & 1374 & 1446 & 1417 & 1456 & \\
\hline $\begin{array}{l}1364 \\
\end{array}$ & 1384 & $\begin{array}{l}1327 \\
\end{array}$ & 1366 & 1371 & 1398 & 342 & 1382 & 367 & \\
\hline 5444 & 522 & 6373 & 5488 & 4425 & 3162 & 2119 & 14 & 1239 & \\
\hline 1109 & 10 & 1118 & 1177 & 1216 & 1100 & 1054 & 1103 & 1129 & \\
\hline & 111 & 1128 & 1146 & 1125 & 1071 & 1055 & 1120 & 1065 & \\
\hline 1002 & 1098 & 1074 & 1044 & 1058 & 1077 & 1101 & 1194 & 1364 & 1623 \\
\hline 944 & 93 & 894 & 932 & 942 & 975 & & 935 & 879 & 872 \\
\hline 1085 & 1322 & 1513 & 1643 & 1711 & 1578 & 1580 & 1528 & 1329 & 1318 \\
\hline 832 & 845 & 829 & 804 & 846 & 788 & 788 & 836 & 851 & 937 \\
\hline 796 & 79 & 742 & & & & & & & 751 \\
\hline 785 & 77 & 762 & 726 & 767 & 769 & 80 & & 784 & \\
\hline 738 & 719 & 754 & 752 & 692 & 736 & 690 & & 744 & 748 \\
\hline 820 & 908 & 944 & 896 & 914 & $8 E$ & 784 & 679 & 646 & 672 \\
\hline & 60 & 612 & & & & & & & \\
\hline & & 55 & 56 & 545 & & & & & 614 \\
\hline 590 & 564 & 508 & 521 & 483 & 524 & 491 & 50 & 487 & 513 \\
\hline 517 & 549 & 594 & 656 & 651 & 661 & 653 & & 548 & $5_{12}$ \\
\hline 471 & 438 & 431 & 438 & 458 & 437 & 440 & 403 & 423 & 410 \\
\hline & 35 & 382 & & & & & & & \\
\hline 37 & 39 & 360 & & & & & & 37 & 353 \\
\hline 367 & 353 & 327 & 337 & 350 & 324 & 350 & 31 & 33 & 359 \\
\hline 331 & 32 & 341 & & 36 & & & & 38 & 369 \\
\hline 64 & 58 & 505 & & 37 & & & & & \\
\hline & & 31 & & 31 & & & & & 322 \\
\hline & 29 & 30 & & 28 & 25 & 303 & & 311 & 285 \\
\hline 54 & 58 & 576 & & 476 & 48 & 419 & & 310 & 306 \\
\hline 26 & 23 & $2 B$ & & & & & & & 310 \\
\hline & & & & & & & & & 254 \\
\hline 28 & 27 & 28 & 27 & 27 & 25 & 22 & & 25 & 261 \\
\hline & & & & & & & & & \\
\hline 29 & 34 & & & & & & & & 314 \\
\hline 28 & & & & & & & & & 279 \\
\hline & & & & & & & & & \\
\hline 27 & 257 & 26 & 26 & 25 & 26 & 26 & & 23 & 260 \\
\hline 253 & & 29 & 3 & 28 & & & & 29 & 284 \\
\hline & & & & & & & & 27 & 30 \\
\hline 29 & 31 & 27 & & & & 2 & & 27 & 262 \\
\hline 26 & 26 & $2 \epsilon$ & & & & & & & 274 \\
\hline 30 & 25 & 27 & & 33 & 29 & & & 28 & 282 \\
\hline 27 & 27 & $2 B$ & & & & & & & 310 \\
\hline 28 & & & & & & & & & \\
\hline 31 & 30 & 3. & 2 & 3 & 31 & & & & 38 \\
\hline 30 & 30 & 31 & 288 & 301 & 299 & 332 & 28 & 304 & 301 \\
\hline 228 & 209 & 205 & 212 & 188 & 168 & 193 & 163 & 161 & $f$ \\
\hline
\end{tabular}

$\begin{array}{rrrrrrrrrr}145 & 161 & 153 & 181 & 161 & 171 & 162 & 153 & 181 & 192 \\ 120 & 129 & 147 & 133 & 125 & 140 & 124 & 117 & 149 & 97 \\ 102 & 100 & 115 & 115 & 106 & 108 & 116 & 106 & 123 & 107 \\ 79 & 101 & 87 & 86 & 93 & 82 & 97 & 97 & 78 & 60 \\ 62 & 63 & 51 & 72 & 58 & 64 & 52 & 44 & 64 & 46 \\ 38 & 45 & 43 & 51 & 52 & 40 & 53 & 51 & 47 & 43 \\ 76 & 73 & 75 & 59 & 62 & 64 & 51 & 52 & 35 & 48 \\ 30 & 36 & 48 & 44 & 39 & 39 & 34 & 27 & 29 & 57 \\ 38 & 34 & 24 & 44 & 51 & 50 & 45 & 38 & 37 & 52 \\ 435 & 547 & 700 & 716 & 754 & 632 & 603 & 565 & 551 & 478 \\ 25 & 16 & 20 & 15 & 20 & 16 & 16 & 19 & 19 & 13 \\ 13 & 14 & 14 & 17 & 5 & 16 & 14 & 10 & 16 & 12 \\ 11 & 12 & 8 & 9 & 14 & 12 & 13 & 15 & 12 & 11\end{array}$


GAMMA RAY SPECTRUM DATA

PRTR ROD FN-86 SPECTRUM N0. 16 DATA TAKEN 9/17/69 LIVE TIME 840.MIN. POSITION 30.IN. ENERGY(KEV)= 1.113*CHAN -9.3 CHAN COUNT COUNT COUNT COUNT COUNT COUNT COUNT COUNT COUNT COUNT

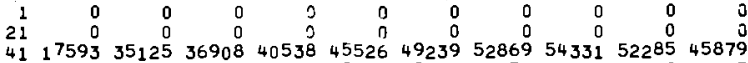
$\begin{array}{lllllllllll}61 & 38349 & 38203 & 38309 & 38792 & 39458 & 39481 & 40155 & 40013 & 40868 & 41415\end{array}$

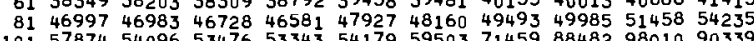

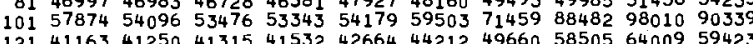
$\begin{array}{lllllllllll}121 & 41163 & 41250 & 41315 & 41532 & 42664 & 44212 & 49660 & 58505 & 64009 & 59423\end{array}$ $\begin{array}{lllllllllll}161 & 39708 & 40322 & 39788 & 39683 & 39850 & 40386 & 40448 & 40051 & 40127 & 39624\end{array}$ $\begin{array}{llllllllllll}181 & 39080 & 39737 & 39427 & 38632 & 38793 & 39120 & 38563 & 38969 & 38717 & 3851\end{array}$ $\begin{array}{llllllllllll}201 & 37569 & 37740 & 37904 & 37631 & 37592 & 37569 & 37395 & 37430 & 37604 & 37393\end{array}$ $\begin{array}{lllllllllllll}221 & 37558 & 37469 & 37588 & 37222 & 37470 & 37043 & 37193 & 37100 & 37218 & 36768\end{array}$ $\begin{array}{lllllllllllll}241 & 36623 & 36897 & 36784 & 36613 & 36817 & 36281 & 36847 & 36759 & 36626 & 37080\end{array}$ $\begin{array}{lllllllllll}281 & 36710 & 36924 & 37228 & 36913 & 36859 & 37022 & 36876 & 37033 & 36972 & 36887\end{array}$

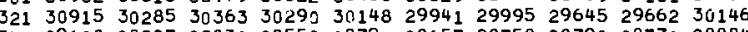
(361 2828228134280652807028169280732783427882279462779 381272822784127835279542786627628277262742279462779 $401263912636426521259152553425302 \quad 25002 \quad 2494224765 \quad 24473$

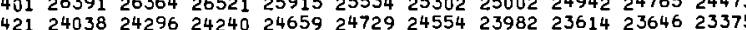
$\begin{array}{lllllllllllll}441 & 19719 & 19454 & 19395 & 18970 & 18754 & 18648 & 18505 & 18507 & 18482 & 18457\end{array}$ $\begin{array}{llllllllllll}481 & 14796 & 14745 & 14796 & 14508 & 14610 & 14358 & 14362 & 14286 & 14299 & 14055\end{array}$

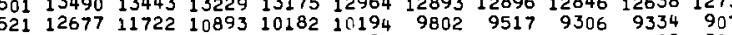
$\begin{array}{rrrrrrrrrrr}541 & 8234 & 8354 & 8176 & 8096 & 8197 & 8698 & 9422 & 10220 & 11529 & 13675 \\ 561 & 10091 & 11201 & 12356 & 15600 & 24723 & 40156 & 51705 & 48360 & 32784 & 18033\end{array}$ $\begin{array}{llllllllllll}581 & 6246 & 6033 & 5731 & 5345 & 5353 & 515 & 5409 & 5399 & 5428 & 5334\end{array}$ $\begin{array}{lllllllllll}621 & 3500 & 3398 & 3434 & 3482 & 3376 & 3367 & 3333 & 3332 & 3397 & 3768\end{array}$ $\begin{array}{rrrrrrrrrrr}661 & 6370 & 4260 & 3370 & 2983 & 2974 & 2874 & 2890 & 2986 & 2886 & 2914 \\ 681 & 3098 & 3182 & 3433 & 4240 & 5632 & 8567 & 11510 & 12602 & 11066 & 8022\end{array}$ $\begin{array}{lllllllllll}701 & 3279 & 2619 & 2432 & 2369 & 2337 & 2373 & 2344 & 2356 & \mathbf{2 2 9 2} & 2316 \\ 721 & 5882 & 7798 & 8554 & 7483 & 5602 & 3852 & 3234 & 3003 & 2861 & 2609\end{array}$ $\begin{array}{lllllllllll}741 & 2160 & 2141 & 2159 & 2154 & 2176 & 2140 & 2153 & 2122 & 2228 & 2104\end{array}$ $\begin{array}{lllllllllll}761 & 2025 & 2053 & 2090 & 1969 & 2020 & 1973 & 1934 & 1907 & 1875 & 1895 \\ 781 & 1715 & 1789 & 1692 & 1765 & 1749 & 1735 & 1690 & 1777 & 1966 & 2230\end{array}$

\begin{tabular}{|c|c|c|c|c|c|c|c|c|c|c|}
\hline 801 & 1689 & 1792 & 1767 & 1653 & 1591 & 1571 & 1537 & 1612 & 1531 & 1539 \\
\hline 821 & 1555 & 1456 & 1511 & 1505 & 1458 & 1487 & 1469 & 1479 & 1418 & 1512 \\
\hline 841 & 1308 & 1328 & 1312 & 1302 & 1289 & 1272 & 1334 & 1322 & 1371 & 1298 \\
\hline 861 & 1210 & 240 & 127 & 1225 & 1200 & 1155 & 1242 & 1189 & 1076 & 1140 \\
\hline 881 & 1067 & 1053 & 1006 & 1065 & 1124 & 1057 & 1049 & 1025 & 974 & 971 \\
\hline 901 & 982 & 1009 & 986 & 1063 & 1014 & 982 & 998 & 1005 & 1055 & 1092 \\
\hline 921 & 940 & 919 & 936 & 879 & 956 & 949 & 982 & 931 & 966 & 955 \\
\hline 94 & 978 & & 93 & 938 & 1009 & 1201 & 1447 & 2063 & 3078 & 4251 \\
\hline 961 & 872 & 862 & 854 & 817 & 757 & 786 & 754 & 745 & 738 & 745 \\
\hline 981 & 770 & 754 & 73 & 76 & 716 & 792 & 78 & 78 & 741 & 759 \\
\hline 00 & 756 & 734 & 732 & 754 & 711 & 749 & 68 & & 738 & 754 \\
\hline & 1583 & 1414 & 1112 & 901 & 726 & 685 & 6 & 622 & 597 & 639 \\
\hline & 626 & 629 & 577 & 601 & 627 & 543 & 592 & 612 & & 775 \\
\hline & 1127 & 1130 & 1003 & 88 & 714 & 652 & 577 & 559 & 550 & 581 \\
\hline 81 & 623 & 591 & 57 & 496 & 510 & 53 & 50 & 534 & 52 & 563 \\
\hline 101 & 489 & 480 & 456 & 480 & 511 & 504 & 456 & 487 & 494 & 503 \\
\hline & 496 & 470 & 462 & 484 & 487 & 488 & 505 & 505 & 481 & 528 \\
\hline & 493 & 463 & 489 & 44 & 458 & 448 & 45 & & 447 & 532 \\
\hline 16 & 403 & 417 & 372 & 394 & 414 & 404 & 343 & 35 & 390 & 379 \\
\hline 18 & 405 & 366 & $40 \overline{8}$ & & 373 & 372 & 40 & 420 & 389 & 375 \\
\hline & 554 & 625 & 80 & & 784 & 681 & 544 & 452 & 376 & 337 \\
\hline 22 & 306 & 320 & 28 & 3. & 325 & 321 & 30 & & & 345 \\
\hline 24 & 269 & 25 & 271 & & 287 & 268 & 28 & & 28 & $3_{12}$ \\
\hline & 282 & & & & 304 & & & & & 257 \\
\hline & 258 & & 23 & & 24 & & & & & 242 \\
\hline & 252 & & 23 & & 21 & & 22 & & & 248 \\
\hline & 230 & & 227 & 24 & 246 & 227 & 24 & 21 & 231 & 228 \\
\hline & 374 & & & & 680 & & & & & 272 \\
\hline & 23 & & & & & & & & 22 & 205 \\
\hline & 17 & & & & & & & & & 200 \\
\hline & 19 & & & & & & & & & 414 \\
\hline & 192 & 1 & 1 & 18 & 18 & 1 & 1 & & & 208 \\
\hline & 180 & 18 & 18 & 17 & 24 & 16 & 18 & & I & 200 \\
\hline & 16 & & & & & & & & & 177 \\
\hline & & & & & & & & & & 166 \\
\hline & 184 & & & & & & & & & \\
\hline & 20 & & & 18 & & 20 & 16 & & & 170 \\
\hline & 16 & & & & & & 15 & & & 159 \\
\hline & 16 & & & & & & & & & 157 \\
\hline & & & & & & & & & & 175 \\
\hline & & & & & & & & & & 17 \\
\hline & 228 & & 203 & & 20 & 17 & 18 & & & 185 \\
\hline & 180 & 179 & 196 & 18 & 17 & 21 & 17 & & & 155 \\
\hline 160 & 196 & 1 & & $i$ & 16 & 18 & 1 & & & 182 \\
\hline 168 & 19 & 1 & 21 & & 19 & 19 & 2 & & & 20 \\
\hline & 19 & 198 & 202 & 195 & 189 & 18 & 19 & & & 198 \\
\hline & 18 & 207 & 17 & & 21 & 20 & 20 & & & 192 \\
\hline 174 & 22 & 21 & 22 & 20 & 21 & 20 & 21 & & & 189 \\
\hline & 177 & & 185 & 161 & 140 & 160 & 132 & 130 & 147 & 126 \\
\hline
\end{tabular}

COUNT COUNT COUNT COUNT COUNT COUNT COUNT COUNT COUNT COUNT

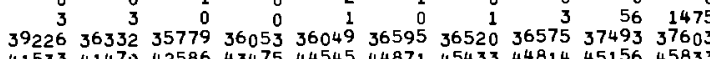
$\begin{array}{lllllllllll}41533 & 41470 & 42586 & 43475 & 44545 & 44871 & 45433 & 44814 & 45156 & 45833\end{array}$ $58296 \quad 6821985485104182110066117377_{135585136512105194} 73153$ $4975943334 \quad 413394038840462406094030740212 \quad 40377 \quad 40530$ $4006839840 \quad 49000 \quad 40217402233986940010399144002839650$ $\begin{array}{llllllllll}39688 & 39572 & 39397 & 39768 & 39161 & 39665 & 39766 & 39026 & 39443 & 39043\end{array}$ $\begin{array}{llllllllll}38337 & 37894 & 38699 & 38112 & 38139 & 38310 & 38105 & 37822 & 37948 & 38070 \\ 37499 & 37478 & 37228 & 37441 & 37312 & 37261 & 37573 & 37078 & 37098 & 37317\end{array}$ $\begin{array}{lllllllllll}37499 & 37478 & 37228 & 37441 & 37312 & 37261 & 37573 & 37078 & 37098 & 37317 \\ 37212 & 36949 & 37079 & 37242 & 37093 & 36847 & 36837 & 36247 & 37103 & 37036\end{array}$ $\begin{array}{lllllllllll}36633 & 36339 & 36402 & 36465 & 36818 & 36423 & 36980 & 36745 & 36541 & 36687\end{array}$ $\begin{array}{lllllllllll}37181 & 36785 & 37337 & 36738 & 36875 & 37379 & 36853 & 36712 & 36923 & 36970\end{array}$ $2953352230413404333180331132102 \quad 316673122231170$ $2890228625284392842928524 \quad 2824628527280372838928613$ $\begin{array}{lllllllllllll}28441 & 27711 & 27913 & 28056 & 27868 & 27667 & 27727 & 27968 & 27658 & 27726\end{array}$ $\begin{array}{llllllllll}29006 & 30137 & 31113 & 30520 & 29216 & 28017 & 27262 & 26995 & 26641 & 26799\end{array}$ $\begin{array}{lllllllllll}24452 & 24376 & 24378 & 24381 & 24296 & 23890 & 24365 & 23939 & 24119 & 23929\end{array}$

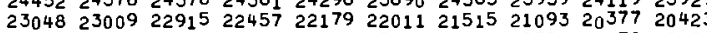
$\begin{array}{llllllllll}18335 & 18232 & 18461 & 18792 & 19267 & 19360 & 18905 & 18334 & 18258 & 18295 \\ 50026 & 27576 & 18567 & 15695 & 15257 & 14743 & 14935 & 14923 & 14856 & 14880\end{array}$ $\begin{array}{llllllllllll}13874 & 13781 & 14189 & 13549 & 13804 & 1357 \% & 13470 & 13595 & 13364 & 13332\end{array}$ $\begin{array}{lllllllllll}12606 & 12826 & 12804 & 13113 & 13108 & 12807 & 12542 & 12559 & 12822 & 1284\end{array}$ $\begin{array}{rrrrrrrrrr}8991 & 8809 & 8515 & 8526 & 8575 & 8500 & 8468 & 8260 & 8275 & 8289\end{array}$ $\begin{array}{rrrrrrrrrr}162562 & 17013 & 151379 & 1590 & 8781 & 5703 & 5958 & 6260 & 6537 & 6431\end{array}$ $\begin{array}{llllllllll}5390 & 5266 & 5390 & 5580 & 5411 & 5745 & 6392 & 7923 & 12869 & 24461 \\ 3756 & 3714 & 3631 & 3672 & 3545 & 3610 & 3510 & 3426 & 3535 & 3528\end{array}$ $\begin{array}{llllllllll}4528 & 6201 & 8113 & 9029 & 7959 & 5894 & 4260 & 3491 & 3206 & 3084\end{array}$ $\begin{array}{rrrrrrrrrrr}4528 & 6201 & 8113 & 9029 & 7959 & 5894 & 4260 & 3491 & 3206 & 3084 \\ 3102 & 3185 & 3185 & 3510 & 4014 & 5454 & 7984 & 10440 & 11128 & 9306\end{array}$ $\begin{array}{rrrrrrrrrr}2982 & 2952 & 2914 & 2931 & 2920 & 3037 & 2996 & 3000 & 2990 & 3092 \\ 6324 & 7464 & 12405 & 22204 & 34453 & 40046 & 33931 & 21669 & 11171 & 5420\end{array}$ $\begin{array}{llllllllll}2349 & 2382 & 2405 & 2354 & 2356 & 2382 & 2390 & 2648 & 3096 & 4071 \\ 2310 & 2286 & 2300 & 2217 & 2278 & 2136 & 2108 & 2301 & 2197 & 2203\end{array}$

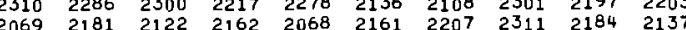
$\begin{array}{llllllllll}1809 & 1711 & 1843 & 1819 & 1689 & 1711 & 1721 & 1722 & 1694 & 1611 \\ 2724 & 3027 & 3117 & 2672 & 2265 & 1799 & 1695 & 1651 & 1557 & 1623\end{array}$

\begin{tabular}{|c|c|c|c|c|c|c|c|c|c|}
\hline 1531 & 1498 & 1545 & 1508 & 1461 & 1555 & 1532 & 1494 & 1587 & 50 \\
\hline 1350 & 1416 & 1404 & 1441 & 1353 & 1341 & 1361 & 1310 & 1342 & 1301 \\
\hline 1338 & 1214 & 1297 & 1189 & 1276 & 1192 & 1181 & 1205 & 1253 & 1173 \\
\hline 117 & 1122 & 1098 & 1085 & 1108 & 1053 & 1069 & 1053 & 1050 & 1054 \\
\hline 1000 & 1030 & 997 & 948 & 1000 & 998 & 1049 & 1025 & 961 & 1003 \\
\hline 1004 & 1009 & 960 & 1010 & 970 & 955 & 899 & 873 & 888 & 905 \\
\hline 890 & 976 & 920 & 855 & 853 & 913 & 944 & 916 & 905 & 934 \\
\hline 4756 & 4341 & 3230 & 2205 & 1426 & 1070 & 87 & 830 & 835 & \\
\hline 762 & 752 & 703 & 732 & 801 & 773 & & 757 & 742 & \\
\hline 740 & 748 & 787 & 800 & 752 & 729 & 757 & 711 & 732 & 719 \\
\hline 695 & 705 & 696 & 752 & 694 & 766 & 853 & 1041 & 1245 & 1494 \\
\hline 610 & 613 & 596 & 604 & 614 & 610 & 604 & 621 & 615 & 613 \\
\hline 1012 & 1135 & 1186 & 1161 & 1000 & 927 & 877 & 887 & 946 & 1111 \\
\hline & .518 & 537 & 531 & 504 & 532 & 566 & 531 & 604 & 616 \\
\hline & 481 & 495 & 507 & 521 & 517 & 472 & 501 & 473 & 511 \\
\hline 500 & 494 & 489 & 510 & 484 & 479 & 468 & 485 & 487 & \\
\hline 446 & 458 & 442 & 492 & 480 & 472 & 460 & 480 & 467 & \\
\hline 53 & 60 & 505 & 523 & 431 & 435 & 44 & 383 & 396 & \\
\hline 373 & 359 & 37 & 38 & 35 & 38 & 38 & & & \\
\hline 391 & 356 & 371 & 372 & 361 & 38 & 434 & 42 & 443 & \\
\hline 310 & 315 & 313 & 343 & 307 & 35 & 299 & 322 & 308 & 327 \\
\hline & & & & 374 & 348 & 324 & 292 & 333 & 304 \\
\hline 291 & 294 & 294 & 29 & 28 & 30 & 27 & & & \\
\hline 216 & 264 & & $2^{3}$ & & & & 235 & 25 & 240 \\
\hline 19 & 261 & & & 25 & 2 & & 240 & 223 & 230 \\
\hline & & 22 & & 23 & & & 24 & 241 & 26 \\
\hline & & & & & & & & 262 & 34 \\
\hline 293 & $2 B$ & & & & & 21 & 22 & 21 & 22 \\
\hline 214 & 23 & 22 & & & & & & & \\
\hline 200 & 20 & & & & 15 & & & 213 & 195 \\
\hline 398 & & & & & & & & 16 & \\
\hline 18 & 18 & 18 & & 18 & 18 & 18 & 22 & 15 & 194 \\
\hline 18 & 16 & 181 & & & 18 & 19 & & 172 & 193 \\
\hline 17 & & 17 & & & 17 & & & 168 & \\
\hline 167 & $1^{8}$ & 16 & 18 & & 17 & 16 & 15 & 151 & 174 \\
\hline 244 & 21 & & & & & & & 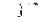 & 15 \\
\hline & 175 & 159 & & & & & & & 176 \\
\hline & 16 & 16 & & & 16 & & 18 & $\therefore$ & \\
\hline 17 & 16 & 176 & & 15 & 18 & & 16 & 17 & \\
\hline 19 & 19 & 194 & 1 & 2 & 18 & 20 & 18 & 19 & 172 \\
\hline & & & & & & & & & \\
\hline & & & & & & & & 17 & 16 \\
\hline 18 & 15 & 202 & 153 & 20 & 10 & 19 & & 171 & 17 \\
\hline & 17 & & 19 & & 2 & 18 & 1 & & \\
\hline 18 & 19 & 21 & 19 & 18 & 18 & 198 & 190 & 188 & \\
\hline 19 & 19 & & & & & & & & \\
\hline & 19 & 227 & 19. & 21 & 21 & 198 & 217 & 204 & 237 \\
\hline & 19 & 189 & 190 & 16 & 17 & 195 & 180 & 202 & 169 \\
\hline 112 & 119 & 108 & 108 & 109 & 120 & 101 & 109 & 100 & 10 \\
\hline
\end{tabular}

$\begin{array}{rrrrrrrrrrr}1781 & 102 & 109 & 121 & 86 & 107 & 105 & 106 & 81 & 102 & 115 \\ 1801 & 111 & 97 & 92 & 76 & 100 & 94 & 91 & 82 & 86 & 77 \\ 1821 & 71 & 68 & 95 & 86 & 87 & 84 & 69 & 77 & 84 & 79 \\ 1841 & 81 & 63 & 70 & 59 & 53 & 51 & 56 & 69 & 81 & 64 \\ 1861 & 59 & 48 & 46 & 44 & 56 & 39 & 48 & 49 & 42 & 38 \\ 1881 & 37 & 30 & 19 & 39 & 39 & 40 & 41 & 35 & 34 & 46 \\ 1901 & 51 & 41 & 56 & 45 & 46 & 60 & 63 & 50 & 32 & 43 \\ 1921 & 22 & 36 & 20 & 30 & 28 & 41 & 31 & 32 & 40 & 22 \\ 1941 & 25 & 21 & 20 & 24 & 19 & 34 & 27 & 22 & 29 & 32 \\ 1961 & 56 & 60 & 57 & 95 & 128 & 178 & 286 & 364 & 466 & 540 \\ 1981 & 15 & 17 & 30 & 18 & 11 & 16 & 16 & 13 & 7 & 9 \\ 2001 & 11 & 12 & 11 & 11 & 7 & 11 & 16 & 14 & 17 & 11 \\ 2021 & 16 & 10 & 8 & 10 & 7 & 7 & 11 & 13 & 11 & 8 \\ 2041 & 9 & 11 & 12 & 15 & 7 & 12 & 13 & 0 & & \end{array}$

$\begin{array}{rrrrrrrrrr}98 & 112 & 102 & 130 & 114 & 102 & 103 & 85 & 81 & 92 \\ 88 & 66 & 82 & 83 & 74 & 90 & 80 & 75 & 93 & 83 \\ 87 & 64 & 82 & 77 & 60 & 66 & 81 & 70 & 67 & 73 \\ 62 & 55 & 66 & 47 & 45 & 75 & 54 & 44 & 17 & 47 \\ 31 & 31 & 39 & 53 & 41 & 36 & 36 & 36 & 37 & 36 \\ 39 & 29 & 30 & 35 & 39 & 30 & 28 & 37 & 25 & 36 \\ 26 & 29 & 23 & 33 & 25 & 31 & 27 & 34 & 26 & 21 \\ 27 & 25 & 23 & 26 & 34 & 17 & 24 & 19 & 22 & 27 \\ 24 & 30 & 37 & 30 & 28 & 39 & 37 & 21 & 23 & 47 \\ 592 & 523 & 514 & 447 & 333 & 186 & 139 & 84 & 49 & 35 \\ 11 & 14 & 11 & 14 & 22 & 8 & 13 & 11 & 9 & 12 \\ 14 & 12 & 19 & 8 & 13 & 17 & 10 & 10 & 17 & 12 \\ 3 & 14 & 11 & 14 & 13 & 14 & 5 & 6 & 10 & 7\end{array}$


GAMMA RAY SPECTRUM DATA

PRTR ROD FE-69 SPECTRUM NO, 13 DATA TAKEN 9/16/69 LIVE TIME 840.MIN. POSITION 30.IN. ENERGY(KEV)=1,109*CHAN -6.9

CHAN COUNT COUNT COUNT COUNT COUNT COUNT COUNT COUNT COUNT COUNT

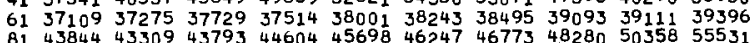

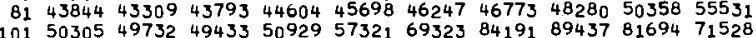
$\begin{array}{lllllllllll}101 & 50305 & 49732 & 49433 & 50929 & 57321 & 69323 & 84191 & 89437 & 81694 & 71528 \\ 121 & 38026 & 38305 & 38476 & 38989 & 41838 & 46978 & 54963 & 58614 & 53768 & 45416 \\ 141 & 37208 & 36785 & 36980 & 36919 & 36738 & 36992 & 36634 & 36461 & 36874 & 36500\end{array}$ $\begin{array}{llllllllllll}161 & 36773 & 36563 & 36522 & 36632 & 36822 & 37041 & 36915 & 36396 & 36268 & 36531\end{array}$ $\begin{array}{lllllllllll}201 & 34817 & 34536 & 34784 & 34332 & 34249 & 33923 & 34020 & 34026 & 34430 & 34391\end{array}$ $\begin{array}{llllllllllll}221 & 34238 & 33753 & 33570 & 33958 & 33674 & 33995 & 33556 & 34024 & 34004 & 33878 \\ 241 & 33234 & 33732 & 33493 & 33466 & 33339 & 33351 & 33047 & 33314 & 33210 & 33170\end{array}$

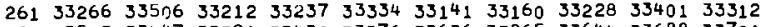
$\begin{array}{llllllllllll}281 & 33509 & 33447 & 33391 & 33430 & 33276 & 33636 & 33865 & 33644 & 33688 & 33701\end{array}$ $\begin{array}{lllllllllll}321 & 33695 & 33527 & 33581 & 33207 & 33427 & 33106 & 33234 & 32954 & 32622 & 32506\end{array}$ $\begin{array}{lllllllllll}341 & 26419 & 26470 & 26121 & 25962 & 26163 & 26076 & 26080 & 26305 & 26227 & 26086\end{array}$

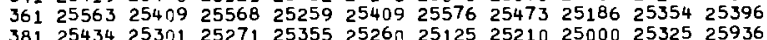
$\begin{array}{lllllllllll}381 & 25434 & 25301 & 25271 & 25355 & 2526 & 25125 & 25210 & 25000 & 25325 & 25936 \\ 401 & 23809 & 23292 & 23349 & 22932 & 23134 & 22473 & 22413 & 22396 & 22155 & 21990\end{array}$ (1) 2247121580210292118820906 $\begin{array}{llllllllllll}441 & 17766 & 17383 & 17138 & 17056 & 16888 & 16768 & 16489 & 16646 & 16559 & 16526\end{array}$ $481132341313112973130712897127041280612748 \quad 1268712350$ $\begin{array}{lllllllllll}481 & 13234 & 13131 & 12973 & 1307 & 12897 & 12704 & 12806 & 12748 & 12687 & 12356 \\ 501 & 11903 & 11929 & 11628 & 11686 & 11644 & 11452 & 11349 & 11182 & 11345 & 11522\end{array}$ $\begin{array}{lllllllllll}521 & 11015 & 10651 & 10089 & 9438 & 9151 & 8824 & 8598 & 8491 & 8257 & 8097\end{array}$ $\begin{array}{rrrrrrrrrrr}541 & 7443 & 7291 & 7336 & 7252 & 7408 & 7938 & 8397 & 9212 & 10021 & 11704 \\ 561 & 8831 & 9947 & 10947 & 13530 & 19925 & 31714 & 43563 & 45519 & 34712 & 20593\end{array}$ $\begin{array}{rrrrrrrrrrr}581 & 5703 & 5271 & 5126 & 5013 & 4991 & 4929 & 4783 & 4726 & 4810 & 4790 \\ 601 & 31776 & 51640 & 65959 & 61195 & 40881 & 20718 & 9206 & 4820 & 3825 & 3460\end{array}$ $\begin{array}{lllllllllll}641 & 2837 & 2755 & 2838 & 2836 & 2734 & 2800 & 2703 & 2689 & 2773 & 2765 \\ 661 & 7547 & 5197 & 3678 & 2924 & 2661 & 2571 & 2678 & 2618 & 2714 & 2585\end{array}$ $\begin{array}{rrrrrrrrrrr}661 & 7547 & 5197 & 3678 & 2924 & 2661 & 2571 & 2678 & 2618 & 2714 & 2585 \\ 681 & 2797 & 2769 & 3045 & 3216 & 4081 & 5609 & 7914 & 10497 & 10907 & 9484\end{array}$ $\begin{array}{rrrrrrrrrrr}681 & 27979 & 2769 & 3045 & 3216 & 408 & 5609 & 7914 & 10497 & 10907 & 9445\end{array}$ $\begin{array}{lllllllllll}721 & 3763 & 5297 & 6655 & 7447 & 6461 & 4858 & 3434 & 2749 & 2664 & 2530\end{array}$ $\begin{array}{lllllllllll}741 & 1917 & 2019 & 1942 & 1960 & 2001 & 2 C 28 & 2035 & 1899 & 1918 & 1907 \\ 701 & 1928 & 1901 & 1879 & 1826 & 1823 & 1838 & 1742 & 1740 & 1735 & 1788\end{array}$ $\begin{array}{lllllllllll}781 & 1576 & 1510 & 1534 & 1509 & 1555 & 1514 & 1586 & 1547 & 1634 & 1696\end{array}$

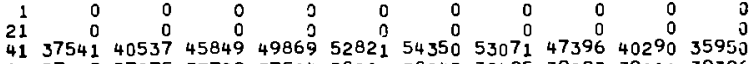
$\begin{array}{lllllllllll}621 & 3135 & 3130 & 3110 & 3113 & 3026 & 3086 & 3003 & 2887 & 3083 & 3337\end{array}$

COUNT COUNT COUNT COUNT COUNT COUNT COUNT COUNT COUNT COUNT $\begin{array}{rrrrrrrrrrr}0 & 0 & 0 & 0 & 0 & 0 & 0 & 0 & 0 & 0 \\ 0 & 0 & 1 & 0 & 1 & 13 & 245 & 1259 & 13378 & 34044 \\ 35245 & 34915 & 35019 & 35471 & 35473 & 35653 & 35706 & 36378 & 36464 & 36556\end{array}$ $\begin{array}{lllllllllll}39817 & 40399 & 41143 & 42299 & 42731 & 42465 & 42577 & 42509 & 43582 & 43725\end{array}$

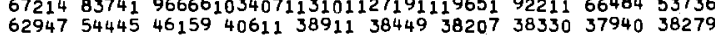
$\begin{array}{llllllllllllll}40248 & 38061 & 37491 & 37235 & 37299 & 37443 & 37288 & 37157 & 37142 & 37275\end{array}$ $\begin{array}{lllllllllll}40248 & 38061 & 37491 & 37235 & 37299 & 37443 & 37288 & 37157 & 37142 & 37275 \\ 36725 & 36755 & 36611 & 36384 & 36533 & 36430 & 36371 & 36675 & 36874 & 36760\end{array}$ $\begin{array}{lllllllllll}36013 & 36060 & 36153 & 36946 & 35803 & 35732 & 36101 & 35695 & 35996 & 35610\end{array}$ $\begin{array}{llllllllll}34083 & 34255 & 34178 & 34128 & 33772 & 34094 & 33891 & 33742 & 33933 & 33853\end{array}$ $339643349034043 \quad 33815 \quad 337653355633500334953366933306$ $\begin{array}{lllllllllll}32898 & 33007 & 32882 & 33285 & 33340 & 33064 & 33416 & 33490 & 33118 & 33021\end{array}$ (3) $\begin{array}{llllllllll}33795 & 33508 & 33875 & 33715 & 33647 & 33432 & 33903 & 33475 & 33772 & 33622\end{array}$ $\begin{array}{lllllllllll}26676 & 26509 & 26707 & 26483 & 26468 & 26631 & 26444 & 26274 & 26519 & 26482 \\ 266080 & 25647 & 25995 & 25700 & 25931 & 25677 & 25769 & 25385 & 25490 & 25970\end{array}$ $\begin{array}{llllllllll}26080 & 25647 & 25995 & 25700 & 25931 & 25677 & 25769 & 25385 & 25490 & 25970 \\ 25698 & 25395 & 25318 & 25139 & 25251 & 25082 & 25143 & 25022 & 24823 & 25142\end{array}$ $\begin{array}{lllllllllll}25698 & 25395 & 25318 & 25139 & 25251 & 25082 & 25143 & 25022 & 24823 & 25142 \\ 27046 & 27631 & 27710 & 26596 & 25273 & 24992 & 24541 & 24145 & 23952 & 23636\end{array}$

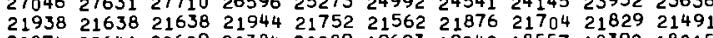

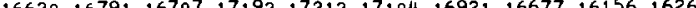
$\begin{array}{lllllllllll}16620 & 1679 & 16707 & 17192 & 17212 & 17104 & 16921 & 16677 & 16156 & 16266\end{array}$ $\begin{array}{llllllllll}12642 & 12470 & 12843 & 12286 & 12213 & 12137 & 12267 & 12077 & 12091 & 11852\end{array}$ $\begin{array}{llllllllll}11377 & 11294 & 11577 & 11520 & 11880 & 11367 & 11173 & 11144 & 11556 & 11495\end{array}$ $\begin{array}{llllllllll}8016 & 7857 & 7791 & 7702 & 7829 & 7573 & 7552 & 7536 & 7492 & 7595\end{array}$ $\begin{array}{llllllllll}14067 & 14867 & 13934 & 11076 & 8628 & 7216 & 6679 & 6637 & 6999 & 7756\end{array}$

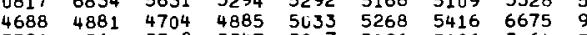
$\begin{array}{llllllllll}3392 & 4881 & 4704 & 4885 & 5633 & 5268 & 5416 & 6675 & 9444 & 17097 \\ 3347 & 3518 & 3217 & 3196 & 3226 & 3064 & 3080 & 3090\end{array}$ $\begin{array}{lllllllllll}3701 & 4712 & 6412 & 7482 & 7824 & 6464 & 4662 & 3441 & 2938 & 2680 \\ 2739 & 2845 & 2938 & 3108 & 3264 & 3995 & 5659 & 7715 & 9629 & 9544\end{array}$ $\begin{array}{llllllllll}2712 & 2700 & 2572 & 2732 & 2720 & 2653 & 2625 & 2758 & 2758 & 2790\end{array}$ $\begin{array}{lllllllllll}7096 & 5775 & 7554 & 13043 & 21918 & 31403 & 34844 & 29228 & 18042 & 8933\end{array}$ $\begin{array}{llllllllll}2155 & 2158 & 2110 & 2130 & 2121 & 2221 & 2199 & 2292 & 2415 & 2940 \\ 2363 & 2179 & 2099 & 2043 & 1965 & 1987 & 1960 & 2006 & 1952 & 1951\end{array}$ $\begin{array}{lllllllllll}1964 & 1904 & 1906 & 1867 & 2025 & 1977 & 1999 & 2051 & 1998 & 2009\end{array}$ $\begin{array}{llllllllll}1609 & 1617 & 1633 & 1669 & 1605 & 1647 & 1622 & 1568 & 1604 & 1551 \\ 1969 & 2418 & 2660 & 2802 & 2606 & 2008 & 1792 & 1595 & 1496 & 1396\end{array}$

\begin{tabular}{|c|c|c|c|c|c|c|c|c|c|}
\hline 1399 & 1394 & 1397 & 1448 & 1420 & 1453 & 1348 & 1487 & 1451 & 1358 \\
\hline 1376 & 1309 & 1259 & 1282 & 1226 & $\begin{array}{l}1247 \\
\end{array}$ & 1306 & 1242 & 1224 & 1264 \\
\hline 7 & 176 & 1169 & 206 & 1147 & 1172 & 094 & 1105 & 1103 & 1110 \\
\hline 043 & 020 & 1096 & 991 & 1029 & 1003 & 994 & 912 & 994 & 992 \\
\hline 88 & 96 & 89 & & & & & & 900 & 28 \\
\hline 90 & 93 & & 924 & & & & & & 878 \\
\hline 819 & $8_{1}$ & 797 & 814 & 821 & 854 & 785 & & 833 & 844 \\
\hline 3189 & 3855 & 4020 & 3523 & 2501 & 1624 & 1114 & 858 & 771 & 761 \\
\hline 703 & 714 & 713 & 715 & 62 & & & & 729 & 694 \\
\hline 724 & 728 & & 68 & 689 & & & & 674 & 684 \\
\hline 649 & & & 684 & 625 & & & & 792 & 1027 \\
\hline $\begin{array}{l}54 \\
55\end{array}$ & & 557 & 531 & 540 & 543 & & & 578 & 555 \\
\hline & 723 & 841 & 1033 & 1010 & 104 & 98 & & 762 & 864 \\
\hline 49 & 501 & 508 & 476 & 46 & 4 & 49 & & 47 & 543 \\
\hline 497 & 456 & 455 & 474 & 43 & 444 & 46 & 460 & 426 & 456 \\
\hline 411 & 448 & 426 & 466 & 408 & 385 & 505 & 440 & 454 & 430 \\
\hline 39 & 40 & 40 & 45 & 42 & 41 & & & 45 & 408 \\
\hline 405 & 463 & 503 & 529 & 49 & & 419 & & & 393 \\
\hline 328 & 387 & 404 & 357 & 34 & & 66 & & & 330 \\
\hline & & & & & & & & & 387 \\
\hline 35 & 34 & & & & & & & & 303 \\
\hline 32. & & & & & & & & & 266 \\
\hline & & & & & & & & & 224 \\
\hline & & & & & & & & & 226 \\
\hline & 21 & & & 23 & & & & & 221 \\
\hline & & & 19 & & & & & & \\
\hline 21 & 219 & 210 & 206 & & 20 & & & & 232 \\
\hline & & & & & & & & & 210 \\
\hline 20 & & & & & & & & & \\
\hline $\begin{array}{l}18 \\
18\end{array}$ & 180 & & 18 & & & & & & 178 \\
\hline 34 & & & & & & & & & 169 \\
\hline 190 & 158 & 190 & 16 & 16 & & 1 & 16 & & 182 \\
\hline 174 & 129 & & & & & & & & 184 \\
\hline 14 & & & & & & & & & \\
\hline 146 & 142 & & & & & & & & 158 \\
\hline 180 & 191 & 217 & & & & & & & 160 \\
\hline 154 & 155 & 130 & 172 & & & & & & 164 \\
\hline 170 & 12 & & & & & & & & \\
\hline 161 & 164 & & & 15 & 15 & & & & 193 \\
\hline 16 & 162 & 144 & 186 & 19 & 17 & & & & 164 \\
\hline 15 & 132 & 15 & & & & & & & 153 \\
\hline & & & & & & & & & 168 \\
\hline 155 & & & 175 & 15 & 16 & & & & 167 \\
\hline 16 & & & 16 & 18 & 17 & 16 & 15 & & 164 \\
\hline 17 & 16 & & 19 & & 1 & & & & 171 \\
\hline 18 & 17 & & & & & & & & 178 \\
\hline 18 & & 21 & & & 17 & & & & 179 \\
\hline 19 & 18 & 187 & 15 & 15 & 19 & 18 & 16 & 203 & 189 \\
\hline 11 & 12 & 100 & 108 & 10 & 11 & 99 & 113 & 89 & \\
\hline
\end{tabular}

$\begin{array}{rrrrrrrrrrr}1781 & 84 & 85 & 106 & 97 & 100 & 88 & 73 & 96 & 88 & 101 \\ 1801 & 112 & 96 & 83 & 74 & 82 & 69 & 82 & 82 & 82 & 67 \\ 1821 & 81 & 75 & 77 & 71 & 65 & 86 & 69 & 57 & 77 & 70 \\ 1841 & 61 & 59 & 61 & 70 & 81 & 59 & 53 & 55 & 73 & 45 \\ 1861 & 45 & 47 & 41 & 44 & 44 & 43 & 40 & 50 & 38 & 38 \\ 1881 & 38 & 34 & 35 & 39 & 38 & 27 & 27 & 34 & 23 & 35 \\ 1901 & 29 & 38 & 32 & 36 & 29 & 39 & 34 & 54 & 50 & 53 \\ 1921 & 28 & 21 & 23 & 24 & 29 & 24 & 27 & 23 & 21 & 30 \\ 1941 & 25 & 23 & 28 & 23 & 21 & 30 & 22 & 23 & 19 & 28 \\ 1961 & 28 & 26 & 33 & 30 & 47 & 54 & 58 & 97 & 114 & 158 \\ 1981 & 131 & 64 & 35 & 25 & 20 & 18 & 11 & 9 & 13 & 18 \\ 2001 & 11 & 8 & 16 & 9 & 6 & 7 & 8 & 20 & 17 & 11 \\ 2021 & 14 & 10 & 10 & 8 & 17 & 12 & 6 & 11 & 9 & 11 \\ 2041 & 10 & 5 & 16 & 7 & 6 & 9 & 12 & 0 & & \end{array}$

$\begin{array}{rrrrrrrrrr}93 & 110 & 101 & 92 & 97 & 102 & 109 & 108 & 92 & 102 \\ 91 & 72 & 76 & 78 & 88 & 73 & 74 & 76 & 69 & 79 \\ 58 & 60 & 58 & 73 & 69 & 70 & 74 & 63 & 69 & 64 \\ 54 & 68 & 53 & 47 & 46 & 53 & 52 & 44 & 59 & 42 \\ 33 & 35 & 30 & 37 & 39 & 36 & 36 & 38 & 38 & 36 \\ 20 & 28 & 21 & 23 & 25 & 34 & 28 & 30 & 28 & 24 \\ 48 & 50 & 43 & 48 & 26 & 29 & 20 & 20 & 28 & 17 \\ 24 & 24 & 24 & 31 & 24 & 24 & 29 & 35 & 22 & 21 \\ 26 & 25 & 26 & 19 & 26 & 28 & 37 & 27 & 32 & 18 \\ 221 & 312 & 372 & 500 & 530 & 543 & 487 & 387 & 315 & 210 \\ 12 & 10 & 16 & 8 & 15 & 8 & 16 & 8 & 18 & 12 \\ 10 & 7 & 14 & 9 & 11 & 9 & 7 & 16 & 13 & 14 \\ 9 & 11 & 11 & 12 & 8 & 12 & 5 & 11 & 9 & 9\end{array}$ 
BNWL-1674

Special Distribution

in Category UC-80

\section{DISTRIBUTION}

No. of

Copies

OFFSITE

1

AEC Chicago Patent Group

G. H. Lee

AEC Division of Reactor Development and Technology

Director RDT

Asst. Dir. for Project Management

Chief, Water Reactors Branch (2)

Asst. Dir. for Reactor Technology

Chief, Reactor Physics Branch (2)

Chief, Fuels Recycle Branch

1

AEC Division of Reactor Licensing

R. E. Baker

3

AEC Technical Information Center

4

Argonne National Laboratory

R. Avery

C. H. Bean

R. E. Machery

W. B. Seefeldt

2

Atomic Energy of Canada, Ltd.

Chalk River Nuclear Laboratory

Reactor Physics Branch

Chalk River, Ontario, Canada

M. Duret

J. Griffiths

$1 \quad$ Babcock and Wilcox

P.0. Box 1260

Lynchburg, VA 24505

D. H. Roy

1

Bhabha Atomic Research Centre

Theoretical Physics Section/RED

Central Complex Building

Trombay, Bombay-85, India

S. R. Dwivedi

Brookhaven National Laboratory

J. Chernick 
No. of Copies

5 Combustion Engineering

P. 0. Box 500

Windsor, Connecticut 06095

W. P. Chernock

R. Harding

R. L. Hellens

S. Visner

H. H. Windsor

1

CNEN - Centro Studi-Nucleaire

Casaccia, Rome, Italy

Ugo Farinel1i

2 Edison Electric Institute

750 Third Avenue

New York, New York 10017

John J. Kearney

George Watkins

2

E. I. du Pont de Nemours \& Co., Inc., SRL

G. Dessauer

H. Honeck

$1 \quad$ ENEL

Via G. B. Martini

(Piazza Verdi)

Rome, Italy

Mr. Paoletti Gualandi

3 General Electric Co., San Jose

Nuc. Dev. and Eng.

175 Curtner Avenue

San Jose, California 95112

D. L. Fisher

S. Levy

R. B. Richards

4 General Electric Co., Vallecitos Atomic Lab.

P. 0. Box 846

Pleasanton, California 94566

B. F. Judson

T. M. Snyder

R. E. Harris

E. Kurtz 
No. of

Copies

1

1

2

1

1

1

2
Idaho Nuclear Corporation

R. Grimsey

Japan Atomic Energy Research Institute TCA-JPDR

Tokaj-Mura, Naka-Gun, Ibaraki-Ken

Japan

Shojiro Matsuura

Nuclear Fuels Services

Suite 600

6000 Executive Blvd.

Rockville, Md. 20852

J. D. Rollins

E. Wick

NUKEM

$\overline{D-645}$, HANAU

POSTFACH 869

Germany

Mr. W. K. L. Jager

Pakistan Institute of Nuclear Sci. \& Tech. P. 0. Nilore

Rawalpindi, Pakistan

M. A. Mannan

Power Reactor \& Nuclear Fuel Development Corp.

9-13, 1-chome, Akasaka

Minato-ku, Tokyo, Japan

Setsuo Kobayashi

S. C. K. - C. E. N.

MOL-DONK

Belgium

Dr. H. Van den Broeck

Gulf United Nuclear Fuels Corp.

Research \& Engineering Center

Grasslands Road

Elmsford, New York 10523

T. B. Holden

J. R. Tomonto 
No. of

Copies

6

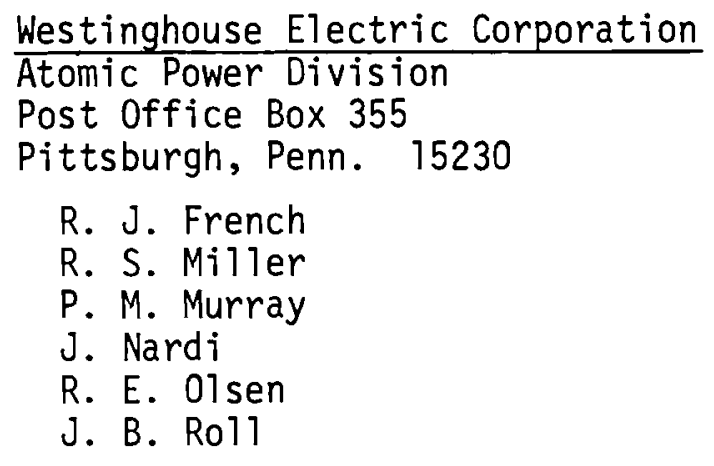

ONSITE - HANFORD

1

1

2

46

AEC Chicago Patent Group

R. M. Poteat

AEC Richland Operations Office

H. A. House

RDT Senior Site Representative

F. Standerfer

Battelle-Northwest
J. L. Carter
N. E. Carter
D. E. Christensen
J. C. Fox
M. D. Freshley
S. Goldsmith
H. L. Henry
U. P. Jenquin
D. A. Kottwitz
J. W. Kutcher
B. R. Leonard
R. C. Liikala

C. W. Lindenmeier

D. F. Newman

D. R. Oden

R. S. Paul

D. L. Prezbindowski

R. H. Purcel1

L. C. Schmid

R. I. Smith

V. O. Uotinen

H. H. Van Tuy 1

N. G. Wittenbrock

W. C. Wolkenhauer

M. G. Zimmerman

BNW Legal (2).

Technical Information (5) 
No. of

Copies

12 WADCO Corporation

J. A. Christensen

E. A. Evans

R. E. Heineman

P. L. Hofmann

R. C. Smith

L. D. Turner

K. L. Young

WADCO Document Control (5) 


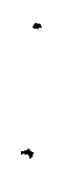

4

$+$

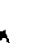
. $\downarrow$ 